\title{
LA VALEUR
}

\section{DES Ressorts et contraintes du marché des idées}

INFORMATIONS

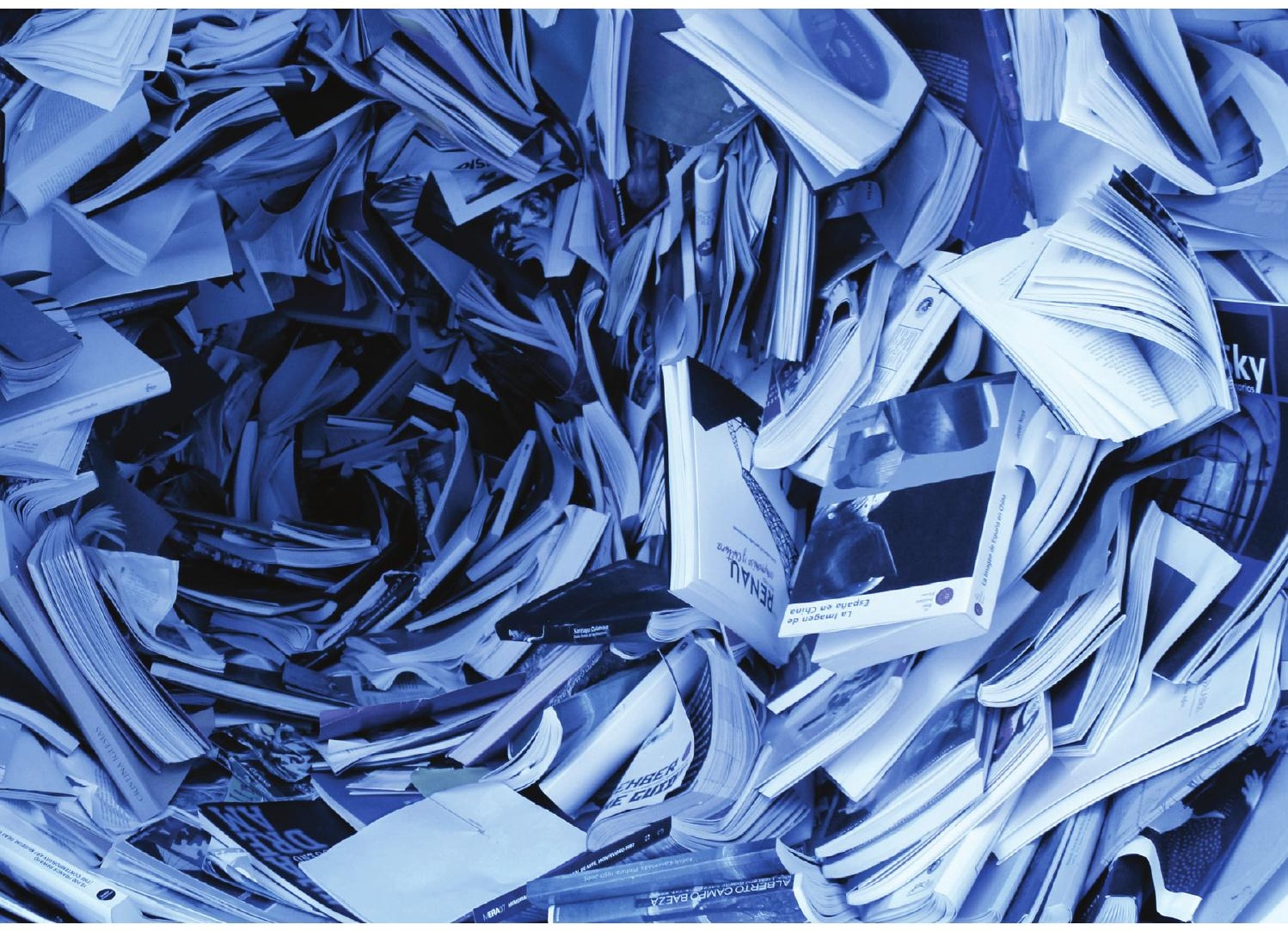

\section{Bertrand Labasse}

Les Presses de l'Université d'Ottawa 


\section{LA VALEUR \\ DES INFORMATIONS}


Page blanche conservée intentionnellement 


\title{
LA VALEUR \\ DES INFORMATIONS \\ RESSORTS ET CONTRAINTES \\ DU MARCHÉ DES IDÉES
}

\author{
Bertrand Labasse
}


Les Presses de l'Université d'Ottawa (PUO) sont fières d'être la plus ancienne maison d'édition universitaire francophone au Canada et le plus ancien éditeur universitaire bilingue en Amérique du Nord. Depuis 1936, les PUO enrichissent la vie intellectuelle et culturelle en publiant, en français ou en anglais, des livres évalués par les pairs et primés dans le domaine des arts et lettres et des sciences sociales.

\section{www.presses.uOHawa.ca}

Catalogage avant publication de Bibliothèque et Archives Canada et Bibliothèque et Archives nationales du Québec

Titre: La valeur des informations: ressorts et contraintes du marché des idées /

Bertrand Labasse.

Noms: Labasse, Bertrand, 1962- auteur.

Description: Comprend des références bibliographiques.

Identifiants: Canadiana (livre imprimé) 20200207334 | Canadiana (livre numérique) 20200207385 | ISBN 9782760331563 (couverture souple) I ISBN 9782760329966 (couverture rigide) | ISBN 9782760331570 (PDF) I ISBN 9782760331587 (EPUB) | ISBN 9782760331594 (Kindle)

Vedettes-matière: RVM: Communication-Aspect social. I RVM: Communication - Aspect psychologique. I RVM: Société informatisée.

Classification: LCC HM561 .L33 2020 | CDD 302.2-dc23

Dépôt légal : Troisième trimestre 2020

Bibliothèque et Archives Canada

Bibliothèque et Archives nationales du

Québec

\section{Équipe de la production}

Révision linguistique

Correction d'épreuves

Mise en page

Maquette de la couverture

Caroline Lavoie

Chantal Ringuet

Nord Compo

Steve Kress
(C) Bertrand Labasse 2020

Il est interdit de reproduire le contenu de la présente publication, de le transmettre sous quelque forme ou par quelque moyen que ce soit, ou de l'emmagasiner dans un système d'extraction de reproduction, sans l'autorisation écrite préalable.

Pour effectuer des photocopies ou tout autre type de reprographie, veuillez obtenir l'autorisation auprès de :

\section{Access Copyright \\ htps://fraccesscopyright.ca \\ 1-800-893-5777}

Droits de traduction et autorisations de reproduction :

www.iprlicense.com

\section{$\mathrm{CRSH} \equiv \mathrm{SSHRC}$}

Ce livre a été publié grâce au soutien d'une subvention de la Fédération canadienne des sciences humaines par l'entremise du Prix d'auteurs à l'édition savante, dont les fonds proviennent du Conseil de recherches en sciences humaines du Canada.

Les Presses de 1'Université d'Ottawa sont reconnaissantes du soutien qu'apportent, à leur programme d'édition, le gouvernement du Canada, le Conseil des arts du Canada, le Conseil des arts de l'Ontario, Ontario créatif, la Fédération canadienne des sciences humaines par l'entremise du programme Prix d'auteurs pour l'édition savante et l'entremise du Conseil de recherches en sciences humaines, et surtout, l'Université d'Ottawa. 


\section{Pour comprendre (enfin ?) les médias}

Q uantité de traités ont déjà été écrits sur la vieille énigme scientifique que constitue la réception de toutes sortes de messages. Encore plus d'essais l'ont été sur les problèmes civiques et professionnels que posent leur emballement numérique et les déboires des filières éditoriales traditionnelles. Pourtant, les ressorts capricieux des préférences culturelles et médiatiques semblent toujours aussi incertains pour les spécialistes qui étudient la production et la réception de ces contenus. Et encore bien plus obscurs pour les naufragés de la société de communication que nous sommes tous :

le professeur tentant de rivaliser avec la forêt des téléphones et ordinateurs portables qui captivent ses étudiants, le politicien confronté aux fluctuations de l'abstention électorale, l'écrivain supputant les minces chances qu'a son premier livre d'obtenir une quelconque recension, le jeune chercheur guettant la moindre citation qui indiquerait que quelqu'un, quelque part, a finalement pris connaissance de son travail. Et, bien sûr, le journaliste accueilli chaque matin par les chiffres d'audience de son émission de la veille ou le nombre d'accès à son article en ligne. (p. 28)

Face à un tel défi, l'approche que Bertrand Labasse explore depuis plusieurs années conduit pas à pas à une réponse étonnamment simple en regard de l'éclairage qu'elle peut offrir à ceux que préoccupe la diffusion des idées dans la société. Un éclairage dont l'intensité pourrait faire penser à un autre livre ontarien qui, 50 ans plus tôt, proposait une façon nouvelle de voir la communication. En réalité, les deux ne s'apparentent ni sur le fond ni sur la forme. On ne trouvera 
pas ici les formules percutantes et les fulgurances prophétiques d'un McLuhan. À leur place, un parcours très stimulant mais méthodique relie des propositions scrupuleusement argumentées, examinées comme si l'auteur se méfiait de ses propres dires et appuyées sur une gamme d'observations et de références scientifiques ou culturelles d'une étendue peu commune.

Cette recherche peut se lire comme une "espèce de conversation " (p. 288) érudite et souvent malicieuse sur nos préférences médiatiques ou littéraires, mais aussi comme une vaste enquête sur l'impasse collective créée par l'empilement des hypothèses discordantes - le «fatras » dirait Thomas Kuhn auquel il se réfère souvent - qu'ont accumulé au fil du temps des disciplines et des écoles rivales. Au terme de l'enquête, aucun coupable mais un constat étonnant : ces travaux, une fois reliés avec le recul nécessaire, contenaient ensemble les clefs d'une clarification aujourd'hui fondamentale qu'aucun d'eux ne pouvait fournir isolément.

Il n'est pas difficile d'imaginer l'agacement que cette synthèse résolument interdisciplinaire pourrait soulever auprès des partisans les plus exclusifs d'une école ou d'une autre : on peut le pressentir à la prévoyance avec laquelle Bertrand Labasse étaye sa démonstration et discute ouvertement de ses points les plus incertains, comme s'il défiait de renverser les plus solides. Ça ne sera pas facile. Si certaines de ses suggestions peuvent en effet se prêter à la discussion, l'aisance avec laquelle ce modèle semble enfin démêler les tensions contradictoires qui jouent sur la production et la réception de contenus très différents est impressionnante. Mais l'auteur a rencontré trop de théories hégémoniques pour prétendre à son tour au monopole de la vérité : il ne propose là, dit-il, qu'un outil conceptuel supplémentaire,

Une construction très résolue, tout à fait confiante dans sa capacité à mieux expliquer une très large gamme de phénomènes, mais pas au point de se prendre pour la réalité de ces phénomènes, ni même pour la seule façon utile de les considérer. (p. 221)

Feinte ou réelle, cette retenue est une des originalités de l'ouvrage, dont la forme détonnera elle aussi dans la littérature savante. Ceux qui, à l'instar des étudiants du séminaire «Information et complexité » qu'il donne chaque année à l'ESJ Lille, ont eu la chance d'assister à l'une de ses conférences reconnaîtront le brio d'un chercheur qui se 
plaît à alterner des considérations théoriques sophistiquées et des digressions insolites, des allusions culturelles raffinées et des saillies hilarantes. Le tout sans jamais, ou presque, perdre le droit fil de sa présentation. Il y a une part de calcul dans cette spontanéité. En spécialiste de la question, Bertrand Labasse sait mieux que nul autre que l'attention doit se mériter ou, pour reprendre son propre cadre, que l'effort cognitif se motive par l'effet produit, quitte à repousser un peu les contraintes de l'expression académique légitime.

Cette attention aux conditions de réception et cette méfiance vis-à-vis des gravités affectées sont toujours à l'œuvre dans ce livre : "l'ennoblir par la dignité d'une écriture hermétique aurait été de la poudre aux yeux» (p. 248). Elles sont même à l'origine d'une innovation éditoriale dont on souhaiterait qu'elle devienne bientôt une norme. Après les « livres dont vous êtes le héros » des années 1980, voici le livre dont vous pouvez choisir le niveau. Pris dans son ensemble, c'est un traité scientifique visant à proposer aux étudiants et aux spécialistes un vaste panorama critique des hypothèses antérieures, un outil analytique de pointe et une brillante série d'études de cas. Si l'on s'épargne ses précisions les plus touffues, chassées non seulement dans les notes de bas de page, mais même dans un complément optionnel à la suite de chaque chapitre, son style et sa clarté le rendent accessible à tous les lecteurs concernés par les enjeux qu'il aborde. Malgré sa passion pour les énigmes théoriques et les paradoxes de communication les plus curieux - dont on trouvera quelques exemples saisissants dans la seconde partie de l'ouvrage - Bertrand Labasse n'a jamais perdu de vue les questionnements concrets des naufragés de la société de communication. Parce qu'il en fait lui aussi partie.

Pierre Savary

Directeur de l’École supérieure de journalisme de Lille 
Page blanche conservée intentionnellement 


\section{À l'abordage d'un « grand mystère »}

Toute situation linguistique fonctionne donc comme un marché sur lequel le locuteur place ses produits et le produit qu'il a produit pour ce marché dépend de l'anticipation qu'il a des prix que vont recevoir ses produits. [...] Un des grands mystères que la socio-linguistique doit résoudre, c'est cette espèce de sens de l'acceptabilité.

Pierre Bourdieu (1980, p. 98, spn.)

Dourquoi les contenus qui nous plaisent nous plaisent-ils ? C'est la 1 formulation la plus simple possible d'un problème qui ne l'est pas. Un « grand » problème, en effet : il obsédait déjà les rhéteurs de l'Antiquité et trône aujourd'hui, presque inchangé, au cœur du bouillonnement de discours commerciaux, politiques, culturels et médiatiques qui forme la société dite «de l'information ». Il a pourtant subi une multitude d'assauts. En 1929, déjà, une recension relevait que «depuis 1900, plus de huit cents études sur les intérêts et habitudes de lecture ont été publiées ", et ce, en ne considérant que les États-Unis (Gray et Munroe, 1929, p. 4). Elles n'ont cessé de se multiplier depuis, dans des perspectives toujours plus éloignées les unes des autres.

Des chercheurs en littérature se sont par exemple penchés sur les déterminants de la réception des œuvres de fiction. Des spécialistes des médias ont étudié les facteurs de sélection des sujets par la presse (newsworthiness), tandis que d'autres examinaient les usages que les lecteurs et spectateurs avaient des informations et les gratifications qu'ils en retiraient. Des didacticiens ont tenté de cerner les paramètres de la lisibilité des manuels scolaires et les stratégies documentaires des élèves. Des consultants ont traqué les variables pouvant susciter l'attrait des sites Internet ou des annonces publicitaires. Des experts en traduction se sont interrogés sur les critères d'appréciation de celles-ci. 
Des spécialistes des bibliothèques et des musées ont étudié les choix des emprunteurs et des visiteurs. Des médecins ont consacré plusieurs centaines de recherches à l'efficacité des dépliants sur la santé ou la clarté des notices pharmaceutiques destinées aux patients. Des ethnologues ont observé comment des groupes différents s'appropriaient différemment les nouvelles technologies de communication et leurs contenus...

Le problème des fondements de la valeur discursive demeure. Ses multiples faces sont largement ébréchées par tant d'efforts profanes ou savants, mais il est essentiellement intact et le simple fait de savoir s'il s'agit bien d'un seul problème - et d'un problème soluble plutôt que d'une multitude de problèmes distincts est encore loin d'aller de soi. C'est pourtant ce que ce livre se propose de montrer. La modestie d'une telle entreprise saute aux yeux : comme on le verra, le défi lancé ci-dessus par Bourdieu l'avait aussi été, pour ne citer qu'eux, par Pascal et avant lui par Platon. En outre, la possibilité d'approcher globalement les phénomènes communicationnels est, pour de solides raisons, considérée depuis plusieurs décennies comme «improbable, voire inconcevable » (Miège, 1995, p. 86). Enfin, le sens commun suggère que si les déterminants de la valeur discursive étaient identifiables, l'arsenal du marketing des industries éditoriales et publicitaires n’aurait pas manqué de les mettre au jour depuis belle lurette. Or, affirme un connaisseur, «s'il y a bien un domaine où il est impossible de théoriser, c'est bien celui des caprices de la lecture publique et des succès de librairie " (Bessard-Banquy, 2017, p. 79).

On se hâte donc de préciser - et on y reviendra - que les ressorts du marché discursif examinés ici en rendent compte sous un angle particulier. La nuance mérite le secours d'une analogie. Sous un angle, un dauphin ressemble à une vache, sous un autre angle à un requin. Mammifère ou prédateur marin ? Aucune de ces façons de voir n'exclut l'autre ou n'est plus pertinente qu'elle : le bon angle est celui qui dévoile le mieux ce que l'on veut comprendre. Aussi un reportage courageux ou un chef-d'œuvre littéraire peuvent-ils être analysés de la même façon qu'une publicité de lessive, une copie scolaire maladroite ou un tweet incendiaire, tout en relevant de registres profondément différents. L'important d'une façon de voir est ce qu'elle permet de voir.

Ce que l'on cherchera à voir dans les pages qui suivent, ce sont donc certaines logiques fondamentales qui sont à l'œuvre dans toutes 
les situations de production et de réception discursive. C'est aussi la façon selon laquelle l'interaction des facteurs en jeu peut être synthétisée dans un modèle général de la fixation des valeurs dans le chaos actuel du marché communicationnel. C'est enfin tout ce qu'un tel modèle peut contribuer à expliquer ou préciser, y compris des paradoxes bien connus ou des notions familières, mais floues (vulgarisation, intelligibilité, intérêt, populisme, représentations...) ou discutables (sensationnalisme, connotation, niveaux de langue...). Et ce sur quoi, ou contre quoi, il permet d'agir : en matière de communication, en effet, la compréhension théorique des phénomènes s'accompagne souvent d'implications très concrètes, économiques, bien sûr, mais aussi pédagogiques, sociales ou politiques. De fait, bien peu des contrats de recherche qui ont partiellement contribué à alimenter la synthèse présentée étaient motivés par un appétit particulier des commanditaires pour la théorie. Il s'agissait d'étudier des questions variées, mais dont les enjeux n'étaient jamais abstraits $^{1}$ : le schéma d'ensemble qui s'en dégage n'est qu'un sousproduit de ces problématiques instrumentales (et de bien d'autres, comme celle de l'enseignement universitaire de la rédaction professionnelle et de celui du journalisme). S'efforcer de dégager clairement les possibles conséquences de ce schéma pour les producteurs discursifs eux-mêmes, et pour les citoyens, sera donc un juste retour des choses.

Devant la complexité insondable du problème auquel elle s'attaque, l'approche proposée se cramponnera aux vieilles ficelles de la démarche analytique : séparer ce qui peut l'être, puis rapprocher ce

1 Saisissons l'occasion pour exprimer bien des remerciements à la Commission européenne (logiques de diffusion de la culture scientifique), la Mission Prospective de la Communauté urbaine de Lyon (variables quantitatives associées à la «société de l'information »), la Fédération nationale de la presse française (évolutions des pratiques et attentes informationnelles) et le Conseil de recherches en sciences humaines du Canada (usages et validité des paramètres psycholinguistiques de la clarté textuelle), mais aussi à tous les chercheurs, tout particulièrement Céline Beaudet, qui m’ont encouragé dans cette aventure, et à ceux dont les objections m'ont enrichi... ou conforté. 
qui doit l'être (les étapes ci-dessous reprennent la succession des chapitres).

1. Dans un premier temps, on prendra succinctement la mesure du marché discursif contemporain, caractérisé comme on le sait par la profusion et la fragmentation toujours croissantes de l'offre de contenus hétérogènes, lesquelles conduisent à une concurrence attentionnelle de plus en plus âpre entre les messages proposés.

2. Qu'est-ce qui peut dès lors conduire à accorder son attention à un contenu plutôt qu'un autre ? L'explication la plus classique repose sur la valeur hédonique prêtée à ce discours, c'est-à-dire le rapport entre l'effort qu'il réclame et la satisfaction que l'on peut en retirer. Cette explication d'abord philosophique (Hume, Bentham...) a dominé durant un siècle les recherches en psychologie avant de se diffuser sous une forme ou une autre dans bien des disciplines. Elle a trouvé sa formulation moderne dans la théorie de la pertinence de Sperber et Wilson. Selon cette dernière, la recherche de l'effet cognitif maximal moyennant l'effort de traitement le plus faible constituerait le moteur fondamental de la sélection et de l'interprétation des informations.

3. Cependant, l'invocation pragmatique de l'« effort de traitement » resterait très vague si les recherches contemporaines ne permettaient de comprendre beaucoup mieux aujourd'hui les principaux facteurs cognitifs de bas et de haut niveau qui contribuent à cet effort et permettent ou non la compréhension des discours. On remarquera au passage que ces données confirment parfois les dogmes des consultants et des manuels pratiques de communication, mais qu'elles contredisent tout aussi souvent certaines de leurs croyances.

4. Les recherches sur les ressorts de l'effet cognitif sont un peu plus confuses, englobant des variables aussi hétéroclites que, par exemple, l'attirance sexuelle, l'utilité personnelle, le suspense ou l'émotion esthétique. On peut cependant en retirer une nomenclature 
raisonnablement solide de l'interaction de ces différentes variables et clarifier ainsi l'attrait considérable des films d'action, des petits chats de YouTube, de la presse tabloïd ou des livres de cuisine.

5. L'examen des facteurs d'effort et d'effet incite à s'attarder sur le rôle essentiel que joue l'imagerie mentale dans le traitement des énoncés. On verra que ce qui semble constituer ses briques fondamentales, les schémas cognitifs, permet non seulement de mieux comprendre le fonctionnement de différents types de discours, mais aussi les stratégies de leurs auteurs. Incidemment, cette approche conduit à voir sous un jour nouveau des phénomènes stylistiques ou génériques bien connus, mais jusqualors décrits plutôt que compris par les doctrines textuelles classiques.

6. Ainsi précisées, les variables qui déterminent la pertinence cognitive des discours semblent remarquablement bien expliquer les dominantes du marché discursif, par exemple celles que traduisent les palmarès des livres ou des films à succès, ou encore les études sur les mots clés que privilégient les internautes. Mais le plus intéressant est ce qui tient cette théorie en échec : très puissante pour élucider certaines manifestations de la valeur discursive, elle est parfaitement incapable de rendre compte d'autres phénomènes tout aussi faciles à constater empiriquement (par exemple le rejet des contenus trop violents ou le dédain pour les produits culturels «vulgaires »). Or, cette limitation n'est pas un défaut de la théorie, mais sa contrepartie obligée : si les préférences discursives sont psychologiquement motivées, elles ne sont pas moins conditionnées par les normes et les valeurs sociales.

7. La régulation sociale de la légitimité des discours met notamment en jeu des contraintes institutionnelles (la recevabilité formelle), positionnelles (la distinction culturelle) ou affinitaires. Cependant, la décohésion des pratiques culturelles, observée en Europe comme en Amérique du Nord, conduit de plus en plus volontiers 
chacun de nous à panacher de façon éclectique des contenus populaires et raffinés de toute nature. Les déterminants traditionnels des préférences, comme le milieu d'origine et le niveau d'étude, ont en effet perdu de leur pouvoir : si la hiérarchisation sociale des goûts continue globalement à s'exercer, elle prédit nettement moins qu'avant les choix de consommation culturelle.

8. Comment, dans ce cas, rendre compte de l'influence des facteurs sociaux dans la réception des discours? La façon la plus sûre de le faire, à défaut d'être la plus subtile, est de distinguer les facteurs d'appréciation et de rejet (le «bien » et le « mal », en quelque sorte), en relevant dans l'un et l'autre cas une gradation similaire de la pression normative, allant de l'approbation ou la réprobation diffuse à l'obligation ou l'interdiction légale.

Ainsi peut-on enfin esquisser un modèle général de la préférence discursive, lequel articule d'un côté les déterminants dépréciatifs et appréciatifs de la pertinence cognitive (l'effort et l'effet) et de l'autre les déterminants dépréciatifs et appréciatifs de la convenance sociale (la proscription et la prescription). À l'usage, une telle articulation rend plus lisibles et cohérents les phénomènes apparemment chaotiques et contradictoires qui agitent le marché discursif.

9. Était-ce donc si simple? Dans les grandes lignes oui, mais dans les détails non, puisqu'il a fallu huit chapitres pour le justifier théoriquement et empiriquement, et encore un chapitre pour préciser les limites de ce modèle et vérifier sa capacité réelle à mieux expliquer les comportements et préférences que ne le pouvait jusqu'à présent le brouhaha des hypothèses partielles disponibles. Mais sous l'angle qui est le sien, et malgré toutes les nuances et les protestations de modestie qu'elle réclamerait, cette façon de voir l'adéquation sociocognitive semble décidément solide.

10. On peut, dès lors, revenir au paysage apocalyptique du débat public contemporain, tel qu'on l'avait contemplé au premier chapitre. L'instrument analytique qu'offre 
l'articulation des facteurs cognitifs et sociaux permet non seulement de compléter un peu les théories classiques de la communication médiatique et culturelle en contribuant à éclairer ce qu'elles peuvent encore laisser dans l'ombre, mais aussi de disséquer les rouages qui caractérisent les discours populistes et expliquent leur inquiétante efficacité, ainsi que celle des contenus distractifs triviaux ou fabulateurs. Mais du même coup, il souligne la responsabilité de tous ceux qui, en négligeant obstinément les logiques de la réception des discours et les conditions d'un débat fondé en raison, ont jusqu'alors abandonné l'espace public aux plus forts et aux plus rusés.

À dire vrai, ce chapitre conclusif ne fait pas partie de l'outil conceptuel proposé : il n'en est que l'une des extensions possibles. En outre celle-ci, s'affranchissant à cet endroit des contraintes de la distanciation théorique, ne fait pas mystère de la perspective condorciste dans laquelle elle est envisagée.

Lorsque l'on commence à envisager la valeur des discours comme le fruit des forces structurées et structurantes de la pertinence cognitive et de la convenance sociale, on en vient rapidement à reconnaître partout ces forces à l'œuvre. La seconde partie de ce livre rassemble ainsi une sélection de microétudes thématiques, consacrées à des questions aussi disparates en apparence que la rhétorique des images publicitaires, les contraintes de la critique culturelle, les problèmes des entreprises de presse, le bon usage de l'obscénité dans les manuels militaires, les dogmes de la rédaction professionnelle, la force du narratif dans la littérature et les médias, ou encore les difficultés théoriques et pratiques de la traduction.

Quelques-unes d'entre elles reflètent telle ou telle des recherches menées au cours des années qui ont permis la construction, l'élargissement, et surtout la mise à l'épreuve du modèle sociocognitif de l'adéquation discursive. D'autres ne sont au contraire que l'ébauche de travaux à venir. Toutes ont en commun, à l'instar des guides de recettes qui accompagnent parfois les appareils ménagers, d'illustrer certaines des applications ou implications concrètes de cet outil. Les théories ne sont pas des révélations éthérées : ce sont des choses. Si les plus ésotériques semblent surtout devenues des objets de culte pour 
des sectes d'adulateurs farouches, d'autres ne valent que pour ce que tout un chacun, chercheur ou praticien, est en mesure de fabriquer avec, en fonction des problèmes qui le concernent. Celle-ci n'a pas d'autre ambition, mais ce n'est pas rien. 


\section{(Précisions)}

Dar définition, cet ouvrage est lui-même soumis au joug des condi1 tions de recevabilité discursive qu'il examine, et en particulier à cette âpre tension entre la clarté et la rigueur des discours que l'on observera plus loin. La tension est d'autant plus douloureuse ici que la grande hétérogénéité des situations abordées, ainsi que la profusion multidisciplinaire des travaux qui les éclairent, appelleraient à chaque ligne un flot accablant de nuances et de précisions : autant qu'un problème épistémologique, l'approche générale des phénomènes communicationnels est un redoutable problème discursif.

Face à celui-ci, l'auteur ne dispose, outre sa témérité, que de deux atouts.

Le premier est de ne rien soutenir ici qui soit intrinsèquement «nouveau ». Les phénomènes sont suffisamment connus et documentés pour que l'on puisse s'y référer assez succinctement (et au besoin superficiellement), les théories aussi, et l'approche proposée ne sort pas du néant : si cette synthèse est originale en soi, les principales pièces dont elle est issue ont déjà été exposées sous leur forme légitime dans des revues ou colloques scientifiques, de même que ses applications à un fatras de questions allant de l'enseignement universitaire de la communication écrite ${ }^{1}$ à l'exécration machinale du « sensationnalisme » journalistique ${ }^{2}$, en passant par la production du jugement artistique et littéraire ${ }^{3}$ ou les contradictions de la communication médicale ${ }^{4}$.

1 Les déterminants cognitifs et sociaux de l'adéquation communicationnelle (Labasse, 2015a).

2 Sexe, sang et physique des particules : le "sensationnalisme" est-il partout... ou nulle part? (Labasse, 2012a).

3 L'art ou le mouchoir? Les facteurs sociocognitifs dans l'appréciation culturelle (Labasse, 2014a).

4 Recevabilité des énoncés dans l'espace public: le cas de l'information nutritionnelle (Labasse, 2016). 
Le second est qu'au cours d'une vingtaine d'années passées à scruter les fondements et logiques de la production discursive et à les enseigner, je n'ai rien trouvé qui impose de maintenir un ton uniforme de la première à la dernière page d'un ouvrage, si sérieux qu'il se veuille sur le fond. Au-delà des usages (dont, comme on le verra, il ne faut pas négliger le poids ${ }^{5}$ ), aucun impératif éditorial, aucune nécessité épistémologique, ne contraignent à choisir entre la forme d'un essai raisonnablement stimulant et celle d'un traité scientifique plus scrupuleux, mais plus fastidieux. C'est pourquoi, empruntant au geste du chef d'orchestre - la main droite donne le tempo, la gauche sculpte les nuances -, on tentera ici une double exposition en accompagnant chaque chapitre d'un complément ("Précisions») où seront repoussés les développements dont un lecteur pressé pourra se dispenser s'il le souhaite : bien que le traitement scientifique d'une question aussi large impose une réflexion constante sur ses conditions de validité et de recevabilité, cette circonspection autoréférentielle peut facilement devenir lassante.

Commençons donc ces précisions par quelques mots sur la méthode (le lecteur pressé peut sauter à partir d'ici). Dans ses grandes lignes, l'approche suivie est très banalement inductivodéductive. Elle procède par la confrontation récursive d'éléments empiriques - qualitatifs, quantitatifs, expérimentaux... - et d'hypothèses scientifiques multidisciplinaires pouvant les expliquer, en vue d'intégrer les uns et les autres dans un modèle général, lequel est à nouveau confronté aux observations empiriques et étendu à d'autres. Cette méthode favorise une grande parcimonie épistémologique en limitant strictement le recours à des hypothèses ou concepts autoproduits. Comme on l'a dit, l'apport que revendique ce travail ne réside pas dans des intuitions inédites, mais dans la façon dont il articule et éclaire, sous l'angle qui lui est propre, une masse considérable d'observations hétéroclites et d'explications antérieurement disponibles, mais peu ou mal rapprochées jusqu'alors : Non nova, sed nove ${ }^{6}$. Cependant, si une telle

5 Même les modestes transgressions typographiques - italiques des citations et séparations des paragraphes - que s'autorise cet ouvrage (pour des raisons que l'on évoquera au chapitre 3) n'ont pas été adoptées sans hésitation.

$6 \mathrm{Ou}$, comme le disait Pascal, "Qu'on ne dise pas que je n'ai rien dit de nouveau : la disposition des matières est nouvelle » (1670/1954, p. 1101). 
approche peut, un peu abusivement, sembler drapée de vertu kuhnienne ${ }^{7}$, elle appelle quelques remarques sur ses deux principales composantes.

Les éléments empiriques présentés ici, y compris les anecdotes les plus triviales, ont principalement été retenus en fonction de leur portée heuristique, laquelle ne préjuge évidemment pas du caractère probant de chacun d'entre eux pris isolément. En d'autres termes, le fait qu'ils soient significatifs à un degré ou à un autre n'implique pas forcément qu'ils soient statistiquement généralisables et ne permet pas de les solliciter au-delà de ce qu'ils représentent. Il s'agit de construire une interprétation unifiée et de vérifier la solidité et l'utilité de celle-ci, mais pas d'exhiber une impossible preuve. Ainsi recourra-t-on non seulement à de nombreux résultats de recherche, de première et de seconde main, mais aussi à des données répondant à des contraintes méthodologiques plus sommaires (statistiques commerciales, sondages...) et donc prises ici pour ce qu'elles sont. De même, les multiples éléments du discours social relevés comme exemples ou comme indices, notamment les articles de presse, ne doivent être considérés que comme des attestations (au sens linguistique du terme) : ils "prouvent» simplement qu'une chose a été énoncée ${ }^{8}$. Par ailleurs, la volonté de ne pas abstraire les phénomènes de leur trame diachronique impose des mises en perspective fréquentes mais succinctes, qui aident à percevoir l'épaisseur et la longueur du tissu historique dans lequel s'insèrent ces phénomènes, mais ne suffisent en aucun cas à en constituer une description historiquement contextualisée : « un trait d'histoire ne prouve pas; un petit conte ne démontre pas ${ }^{9}$. Enfin, toujours dans le souci d'alléger, on se dispensera d'appuyer par des références bibliographiques les situations ou évènements qui semblent suffisamment connus et admis pour pouvoir s'en passer.

7 Dans la mesure où, pour Thomas Kuhn (1983), la connaissance scientifique moderne - en tout cas en sciences de la nature - ne saurait progresser par le livre, celui-ci ne pouvant être que le réceptacle de travaux préalablement soumis à l'examen des pairs.

8 Faut-il préciser qu'il n'entre aucun relativisme dans ce rapprochement d'éléments hétérogènes ? Le fait que tout, anecdotes et analogies comprises, puisse selon nous avoir une valeur indicative ne suggère en rien qu'ils sont épistémologiquement similaires.

9 Malebranche, 1678, p. 159. 
Les éclairages scientifiques mis en regard de ces observations posent des problèmes de légitimité d'une autre nature. Si la communication rassemble des pratiques hétéroclites et bourgeonnantes, on sait qu'elle est aussi un objet d'étude sur laquelle se sont accumulées au fil du temps, et de plus en plus vite au cours du dernier demi-siècle, d'innombrables approches, qui sont toutes pertinentes à un titre ou à un autre, mais pour lesquelles l'adjectif « innombrable » doit être pris au sens littéral. Pourquoi, dès lors, privilégier celle-ci plutôt que celle-là (" celle-là » étant évidemment le paradigme favori du lecteur) ? Au nom de quoi ignorer la main tendue de la grammatologie ou de la linguistique dite « cognitive»? Quels errements scientistes trahit le recours aux propositions de la psychologie expérimentale plutôt qu'à celles, moins naturalistes et donc plus acceptables, de la systémique paloaltienne ou, à l'inverse, à celles des neurosciences si en vogue aujourd'hui ? Outre des considérations épistémologiques que l'on explicitera de temps à autre, on assumera ici une forme modérée d'instrumentalisme théorique : celle visant à employer les outils qui paraissent répondre le plus directement et le plus économiquement au type de difficulté à résoudre, sans contester que d'autres le pourraient aussi par d'autres voies et en soulignant même chaque fois que possible cette convergence, fréquente mais souvent inaperçue. Bien entendu, convenir à une question ne consiste pas à protéger les hypothèses émises à son propos : les travaux pouvant conduire à reconsidérer certaines d'entre elles, voire à les abandonner ont fait l'objet d'une attention particulière, mais ils n'apparaîtront plus forcément ici comme contradictoires, ayant rempli leur œuvre et infléchi le raisonnement en conséquence.

Rappelons enfin qu'un problème aussi pluridisciplinaire requiert des outils qui ne le sont pas moins. Certes, l'interdiscipline est toujours plus ou moins une indiscipline, mais si la multiréférentialité ne sert pas simplement à cautionner des bricolages conceptuels, elle exige que les apports mobilisés le soient avec vigilance, surtout lorsqu'ils sont réinsérés dans une perspective particulière (Bouvard et Pécuchet ne sont jamais loin). Pour autant, elle n'exige pas une sujétion inconditionnelle à l'ensemble du cadre théorique dont provient l'instrument. On n'aura donc aucun scrupule à s'enrichir de telle ou telle proposition sans forcément recourir à tout le 
système qui l'accompagne, acceptant par avance l'anathème des « hommes d'un seul livre ». On jugera l'arbre à ses fruits...

À la vaine litanie des excuses anticipées, ajoutons enfin que l'objet auquel on s'intéresse ici est aussi multifactoriel et complexe qu'il est possible de l'imaginer, mais qu'un texte impose par nature un parcours linéaire. On devra donc, faute de mieux, abuser de ces marques exaspérantes d'anticipation ou de rappel (« comme on l'a vu...», "comme on verra...») qui signalent les liens nécessaires entre des aspects que l'on ne peut tous évoquer simultanément, mais que l'on ne peut pas non plus disjoindre. 
Page blanche conservée intentionnellement 


\author{
PREMIÈRE PARTIE
}

\title{
POURQUOI LES CONTENUS QUI NOUS PLAISENT NOUS PLAISENT-ILS ?
}


Page blanche conservée intentionnellement 


\title{
De quelques convulsions du marché discursif
}

\begin{abstract}
Nos inventions sont d'ordinaire de jolis jouets qui nous distraient des choses sérieuses. Ce ne sont que des moyens améliorés en vue d'une finalité déficiente [...] Nous construisons en toute hâte un télégraphe magnétique du Maine au Texas ; mais il se pourrait que le Maine et le Texas n'aient rien d'important à se dire.
\end{abstract}

Henry Thoreau (1854, p. 57 )

$\mathrm{L}$ es étudiants, en général, n'aiment guère les cours de méthodologie documentaire et de techniques d'expression qui leur sont imposés, exigences pédagogiques souvent fastidieuses qui les séparent des « vraies choses » - l'art de l'ingénieur, du juriste, de l'urbaniste ou de l'enseignant - qu'ils sont venus acquérir. Qui songerait à leur dire que les «vraies choses » ne sont peut-être pas ce qu'ils croient? Au-delà des raffinements disciplinaires et de quelques voies particulières, l'enseignement supérieur n'apprend essentiellement qu'un seul métier, celui d'opérateur sur le marché discursif : l'art de recueillir, traiter et restituer de l'information en lui conférant si possible une quelconque valeur ajoutée ${ }^{2}$. Mais comme tous les emprunts

I Sauf mention contraire, la traduction des citations d'ouvrages référencés en bibliographie sous leur titre anglais original est de mon fait.

2 Une étude menée par l'Université d'État de Caroline du Nord auprès de 600 employeurs publics et privés a ainsi constaté que ceux-ci situaient les aptitudes en communication de ses diplômés (toutes disciplines confondues) au premier rang des compétences attendues, précédant nettement les connaissances techniques et la «capacité à appliquer les principes scientifiques ». Un seconde étude, menée cette fois auprès de 3000 anciens 
au vocabulaire économique, des termes comme marché et valeur présentent - outre leur déplaisant effluve financiariste - une plasticité qui les rend ambigus.

Même s'il génère des centaines de milliards de dollars de revenus directs, et sous-tend plus généralement la totalité de l'activité humaine, le marché auquel on s'intéresse ici n'implique pas nécessairement de transaction marchande ni de coordination structurée. Ce terme commode désigne simplement ici, de la façon la plus large possible, l'ensemble des informations qui s'offrent à être vues, entendues ou perçues d'une façon ou d'une autre, et qui sont à ce titre susceptibles d'être retenues ou rejetées. Si certaines d'entre elles, par exemple le bruit de la pluie qui tombe, ne cherchent pas à avoir du sens, les plus notables sont évidemment celles qui ont été produites ou transformées dans le but de signifier quelque chose : les discours (soit dit, bien sûr, sans la connotation déclamatoire qui déprécie ce terme dans l'usage commun).

La masse bourdonnante formée par ces derniers a été approchée sous de multiples dénominations et perspectives scientifiques (la « noosphère », l'« espace public », l'« univers discursif », le « marché cognitif ${ }^{3} »$, le «discours social »...), mais une conception qui l'est moins, celle de la « société de l'information » exige que l'on s'y arrête brièvement, non seulement parce qu'elle a aisément éclipsé les autres dans l'imaginaire collectif, mais aussi parce qu'elle est seule à annoncer une transformation soudaine et radicale du marché discursif. Celui-ci évolue, bien sûr. Il a toujours évolué. Mais dans quelle mesure et pourquoi ? On se gardera bien de plonger une nouvelle fois ${ }^{4}$ dans

étudiants engagés dans la vie professionnelle, livra le même classement mais en accentuant encore l'écart entre la communication et les connaissances spécialisées (Hoey, 1997).

3 Voir à ce propos la première note des «Précisions" qui suivent ce chapitre.

4 Plusieurs des indications et remarques de ce chapitre introductif font écho à un travail que j'avais antérieurement consacré à cette évolution (Une dynamique de l'insignifiance; Les médias, les citoyens et la chose publique dans la "société de l'information"). Les principales conclusions de ce livre de 2003 n'ont pas forcément trop mal vieilli, mais force est d'admettre que certaines observations fondées sur les données et technologies disponibles à l'époque (celle du web 1.0) gagneraient à être reconsidérées à l'heure des réseaux sociaux. 
les débats nourris qui ont opposé sur ce thème les prophètes des temps nouveaux et leurs contradicteurs, sinon pour soulever un point curieux : la rupture brutale que l'on associe aux nouvelles technologies de l'information s'estompe dès qu'on ne les regarde plus. À la place de l'an zéro d'une nouvelle société n'apparaît qu'une profonde et régulière évolution, dont les conséquences sont tout aussi spectaculaires, mais dont le mouvement est assez différent.

Il faut pour cela s'intéresser à l'offre discursive en faisant un peu abstraction des techniques qui la soutiennent, et même en la considérant sous son aspect le plus trivial : sa quantité. Celle-ci est évidemment difficile à évaluer, aucun indice ne pouvant à lui seul en rendre compte. Ce qui est révélateur en revanche, c'est que tous les indices imaginables racontent la même histoire, celle d'un gonflement continu et de plus en plus rapide de la masse des informations disponibles. Ce n'est pas une surprise : on sait que chaque innovation

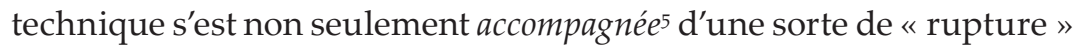
qualitative de l'information offerte, mais aussi d'une inflation quantitative de celle-ci. Ainsi l'imprimerie à caractères mobiles a-t-elle favorisé l'essor de pratiques plus ou moins nouvelles ${ }^{6}$ (dont l'édition de livres profanes, la lecture de divertissement et, plus tard, l'actualité périodique), mais elle a aussi relayé et accéléré une croissance exponentielle déjà amorcée auparavant : la production de livres en Europe serait certes passée de 12 millions d'exemplaires imprimés au cours de la seconde moitié du $\mathrm{xv}^{\mathrm{e}}$ siècle à 628 millions au cours de la seconde moitié du $\mathrm{xvIII}^{\mathrm{e}}$ siècle, mais avant même l'imprimerie, elle avait déjà bondi de quelque 10 ooo manuscrits pendant le vir ${ }^{\mathrm{e}}$ siècle à près de 5 millions au $x^{e}$ siècle (Buringh et Van Zanden, 2009). De même, la mise au point de la presse métallique, puis à vapeur, puis rotative, et celle de la linotype ont autant accompagné l'accélération

5 Contrairement à une idée séduisante, les inventions ne sont ni simplement des causes ni simplement des conséquences des transformations qu'elles accompagnent : les facteurs sociaux, tant politiques que culturels et économiques (ici, l'essor de la démocratie, de l'alphabétisme et de la publicité... entre autres) exercent des influences décisives sur ces évolutions et en subissent réciproquement l'influence. Qui, du remonte-pente et des sports d'hiver, a-t-il «causé » l'autre?

6 Pour un examen moins elliptique de l'évolution de ces pratiques, voir notamment l'Histoire de la lecture dans le monde occidental, dirigée par Guglielmo Cavallo et Roger Chartier (2001). 
et la diversification de la production discursive (populaire, spécialisée...) que la progression spectaculaire de sa diffusion. En 1813, les huit quotidiens français d'information générale totalisaient un tirage de 36 ooo exemplaires. Un siècle plus tard, ils étaient 242 et leur tirage atteignait 9500000 exemplaires (Albert, 1998).

Depuis Platon dénonçant les conséquences apocalyptiques de l'invention de l'écriture (qui « ne peut produire dans les âmes [...] que l'oubli de ce qu'elles savent $\left.{ }^{7} »\right)$ jusqu'à Zola s'inquiétant de celles du "flot déchainné de l'information à outrance » et de "l'état de surexcitation nerveuse » qui en résulterait (1888, p. 1), chacune des nombreuses évolutions qui ont jalonné l'histoire de la communication est apparue à bon droit comme une nette rupture avec le passé. Ainsi que le note James Carey :

Toutes les valeurs prêtées à l'électricité et à la communication électrique, jusqu'à l'ordinateur, le câble et la télévision par satellite, le furent d'abord au télégraphe avec un identique mélange de fantaisie, de propagande et de vérité. (1998, p. 119)

À quand, dans ce cas, faire remonter cette "entrée dans la société de l'information » que célébraient tant les pouvoirs publics dans les années 2000 ? Si l'on entend par là une configuration économique succédant au modèle agricole, puis industriel, alors le tournant paraît bien antérieur à Internet (le Japon, aiguillonné par Yoneji Masuda, disposait déjà d'un Plan for the information society il y a près d'un demi-siècle). Une recherche publiée par le ministère états-unien du commerce a ainsi estimé que la part des emplois du secteur industriel, qui avait dépassé celle des emplois agricoles en 1906, avait à son tour été dépassée par celle des emplois du secteur de l'information... dès $1955^{8}$, c'està-dire à une époque où les très rares ordinateurs existants nécessitaient encore des tubes à vide et des cartes perforées. Ces calculatrices géantes n'étaient manifestement pas pour grand-chose dans l'emballement de la production et de la diffusion des informations. Évidemment, ce que l'on définit comme un emploi du «secteur de l'information»

7 Phèdre (traduction de Mario Meunier, 1922, p. 275)

8 Voir Marc Uri Porat (1977). On notera que dans une optique très différente, mais convergente, Breton et Proulx (1989) situent entre 1940 et 1950 la période où la communication a acquis sa prépondérance symbolique passant du statut de simple commodité à celle de valeur centrale et de clef des problèmes sociaux et économiques. 
pourrait ou non conduire à déplacer cette date de quelques années, mais ce point a peu d'intérêt. Ce qui importe, c'est la tendance générale que montrait cette étude sur une longue période (1860 à 1980). C'est aussi le fait que les indicateurs quantitatifs les plus hétéroclites témoignent du même phénomène.

La consommation de papier d'impression aux États-Unis serait ainsi passée de moins de 2 milliards de tonnes en 1910 à quelque 25 milliards de tonnes en 1990 (Wernick, Herman, et al., 1996). Le nombre de programmes de télévision proposés, une poignée dans les années 1960, se montait à plus d'une centaine trente ans plus tard. Pour leur part, les informations administratives et commerciales (y compris les lois et règlements ${ }^{9}$ ) sont peut-être celles dont le volume a connu la plus forte croissance. Leur progression aurait même été plus rapide au début du $\mathrm{xx}^{\mathrm{e}}$ siècle qu'après la Seconde Guerre mondiale (Schement, 1990) sans pour autant perdre sa vigueur depuis. Anecdotique mais fascinante, l'une des plus remarquables contributions à cette inflation documentaire est le rapport que le bureau de contrôle financier du gouvernement états-unien a consacré, impavide, aux études supplémentaires qui lui sembleraient nécessaires pour "évaluer l'impact des efforts visant à estimer le coût des rapports et études " (U.S. Government Accountability Office, 2012). Quant au livre imprimé, symbole altier des « vieilles » technologies, il n'en a pas moins poursuivi son expansion, Internet ou pas : la production de nouveautés et de nouvelles éditions a pratiquement triplé en France entre 1970 et 2007 (Gaymard, 2009). Plus de 68 ooo nouveaux titres y étaient lancés en 2017, portant le choix offert aux lecteurs à plus de 775000 références (ministère de la Culture, 2018). Au Canada, le nombre de nouveaux titres mis annuellement sur le marché (plus de 12000 en $2016^{10}$ ) a fléchi depuis quelques années, mais reste supérieur à ce qu'il était vingt ans plus tôt.

Une accélération tout aussi ancienne, mais plus prononcée encore, a touché la production même des connaissances humaines.

9 Le Recueil des lois publié annuellement par l'Assemblée nationale française est passé de 620 pages et 912 grammes en 1970 à plus de 2500 pages et de 3,2 kilos en 2004 (Hispalis, 2005) et la production de l'information administrative versée aux archives nationales françaises a progressé de plus de $400 \%$ en 30 ans.

10 Selon les données de Statistiques Canada (table 21-10-0207-01, publiée le 23 mars 2018), rapprochées de données antérieures de la même source. 
Dès les années 1950, un jeune physicien, Derek de Solla Price, s'était avisé que les résultats de recherche publiés dans les revues scientifiques manifestaient «une croissance exponentielle, à un rythme extraordinairement rapide, [...] apparemment universelle et remarquablement persistante » (1986 p. xix). Ses investigations ultérieures confirmèrent cette impression : le nombre des revues savantes semblait doubler tous les dix ans, passant d'une quinzaine vers 1750 à près de 50000 vers 1950, et près de $90 \%$ de la totalité des travaux scientifiques de toute l'histoire de l'humanité étaient le produit de la dernière génération de chercheurs. Conscient des quelques limites de son approche, de Solla Price estimait en tout état de cause peu plausible qu'un tel taux de croissance de la production se maintienne à la fin du siècle. Contre toute attente, c'est pourtant ce que paraît confirmer une recherche récente (Olesen et Von Ins, 2010).

Une conséquence pittoresque de cette prolifération des discours scientifiques est ce que l'on a appelé l'« effet Barnaby Rich », dûment analysé - non sans accroître du même coup la masse des publications savantes - comme la tendance des chercheurs à maintenir « une production élevée d'écrits scientifiques, accompagnée de protestations contre la productivité excessive des autres auteurs " (Tibor et Zsindely, 1985, p. 529). La désignation est judicieuse, Barnaby Rich étant un érudit de la fin $\mathrm{du} \mathrm{XvI}^{\mathrm{e}}$ siècle à qui l'imprimerie avait permis de publier 28 ouvrages, mais qui est surtout resté célèbre pour un commentaire désabusé :

Un des fléaux de cette époque est la multiplicité des livres ; ils surchargent tant le public qu'il ne peut absorber l'abondance de sujets insignifiants qui sont produits et diffusés chaque jour ${ }^{11}$.

Fallait-il pour autant s'arrêter en si bon chemin ? Quitte à souligner la constance historique du boursouflement du marché discursif, l'effet aurait pu être nommé en l'honneur de Sénèque, lequel déplorait quinze siècles plus tôt que «l'abondance de livres dissipe l'esprit ${ }^{12}$ ».

Quoi qu'il en soit, la première chose que l'on peut dire du marché discursif, c'est qu'il gonfle. Il gonfle depuis toujours. Mais il gonfle toujours plus vite.

11 Cit. in Solla Price, op. cit., p. 56.

12 Lettres à Lucilius (trad. J. Baillard, 1914, p. 3). 
Comme il se doit, cette hypertrophie s'accompagne de transformations substantielles, invariablement saluées par un brouhaha d'acclamations et de cris d'alarme.

L'un des plus évidents d'entre eux vise l'accélération du rythme des échanges, dénoncée, par exemple, par Paul Virilo (2009) :

Twitter n'échappe pas à cette règle. Plus on entre dans l'accélération des phénomènes, plus on brouille les repères. On n'a plus d'affrontement entre la vérité et le mensonge, mais une succession toujours plus rapide d'instants irréfutables : des émotions globales, synchrones, instantanées, à l'échelle du monde entier. [...] On croit qu'on défend la démocratie, en réalité, elle est minée. La démocratie s'adresse à un corps social réfléchi, pas à un agrégat d'individus rois faussement unis dans une émotion collective.

La crainte est peut-être fondée, mais elle n'est, en tout cas, pas bien originale. Un péril similaire avait - notamment ${ }^{13}$ - été souligné 120 ans plus tôt :

Dans notre société inquiète et pressée qui n'a plus le temps de lire parce qu'elle a perdu peut-être l'habitude de penser, on ne peut s'astreindre à quelques minutes d'attention. Le livre a été délaissé pour l'article de revue, le périodique a subi la concurrence de la feuille quotidienne, et le journal lui-même, atteint par les dépêches, cherche à attirer l'attention distraite du lecteur en imitant la brièveté du style télégraphique [...] En introduisant dans notre vie deux éléments nouveaux, la hâte et la vitesse, notre pensée náa-t-elle pas acquis une instabilité qui l'a privée de sa force? (Picot, 1889, p. 189)

Le phénomène est souvent associé au traitement de l'actualité, mais il ne s'y limite pas : la durée de vie des nouveaux livres, en tout cas si on l'exprime par le délai pendant lequel ils sont offerts en librairie, se

13 On trouvera par exemple chez Harold Innis (1951 et 1952) d'autres avertissements éloquents sur la destruction de la culture, voire de la civilisation, par la mécanisation du marché discursif et le raccourcissement des temporalités. 
raccourcit très régulièrement. Elle se situe désormais souvent en dessous de la limite des trois mois pendant lesquels le libraire peut retourner les invendus (croissants) à l'éditeur. En sciences sociales, une étude d'Herbert Gans (1992) a souligné la brièveté de la «fenêtre d'attention " (attention span) dont bénéficiaient les recherches antérieures : plus de la moitié des travaux cités dataient de moins de dix ans. C'est, incidemment, dans les publications portant sur les médias que cette «amnésie sociologique » était la plus prononcée (la plus récente décennie captant à elle seule $70 \%$ des citations).

Une seconde tendance manifeste est l'affaiblissement des hiérarchies discursives. Tandis que le rationnement profite aux discours socialement valorisés - par exemple les textes sacrés et les auteurs antiques jusqu'à la Renaissance, puis la haute littérature et les débats politiques, artistiques ou savants - chaque distension du marché discursif ébranle ces privilèges au profit d'un afflux de formes et de pratiques dénoncées comme inférieures et triviales. Ainsi, plusieurs arguments du réquisitoire contre la «littérature industrielle » fulminé par Sainte-Beuve en 1839 pouvaient-ils paraître tout aussi neufs un siècle et demi plus tard ${ }^{14}$ (lui-même notait que «le mal sans doute ne date pas d'aujourd'hui »).

Un autre phénomène à la fois ancien et contemporain est la spécialisation des producteurs et, surtout, des domaines. La division du travail discursif est bien connue, non seulement dans les fonctions «techniques» (par exemple le démembrement du métier jadis unique d'éditeur-libraire-imprimeur), mais aussi dans la création proprement dite. Ainsi, la professionnalisation de la masse indifférenciée des " gens de lettres » conduira-t-elle à la séparation entre les journalistes (d'abord appelés "écrivains périodiques») et les auteurs de fiction (« écrivains littéraires »), tandis que les sciences humaines et sociales

14 «Ce sera de moins en moins un trait distinctif que d'écrire et de faire imprimer. Avec nos mours électorales, industrielles, tout le monde, une fois au moins dans sa vie, aura eu sa page, son discours, son prospectus, son toast, sera auteur. De là à faire un feuilleton, il n'y a qu'un pas. Pourquoi pas moi aussi ? [...] Depuis deux ans surtout, on ne vend plus : la librairie se meurt. On a tant abusé du public, tant mis de papier blanc sous des volumes enflés et surfaits, tant réimprimé du vieux pour du neuf, tant vanté sur tous les tons l'insipide et le plat, que le public est devenu à la lettre comme un cadavre. [...] De nos jours le bas fond remonte sans cesse, et devient vite le niveau commun, le reste s'écroulant ou s'abaissant. ». 
se dégageaient lentement du cocon des « belles lettres » comme, avant elles, les sciences de la nature.

La différenciation la plus spectaculaire concerne cependant l'objet même des discours. Plus il y a à connaître, plus chaque thème tend naturellement à se spécialiser en s'éloignant des autres. C'est, bien sûr, le cas des sciences - où la multiplication des publications évoquée plus haut s'est accompagnée d'un bourgeonnement continu de spécialités, menaçant jusqu'à l'intercompréhension au sein des disciplines constituées ${ }^{15}$ - mais c'est aussi celui de la culture ou encore celui des loisirs. Le développement des magazines spécialisés témoigne de cette diversification accélérée (relayée ultérieurement par celui des sites web et des groupes de discussion). Des domaines comme le ski ou la moto, pour ne mentionner qu'eux, ont connu un éclatement des pratiques qu'a accompagné (suivi, accentué...) une floraison de revues ${ }^{16} \mathrm{~s}^{\prime}$ éloignant de plus en plus du « tronc commun » initial. Dans le cas des sports d'hiver, l'inflation du nombre d'épreuves aux Jeux olympiques - 16 en 1924, 34 en 1964, 61 en 1994, 98 en 2014 - donne une assez bonne idée de l'éclatement des spécialités, donc des connaissances et informations liées à leurs pratiques : à l'instar des amateurs de moto « en général », les amateurs de ski « en général » ne constituent plus des publics cohérents, si l'on entend par là des agrégats partageant un centre d'intérêt et des clefs $\mathrm{d}$ 'interprétation. Quiconque parcourant un magazine dont le thème lui est étranger constatera que ses énoncés et son lexique sont à peine moins déroutants que ceux d'une publication savante. Ainsi des amateurs de ski de fond pourraient-ils avoir du mal à apprécier des pages destinées aux surfeurs des neiges (dont même les tenues diffèrent beaucoup des leurs).

15 Si un physicien et un chimiste pouvaient assez aisément échanger sur leurs travaux au XIX ${ }^{\mathrm{e}}$ siècle, deux physiciens d'aujourd'hui pourraient avoir beaucoup de mal à se comprendre, tant leurs champs de connaissances se sont éloignés en se spécialisant.

Ainsi, les magazines généralistes comme Moto Revue et Moto Journal ont-il ressenti (dès 1974 dans le cas du premier) la nécessité de proposer des alternatives plus spécialisées aux amateurs de tout-terrain, lesquelles ont été suivies plus tard par des magazines encore plus spécialisés, se limitant à une pratique particulière (trial, enduro, moto cross), tandis que d'autres périodiques se concentraient sur les intérêts exclusifs des amateurs de motos anciennes, de motos transformées, etc. 
La question est plus profonde que le seul émiettement de la gamme des loisirs, des formes musicales ou encore des choix gastronomiques. Les connaissances et centres d'intérêt censés être propres à différentes classes sociales ont en effet perdu de leur homogénéité en même temps que se diversifiait l'offre d'information qui leur était proposée. Même si l'on a toujours tendance à prêter au passé plus de cohésion qu'il n'en avait, les paysages mentaux d'un bourgeois ou d'un ouvrier d'aujourd'hui paraissent nettement plus individualisés, hétéroclites, que ne l'étaient ceux de leurs devanciers. Comparativement, la tâche d'un tribun ou d'un journaliste est ainsi beaucoup plus aléatoire que celle de ses prédécesseurs, lesquels, outre qu'ils subissaient moins la concurrence centrifuge d'autres " contenus », avaient moins de doutes sur le socle commun de connaissances et de domaines d'intérêt du public qu'ils visaient. Moins de doutes aussi, comme le remarque Demers (2012), sur la persistance $\mathrm{d}^{\prime}$ « un » public de citoyens éclairés auquel s'adresser. À une large échelle, c'est l'espace collectif du débat public qui, sous sa forme traditionnelle, semble se désagréger progressivement. En témoigne dans une certaine mesure l'érosion de l'audience des journaux télévisés nationaux, ou celle de la presse quotidienne d'information générale qui en une dizaine d'années (1990-2000) avait chuté de près de $10 \%$ au Canada et de plus de $18 \%$ en France ${ }^{17}$ bien avant que Google n'entre en bourse ou que Facebook ne soit créé.

Une dernière tendance bien connue accompagnant l'inflation des informations offertes est, assez logiquement, la dévalorisation de celles-ci. La baisse constante des prix sur le marché discursif (à prendre pour une fois au sens propre) doit beaucoup à l'essor constant de la productivité : un copiste médiéval pouvait difficilement produire cinq pages de manuscrit dans une journée quand une presse moderne débite plus de 5000 livres de poche à l'heure. De même, le cycle audiovisuel numérique (captation, montage et diffusion) est nettement plus efficace et moins onéreux que son devancier analogique. Mais le jeu brutal de l'offre et de la demande est plus impérieux encore. Quand la rareté de l'information faisait sa valeur (au sens propre également), il n'était pas rare que plusieurs familles s'associent pour partager les frais d'un abonnement au journal. Quelques générations plus tard, beaucoup de leurs descendants n'ont aucune envie de

17 Pour les seuls quotidiens nationaux. 
ramasser les quotidiens gratuits qui leur sont proposés, ou même de lire les informations générales que leur prodiguent les pages « actualités » des moteurs de recherche, pas plus que de survoler les livres du domaine public ou autoédités qui s'offrent désormais à eux : la gratuité elle-même ne suffit plus à susciter la demande.

Il ne s'ensuit évidemment pas que celle-ci a disparu. Même dans l'édition littéraire, décrite par un éditeur comme «le seul secteur économique qui réponde à une baisse de la demande par une hausse de $l^{\prime} o f f r e^{18} "$, l'explosion du nombre de titres se traduit par une forte diminution du tirage de ceux-ci, mais l'érosion de leur prix moyen reste modérée, quoique régulière. De même, les quotidiens d'actualité générale payants, bien qu'accablés par la prédation des nouveaux médias sur leur audience et sur leurs revenus publicitaires, préservent une clientèle supérieure à ce qu'annonçaient les prophéties des années 2000. Mais les journaux et les livres, c'est-à-dire les strates supérieures de la hiérarchie discursive traditionnelle, ne s'en trouvent pas moins dilués au sein d'un bouillonnement où domine la gratuité et, corollairement, l'indifférence : la dévaluation réside, en fin de compte, dans la séparation entre la valeur symbolique de l'information - l'importance culturelle, scientifique ou civique qui lui est prêtée et sa valeur d'échange : le prix que l'on est prêt à payer, ou simplement le geste que l'on est prêt à faire pour en avoir connaissance (ramasser un journal gratuit, cliquer sur un lien...), ou encore le temps que l'on accepte de lui consacrer.

Ces problèmes de surabondance et de valeur, que ce soit au sens humaniste ou monétaire, étaient déjà évidents à la fin des années 1960, ce qui avait conduit Herbert Simon (futur « prix Nobel » d'économie) à en dégager un enjeu fondamental, l'attention :

L'autre face d'un problème de surpopulation est un problème de disette, donc un problème d'allocation de ressources. [...] Similairement, dans un monde riche en informations, l'opulence d'information implique une pénurie de quelque chose d'autre ; une disette de quoi que ce soit que l'information consomme. Ce que l'information consomme est assez évident : elle consomme l'attention de ses récepteurs. Ainsi, une abondance d'information

18 Souvent citée, mais probablement orale, cette réflexion est attribuée à Jérôme Lindon, directeur des Éditions de Minuit jusqu'en 2001. 
produit de la pénurie en attention, et une nécessité d'allouer cette attention efficacement au sein de la surabondance de sources d'informations qui peuvent la consommer. (1971, p. 40-41)

Les prémices sont un peu schématiques, mais le raisonnement est séduisant. Il semble, en tout cas, en très bon accord avec l'ensemble des observations rassemblées plus haut. Il l'est aussi, sans ménagement, avec l'expérience quotidienne des producteurs discursifs : le professeur tentant de rivaliser avec la forêt des téléphones et ordinateurs portables qui captivent ses étudiants, le politicien confronté aux fluctuations de l'abstention électorale ${ }^{19}$, l'écrivain supputant les minces chances qu'a son premier livre d'obtenir une quelconque recension, le jeune chercheur guettant la moindre citation qui indiquerait que quelqu'un, quelque part, a finalement pris connaissance de son travail. Et, bien sûr, le journaliste accueilli chaque matin par les chiffres d'audience de son émission de la veille ou le nombre d'accès à son article en ligne.

Envisager la rareté de l'attention comme un déterminant des logiques de production et de réception des discours décentre le regard par rapport aux évidences des ruptures technologiques. Après tout, c'est bien d'elle que parlaient jadis Sénèque, Barnaby Rich et nombre d'autres à toutes les époques ${ }^{20}$. C'est elle ("l'attention distraite du lecteur ») que visait directement Georges Picot dans le propos vieux de plus d'un siècle rapporté plus haut. C'est elle encore qui conduisait Albert Thibaudet à esquisser dès 1923 une théorie comparée de la valeur littéraire et journalistique.

Sauf certains cas exceptionnels, on écrit presque toujours pour le public, avec l'idée d'un public ; à cette idée d'un public déterminé, est incorporée, comme l'une de ses dimensions, la durée d'attention qu'il peut donner à un écrit. Durée d'attention qu'un livre de philosophie a le droit de supposer illimitée. [...] Le style

19 Lesquelles sont directement liées à l'intérêt accordé par les citoyens. Voir notamment à ce propos la synthèse de Dominique Duval, Recension des écrits sur la participation électorale, Québec, Direction générale des élections, 2005.

20 Comme en témoigne le titre éloquent de l'ouvrage d'Ann Blair : Too Much To Know: Managing Scholarly Information Before the Modern Age (Yale University Press, 2010). 
du journal est déterminé par la durée d'attention du lecteur comme le style oratoire, la période, étaient déterminés par la durée d'expiration et d'inspiration des poumons. Ce sont là des durées élémentaires, psychologiques, d'où l'on passerait peu à peu, en une théorie facile à construire, à la durée sociale, celle qui fait dire qu'une œuvre est appelée ou non à durer, à rester.

Pourtant, c'est justement la « révolution » des nouvelles technologies qui a propulsé la question de l'attention au tout premier plan.

Certains, comme le psychologue Daniel Kahneman - lui aussi futur « prix Nobel » d'économie - avaient depuis longtemps tenté de souligner son importance pour la compréhension des processus de traitement de l'information (Attention and effort, 1973). D'autres, beaucoup d'autres, avaient étudié ou dénoncé les effets croissants de la surcharge informationnelle (information overload ${ }^{21}$ ) dans la vie privée ou professionnelle. C'est toutefois de la volonté de penser les transformations associées aux réseaux numériques qu'est provenu un retournement conceptuel inopiné : la «nouvelle économie » dématérialisée pouvait au bout du compte se redéfinir comme « l'économie de l'attention ». L'idée était déjà avancée par Simon ${ }^{22}$, mais elle ne s'est propagée qu'un quart de siècle plus tard, notamment à la suite d'un article publié en 1997 dans une revue consacrée à Internet (une revue en ligne, évidemment). Son auteur, Michael H. Goldhaber, partait d'un postulat de rupture qui ne risquait guère de déplaire à ses lecteurs :

Nous entrons dans une période totalement différente de l'ère ancienne de la production industrielle de masse des objets matériels [...] Il nous faut maintenant raisonner en termes économiques totalement nouveaux, puisque nous entrons dans une sorte d'économie totalement nouvelle.

En examinant à partir de là les «termes économiques totalement nouveaux $»$ qui caractériseraient et expliqueraient le mieux cette nouvelle

21 Pour un bon panorama interdisciplinaire de la masse - elle même écrasante - des recherches publiées à partir de 1970 sur la surcharge informationnelle, voir Eppler et Mengis, 2004.

22 Karl Deutsch, dans la discussion suivant la conférence de Simon et publiée en même temps qu'elle, soulignait ainsi que «l'économie de l'attention est elle-même placée au centre de l'analyse » (ibid., p. 53). 
donne, Goldhaber, après avoir dupliqué (peut-être sans le savoir) le raisonnement sur la profusion et la rareté d'Herbert Simon, avança que «la recherche d'attention pourrait être précisément la finalité que nous essayons de déterminer ", plus que l'information surabondante et dévaluée, mais aussi plus que l'argent lui-même, lequel suivrait l'attention plus qu'il ne la commande : "une grande part de votre activité personnelle est mieux caractérisée comme impliquant des transactions d'attention que des transactions d'argent » (Goldhaber, 1997, s.p.).

Peu après, un article de Thomas Davenport et John Beck dans la Harvard Business Review enfonçait le clou. Là encore, l'argumentation évoquait plus la littérature managériale des librairies d'aéroport qu'un raisonnement académique pointilleux, mais elle a également fait mouche, comme le livre qui l'a prolongé.

Le succès qu'a connu la mutation conceptuelle de l'« économie de l'information » en « économie de l'attention » doit peut-être moins à la floraison d'articles de recherches qui en ont ultérieurement débattu ou qui l'ont approfondi dans des domaines toujours plus nombreux ${ }^{23}$ qu'à l'étonnante démonstration empirique qu'en ont fournie les marchés financiers. En valorisant les entreprises de la nouvelle économie en fonction du nombre de membres ou de visiteurs captés plutôt que de leur capacité à tirer profit de ces publics, ils montraient que cette part d'attention virtuelle pouvait effectivement valoir beaucoup plus cher en bourse que les bénéfices prévisibles ou les produits physiques. Ainsi, la valeur attribuée à chacun des utilisateurs de portails Internet comme Lycos ou Excite dépassait-elle 300 \$ à la fin des années 2000. Deux crises plus tard, les chaotiques introductions en bourses de Facebook et Twitter montrent que cette logique, certes teintée d'un peu de prudence, est toujours à l'œuvre (comparativement, la capitalisation boursière de certains constructeurs automobiles en 2008 est brièvement passée en dessous de la simple valeur totale des voitures produites qu'ils stockaient en vue de leur livraison).

«Économie de l'information» ou "économie de l'attention», voire « société de l'attention » ? Le débat resterait... virtuel si bien des entreprises relevant des médias traditionnels ne tendaient, elles aussi,

23 Dont l'économie et la gestion, mais aussi les sciences politiques, l'éducation, la sociologie des sciences ou de la production culturelle et, bien sûr, plusieurs branches des sciences de la communication. 
à se repositionner dans le second cadre. On se souvient de l'accueil assez réservé qu'avaient reçu les propos sans fard de Patrick Le Lay, alors président-directeur général de la chaîne française $\mathrm{TF}_{1}$ :

[...] pour qu'un message publicitaire soit perçu, il faut que le cerveau du téléspectateur soit disponible. Nos émissions ont pour vocation de le rendre disponible : c'est-à-dire de le divertir, de le détendre pour le préparer entre deux messages. Ce que nous vendons à Coca-Cola, c'est du temps de cerveau humain disponible ${ }^{24}$.

En revanche, les propos non moins explicites d'un ouvrage publié dans le même pays par le syndicat des éditeurs de livres (SNE) n'ont guère soulevé de remous :

Le livre s'inscrit en effet parfaitement dans l'économie de l'attention décrite par le prix Nobel d'économie Herbert Simon. La rareté ne caractérise plus la production des biens, mais l'attention des consommateurs. Comment faire connaître au public cette production croissante de nouveautés ? (Moreau et Peltier, 2015, p. 28)

Serait-ce donc qu'il existe une différence de dignité entre vendre de l'attention pour elle-même et capter de l'attention pour diffuser quelque chose, en l'occurrence des livres ? En réalité, cette opposition entre l'attention en tant que fin et l'attention en tant que moyen est facilement réversible. D'un côté, soutirer de l'attention pour vendre est l'essence de la publicité. De l'autre, au sommet de la hiérarchie discursive, la recherche scientifique a pu être décrite dans l'une de ses plus prestigieuses revues - et presque sans protestations - comme une pure "foire » à l'attention où celle-ci ne serait pas seulement le «mode de paiement », mais aussi le moteur, voire le but ultime.

Le succès en science est récompensé par de l'attention. [...] Obtenir ce «revenu» d'attention est un motif principal pour devenir un scientifique et pour mener des recherches. Afin de maximiser ce revenu, on doit employer sa propre attention de la façon la plus productive possible. (Franck, 1999, p. 53)

24 Entretien avec « Les associés d'EIM » (2004, p. 92). 
Toutefois, comme on le perçoit à travers ces deux derniers exemples, la valeur attentionnelle, pas plus que la valeur monétaire, ne peut à elle seule résoudre le « grand mystère » de la valeur générale des discours.

De même que l'on avait souligné plus haut la divergence croissante entre la valeur symbolique de ceux-ci et leur valeur d'échange, de même la divergence entre leur valeur symbolique et leur valeur attentionnelle est flagrante, en particulier dans l'aristocratie de la production discursive, qu'il s'agisse de littérature ${ }^{25}$ ou de recherche. En témoigne une dissymétrie significative, qui montre que cette puissance apparemment hégémonique n'en est pas moins limitée : la reconnaissance symbolique tend à procurer de l'attention, mais un succès attentionnel ne confère en général aucune reconnaissance symbolique. Bien au contraire, malheur au producteur discursif qui espérerait tirer sa légitimité de l'attention obtenue auprès d'un trop large public. "À quatre cents exemplaires, on a du génie [...] à quarante mille, on décline mais à quatre cent mille, on sera dénoncé comme un crétin doublé d'une imposture littéraire» maugréait ainsi Pierre-Emmanuel Schmitt dans la préface qu'il consacrait à un autre auteur à succès ${ }^{26}$. L'explication est peut-être un peu courte à elle seule, mais cette opposition entre engouement public et reconnaissance qui transparaît dans le champ culturel ou scientifique se remarque aussi dans celui du journalisme ${ }^{27}$ et même dans des domaines censément moins normatifs, comme celui des jeux vidéo (le succès d'Angry birds ou de Call of duty ne leur vaut aucune estime des initiés). Il est, plus généralement, significatif que sur Internet (et ailleurs) l'attention soit si largement et si facilement captée par les contenus « distrayants » alors que la distraction est étymologiquement l'inverse de l'attention : elle est ce qui enlève celle-ci à des objets plus "dignes d'attention", ce que confirme son complice : le divertissement (à la fois «détournement frauduleux » et «ce qui détourne quelqu'un de ses occupations, de ses soucis, de ses peines » selon le Dictionnaire de l'Académie).

25 On trouvera par exemple une vigoureuse illustration de cette divergence dans La littérature à l'estomac de Julien Gracq (1950).

26 À l'occasion de la réédition de Mademoiselle de la Ferté de Pierre Benoit (Albin Michel, 2012).

27 Ainsi, le fait que les tirages des quotidiens à sensation anglais surclassent ceux des journaux plus sérieux ne leur vaut-il guère de respect dans leur sphère professionnelle (même leurs propres lecteurs leur accordent relativement peu d'estime ou de crédibilité). 
Bref, à l'instar de la valeur monétaire, ou même du prestige, la valeur attentionnelle n'est pas la valeur tout court. Elle n'en constitue pas moins une très bonne voie d'accès à celle-ci, pour plusieurs raisons dont chacune pourrait être suffisante.

La première est qu'elle anéantit l'opposition illusoire entre les angles de vue "continuistes » et «discontinuistes » du marché discursif. Beaucoup d'éléments - les seuls que l'on ait repris ici, le reste ayant été suffisamment claironné - permettraient de soutenir que les «nouvelles technologies ${ }^{28}$ » ne provoquent qu'une accélération de logiques et de tendances très anciennes. À l'inverse, nombre d'arguments, surtout depuis l'essor des réseaux sociaux, pourraient tout aussi valablement conforter l'idée d'une "véritable rupture, non seulement technologique, mais encore anthropologique et culturelle »(Rieffel, 2014, p. 15). Mais au bout du compte, ces deux façons de voir, outre qu'elles ne s'opposent pas fondamentalement (ce n'est qu'une question d'angle), conduisent exactement à la même question, celle de l'attention. Privilégier l'une ou l'autre de ces conceptions n'a donc pas tant d'importance que ça. De même, elle force à converger les approches les plus mercantiles de la communication (l'attention comme marchandise) et ses approches les plus critiques (l'attention comme asservissement). Ainsi, l'observation initiale de Guy Debord selon laquelle « la vie des sociétés dans lesquelles règnent les conditions modernes de production s'annonce comme une immense accumulation de spectacles » (1967, p. 15) n'est-elle pas si différente sur ce point de celles publiées dans la Harvard Business Review (la transposition par Debord d'une citation de Marx qui, à l'origine, visait une «immense accumulation de marchandises ", est du reste une autre façon d'affirmer la transition entre les économies matérielle et virtuelle).

La seconde raison, sur laquelle on reviendra plus loin en détail, est qu'une bonne partie des producteurs discursifs professionnels, dont les journalistes et les scientifiques, ont étonnamment mal intégré

28 Plusieurs outils se sont successivement vu attribuer le privilège de transformer radicalement la communication humaine : "Dans les années 70, le téléviseur se voyait attribuer par les futuristes une vocation à devenir le terminal multi-usages autour duquel s'intégreraient les divers moyens de communication » notaient (p. 82) Lacroix, Miège et Moeglin en 1993, époque où l'ordinateur était censé recevoir ce rôle, qui lui a depuis été arraché par le téléphone et la tablette. 
la dimension de l'attention à leurs valeurs et compétences professionnelles. Écartelés entre des normes centenaires et les féroces réalités de la rivalité contemporaine, ils sont non seulement handicapés face à des concurrents externes affranchis de ces normes, mais aussi vulnérables à toutes les transgressions internes des principes qui leur sont enseignés : la lutte pour l'attention est d'autant plus mal maîtrisée qu'elle est simplement occultée ou dénoncée au lieu d'être soupesée et conceptualisée ${ }^{29}$. La question n'est pas seulement déontologique : on montrera que, sur un plan strictement technique, certaines des stratégies discursives les plus largement enseignées aux futurs professionnels sont aussi les plusinadaptées qui soientàl'évolution contemporaine du marché discursif.

La troisième raison, qui pourrait être la première, est que l'attention est évidemment la condition fondamentale de toute prise de connaissance. Malgré la méfiance que l'on doit garder vis-à-vis des interprétations neurobiologiques des pratiques et attitudes humaines, on peut sans grand risque rappeler pour la forme que le système cognitif est avant toute autre chose - et bien avant de servir à penser quoi que ce soit - un dispositif de gestion de l'attention (en soulignant que toute perception repose sur un détachement par rapport à l'ensemble de l'arrière-plan perceptible, la phénoménologie de Husserl dit-elle autre chose ?) Pour ne pas être submergé, le dispositif en question est extraordinairement avare : la quasi-totalité des informations figurant dans le champ visuel est ignorée par les cellules photoréceptrices (dont $95 \%$ ne sont « intéressées » que par les mouvements) et l'essentiel des autres est éliminé avant même leur acheminement à l'aire visuelle primaire du cortex, d'où seule une très faible partie des survivantes parviendra peut-être, fugacement, à la conscience. À l'échelle des discours dans la sphère publique ou privée, les modalités de l'avarice attentionnelle sont évidemment beaucoup plus riches et complexes, mais la primauté de celle-ci n'est pas moins impérieuse. Dans la mesure où la quantité d'attention disponible individuellement

29 En journalisme comme ailleurs, mais peut-être plus qu'ailleurs, la connaissance réfléchie des contraintes est le meilleur moyen de les dépasser. Si les écoles de journalisme chinoises accordent autant de temps à l'étude de la ligne du parti et des règles qui en découlent, c'est peut-être moins pour former des thuriféraires du régime que pour enseigner (implicitement) l'art d'exploiter toutes les failles et de développer tous les degrés de liberté possibles. 
ou collectivement n'est guère extensible, l'allocation d'attention peut plus ou moins être considérée comme un jeu à somme nulle : un discours ne pourra recueillir de l'attention qu'en l'arrachant à tous les concurrents qui se proposeront au même moment. Comme le notait déjà Herbert Simon, les êtres humains "sont des systèmes fondamentalement séquentiels : ils ne peuvent s'occuper que d'une chose à la fois ${ }^{30}$ » (1971, p. 41) Quoi que l'on lise, regarde ou écoute, c'est à la place d'autre chose, d'où le vague sentiment de culpabilité que coûte parfois la consommation de contenus triviaux (sentiment atténué, il est vrai, par l'effritement des hiérarchies discursives). En outre, le gagnant de cette concurrence féroce peut remporter plus qu'une attention immédiate dans la mesure où - pour des raisons que l'on examinera plus loin l'attribution d'attention est généralement cumulative. Plus on en sait sur une question, qu'il s'agisse de la politique monétaire ou de la vie sentimentale des vedettes, plus on est enclin à en apprendre à son sujet, comme en témoigne l'éclatement des domaines d'intérêt et des magazines spécialisés évoqué plus haut. Là encore, Internet, en favorisant un prélèvement de plus en plus personnalisé au sein de la masse discursive, amplifie puissamment une tendance qui était liée à la cognition humaine bien avant l'arrivée du télégraphe.

Voici donc le décor planté. Il n'est nouveau ni sous ma plume ${ }^{31} \mathrm{ni}$ sous bien d'autres, mais se devait d'être examiné tant il conditionne le reste : si judicieuse que soit une proposition politique, si élevée que soit une création artistique, si importante que soit une nouvelle journalistique, elle ne peut «valoir » qu'en accédant à l'existence, et cette existence précaire s'appelle l'attention.

Il faudrait, si l'on voulait aller plus loin, examiner tous les essais qui, comme ceux de Nicolas Carr (2011) ou de Maggie Jackson (2008), prophétisent un effondrement contemporain de la capacité à connaître, ou en tout cas affiner la réflexion sur l'attention à l'aune des travaux - notamment ceux d'Emmanuel Kessous (2012) et d'Yves Citton

30 La capacité de traitement " multitâche » au nom de laquelle bien des adolescents justifient la coprésence sur leur écran de YouTube et du travail qu'ils sont censés faire est non seulement infondée en théorie (on ne fait pas plusieurs choses à la fois, on alterne très vite entre l'une à l'autre), mais aussi très malavisé en pratique (plusieurs recherches ont montré le faible rendement de cette pratique).

31 Voir Labasse, 2002. 
(2014a ; 2014b) - qui ont continué à l'approfondir. On pourrait aussi, pour contourner l'engouement que suscite cette notion, l'envisager sous l'angle de la " visibilité ", comme certains l'ont fait dans des perspectives allant de l'analyse des luttes sociales (Honneth, 2004) à celle du vedettariat (Voirol, 2005, Heinich, 2012). Évidemment, « visibilité » et « attention » ne sont ni tout à fait la même chose ni tout à fait autre chose, mais ça n'a pas vraiment d'importance ici, car il est grand temps d'abandonner ce point de départ : si l'attention détermine une grande partie des stratégies de production et de réception discursive, elle n'explique en rien ce qui la détermine elle-même. 


\section{(Précisions)}

B ien que ce soit un privilège auctorial bien connu, il faut un certain toupet pour faire soi-même ce que l'on reproche à d'autres. Dans quelle mesure peut-on contester le réductionnisme techniciste du modèle hégémonique de la «société de l'information » pour hypostasier à la place l'hypertrophie informationnelle et la crise de l'attention qui en découle comme déterminants fondamentaux du « marché discursif ${ }^{1} »$ ?

1 Locution qui réclame également quelques commentaires. Puisque « marché » il y a, en tout cas dans le sens dilaté dans lequel on emploie ce terme, était-il bien nécessaire de lui accoler une telle épithète - si rébarbative, qui plus est, que l'on a finalement renoncé à l'exhiber en couverture - alors que d'autres (marché des idées, marché cognitif...) étaient déjà disponibles. Il s'agit bel et bien ici du marché « des idées » mais évidemment dans un sens plutôt dilaté lui aussi, désignant comme idée tout ce qui accède à la conscience alors que l'histoire des idées est sensiblement plus exigeante sur ce point, et ce depuis plusieurs siècles. Pour sa part, Gérald Bronner recourt à la notion de «marché cognitif», qui pourrait de prime abord paraitre assez proche du sujet de ce livre : "Le marché cognitif est une image qui permet de représenter l'espace fictif dans lequel se diffusent les produits qui informent notre vision du monde » (2013, p. 23). On ne l'empruntera cependant pas pour diverses raisons. La première est qu'elle met, nolens volens, l'accent sur une seule famille de variables, les déterminants cognitifs, dont on remarquera plus loin l'insuffisance face au problème qui nous occupe. La seconde est que ce qui intéresse cet auteur - «un produit cognitif implique, dans le sens que je lui donne ici, une organisation d'informations en un discours explicite ou implicite sur le vrai et/ou sur le bien » (Bronner, ibid.) - est au bout du compte assez différent de l'objet des pages qui suivent, lesquelles tentent justement de s'affranchir aussi longtemps que possible de toute condition ou considération de cet ordre : l'opinion, qui est au centre des travaux de Bronner et de bien d'autres, n'est 
Même si l'on espère avoir assez martelé que ces deux visions ne s'excluent pas mutuellement - pas plus qu'elles n'excluent l'intrication complexe d'autres facteurs (politiques, culturels, économiques, etc.) tout aussi essentiels - il n'en reste pas moins que ce centrage initial sur la profusion discursive et ses conséquences attentionnelles est tout à fait délibéré.

On a mentionné ci-dessus de nombreux éléments à l'appui de ce point de départ et on en a omis bien d'autres par crainte d'accroître encore la redondance ou l'hétérogénéité du raisonnement, mais certains aspects connexes auraient mérité d'être développés.

Le plus important, que l'on n'a qu'effleuré, est politique : l'effet de la concurrence attentionnelle, d'autant plus impérieux que le pouvoir de gestion de l'attention (l'agenda setting) des médias traditionnels s'est affaibli, constitue à l'évidence une clef de lecture essentielle de la vie démocratique contemporaine, tant en raison de ce (ou ceux) qu'elle peut favoriser que de ce (ou ceux) qu'elle laisse dans l'ombre. La capacité des institutions à taire en montrant - panem et circenses - a été abondamment exposée par la critique philosophique ou sociologique de la communication, mais la dérégulation marchande de l'attention pourrait, dans une mesure qu'il reste à évaluer, redistribuer les cartes en facilitant la visibilité de causes fragmentaires. On gardera cependant cette dimension pour plus tard, la seule invocation de l'attention étant, à ce stade, beaucoup trop rudimentaire pour en rendre compte.

D'autre part, bien sûr, l'accent mis sur le gonflement diachronique de l'offre de contenus ne revient aucunement à nier que les mutations des technologies qui médiatisent la production discursive tout en la diversifiant exercent une influence très significative sur sa réception. Les recherches ethnologiques - par exemple celles de Caron et de ses collègues ${ }^{2}$ - montrent au contraire éloquemment à quel point les objets communicants influent sur les pratiques et les choix des utilisateurs, mais aussi sur le sens qu'ils donnent à ces contenus dont les modes de disponibilité dissolvent les catégorisations traditionnelles.

qu'une variable (certes envahissante, mais une variable tout de même) dans l'étude des préférences discursives.

2 Voir en particulier Caron et Caronia, 2005. 
Un autre point, plus épistémologique, doit être souligné : le gonflement de l'offre discursive mérite d'autant plus d'être examiné qu'il produit lui-même les conditions de son illisibilité scientifique et de celle de la valeur discursive. Penser que la désagrégation accélérée des modalités de production et de diffusion, des publics et des thèmes suffit à elle seule à expliquer la nébulosité globale de la communication publique serait oublier que les recherches dans ce domaine n'échappent pas aux forces centrifuges qui affectent autant leurs objets que leur propre capacité à former "un champ cohérent [...] de discours sur les discours" (Craig, 1999, p. 120).

Il y a quarante ans déjà, l'ouvrage fondateur de Robert Escarpit marquait simultanément, en France, le début et la fin du projet d'une "vue d'ensemble des sciences de l'information et de la communication » considérant sa tentative dans ce sens comme « une dernière chance pour une synthèse de ce genre » (Escarpit, 1976, p. 3) :

Les domaines du savoir deviennent trop nombreux, trop spécialisés pour qu'un seul homme [...] puisse en appréhender ne fût-ce qu'une partie. Certains des exposés qui suivent seront jugés élémentaires, voire contestables par les spécialistes. D’autres seront trouvés difficiles par les non-initiés.

De ce point de vue, on pourrait être tenté d'assimiler simplement lesdits spécialistes, sinon aux amateurs de ski ou de moto rencontrés plus tôt (on recule devant l'effronterie), du moins aux physiciens et chimistes dont Solla Price soulignait le pullulement ou, au bout du compte, aux chercheurs de n'importe quelle discipline, chacune ayant subi et subissant encore l'inéluctable dilatationfragmentation du marché discursif. Ainsi pouvait-on dès la fin du $\mathrm{XIX}^{\mathrm{e}}$ siècle commencer un traité de psychologie de façon tout aussi désabusée :

Le temps approche rapidement où on ne songera pas plus à écrire un livre sur la psychologie en général, qu'on ne s'aviserait d'écrire un livre sur les mathématiques en général. Le sujet peut être approché du point de vue de la physiologie, de la pathologie mentale, de l'ethnologie ou de l'expérimentation psycho-physique. Chacune de ces méthodes possède ses 
propres données et ses façons propres, distinctes et indépendantes, de collecter et d'évaluer les preuves. (Stout, 1896, p. ix)

Toutefois, les spécialistes de la communication se distinguent de toute autre communauté scientifique en rencontrant non pas une, mais deux fois la désagrégation de la sphère discursive, la fragmentation des approches se combinant par définition avec celle des phénomènes étudiés. Sans oublier, bien sûr, la différence des échelles concernées - interindividuelle, organisationnelle, sociétale - qui, elle, ne se dilate pas vraiment, mais dont les nouveaux médias brouillent les délimitations.

D'où la sérieuse éventualité que cette première étape en vue d'aborder globalement une problématique certes partielle, mais néanmoins imposante (il ne s'agit « que » des logiques de la réception des discours et donc, en miroir, de celle de leur production), n'ait abouti qu'à établir l'inanité d'un tel projet. La fragmentation des approches de la valeur discursive en une multitude de problèmes distincts ne serait pas seulement requise, et de plus en plus, par la divergence des pratiques sociales, mais aussi inéluctable du fait de la spécialisation des recherches. Face au Grand Mystère, il ne peut y avoir d'échec collectif si, comme le suggère l'orthodoxie communicationnelle, il n'y a rien à trouver à cette échelle.

On ne s'attend évidemment pas à me voir le concéder si tôt. D'un autre côté, prétendre dès à présent le contraire ne constituerait rien de plus qu'une pétition de principe. On pourrait, à l'instar de Luiz Martino, se demander " pourquoi le désordre du corpus théorique devrait-il être élevé au statut de modèle épistémologique? » (2003, p. 61), mais plutôt que de s'engager dans un débat inégal et complexe (les enjeux de la question en termes de légitimité universitaire dépassent clairement sa dimension strictement scientifique), on préférera prendre appui sur ces avertissements avec reconnaissance : la vertu des mises en garde est de prémunir aussi ceux qui les transgressent.

La prévision d'Escarpit a été particulièrement approfondie par Bernard Miège (1995), dont la démonstration est d'autant plus substantielle qu'elle est largement inductive. Pour juger « improbable et même inconcevable » toute approche globale des phénomènes communicationnels, il s'appuie en effet sur l'examen des 
tentatives successives de « coup de force théorique » (p. 86) dont ce domaine a fait l'objet, tentatives dans lesquels il relève des traits communs : le réductionnisme, l'abstraction, le primat accordé à un paradigme unique, la confusion des instances envisagées, la dérive futurologique, et enfin l'absence ou l'insuffisance des procédures de vérification empirique.

Épistémologiquement parlant, certains de ces critères de contrôle ne peuvent évidemment être que relatifs : pris dans l'absolu, plusieurs définiraient simplement ce qu'est une théorie (c'està-dire une réduction, une abstraction, etc.) et les condamneraient donc toutes, ce qui renouvellerait certainement l'histoire des sciences. Mais de fait, ils éclairent cruellement les limites des entreprises passées... et, peut-être, de toute tentative ultérieure. C'est pourquoi, plutôt que de les mettre en œuvre prématurément, on les conservera précieusement en vue d'évaluer ce parcours au terme de celui-ci.

Convenons en revanche dès à présent que, possible ou pas, une approche élargie des phénomènes communicationnels est épistémologiquement (et nerveusement) coûteuse. Si le premier chapitre témoigne d'une chose, c'est de ce que la double profusion oppressante des discours et des métadiscours tend à imposer le silence par tout ce qu'elle requiert de dire. Il constitue à ce titre une bonne illustration empirique de ce qu'il expose. Le trouve-t-on pléthorique ? Il est, au contraire, le fruit d'une renonciation obstinée, le résultat, en d'autres termes, d'une gestion féroce de l'attention de l'auteur, le conduisant (dans l'espoir de ménager l'attention du lecteur, qu'il imagine généreuse mais pas illimitée) à ne pas remarquer ou à taire.

Prenons trois exemples parmi cent. De «petits» exemples : les autres seraient trop longs. On pourrait notamment remarquer que la tendance, brièvement évoquée, à la division des tâches discursives paraît contredite en partie par le fait que, dans les entreprises d'actualité, des activités antérieurement distinctes (comme celles de photographe, de rédacteur et de secrétaire de rédaction, ou celles de cameraman, de journaliste, de preneur de son et d'éclairagiste) sont fusionnées au nom de la possibilité technique, mais aussi de la rentabilité, voire de la survie économique. Quant au caractère généralement cumulatif de l'attention (plus on en sait 
dans un domaine, plus on est enclin à en savoir), il peut notamment se heurter à un effet de lassitude, ou encore à une modification des déterminants extérieurs (l'attention portée à une matière universitaire chute spectaculairement après l'examen). Enfin, la remise en cause des hiérarchies discursives et du régime d'autorité qui les institue que l'on n'a - pour le moment - associée qu'à l'accroissement de l'offre d'information pourrait l'être en outre à la progression de l'idéal égalitaire depuis les Lumières et même avant, mais aussi... au raccourcissement des temporalités (également évoqué, mais seulement en rapport avec ce gonflement informationnel). En effet, comme le remarque notamment Eirick Prairat, la durée est elle-même une source majeure de l'autorité : «lorsque le présent et ce qui l'accompagne [...] s'imposent aussi fortement, la culture des pères cède le pas devant le culte des pairs » (2012, p. 177). L'explication de la décohésion des préférences discursives par la concurrence des nouveaux venus sur le marché discursif, pour fondée qu'elle soit, aurait donc gagné à être affinée. Elle le sera d'ailleurs au chapitre 7 , mais de toute façon moins qu'il ne le faudrait.

Importait-il de débattre de ces points? Non. Ni de ça ni de toutes les autres choses qu'un lecteur attentif au mauvais moment serait en droit de relever. Si une théorie élargie doit rendre compte homothétiquement de la complexité du réel et se protéger de toutes les embuscades qu'elle détecte, alors la preuve est faite qu'une telle théorie est effectivement impossible dans le cas qui nous occupe (et probablement dans les autres). Certains renoncements, évidemment, sont plus pénibles que d'autres, tant ils exposent à des objections légitimes, quoique de portée relative. On s'en est déjà expliqué. Il ne reste qu'à assumer à regret, sans pour autant s'absoudre ${ }^{3}$.

3 Relisons tout de même Escarpit: « Certains des exposés qui suivent seront jugés élémentaires, voire contestables par les spécialistes. D'autres seront trouvés difficiles par les non-initiés » et même, un peu plus loin : «Une théorie n'est jamais qu'une façon de voir les choses ». Là où s'exposait le mal se trouvait aussi le remède. 


\section{La pertinence des discours}

Or voici de quelle manière j'ai changé mon plan, pour tâcher d'attraper mieux le goût du public. J'ai divisé ma composition en deux parties [...] en un mot, assez de variété pour pouvoir croire que par un endroit ou par un autre chaque espèce de lecteur trouvera ce qui l'accommode.

Pierre Bayle (1647/1820, p. 2)

"Dourquoi les contenus qui nous plaisent nous plaisent-ils? » Le pro1 blème est simple à formuler, mais toute réponse semble condamnée à s'enchevêtrer dans l'écheveau des valeurs et contraintes sociales, raison pour laquelle les sondages en la matière sont si douteux. Les questions sur les goûts musicaux, par exemple, propulsent l'amour de la musique classique à des sommets dont aucun chef d'orchestre n'oserait rêver et les questions sur les préférences télévisuelles dotent les chaînes culturelles d'une séduction assez éloignée de leurs résultats d'audience. Les hiérarchies discursives sont peut-être affaiblies, mais elles sont loin d'être mortes. Comment, dans ce cas, comprendre que des chatons jouant du piano sur YouTube puissent si aisément surclasser en popularité les retransmissions de conférences littéraires ?

Pour avancer, rien n'interdit d'occulter provisoirement l'angle des contraintes et valeurs sociales comme on l'avait fait plus tôt pour celui des technologies. L'expérience est incongrue, un peu inconfortable, mais elle ne requiert pas forcément beaucoup d'abstraction. Illustrons-la par un exemple vécu. Invité lors du congrès national d'une fédération d'enseignants du secondaire à parler du « rôle » des médias dans la diffusion des connaissances, j’avais par curiosité demandé aux participants comment ils prépareraient leurs cours si chaque élève était libre d'y assister ou non, sans conséquence directe pour sa scolarité et sa carrière ultérieures. Regards effarés, silence éloquent : retirez aux 
discours scolaires leurs leviers institutionnels et les problèmes pédagogiques ressembleront tout à coup furieusement à des problèmes journalistiques. Faites plus généralement (mais non moins artificiellement) abstraction des contraintes et valeurs sociales, et ce qui restera sera la pure question de la valeur cognitive. Celle-là même qui peut conduire à préférer une émission pourtant perçue comme idiote à un excellent traité de philosophie ou de mathématiques.

Aborder le problème sous cet angle débouche directement sur l'une des plus vieilles et des plus élémentaires explications des préférences humaines, la théorie hédonique, c'est-à-dire l'idée selon laquelle nos comportements sont motivés par la recherche du bien-être et l'évitement de la souffrance. Il s'agit moins là d'un corps de doctrine homogène que de l'un de ces axiomes aux mille visages sur lesquels - à l'instar de la question de l'attention - se croisent sans forcément se regarder des auteurs de toute provenance. Depuis Démocrite, l'idée a été caressée ou embrassée par bon nombre de philosophes, en particulier David Hume :

Le ressort principal, ou principe activateur de l'esprit humain est la souffrance et le plaisir; et quand ces sensations sont retirées tant de nos pensées que de notre perception, nous sommes dans une grande mesure incapables de passion ou d'action, de désir ou de volition. Les effets les plus immédiats du plaisir et de la souffrance sont les mouvements d'attirance ou de répulsion de l'esprit [...] (1740/1888, p. 574)

Si ce « ressort principal » a été invoqué pour comprendre les comportements (motivation), il l'a aussi été pour les justifier (éthique), voire les prescrire (philosophie politique) :

La nature a placé l'humanité sous la gouvernance de deux maîtres souverains, la souffrance et le plaisir. Il n'appartient qu'à eux de montrer ce que nous devons faire, aussi bien que de déterminer ce que nous ferons. (Bentham, 1780/1879, p. 5 ; italiques dans l'original)

Appliquée à la question de l'attention, la perspective hédoniste peut revendiquer de lointaines racines évolutionnistes ${ }^{1}$. Après tout, le

1 Ainsi Davis et McLeod assènent-ils, de façon un peu radicale, que l'intérêt pour la presse à sensation «n'est pas socialement construit » mais repose 
filtrage attentionnel du champ visuel, dont on rappelait plus haut qu'il est surtout « intéressé » par le mouvement, s'explique facilement par le fait que le mouvement était essentiellement annonciateur de souffrance (si l'on était une proie) ou de satisfaction (si l'on était un prédateur). La même dualité, si elle demeure l'objet de vifs débats², a accompagné au $\mathrm{XIX}^{\mathrm{e}}$ et au début du $\mathrm{xx}^{\mathrm{e}}$ siècle tout le développement théorique et expérimental de la psychologie moderne (et, incidemment, celui de la psychanalyse3). Comme le résumait dans les années 1950 le futur président de l'Association canadienne de psychologie, dont les recherches sur l'attention faisaient autorité :

[...] la plupart des auteurs de cette période conçoivent l'intérêt comme quelque chose lié de près avec l'attention et la sensation plaisir-souffrance. [...] Pour Stout, l'intérêt n'est pas identique à l'attention, mais est la «tonalité hédonique de l'attention». [... Pour Arnold], il y a une conscience subjective des conséquences plaisantes ou déplaisantes qui peuvent découler de ce qui est ressenti sur le moment, mais il y a simultanément une détermination d'agir sur cette base pour concrétiser la récompense attendue ou éviter la souffrance attendue. Il peut être utile de remarquer à quel point ceci s'accorde bien avec les travaux des théoriciens contemporains. (Berlyne, 1949, p. 185)

«Ceci » allait tout aussi bien s'accorder avec l'essor ultérieur de la psychologie behavioriste. Bien que celle-ci ait rejeté toute explication du psychisme, qu'elle ne voulait connaître qu'à travers ses réactions aux stimuli négatifs ou positifs, elle s'accommodait à merveille de la polarisation récompense-punition, dont elle tirait ses outils de conditionnement ou de dressage.

D'où l'incrédulité que l'on peut éprouver devant la désinvolture avec laquelle Kahneman et ses collègues annonçaient en 1999 la découverte d'un " nouveau champ de la psychologie » :

sur « des catégories d'information qui accroissent l'adaptation évolutive » (2003, p. 208).

2 Sur ces débats, voir la convaincante « défense de l'hédonisme psychologique » proposée par Lemaire (2007).

3 On songe évidemment au "principe de plaisir » spéculé par Freud, qui plaçait lui aussi la recherche du plaisir et l'évitement de la souffrance au cœur de l'activité psychique. 
La psychologie hédonique - on pourrait l'appeler ainsi - est l'étude de ce qui rend les expériences et la vie plaisantes ou désagréables. Elle se consacre aux sentiments de plaisir et de souffrance, d'intérêt et d'ennui, de joie et de tristesse et de satisfaction et d'insatisfaction. Bien que l'adjectif « hédonique » soit souvent utilisé pour se référer seulement au plaisir, la psychologie hédonique couvre le spectre complet du plaisant au déplaisant [...] (p. ix)

Cependant, malgré la richesse de cet ouvrage, ses contributeurs étaient peu concernés par l'appréciation hédonique des discours et, en retour, la renseignaient assez peu.

C'est au fondateur des sciences de la communication en tant que discipline universitaire, Wilbur Schramm, que revient sans doute, dès les années 1950, la transposition de la dualité hédonique au problème de la valeur discursive. Son approche reste imprégnée du vocabulaire, alors hégémonique, de la psychologie behavioriste. Cherchant à préciser les termes de l'« équation de sélection », c'est-à-dire de l'évaluation qui conduit à préférer tel ou tel contenu médiatique (c'est là une autre formulation du Grand Mystère), Schramm avança que celle-ci correspondait simplement à la « récompense attendue » divisée par l'« effort requis »:

On peut accroître la valeur de cette équation soit en accroissant le dividende, soit en réduisant le diviseur, ce qui revient à dire qu'un individu aura plus tendance à sélectionner une certaine communication si elle lui promet plus de récompense ou requiert moins d'effort qu'une communication comparable. Vous pouvez constater comme ceci fonctionne en fonction de votre propre expérience. (1954, p. 19)

Effectivement... La « propre expérience » de beaucoup d'entre nous est, par exemple, qu'une information trouvable en un clic sur Google est beaucoup plus appréciable qu'une information peut-être plus solide, mais nécessitant d'aller à la bibliothèque.

Une version ultérieure de l'équation de sélection (1971) conduisit Schramm à développer le numérateur (la « récompense attendue » devenant « la force de la récompense perçue moins la force de punition perçue »), mais aussi à nuancer le caractère implicitement rationnel d'un tel calcul. Toutefois, soulignait-il, cette équation permettait d'expliquer bien des choses, telles que la concurrence entre la télévision et le 
cinéma, l'écoute des radios clandestines, malgré le brouillage des ondes hertziennes, ou encore - justement - l'usage des bibliothèques.

Cependant, l'hypothèse coût-bénéfice de Schramm n'a soulevé qu'un intérêt relativement modéré, ce qui pourrait s'expliquer par de nombreuses raisons. La première est qu'elle ressemblait beaucoup à un truisme. À l'instar de sa sœur et rivale, la « loi du moindre effort » (promise, elle, à un beau succès populaire, mais dont on a oublié la paternité scientifique $\left.{ }^{4}\right), l^{\prime}$ « équation de sélection » semble une explication trop simple et évidente pour justifier beaucoup d'attention, y compris de la part de son auteur qui s'y est peu attardé. Une autre raison est que les termes de l'équation sont assez généraux et donc difficiles à évaluer précisément. Une autre encore est que le fossé entre ses deux composantes n'a cessé de s'accroître au fil du temps : d'un côté, des chercheurs se sont focalisés, à la suite de Zipf (1949), sur le coût ou l'effort comme déterminant des choix informationnels. De l'autre, une large mouvance, le courant dit des « usages et gratifications", s'est notamment efforcée de dresser un catalogue assez composite de tout ce qui pouvait rendre l'information utile ou désirable (distraction, développement personnel, etc.) sans vraiment mettre en regard l'effort qu'elle pouvait réclamer.

C'est au bout du compte d'un champ de recherche totalement différent, celui de la pragmatique inférentielle, qu'a ressurgi la formulation la plus élégante et la plus prometteuse de l'hypothèse hédonique. Le problème central de ce champ était le traitement des ambiguïtés du langage naturel, exercice que les humains réussissent généralement très bien et les machines, assez mal. Les premiers percevront par exemple facilement que «pouvez-vous me passer le sel ? n'est pas une question, mais une requête. Pour l'expliquer, le philosophe Paul Grice (1975) supposa que les humains interprétaient l'information en fonction d'un « principe de coopération » tacite. Celui-ci impliquerait un ensemble de règles (de quantité, de qualité, de relation et de manière) dont la violation commanderait une réévaluation de l'énoncé. Ainsi, dans un dialogue comme «-Veux-tu sortir ? - Il pleut ! », le fait que la réponse n'ait, au premier degré, aucun rapport avec la question conduira à la réinterpréter de façon à ce qu'elle ait un sens : ce que dit le répondant est formellement incohérent, mais ce qu'il veut dire ne l'est pas.

4 Guillaume Ferrero (1894). "L'inertie mentale et la loi du moindre effort ", Revue Philosophique de la France et de l'Étranger, 37, p. 169-182. 
Percevant la portée de cette approche («avant Grice, l'importance de ce truisme a été ignorée ») un anthropologue et une linguiste, Dan Sperber et Deirdre Wilson, conclurent que toutes ces règles implicites pouvaient se ramener à une seule condition - la pertinence - et que celle-ci était la clef de l'interprétation du langage : dès lors qu'il relève d'une intention communicative, tout énoncé communique d'abord la présomption de sa propre pertinence (il s'engage à signifier) et c'est en fonction de cette présomption que l'on peut savoir à quel degré et de quelle façon le comprendre, y compris lorsqu'il contrevient ostensiblement à cet engagement ${ }^{5}$. Mais, pour Sperber et Wilson, si l'attente de pertinence régit l'interprétation du langage, c'est qu'elle constitue un principe cognitif encore plus fondamental :

\begin{abstract}
Nous soutenons que tous les êtres humains visent automatiquement à maximiser l'efficacité de leur traitement de l'information, qu'ils en soient conscients ou non; en fait, leurs intérêts conscients, divers et changeants, résultent de la poursuite permanente de ce but dans des conditions variables. Autrement dit, le but cognitif particulier que poursuit un individu à un moment donné est toujours un cas particulier d'un but plus général : maximiser la pertinence de l'information traitée. (1989, p. 80)
\end{abstract}

Encore fallait-il préciser ce que pourrait bien être cette pertinence. Après avoir considéré, puis rejeté, une approche classificatoire (c'est-à-dire un critère absolu déterminant la pertinence ou la non-pertinence), Sperber et Wilson ont démontré avec les méthodes de leur champ ${ }^{6}$ qu'une définition comparative était plus appropriée : toutes choses étant égales par ailleurs, plus un énoncé demande d'effort de traitement, moins il est pertinent et plus il produit d'effet contextuel, plus il est pertinent.

Voilà donc la pertinence définie comme le rapport entre l'effet

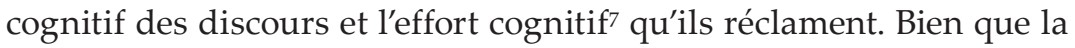

5 À une question comme «Ton examen s'est-il bien passé? », la réponse « Il fait beau aujourd'hui » signifie par son incohérence manifeste que l'on ne veut pas répondre et donc que l'examen s'est probablement mal passé.

6 Principalement l'examen comparatif de situations ou d'énoncés simulés (comme les dialogues présentés ci-dessus).

7 L'« effet contextuel » n'est pas exactement la même chose que l'effet cognitif, mais il est suffisamment similaire pour qu'on le considère comme tel, ainsi que ces auteurs le feront eux-mêmes plus tard. 
logique générale de cette relation coût-bénéfice ne soit pas exactement inédite, l'hypothèse ainsi formulée n'en possède pas moins de sérieux atouts par rapport à ses devancières. Il ne s'agit plus d'une conjecture surannée ou d'une remarque succincte en bas de page, mais d'une construction minutieuse, dont la validité et la fécondité sont largement reconnues au sein de son domaine scientifique, conduisant même à souligner que «la théorie la plus représentative de la pragmatique cognitive est la théorie de la pertinence de Speber et Wilson » (Moeschler et Reboul, 1994, p. 91). Une autre de ses vertus est la clarification terminologique et conceptuelle qu'elle apporte. Plutôt que de recourir à des sensations équivoques comme l'« intérêt », le "plaisir» ou la « souffrance », elle propose des désignations analytiques moins subjectives, plus opératoires, et clairement articulées entre elles. Pourrait-il donc s'agir de la réponse à la question de la valeur discursive?

Envisager la pertinence comme un ressort fondamental du marché discursif suppose d'étendre sa portée bien au-delà du problème des inférences conversationnelles qui a donné naissance à cette théorie et même du raisonnement qui la soutient. C'est cependant conforme à l'ambition de ses auteurs, qui l'envisagent explicitement (p. 13) dans la perspective d'« une théorie générale de la communication ». Ça l'est aussi à l'attention que lui ont portée dans le champ de la communication quelques auteurs comme Meunier et Peraya (2010), quoique leur examen judicieux de ce cadre théorique ne soit pas allé jusqu'à réévaluer et élargir ses fondements mêmes.

Si un tel élargissement est séduisant, c'est qu'une généralisation $\mathrm{du}$ concept de pertinence en tant qu'opposition dynamique entre effort cognitif et effet cognitif permet (au prix d'une certaine dilution) de lui attribuer tout l'héritage des suggestions antérieures qui vont à peu près dans le même sens. Par exemple - outre la plupart de celles mentionnées plus haut (Schramm, etc.) - la théorie pédagogique de John Dewey (1913) mettant en relation l'« intérêt » et l'« effort » que l'on peut obtenir des élèves ou encore, il y a presque 2000 ans, l'observation de Quintilien ${ }^{8}$ pour qui «plus le sujet traité est naturellement sévère, plus, pour le faire passer, il faut y répandre de charmes ». De même, l'aphorisme quasiment identique de Nietzsche "plus abstraite est la vérité que tu veux enseigner, plus tu dois en sa faveur séduire les sens » (1866/1990, p. 622), pourrait aisément se reformuler selon les termes de la

8 Institution oratoire (Trad. H. Bornecque, 1933, p. 71). 
pertinence: "plus la vérité que tu veux enseigner réclame d'effort cognitif, plus tu dois en sa faveur produire d'effet cognitif ».

Comme toutes les successions inattendues, il s'agit là d'un héritage sans filiation : loin d'être transmise et enrichie de génération en génération, la dialectique de l'effort et de l'effet est, comme on l'a vu, de ces choses qui s'égarent et se retrouvent sans cesse. Mais c'est justement cette étonnante convergence transcendant les siècles et les écoles de pensée (convergence d'autant plus étonnante que nul ne s'en est apparemment avisé) qui peut conduire à soupçonner que, truisme ou pas, le rapport effort-effet est bel et bien une articulation fondamentale de la communication et constitue à ce titre une clef de la valeur discursive. Cependant, cette curiosité épistémologique ne serait guère plus qu'un argument d'autorité (puisque tant de maîtres l'ont dit, ça doit être vrai) si l'héritage ne comprenait pas en outre une masse imposante de confirmations empiriques.

On ne s'attardera pas ici sur toutes les observations des comportements animaux et humains qui avaient conduit au succès de l'hypothèse hédoniste au $\mathrm{xIx}^{\mathrm{e}}$ siècle, ni sur la masse considérable des recherches, ponctuées de prix Nobel, qui ont analysé la rationalité des choix économiques ou politiques en fonction de l'effort requis pour s'informer'. Plus concrètement, d'innombrables travaux sur les pratiques documentaires des utilisateurs de bibliothèques (traditionnelles ou en ligne) ou des gestionnaires et ingénieurs au sein des organisations témoignent de stratégies de sélection où le rapport entre l'effort et la satisfaction est déterminant. Le même rapport, comme on s'en doute, joue un rôle encore plus capital dans la lecture de presse. Ainsi, le directeur d'un quotidien a-t-il pu se forger sa propre théorie en fonction des résultats de toutes les études de lectorat qu'il avait passé sa carrière à commander :

... la lecture obéit [...] à la loi universelle du moindre effort. Cette loi est telle qu'elle conduit le lecteur à refuser de payer son information de plus d'effort perceptif qu'elle ne lui promet de profit. Cependant, la lecture, étant la forme la plus élaborée de notre

9 Notamment les modèles d'«ignorance rationnelle » d'Antony Downs, de " rationalité à faible information » de Samuel Popkin, de "rationalité limitée » d'Herbert Simon et de «biais heuristiques » de Daniel Kahneman, sur lesquels on reviendra (brièvement) plus loin. 
quête perpétuelle d'information, est aussi probablement celle qui consomme la plus grande énergie neurocérébrale. Il n'est donc pas étonnant qu'elle nous inspire le souci le plus minutieux d'auto-économie et que, dans cette perspective, elle soit à tout instant précédée d'une comparaison prévisionnelle de son coût et de son profit. (Douël, 1981, p. 38)

Un autre domaine concerné par les pratiques et attentes du public est celui des musées, confronté de surcroît à un syndrome d'épuisement et de confusion identifié dès 1916, la «fatigue muséale » (museum fatigue). Les études sur cette sensation et, plus généralement sur les parcours des visiteurs d'exposition, ont conduit une équipe américaine à découvrir un principe auquel, dans la meilleure tradition, elle a attribué un nouveau nom (l'« Attention value model »). Celui-ci repose avec perspicacité sur l'hypothèse selon laquelle

le rapport entre les bénéfices et les coûts prédit les choix des visiteurs dans l'environnement muséal. Ainsi, accroître le nombre de mots sur un cartel d'exposition (accroître le coût) est censé réduire sa lecture par les visiteurs. La question demeure, cependant, de savoir si réduire les coûts est plus important que de satisfaire les intérêts (les bénéfices perçus). (Bitgood, Dukes et Abby, 2006, p. 5)

Différente encore était la perspective des chercheurs et consultants qui s'attaquèrent tout au long $\mathrm{du} \mathrm{xx}^{\mathrm{e}}$ siècle à l'évaluation quantitative de la difficulté des livres scolaires, et bientôt d'une foule d'autres textes. Le plus fameux d'entre eux, Rudolf Flesch, multiplia les expériences à cette fin, ce qui le conduisit non seulement à proposer une échelle de lisibilité (composante de l'effort), mais aussi à s'aviser qu'une telle quantification réclamait en regard un autre type de quantification, celle de l'« intérêt humain » (effet), qu'il s'efforça également de mesurer.

Contribuer à l'écrasant catalogue des constats sur le rôle de l'effort et de l'effet dans les préférences discursives est, au bout du compte, à la portée de chacun. Il suffit par exemple pour cela de relever sur Internet la liste des plus grands succès cinématographiques du $x x^{\mathrm{e}}$ siècle en nombre d'entrées ou en revenus, et d'estimer l'effort cognitif qu'ils réclament et l'effet cognitif qu'ils promettent (dont ces 
« effets » spéciaux qui portent si bien leur nom) par rapport à la liste des films primés dans les grands festivals, ou a fortiori par rapport à des œuvres plus confidentielles. On pourra aussi, comme le proposait Schramm, faire appel à sa "propre expérience». Bien que ce type d'introspection « dans un fauteuil » (armchair psychology) soit précisément ce contre quoi s'est construite la psychologie scientifique moderne, on sait que l'on peut consacrer un temps d'écoute stupéfiant à une émission télévisée triviale avant de s'aviser qu'elle n'avait pas d'intérêt. Si elle offrait si peu d'effet cognitif, pourquoi donc l'avoir préférée à un roman qui en promettait beaucoup plus ? C'est que, contrairement à ce dernier, cette quasi-hypnose ne réclamait qu'un effort cognitif minimal. Dès lors, elle n'avait pas besoin de beaucoup d'effet pour que sa valeur cognitive nette (pertinence) dépasse celle du livre. De fait, de nombreuses recherches utilisant les protocoles les plus divers - analyse des expressions et fixations oculaires, utilisation de stimuli perturbateurs, questionnaires et même électro-encéphalogrammes - ont démontré depuis longtemps ${ }^{10}$ que l'attention accordée aux programmes télévisés était remarquablement basse et parfois quasi-nulle : en réalité, TFı ne vend pas aux publicitaires l'attention de ses téléspectateurs, elle leur vend de la croyance (assez malavisée) en cette attention.

On ne conclura pas de la masse des observations évoquées jusqu'ici qu'elle "prouve» la validité de l'hypothèse de Sperber et Wilson et de leurs devanciers. Une preuve a peut-être un intérêt dans les palais de justice, mais c'est une notion plus que douteuse en sciences (et $a$ fortiori en sciences humaines et sociales). En revanche, on peut soutenir que cette hypothèse explique infiniment mieux l'ensemble de ces phénomènes que toute hypothèse concurrente, ce qui est le critère déterminant, mais qui impose au moins trois tests successifs.

1. Les phénomènes en question sont-ils réellement apparentés ou leur similitude ne provient-elle que d'un rapprochement plus ou moins artificiel? Ici ressurgit la question de la diversité et de l'homologie des situations communicationnelles évoquée en introduction. En l'occurrence, si l'on met à part les classes d'observations plus

10 On trouvera par exemple un bon bilan de ces travaux dans Chaffee et Schleuder, 1986. 
éloignées (les observations sur l'animal, les choix économiques et politiques...) qui n'avaient été évoquées que pour mémoire, toutes peuvent strictement se réduire au choix d'allouer son attention à un type ou un autre d'offre discursive et sont à ce titre comparables dans notre perspective, même si - comme on le soulignera un peu plus loin - les variables retenues par leurs auteurs sont aussi fluctuantes que les noms qu'ils leur attribuent.

2. En admettant que ces observations soient assimilables sous cet angle, en existe-t-il une explication générale plus économique et plus convaincante que l'opposition de l'effort et de l'effet? Le nier revient évidemment à courir le (faible) risque d'être démenti un jour ou l'autre, mais il semble, en tout cas, assez difficile d'en trouver une.

3. À l'inverse, ces constats empiriques en excluent-ils d'autres dont la pertinence ne puisse pas rendre compte, ou dont elle rende compte plus difficilement? Pas le moins du monde : comme on le verra plus loin, cet outil analytique est étonnamment puissant dans certains cas, mais l'est beaucoup moins dans d'autres. Pour ne pas entrer prématurément dans les détails, on se contentera à nouveau d'une analogie. De la fenêtre de mon bureau, on peut distinguer une barrière solide que les services de la voirie, quoique peu enclins à l'abstraction, ont érigée contre un axiome non moins solide (d'ailleurs un cousin de la pertinence) : les individus tendent à choisir le chemin le plus direct entre deux points, même si ce chemin traverse en diagonale un croisement à fort trafic. Sur mon fond d'écran, on peut voir la face nord du mont Blanc et trois de ses voies d'accès, dont la plus directe - la voie historique - est de loin la moins empruntée. Le témoignage du second paysage ne contredit pas pour autant l'axiome qu'illustrait si bien le premier. Plus généralement, le goût pour la haute montagne ne réfute en rien la règle bien établie selon laquelle les individus tendent à marcher le moins possible. Il rappelle simplement que les préférences humaines peuvent difficilement être expliquées par une seule variable : le fait qu'elle soit déterminante n'interdit pas que d'autres le soient aussi. Celles-là nous intéresseront plus tard. 
Reste le fait que les observations invoquées à l'appui de l'hypothèse de Sperber et Wilson varient dans ce qu'elles englobent en tant qu'effet ou qu'effort. Faudrait-il, par exemple, compter l'effort physique (aller à la bibliothèque...) ou l'effort financier (acheter un produit culturel) parmi les facteurs à l'œuvre? Le bon sens l'exigerait. Il a tort. Considérer comme tel tout ce qui s'apparente à un coût sous une forme ou sous une autre conduit non seulement à entretenir l'imbroglio qui obscurcit depuis si longtemps cette question, mais aussi, ce qui est pire, à la dissoudre dans une tautologie rédhibitoire : dire que la valeur discursive est égale à tout ce qui donne de la valeur moins tout ce qui en enlève revient simplement à dire que la valeur est égale à la valeur, donc à ne rien dire du tout.

D'autre part, les relations de causalité en jeu ne sont pas de même nature. Comme on le remarquait plus haut, la gratuité et l'accessibilité ne suffisent pas à provoquer un fort engouement pour l'actualité générale. Une caractéristique du marché (économique) des produits culturels est justement le fait que la concurrence n'y est pas directement liée au prix de vente : un studio de cinéma, par exemple, n'essaye pas d'inonder le marché en vendant ses films moins cher que ses rivaux. Les grands éditeurs s'affrontent pour les prix littéraires, pas sur les prix de vente. Bien sûr, des facteurs plus complexes d'uniformisation sont aussi à l'œuvre, mais on n'irait pas voir un long métrage rébarbatif à la place d'un autre plus séduisant en considérant seulement que le prix d'entrée du premier est plus bas. Les journées d'appel à coût réduit ou nul des musées et cinémas dynamisent certes leur fréquentation, mais, à l'instar des fournisseurs comme Netflix, elles ne modifient pas la concurrence interne entre les contenus proposés par ces instances. De même, l'accessibilité en ligne des grands classiques de la littérature entrés dans le domaine public leur offre certainement un nouveau lectorat, mais pas au point d'affecter l'attractivité des nouvelles parutions, qu'il faut pourtant commander ou aller retirer : celles-ci parviennent donc - dans une certaine mesure, certes - à défier cette " culture de la gratuité » que déplorent les producteurs discursifs.

Les choix de consommation culturels deviennent plus lisibles lorsque l'on considère les facteurs extradiscursifs tels que le prix et l'accessibilité comme des variables interagissant avec la pertinence discursive plutôt que comme des composantes de celle-ci. Ainsi, la queue à l'entrée d'un cinéma peut-elle dissuader d'aller voir le film 
prévu, mais elle ne changera rien à la pertinence qu'on lui prêtait : elle conduira seulement à se contenter d'un film supposément moins pertinent. Bref, en première analyse (on raffinera plus tard), c'est bien la pertinence qui peut conduire à allouer du temps, de l'argent et du mouvement à la consommation d'un produit culturel. On notera que, si la première variable reste révocable tant que l'on peut abandonner cette consommation, les deux dernières constituent un pari pour lequel il est difficile de récupérer sa mise (les spectateurs qui hurlent «remboursez! » ne s'attendent pas à être pris au mot).

La portée analytique de l'axiome reformulé par Sperber et Wilson réside largement dans le fait que, contrairement à la fraction de sélection de Schramm, il permet de caractériser des facteurs délimités et, donc, comme on le proposait plus haut, de se rapprocher de la pure question de la valeur cognitive en la distinguant d'autres déterminants. Mais cet axiome trouve à son tour ses limites dès que l'on cherche à préciser les notions d'« effort cognitif» et d'« effet cognitif». Dans la perspective inférentielle des auteurs, ces notions pouvaient sans trop d'inconvénients être réduites à des évocations assez approximatives :

Le traitement de l'information par les êtres humains leur demande un certain effort mental, et produit en eux un certain effet cognitif. L'effort demandé est un effort d'attention, de mémoire et de raisonnement. L'effet produit consiste en une modification des croyances de l'individu : l'addition de nouvelles croyances, l'élimination de croyances antérieures, ou simplement un affaiblissement ou en renforcement de croyances antérieures. (1992, p. 228)

Cependant, de telles définitions sont à la fois trop vagues et trop fragiles pour que l'on chemine avec elles vers les sources de la valeur discursive. Il avait par exemple été suggéré plus haut que la télévision (ou d'ailleurs, la vidéo en ligne) réclamait moins d'effort cognitif que le texte d'un roman (ou d'un journal), mais cette suggestion restera une affirmation gratuite tant que l'on n'aura pas explicité ce qui permettrait de soutenir et d'expliquer cette différence. Invoquer pour cela l'«attention» serait tautologique et se référer vaguement à la « mémoire » et au «raisonnement » ne conduirait pas bien loin. 
Quant à l'idée selon laquelle l'effet produit se résume à «une modification des croyances", elle n'est pas seulement insatisfaisante en théorie, elle est aussi - et plutôt brutalement - contredite par les réalités prosaïques du marché discursif dès que l'on s'éloigne du cadre restreint des ambiguïtés conversationnelles. Au tournant du millénaire, alors que les autorités publiques s'enthousiasmaient encore pour la société $d u$ savoir à laquelle mèneraient les autoroutes de l'information, les études sur les traces $(\log s)$ des moteurs de recherche indiquaient pour leur part que ces autoroutes menaient surtout vers les mots clefs «sex", «nude » et «free » (Spink, Wolfram et al., 2001). Le dernier terme porte sur l'un des facteurs extradiscursifs que l'on vient d'évoquer, mais les deux premiers sont plus embarrassants : quoi qu'ils puissent sans doute amener occasionnellement à découvrir la possibilité d'une position exotique, il est difficile de croire qu'ils soient avant tout motivés par la soif de connaissance ou le désir d'une « modification des croyances ». À moins, bien sûr, de distendre le sens de ces termes, ce qui conduirait à une nouvelle tautologie : l'effet produit réside dans le fait de percevoir quelque chose.

Bref, à la question "Pourquoi les messages qui nous plaisent nous plaisent-ils?", le jeu de l'effet cognitif et de l'effort cognitif paraît constituer une sérieuse piste de réponse, mais elle réclame à l'évidence un examen plus approfondi de ces facteurs. On ne fait, du reste, que suivre en cela la logique qui avait conduit Sperber et Wilson à prolonger la théorie de Grice en remarquant que dans cette dernière "plusieurs des concepts essentiels [...] ne sont pas définis " et en particulier que celui de pertinence « revient à faire appel à notre intuition en de nouveaux termes » (1989, p. 61). 


\section{(Précisions)}

T 'influence de l'effort et de l'effet sur les préférences discursives Lemble attestée par une telle redondance d'observations, et explique si facilement celles-ci, qu'il est difficile de penser qu'à l'instar de la lettre volée d'Edgar Poe, la possible universalité de ce qui paraît être un rouage communicationnel fondamental ait pu rester masquée par sa propre évidence ou son ubiquité («Arrêtons d'opposer plaisir et effort à l'école», s'exclamait par exemple un ministre français de $l^{\prime}$ Éducation ${ }^{1}$ ).

En première hypothèse, on peut n'y voir qu'une marque de plus de l'éclatement des communautés discursives : dans un contexte d'âpre concurrence attentionnelle, la spécialisation des intérêts frappe sévèrement les disciplines des sciences humaines et sociales, où la colonisation de « nouveaux » territoires conceptuels piétine volontiers les traces d'occupations antérieures. Cette superposition de discours théoriques ou empiriques éminemment respectables, et dont chacun peut se targuer d'un type de validité interne, aboutit donc nécessairement à un fatras global. Il se manifeste dans l'intrication des notions (comme l'attention, l'intérêt ou la pertinence), mais aussi dans la connexité de traditions siamoises, comme celle qui considère le bénéfice comme une constante et ne s'intéresse qu'au coût (la mouvance du moindre effort) et celle qui s'intéresse à l'interaction optimale de ces deux variables (appelons-la la mouvance arbitragiste). Le fait que, contre toute évidence, la première ne se soit pas depuis longtemps diluée au sein de la seconde en dit long sur l'état de la question.

1 Vincent Peillon : "Arrêtons d'opposer plaisir et effort à l'école ». Propos recueillis par Maryline Baumard et Mattea Battaglia, Le Monde, 8 février 2014, p. 10. 
Encore se situe-t-on ici à l'intérieur du périmètre dans lequel on a un peu arbitrairement circonscrit la conception hédoniste de la communication. Dès que l'on s'approche de sa périphérie, la concurrence attentionnelle à l'œuvre se manifeste dans toute sa brutalité. Ainsi, Sperber et Wilson peuvent-ils traiter de « communication et cognition » (sous-titre de leur ouvrage) en se souciant assez peu de la seconde, et encore moins de la première. Tout dépend évidemment de ce qu'on appelle communication, simple processus langagier ou interaction sociale multiscalaire. Cette ambivalence conduit ces auteurs à une remarque plutôt inattendue : "tout le monde semble considérer qu'une théorie générale de la communication est possible et nécessaire » (p. 13), aussitôt dépassée par une assertion encore plus renversante : "D'Aristote aux sémioticiens modernes, toutes les théories de la communication ont été fondées sur un seul et même modèle, que nous appellerons le modèle du code ${ }^{2} »$. Toutes... sauf la quasi-totalité des sciences de la communication modernes, dont le rejet du modèle du code est souvent considéré comme un principe fondateur et est en tout cas l'un des principaux dénominateurs communs depuis près d'un demi-siècle.

Si la question n'a guère d'importance dans certains domaines - la même affirmation se trouve presque mot pour mot dans l'ouvrage d'un neurobiologiste célèbre (Changeux, 2008, p. 243) - on conçoit qu'elle en ait pour le champ de savoirs ainsi ignoré : autant que n'importe quelle considération de méthode ou d'objet, l'attention accordée ou refusée est l'une des principales forces structurant les sciences de l'homme et de la société, où elle façonne l'inclusion et l'exclusion disciplinaire (et récursivement se conforme à elles). Il est donc logique que toute théorisation communicationnelle dont il serait "facile de montrer qu'elle repose sur l'ignorance de pans entiers des connaissances produites antérieurement» (Miège, 1995, p. 85) reçoive un accueil mitigé au sein des pans en question.

2 On ne débattra pas ici de la possibilité de réduire ainsi la pensée communicationnelle d'Aristote (notamment la question de la réception qui sous-tend toute la Rhétorique) à une théorie du code : contentons-nous d'exprimer de sérieuses réserves sur ce point, en concédant cependant que, comme le remarquait Michel Meyer (1993), la tripartition ethos-logos-pathos peut sous un certain angle, s'y apparenter. 
Pour autant, l'inattention obstinée qui frappe plus généralement le jeu de l'effort et de l'effet cognitif dans les approches réellement communicationnelles (à la notable exception de Schramm) pourrait n'être pas seulement due aux nécessités pratiques de délimitation du soi et du non-soi universitaire, ni même à la surabondance des discours savants en général. Même si l'on ne peut pas s'intéresser à tout, il n'est pas si facile de se désintéresser d'une relation si plausible et visible sans au moins l'avoir réfutée sérieusement ${ }^{3}$, d'autant que, comme on le verra, le coût épistémologique (et sociétal) d'une telle zone obscure est plutôt élevé.

On doit donc risquer une possible explication complémentaire : la dialectique effort-effet est réputée inexistante - en fait, elle n'est même pas réputée quoi que ce soit - parce qu'elle est détestablement naturaliste. D'où le vif intérêt qu'elle a suscité auprès des psychologues et des économistes. Mais dans un champ traditionnellement obnubilé par la question de l'opinion, que ce soit pour la manipuler (depuis la rhétorique antique jusqu'à l'ingénierie discursive politique et commerciale) ou, plus souvent, pour en dénoncer la manipulation, un axiome aussi désinvoltement asocial, aussi indifférent aux convictions individuelles et collectives, est inconcevable ou en tout cas indicible. Le modèle du code - qui du moins proposait des instances différenciées - avait eu droit à un procès en bonne forme et à une exécution publique. Pour l'axiome hédonique, une disparition pouvait suffire.

Si telles sont effectivement les raisons de cette inattention scientifique, alors ce sont a priori de saines motivations. Dont on montrera cependant qu'elles ont conduit à une très mauvaise solution.

3 Bien sûr, la trivialité de l'hypothèse hédonique sous sa forme élémentaire ne saurait être réellement en cause, d'abord parce que la trivialité ne se présume pas (elle se démontre), ensuite parce que bien des remarques triviales de prime abord (par exemple l'idée que l'on peut «agir» avec des mots, ou que la perception collective du réel est socialement construite) ont fait preuve d'une remarquable fécondité scientifique. 
Page blanche conservée intentionnellement 


\section{L'appel des choses simples}

En quoi vous serai-je utile si ma parole ne vous apporte ni révélation ni connaissance [...] Vous de même ; si votre langue n'exprime pas des paroles intelligibles, comment comprendra-t-on ce que vous dites? Vous parlerez en l'air.

Première épître aux Corinthiens (14:6-9)

R ien avant d'être un problème scientifique, la valeur discursive

Était la pierre philosophale de tous ceux qui, depuis l'Antiquité, se sont efforcés de trouver le secret de l'efficacité argumentative ou de la réussite littéraire. Beaucoup d'entre eux n'ont pas manqué d'identifier la captation de l'attention comme la condition première de ce succès et, dès lors, d'envisager les ressorts cognitifs de celle-ci. Ainsi, l'auteur de la Rhétorique à Hérennius :

L'exorde est le début du discours ; il dispose l'esprit de l'auditeur à l'attention. [...] Nous pourrons captiver son intérêt si nous savons exposer rapidement le fond de la cause, et fixer son attention ; car c'est nous témoigner de l'intérêt que de consentir à nous écouter. Nous commanderons l'attention en promettant de parler de choses importantes, nouvelles, extraordinaires, ou de faits qui regardent l'État ${ }^{1}[\ldots]$

On voit que l'appétit pour la concision (moins d'effort) et le sensationnel (plus d'effet) que l'on attribue souvent à la communication moderne n'est pas si récent. Le problème, évidemment, est que les préceptes de ce genre, s'ils peuvent être appropriés dans certains cas, peuvent tout autant ne pas l'être dans d'autres : la concision est

1 Rhétorique, à C. Hérennius (trad. Désiré Nisard, 1875, p. 4-5). 
rarement le principal critère de choix d'un magazine. Quant à l'appétit supposé pour les «faits qui regardent l'État »... En 2010, le président Obama a dû reporter la date prévue pour son discours annuel sur l'état de l'Union, ses conseillers ayant judicieusement estimé qu'il ne pourrait concurrencer le dernier épisode de la série télévisée Lost programmé au même moment.

À l'inverse, on serait bien en peine de déterminer lequel des facteurs évoqués peut s'appliquer aux animaux amusants d'Internet (auxquels on ne se référera jamais trop). Il est vrai que, dans l'optique délibérative de la rhétorique gréco-romaine, un petit chat constituait un argument délicat à faire valoir devant une assemblée, mais il n'en reste pas moins, anachronismes mis à part, que toute énumération de recettes débouche fatalement sur des écueils de ce type dès lors qu'elle est dépourvue de justification d'ensemble.

C'est la raison pour laquelle Platon avait, quatre siècles auparavant, condamné les traités de rhétorique. En fait, la raison était surtout la concurrence entre rhéteurs et philosophes sur le marché des idées et - déjà - la primauté que ces derniers entendaient conférer aux considérations morales dans ce domaine. Cependant, si Socrate ne manquait pas d'interpeller ses adversaires sur leur sens du bien et du mal, ses coups les plus douloureux étaient bien ceux qui visaient les fondements mêmes de leurs propos.

\begin{abstract}
...il est au contraire évident que pour enseigner à discourir avec art, il faut savoir exactement indiquer l'essence de la chose à laquelle se rapporte l'art de la parole; cette essence, c'est l'âme. [...] Il est donc évident que Thrasymaque, ou tout autre qui voudrait enseigner avec soin l'art de la rhétorique devra d'abord, avec toute l'exactitude possible, décrire l'âme [...] Il décrira en second lieu, comment et sur quoi elle agit, comment et par quoi elle peut être affectée ${ }^{2}$.
\end{abstract}

Revoici le Grand Mystère ("qu'est-ce qui plaît? »). Comme souvent, il est soudé à son double maléfique («qu'est-ce qui persuade?») - lequel ne nous intéresse pas directement ici -, mais du moins se trouve-t-il disjoint des considérations morales au profit d'une exigence scientifique claire : sans théorie cognitive de la réception (la «psychagogie »

2 Platon, Phèdre ou De la beauté des âmes (trad. Mario Meunier, 1922, p. 270-271). 
que Socrate appelait de ses vœux), la prétention à l'expertise dans ce domaine ne masque pour lui qu'«une activité sans rien de technique, mais qui relève d'un tempérament intuitif, intrépide et très doué pour les relations ${ }^{3}$. »

Face à l'échec ultérieur de la rhétorique, réduite au fil du temps à un interminable catalogue de figures de style, le défi sera réitéré par Pascal, appelant à " un art de dire les choses de telle façon que ceux à qui l'on parle puissent les entendre sans peine et avec plaisir " (on y reconnaîtra, soulignée par nous, une autre formulation frappante du jeu de l'effort et de l'effet...), mais soulignant qu'un tel art « suppose qu'on aura bien étudié le cœur de l'homme pour en savoir tous les ressorts ». (1670/1954, p. 1094)

Malheureusement pour les producteurs discursifs (et, comme on le verra, pour leurs destinataires, voire pour la chose publique en général), lesdits ressorts semblaient, à la fin du $x^{e}$ siècle, presque aussi obscurs que dans la Grèce antique ou au temps de Pascal ${ }^{4}$.

Ce n'est pas le genre de choses qu'il convient d'affirmer à la légère, surtout si l'on considère le nombre étonnant de cours de «techniques de communication» qui se donnent dans toutes les branches de l'enseignement supérieur (on recrute beaucoup plus d'enseignants-chercheurs à ce titre que pour des domaines comme l'astronomie-astrophysique).

Afin d'en avoir le cœur net, j'avais entrepris dans les années 2000 de dresser un inventaire méthodique de l'ensemble des connaissances et préceptes techniques que pouvaient receler cinquante manuels de rédaction professionnelle francophones ou anglophones consacrés à trois domaines : le journalisme, la rédaction scientifique, technique et médicale, ainsi que la communication écrite en général (Labasse, 2006). Il semblait en effet raisonnable, compte tenu de la

3 Platon, Gorgias (trad. Jacques Cazeaux, 1996, p. 43-44).

4 On notera que, comme Bourdieu, Pascal a esquissé sa propre réponse au problème qu'il soulevait, en l'occurrence celle d'une approche individualisée (ce qu'on nommera à l'époque moderne l'armchair psychology) : faute de pouvoir connaître l'esprit et les goûts de tous les hommes, du moins peut-on essayer de comprendre ceux de celui à qui l'on s'adresse. La distinction qu'opérait Pascal entre les voies du cœur et celles de l'esprit devrait aussi être précisée, mais ce n'est pas le lieu, pas plus que ce n'est celui de revenir sur l'origine de la pensée citée (discutée par Brunschvicg). 
taille d'un tel corpus, de penser qu'aucun fondement significatif de $l^{\prime}$ « art de dire les choses » n'aurait pu échapper à tant d'auteurs, majoritairement enseignants ou formateurs. Le dépouillement systématique de ces dix mille pages a d'abord permis de constater un consensus écrasant sur un point central : l'essence de la communication écrite - par opposition à l'expression écrite des apprentissages scolaires tient à ce que l'on pourrait appeler son lectocentrisme ${ }^{5}$ : la valeur des discours ne réside pas dans leur style mais dans leur adéquation à leurs destinataires, donc dans leur réception. Les rhéteurs et leur adversaire ne disaient rien d'autre et Pascal encore moins.

Le problème commence évidemment lorsque l'on doit passer de l'adage fondamental "écrivez pour votre lecteur", réitéré livre après livre, à son application pratique. Pour ne pas reproduire les fastidieux détails de cette analyse, déjà publiés et republiés ${ }^{6}$ (mais dont on devra reprendre quelques éléments plus loin), contentons-nous de dire que l'étude a confirmé sans ambiguïté qu'aucun de ces manuels n'échappait à la critique socratique assénée par Platon vingt-quatre siècles plus tôt. Bien qu'il soit un peu injuste de résumer de la même façon abrupte des ouvrages mûrement réfléchis et d'autres étonnamment farfelus, il ne l'est pas de remarquer par exemple que, judicieux ou non, les quatre préceptes essentiels sur lesquels ils s'accordent - dire l'important d'abord / faire des phrases courtes / utiliser des mots courts / simplifier les idées - se passent généralement de démonstration élaborée et sont invariablement dépourvus de socle théorique plausible. Fort éloignés de la "psychagogie » de Socrate, les secrets de la valeur discursive évoquent donc bel et bien ce qu'il assimilait à une «cuisine» :

[...] elle ne peut rendre raison ni du destinataire ni du contenu de ses ordonnances selon leur nature effective, et la cause ne peut pas, élément après élément, figurer dans leur énoncé. Or, moi, je ne donne pas le nom de technique à quelque chose d'irraisonné. (p. 48)

5 Les anglophones parlent pour leur part d'une logique «reader-centered». On pourrait préférer destinocentrisme pour tenir compte du fait que les discours peuvent aussi avoir des auditeurs ou des spectateurs, mais il n'est pas certain que ce détail exige de recourir à un terme aussi biscornu.

6 From linguistics to communication's didactics: The case of lexicology (Labasse, 2001), L'écrit professionnel : ambiguittés et identités d'un objet académique indistinct (Labasse, 2009), etc. 
Mais ces manuels sont-ils vraiment «irraisonné[s] » ? Si les recettes de cette cuisine fonctionnent, si, en d'autres termes, l'alchimie apporte effectivement la pierre philosophale, alors la rationalité instrumentale des guides d'optimisation de la valeur discursive ne peut être mise en doute. Or, à l'instar de la Rhétorique à Hérennius, les dogmes de l'efficacité communicationnelle fonctionnent bel et bien... sauf quand ils ne fonctionnent pas du tout. Et pour en comprendre la raison, il faut plus que du savoir-faire pratique ou de l'intuition : il faut affronter le vieux défi.

On remarquera d'abord que le "concentré de manuels", les quatre préceptes hégémoniques auxquels, distillation après distillation, on peut réduire les règles de la communication efficace, est étonnamment homogène. Bien qu'ils portent respectivement sur l'introduction, sur les mots, sur les phrases et sur les idées, tous les quatre s'avèrent être - en parfait accord avec la théorie de la pertinence - des moyens concourant à la même fin : minimiser l'effort de traitement réclamé au destinataire. Ils se rapprochent notamment sur ce point des nombreux consultants politiques, publicitaires ou éditoriaux dont la passion pour la brièveté et de la simplicité a été résumée dans l'acronyme Kiss (Keep It Simple and Stupid).

Résistons à la tentation de se demander «est-ce donc là ce qu'on aime vraiment? " (l'imbroglio des motivations n'attend que ça pour submerger la question et l'étouffer à nouveau). Partons plutôt de l'hypothèse que l'expertise pratique de la communication développée depuis 2500 ans n'est pas à négliger. L'argument, bien sûr, est plutôt court en soi (il n'a jamais permis à un acupuncteur de m’approcher avec une épingle), mais il est plus que conforté par la multitude des recherches démontrant la corrélation entre l'accessibilité des discours et leur efficacité, non seulement auprès du grand public, mais aussi de spécialistes supposés rodés à la lecture de textes difficiles, comme les analystes financiers, les juristes ou même les universitaires. Dans ce domaine, par exemple, les chances de recevoir des crédits de recherche, d'obtenir des citations (donc de l'attention) ou encore d'être publié dans des revues savantes paraissent dépendre significativement de l'effort cognitif réclamé par le texte. Ainsi, les étudiants que des maîtres à la triste figure auraient persuadés de la magie du jargon risqueraient-ils d'être déçus : une expérience a par exemple montré que si plus de $85 \%$ d'entre eux (en l'occurrence un échantillon de 110 étudiants de Stanford) admettaient «changer les mots dans un 
travail universitaire pour le faire paraître plus solide ou intelligent en utilisant un langage compliqué ", cette stratégie appliquée à des demandes d'admission en second cycle obtenait l'inverse de l'effet escompté (Oppenheimer, 2006). Plus curieusement, l'effet de la difficulté a aussi été observé chez les biologistes et même les physiciens, qui semblent ne pas aimer tant que ça les textes farcis de formules mathématiques : la densité d'équations des publications serait négativement corrélée à leurs chances d'être citées (Fawcett et Higginson, 2012).

Bien entendu, ce genre de conclusion pourrait, comme toujours en pareille matière, être contredite par d'autres travaux, certes bien moins nombreux mais cependant intrigants. Des spécialistes de la psychologie expérimentale s'en alarment même : "Comment la clarté peut-elle être si séduisante dans certaines recherches et ne pas l'être dans d'autres ? "(Galak et Nelson, 2011, p. 250). Leur hypothèse est que ces divergences résultent de la variété des façons de concevoir et de mesurer la facilité du traitement cognitif. On a trop souvent traversé des chaos terminologiques du même genre au fil des pages qui précèdent (et on en croisera d'autres) pour ne pas appuyer cette supposition avec enthousiasme. Mais on doit lui adjoindre une autre hypothèse. Le problème que soulèvent les recherches en question n'est pas différent de celui que rencontraient ces préceptes rhétoriques ou communicationnels qui fonctionnent dans bien des cas et pas du tout dans d'autres : en l'absence d'un schéma d'ensemble permettant de percevoir les interactions des différentes variables à l'œuvre, chaque facteur pourra isolément paraître agir de façon imprévisible.

Dans les deux hypothèses, il est essentiel de se demander comment différencier les facteurs d'effort cognitif (non sans vérifier au passage s'ils confirment ou non les quatre piliers de l'efficacité communicationnelle des manuels). D'où la nécessité, qu'on le veuille ou non, de se plonger brièvement dans les rouages un peu rébarbatifs des processus psycholinguistiques?

L'idée même que l'effort de traitement requis pour comprendre un discours découle de plusieurs processus distincts, et non d'un processus unique, est aussi établie scientifiquement qu'elle est facile à

7 On parle évidemment ici de la psycholinguistique expérimentale contemporaine et non de la théorie de la grammaire générative que Chomsky avait avancée sous le même nom. 
percevoir intuitivement : quiconque est sur le point de s'endormir ou préoccupé par un problème personnel peut lire un récit extrêmement simple avant de s'aviser qu'il n'a rien retiré de la page dont il vient de pourtant parcourir tous les mots. Si l'on peut ainsi lire sans lire, c'est que l'acte de lecture (ou d'audition, etc.) ne se réduit pas à une activité indivisible, mais résulte de plusieurs processus dont certains peuvent échouer quand d'autres se poursuivent.

La façon la plus simple de les considérer est de partir de l'une des distinctions les plus élémentaires de la psychologie du langage, la gradation entre les processus cognitifs de bas niveau, ou «ascendants » (bottom-up), guidés par les données perçues, et les processus de haut niveau, ou «descendants » (top-down), guidés par les connaissances préalables. Cette répartition classique des niveaux de traitement est, à dire vrai, un peu schématique : l'esprit humain n'est pas une usine d'assemblage où chaque atelier transmettrait sa production au suivant ${ }^{8}$. Elle permet cependant de distinguer quatre " groupes de tâches " principaux associés à l'effort cognitif.

$\mathrm{Au}$ plus «bas niveau » se trouve l'acquisition visuelle ou auditive des formes signifiantes. Quoiqu'il s'agisse d'un processus réflexe, quiconque penserait qu'il ne réclame pas d'effort n'a jamais eu à chausser des lunettes, renforcer l'éclairage ou tendre l'oreille. Et quoiqu'il soit le plus humble dans la hiérarchie des choses de l'esprit, il n'en conditionne pas moins toutes les autres. Indépendamment de leur contenu, la valeur des discours est d'abord déterminée par la possibilité sensorielle d'en prendre connaissance. De même qu'à la radio une retransmission séduisante, mais noyée sous les parasites à la limite de portée des émetteurs FM sera vite abandonnée au profit d'un contenu plus audible, un texte à la typographie défaillante (caractères trop petits, flous, peu contrastants, manuscrits ou recourant à une police excentrique) abaisse et souvent annihile la valeur de son contenu. Ce facteur premier, la « légibilité », est si évident qu'il a suscité de nombreuses recherches au cours du dernier demi-siècle - notamment, celles, désormais classiques, de Tinker (1963). Il continue, sous sa forme la plus triviale (« faut-il choisir des caractères avec ou sans empattement?»), à fasciner la sphère des concepteurs de sites

8 Comme en tout ce qui concerne l'être humain - seul ou en société - les nécessités analytiques exigent de séparer des phénomènes étroitement mêlés, en essayant seulement de les mutiler le moins possible. 
Internet. Hélas pour eux, ce que l'on sait de la question n'a rien pour favoriser la créativité graphique : en termes de contraste, de typographie, etc., les normes les plus classiques, celles de l'imprimerie, sont, à quelques aspects près', celles qui obtiennent les meilleurs résultats (quant à la querelle des empattements - accroissent-ils ou non la légibilité ? - elle n'a pu être tranchée de façon convaincante, ce qui suggère que cette question n'est pas aussi déterminante que ça).

La perception des indices graphiques ou sonores ouvre la voie à l'accès lexical (mettre en relation la forme identifiée avec un mot connu) et au traitement syntaxique (lui attribuer un statut dans la phrase). Sans entrer dans les questions théoriques sous-jacentes, que l'on trouvera dans tout bon manuel de premier cycle, on notera que la difficulté de traitement des mots est clairement corrélée à leur longueur. Ainsi, une expérience, représentative de plusieurs autres, a-t-elle confirmé que le temps de lecture des mots de cinq syllabes était en moyenne $50 \%$ plus long que celui des mots de deux syllabes et leur taux de mémorisation était $30 \%$ plus faible (Baddeley, 1992). Ceci pourrait s'expliquer, outre les limitations propres au champ visuel $^{10}$, par le fait que, comme l'a montré Zipf (le théoricien du «moindre effort» rencontré plus haut), les mots longs sont statistiquement moins fréquents que les mots courts, ce qu'il attribuait à une tendance naturelle à raccourcir des mots les plus utilisés (Cinématographe/Cinéma/Ciné). Or, d'autres recherches ont établi que la facilité d'activation d'un mot dans le lexique mental, à supposer que ce mot soit connu, était inversement proportionnelle à la fréquence de

9 Si le noir sur fond blanc offre une légibilité presque optimale, il est cependant surpassé par le noir sur fond jaune (il ne reste aux esthètes qu'à espérer que les graphistes ne l'apprendront pas). Par ailleurs, la séparation explicite des paragraphes est un facteur essentiel de la facilité de lecture (elle favorise la gestion du buffer mnésique) : le monde de l'imprimerie la manifeste par un alinéa rentrant, ce qui est un peu mieux que rien, mais les lignes vides qu'emploient notamment les sites web sont beaucoup plus efficaces.

1o L'empan fovéal, en l'occurrence le nombre de lettres qu'il est possible de percevoir clairement en une fixation oculaire se limite à 6 à 8 caractères environ, auxquels s'ajoutent d'autres signes perçus de façon vague (empan parafovéal). On se souvient que l'essentiel du champ visuel ne $\mathrm{s}^{\prime}$ « intéresse » qu'à des stimuli très frustes, en particulier le mouvement : sa partie analytique centrale est extrêmement réduite. 
son usage. Évidemment, il s'agit toujours là de probabilités - l'ache, plante ombellifère, est moins connue que l'acharnement ou l'acheminement -, mais en moyenne statistique, la corrélation est solide et même étonnamment régulière ${ }^{11}$ (Labasse et Thoiron, 2002).

La question de l'effort de traitement syntaxique est plus compliquée. Outre que la définition même de ce qu'est une " phrase » (ou un « mot») est si problématique que les spécialistes ont la prudence d'éviter l'un et l'autre terme, les difficultés cognitives liées à l'interprétation de ces assemblages grammaticaux dépendent de multiples facteurs, dont leur longueur mais aussi leur complexité (tournures passives, doubles négatives, nombre de subordonnées et d'incidentes, distance sujet-prédicat, structuration...) Cependant, la longueur des phrases constitue un indice approximatif de la plupart de ces problèmes : outre que le nombre de mots à gérer représente en soi une charge mentale importante, il est généralement lié à la complexité de la phrase.

On retrouve là de vieux amis des professionnels de la communication et de leurs formateurs : à première vue, mais on y reviendra, les règles d'or "préférez les mots courts » et "faites des phrases courtes " ne semblent pas dépourvues de justifications psycholinguistiques, d'autant que l'évolution des habitudes de lecture va dans le même sens. Ainsi, une étude sur les mutations des styles littéraires portant sur 240 romans anglophones (20 par tranche de 20 ans entre 1740 et 1979) a-t-elle montré que leur longueur lexicale et syntaxique n'avait cessé de fondre : leurs phrases sont, en moyenne, passées de 41,5 mots au $\mathrm{XVIII}^{\mathrm{e}}$ siècle à 15,2 mots au $\mathrm{Xx}^{\mathrm{e}}$ siècle, tandis que leur taux de mots de plus de 9 lettres descendait de 4,7\% à 2,8\% (Danielson et Lasorsa, 1989). D'où les difficultés - du moins une partie des difficultés - que peuvent éprouver des collégiens contemporains face à la langue des œuvres classiques. D'où aussi les tentatives des éditeurs pour " moderniser » celle-ci et les polémiques que suscitent parfois ces réécritures, échauffourées typiques de l'affrontement entre valeur cognitive et valeur symbolique (on en trouvera dans la seconde partie de l'ouvrage une étude plus détaillée, consacrée au cas des retraductions d'Enid Blyton).

11 Cette relation inverse entre la longueur moyenne des mots et leur fréquence dans le langage courant, que Zipf supposait non linéaire, peut en réalité être caractérisée par une formule logarithmique tout à fait linéaire (on en trouvera les détails dans Labasse et Thoiron, 2002). 
La question du poids des mots et des phrases n'est pas nouvelle. On se souvient par exemple de Buffon appelant à condenser l'expression des idées : "si on les serre, le style devient ferme, nerveux et concis » (1753/1818, p. 599). C'est toutefois au $\mathrm{xx}^{\mathrm{e}}$ siècle que ces problèmes de poids sont devenus une obsession collective. Soucieux de rationaliser l'adéquation entre l'offre et la demande sur le marché discursif, un nombre croissant de secteurs, d'abord l'éducation, puis l'administration (en particulier l'armée) et les médias (notamment les grandes agences de presse anglophones) ont suscité un foisonnement de recherches en vue de mesurer la « lisibilité » des textes, c'est-à-dire leur facilité de lecture et - croyait-on - de compréhension pour des lecteurs de différents niveaux d'études. Des centaines de formules de calcul ont ainsi été élaborées et testées. Certaines comportaient une dizaine de variables, mais les seules à avoir connu un réel succès ne mesuraient au bout du compte que deux paramètres : la longueur des mots et la longueur des phrases. C'est notamment le cas de la formule de lisibilité de Rudolph Flesch, dont une version est intégrée au logiciel MS Word.

En dépit d'un bon nombre d'objections théoriques ou empiriques, ces formules ont acquis un rôle non négligeable : aux ÉtatsUnis, en particulier, la législation de plus de quinze états requiert que les contrats d'assurance atteignent un score de lisibilité spécifié avec l'une de ces formules et, selon une estimation, plus de $40 \%$ des commissions scolaires utiliseraient ces procédés pour sélectionner les livres de classe. Le monde médical est également un grand utilisateur de ces formules, notamment afin de s'assurer que les formulaires de « consentement éclairé » par lequel les patients acceptent de se soumettre à des opérations ou des expériences soient compréhensibles. Un examen systématique de la littérature scientifique (avec l'aide de Yann Rucar, assistant de recherche) m'a permis de relever entre 2000 et 2009 plus de 400 publications médicales se référant à la lisibilité, dont plus des trois quarts assimilaient purement et simplement la lisibilité à l'intelligibilité, $3 \%$ à peine distinguant clairement les deux notions (Labasse, 2012a). Or, la lisibilité ne correspond pas du tout à la clarté textuelle. Elle peut dans certains cas être l'indice de difficultés de plus haut niveau, mais la seule chose qu'elle traduise directement est l'effort lié au traitement lexico-syntaxique. En d'autres termes, des phrases et des mots longs peuvent être obscurs (ou pas), mais ils sont dans tous les cas fatigants à lire. 
Prenons un texte quelconque et mélangeons ses phrases au hasard. Mélangeons aussi les mots au sein de chaque phrase. Les moyennes lexicales et syntaxiques étant préservées, son score de lisibilité ne changera pas, bien que son sens ait disparu...

Ce qui a changé relève d'un troisième niveau d'effort cognitif : l'établissement de la cohérence discursive. On peut, pour simplifier, regrouper sous cette appellation les différents processus permettant de reconnaître les liens logiques entre les informations fournies par le texte. À l'échelle de la phrase, il s'agit des relations sémantiques entre les mots, notamment par la résolution des anaphores. Ainsi, en lisant dans un article en ligne qu'un accusé « adorait cette belle-mère qui avait épousé son père en 1976, alors qu'il n'avait que 12 ans ${ }^{12}$ » on peut deviner que la victime n'avait pas épousé son propre père, et que ce dernier n'avait pas 12 ans au moment de ses noces. Ceci réclame toutefois un effort, inconscient mais non nul, et parfois vain lorsque le contexte ne permet pas de résoudre l'ambiguïté. Cet effort a même été envisagé comme le critère régissant la mise en cohérence des constituants du texte : selon l'hypothèse de Frazier et Fodor (1978), le module de traitement choisirait en effet de privilégier l'interprétation « qui lui coûte le moins d'effort » (coût qu'ils lient, du reste, à la longueur des segments de phrase à manipuler mentalement).

En tout état de cause, la quantité d'inférences que réclame à tout moment la compréhension d'un discours - par exemple le fait que la «victime » évoquée plus haut devait être la «belle-mère » mentionnée dans la citation - est telle que le traitement du langage a pu être résumé, selon la formule classique de Goodman (1967), comme «un jeu de devinette psycholinguistique ». Ce jeu, généralement peu amusant, porte aussi sur les liens entre les énoncés. Ainsi, le rapport entre deux propositions comme "Il était en retard. Il avait manqué le bateau» est peut-être évident pour celui qui les formule, mais ne l'est pas pour son destinataire. Il peut s'agir d'une conséquence (« ...donc il avait manqué le bateau »), d'une cause (« ... car il avait manqué le bateau»), de deux informations distinctes (« ... par ailleurs...»), voire d'une opposition $(« \ldots$ mais...»), d'une alternative («...ou...»), etc. Moins les interlocuteurs partagent de connaissances communes, plus il est difficile de produire les inférences assurant la cohérence entre les éléments

12 Ian Hamel (2012). "L'enquête à charge contre un chercheur français ». Le Point (lepoint.fr), 22 mai 2012. 
d'information exprimés. On comprend donc que, face au gonflement de l'offre discursive, la fragmentation des connaissances des spécialistes et a fortiori du « grand public » (notion de plus en plus douteuse) tende à accroître la difficulté de compréhension des sujets non familiers, et donc à réduire leur pertinence, donc leur familiarité, et ainsi de suite. C'est ainsi qu'une grande partie des sujets politiques, économiques, scientifiques, etc. évoqués à la télévision par des journalistes qui voient le rapport entre leurs énoncés, puisse de plus en plus ressembler à ce que dénonçait déjà Sarcey en 1866, «un amas informe, indigeste de petits faits, qui tombent les uns par-dessus les autres, sans qu'aucun ferment d'idées mette en jeu et fasse lever cette pâte ${ }^{13}$ ».

Enfin, la pénible construction de la cohérence discursive se joue également à l'échelle globale du texte : "pourquoi me parle-t-on de ça?", "où veut-on en venir ?». Compte tenu de la question bourdonnante et foisonnante à laquelle se consacre ce livre, on peut craindre qu'il ne constitue lui-même, par la force des choses, un remarquable exemple de ce genre de problème, d'où procède notamment la règle « dites l'important d'abord» (conseil un peu trop rudimentaire pour m'être d'un grand secours en l'espèce).

Le quatrième niveau de l'effort cognitif, qui interagit avec le troisième comme le troisième interagit avec le second, n'est pas de nature propositionnelle mais représentationnelle. S'il est un bilan à tirer des recherches des dernières décennies sur la compréhension du langage, c'est d'abord l'idée que cette compréhension revient à construire un modèle mental figurant l'état du monde évoqué dans le texte. Il en existe, selon les écoles, différentes variantes et appellations («modèle de situation", "représentation particularisée ", "simulation perceptuelle »...), mais les résultats expérimentaux et leurs différentes interprétations théoriques convergent globalement sur le caractère figuratif de la compréhension. Pour le dire plus simplement (peut-être trop...), on « voit » ou on ne « voit pas » ce que quelque chose signifie.

Présenté ainsi, ça ne semble pas une découverte très impressionnante, mais elle ne va pas de soi. Une conception alternative et un temps dominante, celle de la linguistique computationnelle, supposait au contraire un encodage propositionnel abstrait, régi par des règles similaires à celles de la logique formelle (conjonction, disjonction,

13 Cit. in L'année littéraire et dramatique, 1867, p. 533. 
implication, etc.) Une telle approche était d'autant plus séduisante qu'elle promettait un succès rapide pour l'intelligence artificielle, que les pionniers des sciences cognitives promettaient dans les années 1960 de mettre au point en «moins d'une génération »: une fois les règles éclaircies, il serait facile de les apprendre aux ordinateurs. La suite montra en pratique qu'il n'en était rien, ce que confirmèrent en théorie des expériences cinglantes comme celle de Wason et Johnson-Laird : la compréhension humaine est plus analogique que logique. Il lui faut construire un modèle mental (Johnson-Laird, 1983). Celui-ci peut porter sur des éléments concrets, à l'instar des personnages et accessoires dont on compose une scène, mais aussi des notions abstraites, par exemple des flux ou des relations structurelles entre des entités. Les diagrammes que l'on projette dans une réunion d'affaires, ou les réseaux criminels dont les policiers décorent leurs murs, ne sont qu'une façon d'économiser sur l'effort que réclamerait la construction d'un modèle mental à partir d'un texte (une image ne "vaut » pas mille mots, elle coûte simplement moins cher en termes cognitifs).

L'effort cognitif que réclame la construction d'un modèle mental est si bien perçu intuitivement que chacun s'empresse de l'éviter lorsque sa pertinence ne le justifie pas. C'est le cas de ces longues descriptions dans lesquels l'auteur s'efforce de fournir une abondance de détails pour permettre à ses lecteurs de se représenter exactement la scène qu'il a en tête. Ces derniers, quant à eux, ne manqueront pas de s'en dispenser : pourquoi se donner la peine de se figurer précisément le salon de la duchesse ou son accoutrement lorsque l'on attend simplement qu'elle soit assassinée ou séduite ? On glissera donc sur les mots sans rien en faire, jusqu'au moment où ils voudront bien s'animer. À moins, bien sûr, que le talent de l'auteur justifie cette tâche, au moins pour ses lecteurs les plus littéraires. Comme la marche en montagne, la lecture littéraire peut justement transcender les logiques élémentaires des préférences humaines : après tout, la lisibilité lexico-syntaxique de Proust est calamiteuse et les digressions de Balzac sont des modèles d'incohérence (pour ne pas parler du théâtre de Ionesco, qui joue justement sur celle-ci).

Il n'en reste pas moins que les quatre niveaux d'effort de traitement que l'on vient d'examiner brièvement, la légibilité perceptive, la lisibilité lexico-syntaxique, la cohérence (locale ou générale) et la figurabilité, semblent former la nomenclature la plus appropriée pour comprendre l'influence de l'effort dans l'appréciation de la valeur 
discursive. Une caractérisation plus rudimentaire de l'effort, par exemple celle de Sperber et Wilson examinée plus haut, n'aurait aucune utilité analytique. À l'inverse, une caractérisation plus détaillée et plus complexe, scientifiquement plus proche sur le fond et la forme des publications de référence, dépasserait largement les nécessités du problème qui nous occupe. En d'autres termes, elle ne compenserait pas par un gain significatif l'effort cognitif bien supérieur qu'elle réclamerait.

\begin{tabular}{|c|l|l|}
\hline \multicolumn{2}{|c|}{ Type } & Facteurs impliqués \\
\hline \multirow{2}{*}{$\begin{array}{c}\text { Bas niveau } \\
\text { cog. }\end{array}$} & Perceptif & Légibilité (typographique, etc.), audibilité \\
\cline { 2 - 3 } & Acquisitif & $\begin{array}{l}\text { Lisibilité (lexico-syntaxique) et } \\
\text { facteurs assimilés }\end{array}$ \\
\cline { 2 - 4 } $\begin{array}{c}\text { Haut niveau } \\
\text { cog. }\end{array}$ & Logique & $\begin{array}{l}\text { Cohérence locale (cohésion, connexité) } \\
\text { et générale }\end{array}$ \\
\cline { 2 - 4 } & Figuratif & Représentabilité (modèle mental) \\
\hline
\end{tabular}

Figure 1. Les principales composantes de l'effort cognitif

Telles que regroupées ici, les quatre familles de facteurs d'effort suffisent par exemple pour affiner l'observation évoquée plus haut : le fait que la télévision et la vidéo en ligne attirent plus facilement que le texte, même lorsque leur contenu apporte moins. En effet, le traitement cognitif du message audiovisuel requiert moins d'effort de traitement de bas niveau (le déchiffrage), mais il dispense surtout de l'essentiel de l'effort de haut niveau : au lieu d'exiger la construction d'un modèle mental de l'état du monde évoqué, il fournit directement ce modèle. La contrepartie est, comme le montrent les expériences, qu'en épargnant ce travail d'intégration, le discours audiovisuel est moins bien mémorisé et moins susceptible de conférer des connaissances durables que le texte. On n'a rien sans rien.

D'autre part, cette dissociation des facteurs d'effort permet de comprendre certains dysfonctionnements des règles d'or censées optimiser la valeur discursive. Ainsi, «faites des phrases courtes" peut-il négliger le fait que le raccourcissement des phrases expose à un autre danger, celui de la parataxe. Les phrases se découpent comme les poulets, à leurs jointures internes, donc à l'endroit des connecteurs logiques qui en lient les propositions. En remplaçant par exemple «Il est en retard car il a manqué le bateau » par «Il est en retard. Il a manquéle 
bateau », on ne réduira quasiment pas l'effort de traitement lexicosyntaxique, déjà négligeable dans la phrase initiale, mais on accroîtra considérablement l'effort que réclame l'établissement de sa cohérence (a fortiori si le sujet est peu familier).

De même, le précepte " préférez les mots courts » oublie une caractéristique essentielle des mots, leur valence d'imagerie, c'est-à-dire leur capacité à évoquer des images mentales. Or, celle-ci est évidemment cruciale pour établir les relations entre les éléments d'information (cohérence) et pour construire un modèle mental (figurabilité). Ainsi, "Un homme entra dans la maison» ne signifie-t-il pas grand-chose comparé à "Un chevalier se précipita dans la chaumière ", énoncé beaucoup plus riche permettant de multiples inférences (épée brandie, paysans terrifiés, etc.) : la première formulation pourrait être suivie par n'importe quelle proposition, la seconde peut difficilement l'être par «... et proposa une partie d'échecs» ou « ... et décrocha le téléphone». Déférence gardée envers Paul Valéry ${ }^{14}$, les moindres mots ne sont pas forcément les moindres maux. Comme toujours, il n'y a rien là de bien nouveau. Sans laboratoires ni protocoles expérimentaux, les rhéteurs avaient depuis longtemps souligné l'importance de l'hypotypose, peinture des mots permettant de former dans l'esprit de l'auditoire une vive image de ce que l'on évoque (l'enargeia, ou force de l'évidence).

Pour autant, la valence d'imagerie d'un terme ne correspond pas tout à fait à son caractère concret (concreteness) ni même à la densité de sens ressentie (meaningfulness). Ainsi a-t-on vérifié depuis longtemps que la valence d'un terme comme « jalousie » est supérieure à celle de «bivouac» (Paivio, Yuille et Madigan, 1968). Selon cette étude, l'indice de corrélation entre l'imagerie de 925 mots anglais et leur caractère concret est de 0,83 tandis que la corrélation entre imagerie et sens perçu n'atteint que 0,72 (soit une relation forte, mais loin d'être absolue). On pourrait l'expliquer en faisant appel à la théorie des schémas cognitifs, qui propose une explication élégante et a priori convaincante de la structuration mentale des connaissances et de leur rôle dans la compréhension des discours. Cependant, l'invocation des schémas cognitifs exigerait des développements un peu longs : d'une part,

14 "Conseil à l'écrivain : Entre deux mots, il faut choisir le moindre», écrivait Paul Valéry (1929, p. 555). Mais qui réduirait la poétique et, pourrait-on dire, la psycholinguistique de Valéry à ce pauvre calembour sousestimerait gravement la perspicacité de celles-ci. 
cette question fait l'objet de débats scientifiques que l'on ne peut occulter, d'autre part ses enjeux considérables pour certaines des impasses théoriques que hantent des problèmes discursifs en déshérence (la nature de la "vulgarisation », la validité de l'opposition dénotationconnotation, la disqualification des clichés et stéréotypes, etc.) requiert un examen attentif. Dans la mesure où ce dernier n'est pas vraiment indispensable pour percevoir les différents degrés de l'effort cognitif, on peut le repousser pour le moment.

Une autre dimension essentielle sur laquelle il convient de glisser - et cette fois sans aucune promesse d'y revenir plus tard - est l'incidence de la mémoire dans l'effort de traitement. On précisera un peu plus loin (voir les « Précisions » de ce chapitre) pourquoi cet aspect n'est pas nécessaire ici. Il est aussi possible de le percevoir intuitivement ${ }^{15}$ en remarquant que les niveaux d'effort cognitif indiqués plus haut correspondent bien au ressenti de l'effort que réclame un discours : je n'arrive pas à le lire/l'entendre (légibilité) ; le texte est fatigant (lisibilité) ; je ne vois pas le rapport (cohérence) ; je ne vois pas de quoi on parle (figurabilité). En revanche, je n'arrive pas à retenir les phrases, ou ce livre est difficile à mémoriser serait une façon plus inattendue d'exprimer sa valeur discursive. On dit souvent que l'on n'a rien retenu d'un film ou d'un livre, mais généralement avec indulgence, et toujours a posteriori (forcément). En outre, les jugements métamnésiques sur la valeur discursive - ce récit est inoubliable - portent essentiellement sur la préservation en mémoire à long terme, or celle-ci est une conséquence, plutôt qu'une cause, et elle est largement due... à des facteurs très éloignés de l'effort cognitif (illustrant à nouveau que l'on ne peut pas comprendre la communication humaine sans une cartographie générale des variables en jeu).

Pourquoi un récit serait-il plus inoubliable qu'un autre? Poser la question conduit aussitôt à percevoir que la vieille « loi du moindre effort ", à laquelle certains prêtaient tant de pouvoir, reste absolument muette sur ce point. Mais la hiérarchie des tâches que l'on vient d'expliciter est tout aussi impuissante. C'est là une excellente chose : la meilleure façon de comprendre ce qui relève de l'effort cognitif est justement de considérer ce qu'il n'explique pas. Or, le propre de l'effort cognitif est que son influence sur la valorisation des discours est dépréciative et non appréciative.

15 Non sans rappeler que ce n'est plus une forme de raisonnement scientifiquement valide depuis un bon siècle. 
Voici un voyageur, disons un homme d'affaires, choisissant un livre avant de monter dans son train. Selon une prédiction fondée sur le moindre effort cognitif, son choix devrait se porter sur un livre pour enfants. Comment résister par exemple à Oui-oui et son cerf-volant (Blyton, 1967)? Sa typographie est remarquablement légible, son indice de charge lexico-syntaxique (CLS) n'est que de 21,1 (contre 43,4 pour Zola, 59,6 pour Durkheim et 71,7 pour Husserl ${ }^{16}$ ) et sa difficulté conceptuelle ne semble pas insurmontable. Certes, il serait assez peu probable que le voyageur se rue à la place sur la Fondation de la métaphysique des mours (CLS : 131,0) si la boutique de la gare l'avait en rayon, mais on voit ici qu'en matière d'appréciation discursive, le « goût des choses simples » ne conduit nulle part ${ }^{17}:$ impliquant essentiellement des variables négatives (qui repoussent plutôt qu'elles n'attirent), il exige de ce fait l'existence symétrique de variables positives sans lesquelles aucun discours ne pourrait être pertinent.

16 Ces évaluations, plus fiables que les calculs de lisibilité classiques mais encore limitées en termes de représentativité du corpus, ont été faites en vue d'étalonner un projet pilote (non publié) commandité par le Syndicat de la presse hebdomadaire régionale.

17 Laissons de côté, bien sûr, la littérature des petites choses, dont les « plaisirs minuscules » (Philippe Delerm) éveillent des échos d'une tout autre nature. 
Page blanche conservée intentionnellement 


\section{(Précisions)}

T a typologie des facteurs d'efforts cognitifs que l'on vient d'exa$\amalg$ miner suit une gradation allant de l'étape amont la plus éloignée de la compréhension (la perception des signes) jusqu'à la compréhension elle-même. On pourrait donc être tenté de la prendre pour un modèle procédural de la lecture. Ce n'est pas vraiment le cas. Il ne s'agit pas du tout ici de contribuer aux théorisations des modalités de la lecture et de la compréhension, mais simplement de prendre acte des travaux conceptuels et expérimentaux qui les nourrissent, et ce dans la seule perspective qui nous concerne. Puisque le problème est de clarifier les coûts cognitifs du traitement de l'information, cette perspective n'est, en quelque sorte, que comptable. On pourrait par exemple expliquer la genèse des œuvres cinématographiques en débattant des interactions complexes des scénaristes, producteurs, réalisateurs, acteurs, etc. : un regard pragmatiquement comptable y reconnaîtra invariablement des coûts de structure, de main-d'œuvre, de consommables, de location et autres.

L'indifférence que suggère cette analogie est cependant exagérée. En l'espèce, on remarquera aisément que, si la première dimension évoquée est infralinguistique, les trois autres pourraient être rapprochées des niveaux de représentation textuelle identifiés par van Dijk et Kintsch (1983) - respectivement : la structure de surface, la base propositionnelle (microstructure et macrostructure) et le modèle de situation - niveaux qui sont aujourd'hui assez largement acceptés (Graesser, Millis, Zwaan, 1997 ; Radvansky, Zwaan et coll., 2001). Mais ils pourraient l'être aussi, mutatis mutandis, aux trois niveaux de traitement (représentationnel, associatif et référentiel) que prévoit la théorie alternative du «codage double » (Paivio, 1991 ; Sadoski \& Paivio, 2007) et sans doute à d'autres : l'un des avantages d'une nomenclature en niveaux de tâches est qu'elle 
demeure agnostique sur tout ce qui ne la conditionne pas, notamment la nature des processus en jeu, leurs délimitations et leur interprétation modulaire ou connexionniste, etc.

Ainsi, comme on l'avait d'ailleurs souligné, l'indication selon laquelle la clef ultime de la compréhension réside dans la construction d'une représentation mentale (et non d'une structure propositionnelle) de l'état du monde présenté par le texte reflète globalement la convergence des recherches théoriques et expérimentales dans ce domaine ${ }^{1}$, mais cette mention ne s'appesantit pas sur les différences séparant les diverses conceptualisations de cette figuration mentale, dont témoigne la variété de ses désignations scientifiques («modèle mental» pour Jonshon-Laird, 1983; " modèle de situation » pour van Dijk et Kintsch, 1983, "représentation particularisée » pour Richard, 1990, "simulation perceptuelle » pour Barsalou, 1999, "représentation mentale » pour van den Broek et Helder, 2017...). Ces différences peuvent être considérables du point de vue des écoles et courants de recherche. Par exemple, l'intérêt actuel pour la "cognition incarnée » (embodied cognition), qui envisage la compréhension d'un texte par la relation qu'il établit avec les expériences sensorielles qu'il évoque (visuelles, auditives, motrices...), est parfois posé en opposition à la modélisation mentale (voir Sadoski, 2018) alors même que cette approche fait appel à des simulations perceptuelles qui ne sont pas phénoménologiquement incompatibles avec une modélisation analogique, voire requièrent une telle modélisation.

On trouvera par exemple dans Zwaan (2016) une revue récente de cette question assez complexe, mais ce niveau de précision scientifique n'est pas pertinent dans la perspective de ce livre, n'ayant guère d'incidence sur la sobre division « comptable » (en familles de tâches) que l'on a adoptée. Notons cependant qu'il aurait pu être intéressant - sous un autre angle que celui adopté ici - d'explorer de possibles convergences entre les

1 C'est une hypothèse, bien sûr - au même titre que le big bang est une hypothèse en physique - mais elle fait l'objet d'un assez large consensus à ce niveau de généralité et, en tout état de cause, on voit mal ce qui pourrait rendre compte de toutes les observations disponibles sans recourir d'une façon ou d'autre à un tel processus de modélisation mentale. 
recherches sur la "cognition incarnée » et certaines approches communicationnelles, notamment celle de Gloria Awad (2016) qui s'intéresse au caractère sensoriel et même « haptique » de la réception de l'information.

De tous les silences que requiert ce chapitre, le plus manifeste est peut-être celui qui concerne le coût mnésique (l'effort de mémoire) du traitement discursif. Ce n'est pas que ce coût soit négligeable : il est au contraire prépondérant puisque, par définition, la compréhension résulte d'échanges constants entre la (ou les) mémoire(s) et le texte. L'accès lexical, le traitement syntaxique (a fortiori celui des phrases longues!), la production des inférences et la construction d'un modèle mental reposent tous sur différentes formes de mémorisation fugace ou durable. C'est d'ailleurs la façon courante d'étudier le traitement du langage en psychologie. Toutefois, cette perspective n'est ni la plus claire en l'état actuel des choses ni la plus pertinente dans le strict cadre du problème de la valeur des discours. Le constat de l'omniprésence de la mémoire est en effet réversible : si tous les degrés d'effort de traitement peuvent être rattachés au coût de différents processus mnésiques, l'inverse est également vrai. Quel que soit l'angle sous lequel on aborde les mêmes phénomènes, on ne peut les compter deux fois : le traitement syntaxique par exemple correspond à une charge de la mémoire de travail ${ }^{2}$, il ne s'ajoute pas vraiment à celle-ci.

En somme, la rusticité empirique de cette typologie est gage de sa robustesse : quel que soit le modèle théorique envisagé, les coûts cognitifs, largement attestés, seront toujours là. Leur ventilation générale gagnerait peut-être à être affinée : on se doute, par exemple, que l'établissement de la cohérence relève de processus psycholinguistiques très différents selon qu'elle concerne les unités élémentaires (cohésion), les propositions (connexité) ou la structuration globale du discours (cohérence stricto sensu). De même, la distinction pratique entre les efforts de traitement liés à la syntaxe et ceux liés à la cohérence anaphorique (etc.) pourrait être débattue. Toutefois, comme on l'a dit, l'effet de ces gains descriptifs ne paraît pas justifier ici le surcroît d'effort - et de pages que réclamerait une typologie plus détaillée, d'autant que toute

2 Ou de la mémoire à court terme selon les auteurs, à moins qu'il ne s'agisse de la même chose, ou qu'elles ne se recoupent en partie... 
précision en appellerait d'autres comme l'abîme appelle l'abîme. On soutiendra donc que, pour rendre compte globalement des déterminants de la valeur discursive, cette nomenclature est fondée, suffisante, et même tout à fait rigoureuse dans ce cadre, sans forcément être immuable.

Indépendamment de son caractère pratique, elle souligne aussi par l'exemple que les apports possibles de la psychologie contemporaine à la compréhension des questions communicationnelles n'imposent pas l'alternative «tout ou rien » qui sous-tend les discours de certains spécialistes, comme si les travaux sur les problèmes de réception (des médias, de la littérature, etc.) n'avaient d'autres choix que de se convertir aux rationalités propres à la recherche expérimentale ou se réfugier aussi longtemps que possible derrière un farouche rejet de ses éclairages.

On connaît certes plus d'un exemple de recettes pratiques abusivement appuyées sur des bricolages pseudo-psychologiques éhontés, mais ces produits de contrebande sont aussi la conséquence des tentations protectionnistes qui préviennent, ici l'exportation raisonnée, là l'importation mesurée de tous les éléments scientifiques nécessaires à l'appréhension de phénomènes intrinsèquement transdisciplinaires (on y reviendra). 


\section{Le trouble jeu de l'effet cognitif}

Il y a des sujets qui ont du génie. Ils en ont même pour deux : l'auteur n'en a plus besoin; ainsi l'Himalaya, Landru, la stratosphère, ou les sources del'Amazone, les mœurs du phoque, Hiroshima [...] Le lecteur aime se trouver soudain tête à tête avec un requin. Il aime rester pendu par le fond de sa culotte à trois mille mètres au-dessus de l'abîme géographique. Il aime bien moins s'asseoir tête à tête avec l'homme ou rester suspendu au-dessus de l'abîme humain.

Alexandre Vialatte (2000, p. 259-26o)

T e matin du 27 février 2015, une grave affaire agitait les réseaux sociaux. Son enjeu était de déterminer la juste couleur d'une robe, d'ailleurs assez laide, sur laquelle un internaute et son amie n'avaient pu s'accorder la veille. Peu après, une allusion ironique dans un cours de troisième année me valut une attention inaccoutumée : enfin quelque chose d'intéressant. Intéressant pour moi également : combien d'étudiants avaient-ils pu saisir l'allusion? Vingt-quatre sur vingt-neuf levèrent la main, les yeux brillants. Le cours étant consacré aux rapports entre journalisme et littérature ${ }^{1}$, on pouvait en effet s'attendre à ce que ses participants portent une certaine attention aux grands débats publics. Combien étaient en mesure d'indiquer le nom ou au moins le poste d'un ministre venant de démissionner (l'actualité fédérale et provinciale canadienne offrait cette semaine-là l'embarras du choix)? Trois mains se levèrent avec hésitation. Peut-être la barre était-elle trop haute. Combien d'étudiants pouvaient-ils mentionner

I Il y a dix ans, j'aurais pu traiter le sujet en une phrase («Il n'y en a pas »). Aujourd'hui, trois mois de cours me permettent à peine d'en effleurer la richesse et les contradictions. 
l'un des sujets, n'importe lequel, susceptibles de figurer à la une de l'un des quotidiens du jour? Trois également. Sur vingt-neuf.

Ce pourrait être le moment d'emboucher les antiques trompettes de la déploration générationnelle, ou, mieux encore, de se rappeler la frappante distinction que Neil Postman opérait trente ans plus tôt (le web n'existait même pas...) entre Le meilleur des mondes et 1984:

Huxley et Orwell ne prophétisaient pas la même chose. Orwell prévenait que nous serions dominés par une oppression imposée de l'extérieur. Mais dans la vision de Huxley, aucun Big Brother n'était nécessaire pour priver les gens de leur autonomie, de leur maturité, de leur histoire. Selon sa perspective, les gens en viendraient à aimer leur oppression, à adorer les technologies qui annulent leurs capacités à penser. Ce que craignait Orwell, c'était ceux qui interdiraient les livres. Ce que craignait Huxley était qu'il n'y aurait pas de raison d'interdire un livre, car il n'y aurait personne qui désirerait en lire. (1986, p. vii)

Convenons-en, les perspectives actuelles ne rassureraient pas Postman. Le temps semble bien loin où Condorcet, indifférent à la contradiction que constituait sa propre condamnation à mort, poursuivait l'écriture de son "tableau historique des progrès de l'esprit humain ». Toute néophobie mise à part, les évolutions du marché discursif évoquées plus haut - surabondance informationnelle, relativisme, crise de l'attention... - semblent inexorablement confirmer l'hypothèse soutenue par Tichenor, Donohue et Olien $\left(1970^{2}\right)$ d'un effet de knowledge gap, fossé de connaissances que tout afflux d'information tendrait à élargir entre l'aristocratie culturelle capable de s'y intéresser et la vaine populace.

Mais avant de se réfugier du bon côté de ce fossé, abandonnant le reste des terres aux zombies du divertissement virtuel, puisons à nouveau dans nos souvenirs pour revisiter un de ces lieux où règnerait encore l'esprit. Un colloque international d'universitaires ${ }^{3}$, public qui n'est pas connu pour la frivolité de ses préférences discursives (c'est un stéréotype, mais les stéréotypes sont des outils de réduction

2 Donc, là encore, bien avant l'arrivée d'Internet.

3 Colloque France-Québec : l'écriture dans tous ses états. Poitiers, 12 au 15 novembre 2008. 
de l'effort cognitif face à la complexité du réel). L'orateur - ce n'était pas moi - déroule son exposé et présente un exemple lexicologique, tiré d'un récit de Samuel de Champlain :

Dieu vint à cest homme, \& luy demanda où estoit son petunoir, l'homme print son petunoir, \& le donna à Dieu, qui petuna beaucoup ; Apres avoir bien petuné, Dieu ro[m]pit ledict petunoir en plusieurs pieces, \& l'homme luy demanda, Pourquoy as-tu rompu mon petunoir?

Des yeux s'ouvrent dans l'assistance, jusque-là écrasée par tant d'érudition. Inconscient de ce frémissement, l'orateur poursuit. N'y tenant plus, un participant (pas moi non plus) finit par l'interrompre. « Mais pourquoi? - Pourquoi quoi donc? - Pourquoi Dieu a-t-il fait ça? - Oh, je ne sais pas, c'était juste un exemplet. » Les chercheurs de deux continents lâchent un gémissement de frustration : cette bouffée soudaine d'effet cognitif ne tenait pas la promesse de pertinence qu'elle avait brièvement fait miroiter. Mais pendant une minute, la majorité des participants (moi compris) avait été cognitivement indistinguable d'une classe de maternelle attendant, bouche ouverte, le dénouement d'un conte. Et j'aime à penser que le lecteur, lui aussi, aimerait en connaître la fin. On voit ici la force de l'effet cognitif, capable de légitimer dans une enceinte scientifique une question parfaitement incongrue dans un tel contexte. On peut même soupeser cette force : quoique ladite question mait taraudé depuis lors (Pourquoi Dieu a-t-il donc cassé la pipe de ce malheureux ?) elle n'a jamais eu la puissance nécessaire pour me traîner vers la bibliothèque. Les zombies, c'est nous tous : les louches séductions de l'effet cognitif ne sont pas l'apanage des nouvelles générations ou des fractions les moins diplômées du corps social. Elles sont plus profondes que ça.

Les difficultés commencent lorsque l'on tente de les caractériser. Comme toujours, la question est simple à formuler - «qu'est-ce qui suscite notre intérêt ? »-, mais, on s'en doute, la réponse l'est moins. En comparaison, le problème de l'effort ressemble même, rétrospectivement, à un modèle d'ordonnancement conceptuel et de consensus scientifique. On distingue bien une sorte de consensus à propos de

4 L'échange étant reconstitué de mémoire, les mots n'étaient peut-être pas exactement ceux-ci, mais le sens était le même. 
l'intérêt, mais c'est celui qui, sous les plumes les plus diverses, déplore depuis un demi-siècle au moins la confusion et l'insuffisance des recherches sur cette question. Ou, plutôt, ces questions, car chaque angle d'approche construit par définition un objet différent. Pour les courants les plus classiques ou les mieux nourris par une demande institutionnelle d'expertise, l'intérêt se confond plus ou moins avec la motivation scolaire (psychopédagogie) et avec la disposition pour un travail (orientation professionnelle), quand il ne concerne pas plutôt la psychologie clinique (obsession, asthénie...). Mais c'est aussi une attitude (curiosité, attention), ou un sentiment (affinité), une émotion ou encore bien d'autres choses (implication, besoin), à moins qu'elles n'appartiennent à l'une des catégories précédentes, en supposant que celles-ci ne soient pas les mêmes.

Le brouhaha s'estompe un peu si l'on se replie obstinément sur le seul traitement cognitif des discours. Le thème de l'insuffisance des recherches est toujours là : la quantité d'intérêt (interestingness) est ainsi présentée comme "une variable négligée dans le traitement des discours » (Hidi et Baird, 1986). Mais quelques points importants se remarquent. Le principal, amplement démontré expérimentalement, est surtout une confirmation : l'intérêt que l'on ressent pour un discours est lié aux connaissances dont on dispose déjà sur son thème (décidément, il faudra que l'on examine en détail le rôle des schémas cognitifs préalables). Malheureusement, on peut aussi démontrer l'inverse : quelque chose d'inconnu (surprenant, inquiétant, incongru...) pourra obtenir beaucoup plus d'attention qu'une information prévisible. D'où la nécessité, admise par tous, de tenter de cerner les différents facteurs en jeu et leurs interactions. Mais cette volonté a surtout conduit à accumuler "une longue liste d'épicerie 5 des variables affectant l'intérêt » (Silvia, 2006, p. 78), dans laquelle on peut entre autres relever des facteurs aussi tautologiques que «le caractère impliquant» du discours, mais aussi un bon nombre de variables... dépréciatives, par exemple la «cohérence », la « simplicité du vocabulaire » et même la « facilité de compréhension » (notion dont on appréciera la précision) ; comme on vient de le voir à propos de Oui-oui et son cerf-volant, ce genre de critères suscite rarement l'excitation. Bref, ce que ces travaux établissent surtout, outre la remarquable capacité des angles d'approche

5 La métaphore originelle est "laundry list », peut-être moins parlante en français. 
restreints à réorganiser le réel dans leur perspective propre, c'est la nécessité de distinguer, en quelque sorte, l'intérêt global de l'intérêt intrinsèque. Le premier, qui intégrerait la "facilité de compréhension" (sic), correspond à peu près à ce que l'on a désigné jusqu'à présent comme la pertinence; on peut donc se débarrasser sans regret de ce doublon (dès lors qu'il comporte à la fois des «facteurs d'intérêt » positifs et négatifs, ce n'est plus qu'une appellation alternative assez confuse). Gardons le second.

Une distinction plus importante a été proposée entre l'intérêt «personnel ", lié aux dispositions préalables du destinataire et l'intérêt "situationnel ", lié au discours lui-même. Comme personne ici n'a sans doute envie de s'enfoncer encore plus profondément dans une interminable discussion des propositions scientifiques actuellement sur le marché, on se contentera de souligner que cette distinction, ainsi que ses différentes variantes ultérieures, peuvent être tout à fait judicieuses s'il ne s'agit que de faire avancer la psychologie de l'intérêt pour elle-même, mais qu'à l'échelle générale de l'appréciation de la valeur discursive, elles ne font que repousser ou réduire des problèmes d'une tout autre nature. En un mot, si les dispositions personnelles englobent par exemple les "gouts", alors aucun protocole expérimental ne suffira à les élucider. Et si elles ne les englobent pas, alors ce ne sont pas des dispositions. Toujours le même genre de problème...

Il existe heureusement une nomenclature minimale répondant au triple critère de la reconnaissance scientifique intradisciplinaire, de l'utilité analytique et de l'économie conceptuelle. On la trouve notamment dans le bilan des connaissances sur le traitement cognitif du discours qu'a dressé le chercheur le plus influent de ce domaine au $\mathrm{xx}^{\mathrm{e}}$ siècle. Un bilan de quelque 460 pages. La question de l'intérêt $\mathrm{y}$ bénéficie... d'une vingtaine de lignes ${ }^{6}$ :

Certaines choses sont intrinsèquement intéressantes, comme le sexe et la violence. Tout ce qui a à voir avec la sphère personnelle, en fait, est dans cette catégorie. [...] L'autre classe de choses intéressantes sont celles qui ne sont ni trop familières ni trop

6 En 1959, les traités français de psychologie «consacraient 0,1 \% de leurs pages [à] l'étude des intérêts » contre $7 \%$ pour les traités américains, notait Donald Super (1964, p. 1). 
étranges [...] Ce sont les choses qui éveillent notre curiosité, sans être nécessairement liées à notre propre personne. (Kintsch, 1998, p. 418-4197)

La proposition n'est pas incompatible avec celle évoquée un peu plus haut, tout en étant plus compatible avec une perspective élargie. Synthétisant de facto les enseignements d'un grand nombre d'observations, expérimentales ou non, elle pourrait à ce titre être plus ou moins considérée comme le plus petit dénominateur commun à la plupart de ces travaux depuis ceux de Berlyne (1949). Or, cette typologie, quoiqu'un peu vague, permet de distinguer un agencement agréablement familier : le même que celui qui, dans le cas de l'effort, soulignait l'interaction de facteurs de bas niveau (instinctifs, guidés par les données) et de facteurs de haut niveau (construits, guidés par les connaissances).

Dans une perspective similaire, une nomenclature un peu plus détaillée (Schank, 1979) avait proposé plusieurs familles :

- les éléments absolus, comme «le danger; le pouvoir, le sexe, l'argent, la destruction [...]";

- les éléments inattendus, surprenants ;

- les éléments reliés à l'existence personnelle ;

- les éléments "qui ne sont pas nécessairement intéressants, mais dont la juxtaposition l'est», comme l'humour, les dilemmes ou les paradoxes.

Indépendamment de la question de l'humour, lequel est trop multifactoriel pour constituer malgré sa puissance un élément autonome ${ }^{8}$, on

7 Le passage résume pour l'essentiel une distinction proposée une vingtaine d'années plus tôt par le même auteur (Kintsch, 1980), ce qui ne témoigne pas d'une problématique en pleine effervescence.

8 On ne se risquera pas à improviser une théorie de l'humour après que Bergson et bien d'autres s'y soient essayés avec plus ou moins de bonheur, mais force est de remarquer que cette notion recouvre une gamme d'effets qui s'étage du plus bas au plus haut niveau cognitif. On signalera en outre plus loin qu'elle met souvent en jeu des déterminants sociaux beaucoup plus larges que ceux du traitement cognitif des discours, mais même dans ce cadre restreint, certaines formes d'humour - pas les meilleures - reposent sur des effets de brutalité (peaux de bananes et bâtons 
notera que, là encore, la dernière classe de variables n'est pas un modèle de précision analytique. À l'instar de celle proposée par Kintsch, on pourrait la définir comme «tout le reste». En l'espèce, cependant, de telles approximations sont des qualités plutôt que des défauts, justement parce que cette indétermination prudente laisse ouverte la question de son périmètre. Une explicitation plus rigide pousserait à considérer arbitrairement comme «psychologiques » l'ensemble des variables culturelles, auquel cas l'effet cognitif, qui seul nous intéresse pour le moment, se diluerait dans l'effet en général et les expérimentalistes devraient, entre autres, appeler à leur secours les sociologues et historiens de l'art (la réception des discours variant selon les lieux et les époques, ce qui n'est pas évident à observer en laboratoire).

Quoi qu'il en soit, il faudra se contenter de ce que l'on a. Pour respecter la promesse de parcimonie épistémologique de ce livre (recourir autant que possible à l'état des connaissances scientifiques publiées plutôt qu'à l'imagination de l'auteur), on ne peut au bout du compte esquisser plus que quelques grandes familles d'effet cognitif, assez générales mais tout à fait suffisantes ici et relativement consensuelles.

La première - la plus incontestable de toutes - regroupe les facteurs spontanés que l'on peut considérer, sans le moindre jugement de valeur, comme le plus bas niveau de l'effet cognitif : le spectacle du sexe, de la violence, etc.

On peut ensuite considérer sans trop de risques qu'interviennent des facteurs égocentriques ou utilitaires (ceux qui concernent la « sphère personnelle»). C'est peut-être aussi le cas des facteurs stochastiques (inattendus, surprenants), mais on pourrait aussi estimer que cette dernière catégorie ne forme qu'une variable générale qui renforce ou atténue toutes les autres. D'un côté, la théorie mathématique de l'information - issue de l'ingénierie des télécommunications - assimile purement et simplement la valeur élémentaire d'une information à son imprévisibilité9. De l'autre côté, de la neige sur un écran de télévision

de dynamite), d'autres sur la surprise (ce seraient donc des effets inatten$d u s)$, d'autres sur la mise en jeu des connaissances de haut niveau ("Combien de psychanalystes faut-il pour changer une ampoule? Un seul, mais il faut qu'elle désire vraiment changer. »)

9 Non seulement la répétition monotone d'un caractère, par exemple 300 ooo fois la même lettre, comporte infiniment moins d'information 
brouillé est tout à fait aléatoire, donc imprévisible, mais elle est moins intéressante que le scénario, pourtant assez prévisible, du western qui devait être retransmis à la place. En d'autres termes, on peut supposer que l'imprévisibilité produit de l'effet cognitif, non pas en tant que telle, mais en s'appliquant à quelque chose digne d'attention par ailleurs.

Enfin, au plus haut niveau de l'effet cognitif, la dernière catégorie, aussi incontestable que la première, est... " tout le reste ", c'est-àdire l'ensemble des facteurs construits, pour autant qu'ils relèvent de processus psychologiques. Ceux-ci intègrent fort probablement, mais sans s'y limiter, quelque chose comme ce qu'Aristote appelait le désir naturel de connaître (rappelons qu'on ne parle pas pour le moment du désir socialement déterminé de connaître) :

Tous les hommes ont un désir naturel de savoir, comme le témoigne l'ardeur avec laquelle on recherche les connaissances qui s'acquièrent par les sens. On les recherche en effet pour ellesmêmes et indépendamment de leur utilité, surtout celles que nous devons à la vue ; car ce n'est pas seulement dans un but pratique, c'est sans vouloir n'en faire aucun usage, que nous préférons en quelque manière cette sensation à toutes les autres ; cela vient de ce qu'elle nous fait connaître plus d'objets, et nous découvre plus de différences ${ }^{10}$.

Il est difficile d'être intuitivement plus simple que cette hiérarchisation élémentaire des niveaux cognitifs (et plus réducteur, bien sûr, mais ce n'est pas un problème à ce stade) : pour schématiser grossièrement, plus un facteur est commun à l'ensemble du règne animal, plus il est spontané, et donc de bas niveau, plus il est propre aux hominidés, et ultimement aux humains, plus il est construit, et donc de haut niveau (on ne spéculera pas ici sur les degrés de différence possibles entre la curiosité animale et l'éventuel « désir de connaître ", plus abstrait, prêté aux humains).

Pourrait-on développer cette nomenclature en multipliant les facteurs intermédiaires? Peut-être, mais ça n'a guère d'importance

qu'un roman de 300 ooo signes, mais dans une phrase comme « il gobe de l'ai_. » la dernière lettre sera beaucoup plus informative car imprévisible (air ou ail ?) que dans «il gobe un œu_" (forcément $œ u f$ ).

10 Métaphysique (trad. Victor Cousin, 1838, p. 121). 
ici : si l'avancée des recherches sur la psychologie de l'intérêt discursif en établit d'autres, on les accueillera avec d'autant plus de plaisir qu'ils affineront le schéma général mais n'en changeront probablement pas l'économie globale, laquelle semble très solide. Elle s'accommode par exemple fort bien du fait que la puissance de l'humour puisse se répartir sur différents niveaux d'effet cognitif (voir note supra), mais que l'on puisse, pour des raisons pratiques, préférer la considérer à l'occasion comme un facteur en soi, de niveau intermédiaire.

En épluchant la «longue liste d'épicerie des variables affectant l'intérêt » dressée par Silvia, on ne peut y trouver - une fois éliminées celles qui relèvent en réalité de l'effort cognitif - que deux variables ne s'intégrant pas de façon évidente à l'une de ces familles : l'émotion et le suspense. La première se réfère à un état mental large et transversal, qui paraît plus être une résultante des facteurs ci-dessus (on est ému par la violence, par les risques personnels ou par d'«autres choses » de plus haut niveau) qu'une cause première circonscrite sérieusement. Oublions-la. Quant au suspense, on pourrait lui aussi le rapprocher du premier groupe (un film à suspense tourne plus ou moins autour de la question «Mourra-t-il ou pas ?", quoiqu'en général il ne meure pas $\left.{ }^{11}\right)$, mais aussi, et surtout de l'imprévisibilité qui caractérise les facteurs stochastiques (que va-t-il se passer ?), sans exclure la possibilité d'« autres choses » (voir Baroni, 2007). Ainsi remarquera-t-on que la couleur de la robe évoquée plus haut, de même que la propension de Dieu à briser les pipes qu'on lui prête, génèrent une incertitude stochastique qui pourrait en expliquer la pertinence cognitive, comme elle pourrait contribuer à expliquer l'efficacité des récits, non seulement en littérature, mais aussi en matière d'actualité et même dans la communication politique ou commerciale (d'où l'essor du storytelling, sur lequel on reviendra dans la seconde partie de ce livre).

Et les petits chats d'Internet? Bien qu'il s'agisse moins d'une composante quantitativement significative du marché discursif que d'un symbole commode (et d'un cliché), on remarquera que beaucoup d'entre eux paraissent faire des choses incongrues, donc surprenantes, drôles... Ou arborer des expressions incongrues. Mais pas tous. D'autres sont simplement mignons (les plus intéressants sont incongrus et mignons). Voilà une caractéristique qui n'est pas sur la

11 Games of Throne étant la fascinante exception qui confirme la règle, d'où l'effet cognitif peu ordinaire que produit George R. R. Martin en massacrant successivement tous ses héros. 
«liste d'épicerie ${ }^{12}$. » On peut, sans trop d'hésitation, adoucir grâce à elle la brutale famille des facteurs de bas niveau. Comme ces derniers, elle est essentiellement instinctive et très peu dépendante des connaissances préalables : un profil de phares et de calandre suffit à donner à une voiture l'air mignon (ou méchant), même si l'on sait bien qu'il ne s'agit que d'une machine. Les éthologues attribuent même à la sensibilité à la «mignonnerie » (cuteness) de profondes motivations évolutionnistes, à l'instar des autres pulsions fondamentales : elle serait - pour résumer - ce qui poussait nos lointains aïeux à nourrir leurs enfants plutôt qu'à les manger (Glocker, Langleben et coll., 2009). Ne voir dans les calendriers et cartes postales de chatons que les fossiles psychologiques des impératifs de préservation de l'espèce serait assez réducteur, comme toute explication purement évolutionniste du comportement humain (voir supra l'exemple de Davis et McLeod, 2003). La mignonnerie a sans doute un caractère rassurant et plausiblement une surdétermination sociale : on sait par exemple combien il est périlleux de ne pas trouver un bébé mignon. Mais au risque d'indigner les fabricants de peluches, il est difficile de ne pas la considérer comme un facteur d'effet de bas niveau, au même titre que le sexe. Toujours sans jugement de valeur, bien sûr.

Si l'on devait chercher des facteurs d'effet plus complexes, on pourrait songer à l'empathie (laissons donc l'évolution en dehors de ça, quelles qu'aient pu être la solidarité des préhominidés ${ }^{13}$ ou la sympathie mutuelle des vertébrés primitifs), ou encore à la perception de la beauté, en se limitant à la fragile partie de cette sensation qui n'a pas trop de ressorts copulatoires, disons la beauté d'un paysage ou d'un adagio. Sur ce dernier point, le philosophe Jean-Marie Schaeffer a développé une argumentation imposante à l'appui de la thèse selon laquelle «l'expérience esthétique fait partie des modalités de base de l'expérience commune du monde et [...] exploite le répertoire commun de nos ressources attentionnelles, émotives et hédoniques, mais en leur donnant une inflexion non seulement particulière, mais bien singulière » (2015, p. 13, c'est moi qui souligne). Parmi ses mérites, elle compte celui de « séparer clairement l'esthétique de l'artistique» (p. 32), le premier ne correspondant

12 À moins qu'elle ne fasse partie du «caractère émouvant », vaste et flou sentiment évoqué un peu plus tôt.

13 Mais l'on pourra sur ce point se référer par exemple à Frans de Waal (1997). 
qu'à une expérience cognitive, indépendamment de la dignité attribuée ou non à son objet. On converge plus ou moins là avec David Hume qui attribuait le sentiment esthétique au « ressort principal de l'esprit humain ", la dualité peine/plaisir : "l'objet qui est qualifié de beau ne plaît que par sa tendance à produire un certain effet » (1888/1740, p. 574-576).

Faut-il pour autant voir dans la beauté (ou l'empathie) un déterminant supplémentaire de l'effet cognitif, ou encore l'intégrer aux facteurs déjà explicités ? Le travail de Schaeffer montre que la première option est tout à fait plausible. Cependant, bien qu'il se fonde sur quelques apports théoriques et empiriques des sciences cognitives, les rapprochements intéressants qu'il opère entre eux n'emportent pas les hésitations que l'on peut avoir ici à subdiviser encore plus une nomenclature dont la robustesse, plutôt que la finesse, est le critère premier. C'est ici que le vague fourre-tout de la catégorie supérieure (les « autres choses ») prend toute sa valeur épistémologique, d'autant que, comme on l'a dit, ses bords sont assez flous pour ne pas avoir à statuer trop abruptement sur ce qui relève ou non de la psychologie. La prudence scientifique et mon penchant naturel pour la facilité se conjuguent donc pour ranger, en tout cas jusqu'à nouvel ordre, ces variables du côté des «autres choses ", sans contester que l'on puisse raffiner cette catégorie.

C'est l'occasion de souligner, au cas où ça ne serait pas évident, que les familles de facteurs explicitées (plus ou moins) ci-dessus sont des repères - des outils conceptuels - et non des catégories "naturelles » qu'imposerait une quelconque structure fondamentale de la réalité humaine : il n'existe pas plus «trois» niveaux d'effet cognitif qu'il n'existe « cinq » sens ou " trois » niveaux de langage ${ }^{14}$. Prenons le cas de l'une des variables les plus ambivalentes qui soient, celle de la « familiarité », laquelle se joue mieux qu'une autre des petites boîtes dans lesquelles on tenterait de l'enfermer. Comme le trahit l'allusion un peu embarrassée de Kintsch à ces «choses intéressantes [...] qui ne sont ni trop familières ni trop étranges ", la familiarité peut contribuer à l'effet cognitif

14 À tout prendre, parler de trois niveaux d'effet est plus légitime que de parler des cinq sens (nous en avons beaucoup plus) ou des trois, ou quatre, ou cinq niveaux de langage (ce ne sont que des gradations arbitraires dans une variation continue). Mais toutes les segmentations de ce type, y compris la fragile dichotomie nature-culture, n'en sont pas moins utiles pour organiser conceptuellement et pédagogiquement le réel, c'està-dire réduire l'effort cognitif que réclame sa complexité. 
par sa présence (dans une certaine mesure), comme par son absence (dans une certaine mesure). Ça n'est pas très pratique, mais c'est ainsi.

D'une part, il est solidement établi que l'on s'intéresse d'autant plus à une information qu'elle concerne plus le paysage qui nous est familier. Cet axiome se traduit par exemple par le caractère autocumulatif de l'attention, déjà évoqué (plus on en sait sur un thème, plus on est enclin à en apprendre à son sujet) bien que cette pertinence soit aussi liée à l'effort cognitif plus faible que réclame le traitement d'un sujet connu. Il se traduit aussi par l'un de ces adages professionnels qui tantôt marchent tantôt ne marchent pas, le «principe de proximité », en vertu duquel un évènement serait d'autant plus intéressant qu'il est spatialement ou socialement proche du lecteur. Comme on le voit, cet adage tient largement à l'interaction avec la sphère personnelle. Selon sa version la plus brutale, la « loi du mort/km », un accident dans les environs équivaut à un désastre de plus grande ampleur dans une région voisine, ou à une catastrophe majeure à l'autre bout du monde. Et, bien sûr, ce qui touche les proches, famille ou amis, ou simplement les homologues socioprofessionnels (les boulangers s'intéressent à ce qui arrive à un boulanger, les étudiants à un étudiant...) ne peut manquer de capter l'attention.

Mais, d'autre part, il est solidement établi que ce qui est familier, connu, habituel, est mortellement ennuyeux. Selon un autre adage professionnel de base, un train qui arrive à l'heure n'est pas une nouvelle, et un chien qui mord un passant est moins intéressant qu'un passant qui mord un chien. De fait, on tourne plus de documentaires dans la savane africaine que dans les cours de fermes (le dépaysement est par ailleurs une motivation touristique première) et un scénario que l'on pourrait résumer par «Des gens ordinaires à qui il arrive des choses ordinaires » a plus de chances d'être celui d'un film d'auteur que d'une superproduction hollywoodienne. Cependant, l'extra-ordinaire n'est pas l'apanage des productions commerciales de masse. Si l'on prête à Samuel Goldwyn (celui de la Metro-Goldwyn-Mayer) la maxime ${ }^{15}$ selon laquelle un bon scénario "doit commencer par un tremblement de terre et aller crescendo jusqu'à l'apothéose finale », on peut à l'opposé $^{16}$ rappeler qu'une théorie de l'effet artistique, celle de Victor

15 On l'attribue aussi à Cecil B. DeMille, qui fut un temps son associé.

16 L'opposition n'est que partielle : un superhéros aux pouvoirs extraordinaires peut se trouver devant des dilemmes qui ne sont que des 
Shklovsky, fait justement reposer la valeur culturelle sur la défamiliarisation (ostranénie) que provoque une véritable œuvre, sa capacité à nous projeter hors de la perception familière du réel (voir dans le même sens Wolfgang Iser, 1970/2012). De même, quoique fort différemment, l'esthétique de la réception d'Hans Robert Jauss voit dans l'écart entre les écrits prévisibles et les œuvres inattendues une clef de l'effet que celles-ci produisent lors de leur parution ${ }^{17}$.

En somme, ce qui est familier est intéressant (impliquant) de même qu'inintéressant (banal), tandis que ce qui n'est pas familier est inintéressant (indifférent), ainsi qu'intéressant (captivant). En particulier pour les productions les plus commerciales. De même que pour les créations littéraires ou artistiques les plus exigeantes...

L'éthologie et la neurologie, qui règnent sur les facteurs de bas niveau, bafouillent elles aussi ici. On sait notamment que la perception animale ignore rapidement l'odeur, la vue ou le bruit auquel elle s'accoutume. Mais aussi qu'elle se détourne assez vite d'un objet inconnu, inclassable, dès lors qu'il ne présente pas de danger ou d'intérêt gastronomique (il devient de ce fait à la fois étrange et familier, sans rester le moins du monde intéressant).

Tout ceci revient au bout du compte à indiquer que la familiarité/ défamiliarité, en tout cas dans l'acception large et vague qu'illustre l'hétérogénéité volontaire des exemples ci-dessus, n'est pas en soi un facteur d'effet, mais plutôt une modulation d'autres facteurs. Ainsi comprend-on qu'elle puisse, selon les cas, jouer au plus bas niveau cognitif $^{18}$, mais aussi, voire surtout concerner la sphère personnelle (famille, etc.) et, bien sûr, la dimension stochastique (imprévisibilité). Sans oublier, sans doute, beaucoup d'« autres choses » de plus haut niveau.

La pluralité de ses voies se manifeste en particulier dans ce qui est apparemment l'un des sujets les plus intéressants qui soient : la vie

clichés rebattus, tandis qu'un personnage banal peut susciter des perceptions qui transcendent la banalité de l'existence.

17 On reviendra dans l'annexe de ce chapitre («Précisions») sur quelques apports et limites des approches classiques de la valeur littéraire.

18 La mort, le danger, le sexe, etc. ne sont « de bas niveau » qu'en tant que stimulis instinctifs : l'examen scientifique d'un risque ou la réflexion ontologique aiguillonnée par la finitude de l'existence humaine sont d'une autre nature. 
privée des célébrités. Si les amours tumultueuses des vedettes sont suivies avec tant d'attention, toute considération sexuelle mise à part, c'est plausiblement parce que celles-ci sont familières par définition (être une vedette, c'est être « connu ») et s'intègrent même à la sphère personnelle de ceux qui les suivent (on les désigne souvent par leurs prénoms), tout en étant extraordinairement exotiques par le mode de vie fascinant qu'on leur prête : ce sont, comme le résume Richard Schikel (2015), des "étrangers intimes », vis-à-vis de qui Nathalie Heinich (2012) a souligné le caractère très personnel de l'attachement (identification, désir de proximité, voire d'intimité). Quant aux incidents ou autres qui les affectent, ils sont stochastiques par définition (être un incident, c'est être inattendu). Mais le personnage de Chesterton pour qui le journalisme "consiste largement à dire "Lord Jones" est mort à des gens qui n'avaient jamais su qu'il était vivant » (1914, p. 164) se méprend sérieusement sur son métier : les Lord Jones inconnus de ce monde mourront inconnus, à moins, bien sûr, de connaître une fin extra-ordinaire.

Quoi qu'il en soit, ce genre de spéculation n'est pas indispensable. En l'état, la nomenclature minimale des niveaux d'effet cognitif proposée plus tôt par Walter Kintsch reste au bout du compte la plus fiable, même s'il semble opportun, comme on l'a vu, de l'assortir de modulations.

\begin{tabular}{|c|c|c|c|}
\hline \multicolumn{2}{|r|}{ Déterminants } & \multicolumn{2}{|c|}{ Modulations } \\
\hline \multirow{3}{*}{ Bas niveau cog. } & $\begin{array}{l}\text { Spontanés } \\
\text { (mort, sexe, mignonnerie...) }\end{array}$ & \multirow{3}{*}{ 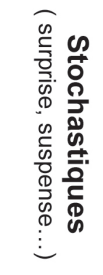 } & \multirow{3}{*}{ 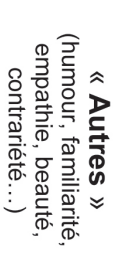 } \\
\hline & $\begin{array}{l}\text { Égocentriques / utilitaires } \\
\text { (personne, proches, homologues...) }\end{array}$ & & \\
\hline & $\begin{array}{l}\text { Élaborés } \\
(\text { («autres »..) }\end{array}$ & & \\
\hline
\end{tabular}

Figure 2. Les principales composantes de l'effet cognitif

Quoique ces modulations s'apparentent assez à des facteurs d'effet pour que l'on puisse si l'on préfère les considérer comme tels, on peut plausiblement les en distinguer en fonction de deux critères, la conjonction et la réversibilité :

- Alors que les trois familles élémentaires de déterminants sont relativement autonomes, les modulations 
tendent plutôt à se conjuguer avec les précédentes, quel qu'en soit le niveau. La mort, le sexe, l'entourage, etc., sont intéressants «en soi », alors que des propriétés comme inattendu, nouveau, mais aussi drôle, familier, poignant, beau, étonnant, etc. s'appliquent généralement à quelque chose (par exemple une mort surprenante, ou affligeante, ou même drôle $\left.{ }^{19} . ..\right)$.

- Alors que les déterminants des catégories élémentaires sont relativement monovalents, les modulations sont généralement réversibles, c'est-à-dire que leur opposé produit également de l'effet : ce que l'on a remarqué pour l'ambivalence familiarité/étrangeté pourrait l'être aussi pour le couple beauté/laideur ou empathie/antipathie (ce qui est beau ou sympathique est peut-être intéressant, mais ce qui est difforme ou détestable peut l'être tout autant). Ce point incite à ajouter une autre variable dont le caractère paradoxal a été relevé avec un peu d'embarras par les travaux sur la «valeur informative» (voir «Précisions » ci-dessous) : le degré de contrariété d'une information, c'est-à-dire le bilan négatif ou positif de ce qu'elle rapporte. Ainsi, les médias et leurs publics sont-ils réputés privilégier les mauvaises nouvelles, quoiqu'ils aiment aussi les bonnes. De même, le théâtre exige une fin heureuse (comédie) ou tragique (drame). Dans tous les cas, les informations neutres paraissent avoir moins de valeur que celles qui penchent clairement dans un sens ou dans l'autre.

La nomenclature qui résulte de cette exploration de l'effet cognitif n'est pas aussi raffinée que l'on pourrait la rêver, surtout si l'on considère que deux cases sur cinq sont intitulées « autres » (insistons sur le fait qu'il n'y a là aucune désinvolture : la désinvolture serait de prétendre légiférer en la matière). On verra cependant qu'elle est tout à fait suffisante dans notre perspective, non seulement parce qu'elle est beaucoup plus précise que la «modification des croyances de l'individu » évoquée par Sperber et Wilson, mais aussi parce qu'elle paraît empiriquement plus solide et analytiquement plus opérante.

19 Comme celles des malheureux récipiendaires des «Prix Darwin » qui récompensent les façon les plus stupides de trépasser. 
Ainsi rend-elle assez bien compte de certaines des dynamiques caractéristiques de l'offre et de la demande sur le marché discursif. En 2013, par exemple, les chroniqueurs ont relevé avec effarement que le prix du public du Salon du livre de Montréal, catégorie Essais, avait été attribué à un ouvrage intitulé La mijoteuse : De la lasagne à la crème brûlée. Sans débattre ici de l'importance à accorder aux recettes de lasagnes dans l'histoire de la pensée humaine, l'incident force à rappeler que les hautes castes de la hiérarchie discursive ne représentent qu'une assez mince fraction de la production livresque vendue et consommée ${ }^{20}$ : les guides pratiques et autres ouvrages concernant directement la sphère personnelle y occupent une place à peu près équivalente à celle de la fiction et très supérieure à celles des sciences humaines.

C'est toutefois sur le marché des nouvelles que l'influence des grandes familles d'effet cognitif se fait le plus directement sentir. Les facteurs de bas niveau, bien sûr, s'y exercent de longue date. Avant même la parution au XviI siècle du premier journal francophone, des parutions irrégulières rapportaient des crimes abominables ou des histoires de monstres anthropophages, toujours présentées comme «très véridiques». Mais la naissance du journalisme moderne et la concurrence entre les quotidiens de masse ont occasionné dans la seconde moitié du XIX siècle un regain d'appétit pour les façons intéressantes de trépasser, telles que les détaillait très méthodiquement une note de service ${ }^{21}$ envoyée en 1883 par l'agence de presse Reuters à ses correspondants :

En conséquence de l'intérêt croissant qu'accordent la presse de Londres et la presse de province anglaise aux désastres, etc., de toutes sortes, il est demandé aux agents et correspondants d'avoir, dans l'avenir, la bonté de signaler [au siège de Londres] tous les cas de ce genre. Les évènements qui suivent font partie de ceux qui devraient être compris dans ce service: les incendies, les explosions, les inondations, les accidents de chemin de fer,

20 En France, par exemple, les sciences humaines et sociales représentaient $4,3 \%$ des livres vendus en 2014 et la littérature générale $15,6 \%$, dont seulement 1,4\% pour les œuvres classiques (Syndicat national de l'édition, 2015).

21 Reproduite in Read, 1999. 
les tempêtes destructrices, les tremblements de terre, les naufrages avec pertes humaines, les accidents à des vaisseaux de guerre anglais et américains ou des vaisseaux postaux, les combats de rue ayant un caractère grave, les désordres engendrés par des grèves, les duels entre - et les suicides de - personnalités politiques ou sociales, et les meurtres présentant un caractère spectaculaire ou atroce.

Le second degré d'effet cognitif, l'« interaction avec la sphère personnelle », accompagnait lui aussi la presse depuis ses origines : pour justifier le lancement de la Gazette en 1631, Théophraste Renaudot mettait ainsi en avant l'« utilité qu'en reçoivent le public et les particuliers », chacun, grâce aux nouvelles, "ajustant volontiers ses affaires au modèle $d u$ temps [...] sans parler du soulagement qu'elles apportent à ceux qui écrivent à leurs amis » $(1632$, p. 5). Toutefois, c'est à nouveau la concurrence attentionnelle, cette fois-ci celle de la radio et de la télévision, qui a conduit les quotidiens à accentuer le recours à ce ressort, rebaptisé « information-service». C'était là une francisation du concept de «news you can use » développé en 1979 par l'association américaine des éditeurs de presse après qu'une étude marketing de grande ampleur eut souligné "l'importance $d u$ "moi" » dans l'évolution des attentes des lecteurs. Quoique sa partie la plus trivialement utilitaire - par exemple les horaires des cinémas ou des pharmacies - ait assez mal résisté à la disponibilité des informations pratiques sur Internet, cette dimension personnelle a conservé toute son importance dans l'offre discursive des entreprises de presse actuelles. En témoignent non seulement les sections dédiées à la santé, au bien-être ou encore à la gastronomie (équivalents journalistiques des livres évoqués plus haut), mais aussi celles portant sur la finance ou la météo, et, bien sûr, les couvertures inlassablement consacrées au salaire des cadres et aux prix de l'immobilier, toutes choses dont l'intérêt (ou non) est directement lié au vécu du lecteur.

Il va toutefois de soi, à moins d'adhérer aux critiques les plus caricaturales et condescendantes de la production journalistique, que celle-ci est loin de se réduire à ces effets puissants : son contenu fait très largement appel à des facteurs de haut niveau, ceux qui mettent le plus directement en jeu les connaissances sur le monde. Même le magazine Play Boy était réputé non seulement pour l'intérêt de ses photos, mais aussi pour celui de ses grandes interviews et des textes 
qu'il commandait à des auteurs comme Saul Bellow, John Irving ou Margaret Atwood ${ }^{22}$. Du reste, la hiérarchie cognitive des facteurs d'effet, bien qu'elle soit par nature dépourvue de tout jugement de valeur, paraît étonnamment parallèle à la hiérarchie symbolique du journalisme. Plus importante est la place que les médias imprimés ou audiovisuels - par exemple les stations de télévision locale étatsuniennes - allouent aux facteurs de bas niveau cognitif (crimes, accidents, etc.), plus bas est le statut qui leur est accordé dans le champ de l'information.

Il va aussi de soi que ces variables ne sont pas des « choses » qui s'exercent mécaniquement et uniformément : de même que le sens n'est pas à l'intérieur du discours, mais résulte de l'interaction entre ce discours et son destinataire, de même les niveaux d'effet cognitif sont universels dans leur influence - nul n'y échappe-, mais non dans leur intensité. C'est essentiellement le cas des facteurs de plus haut niveau, dont l'attractivité varie dans une certaine mesure d'un individu à l'autre. Ainsi, l'usage intensif des réseaux sociaux est-il négativement corrélé non seulement aux résultats universitaires, mais aussi au « besoin de connaître » évoqué plus haut (Zhong, Hardin et al., 2011). D'autre part, l'intérêt n'est pas simplement produit par le thème du discours : il réside aussi - comme on le verra plus loin - dans l'habileté ou non avec laquelle les différents facteurs d'effet sont exploités par les producteurs discursifs.

Il va enfin de soi que ces variables ne s'excluent pas mutuellement. Au contraire, leur combinaison est généralement ce qui confère le plus d'intérêt à une information. Ainsi, l'accident nucléaire de Fukushima a-t-il mobilisé l'attention d'autant plus fortement qu'il associait (outre l'imprévisibilité qui est en théorie[ $\mathrm{s}^{23}$ l'essence de toute nouvelle) la violence meurtrière de la catastrophe qu'il couronnait, mais aussi l'inquiétude personnelle que suscitait la possible dissémination planétaire des particules radioactives ainsi que le désir de

22 Bien entendu, il y avait une forte dimension de légitimation dans le choix de ces contenus, mais laissons ça pour le moment.

23 C'est-à-dire à la fois dans la théorie mathématique de l'information (abhorrée en sciences de la communication pour son réductionnisme et pour d'autres raisons plus troubles) et dans la théorie praticienne du journalisme, dont l'essence réside selon les manuels professionnels dans le fait de rapporter ce qui est nouveau. 
comprendre, entre autres, l'enchaînement technique d'un accident réputé impossible.

C'est ici qu'il serait opportun, pour dépasser ce genre d'illustration anecdotique, de s'appuyer sur des données qualitatives et quantitatives solides. Les données ne manquent certes pas, en particulier toutes celles qui ont été collectées dans l'espoir de percer le secret de la valeur journalistique des informations ("news value» ou "newsworthiness »). Malheureusement, leur bilan n'est pas exactement ce que l'on pourrait qualifier de solide. Il est même si problématique d'un point de vue théorique ou méthodologique qu'il vaut sans doute mieux en repousser l'examen dans une section optionnelle. 
Page blanche conservée intentionnellement 


\section{(Précisions)}

Tly a décidément plus d'une façon de poser la question de l'intérêt. On se souvient par exemple que Rudolf Flesch (1948), confronté à la logique de la pertinence cognitive, avait été contraint d'équilibrer sa formule de lisibilité (qui quantifiait l'effort de traitement lexico-syntaxique) par une autre formule calculant cette fois l'« intérêt humain » des textes. Habitué à mesurer des mots et des phrases, il avait conservé ces unités dans sa formule d'intérêt humain, laquelle reposait sur deux variables : le pourcentage de "mots personnels », soit tout ce qui ne relève pas du genre neutre en anglais (he, her, mais pas it), et le pourcentage de «phrases personnelles » (soit toutes celles contenant un dialogue, une interrogation, une injonction ou une exclamation). Mais bien que cette formule paraisse relativement sensée, à défaut d'être très subtile, sa fiabilité était pour le moins incertaine.

Pour sa part, Abraham Moles avait dans les années 1960 abordé la question de façon plus théorique et envisagé l'intérêt comme le rapport avec le «taux d'abstraction » (ce qu'on appellerait ici l'effort cognitif) et la «distance à l'individu », laquelle ressemble un peu à ce que l'on désignait comme l'interaction avec la sphère personnelle. Mais une fois ajouté un troisième axe plus ou moins psychanalytique, la « couche de profondeur de l'individu », la tentative de quantification qui en résultait devenait assez difficile à faire coïncider avec le marché discursif, des informations proches par leurs thèmes pouvant se retrouver à des emplacements très éloignés de ce complexe tableau tridimensionnel (Moles, 1967).

D'autres ${ }^{1}$, à la suite d'Harold Lasswell, ont tenté d'établir globalement, mais un peu arbitrairement, les « fonctions » essentielles

I Il serait bien trop long, redondant et imprudent d'essayer d'exposer ici dans le détail la multitude des théories communicationnelles de la 
que rempliraient les médias de masse : trois fonctions au départ - la surveillance de l'environnement, la coordination des composantes de la société et la transmission de l'héritage culturel - auxquelles on s'avisa bientôt de rajouter, entre autres, la distraction. Il s'agissait plutôt là de rôles organiques attribués aux médias dans le fonctionnement et la préservation d'une machinerie sociale idéale (ou cauchemardesque). Le courant ultérieur, dit des « usages et gratifications ", a renversé cette perspective pour adopter celle des publics, mais sans vraiment abandonner ce type de composantes. Ainsi, Denis McQuail (2010, p. 356) a-t-il résumé comme suit les "principales " gratifications qui seraient recherchées ou obtenues des médias :

Information et éducation
Orientation et conseils
Distraction et relaxation
Contact social
Renforcement des valeurs
Satisfaction culturelle
Relâchement émotionnel
Formation et confirmation de l'identité
Expression du mode de vie
Sécurité
Stimulation sexuelle
Passe-temps

L'examen de cette nouvelle "liste d'épicerie », synthétisant pourtant des recherches de terrain très substantielles, montre à nouveau les problèmes que rencontre tout inventaire faisant appel à des désignations englobantes et dépourvues de hiérarchisation : non articulées en théorie, elles sont peu opératoires en pratique. Quoiqu'elles soient toutes très plausibles, le recouvrement de ces catégories est tel qu'elles semblent à chaque instant sur le point de se dévorer

réception : outre les nombreux ouvrages qui l'abordent mieux que l'on ne peut l'envisager ici, dont celui de Breton et Proulx déjà évoqué (1989), on en trouvera un examen collectif approfondi dans le numéro 11-12 que la revue Hermès a consacré sous la direction de Daniel Dayan, au thème "À la recherche du Public : Réception, Télévision, Médias » (1993). 
mutuellement et que le moindre fait divers pourrait s'inscrire dans la moitié d'entre elles. Certes, leur angle n'était pas la question «pourquoi ce qui nous plaît nous plaît-il ? : elles visaient plutôt à répondre à sa cousine "qu'est-ce que les gens retirent des médias?" Mais même dans cette perspective, un modèle structuré comme la pyramide des besoins de Maslow pourrait, à tout prendre, s'avérer plus éclairant, bien que sa validité empirique soit pour le moins sujette à débat et qu'il n'ait pas été conçu à cette fin.

Gardons-nous bien de jeter le bébé avec l'eau du bain. Outre que la perspective des "usages et gratifications» a apporté une bouffée d'oxygène à un domaine de recherche asphyxié par la question hégémonique des effets des médias sur l'opinion ${ }^{2}$, ses prémisses gardent toute leur pertinence et nombre de ses apports scientifiques ou conceptuels seraient d'autant plus difficiles à contester (de bonne foi) que l'essor des médias sociaux ne cesse apparemment de les conforter. Mais, outre son ancrage sociologique assez mince 3 , la composante psychologique que voulait intégrer ce programme demeure un échec patent, ce rapprochement n'ayant guère évolué depuis le constat désabusé de ses promoteurs dans les années 1970 :

L'étude de l'usage des mass medias souffre actuellement de l'absence d'une théorie pertinente des besoins sociaux et psychologiques. Ce qui manque est moins un catalogue de besoins qu'un regroupement d'agrégats de besoins, un classement des différents niveaux de besoins, et une formulation d'hypothèses reliant des besoins particuliers avec des gratifications médiatiques particulières. (Katz, Blumer et Gurevitch, 1973, p. 513)

2 Question dont l'importance scientifique et politique est suffisamment évidente pour n'avoir pas besoin d'être soulignée tant que sa prépondérance ne sature pas la pensée dans ce domaine. En 1959, déjà, Katz remarquait que la réduction de la recherche en communication à la problématique de l'influence pouvait la faire percevoir comme « morte ou mourante » alors qu'il "y a plus, beaucoup plus dans les médias de masse que la persuasion » (1959, p. 1-2).

3 Comme en convenait d'ailleurs Blumer (1979), théoriser les origines sociales des besoins et usages médiatiques empiriquement catégorisés restait un sérieux défi. 
On aurait mauvaise grâce à le leur reprocher, l'état des connaissances sur le traitement cognitif des discours étant loin de permettre d'aborder ce genre de question à cette époque (et encore moins à celle de Flesch ou de Moles, ou a fortiori de Pascal ou de Platon). Il l'est tout juste assez aujourd'hui pour que l'on tente de s'attaquer à ce qu'ils identifiaient eux aussi comme un défi essentiel, le " problème de longue date de la science sociale et psychologique: comment [...] tirer un système des longues listes des besoins humains et sociétaux » (id., p. 514).

En outre, et de façon plus générale, les enquêtes inductives menées auprès des publics - non seulement dans le cadre des travaux sur les "usages et gratifications", mais plus encore dans celui des études marketing des industries culturelles - se heurtent aux limites bien connues de la confiance à accorder aux affirmations des répondants dans ce domaine sensible. En supposant contre toute vraisemblance que chacun connaisse réellement ses attentes et comprenne leurs motivations, serait-il vraiment prêt à les révéler?

Pour contourner cette difficulté, on peut aussi aborder le marché discursif dans l'autre sens, c'est-à-dire en partant non de la demande, mais de l'offre éditoriale. Celle-ci est par nature «sincère » dans sa globalité, si l'on entend par là qu'elle s'efforce en général de sélectionner les discours en fonction de la valeur qu'elle leur prête sur le marché. On verra plus loin que ce jugement - outre qu'il est tempéré par des principes professionnels - est très loin d'être infaillible, mais il n'est pas pour autant dépourvu de fondements empiriques : à la bourse des valeurs informationnelles, la cote est après tout fixée sur la base d'un ou deux siècles de succès et d'échecs éditoriaux. D'où l'intérêt de s'interroger sur les critères selon lesquels les professionnels évaluent cette valeur, d'autant que, comme le soulignait Gans (1979), les jugements de ceux-ci sont souvent proches.

La nomenclature la plus connue dans ce domaine est celle publiée par les Norvégiens Galtung et Ruge en 1965. Se fondant sur une "psychologie de la perception» (sic) de leur cru, apparemment inspirée de la théorie mathématique de l'information, elle comporte douze variables : 
- huit universelles : la fréquence (c'est-à-dire la durée : les évènements se déroulant lentement ont moins de valeur informative que ceux qui s'insèrent entre deux éditions); l'amplitude (c'est-à-dire la magnitude : plus les conséquences sont importantes, plus l'évènement l'est) ; la non-ambiguïté (clarté du signal) ; l'interprétabilité (proximité culturelle et «pertinence») ; la consonance avec une image mentale préalable; le caractère inattendu et la continuité dans le temps ;

- quatre culturellement déterminées : l'intégration au mélange global d'information offert par le média ; la référence aux nations dominantes; la référence aux célébrités, la référence aux individus (personnification), et enfin la référence aux situations indésirables (négativité).

Non sans être conscients de quelques-unes des tensions internes de cette liste ("la sixième hypothèse apporte une correction à la quatrième et la cinquième »), Gatlung et Ruge l'ont appliquée à l'analyse $\mathrm{du}$ traitement de trois crises internationales dans quatre quotidiens norvégiens et ont constaté que, comme il se devait, ce corpus confirmait leurs hypothèses.

Ceci n'a pas empêché d'autres auteurs d'en souligner certains problèmes ou de proposer des listes complémentaires ou alternatives.

Remarquant que l'un de ces problèmes résidait dans le fait d'avoir sélectionné des évènements de même nature (les crises internationales), Harcup et $\mathrm{O}^{\prime} \mathrm{Neill}$ ont entrepris une démarche inverse : ils ont analysé sans les filtrer a priori les quelque 1300 articles principaux publiés durant un mois par trois quotidiens britanniques représentatifs du spectre journalistique (populaire, intermédiaire et «de qualité »). Ainsi ont-ils constaté que tous ces articles pouvaient être rattachés d'une façon ou d'une autre à un ou plusieurs critères de Galtung et Ruge, quoiqu'« il soit rapidement devenu manifeste que ces facteurs ne pouvaient être identifiés dans les pages des quotidiens qu'au moyen d'une copieuse quantité d'interprétation subjective ». En outre "nous étions fréquemment confrontés à des questions comme "qu'est-ce qu'un évènement non ambigu" ou "la référence à quelque chose de négatif pour qui ?" » (Harcup et O’Neill, 2001, p. 268). Mais les chercheurs remarquèrent surtout qu'un bon 
nombre d'autres aspects jouaient un rôle essentiel, par exemple la disponibilité ou non de photos, la dimension sexuelle, la drôlerie et même, la référence aux situations positives.

En vertu de quoi ils proposèrent une nouvelle liste, à la fois élargie et simplifiée, des valeurs informationnelles :

- les puissants (individus, organisations ou institutions) ;

- les célébrités ;

- le distrayant (sexe, show-business, intérêt humain, animaux, humour, présence de photos...) ;

- le surprenant;

- les mauvaises nouvelles ;

- les bonnes nouvelles (sauvetages, traitements...) ;

- la magnitude (nombre des personnes impliquées ou impact potentiel) ;

- la pertinence (sujets, groupes ou nations perçues comme pertinents pour le public) ;

- la persistance (prolongation de sujets déjà dans l'actualité) ;

- la concordance avec les perspectives du journal.

Quinze ans plus tard, Harcup et $\mathrm{O}^{\prime}$ Neil se sont remis à la tâche sur un nouveau corpus, afin de prendre en compte les travaux ultérieurs, mais aussi les bouleversements associés à l'essor des réseaux sociaux. Ce réexamen les a conduits à confirmer leur liste (Harcup et $\mathrm{O}^{\prime} \mathrm{Neill}$, 2017), en l'enrichissant de quelques critères :

- l'exclusivité (scoops, sondages...) ;

- le caractère conflictuel (controverses, grèves et manifestations, guerres...) ;

- le support audiovisuel (disponibilité d'images, de vidéos, possibilité d'infographie...) ;

- la dramaturgie (trame narrative) ;

- la partageabilité (potentiel de retransmission sur les réseaux sociaux).

Bien sûr, les recherches sur la valeur médiatique n'entendent pas plus que celles sur les «usages et gratifications» répondre à la question «pourquoi ce qui nous plaît nous plaît-il ? » : elles visent 
une autre de ses cousines "pourquoi ce qui plait aux professionnels des médias leur plaît-il ? " (pour mesurer la différence considérable entre les deux, voir Lee et Chyi, 2014). Dans cette optique, elles semblent présenter une certaine valeur prédictive : plus un sujet embrasse ces différents critères, plus il a généralement de chances d'être publié. Cependant, il faut un peu d'agilité pour expliquer au moyen de ces variables l'enthousiasme avec lequel la presse a, par exemple, salué la résolution mathématique du dernier théorème de Fermat à la fin des années 1990. Ce qui incite à se demander si la longueur d'une « liste d'épicerie » qui voudrait vraiment prévoir tous les motifs de publication possibles ne serait pas infinie. À moins d'user de tautologies, comme le font déjà craindre les références ci-dessus au «distrayant» et au «pertinent». Encore ne s'agit-il ici que des articles d'actualité et non des autres composantes de l'offre médiatique (pour ne pas parler de l'offre littéraire, cinématographique ou de la communication interne ou externe des organisations) : même en s'en tenant à la presse quotidienne, les chercheurs ont choisi explicitement - et à juste titre compte tenu de leur perspective - de faire abstraction de textes tels que les éditoriaux, les chroniques, les critiques, etc., qui, selon les cas, peuvent ou non avoir d'autres raisons d'être publiés et lus. Plus généralement, souligne Pamela Shoemaker, le concept de valeur de nouvelle (newsworthiness) "ne peut jamais entièrement prédire ce qui deviendra de l'actualité, et pourquoi le pourrait-il ? La valeur de nouvelle est une réalisation mentale, une pensée ou un jugement, alors que l'actualité publiée est un artefact social, une chose" (2006, p. 110).

Ainsi, les multiples recherches empiriques (inductives) sur la valeur prêtée aux informations médiatiques permettent difficilement en l'état d'affiner un modèle théorique des différents facteurs cognitifs en jeu, et encore moins de l'établir. Ce n'est pas comme ça que l'on résoudra le "problème de longue date de la science sociale et psychologique " (Katz et al., supra). Mais au minimum, ces éclairages peuvent servir de liste de contrôle à un tel modèle. Or, une fois mis de côté tout ce qui ne concerne pas la cognition au sens strict, le reste des variables proposées converge clairement avec la grille proposée dans le chapitre précédent. On pourra cependant en retenir une ou deux modalisations supplémentaires, comme le caractère positif ou négatif des 
informations et éventuellement les enjeux de pouvoir, que Schank rangeait déjà, comme on l'a vu, parmi les facteurs de bas niveau.

En échange, un modèle théorique de la pertinence cognitive pourrait notamment apporter aux travaux empiriques sur la valeur de nouvelle une plus grande attention à l'influence de l'effort cognitif, plus ou moins présente dans la liste originelle de Galtung et Ruge, mais très curieusement négligée par Harcup et $\mathrm{O}^{\prime}$ Neill : on verra plus loin que la clarté est un critère très important de sélection éditoriale ${ }^{4}$.

Notons par ailleurs que de nombreux autres travaux, centrés pour leur part sur les attentes documentaires des personnes recherchant des informations spécifiques, ont également produit des observations et hypothèses d'un grand intérêt. Seule l'obligation de ne pas laisser ce livre gonfler indéfiniment contraint à remettre à plus tard l'examen des liens manifestes que l'on pourrait établir dans un sens et dans l'autre entre son propos et ces diverses approches (voir en particulier, sur la question de la pertinence documentaire, le panorama qu'en dresse Simonnot, 2008).

Dans un tout autre ordre d'idées, et un cadre théorique et méthodologique radicalement différent, on ne peut manquer, puisque l'on parle d'effet, de mentionner la masse imposante et croissante des recherches neurobiologiques portant sur le « circuit de la récompense " (medial forebrain bundle, etc.) activé par la satisfaction d'un besoin quelconque : ce qui est vrai pour la consommation d'aliments ou de psychotropes l'est aussi pour la consommation des textes de toutes sortes. Cette approche explicitement naturaliste, qui relie des hypothèses évolutionnistes (pour expliquer l'origine de ces besoins) à des techniques de neuroimagerie fonctionnelle (pour observer les zones cérébrales concernées) a notamment permis le développement de ce que Jaak Panksepp (1998) a baptisé les «neurosciences affectives ». Bien que ces travaux paraissent pour l'essentiel conforter sous un autre

4 Plus exactement, l'effort de traitement que réclameraient aux lecteurs les sujets compliqués est l'une des deux principales raisons de rejet éditorial exprimées par les professionnels, l'autre facteur le plus commun étant... le manque d'effet que produit la nouvelle (Pew, 2000). 
angle les éléments présentés plus haut - réaffirmant notamment la dimension hédonique (quasi-behavioriste dans leur perspective) des motivations et identifiant des circuits associés à différents niveaux de besoins (compatibles avec les facteurs d'effets primaires, utilitaires et supérieurs) - on ne s'y attardera pas ici pour des raisons qui n'ont rien à voir avec leur importance scientifique intrinsèque.

La première est que recourir à cet angle de vue supplémentaire impliquerait de traverser un corpus de recherches considérable pour n'en retirer - une fois dédupliquées les indications plus ou moins redondantes avec tout ce qui précède - qu'assez peu d'éléments réellement pertinents et nouveaux dans le cadre spécifique de la question posée. Il serait par exemple légitime de souligner le rôle essentiel de la dopamine derrière les choix discursifs, ou l'implication de l'aire tegmentale ventrale et du noyau accumbens, mais il n'est pas certain que ces mentions impressionnantes se justifieraient par l'éclairage qu'elles apporteraient à l'heure actuelle au problème général de la valeur discursive. La seconde raison, qu'illustrent nombre d'essais s'appuyant sur les neurosciences, est que ce champ de recherches, à l'instar de la génétique, peut très facilement conduire à des surinterprétations échevelées (attribuant par exemple à une ou quelques variables des phénomènes profondément multifactoriels) : on peut dans une certaine mesure expliquer Michel-Ange par les pigments qu'il utilisait et les fragments de marbre qu'il retirait d'un bloc, mais seulement dans une certaine mesure. À l'inverse, nombre de spécialistes - dont Panksepp lui-même - conviennent que " nous en sommes encore aux premières étapes de l'analyse de la psychobiologie des émotions, et de nombreuses générations supplémentaires de travaux méticuleux seront nécessaires avant que nous n'en ayons des cartes précises » (1998, p. viI).

Bref, on n'ira pas dans ce livre au-delà de quelques allusions à ce domaine de recherches, sans contester que ce champ en constante évolution puisse au fil du temps apporter des éléments plus précis et spécifiques au problème de la valeur discursive, mais sans avoir de raisons de penser (jusqu'à nouvel ordre...) que de telles indications bouleverseront les composantes générales de cette valeur. Et encore moins qu'elles pourront à elles seules en fournir une compréhension globale : comme l'ont montré depuis si 
longtemps tant d'autres recherches dans tant d'autres branches du savoir, ce problème buissonnant excelle à déjouer les tentatives restreintes à une perspective disciplinaire exclusive.

C'est notamment ce qu'ont illustré, fort loin des méthodes de laboratoire (si ce n'est à l'opposé), les travaux consacrés dans les années 1970 et 1980 à l'effet produit par les textes de fiction. Leur histoire est suffisamment édifiante pour justifier une ultime digression.

Le problème de la réception des œuvres littéraires, bien sûr, ne sortait pas du néant: "On éprouve tous les jours que les vers et les tableaux causent un plaisir sensible, mais il n'en est pas moins difficile d'expliquer en quoi consiste ce plaisir » soulignait par exemple l'abbé Dubos au début du xviII ${ }^{\mathrm{e}}$ siècle (1719, p. 1). Mais malgré les observations de Dubos, Batteux et quelques autres, cette version culturelle du Grand Mystère avait été prudemment maintenue à la porte des études littéraires, lesquelles avaient eu, au cours du XIx siècle, la sagesse de se construire autour du texte (son écriture, son interprétation...) et de l'auteur (sa vie, son temps...). Leur épanouissement académique remarquable - quoique non dépourvu d'arrières-pensées apologétiques ${ }^{5}$ - présentait néanmoins un inconvénient : la valeur littéraire des textes et leur interprétation légitime étaient surtout celles que leur attribuaient des spécialistes raffinés.

Louise Rosenblatt sonna l'alerte dès 1938 : ces textes n'existaient qu'à leurs yeux et à ceux des connaisseurs qui leur ressemblaient ${ }^{6}$. Mais l'idée d'accorder aux côtés de l'œuvre et de l'auteur une place au rôle du lecteur ordinaire dans la construction du

5 Il s'agissait de comprendre, mais aussi de célébrer la " grande » littérature face aux fictions plus légères que dévoraient les classes populaires.

6 "Des termes tels que le lecteur, l'étudiant, l'auvre littéraire [...] sont des fictions quelque peu trompeuses, bien que pratiques. Il n'existe pas de lecteur générique ni d'auvre littéraire générique; il n'y a en réalité que des millions de lecteurs potentiels d'œuvres littéraires individuelles. Le roman, le poème ou la pièce n'existe, après tout que par l'interaction avec des esprits spécifiques. La lecture de tout ouvrage littéraire est par nécessité une situation individuelle et unique impliquant l'esprit et les émotions d'un lecteur particulier » (Rosenblatt, 1938, p. 32). 
sens du texte et dans son appréciation fut repoussée à plus tard. Le plus tard possible. Presque trop tard, s'avisa-t-on soudain dans les années 1970 : "l'histoire de la littérature est tombée dans un discrédit toujours plus grand, et qui n'est nullement immérité. [...] Ses plus grandes réussites remontent toutes sans exception au XIX $X^{e}$ siècle» (Jauss, 1972/1978, p. 23). Il s'agissait désormais de préserver non seulement ce que l'on pouvait de la philologie, mais même des fondements de la critique littéraire universitaire : "une théorie des textes littéraires semble bien ne plus pouvoir se passer de lecteur" (Iser, 1976/1985, p. 69).

On ne dira rien ici des nombreux lecteurs génériques7 qui se bousculèrent bientôt dans les colloques et les thèses de littérature (ce serait trop et pas assez : il existe sur ce sujet d'excellentes synthèses, sans compter les écrits originaux). Rien, si ce n'est, pour rester très schématique, qu'au-delà des multiples différences de ces lecteurs imaginés, ils s'apparentaient vaguement sur un point - outre leur caractère très spéculatif - celui d'être bien décidés à collaborer avec l'auteur en relevant avidement les indices que celui-ci disséminait à leur intention. Le «lecteur qui s'en fiche un peu » ou le «lecteur qui est obligé de lire ce foutu roman avant l'examen » restent à conceptualiser, quoique Stanley Fish les ait laissés entrevoir et que d'autres, de Norman Holland à Michel Picard, s'y soient plus ou moins essayés (mais en se fiant à un outillage psychanalytique pour le moins discutable).

Bref, à l'instar du « suffisant lecteur » de Montaigne (1588/2001, p. 195), les figures habituellement invoquées pour expliquer la réception des textes littéraires étaient plus ou moins celles de détectives lancés individuellement ou collectivement à la poursuite du sens, voire de la beauté de ces œuvres. De tels lecteurs existent fort heureusement ${ }^{8}$, comme il se trouve des amateurs de

7 Le «lecteur informé » de Fish, le «lecteur visé » de Wolff, l'« archilecteur » de Riffaterre, le «lecteur implicite» d'Iser, le «lecteur abstrait» de Lintvelt, le lecteur trinitaire («liseur», «lu » et «lectant») de Picard, le «lecteur modèle » d'Eco, sans oublier le «public » de Jauss.

8 Ce sont ces «lecteurs "à part entière" » dont Escarpit (1966, p. 354) soulignait le caractère minoritaire dans la consommation livresque. Mais plus d'un demi-siècle après ses travaux pionniers en sociologie de la littérature, l'idée de "réintégrer le fait littéraire dans l'ensemble des 
marche en montagne au sein d'une population qui préfère les ascenseurs aux escaliers, mais la capacité de ces montagnards à représenter la pratique normale de la bipédie pourrait prêter à discussion.

Ainsi, voulant faire place à la contribution du ou des lecteur(s) dans ce qui leur incombait jadis, l'interprétation et l'appréciation des textes, les savants ont suscité des créatures qui leur ressemblaient furieusement. Cette curieuse similitude de vues a souvent été soulignée (notamment par Bourdieu, 1998, Jouve, 1993, Parmentier, 2017, etc.) et plusieurs auteurs en ont convenu. De bonne grâce, certes, mais de façon un peu paradoxale si l'on considère par ailleurs que ces créateurs de lecteurs génériques nont jamais manqué de préciser que leurs créatures théoriques n'avaient absolument rien à voir avec des lecteurs réels.

Cependant, l'ambiguïé de tels modèles de lecture, notamment ceux associés à l'« école de Constance», tient surtout au fait que leur mission explicite (expliquer l'effet esthétique ou l'évolution de la réception des textes) était sous-tendue par des préoccupations épistémologiquement plus délicates : justifier à rebours la perspective qui leur a donné naissance - « Le lecteur a bon dos dans cette théorie. Grâce à lui l'histoire littéraire est de nouveau légitime » ironise Compagnon (1998, p. 258) à propos de Jauss - mais aussi, dans la plus haute tradition apologétique de ce champ, préserver le statut à part de l'œuvre littéraire et de sa lecture. Au prix, lorsque nécessaire, de quelques coups de hache ${ }^{9}$.

phénomènes de communication dont il n'est qu'un des aspects » (ibid., p. 357) paraît toujours inconfortable, au point de devoir encore susciter des manifestes en ce sens (par exemple Vaillant, 2003) : si le corpus légitime s'est largement étendu - notamment à la « paralittérature » (sic) son approche théorique a moins vite évolué.

9 Là comme ailleurs, il faudrait nuancer si ce n'était si long. D'un côté, les travaux de l'école de Constance visaient subtilement à faire la part des choses en recherchant un compromis acceptable entre l'essentialisme ancien et le relativisme qui menaçait. De l'autre, ils ne rechignaient pas à asséner en cas de besoin des pétitions de principe plutôt péremptoires. Par exemple la réduction spectaculaire des œuvres littéraires aux seuls textes de fiction opérée ex abrupto par Iser (1970/2012), en vertu d'un a priori typique de ce domaine, mais passablement désuet (voir l'abjuration de Genette, 2001, au nom de la narratologie). 
D'où, aujourd'hui, un écart de plus en plus curieux entre la logique originelle de ces outils analytiques sophistiqués et l'usage que l'on peut en faire : une fois enseignés par des professeurs qui n'y recourent pas tant que ça eux-mêmes ${ }^{10}$, ils sont appliqués avec enthousiasme par les étudiants des cycles supérieurs à des objets de plus en plus éloignés des œuvres légitimes dont ils devaient élucider le caractère ineffable.

En effet, les programmes universitaires de lettres, eux aussi offres discursives sur un marché hyperconcurrentiel, et nettement désavantagés sur ce marché en proie à l'utilitarisme, se sont découvert le plus vif intérêt pour tous les thèmes d'enseignement ou de recherche susceptibles de renforcer leur pertinence hédonique auprès des nouvelles générations. Ainsi se sont multipliées les dissertations et thèses scrutant l'« horizon d'attente » ou le « lecteur implicite » (etc.) de bandes dessinées, de paroles de rap, de romans d'horreur et de toute autre chose propre à séduire le public contemporain.

Les esprits chagrins pourront toujours spéculer sur ce que Jauss, par exemple, penserait de ces transpositions d'un modèle qui tendait justement à disqualifier le "domaine de l'art "culinaire" $d u$ simple divertissement» (p. 6o). L'extension des divers modèles abstraits de la lecture et de la réception n'en constitue pas moins un bel hommage, et fort mérité, à leur utilité pratique (et symbolique). Un hommage un peu ironique cependant puisque leur inattention - disciplinairement motivée - à l'influence d'une large part des déterminants sociaux ${ }^{11}$ et cognitifs $^{12}$ de la consommation culturelle réelle empêche ces modèles d'expliquer leur propre

10 "l'École de Constance est tombée en désuétude. Plus personne, semble-t-il, ne la pratique ni ne se réclame encore d'elle » affirme même Fabien Pillet (2011, p. 764) de façon un peu brutale, même si l'intention de cet auteur est de souligner par contraste l'utilité que pourraient encore présenter ces travaux au prix d'une réactualisation.

11 Voir (entre autres) la féroce critique de Bourdieu, 1988.

12 Les figures de lecteurs surmotivés construites par les théoriciens sont, contrairement au commun des mortels, étonnamment insensibles à la plupart des facteurs d'effet de bas niveau cognitif, mais aussi remarquablement généreux de leurs efforts mentaux (lesquels sont fugitivement évoqués ici ou là, mais sans paraître constituer un frein à la lecture). Cependant, ce qui limite la portée générale de ces théories est aussi ce qui peut les rendre précieuses pour examiner 
évolution de carrière et limite sensiblement, sans l'abolir, leur capacité à rendre compte de la réception des produits éditoriaux de plus en plus variés qu'on leur donne à analyser.

En somme, conclut un bilan de ces travaux,

Outre la délicate question, qu'à notre avis aucun des auteurs ne parvient à résoudre, de la réception effective, il semble aussi très difficile d'obtenir un modèle de récepteur capable de prendre en compte toutes les dimensions (psychologique, historique, culturelle, sociale) de l'individu. Mais il apparaît également que construire un tel modèle constitue un passionnant défi pour la recherche contemporaine, car connaître le lecteur revient à mieux connaître la littérature. (Pillet, 2011, p. 781)

spécifiquement certaines des composantes les plus élevées de l'effet cognitif. À condition de ne pas prendre cette partie pour le tout. 


\section{Des images plein la tête ${ }^{1}$}

Sans avoir l'idée d'une chose, c'est-à-dire sans en avoir une représentation mentale, vous ne sauriez la concevoir, ni en parler.

Cicéron ${ }^{2}$

$\mathrm{Q}$ uelles que soient les raisons pour lesquelles on accorde de la valeur et donc de l'attention à un discours, toutes ${ }^{3}$ passent par un même objectif intermédiaire : en retirer du sens, c'est-à-dire établir un lien entre l'état du monde présenté et les connaissances que l'on détient déjà. Même les processus de bas niveau cognitif, qu'il s'agisse d'effort ou d'effet, impliquent une certaine interaction avec ces connaissances. Présentez un texte à un poisson : il n'y verra pas de signes interprétables, même si l'on a accordé le plus grand soin à la typographie. Projetez-lui un film érotique (un film d'humains, bien entendu) : il restera de marbre.

Si la valeur des discours repose sur leur interaction avec les connaissances préalables, il faut que les uns et les autres aient un dénominateur commun, une sorte de point de rencontre ou de monnaie d'échange. La conception la plus élégante et la plus convaincante de celui-ci repose sur ce que Kant (1781/1869, p. 202) appelait un «art caché dans les profondeurs de l'âme humaine »: le «schématisme de l'entendement».

1 Ce chapitre reprend très largement les éléments d'un article paru en 2016 sous le titre «Le statut des schémas cognitifs dans la production et la réception discursives » dans le numéro 171-172 de la revue Pratiques.

2 De la nature des Dieux (trad. D. Nisard, 1848, p. 89).

3 Ou presque... On peut, par exemple, être fasciné par des litanies, harangues ou spectacles incompréhensibles, mais ce cas qualitativement et quantitativement marginal demeure aux limites extrêmes du traitement du discours. 
Le concept du chien, par exemple, désigne une règle d'après laquelle mon imagination peut se représenter d'une manière générale la figure d'un quadrupède, sans être astreinte à quelque forme particulière que m'offre l'expérience [...]

Outre son importance phénoménologique (voir Schütz, 1962, 2011, etc.), l'hypothèse des schémas mentaux a fait assez tôt l'objet de travaux en psychopédagogie (Piaget, 1923) et même d'expériences sur le traitement des récits (Bartlett, 1932), mais c'est avec l'essor de la psychologie cognitive dans les années 1970 que les recherches à leur propos sont devenues foisonnantes, entraînant comme il se doit une multiplication d'appellations : «frames » (Minsky, 1975), «scripts » (Shank et Abelson, 1977), "story grammar» (Mandler et Johnson, 1977)... Malgré (ou à cause de) cette diversité, Rumelhart était dès 1980 en mesure d'élaborer une définition générale des schémas consacrant l'arrivée à maturité de cette théorie :

Selon la «théorie des schémas », la totalité de la connaissance est emboitée dans des unités. Ces unités sont les schémas. Incorporées avec ces groupements de connaissances se trouvent, en plus de la connaissance elle-même, des informations sur la façon d'employer cette connaissance. Ainsi, un schéma est-il une structure de données pour représenter les concepts génériques stockés en mémoire. Il existe des schémas représentant notre connaissance pour tous les concepts : ceux qui sous-tendent des objets, des situations, des évènements, des actions et des séquences d'actions. (1980, p. 4-5)

En d'autres termes, évoquer par exemple un pique-nique active un schéma générique de pique-nique, un peu vague et différent pour chacun, mais impliquant généralement des sandwiches (et souvent une nappe à carreaux, bien qu'elle corresponde rarement à l'expérience personnelle), mais jamais une enclume ou une machine à coudre.

De nombreuses recherches expérimentales avaient conduit au cours de la décennie à mettre en évidence le rôle crucial de ces structures mentales dans le traitement du discours. Ainsi, la description d'une procédure aussi courante que faire la lessive, mais rédigée de façon trop abstraite pour activer un schéma, ne permet-elle pas aux sujets de produire les inférences qu'exige la compréhension d'un texte (Bransford \& Johnson, 1972). De même, des ambivalences textuelles, par 
exemple celle d'un récit pouvant s'appliquer aussi bien à une partie de cartes entre amis qu'à la répétition d'un quatuor à vent, sont invisibles à la plupart des lecteurs, ceux-ci l'interprétant exclusivement en fonction du schéma qu'ils ont sélectionné a priori (Anderson, Reynolds et al., 1977). Les schémas activés guident par ailleurs l'attention accordée aux diverses informations contenues dans un texte et conditionnent leur mémorisation et leur oubli (Pichert et Anderson, 1977) ou conduisent même à distordre celles-ci a posteriori (Snyder et Uranowitz, 1978).

L'interprétation de telles expériences a permis de préciser certaines propriétés des schémas (Adams et Collins, 1977; Anderson, Reynolds et al., 1977 ; Rumelhart et Ortony, 1977 ; Anderson, Spiro et al., 1978 ; Rumelhart, 1980 ; Anderson et Pearson, 1984).

- Les schémas sont des assemblages de connaissances plus ou moins informelles (ce ne sont pas des définitions) structurées par enchâssement : le schéma générique de «maison » est par exemple un constituant du schéma générique "village », et il englobe pour sa part les schémas «fenêtre ", " cuisine », etc.

- Ces connaissances sont hiérarchisées, de telle sorte que le niveau le plus élevé d'un schéma correspondrait aux propriétés les plus symboliques, abstraites et générales de ce qu'il décrit et les niveaux inférieurs à des caractéristiques plus spécifiques.

- Les schémas comportent des sortes de cases («slots») qui peuvent rester vacantes, recevoir une valeur par défaut ou accepter des valeurs instanciées par le texte (par exemple, le toit de la maison peut s'avérer "gris », "rouge», " en tuiles », « en tôle », etc., ou rester indistinct).

- Par les variables qu'ils autorisent ou non, les schémas spécifient de façon contraignante les relations probables ou possibles entre leurs composants internes (la porte ne peut pas se trouver sur le toit) ainsi que celles qu'ils peuvent avoir avec d'autres schémas (la maison ne peut pas rentrer dans la voiture). La violation de ces contraintes, si elle est perçue ${ }^{4}$, entraînera un réexamen du texte ou la

4 Les incohérences mineures peuvent échapper à l'attention, étant masquées par les représentations «normales » imposées par le schéma. 
substitution d'un nouveau schéma, plus compatible que le précédent avec les indications textuelles fournies.

- Ces spécifications permettent notamment de générer les inférences qui compensent le caractère fondamentalement lacunaire de tout discours : "Il prit la clef et entra dans la maison" dispense ainsi de préciser (à un être humain) qu'«il introduisit la clef dans une serrure à mihauteur de la porte, puis la tourna afin de déverrouiller cette dernière avant de la faire pivoter vers l'intérieur ", toutes les informations omises étant implicitement fournies par le schéma actif.

- Le traitement et la mémorisation sélective des informations tendent normalement à filtrer les propriétés de niveau hiérarchique inférieur et les variables perçues comme non pertinentes (lu : «il se glissa sans hâte sur le siège en tissu de sa petite voiture japonaise »; retenu : «il monta dans sa voiture »).

C'est ainsi que Rumelhart (1980) a pu comparer simultanément un schéma à une pièce de théâtre (qui peut être jouée par différents acteurs dans différents décors sans que ces instanciations ne changent sa structure), à une théorie (qui synthétise la conception que le lecteur se fait d'une classe d'objets ou de situations), à une procédure (qui détermine comment associer, interpréter et retenir ce qu'il lit), et enfin à un répartiteur (qui analyse les éléments conceptuels du message et leur attribue un rôle).

Quoique la théorie des schémas ait donné lieu à des débats scientifiques animés (voir ci-dessous la section «Précisions»), les éclairages qu'elle peut apporter à la logique des échanges discursifs sont considérables.

Le plus évident d'entre eux concerne l'effort cognitif, dont on avait souligné qu'une bonne partie - celle qui échappe aux formules de lisibilité lexico-syntaxique ${ }^{5}$ - repose sur la gestion de la cohérence,

5 En fait la longueur lexicale, étant corrélée à la fréquence d'usage, pourrait dans une certaine mesure refléter la rareté des mots, et donc leur moindre capacité à activer un schéma mental. Mais d'un autre côté, les mots les plus courts et les plus fréquents sont aussi les plus dépourvus de cette capacité. Ainsi, les quinze mots les plus fréquents en français, qui 
c'est-à-dire l'établissement des relations logiques entre les éléments d'information afin de construire un modèle mental provisoire de l'état du monde évoqué par le discours. Or, la plupart de ces relations sont implicitement fournies par les schémas activés. Comme le notait plus généralement Eco,

... toute fiction narrative est nécessairement, fatalement rapide, car - lorsqu'elle construit un monde, avec ses évènements et ses personnages - il lui est impossible de dire tout ce monde. Elle mentionne, et pour le reste, elle demande au lecteur de collaborer en comblant une série d'espaces vides. Du reste, ainsi que je l'ai déjà écrit, tout texte est une machine paresseuse qui prie le lecteur d'accomplir une partie de son propre travail. Gare si un texte disait tout ce que son destinataire doit comprendre : il n'en finirait plus. Si je vous téléphone en vous annonçant « je prends l'autoroute, j'arrive dans une heure », il est implicite que, en même temps que l'autoroute, je prends ma voiture. (1996, p. 9, spn.)

Il est intéressant que cette réflexion centrée sur la littérature de fiction s'élargisse aussitôt au traitement des discours en général, comme est intéressante la mention du «travail » (l'effort cognitif) du lecteur dans l'établissement de la signification. Et plus intéressant encore le fait que le spécialiste de la sémiologie littéraire choisisse peu après de se référer à un travail de Roger Shank (le même Schank dont provient partiellement la nomenclature de l'effet cognitif exposée plus haut); décidément, les frontières entre le pinacle de la littérature et les humbles rouages des processus psycholinguistiques sont bien moins étanches qu'on ne le pense.

Pour s'en tenir temporairement à ces rouages, on notera que la notion de schéma cognitif s'avère plus riche que celle de "valence d'imagerie » utilisée notamment en neurolinguistique. Alors que celle-ci doit, comme on l'a vu, être assortie de variables complémentaires (le caractère concret, ou concreteness, et la densité de sens, ou meaningfulness), les schémas mentaux, porteurs de sens par définition,

représentent à eux seuls le tiers des termes utilisés à l'écrit ou à l'oral (il suffit même d'une liste de 50 mots pour englober la moitié de toutes les occurrences rencontrées) sont dans l'ordre : de, le, la, être, un, à, et, les, avoir, $i l$, des, que, ne, en, et se. 
sont normalement imagés et concrets, mais ne le sont pas obligatoirement : ainsi s'explique qu'un terme comme «jalousie » puisse avoir une plus forte valence d'imagerie que « bivouac ». En d'autres termes, «jalousie », lourde d'inférences possibles (cris, etc.) évoque bien une sorte d'image, mais ce n'est pas une image très nette.

En ce qui concerne l'effort discursif, la différence conceptuelle est parfois minime. La théorie des schémas confirme par exemple ce que l'on avait déjà souligné quant à la futilité d'une conception purement lexicale de la compréhension. Se demander si les lecteurs visés « connaissent ce mot » méconnaît le fait que la connaissance d'un individu ne ressemble pas à celle d'un dictionnaire, mais "reflète la tolérance humaine pour le flou, l'imprécision et les quasi-contradictions » (Rumelhart et Ortony, 1977, p. 111). Il importe moins de savoir si un lecteur est capable de définir précisément ce qu'est spécifiquement un teckel ou un carburateur que de s'interroger sur les schémas, plus ou moins vagues et génériques, que ces termes peuvent activer. Le plus souvent, subodorer qu'il doit s'agir d'une sorte de chien ou d'une quelconque pièce de moteur suffira parfaitement à assurer la compréhension du texte, tout en permettant à des destinataires plus savants de retirer plus d'information de celui-ci. En d'autres termes, il aura autant de valeur pour les non-initiés et en aura plus pour les connaisseurs.

Si de nombreuses séries télévisées, qu'elles soient médicales ou de science-fiction, peuvent faire appel à un vocabulaire abscons sans décourager le moins du monde leurs spectateurs, c'est bien parce que la connaissance précise de chaque mot ne conditionne pas forcément le sens du récit. En revanche, des termes usuels mais peu évocateurs dégradent sensiblement la valeur d'un énoncé, comme l'illustre par exemple cet article :

Plusieurs méfaits se sont récemment produits sur l'heure du midi autour de l'école [...]. « Parmi les jeunes qui sortent sur l'heure du midi, quelques-uns commettent des actes incompatibles avec la mission éducative de l'école (vols, vandalisme, attroupements, menaces) », peut-on lire dans la lettre aux parents. Des plaintes ont été adressées par des commerçants et des citoyens.

«Finalement, certains de nos élèves ont commis des gestes imprudents aux abords de la rivière des Prairies ", souligne également le message, signé par le directeur de l'école et une agente 
sociocommunautaire du Service de police de la Ville de Montréal. Des élèves rencontrés mercredi autour de l'école nous ont expliqué que des jeunes s'amusaient notamment à grimper sur la structure du pont Viau, près de l'école ${ }^{6}$.

Dans le premier paragraphe, les auteurs du message officiel ont perçu la stérilité cognitive de la mention d'«actes incompatibles... » et en ont renforcé la pertinence par des précisions plus évocatrices. Ils se sont en revanche contentés d'une allusion à des "gestes imprudents", bien trop vague pour le journaliste de La Presse qui a donc dû aller se renseigner lui-même auprès des élèves.

Bien entendu, les schémas mobilisés dans la transmission de l'information journalistique (ou autre) ne sont pas des objets figés que l'on diffuserait comme des marchandises : ils se transforment dans leur confrontation avec ceux dont disposent le lecteur et, en retour, peuvent contribuer à modifier ceux-ci (voir notamment McQuail, 2010 et Graber, 1984).

Des schémas assez développés seraient souvent requis pour produire les inférences que réclame la compréhension des messages politiques, techniques ou administratifs, ce que leurs auteurs - parce que ces schémas leur sont familiers - ne réalisent pas nécessairement. C'est même le cas d'une grande partie des informations médiatiques (économie, politique, diplomatie...), dont les professionnels de l'actualité sont pourtant censés assurer la clarté pour leur public, mais dont les études montrent qu'elles peuvent poser de sérieux problèmes d'interprétation (Findahl et Hoijer, 1985 ; Hoijer, 1992 ; Mathieu, 2009). Il est vrai que les journalistes eux-mêmes peuvent mal percevoir une information lorsqu'ils l'interprètent en fonction d'un schéma inapproprié (Labasse, 2004).

Le jeu des schémas explique par ailleurs facilement le caractère autocumulatif de la sélection de l'offre discursive. Pas totalement, bien sûr, mais très largement? . Parcourons par exemple cette présentation d'un nouveau modèle de voilier dans un magazine de loisirs spécialisé :

6 C'est moi qui souligne (article d'Hugo Meunier dans La Presse du 11 juin 2015, p. 14).

7 Le reste de l'explication relève des affinités sociales, hors sujet pour le moment. 
[...] on remarque aussi le rail d'écoute circulaire qui autorise l'absence de hale-bas. Un épaulement du rouf permet de placer très à l'intérieur le réglage de point de tire du solent. [...] Le piano est centré sur le rouf [...] Et surtout, le bateau aura déjà un palan fin pour le pataras ou des outriggers pour contrôler le bras du boutdehors orientable ${ }^{8}$.

Ces précisions qui captiveront assez certains pour qu'ils leur accordent de l'attention - et incidemment le prix du numéro ou de l'abonnement apparaîtront comme un effroyable ramassis de jargon pour les amateurs d'autres loisirs, quel que soit leur niveau d'études. Dépourvus des schémas nécessaires, ils seront incapables d'établir la cohérence de ces énoncés et de construire un modèle mental de ce voilier. Quand bien même s'y efforceraient-ils que - faute de savoir qu'un piano est, dans ce contexte, une rangée de taquets permettant de régler les cordages leurs schémas usuels les amèneraient à imaginer un instrument de musique assez incongru sur un voilier de compétition. Mais il est peu probable qu'ils se livrent à un tel effort, cette absence de sens impliquant évidemment une absence d'effet cognitif (pas de schémas disponibles, donc pas d'interaction avec les connaissances), tandis qu'à l'inverse, l'enrichissement des schémas des connaisseurs les rendront encore plus disponibles pour des informations encore plus spécifiques.

On perçoit, incidemment, un possible lien avec les modalités familier/étrange ou surprenant/habituel de l'effet cognitif évoquées plus haut (chap. 4) : un énoncé qui n'a aucun schéma auquel s'accrocher ne pourra avoir de valeur, mais un énoncé correspondant trop exactement à un schéma préalablement disponible (une information connue ou banale) n'en aura pas non plus?.

Sur un plan plus théorique, la théorie des schémas peut apporter (et recevoir) d'intéressants éclairages sur des problèmes ou des concepts issus d'autres traditions scientifiques. On signalera un peu plus loin ses convergences possibles avec des notions telles que celles de stéréotype, de représentation sociale, d'idéal-type, de cadre de l'expérience, de paradigme, etc. C'est toutefois sur l'analyse des logiques

8 «Nacira 6.50 : Un mini pour gagner». Voile Magazine $\mathrm{n}^{\circ} 144$ (déc. 2007), p. 28.

9 C'est donc assez proche de ce que Clark et Haviland appelaient le connunouveau (given-new) dans les processus de traitement discurisif. 
et effets des formes discursives (poétique, rhétorique, genres) que ses implications sont les plus manifestes.

Au-delà de l'interminable catalogue des figures de style, les tropes, en particulier pourraient se comprendre (voire se définir) comme des opérations jouant sur l'activation des schémas cognitifs. Ainsi, les métonymies et figures apparentées exploitent-elles les propriétés de hiérarchisation et d'enchâssement qui seraient, comme on l'a vu, une caractéristique fondamentale de ces schémas. De même et surtout, l'ensemble des figures d'analogie (métaphore, comparaison...) paraissent se réduire à un même procédé fondamental : transférer à un schéma les propriétés d'un autre schéma ${ }^{10}$.

A priori, il n'y a rien là que l'on ne pouvait déjà trouver au XviII ${ }^{\mathrm{e}}$ siècle dans le traité des tropes de Du Marsais (lequel envisageait même le terme... "schemata» pour désigner les figures de pensée $\left.{ }^{11}\right)$ : "Un des plus fréquens usages des tropes, c'est de réveiller une idée principale, par le moyen de quelque idée accessoire » (Du Marsais, 1730, p. 26). Mais ce point de départ psychologique n’a pas détourné Du Marsais et ses homologues de considérer les signes mis en œuvre (le code) plutôt que les opérations mentales en jeu, cédant à ce que Genette dénonce comme une "rage de nommer qui est façon de s'étendre et de se justifier en multipliant les objets de son savoir » (1966, p. 214).

Or, il semble moins pertinent, tant d'un point de vue analytique qu'opératoire, de se demander comment la figure se classifie formellement (synecdoque ou métalepse ? comparaison ou métaphore? in praesentia ou in abstentia? etc.) que de s'intéresser à la dynamique cognitive qui la sous-tend. Quel que soit, en effet, le procédé langagier sur lesquelles elles reposent, les figures d'analogie peuvent être caractérisées en fonction de la disponibilité cognitive des schémas qu'elles relient.

Il est, d'un côté, fréquent que l'«idée principale » et l'« idée accessoire » mises en relation activent toutes deux un schéma disponible, ce qui est généralement le cas des tropes ayant un rôle esthétique ou rhétorique, celui de rendre «plus vives, ou plus nobles, ou plus agréables

10 Pour une inversion intéressante, quoique spéculative et très débattue, de cette relation de causalité, voir Lakoff et Johnson, 1980.

11 "On divise les figures en figures de pensée, figurae sententiarum, schemata ; et en figures de mots, figurae verborum » (Du Marsais, 1730, p. 30). 
[...] les manières de parler » (Du Marsais, 1730, p. 11). Ce qui revient à dire que leur pertinence réside dans l'effet cognitif qu'elles produisent (" elle se mit à tousser comme une étoffe de soie qui se déchire $e^{12}$ ), y compris lorsque cet effet vise un but argumentatif («ce que le lion n'eût pas osé, le singe l'a fait' ${ }^{13}$ ! »).

En revanche, le cas également fréquent où aucune «idée principale» ne préexiste à l'«idée accessoire» renvoie à de toutes autres logiques discursives.

C'est en particulier le cas des textes de vulgarisation, dont on a souvent remarqué la densité d'analogies (par exemple Jacobi, 1986 ; Jeanneret, 1992), mais sans nécessairement en tirer toutes les conséquences, d'où l'échec des tentatives de caractériser ce type de discours : "La question la plus importante reste posée: "qu'appelle-t-on vulgarisation"? [...] Les réponses à cette question ne peuvent être que subjectives" (Jeanneret, 1994, p. 9-10). Il semble cependant possible de définir la vulgarisation - indépendamment de son sujet - comme étant toute pratique discursive non curriculaire qui vise à permettre à ses destinataires de créer de nouveaux schémas cognitifs. Au sein de la production médiatique, par exemple, les textes (ou les passages de texte) «vulgarisateurs " se distingueraient des textes strictement «informatifs » par le fait que les textes informatifs se contentent d'activer et d'instancier - on pourrait dire ici d'actualiser - des schémas déjà connus du lectorat (ou supposés tels) quand les textes ayant une dimension vulgarisatrice impliqueraient nécessairement un dispositif favorisant la construction de nouveaux schémas et autant que possible leur mémorisation.

Si les textes curriculaires tels que les manuels scolaires, quoiqu'ils visent eux aussi à transmettre de nouveaux schémas, ne sont pas inclus dans cette caractérisation, ce n'est pas en vertu de la longue tradition qui distingue ces discours légitimes de l'effrontée vulgarisation, mais bien en raison de leur économie discursive différente, que démontre justement le fait que ces écrits sont étonnamment dépourvus d'analogies, et en particulier de métaphores.

[...] l'observation des manuels scolaires montre que les métaphores en sont absentes. Par contre, elles sont omniprésentes

12 Vian, 1947/2007, p. 143.

13 Hugo, visant « Napoléon le petit» (1893, p. 356). 
dans les textes de vulgarisation scientifique à destination des lecteurs appartenant aux mêmes tranches d'âge. Comment expliquer cette différence? (Béguin, 1996, p. 75)

L'explication se trouve tout simplement dans l'économie des échanges communicationnels. La construction de nouveaux schémas requiert un effort cognitif nettement plus lourd que l'activation de schémas existants (raison pour laquelle, comme on l'a vu, la plupart des gens s'intéressent de préférence aux thèmes qu'ils connaissent déjà). Or, le recours aux analogies est la façon la moins coûteuse d'y parvenir, puisque le nouveau schéma s'obtient en adaptant un schéma disponible plutôt qu'en partant de zéro. Mais ce bricolage n'est pas sans conséquences. D'une part, l'analogie est toujours approximative (ce serait, sinon, une similitude pure et simple) et donc épistémologiquement imparfaite. D'autre part, la construction du nouveau schéma, même ainsi facilitée, demeure cognitivement coûteuse : pour assurer la pertinence de son discours, il incombe donc au vulgarisateur de justifier un tel effort en offrant un effet cognitif au moins équivalent, d'où le «sensationnalisme » dont sont souvent accusés les textes de vulgarisation (voir Labasse, 2012b). Pour leur part, les auteurs de manuels scolaires, qui s'adressent par définition à des lecteurs contraints, n'ont pas besoin de recourir à de tels procédés - «la certitude d'être non seulement écouté, mais entendu [...] est le propre de tout langage d'autorité » remarquait Bourdieu (1980, p. 96) - et ils n'auraient en tout état de cause pas la liberté requise pour assumer l'approximation épistémologique qu'ils requièrent. Ainsi, la spécificité générique de la vulgarisation, sa posture ambiguë (suspectement journalistique pour les enseignants, suspectement didactique pour les journalistes) et ses stratégies discursives peuvent-elles pareillement s'expliquer par la dynamique des schémas en jeu et plus généralement celle de la pertinence.

Ses errements le peuvent aussi. Le cas de la description de la structure de l'ADN en fournit un exemple presque canonique : si évoquer à son propos « une double hélice enroulée sur elle-même » ne semble plus guère poser de problème aujourd'hui, c'est que cette expression est désormais en mesure d'activer directement un schéma spécifique, acquis à force de répétitions et d'illustrations (Labasse, 2015a). La situation était tout à fait différente il y a quelques décennies, où la même description ne pouvait activer, pour le commun des mortels, 
qu'un double schéma d'hélice usuelle (bateau, ventilateur...). Or, il était impossible de construire à partir de ce schéma un modèle mental de la situation présentée dans le texte, les propriétés typiques d'une hélice ne prévoyant en rien que deux de ces objets puissent être assez souples pour être enroulés sur eux-mêmes.

La nature de la vulgarisation n'est pas le seul de ces problèmes définitionnels persistants auxquels la notion de schéma paraît apporter une solution ou, à tout le moins, un éclairage. Il en va ainsi de la connotation, dont «les linguistes [...] ne sont pas parvenus à préciser de façon entièrement satisfaisante le statut " (Gandon, 1980). Sous l'angle offert par la théorie des schémas, dénotation et connotation n'apparaissent plus comme des dimensions discursives qualitativement distinctes, mais simplement comme des variations d'étendue et d'effet dans un même processus d'activation schématique. En d'autres termes, la distinction entre dénotation et connotation peut avoir un intérêt analytique, et en tout cas pédagogique, mais, comme toujours dans de tels cas, la «réalité » de ce qu'elle... dénote est plus que discutable.

Il en va de même pour les « niveaux de langage », dont on a déjà signalé le caractère un peu arbitraire, mais qui posent un autre problème intéressant. Une idée commune veut en effet que les tournures vulgaires soient désobligeantes et que le style soutenu soit intrinsèquement plus délicat. Comment comprendre, alors, que traiter quelque chose (ou quelqu'un...) de «merde» soit sensiblement moins brutal que d'utiliser à son encontre des termes aussi raffinés qu'«étron»ou " excrément »? La différence ne réside nullement dans leur sophistication respective, mais dans le fait que le premier, lessivé par l'usage, active un schéma beaucoup moins vif que les deux autres.

Bien d'autres éclairages pourraient éventuellement être dérivés de la théorie des schémas, sur des questions aussi variées que les procédés humoristiques (tels qu'activer trompeusement un schéma et lui substituer brusquement un autre schéma, transformant ainsi le modèle mental qui en résulte), les clichés (qui peuvent être définis comme des tropes n'activant plus aucun schéma source $\left.{ }^{14}\right)$, et, bien

14 Le statut du cliché a souvent été abordé par les spécialistes, mais il serait utile de s'interroger davantage sur son économie cognitive curieusement inversée. D’un côté, en effet, les clichés courants (c'est «la cerise sur le 
sûr, la publicité (domaine qui, pour des raisons évidentes, s'est passionné plus que d'autres, et plus tôt, pour cette théorie).

Mais ce serait à nouveau s'engager dans un labyrinthe hétéroclite de " choses déjà dites » sous d'autres angles. Souligner que bien des plaisanteries reposent sur l'incohérence avec un schéma préalable (absurdité) ou son accentuation outrancière (caricature) n'est pas nouveau en soi, le rapport entre les clichés et les images ne l'est pas non plus, et la manipulation publicitaire des représentations encore moins.

En somme, la notion de schéma, dans son sens le plus général, semble constituer, à l'instar de la conception hédonique de la communication (effort-effet), un autre de ces carrefours principaux où se croisent sans se voir de multiples approches. C'est par exemple le cas de ce que l'on désigne confusément ${ }^{15}$ comme des stéréotypes. Ainsi, deux phrases comme : "Le vagabond prit les gâteaux sur le bord de la fenêtre » et "Le pâtissier prit les gâteaux sur le bord de la fenêtre ", strictement identiques d'un point de vue linguistique, conduiront à construire des modèles mentaux très différents, seule la première suscitant l'inférence selon laquelle les gâteaux ont été volés. Le lien entre la notion de schéma et celle de stéréotype avait été relevé dès l'origine : "Les schémas, dans un certain sens, représentent des stéréotypes de ces concepts » (Rumelhart et Ortony, 1977, p. 101). Réciproquement, "les stéréotypes paraissent fonctionner comme des schémas qui conduisent automatiquement à l'élaboration d'inférences dans la compréhension des textes » notent Whitney et al. (1995, p. 148), qui évoquent également les «archétypes». Mais il paraît tentant, voire inévitable, de rapprocher plus généralement la notion de schéma cognitif de toutes celles construites au sein d'autres champs disciplinaires pour rendre compte de structures apparemment similaires ou voisines.

C'est en particulier le cas de la théorie des «représentations sociales» qui, bien que méconnaissant la somme des travaux

gâteau », " un pavé dans la mare »...) sont trop délavés pour activer des schémas produisant de l'effet cognitif. Mais de l'autre, ils permettent souvent d'épargner l'effort de traitement des lecteurs en leur évitant des périphrases plus longues (on évoquera par ailleurs plus loin la réprobation qu'ils suscitent chez les connaisseurs : c'est là une question d'un tout autre ordre).

Voir Amossy (1989, 1991) pour une tentative méritoire (quoique psychologiquement lacunaire) de trier le fatras des écrits sur ce thème. 
antérieurs en psychologie expérimentale, paraît converger avec eux par de multiples aspects. Ainsi, le modèle de représentation sociale développé, à la suite de Moscovici, par Abric et ses collègues supposet-il un noyau central invariant, des éléments périphériques plus fluctuants, des relations d'emboîtement, des degrés d'activation plus ou moins prononcés, des réseaux d'activation, etc. Ces analogies ne font évidemment pas de «représentation sociale» un synonyme de « schéma cognitif » : outre que cette notion est loin de se réduire à ces rapprochements approximatifs, elle procède d'une problématique différente (les perceptions partagées par un groupe social plutôt que celles d'un lecteur individuel), d'intérêts différents (les opinions et conceptions plutôt que les processus de traitement psycholinguistiques et mnésiques) et de méthodes différentes (données déclaratives plutôt qu'expérimentales). Mais justement parce qu'ils n'observent pas la même chose sous le même angle, les travaux sur les représentations sociales et sur les schémas cognitifs seraient en mesure d'échanger des résultats et des questionnements particulièrement féconds : telle propriété des représentations s'applique-t-elle aux schémas? (et réciproquement).

Un tel rapprochement, si les tropismes disciplinaires ne le rendaient pas improbable, serait peut-être l'un des chantiers les plus importants de l'histoire des sciences humaines et sociales. Celui-ci semble du reste s'esquisser aux États-Unis, où des sociologues comme Paul Dimaggio (1997) ou Eviatar Zerubavel (1999) n'hésitent même plus à recourir à la théorie des schémas pour approcher des phénomènes culturels, mais il dépasse évidemment de beaucoup l'optique de ce livre, de même que les interrogations qui pourraient par la même occasion porter sur des notions moins directement liées telles que les idéaux-types ${ }^{16}$ ou les paradigmes (lesquels, comme les schémas cognitifs, filtrent la perception générique du réel et guident les inférences et questionnements à son propos).

En fait, la diversité des travaux qui font appel sous une forme où une autre à des structures évoquant de près ou de loin les schémas

16 Une différence considérable est que les idéaux-types weberiens ou les paradigmes kuhniens sont des constructions délibérées, dont l'utilité interprétative est soigneusement soupesée, mais ce caractère réflexif ne les empêche pas de se comporter également comme des schémas cognitifs et de partager un bon nombre de leurs propriétés. 
cognitifs - Goffman $\left(1991^{17}\right)$ et les "cadres de l'expérience", Paveau (2006) et les "prédiscours », Schütz (passim ${ }^{18}$ ) et les «typifications» et "schèmes de pertinence", également abordés par Berger et Luckmann (1966), Sperber (1996) et les "représentations culturelles », etc. - paraît appeler désespérément un examen d'ensemble.

Peu enclin à se lancer dans une croisade aussi incertaine, périlleuse de surcroît (chaque école conceptuelle surveille ses notions comme une mère ours ses petits), on se bornera ici à souligner que l'influence des schémas cognitifs constitue décidément un aspect essentiel de la production et de l'appréciation de la valeur discursive.

17 La distinction opérée par Goffman entre les «cadres naturels» et les «cadres sociaux » est particulièrement éclairante dans le cas du traitement journalistique des crises et des risques (Labasse, 2004).

18 En fait, une grande partie de la pensée phénoménologique de Schütz - dont évidemment celle relative à la "pertinence» (voir notamment Schütz, 1962 et 2011) - serait à considérer ici si on le pouvait. 
Page blanche conservée intentionnellement 


\section{(Précisions)}

$\mathrm{D}$ ans quelle mesure peut-on vraiment se fier à la théorie des schémas ? Si une hypothèse qui n'apporte rien est facile à écarter, une théorie qui, à l'inverse, semble expliquer si facilement et si bien tant de choses requiert une vigilance à la mesure de ses promesses, d'autant que la notion de schéma, si elle excelle apparemment à en redéfinir d'autres, n'est pas irréprochable quant à sa propre définition.

Dans son sens le plus général, le concept de schéma est si prégnant que des auteurs de divers horizons ont pu développer intuitivement des propositions spéculatives convergentes avec celles de la psychologie moderne sans se fonder sur son cadre théorique ni apparemment connaître les propriétés qui lui ont été associées par les spécialistes. C'est par exemple le cas d'Estivals, fondateur de la «Société de bibliogie et de schématisation » (voir Estivals, 1993), de Pottier "supposant» (sic) l'existence de "noèmes » ("figure de sens élémentaire, abstraite et universelle », 2000, p. 14), de Lakoff $^{1}$ (1980, 1987, etc.) ou encore de Grize (1996), qui situe une «schématisation discursive » de son cru au cœur des logiques communicationnelles.

$1 \quad$ Le cas de Lakoff (et de ses associés) est un peu particulier dans la mesure où ce linguiste connaissait apparemment les travaux dans ce domaine, mais a opté pour la conquête d'un territoire conceptuel qui lui soit propre, ce qui l'a conduit à conférer, non sans brio, à des entités langagières, les métaphores, ou à des «modèles cognitifs idéalisés »- on ne saurait mieux dire - des propriétés similaires à celles attribués aux schémas par les recherches expérimentales (voir notamment Lakoff et Johnson, 1980 ; Lakoff, 1987, etc.) Si les hypothèses de Lakoff ont inspiré nombre de chercheurs dans des disciplines variées, elles ont aussi suscité " un solide lot de critiques, s'étendant du sain scepticisme au rejet absolu » (Thay, 2014, p. 56), notamment de la part de spécialistes de la psychologie cognitive comme Steven Pinker (2006). 
Mais même dans l'acception scientifique originelle des schémas, il n'apparaît pas que les divers efforts de caractérisation soient totalement parvenus à contrecarrer la malléabilité originelle de cette notion. D'un côté, «le concept de schémas a été un concept théorique central de la psychologie cognitive et développementale» (Steel, 2012) - il joue du reste un certain rôle dans la notion d'effet contextuel de Sperber et Wilson ${ }^{2}$ - et il alimente encore aujourd'hui des centaines, voire des milliers de publications. De l'autre, ces publications constituent pour l'essentiel des applications de ce paradigme à des problématiques allant de l'enseignement d'une langue seconde (Al-Issa, 2011 ; Jahangard et al., 2012 ; Zhao et Zhu, 2012) aux erreurs de jugement des pilotes d'avion (Plant et Stanton, 2012) ou à celles des managers (Chung et Lee, 2011), et, plus rarement à des questions communicationnelles (Meunier et Peraya, 2010), sans pour autant que la théorie des schémas en tant que telle se soit sensiblement développée depuis les années 1980 ou qu'elle ait joué un rôle de premier plan dans les avancées récentes de la psycholinguistique cognitive. Une nouvelle vague de travaux dans le cadre de la neurologie tend cependant à revisiter la théorie des schémas avec un intérêt croissant ces dernières années (voir Ghosh et Giboa, 2014, pour une revue).

Comme tout candidat au statut de paradigme scientifique, la notion de schéma a, dès son avènement dans les années 1980, suscité sa part de perplexités et de critiques. En l'espèce, celles-ci ne manquaient pas de substance. La plus évidente est le risque tautologique d'une théorie que ses promoteurs, et plus encore ses utilisateurs, tendaient à élargir de façon un peu vague à tous les processus liés à la mémoire humaine :

Bien que les théories des schémas, soit orientent, soit sont utilisées pour expliquer une portion considérable des recherches actuelles, il est largement admis (par ex. Brewer \& Treyens, 1981; Taylor \& Crocker, 1981), que le terme schéma n’a pas de définition définitive. Il est le plus souvent utilisé pour évoquer la connaissance générale qu'une personne possède d'un domaine particulier. Un schéma permet l'encodage, le

2 Sous la désignation de «bloc d'information encyclopédique » (Sperber et Wilson, 1989, p. 209). 
stockage et la récupération de l'information liée à ce domaine.

(Alba et Hasher, 1983, p. 203)

De ce fait, le terme, lorsqu'il est employé inconsidérément, pourrait être simplement remplacé par « connaissance » (comme « cognitif » pourrait l'être par «mental » chez bien des essayistes) sans perdre beaucoup de spécificité dans un bon nombre de textes. Même s'il s'est rapidement dilaté à l'usage, le concept était déjà passablement large à l'origine, puisque, selon les cas, il englobait non seulement les agrégats génériques de la mémoire à long terme, tant statiques (comme «maison ») que procéduraux (alias scripts, comme « prendre de l'essence ) ou narratifs (alias grammaires de récits, comme "conte de fées $»)$, mais aussi la situation particulière que le texte évoque et que le lecteur construit à sa lecture (le modèle mental). De surcroît, les propriétés de hiérarchisation et d'enchâssement attachées à la notion de schéma ne facilitent pas la détermination de ce qui constitue ou non une telle structure, tout objet sémantique pouvant être considéré comme un cas particulier d'un concept plus générique ou, à l'inverse, être divisé en constituants plus restreints ou plus spécifiques.

Au-delà de ces difficultés définitionnelles manifestes, plusieurs revues critiques ont examiné les inductions communes aux textes fondateurs de la théorie des schémas, ainsi que leurs prédictions et leurs fondements expérimentaux (notamment Alba et Hasher, 1983 ;Fayol, 1985 ; Fiske et Linville, 1980 ;McVee, Dunsmore et Gavelek, 2005 ; Nassaji, 2007 ; Sadoski, Paivio et Goetz, 1991 ; Sadoski 2018 ; Thorndyke et Yekovich, 1980 ; Whitney et al., 1995).

Sans prétendre les résumer, on en retiendra principalement ici, d'une part, que la notion de schéma ne rend qu'imparfaitement compte de toute la complexité des processus mnésiques (par exemple le niveau de détail de certains souvenirs paraît exclure qu'ils aient fait l'objet d'une abstraction schématique³) et, d'autre part, qu'aucune des nombreuses expériences sur lesquelles ils se fondent n'est individuellement à l'abri d'une contestation méthodologique ou d'une quelconque explication alternative. Dès 1980, Thorndyke et Yekovich remarquaient ainsi :

3 De même que la mémorisation verbatim de certaines séquences textuelles («Entre ici, Jean Moulin... ») exclut que le code linguistique soit invariablement oublié au profit des schémas. 
Les théories récentes, construites sur la notion de schéma ont expliqué de nombreux résultats concernant la compréhension humaine, le rappel et la condensation de la prose. Bien que la théorie des schémas fournisse un cadre plausible et descriptif pour comprendre le traitement des connaissances humaines, elle est faiblement circonscrite et fournit peu de propositions détaillées sur les processus. Ce manque de contraintes permet assez de souplesse pour s'adapter a posteriori à beaucoup de résultats empiriques. Toutefois, en raison de cette souplesse, la théorie n'a qu'une valeur prédictive limitée et n'est pas testable scientifiquement sous sa forme actuelle. (Thorndyke et Yekovich, 1980, p. 23)

Mais, s'il est clair que la théorie des schémas peine en l'état à remplir les stricts critères de réfutabilité expérimentale de la psychologie moderne, il est d'autant plus frappant de remarquer que beaucoup de ceux qui soulignent ses limites (Fayol, 1985 ; Fiske et Linville, 1980 ; Thorndyke et Yekovich, 1980 ; Whitney et al., 1995) n'entendent pas renoncer pour autant à cette théorie, et que même ceux qui appellent vigoureusement à son remplacement proposent des alternatives qui ne sont pas foncièrement incompatibles avec la notion de schéma. Alba et Hasher (1983) et Nassaji (2007) se tournent ainsi vers le modèle Construction-Intégration de Kintsch (19884)' Sadoski, Paivio et Goetz (1991) avancent pour leur part une hypothèse de leur cru, la théorie du double encodage, mais, dans les faits, ni les uns ni les autres n'éliminent la possibilité, et même la nécessité, de structures de connaissances génériques. Ce que réfutent surtout leurs textes, c'est la prétention originelle de la théorie des schémas à expliquer seule et totalement la globalité des processus de traitement et de mémorisation de l'information.

Le débat autour des schémas peut s'interpréter (à l'instar de Whitney et al., 1995) comme l'expression d'un conflit plus vaste, mais de moins en moins pertinent, celui qui opposait les conceptions de la compréhension humaine dites «bottom-up » (dirigée par les données) et «top-down» (dirigée par les connaissances).

4 Léger anachronisme commis au nom de la fluidité du texte : en réalité, Alba et Hasher (1983) se réfèrent à une version plus ancienne (Kintsch \& van Dijk, 1978) de ce qui deviendra le modèle de Kintsch. 
Les théoriciens des schémas s'opposaient avant tout à la conception «atomistique » (Brewer et Nakamura, 1984), jadis prévalente, selon laquelle la compréhension était dirigée de façon ascendante par les unités linguistiques concentriques - mots, syntagmes, phrases... - contenues dans le texte :

La compréhension des mots, des phrases et du discours ne peut être simplement une question d'application des connaissances linguistiques. [...] On en conclut, dès lors, que les structures de connaissances que le lecteur apporte au texte sont plus importantes que les structures se trouvant, si l'on peut dire, «dans» le texte. (Anderson, Reynolds et al., 1977, p. 369)

D'où leur défense un peu radicale d'une conception essentiellement descendante, dirigée par les connaissances du lecteur. Cependant, la psycholinguistique cognitive moderne et les expériences qui la soutiennent ne s'accommodent plus d'un choix dichotomique entre ces deux extrêmes, les données langagières et les connaissances contribuant nécessairement à la compréhension du texte. Ainsi, la théorie dominante, le modèle ConstructionIntégration de Walter Kintsch,

décrit l'interaction entre les processus top-down et bottom-up dans la compréhension : comment les processus top-down guident la compréhension et comment les processus bottom-up la contraignent. À tous les niveaux d'analyse - depuis le traitement linguistique de base jusqu'à l'intégration des connaissances - les processus top-down et bottom-up déterminent en commun la nature des représentations mentales formées durant la compréhension. (Kintsch, 2005, p. 125)

Pour sa part, le modèle également séduisant de «double codage » développé par Paivio et ses collègues "est une théorie interactive, rendant compte des processus bottom-up, dirigés par ce qui est imprimé, et des processus top-down, dirigés par la mémoire" (Sadoski et Paivio, 2012, p. 8).

La théorie des schémas pourrait-elle, en réduisant ses revendications, s'intégrer à ce nouveau paysage? Il ne s'agit pas 
simplement là d'une question de partage d'influence sur les processus de lecture. Sous ses formes originelles, l'assemblage nommé théorie des schémas associe des propositions de diverses natures, dont quelques-unes ont perdu leur intérêt quand d'autres semblent préserver toute leur importance. D'un côté, la principale convergence des recherches actuelles sur les processus de lecture, selon laquelle comprendre un texte consiste à construire progressivement une représentation figurée de la situation spécifiquement décrite dans ce texte, diverge de l'hypothèse originelle d'un schéma préalable dominant, qui devrait être activé dès le début du texte pour contrôler assez rigidement l'acquisition et l'interprétation du contenu de celui-ci. La théorie d'un schéma narratif global, comme l'a montré Fayol (1985) de façon très concluante, rencontre des difficultés quasi-insumontables. Mais d'un autre côté, aucun modèle théorique ne peut se dispenser de considérer que la compréhension du texte implique l'activation de connaissances génériques instanciables 5 . En d'autres termes, s'il est plus que douteux que la lecture du récit d'une partie de pêche soit globalement contrôlée par l'activation initiale d'un schéma global «aller à la pêche » dont le texte ne serait qu'une variation, il est en revanche plus que plausible que la construction d'une représentation du sens de ce texte réclame l'activation de schémas génériques comme "poisson», «barque» ou « rivière » pouvant être enchâssés (comme « écaille » dans « poisson ») instanciés par le texte (" barque rouge », « rivière en crue ») et dont les propriétés typiques permettent une partie des inférences (« les poissons ont probablement fui à cause du bruit »). Si les schémas, structures de connaissances permanentes, ne peuvent pas, ou plus, être assimilés au modèle mental plus ou moins éphémère que le lecteur met en scène à la lecture du texte, ils n'en sont pas moins des constituants essentiels de ce modèle.

Dans cette perspective, la distinction entre les schémas et les modèles de situation peut être conceptualisée comme la différence entre des types (les schémas) et des symbolisations (les modèles de situation). Pour aller plus loin, nous voudrions

5 Même Sadoski et Paivio, les plus vigoureux pourfendeurs de la théorie des schémas, supposent la mise en jeu de « représentations », tant verbales que visuelles. 
appuyer la proposition faite par van Dijk et Kintsch (1983) que les schémas peuvent être employés comme des briques pour la construction des modèles de situation. (Zwaan et Radvansky, 1998, p. 162)

La dépréciation de la théorie des schémas en tant que cadres préalables du récit global n'affecte donc en rien la légitimité de la notion de schéma en tant qu'unité de connaissance générique, composante assemblée à d'autres pour contribuer à construire un modèle de ce que dit le texte. De ce point de vue, la plupart des propriétés postulées originellement paraissent assez bien s'appliquer à ces composantes cognitives, sauf sans doute la propension systématique à " abstraire » prêtée aux schémas. Les travaux des dernières décennies sur l'imagerie mentale ont en effet établi l'importance de la dimension perceptive (en particulier visuelle) dans la cognition et ce que les pionniers de la théorie des schémas désignaient comme la tendance de ceux-ci à "abstraire » doit plutôt se comprendre comme une tendance à "généraliser", c'est-à-dire à ramener les énoncés à des objets génériques, résumés par leurs caractéristiques typiques les plus saillantes : le terme " poisson », par exemple, n'active pas une notion "abstraite», mais une représentation générique, un peu indistincte mais néanmoins figurable.

Toutefois, selon les normes de la psychologie moderne, le substrat de ces schémas (réseau connexionniste?), la nature de leur encodage (perceptif? propositionnel ? verbal ? voire, plausiblement, plusieurs de ces modalités) et les modalités de celui-ci demeurent assez indéterminés et difficiles à tester. Même les recherches en neurologie, dont on a évoqué plus haut l'intérêt renouvelé pour cette notion, n'ont pas - ou pas encore - contribué à établir exactement ce qu'elle recouvre. Il est vrai que, comme le soulignent Ghosh et Giboa, «les récentes études sur l'infrastructure neuronale des schémas ont émergé de différentes interprétations de ce qui est signifié par le terme schéma » (2014, p. 113).

Dans une perspective appliquée, mentionner cette imprécision n'a cependant qu'un intérêt anecdotique. Outre que la plupart des théories qui ont cours en sciences humaines et sociales résisteraient infiniment moins bien que les schémas aux stricts critères popperiens des sciences expérimentales, il faudrait une certaine candeur épistémologique pour croire - même en sciences de la 
nature - que la précision absolue des concepts est nécessairement la condition de leur valeur analytique. De même que les équations de Newton demeurent largement utilisées dans le domaine aérospatial, que la vieille conception planétaire de l'atome garde une certaine utilité en chimie et qu'une terre plate constitue une approximation souvent admise en géologie, la notion de schéma cognitif peut être considérée comme scientifiquement légitime et opératoire dès lors qu'elle est, à son niveau de précision, compatible avec l'état des connaissances empiriques et théoriques, ce qui est le cas (voir Butcher et Kintsch, 2003 ; Whitney et al., 1995, Zwaan et Radvansky, 1998, Gosh et Giboa, 2014), et qu'elle rend compte de façon satisfaisante de phénomènes sans méprendre sur la complexité réelle de ce qu'elle modélise (complexité qui, en tout état de cause, est loin d'être percée par les recherches actuelles).

Ainsi, que l'on considère ces représentations comme des structures fondamentales de la cognition ou comme des concepts heuristiques, leur importance pour l'analyse de la production et de la réception discursive est considérable. 


\title{
Triomphe et déroute de l'homo pertinensis
}

La culture tient à la nature, la nature tient à la culture, comme sa bigarrure au tigre.

Confucius $^{1}$

\begin{abstract}
A u terme de ce parcours dans le maquis de l'effort et de l'effet cognitif, il devient possible d'esquisser un modèle général des principaux facteurs susceptibles de jouer sur la pertinence des informations. Celui-ci (voir p. 142) peut sembler un peu rébarbatif de prime abord, mais, si l'on fait abstraction des quelques précisions de la partie droite, sa structure essentielle - c'est-à-dire sa colonne centrale - est d'une très grande simplicité, se contentant au bout du compte de situer et d'articuler des variables intuitivement assez banales (hélas²).

Lorsqu'on y prête attention, on peut remarquer l'influence considérable de ces forces dans les périodes et domaines les plus variés. C'est par exemple le cas de la production livresque avant même la naissance de l'imprimerie. Les multiples innovations graphiques qui, au fil des siècles, conduisent à la forme parachevée des manuscrits médiévaux peuvent en effet être considérées à plus d'un égard comme une gestion éditoriale de plus en plus fine de la pertinence cognitive. La plupart de ces évolutions visent à alléger l'effort
\end{abstract}

1 Cette traduction (Ryckmans, 1987, p. 66-67) a été contestée par P.-E. Dauzat... mais elle est trop jolie pour que l'on y renonce si facilement.

2 Une conception de la démarche scientifique, soutenue notamment par Gaston Bachelard (1938), la considère à l'aune de la «rupture épistémologique » qu'elle introduit par rapport à la perception commune des phénomènes. Mais cette approche, pour inspirante qu'elle soit, ne saurait me conduire à rompre pour le plaisir de rompre. 


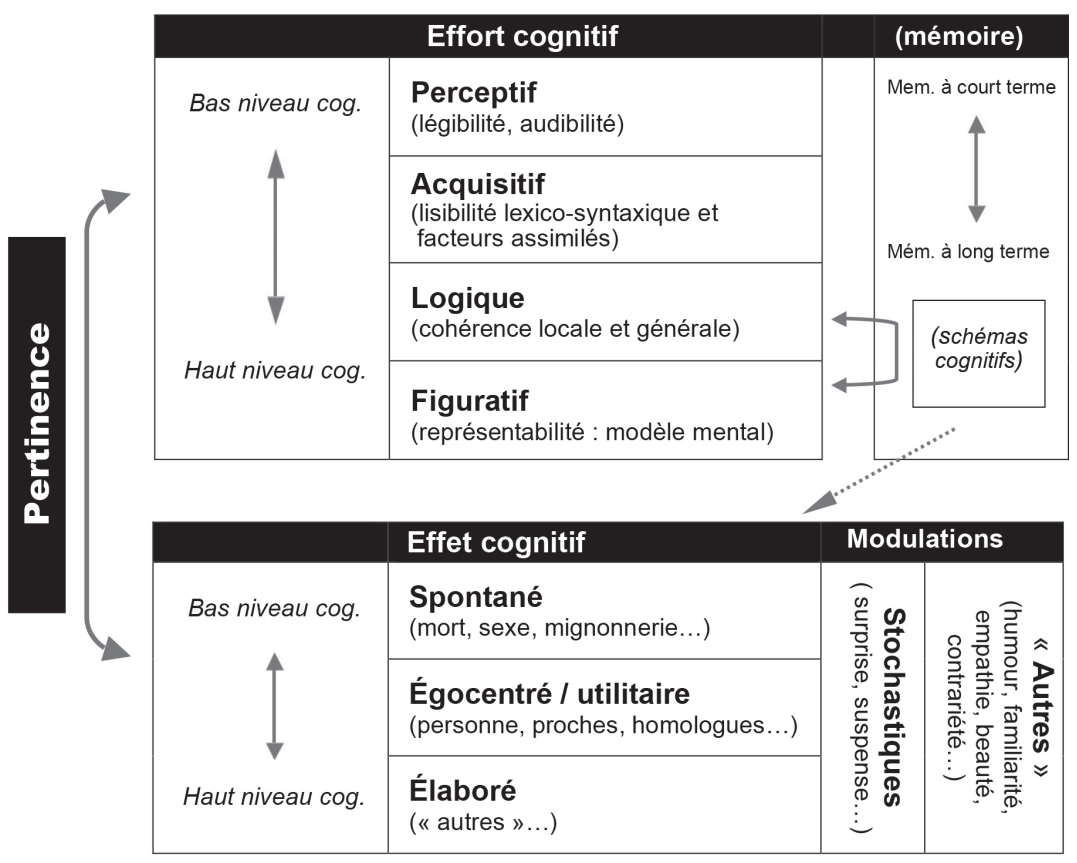

Figure 3. Un modèle de la pertinence cognitive

de traitement de bas niveau. Outre la disposition du texte en colonnes (composition cognitivement optimale, qui remonte aux rouleaux de papyrus mais ne survit que dans les journaux et les ouvrages utilitaires), l'utilisation des minuscules et l'insertion d'espaces entre les mots apportent un gain de légibilité considérable par rapport aux austères inscriptions latines en majuscules non espacées. De même, l'usage des capitales initiales ainsi que le développement de la ponctuation favorisent le traitement lexico-syntaxique du texte (et donc sa lecture silencieuse). Les lettrines et la séparation en paragraphes, de même que l'usage de rubriques (titres de sections) facilitent également le traitement de sa cohérence, tandis que les illustrations renforcent sa figurabilité. Mais, à l'instar de la couleur des majuscules initiales et des décorations enluminées, elles tendent tout autant à accroître l'effet cognitif de l'information que son repérage et sa mémorisation : œuvres d'art et de piété ou de savoir, les manuscrits sont aussi des produits cherchant dans toute la mesure du possible à valoir - dans tous les sens du terme, bien sûr - sur le marché discursif. 
Si le graphisme est l'aval de la production, le choix des sujets est son amont, et des logiques similaires s'y remarquent à des époques et dans des contextes très différents. On avait par exemple évoqué un peu plus haut la question de ce choix à propos de la valeur journalistique des informations qui sont publiées, mais les nouvelles qui ne le sont pas méritent également que l'on s'y arrête. Une organisation états-unienne a ainsi demandé à 377 professionnels des médias les raisons pour lesquelles ils étaient susceptibles de ne pas publier une information dont ils étaient pourtant convaincus qu'elle devrait l'être (PEW, 2000). Les deux motifs qui se détachaient clairement étaient le sentiment qu'une information est « importante mais ennuyeuse » $(77 \%)$ ou qu'elle est «trop complexe» $(52 \% 3)$. Serait-ce que les journalistes tendent à occulter d'autres raisons, comme la crainte de représailles internes ou externes (par exemple celle de déplaire à des amis de la direction : $18 \%$ ) ? Qui connaît les débats des organisations professionnelles dans ce champ sait qu'ils les dénoncent au contraire volontiers, a fortiori lorsque leur anonymat est protégé. D'autant que, contrairement aux pressions, les deux principales raisons avouées sont des aveux d'impuissance qui mettent directement en question les compétences professionnelles des répondants ${ }^{4}$.

Ce que disent les journalistes est bien ce que sont les journalistes : des professionnels de la pertinence. Ainsi, les deux raisons avancées peuvent-elles très précisément se reformuler dans ce cadre : la première revient à dire "l'information ne produit pas assez d'effet cognitif » et la seconde "l'information exige trop d'effort cognitif ». Tout l'intérêt de cette transposition est qu'elle substitue à des critères isolés et inertes des déterminants articulés qui interagissent selon une logique d'ensemble. La complexité ou l'insipidité ne sont pas des

3 On n'aditionne ici que les réponses «généralement» et «occasionnellement » : si l'on ajoute « rarement », seuls $7 \%$ des répondants disent n'avoir jamais été concernés par l'autocensure d'une information «importante mais ennuyeuse » et $15 \%$ par celle d'une information «trop complexe » (et encore... les données publiées cumulent les réponses « jamais » et « ne sait pas »).

4 Croire que la valeur de l'information réside nécessairement dans l'information revient à réduire le traitement journalistique à un travail de convoyage et à nier sa possible valeur ajoutée : rendre intéressant ou clair un sujet qui ne l'est pas à l'origine est en principe une aptitude journalistique fondamentale. 
dimensions indépendantes : une information complexe sera néanmoins publiée si cette difficulté est compensée par son effet cognitif (par exemple les modalités biologiques d'une épidémie) et, à l'inverse, une information peu intéressante (une nouvelle de routine) sera jugée acceptable si elle est cognitivement peu exigeante.

Cependant, les deux exemples ci-dessus présentent un second intérêt. Tout en illustrant ce que la notion de pertinence permet de mieux comprendre, ils exposent crûment ce que cet outil conceptuel ne permet pas du tout d'expliquer. Ainsi, les copistes médiévaux qui, au cours du XII ${ }^{\mathrm{e}}$ siècle s'avisèrent de remplacer les lettres carolines par les illégibles caractères gothiques posent-ils un sérieux problème. À contre-courant des autres évolutions, cette typographie qu'Émile Javal, pionnier de la psychologie de la lecture (et avocat, bien avant Zipf, de la théorie $\mathrm{du}$ 《 moindre effort » cognitif), jugeait à juste titre «détestable ", est parfaitement incompatible avec l'hypothèse selon laquelle le marché discursif serait régenté par la seule recherche de la plus grande pertinence possible. Il en va plus généralement de même pour la sélection des ouvrages à la reproduction desquels les copistes consacraient tant de temps. Si une petite partie de la production concernait des discours plaisants (amour courtois, fabliaux...), sans compter les illustrations parfois obscènes qui rôdaient dans les marges ${ }^{5}$, l'essentiel de celle-ci, consacré à la dévotion ou l'érudition, n'était manifestement pas guidé par l'arbitrage hédonique du plaisir et de l'effort.

C'est tout aussi vrai en ce qui concerne le journalisme, dont le jugement de pertinence explique parfaitement certains choix de sujets, et pas du tout d'autres (problèmes internes de pays non stratégiques, manifestations culturelles, découvertes scientifiques sans enjeux utilitaires, etc.).

On remarquait plus haut qu'une conception de la valeur discursive fondée sur la « loi du moindre effort » conduirait les librairies de gare à ne proposer que des livres et journaux pour enfants, dont la légibilité, la lisibilité, la cohérence et la figurabilité sont généralement optimales. Mais un libraire qui serait pour sa part convaincu par Sperber et Wilson que

5 «La complaisance devient certaine lorsque les caricatures de la sexualité s'exhibent dans le Roman de Renart ou les fabliaux : c'est brutal, c'est sale, mais on ne s'en lasse pas » (Wirth, Engammare et al., 2008 p. 251). 
les êtres humains cherchent automatiquement, dans toute leur activité cognitive, à obtenir la pertinence la plus grande possible, c'est-à-dire l'effet cognitif le plus grand, pour l'effort de traitement le plus faible (1992, p. 228)

ne mettrait en vente que des quotidiens de faits divers, des magazines salaces et des livres garantissant également un effet élevé pour un effort minimal. Or, ceux-ci sont manifestement loin de représenter la totalité de l'offre et de la demande éditoriale, y compris dans les points de vente les plus liés à la lecture distractive : il n'y a pas que de la littérature de supermarché dans les supermarchés. En outre, "l'effet cognitif le plus grand » n'est pas forcément le plus désirable, comme en témoigne notamment le fait que des récits ou des images puissent être qualifiés d'«insoutenables » et être effectivement évités. Une tentative avortée d'esquisser une théorie ressemblant à la pertinence avait même proposé dès le $\mathrm{xI}^{\mathrm{e}}$ siècle d'articuler l'«économie des énergies mentales » (soit l'effort) avec... l'«économie des sensibilités mentales », soit, plus ou moins le contraire de l'effet maximal (Spencer, 1852).

Pourtant, ces constats ne remettent nullement en cause la validité de l'axiome fondamental de la pertinence : ils soulignent simplement que ce qui fait sa force marque aussi les limites de sa portée. Si l'affinité ou l'aversion sont psychologiquement déterminées, elles sont par ailleurs culturellement surdéterminées. Les êtres humains ne sont pas seulement des créatures psychologiques, ce sont aussi des créatures sociales. Voilà une bien mince révélation, surtout après avoir passé plusieurs chapitres à esquiver ostensiblement toutes ces choses compliquées (les goûts, les valeurs, les normes...) qui cherchaient inlassablement à s'emmêler dans les lisses et sobres rouages du traitement psycholinguistique des discours.

Or, la conception de la pertinence comme un arbitrage hédonique, que ce soit sous sa forme originelle ou selon l'explicitation que l'on en a proposée, ne peut intégrer les déterminants sociaux sans se dénaturer. Même en tenant compte des observations ultérieures de ses auteurs sur ce point (on y reviendra), elle est, dans ses fondements, une théorie psychologique naturaliste. Étendez-la à l'ensemble des valeurs socialement déterminées et elle ne signifie plus rien («Les êtres humains cherchent automatiquement, dans toute leur activité cognitive, à obtenir ce qui leur plaît»). Retranchez-en les valeurs sociales et elle retrouve sa portée analytique, mais ne peut plus espérer devenir la 
théorie générale de la communication qu'envisageaient Sperber et Wilson, une théorie asociale de la communication ne pouvant guère être plus qu'une contradiction dans les termes.

On peut, du reste, observer la même alternative sous un tout autre angle. Psychologique ou pas, l'axiome hédonique de la pertinence est, à l'instar de ses devanciers et par définition, une conception coûts-bénéfices des préférences humaines. À l'instar de l'homo œconomicus prétendument rationnel de l'économie néo-classique, l'homo pertinensis ne viserait qu'à maximiser la satisfaction qu'il retire de l'information qu'il consomme. Cette parenté est moins fortuite que l'on ne pourrait le penser, Sperber et Wilson admettant eux-mêmes une telle source d'inspiration :

À un niveau très général, on pourrait comparer le concept de pertinence avec des concepts comme ceux de productivité ou de rendement, concepts qui s'analysent en termes de coûts et de bénéfices [...] le parallèle entre la productivité et la pertinence est clair. (1989, p. 188-189)

Il est très clair, en effet. Aussi clair que l'est la presque ${ }^{6}$ similitude entre les problèmes de l'homo œconomicus et ceux de l'homo pertinensis.

En effet, la prise en compte de valeurs morales peut influencer le comportement économique dans un sens différent de celui de la recherche de la maximisation de l'intérêt personnel, comme il est patent dans des domaines comme le marché du travail, la distribution des revenus, la croissance à long terme ou la production de biens publics. Et négliger la nature spécifique de cette influence mine la capacité explicative et prédictive des modèles.

6 Paradoxalement, certaines des réfutations de l'homo cconomicus sont justement de nature psychologique et s'apparentent même à ce que prédit la théorie de la pertinence : la maximisation de l'intérêt personnel par les agents économiques supposerait qu'ils soient capables de calculer parfaitement les conséquences de leurs choix, ce qui impliquerait un effort de documentation et de réflexion disproportionné par rapport au bénéfice retiré d'une telle optimisation (par exemple, pour acheter de la nourriture, vérifier aliment par aliment les prix de toutes boutiques, puis les corriger en fonction de la dépense d'essence nécessaire pour atteindre chaque point de vente et en retirer le trajet idéal pour faire ses courses). 
La question est donc de savoir si l'intégration des valeurs morales dans la délibération des agents n'implique pas de renoncer à l'unidimensionnalité des préférences [...] (Lopes, 2005)

À l'instar du modèle économique des choix rationnels, le modèle de la pertinence cognitive n'est pas "faux", pas plus que n'était faux l'axiome mentionné plus haut selon lequel les humains tendent à choisir le chemin le plus court pour marcher d'un point à l'autre. L'homo œconomicus et l'homo pertinensis sont non seulement utiles, mais aussi tout à fait appropriés pour aborder de façon approximative un bon nombre de phénomènes. Ils ne deviennent des monstres que lorsque l'on prend ces réductions schématiques pour des explications fidèles, ou même suffisantes, de la complexité des conduites humaines.

$\mathrm{Si}$, comme le soulignait Lopes, les « valeurs morales » influencent le comportement économique, elles influencent autant et sans doute plus la sélection des informations. Imaginons un accident sanglant : un passant, appliquant inconsidérément le principe du trajet le plus court, vient de disparaître sous les roues d'un autobus. Quiconque affirmerait que ce spectacle ne l'intéresse pas se méprendrait sérieusement sur son propre compte. Un tel drame est hautement pertinent, et il est pertinent pour tous : le voyeurisme est aussi naturel cognitivement que la soif ou la faim le sont physiologiquement. Pourtant, certains passants refuseront de se mêler aux badauds qui se bousculent autour de la scène, non parce qu'elle ne les attire pas, mais parce que leurs valeurs morales (notamment la réprobation dudit voyeurisme) luttent contre le puissant effet de premier niveau qui confère sa pertinence à l'accident.

Le poids des valeurs sociales est bien connu, que ce soit intuitivement («je ne regarde pas ce genre de films ») ou dans l'abondante littérature scientifique consacrée aux goûts et aux normes (on y reviendra, bien sûr). Mais il est rarement envisagé en tant que polarité contrebalançant celle de la pertinence pour assurer l'équilibre général de la valeur de l'information. On peut pourtant remarquer partout les traces empiriques de cette opposition.

C'est par exemple le cas du silence obstiné avec lequel la presse française a couvert les frasques extraconjugales de plusieurs présidents de la République. Cette tradition, qui n'a pris fin qu'avec la liaison de François Hollande, n'était évidemment pas liée à la pertinence 
de ces informations, particulièrement élevée en l'espèce. Elle était le fruit de normes culturelles locales dont on ne trouve pas l'équivalent dans le journalisme anglo-saxon, même de haute tenue, qui n'hésite pas à dévoiler les frasques des dirigeants. À l'inverse, celui-ci est, encore aujourd'hui, paralysé à l'idée de rapporter les termes inconvenants qu'emploient parfois les personnages publics, malgré l'évidente pertinence de telles citations. Ainsi, un journal comme le New York Times recourt-il à une vaste gamme d'euphémismes (" un terme expressif», «une formule obscène »...) pour évoquer aussi abstraitement que possible le genre d'expressions que les journaux français se font une joie de reproduire mot pour mot, par exemple "Que l'UE aille se faire foutre " (Libération, 08.02.2014, p. 9) ou même "Va te faire enculer, sale fils de pute » (L'Équipe, 19.06.2010, p. 1).

Ces normes discursives locales relèvent-elles primitivement de principes professionnels ou d'exigences imposées par les publics visés? La métaphore du marché discursif suggère à point nommé qu'il serait risqué de leur attribuer une origine unilatérale, un marché étant par nature le lieu d'un ajustement approximatif entre production et réception :

En matière de biens culturels - et sans doute ailleurs - l'ajustement entre l'offre et la demande n'est ni le simple effet de l'imposition que la production exercerait sur la consommation ni l'effet d'une recherche consciente par laquelle elle irait au-devant des besoins des consommateurs mais le résultat de l'orchestration objective de deux logiques relativement indépendantes, celle des champs de production et celle du champ de consommation [...] (Bourdieu, 1979, p. 255)

C'est ce que traduit particulièrement bien une curieuse évolution des romans sentimentaux, branche éditoriale soumise plus qu'une autre aux diktats des études marketing. Au tournant du siècle, les grands éditeurs ont en effet renoncé aux fameuses " clinch covers", les couvertures très colorées sur lesquelles s'étreignaient traditionnellement des couples éperdus d'amour (Olivier, 2007). Les ont remplacées des images quelconques, paysages ou fleurs, ostensiblement dépourvues de la moindre pertinence (y compris au sens commun du terme, ces vues n'ayant souvent aucun rapport avec le récit). Les études menées auprès des lecteurs, en l'occurrence des lectrices, n'avaient pourtant 
pas révélé que les corps furieusement enlacés avaient perdu leur effet cognitif et que les acheteuses potentielles entendaient désormais s'intéresser aux paysages ou aux fleurs. Ce qu'elles avaient montré, c'est que celles-ci lisaient beaucoup en transport en commun et qu'elles n’appréciaient guère que la couverture expose leurs préférences littéraires aux autres voyageurs. Si séduisantes que puissent être pour elles les caresses tropicales, elles n'étaient pas de taille à rivaliser avec la réprobation sociale réelle ou supposée. Estimant gagner plus en légitimité que ce qu'ils perdaient en pertinence, les éditeurs optimisaient donc la valeur de leurs produits sur le marché discursif.

Pour rester dans l'optique suivie jusqu'à présent - rechercher de possibles régularités derrière les situations les plus hétérogènes en apparence - visitons donc quelques expositions. La première, organisée en 2012 à Paris (Palais de la Découverte), traitait de la reproduction et de la sélection évolutive dans le monde animal, sujet propre à exciter les amateurs de sciences, mais à qui un coup de pouce pouvait permettre de capter une plus large attention. Le titre retenu à cette fin, «Bêtes de sexe », était à cet égard une merveille d'optimisation discursive, exploitant plusieurs niveaux d'effet sans franchir les limites de l'acceptabilité sociale contemporaine. Encore fallait-il l'illustrer. Une énergique copulation entre deux bestioles aurait eu le mérite de la clarté, mais pas forcément celui de la recevabilité normative. Les organisateurs ont donc opté pour la photo d'un seul gorille, lascivement étendu dans la pose du macho satisfait, ce qui leur permettait de jouer habilement sur une propriété saillante - si l'on ose dire - du schéma cognitif « gorille» (la vigueur sexuelle que chantait Brassens). On imagine cependant que cette idée a suscité quelques hésitations, puisqu'elle revenait à malmener au nom de la pertinence un autre type de contrainte normative, la mission pédagogique des musées scientifiques, censés dissiper les mythes scientifiques et non les alimenter (on sait que le gorille est en réalité pourvu d'un organe sexuel minuscule, d'ailleurs presque invisible sur l'affiche).

S'il s'agissait en l'espèce de soutenir la pertinence cognitive d'une exposition socialement légitime, les gestionnaires de la valeur discursive peuvent être amenés dans d'autres cas à tenter une opération inverse : renforcer la légitimité sociale d'une exposition par ailleurs pertinente. C'est typiquement le cas des exhibitions de cadavres écorchés et plastinés (« Our body», «À corps ouvert »...) qui sillonnent la planète. Il n'est guère nécessaire de souligner l'extrême pertinence 
de ces dépouilles, qui requièrent très peu d'effort pour toucher tous les registres de l'effet (avant tout le premier niveau, bien sûr, mais pas seulement lui). Conscients que la pertinence ne suffit pas à assurer la recevabilité d'une offre discursive face aux normes sociales, les organisateurs se sont toujours efforcés de mettre en avant les vertus pédagogiques de leur monstration. L'argument est défendable, du reste, comme aurait pu l'être celui de la valeur artistique, mais souvent insuffisant pour contrecarrer la réprobation soulevée par la mise en scène de cadavres d'origine suspecte.

L'interdiction de la version française de cette exposition a eu ceci d'intéressant qu'elle a dû tenir compte d'une grande différence entre les dimensions cognitive et sociale de l'appréciation discursive. Alors que la première est relativement stable dans l'espace et dans le temps, la seconde est au contraire extrêmement variable, tant dans l'espace (voir plus haut les normes de convenance des quotidiens états-uniens et français) que dans le temps. Ainsi, un intitulé comme «Bêtes de sexe " aurait-il été tout à fait inacceptable il y a un ou deux siècles, tandis que l'exhibition de cadavres difformes dans des collections privées (dont le nom, " cabinets de curiosité », est éloquent) ne l'était pas plus que l'exposition de «monstres » sous les chapiteaux de foire.

Le glaive de la justice a donc dû s'abattre avec précision afin de retrancher de l'espace public les nouvelles initiatives marchandes sans pour autant toucher les momies, pièces anatomiques et autres têtes réduites conservées dans un bon nombre de musées, ou encore les morceaux de saints dont s'enorgueillissent les cathédrales. Soulignant ainsi qu'il demeure possible « de donner à voir aux visiteurs d'un musée des momies extraites de leur sépulture, voire d'exposer des reliques, sans entraîner d'indignation ni de trouble à l'ordre public ", la Cour d'appel s'est exclusivement fondée sur l'absence de preuve de don bénévole des corps exposés, ce qui peut cependant laisser un doute (le consentement des ex-occupants des têtes réduites et autres pièces des collections ethnologiques n'étant pas plus assuré).

Un point, en tout cas, ne laisse guère de doute : que l'on considère les manuscrits médiévaux ou les unes des quotidiens, les couvertures de romans à l'eau de rose ou les affiches d'expositions, l'approche des déterminants de la valeur discursive ne peut ni faire abstraction

7 Cour d'appel de Paris, arrêt o9/09315 du 30 avril 2009. 
des facteurs cognitifs, ni s'en contenter en ignorant les déterminants sociaux. En revanche, envisager cette valeur comme le produit de la tension entre ces deux dimensions permet bel et bien d'explorer une très large gamme de phénomènes avec un référentiel commun. En s'inspirant de Sperber et Wilson, il devient ainsi possible de formuler une nouvelle proposition :

Les êtres humains cherchent automatiquement, dans toute leur activité communicationnelle, à obtenir l'adéquation sociocognitive la plus grande possible, c'est-à-dire la pertinence cognitive la plus élevée au regard des contraintes sociales éprouvées.

Cet axiome (un peu plus qu'un axiome, en fait) se laisse même dessiner assez facilement :

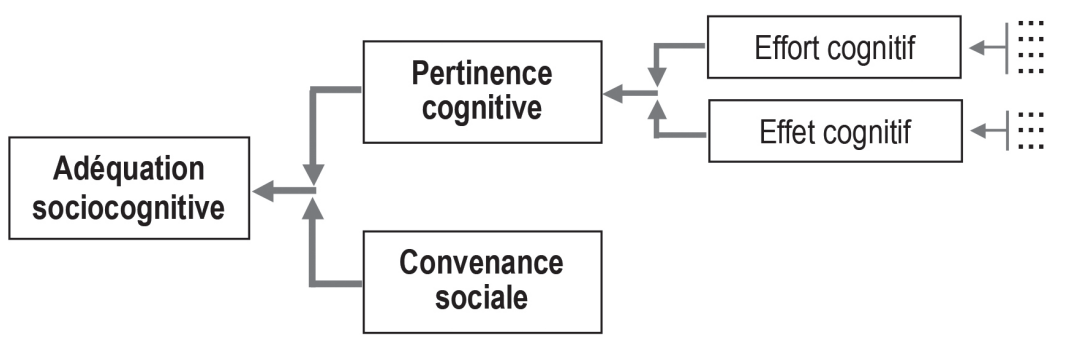

Figure 4. L'articulation de l'adéquation discursive

Il va sans dire que la distinction entre ce qui relève du cognitif et du social, quoiqu'inévitable, est assez arbitraire, au même titre que toutes les catégorisations qui permettent de fonctionner en tant qu'être humain. Notamment en tant qu'universitaire, puisqu'elles définissent les démarcations pratiques entre les disciplines ou les départements. Mais articuler explicitement ces deux "dimensions » parait largement préférable aux alternatives traditionnelles : soit ignorer obstinément l'une ou l'autre, soit au contraire amalgamer le tout dans une confusion aussi inopérante en théorie qu'en pratique (d'autant que, comme noté plus haut, «cognition » n'y est souvent qu'un synonyme pédant de «pensée » ou « connaissance »).

Ainsi la perspective intégrée de l'adéquation sociocognitive interdit-elle, par exemple, de réduire les journalistes à « des professionnels de la pertinence » comme on l'avait fait un tout petit peu plus tôt à 
propos des informations rejetées. Certes, les facteurs cognitifs suffisent pour réaliser que les deux principaux motifs de rejet indiqués par eux sont en réalité les deux faces de la même dynamique (trop d'effort en fonction de l'effet ou pas assez d'effet en fonction de l'effort), mais ils occultent le fait que le jugement éditorial est par ailleurs conditionné par un empilement de normes internes ou externes, qui tantôt s'opposent à ces facteurs, tantôt les renforcent : on évoquera parfois même le « devoir » de parler de ceci ou de taire cela. Les journalistes ne sont pas des professionnels de la pertinence, ce sont des professionnels de l'adéquation, soucieux de livrer sur le marché discursif les nouvelles les plus pertinentes possible, mais seulement en fonction des critères publics de convenance et de leurs propres critères professionnels. Si tel n'était pas le cas, l'essentiel de la production éditoriale se réduirait aux recettes de la presse tabloïd et des sites Internet les plus racoleurs. Or la hiérarchie symbolique du journalisme, soulignée notamment par Rieffel (2005), est plus ou moins l'inverse de celle qu'impliquerait le seul jeu de la pertinence cognitive (et ne relève pas simplement d'une répartition des niches du marché discursif de l'actualité).

Le rapport pertinence-convenance permet en outre d'éclaircir certains des verdicts les plus obscurs de l'évaluation discursive. Plus d'un élève (dont l'auteur de ces lignes) a pu découvrir en marge d'une dissertation une curieuse annotation au stylo rouge : «style trop journalistique». Le correcteur voulait certainement dire quelque chose, mais quoi ? On serait malavisé d'agacer son professeur en lui demandant de définir plus précisément en quoi consiste donc ce «style journalistique", puisque l'adjectif englobe a priori des écritures aussi différentes que celles des revues satiriques, des périodiques culturels et des magazines sportifs, en passant par la forme sèche des dépêches d'agence et la variété infinie des articles de quotidiens. L'approche stylistique traditionnelle s'épuiserait en vain à chercher dans ces textes le genre de régularités apparentes qui, une fois étiquetées, alimentent justement les cours de français : le «style journalistique » n'est pas du domaine du code $e^{8}$. Létrange annotation exprime plus qu'un jugement linguistique : elle est un pur jugement d'adéquation sociocognitive, condamnant un passage qui s'avère trop pertinent (trop

8 Mis à part le cas particulier de la pyramide inversée, qui sera examiné plus loin. 
simplifié ou trop énergique) en fonction des contraintes propres à un travail scolaire.

La retenue expressive que requiert dans ce cas la valeur discursive est toutefois susceptible de la desservir dans d'autres. Ainsi, le même enseignant pourrait-il peu après, et toujours à juste titre, grommeler contre le "style administratif» d'une lettre ou d'une circulaire déposée dans son casier. Là, sa formation lui serait un peu plus utile, l'ayant sensibilité aux tournures impersonnelles (diathèse passive, pronoms indéfinis...), aux signifiants abstraits et aux hyperonymes. Mais elle l'aiderait moins à expliciter la logique de leur emploi et, surtout, le déplaisir qu'il en éprouve. Si, comme l'estime un spécialiste, "l'Administration a et aura généralement "une manière particulière d'écrire et d'exprimer sa pensée" " (Catherine, 1996, p. 14), cette écriture distanciée revient typiquement à produire peu d'effet (en particulier l'interaction avec la sphère personnelle) et à réclamer souvent un effort considérable pour construire le modèle mental très précis évoqué par le texte. Cette exigence n'est possible qu'en vertu d'un statut attentionnel privilégié du « langage d'autorité » (l'administration s'attend à ce qu'on l'écoute) et d'une certaine indifférence à l'adéquation (elle ne cherche pas à plaire $\left.{ }^{9}\right)$. Du reste, une circulaire qui commencerait par exemple par «Écoutez les gars, ça commence à bien faire... » obtiendrait certainement une haute valeur attentionnelle, mais cette transgression des usages ne se convertirait probablement pas en appréciation discursive : pour les destinataires aussi, la convenance sociale contrebalance toujours l'efficacité cognitive.

Il est cependant embarrassant de se contenter d'évoquer vaguement la «convenance» ou les «contraintes» comme une sorte de polarité indistincte qui ne servirait en pratique qu'à équilibrer la pertinence. Outre que cette approximation laisse un blanc assez disgracieux dans un coin de l'élégant schéma esquissé plus haut (hic sunt leones), elle recouvre, comme on l'a signalé, un enchevêtrement de modalités et de désignations - goûts, opinions, règles, valeurs, attitudes, normes, usages, principes, et on en oublie - dont n'importe quelle recherche de la valeur discursive requiert un examen attentif.

C'est ici que les vraies difficultés commencent...

9 Mais du moins accorde-t-elle, notamment au Québec, de plus en plus d'attention à l'effort cognitif qu'elle réclame et à la relation qu'elle institue (voir Clerc et Kavanagh, 2005). 
Page blanche conservée intentionnellement 


\section{(Précisions)}

T a théorie hédonique de la pertinence dont on vient d'examiner le $\amalg$ périmètre de validité, large mais pas illimité est-elle encore la Théorie de la pertinence de Sperber et Wilson ? Il serait à craindre, en effet, que la nécessité de densifier les notions d'effet et d'effort évoquées assez désinvoltement par ces auteurs n'ait conduit à renforcer son ancrage dans la psychologie cognitive expérimentale, et du même coup à accentuer sa distance vis-à-vis des déterminants sociaux.

Ce n'est, à vrai dire, crucial ni pour la proposition originelle, ni pour son avatar. La première, qui répondait aux problèmes spécifiques de la pragmatique inférentielle, n'a guère besoin de telles précisions pour y jouer le rôle éminent que l'on sait (rôle qu'à l'inverse ces précisions n'affectent guère, celles-ci ne concernant qu'une partie d'un système pragmatique plus vaste). Quant au second, le considérer comme un développement de l'axiome de Sperber et Wilson ou comme une transposition libre de celui-ci ne changerait pas grand-chose aux éléments dont il décrit l'articulation (et dont il souligne la convergence diachronique).

La question n'en est pas moins intéressante puisque, indépendamment de l'inspiration dont on leur est en partie redevable (comme eux-mêmes l'étaient à Grice), la perspective de Sperber et Wilson demeure de loin la plus aboutie des théories hédoniques (peine-plaisir ou coût-bénéfice) de la communication ${ }^{1}$.

Comment ces auteurs, dont un anthropologue, envisageaient-ils eux-mêmes les rapports entre le socle explicitement cognitif de leur modèle et la surdétermination sociale des processus communicationnels? Le tronc central de la théorie, centré sur la production

1 La théorie de Schramm, beaucoup plus communicationnelle par essence, n'avait été qu'esquissée. 
automatique des inférences dans les échanges conversationnels, ne s'en préoccupe pas vraiment. On pourrait même dire qu'il s'éloigne en cela de Grice, lequel se référait à des normes minimales partagées et respectées par les locuteurs, alors que pour Sperber et Wilson, «les communicateurs ne "suivent" pas le principe de la pertinence, et ils ne pourraient pas le violer, même s'ils le voulaient » (1989, p. 244).

Une réponse de Sperber et Wilson (1997) aux critiques visant le silence de la théorie de la pertinence sur la dimension sociale des interactions langagières est particulièrement intéressante. Cet article, même s'il persiste et signe dans la méconnaissance des recherches communicationnelles ${ }^{2}$ que manifestait le livre, assume volontiers le fait que celui-ci et les travaux qui l'ont suivi ont « largement ignoré les aspects de la communication débattus dans la littérature sociologique [... se focalisant...] sur des questions typiquement débattues en psychologie». Toutefois, soulignent les auteurs, il ne s'agit là que d'un point de départ, destiné à éclaircir les soubassements fondamentaux de la communication qui demeuraient une impasse pour les sciences sociales. Si tel est bien son objet, alors on peut considérer que l'intégration de son axiome primordial dans un cadre élargi aux déterminants sociaux ( $l^{\prime}$ «adéquation » sociocognitive évoquée ici) s'inscrit fort bien dans la perspective évoquée par ces auteurs.

Un élargissement beaucoup plus spectaculaire a d'ailleurs été opéré par le travail ultérieur de Dan Sperber (1996) sur la " contagion des idées ", qu'il présente sans détour comme une "théorie naturaliste de la culture » et qu'il explique également par le rapport entre l'effort mental et l'effet cognitif.

Du point de vue des objets considérés, le bond est vertigineux entre les bribes de conversations forgées, énoncés minimalistes auxquels se consacrait la théorie de la pertinence, et la culture dans son ensemble, abordée par Sperber à partir d'une métaphore épidémiologique ostensiblement matérialiste (envisager la « contagion »

2 Réduites à nouveau au «modèle du code », alors même que l'on pourrait objecter que la théorie de la pertinence, focalisée sur le traitement du langage, s'apparente beaucoup plus à ce modèle (qu'elle affine et étend aux inférences) que les recherches sur la production et la réception discursives du dernier demi-siècle. 
des idées comme celle des microbes). Par rapport au problème communicationnel qui nous intéresse - qui, tout compte fait, n'est pas aussi large que ça en comparaison - la première salve de Sperber était un peu courte et la seconde beaucoup trop longue. On notera cependant que celle-ci érige cette fois en manifeste épistémologique ce qui n'était que sous-jacent dans la première : un positionnement à l'opposé de l'approche sociologique exclusive de la tradition de Comte, Durkheim, Bourdieu (etc.). Disons, pour simplifier abruptement, que si le dogme sociologique traditionnel exige que l'on n'explique le social que par le social, ses challengers se proposent, en quelque sorte, d'expliquer le social sans le social. Là aussi, les deux salves encadrent notre position, tout en tombant en principe assez loin d'un côté et de l'autre de celle-ci pour ne pas la concerner.

Cependant, n'être aucunement partie prenante dans le stérile affrontement du sociologisme et du psychologisme ${ }^{3}$ (comme le souligne le chapitre précédent) ne protège pas forcément de ses dommages collatéraux. On discutera plus loin des inconvénients qu'entraîne en pratique le fait de décliner l'alternative clanique entre l'une ou l'autre bannière, mais il convient de s'arrêter dès maintenant sur un tir passé trop près pour qu'on l'ignore. Commentant avec assez peu d'enthousiasme les travaux anthropologiques qui se réclament des sciences cognitives, Bernard Lahire (2008, p. 90) a en effet soulevé avec acuité un problème important : le risque des invariances artificielles :

L'ensemble des thèses naturalistes repose sur l'hypothèse de l'existence d'invariants culturels, qui s'expliquerait par les contraintes cognitives du cerveau humain. Or, il faut se méfier de la propension à «fabriquer» artificiellement de l'invariance ou à faire apparaître des « principes sous-jacents » en se plaçant à un niveau d'abstraction tel que deux phénomènes qui paraissaient significativement différents jusque-là puissent révéler leurs propriétés communes. [...] C'est ainsi que Pascal Boyer peut concevoir les structures mentales communes à un utilisateur d'ordinateur et à un navigateur

3 On simplifie, bien sûr, notamment en omettant d'autres baronnies, dont celle - linguistique - du «philologisme» dénoncé par Bourdieu (1977, p. 18). 
mélanésien. [...] Et plus on augmente le niveau d'abstraction auquel on se place en faisant en sorte que tout se ressemble, plus on accroît les chances de mettre en évidence des éléments communs qu'on convertira ensuite en «principe sous-jacents ».

Humm... Faudrait-il se sentir visé par anticipation ? Évidemment, on ne propose pas ici d'expliquer l'esprit humain, la culture ou le sens de la vie. Pas même de contribuer le moins du monde à l'un de ces vieux débats (connexionnisme contre modularisme, nature contre culture, etc.) qui se situent loin en amont de notre problème. Mais ce qui s'applique au rapprochement un peu hasardeux entre les utilisateurs de pirogues et de claviers pourrait-il s'appliquer, disons, à certaines similitudes entre les logiques des copistes médiévaux, des journalistes, des éditeurs de romans sentimentaux et des organisateurs d'expositions?

En matière d'abstraction, comme le suggère Lahire en parlant de «niveaux », c'est la dose qui fait le poison. Ainsi qu'on le remarquait à propos d'un avertissement antérieur et non moins valide (Miège, 1995), l'abstraction - mais aussi la quête des invariances ou régularités et des principes sous-jacents - est la finalité de la recherche, laquelle se réduirait sinon à un amoncellement de descriptions inconséquentes (aboutissement possible de l'idéalisation contemporaine du terrain contre le travail de théorisation des phénomènes). Du reste, en d'autres circonstances, Lahire n'est pas le dernier à défendre l'induction théorique :

Le risque du dénigrement théorique (qui peut devenir un véritable chic théorique inversé) est alors de laisser implicites et confus des problèmes qu'il est justement du ressort de l'activité théorique d'élucider, de délimiter, de clarifier, de débrouiller, de généraliser, etc. (2004, p. 120).

Par ailleurs, nul ne se propose probablement de nier que les personnes, contrairement aux protons, présentent toutes des différences irréductibles, ni que, comme eux, elles présentent des invariances susceptibles d'abstraction. "Les êtres humains boivent tous les jours » est une généralisation inductive, une abstraction : elle ne se réfère pas à des individus particuliers (ni même à des 
données empiriques spécifiées) et supporte des exceptions, quoique celles-ci se terminent généralement mal pour les personnes concernées.

Dès lors que, pour reprendre l'aphorisme de Paracelse, la dose fait le poison, ce qui importe est de déterminer la dose à partir de laquelle la «bonne » abstraction devient dangereuse. Mais contrairement à celle des poisons biologiques, cette dose dépend souvent de ce qui conforte ou non les arguments de l'évaluateur. Il suffit, en outre, de feuilleter tel ou tel auteur majeur pour s'aviser que l'abstraction ne semble nuisible, voire fatale, qu'aux ouvrages des autres. Évidemment, la question est un tout petit peu plus compliquée que ça (en 2006 et 2007, l'Année sociologique ne lui a pas consacré moins de deux numéros consécutifs sans l'épuiser), mais on ne peut ici ni la creuser ni l'ignorer. En revanche, on peut essayer de préciser ses propres critères. Un filtre possible, à l'image de celui que l'on avait déjà employé au chapitre 2, pourrait être :

- d'expliciter (lorsque ça ne va pas de soi) en vertu de quoi et dans quelle mesure on peut estimer que les phénomènes sont comparables, en amont des invariances que l'on pense y trouver. Par exemple, les copistes, journalistes, éditeurs de romans et organisateurs d'expositions peuvent être considérés comme comparables (mais pas similaires, bien sûr) dans le fait qu'ils mettent sur le marché une offre discursive, mais pas dans leurs préférences entre fromage et dessert, mer et montagne ou inhumation et crémation. De même, les spectateurs, lecteurs, visiteurs, etc. évoqués plus haut étaient comparables (mais pas similaires) dans le fait d'effectuer un choix sur ce marché. En revanche, les piétons qui tantôt traversent les carrefours tantôt gravissent les cimes ne pouvaient avoir, comme on l'a souligné, qu'un intérêt heuristique ;

- de se demander si d'autres hypothèses peuvent expliquer de façon plus économique ou plus convaincante (idéalement les deux) les observations des phénomènes examinés ;

- de préciser autant que possible ce dont on estime que les invariances relevées rendent compte et ce dont on 
considère qu'elles ne rendent pas compte, voire de signaler les exaspérantes exceptions occasionnelles (lorsqu'elles en valent la peine).

Ayant ainsi défini des règles appropriées, l'auteur s'avise non seulement qu'elles conviennent à merveille à l'argumentation qu'il a développée jusque-là, mais aussi qu'elles le lavent du soupçon qui visait les anthropologies naturalistes (tout en condamnant au passage quelques essais à succès sur la communication), et surtout qu'elles justifient la suite, puisque c'est précisément la limite de validité des invariants naturalistes de la cognition, longuement soulignée au chapitre précédent, qui oblige à s'intéresser maintenant à ce dont ils ne peuvent rendre compte. 


\section{Les errances de la convenance}

L'offense à la morale publique est dans les tableaux lascifs que je mettrai sous vos yeux, l'offense à la morale religieuse dans des images voluptueuses mêlées aux choses sacrées. J'arrive aux citations. $[\ldots]$ « Le froid de la nuit les faisait s'étreindre davantage, les soupirs de leurs lèvres leur semblaient plus forts, leurs yeux, qu'ils entrevoyaient à peine, leur apparaissaient plus grands, et au milieu du silence il y avait des paroles dites tout bas qui tombaient sur leur âme avec une sonorité cristalline et qui s'y répercutaient en vibrations multipliées. » Connaissez-vous au monde, messieurs, un langage plus expressif? Avez-vous jamais vu un tableau plus lascif?

Procureur impérial Pinard

$\mathrm{F}$ ace à l'essaim bourdonnant et foisonnant des contraintes sociales qui surdéterminent l'adéquation ou l'inadéquation des discours, il est difficile de ne pas céder au découragement.

Considérons à nouveau le tollé qui avait suivi en 2004 le propos du président-directeur général de $\mathrm{TF}_{1}$ (souvent cité, rarement lu en contexte) : "Ce que nous vendons à Coca-Cola, c'est du temps de cerveau humain disponible. " À l'évidence, cette formulation brève, imagée et forte, offre un ratio effort-effet remarquable. Mais cette pertinence cognitive ne suffit pas à expliquer qu'elle ait pu capter à elle seule la quasi-totalité de l'attention allouée à l'ouvrage où elle figurait, lui attirant au passage plus d'attention qu'il ne pouvait en rêver.

1 Tribunal correctionnel de Paris, $6^{\mathrm{e}}$ Chambre. Audiences des 31 janvier et 7 février 1857, Ministère public contre M. Gustave Flaubert. 
De l'autre côté, celui des valeurs, ce qu'exprimait fondamentalement l'énoncé n'avait rien d'inédit (il s'agit de «livrer des publics aux annonceurs » dit-on aux États-Unis ${ }^{2}$ ) et ne semble ni plus scandaleux en soi ni plus nouveau que si Patrick Le Lay avait lâché «Nos revenus proviennent de la publicité », ce qui aurait signifié à peu près la même chose et aurait même pu se couler dans la syntaxe originale («Ce que nous vendons aux marques, c'est de l'espace publicitaire»). Évidemment, cette version aurait été plus appropriée puisque, comme on l'a vu, le cerveau en question est en réalité loin d'être disponible. Mais il ne semble pas que ce soit là le point qui a déchaîné l'indignation générale.

Par ailleurs, le propos en cause n'enfreignait aucune loi. Sa retranscription n'écornait même pas les règles ortholinguistiques dont la violation par un personnage public suscite périodiquement les foudres des commentateurs" ("Se que nous vendont à cocacola, s'ait du tant de servau humin disponible »). Il ne tombait pas non plus dans le type d'irrespect identitaire, par exemple le sexisme, qui aurait condamné un énoncé presque équivalent dans l'absolu, mais plus malvenu ${ }^{4}$ " $\mathrm{Ce}$ que nous vendons à L'Oréal et Palmolive, c'est du temps de cerveau féminin disponible »). Il était, certes, assez direct, mais moins que si son auteur, inopinément converti à la doxa du militantisme antilibéral, avait soudain emprunté son lexique ("Ce que nous vendons à l'institution, c'est du conformisme social » ou « de la diversion idéologique », etc.)

La réception aurait-elle été plus favorable si au contraire Le Lay, se souvenant du statut de «mieux-disant culturel» revendiqué lors de l'attribution de la concession de TFı en 1987, avait risqué : « Ce que

2 L'expression n'est pas ironique, assure Ien Ang qui la rapporte : dans un secteur "dont l'assise économique repose dans le fait de "livrer des publics aux annonceurs" [...] les gens prennent cette tâche très au sérieux et comme allant de soi » (1991, p. ix).

3 Ainsi, les huées qui ont salué des messages de la ministre québecoise de la Famille, Francine Charbonneau ( 13 «fautes » en 115 mots) ou de la ministre française de la culture, Aurélie Filippetti ( 5 «fautes » en 15 mots).

4 Du reste, la mythique « ménagère de moins de cinquante ans » que courtisaient tant de supports publicitaires au $x^{\mathrm{e}}$ siècle n'est plus leur cœur de cible explicite, non seulement en raison du caractère embarrassant de cette désignation, mais aussi à cause de la dilution des responsabilités de consommation dans les structures familiales actuelles. 
nous vendons, c'est l'accès à la culture pour tous " ? On peut en douter, et l'intervention d'un spécialiste à l'occasion d'une autre audition du président de la chaîne le confirme :

Je vous reconnais, M. Le Lay, le mérite de défendre en toute honnêteté et en toute transparence ce qu'est la logique économique d'une télévision commerciale. Vous venez de nous l'expliquer; vous seriez venu nous expliquer la logique d'abord et avant tout culturelle de $\mathrm{TF}_{1}$, on vous aurait jugé malhonnête. (Coulangeon, 2006, p. 145)

N'ergotons donc pas sur ce que vend effectivement TFi. Outre que Patrick Le Lay l'a déjà très (trop ?) bien résumé en pratique, le fondement de son métier est remarquablement évident si on le considère dans la perspective de l'adéquation sociocognitive telle que l'on vient de l'exposer : TFi vend aux annonceurs de l'attention, qu'il achète aux spectateurs en leur proposant la pertinence cognitive la plus élevée possible en fonction de contraintes sociales âprement débattues par les uns et les autres (syndicats de journalistes, associations familiales, etc.). La page suivante du même ouvrage témoigne du reste de ce genre d'arbitrage permanent (et toujours provisoire...) : "Nous avons décidé de ne pas faire le Loft : cétait un programme qui ne nous semblait pas coller avec l'image familiale de TF1. »

Le plus curieux n'est pas le fait que l'on puisse considérer avec le même modèle analytique la recevabilité d'une simple phrase de quatorze mots et la stratégie globale d'une grande chaîne de télévision : c'était, après tout, l'hypothèse initiale de ce livre. Ce qui est plus frappant, c'est qu'il n'existait apparemment aucun énoncé permettant, à pertinence et longueur égales, d'exprimer de façon « convenable » la pensée du président-directeur général de TFi. La seule solution inoffensive aurait été de réduire sa pertinence cognitive (invoquer «de l'espace publicitaire», moins figuratif que «du temps de cerveau»). En d'autres termes... de réduire sa valeur discursive pour assurer sa valeur discursive : la contradiction montre que l'on rencontre là un sérieux problème.

Ce n'est pas le seul. Quoiqu'aucune des reformulations essayées plus haut ne soit mensongère (ce sont des «façons de voir» qui ne s'excluent pas réellement), les convenances que chacune violait à sa façon sont de nature si différente - respect de la "mission", de la 
grammaire, des sensibilités identitaires, des légitimités culturelles, etc. - et souvent si contradictoires - paraître cynique ou hypocrite - qu'elles laissent peu d'espoir de mettre la main sur une typologie raisonnablement simple et cohérente. D'autant que, bien sûr, elles n'ont rien d'absolu. Elles varient non seulement en fonction du destinataire, mais aussi selon l'image de celui qui s'exprime (l'éthos de l'ancienne rhétorique) : ce qui a été perçu - sincèrement ou non - comme inconvenant dans la bouche du dirigeant n'aurait pas choqué ${ }^{5}$ ou même surpris dans celle d'un penseur de la contre-culture.

À cela s'ajoute une caractéristique particulière des discours, que l'on pourrait appeler leur caractère autojuridictionnel. Si Sperber et Wilson ont bien montré que ceux-ci communiquent d'abord la présomption de leur pertinence, ils communiquent aussi, du point de vue de l'adéquation sociocognitive, les critères particuliers selon lesquels ils s'offrent au jugement. Pour simplifier, un texte qui se présente et est perçu comme "poétique », "scientifique », "journalistique ", " humoristique ", etc., soumet du même coup l'appréciation de sa valeur à un ensemble spécifique de normes implicites et explicites.

C'est d'ailleurs si manifeste que bien des auteurs se sont épuisés à définir, entre autres, des " genres » textuels en s'efforçant d'en collecter les indices épars. D'autres l'ont abordé sous un angle moins descriptif, celui de la réception, en avançant diverses hypothèses («contrat » de lecture ${ }^{6}$, « horizon d'attente »...) que l'on ne développera pas ici. La notion d'«identité discursive » esquissée par Rémy Rieffel (2005) illustre bien sa prégnance dans le cas des médias. Dans celui de la fiction, note Vincent Jouve, "Tout roman, d'une certaine manière, propose à la fois une histoire et son mode d'emploi : une série de signaux indiquent

5 Sauf les salariés de la chaîne, qui pourraient à bon droit souligner, non pas la fausseté de cette façon de voir, mais son caractère réducteur, de même que peu de metteurs en scène conviendraient sans doute que leur métier consiste à vendre des places de théâtre.

6 Notion elle-même extraordinairement fluctuante en fonction des discours visés, notamment littéraires (voir Jouve, 2010) ou médiatiques (voir Charaudeau, 2005), mais aussi des dispositions que l'on prête à ce « contrat », terme évidemment problématique (voir Jeanneret et PatrinLeclère, 2004 ; Wagner, 2012...) dont les conceptions, parfois simplement gricéennes, peuvent aussi être nettement coercitives. 
selon quelles conventions le livre demande à être lu» (2010, p. 9). Ainsi le succès d'un ouvrage est-il aussi celui des conditions qu'il propose ou renouvelle («toute œuvre légitime tend en fait à imposer les normes de sa propre perception » remarque Bourdieu, 1979, p. 29). À une échelle plus générale, Marc Angenot (1989) observe que chaque discours participe d'un système (ou répertoire) spécifique qui en détermine la recevabilité sur le fond et la forme. D'autres encore, dans le domaine de l'ergonomie, ont développé le concept d'«affordance» pour évoquer la capacité d'un objet à suggérer la façon selon laquelle on peut l'utiliser (lequel renvoie du reste à une appréciation coût-bénéfice qui n'est pas sans évoquer la pertinence ${ }^{7}$, mais qui, comme elle, est peu concerné par les normes sociales). Et, en fait de normes et de jugements, la jurisprudence des tribunaux supérieurs a depuis longtemps établi que le type d'engagement d'un discours - satirique, factuel, littéraire, etc. - déterminait ce qu'il pouvait ou non avoir de condamnable.

Pour s'en tenir ici à l'exemple de Patrick Le Lay, son propos avait été recueilli dans un ouvrage de gestion consacré aux stratégies des dirigeants d'entreprises. Il ne pouvait devenir totalement ${ }^{8}$ scandaleux qu'en l'arrachant à cette juridiction discursive, même s'il fallait pour ça l'amputer sans vergogne de ses deux premières phrases, dont chacune revendiquait justement ce cadre de jugement :

Il y a beaucoup de façons de parler de la télévision. Mais dans une perspective «business », soyons réalistes : à la base, le métier de TF1, c'est [...] (p. 92)

Toutes ces questions sont bien connues sous une forme ou sous une autre. Depuis plus de 2000 ans pour un bon nombre d'entre elles : le simple fait d'examiner sérieusement ce qui a trait aux conditions de convenance des discours dans la Rhétorique et la Poétique d'Aristote, sans compter celles que l'on dériverait de son Éthique, pourrait suffire à remplir un livre de bonne taille. En fait, si l'on considère que la communication est la base des sociétés humaines (et réciproquement), tout ce qui parle de celles-ci parle plus ou moins de celle-là.

7 Voir Morineau (2001) et, pour une extension à la réception des discours textuels, Kavanagh et Sperano (2016).

8 L'idée de "préparer » le spectateur à la publicité pourrait évidémment choquer dans n'importe quel contexte. 
C'est peut-être le moment de faire marche arrière, en repartant de la question qui avait déjà fourni un point de départ :

Toute situation linguistique fonctionne donc comme un marché sur lequel le locuteur place ses produits et le produit qu'il a produit pour ce marché dépend de l'anticipation qu'il a des prix que vont recevoir ses produits. [...] Un des grands mystères que la socio-linguistique doit résoudre, c'est cette espèce de sens de l'acceptabilité. (Bourdieu, 1980, p. 98)

La sociolinguistique? Il n'est pas certain que cette discipline accepterait la périlleuse mission que lui assignait Bourdieu, même s'il la limitait à l'acceptabilité formelle, ou le défi encore plus vaste qu'impliquait inévitablement sa métaphore du marchés. Solidement ancrée dans l'étude de terrain des conversations courantes (ou parfois celle de textes), elle excelle à décrire les caractéristiques et les variations des parlers ordinaires, ou à exposer quantitativement les stratifications sociales de la prononciation, du lexique et de la syntaxe, mais elle ne s'aventure pas à en induire des modèles théoriques élargis. Ainsi Labov, un demi-siècle exactement après son étude fondatrice sur les façons de parler des clientèles populaires et huppées des grands magasins new-yorkais (1966), soulignait-il sa « longue résistance » vis-à-vis des généralisations théoriques : "mon approche a été de viser une question linguistique particulière, par exemple la motivation sociale d'une variation phonétique à Martha's Vineyard et d'arriver à une réponse [...]» (2016, p. 595).

En revanche, Bourdieu lui-même n'a pas manqué de répondre à la question qu'il soulevait. On aurait peut-être pu le signaler plus tôt, mais ça ne devient réellement pertinent qu'ici. Il y a même répondu plusieurs fois : dans son sens le plus restreint (l'adéquation langagière) comme dans son sens le plus étendu (la légitimité des produits culturels $\left.{ }^{10}\right)$.

9 Saisissons l'occasion pour souligner qu'il n'importe pas ici de savoir si Bourdieu « voulait » parler de la valeur discursive en général ou de la seule acceptabilité formelle du langage en situation scolaire. Aucune herméneutique ne peut enchaîner la notion mise en jeu : puisque c'est du «marché » qu'il était question, c'est du «marché » que l'on traitera, et en particulier « de l'anticipation [...] des prix que vont recevoir ses produits ».

Io Sans compter, bien sûr, que toutes ses approches de champs spécifiques, tels que le monde universitaire (1984), la création littéraire (1992) ou le 
La première perspective, dans laquelle s'inscrivait la citation en exergue, visait essentiellement les modalités de régulation du langage parlé, en l'occurrence au sein de l'institution scolaire (1980) mais aussi dans des situations plus générales $\left(1977,1982^{11} \ldots\right)$. Après avoir exécuté sans grande difficulté toute prétention de la linguistique (tant classique que structurale) à rendre compte de l'acceptabilité sociale des énoncés, Bourdieu ramène celle-ci à quatre critères familiers, voire banals ${ }^{12}$ :

Pour récapituler de façon abstraite et rapide, la communication en situation d'autorité pédagogique suppose des émetteurs légitimes, des récepteurs légitimes, une situation légitime, un langage légitime. (1980, p. 103)

La même grille peut s'appliquer hors du cadre scolaire :

On peut ainsi énoncer les caractéristiques que doit remplir le discours légitime, les présupposés tacites de son efficacité : il est prononcé par un locuteur légitime, c'est-à-dire par la personne qui convient - par opposition à l'imposteur - (langage religieux/ prêtre, poésie/poète, etc.) ; il est énoncé dans une situation légitime, c'est-à-dire sur le marché qui convient (à l'opposé du discours fou, une poésie surréaliste lue à la Bourse) et adressé à des destinataires légitimes; il est formulé dans les formes phonologiques et syntaxiques légitimes (ce que les linguistes appellent la grammaticalité), sauf quand il appartient à la définition légitime du producteur légitime de transgresser ces normes. (1977, p. 21)

journalisme (1996), " parlent » nécessairement beaucoup des régulations des discours propres à ces champs.

11 Ainsi que dans Bourdieu (1980, etc.). Pour tenter de limiter les confusions que peuvent engendrer la reprise en recueils de certains articles, puis leur développement ultérieur dans d'autres livres, parfois sous des titres similaires (et dans la mesure où l'on n'en retient ici que quelques repères essentiels, sans prétention exégétique) on préférera dans la mesure du possible, se référer à des parutions de dates différentes et à leur expression la plus synthétique (souvent issue d'interventions orales).

12 En ce sens qu'ils reprennent les repères les plus intuitivement évidents et scientifiquement débattus de la communication (émetteur, récepteur, canal, code). Mais, comme on s'en doute, la ressemblance avec le modèle mécaniste de l'encodage-décodage s'arrête à l'homologie de ces instances. 
De fait, même en les généralisant au-delà de l'oral, ces conditions renvoient à des aspects essentiels de la formation des prix sur le marché discursif : la valeur découlant du locuteur lui-même (l'ethos évoqué plus haut dans son sens rhétorique $\left.{ }^{13}\right)$, celle qui s'apprécie de façon contextuelle (soit, plus ou moins, le caractère autojuridictionnel des discours), le rapport aux destinataires (sans lequel la communication n'est qu'expression), et enfin cette obsédante correction formelle pour laquelle les universités multiplient aujourd'hui les cours de remédiation orthographique et grammaticale.

En transposant ainsi ces catégories, on les dépouille de ce qui faisait la spécificité de la pensée de Bourdieu. Sa densité analytique, cela va sans dire, mais aussi sa perspective (sa «façon de voir ») : la recherche inlassable des mécanismes de domination symbolique. Si la question de la valeur des discours se trouve réduite à celle de leur légitimité, vue comme la source de leur autorité, alors la question est d'une certaine façon réglée. Mais elle n'est réglée que d'une certaine façon, les autres aspects n'ayant dès lors plus lieu d'être considérés.

Dans une perspective parallèle et un même environnement historique (la fermentation politique et intellectuelle des années 1960 à 1980), Foucault avait similairement développé l'idée selon laquelle
[...] dans toute société la production du discours est à la fois contrôlée, sélectionnée, organisée et redistribuée par un certain nombre de procédures qui ont pour rôle d'en conjurer les pou- voirs et les dangers [...] (1971, p. 10)

Ces formes de contrôle social, dont Foucault avançait pour sa part dix mécanismes en trois groupes (modalités externes au discours, internes à celui-ci et régulant son accès) ne contredisent pas celles exposées par Bourdieu. Elles s'inscrivent dans un autre cadre théorique et méthodologique (l'introspection philosophique contre la posture sociologique), mais elles exposent beaucoup de rouages communs - par exemple, le «rituel ", qui impose au locuteur une qualification et un protocole - et, surtout, elles décrivent le même objet sous le même angle. L'objet est la production du discours (plutôt que sa réception) et l'angle est celui de l'encadrement social d'un marché discursif

13 Bourdieu utilise pour sa part ce terme dans une acception qui lui est propre. 
sévèrement régulé. Un marché où les chats jouant du piano n'ont pas de place. Un marché où un aventurier s'essayant à la politique n'arrache pas la Maison-Blanche à l'establishment politique. Un marché où un auditoire scientifique n'interrompt pas un conférencier pour réclamer la fin d'un vieux conte populaire. Un marché où un chanteur de folk misanthrope ne saurait obtenir l'onction suprême du Nobel de littérature. Bref, un marché où la légitimité règne d'en haut, et où l'attention ne joue aucun rôle.

Certes, le poids des critères institutionnels qui régissent l'acceptabilité des discours est considérable - on tendra par exemple souvent (quoique de moins en moins sur les réseaux) à accorder plus de valeur aux propos d'un locuteur "faisant autorité » qu'à un béotien -, mais les dominations symboliques peuvent difficilement ${ }^{14}$ expliquer à elles seules la fixation des valeurs sur le marché discursif contemporain. On peut même douter qu'elles suffisaient jadis, si l'on en croît par exemple Corneille :

[...] notre premier but doit être de plaire à la cour et au peuple, et d'attirer un grand monde à leurs représentations. Il faut, s'il se peut, y ajouter les règles, afin de ne déplaire pas aux savants, et recevoir un applaudissement universel; mais surtout gagnons la voix publique ; autrement, notre pièce aura beau être régulière, si elle est sifflée au théâtre, les savants n'oseront se déclarer en notre faveur [...] (1637/1862, p. 119)

L'évocation des savants n'est pas anodine, ce propos ayant été rédigé au moment même où l'Académie française, érigée en tribunal littéraire, soupesait la valeur discursive du Cid. Mais en mettant en balance le respect des règles (en amont de la production) et l'agrément du public (en aval de celle-ci), Corneille n'illustre pas moins une opposition tout à fait intemporelle, celle qui confronte les normes et les goûts. Une opposition entre parents : dans une certaine mesure, les goûts ne sont guère moins sociaux que les normes. S'ils n'étaient que spontanés, "naturels ", ils se réduiraient à l'application pure et

14 Convenons qu'un exégète déterminé, et entraîné aux acrobaties conceptuelles les plus audacieuses, pourrait peut-être parvenir à tout expliquer sous cet angle (chatons compris) : on salue par avance l'exploit avec l'admiration qu'il mérite, mais on ne le tentera pas. 
simple des critères de la pertinence cognitive. Mais pour Bourdieu, à l'inverse, ils sont essentiellement le fruit et le garant des hiérarchies sociales. C'est là son autre réponse (1979) à la question de la valeur discursive, toujours en termes de légitimité, mais cette fois-ci en partant de la réception.

Dans cette perspective, ce qui nous plaît dépendrait avant tout de notre position sociale, que ce soit au sein de la classe culturellement dominante (soucieuse, dans sa célébration arbitraire du raffinement esthétique de se distinguer des autres), de la petite bourgeoisie culturelle (anxieuse d'imiter la première, mais pathétiquement maladroite dans ses tentatives), ou encore de la classe populaire (aliénée et stigmatisée jusque dans la vulgarité de ses goûts). Il serait pathétiquement maladroit, si ce n'est vulgaire, de prétendre résumer en quelques lignes les 670 pages de La Distinction, mais on en a dit assez pour que l'on reconnaisse effectivement là un déterminant majeur de la valeur discursive. Y compris, soyons sincères, les choix culturels de chacun de nous, reflets de ce conflit permanent entre l'agrément hédonique et la légitimité culturelle.

Le conflit est un peu masqué, comme on l'a amplement souligné plus haut, par le caractère autocumulatif de la familiarité : dans la mesure où ce que l'on connaît déjà - mangas, gastronomie, art moderne ou opéra baroque - favorise l'acquisition de nouvelles connaissances $^{15}$ sur le même sujet (moins d'effort cognitif pour plus d'effet combinatoire), on peut devenir « sincèrement » passionné par

15 Même Bourdieu, que l'on ne tient pas pour un grand amateur de psychologie expérimentale, remarque le rôle de structures mentales correspondant plus ou moins aux schémas cognitifs : "L'œuvre d'art ne prend un sens et ne revêt un intérêt que pour celui qui est pourvu du code selon lequel elle est codée. La mise en cuvre consciente ou inconsciente du système de schèmes de perception et d'appréciation plus ou moins explicites qui constitue la culture picturale ou musicale est la condition cachée de cette forme élémentaire de connaissance qu'est la reconnaissance des styles. Le spectateur dépourou du code spécifique se sent submergé, "noyé", devant ce qui lui apparaît comme un chaos de sons et de rythmes, de couleurs et de lignes sans rime ni raison » (1979, p. II). De même, aux États-Unis, les données statistiques analysées par DiMagio et Useem (1978) montrent clairement que la «possession des codes culturels » est un bien meilleur prédicteur de préférences culturelles élevées que les revenus disponibles, l'éducation jouant ainsi sur «la mesure selon laquelle les individus considèrent les arts comme compréhensibles et intéressants ». 
les domaines d'intérêt les plus abscons. Mais cet intérêt ne naît pas de rien (sauf, bien sûr, en ce qui concerne les facteurs élémentaires d'effet cognitif) : la main invisible qui pousse les vacanciers dans les musées d'art étrangers est, en tout cas en partie, celle de leur entourage, aux yeux de qui le statut de leurs choix touristiques confirmera ou non leur propre statut socioculturel. Et, en vacances ou non, les recherches sur les visiteurs des musées, dont celle de Bourdieu et Darbel (1966) confirmaient le rapport étroit entre la stratification sociale (niveau d'études, profession) et la fréquentation des institutions culturelles. Le constat contraste assez plaisamment avec celui des recherches mentionnées au chapitre 2, lesquelles, à propos de la fatigue muséale, montraient tout aussi clairement l'influence de l'effort et de l'effet sur les parcours de ces mêmes visiteurs de musées, mais il n'est pas moins solide et, dans ses grandes lignes, pas plus surprenant.

Chacun pourrait reconnaître ces déterminants dans son expérience personnelle, mais il n'est pas indispensable d'être sociologue pour échapper à la spéculation introspective : les données empiriques les plus rudimentaires témoignent de l'opposition entre la pertinence hédonique et la légitimité culturelle de l'offre discursive, y compris chez ceux qui n'en goûtent pas les strates les plus raffinées. Un sondage Ifop publié par le magazine Classica en février 2001 indiquait ainsi que $39 \%$ des répondants français citaient la musique classique parmi leurs préférences musicales, derrière la variété (58\%), mais devant le pop rock (30 \%), le jazz (19\%), la musique techno (10 \%) ou le rap (9\%). Sans entrer dans la critique méthodologique des sondages d'opinion, un coup d'œil aux ventes réelles suffit pour apprécier l'écart entre la valeur symbolique et la valeur hédonique des œuvres : à l'époque de ce sondage, la musique classique ne représentait que $7 \%$ de la musique effectivement vendue (ministère de la Culture et de la Communication, 2001). Et le même genre d'écart se manifeste lorsque l'on interroge, toujours par exemple, les spectateurs sur leurs chaînes de télévision "préférées ", lesquelles, selon les chiffres d'audience ${ }^{16}$ ne sont pas celles qu'ils regardent.

16 À peine moins discutables que les sondages d'un point de vue méthodologique, mais ce n'est pas la question ici, ces diverses approximations suffisant amplement à ce niveau d'analyse. Incidemment, le fait de mélanger aussi abruptement la sociologie des pratiques culturelles et la sociologie des goûts et valeurs n'est pas plus gênant à ce niveau. 
Évidemment, la société strictement hiérarchisée que Bourdieu se plaisait à dépeindre évoque un peu les clichés en noir et blanc de Doisneau ou Cartier-Bresson ${ }^{17}$ : chaque décennie qui passe souligne un peu plus le caractère historiquement situé de ce contexte de domination culturelle (et celui de son analyse). Mais si le combat pour la distinction transcende toujours plus largement la vieille stratification des classes sociales, il n'a pas perdu son actualité. Les forums de discussion où des aficionados des jeux vidéo manifestent leur mépris des titres à succès, comme ceux où des connaisseurs de rock rejettent dans l'ignominie de la pop music des groupes jadis encensés, ne cessent de l'illustrer. Ce qui nous distingue (ou nous assimile à un groupe « distingué ») nous plaît. Ou, plutôt, ça nous plaît aussi.

Il reste cependant que les listes des succès musicaux, cinématographiques ou éditoriaux du moment ne témoignent décidément pas d'un souci de distinction très prononcé (ça serait d'ailleurs une sérieuse contradiction). La réussite commerciale des productions culturelles de masse peut évidemment être interprétée comme la preuve de l'aliénation des basses castes, réduites à une pseudoculture de pacotille, mais cette vision surplombante se heurte au fait que les consommateurs respectent assez peu - et de moins en moins - ce genre de stratification. Outre les «braconnages » culturels par lesquels, notait Michel de Certeau (1980), les individus détournent et réinventent le sens des contenus qui leur sont offerts (voir aussi Hoggart, 1970), la sélection même qu'ils opèrent parmi ces contenus transgresse allègrement les hiérarchies légitimes. Ainsi, aux États-Unis, DiMaggio et Useem (1978) pouvaient-ils remarquer dès la fin des années 1970 que la production culturelle populaire, par exemple le rock, était consommée à un taux équivalent dans toutes les classes sociales. Sur un échantillon de plus de 11 ooo personnes interrogées en 1982, Peterson et Kern ne purent repérer que dix répondants assez snobs pour affirmer n'aimer aucune des productions culturelles associées aux classes moyennes ou populaires, et ils n'étaient plus que trois lorsque l'enquête fut réitérée dix ans plus tard (Peterson et Kern, 1996).

17 D'accord... Toutes les photos de Cartier-Bresson et de Doisneau (par exemple celles de Saint-Germain des prés) ne représentent pas la France archétypique des années 1960, mais nous étions d'accord pour ne pas entrer dans ce genre de détails, non? 
En d'autres termes, la consommation de la « haute culture », par exemple l'opéra, reste surdéterminée par les hiérarchies sociales, mais la consommation de la culture populaire l'est beaucoup moins. Encore faut-il rappeler à quel point la notion même de « haute culture » est malléable. On sait que La Flûte enchantée a été composée par Mozart pour le public plébéien d'un théâtre populaire de la banlieue viennoise (on parlerait peut-être aujourd'hui d'un cabaret) et les multiples artifices de cet opéra - péripéties, effets spéciaux, scènes comiques, monstres et magie - témoignent des solides exigences de l'assistance en matière de distraction. Aux États-Unis, affirme Levine (1988), la distinction entre la culture légitime et la culture populaire ne s'est produite qu'au milieu du XIX ${ }^{\mathrm{e}}$ siècle. Mais si la sacralisation de la « haute culture » a pu se produire à des époques et dans des contextes assez différents d'un côté et de l'autre de l'Atlantique, sa désacralisation dans la seconde moitié du $\mathrm{xx}^{\mathrm{e}}$ siècle semble universelle. Peterson (1992) et ses collègues ont ainsi documenté le basculement d'une hiérarchie culturelle opposant l'élite et la masse à une hiérarchie distinguant les «omnivores » et les «univores ». Au sommet de la pyramide culturelle, les classes les plus instruites se caractériseraient aujourd'hui par l'éclectisme de leurs goûts, associant librement des créations raffinées et des produits culturels de grande consommation, tandis qu'à la base de cette pyramide, les moins instruites se cantonneraient à une monoculture d'œuvres populaires. En d'autres termes, ceux qui aiment Brahms ne dédaignent pas forcément Elvis, mais ceux qui adulent Elvis ont peu de chances d'apprécier Brahms.

Assez curieusement, j'avais pu observer une dissymétrie du même genre à partir d'un angle très différent en étudiant sommairement le rapport à l'information des pays européens en fonction des données des enquêtes Eurobaromètre : on sait que les pays du nord de l'Europe lisent beaucoup plus les journaux que les pays du Sud, mais aussi qu'ils se distinguent dans la plupart des indicateurs culturels, éducationnels et civiques (Labasse, 2003). Il serait évidemment vain d'expliquer cet étrange gradient Nord-Sud par le genre de déterminisme climatique que supposait Montesquieu. Par ailleurs, les questions Eurobaromètre sur l'intérêt accordé à l'actualité ne montraient aucune différence globale quant à l'attention que les répondants suédois ou grecs affirmaient lui accorder, bien que les premiers obtiennent des résultats très supérieurs aux questions testant leurs connaissances réelles. Cependant, une différence frappante semblait se manifester 
lorsque l'on étudiait la dispersion (écart type) de l'intérêt déclaré vis-à-vis de divers domaines de l'actualité (sports, politique, sciences, culture, économie...). Celle-ci était deux fois plus élevée dans les pays les moins informés que dans les pays les plus informés. Pour schématiser, les Français, les Portugais ou les Grecs tendaient à s'intéresser soit aux sports, soit à la politique, soit aux sciences (etc.), quand les Finlandais, Suédois ou Allemands s'intéressaient aux sports et à la politique et aux sciences (etc.). Le même type d'écart entre omnivores et univores se manifestait lorsque l'on considérait, non les nationalités mais les catégories socioprofessionnelles des répondants, tous pays confondus : l'écart type était de 3,17 pour les cadres, mais de 10,81 pour les professions manuelles. Cependant, malgré la sympathie - évidemment désintéressée - que je peux éprouver vis-à-vis de tout ce qui tend à suggérer un lien entre l'étendue de la gamme des références et la richesse de la pensée (Tetlock, 2005, l'a aussi montré de façon frappante dans le domaine des jugements d'experts), la nature des agrégats de données utilisés ne permettait pas d'avancer sérieusement des conclusions formelles en l'état, d'autant que l'idée même de traiter des pays comme des individus avait quelque chose, au mieux de perturbant, au pire d'absurde.

En revanche, c'est bien des individus, et même de beaucoup d'entre eux (près de 30 ooo répondants), que parlaient les données analysées de façon plus systématique quelques années plus tard pour l'une des plus larges études quantitatives jamais consacrées au lectorat de la presse écrite (Labasse, 2008). Or, celles-ci indiquaient des phénomènes assez similaires. Elles manifestaient tout d'abord la corrélation statistique prévisible entre le niveau d'études et les choix de consommation culturelle ${ }^{18}$. Plus concrètement, l'indice reflétant les

18 La corrélation (Rho de Spearman) entre le niveau d'études (cinq tranches) et les autres variables se monte à 0,267 pour la consommation culturelle «légitime» (définition ci-dessous), de -0,059 pour la consommation «populaire », de 0,189 pour l'intérêt pour l'actualité et de 0,207 pour la lecture de la presse quotidienne nationale. Tous ces tests dépassent très largement le seuil de signification $(\mathrm{p}<\mathrm{0}, \mathrm{OO1})$, ce que l'on peut rapprocher de la grande taille de l'échantillon, soit 22626 individus après suppression de réponses incomplètes.

L'indice « légitime » regroupe cinq types de pratiques : lecture régulière de livres (genre indifférent), visite de musées, sorties musicales (concert classique, ballet ou opéra) et écoute de radios culturelles (France musique 
pratiques «légitimes » déclarées (voir la note précédente) s'étageait très régulièrement depuis les personnes sans diplôme $(0,85)$ jusqu'à celles ayant un diplôme de second ou troisième cycle $(1,77)$. Mais cet écart du simple au double n'était pas si large si l'on considère que les non-diplômés déclaraient en moyenne près d'une activité « légitime », quand les plus instruits en revendiquaient près de deux. Parallèlement, un indice regroupant diverses pratiques «populaires » indiquait une décroissance, mais moins régulière et moins prononcée, entre les nondiplômés $(1,37)$ et les plus diplômés $(1,24)$, montrant que ces derniers ne dédaignaient pas les fêtes foraines ou les concerts et radios de rock. Et, confortant l'hypothèse d'un lien entre le degré de formation et la diversité des appétences, l'intérêt déclaré pour huit domaines différents de l'actualité progressait régulièrement (mais pas spectaculairement) de 3,5 à 4,4 en fonction du niveau d'études. Cependant, le contrat de recherche de la Fédération nationale de la presse française ne prévoyait nullement de s'attarder à ce genre de question : de nombreuses autres variables, tout à fait fascinantes mais un peu hors sujet ici, recelaient des éclairages beaucoup plus stratégiques sur les mutations de la lecture de presse et les évolutions éditoriales qui permettraient d'y faire face. En outre, les données déclaratives analysées dans ce cadre ne pouvaient livrer qu'une image quantitative assez stéréotypée des préférences culturelles réelles.

Spécifiquement consacrée à celles-ci, la recherche beaucoup plus substantielle de Bernard Lahire (2004) ne risquait pas ce genre de problème : exploitant les données des enquêtes périodiques du ministère français de la Culture, il se fondait en outre sur un important travail de terrain (et sur une visée sociologique, plutôt que sociographique). Cette recherche lui a permis d'observer en France la même tendance à l'hybridation des goûts populaires et raffinés qu'avaient relevée aux États-Unis Peterson et ses collègues. Toutefois, le tableau plus approfondi qu'il en dresse est aussi plus nuancé. D'une part, la propension à l'éclectisme concerne une large partie du public, et pas seulement sa fraction distinguée : plus des trois quarts des répondants $(75,8 \%)$

et France culture). L'indice « populaire » inclut pour sa part les sorties en discothèques, les fêtes foraines, les concerts de variété, jazz ou rock et l'écoute des radios NRJ ou Skyrock (le jazz n'est plus vraiment une musique "populaire », mais il était associé au rock dans le choix offert aux répondants). 
manifestent un certain degré de dissonance culturelle (c'est-à-dire d'association de registres légitimes et illégitimes) et les profils non dissonants se retrouvent aux deux extrémités du spectre : l'étroitesse des préférences touche aussi bien les béotiens les plus démunis que les connaisseurs les plus exclusifs. D'autre part, et même s'il est indéniable que "globalement, l'intensité de la foi en la culture artistique et littéraire légitime a sensiblement diminué au cours des trente dernières années " (p. 562), le mélange des préférences n'implique pas la confusion des valeurs symboliques. Si l'on peut s'adonner à des consommations culturelles triviales, et même les revendiquer, c'est généralement en pleine reconnaissance de leur faible statut dans la hiérarchie de la légitimité. Comme on l'avait remarqué à plusieurs reprises, «ce qui nous plaît » (la valeur discursive en général) ne se confond pas avec " ce que nous jugeons digne de plaire " (la valeur symbolique) même si l'un et l'autre se recoupent heureusement.

Au bout du compte, l'importance des stratégies de distinction et de la (ou des) légitimité(s) dans l'appréciation de la valeur discursive se trouve relativisée plutôt qu'invalidée ${ }^{19}$ par l'approfondissement contemporain des modalités de la construction et de l'expression des préférences culturelles. Il s'accorde en outre bien à ce que, sous l'angle limité de la profusion de l'offre discursive, l'on avait entrevu au chapitre premier : l'élargissement des choix de consommation culturelle, la désagrégation des légitimités discursives les plus traditionnelles et pourtant le maintien d'une certaine perception hiérarchisée du noble et du commun («I know, it's only rock'n roll (but I like it $\left.\left.{ }^{20}\right) »\right)$. Du reste, cet étagement se reproduit à l'intérieur même des catégories habituelles : comme le remarquait Line Grenier (1986), même les travaux se consacrant à la musique populaire sont tentés de privilégier les chansons « à texte » et à négliger les succès commerciaux.

Mais, si prégnantes que puissent être les logiques de distinction, elles ne peuvent rendre compte que d'une partie des préférences discursives socialement déterminées (pour ne pas parler, bien sûr, des préférences cognitivement déterminées). Il est facile de trouver des aspects de l'appréciation discursive qui paraissent ne résulter directement ni de la pertinence cognitive ni de la distinction sociale. C'est

19 Sur cette pondération, voir Coulangeon, 2014.

20 The Rolling Stones (1974). It's Only Rock'n Roll (But I like it). Amsterdam, Promotone (souligné par moi). 
par exemple le cas des sensualités ritualisées dont Antoine Hennion (2004) a montré l'importance pour les amateurs engagés dans une pratique culturelle soutenue (classer et parcourir sa discothèque, se préparer à la dégustation d'un grand cru...) et de la jouissance qu'ils retirent de cette pratique. Ainsi est-il conduit, par une autre voie - l'ethnographie des aficionados - à souligner à nouveau la diversité des déterminants de l'attachement culturel.

Le goût n'est ni le conséquent (automatique ou éduqué) des objets goûtés eux-mêmes, ni une pure disposition sociale projetée sur les objets ou le simple prétexte d'un jeu rituel collectif, c'est un dispositif réflexif et instrumenté de mise à l'épreuve de nos sensations.

Dans la perspective méthodologique et théorique qui leur est propre, ces observations sont plus attentives à certains aspects (la matérialité des objets culturels, les réseaux affinitaires) et beaucoup moins à d'autres (la cognition), mais elles sont compatibles avec les distinctions opérées plus haut, dont la coprésence de facteurs hédoniques, sociaux et extradiscursifs.

Pour s'en tenir ici à la dimension sociale des préférences discursives, une tout autre approche, traditionnelle et très générale, est celle qui souligne l'influence des "valeurs », qu'elles soient réputées individuelles, collectives ou les deux à la fois.

Les valeurs... Du nouveau foisonnement qui s'annonce, on hésite à discuter, si ce n'est pour mentionner à titre d'illustration l'une des nomenclatures les plus communément utilisées, celle de Schwartz (2006), laquelle est présentée comme relativement homogène à l'échelle de la planète. Certaines de ses composantes ne manquent pas d'évoquer des facteurs déjà rencontrés comme le " pouvoir » et la «conformité» (voir ci-dessus, Bourdieu) ou encore... l'« hédonisme» (voir ci-dessus, Sperber et Wilson), mais bien d'autres, comme l'«autonomie », la «bienveillance», ou l'« universalisme » semblent plus difficilement relever des rouages de la domination symbolique ou de la pertinence cognitive. Or, il est également difficile de ne pas voir que les valeurs de ce type jouent largement sur l'évaluation de l'offre discursive. Contentons-nous ici de rappeler l'indignation suscitée par le provocant Éloge littéraire d'Anders Breivik de Richard Millet (2012), et en particulier au texte où Annie Ernaux, appuyée par une centaine 
d'écrivains, dénonçait « des propos qui exsudent le mépris de l'humanité et font l'apologie de la violence » et «un texte porteur de menaces pour la cohésion sociale » (2012, p. 20). À l'inverse, on sait que la célébration des valeurs morales suscite rarement l'appréciation des connaisseurs comme en témoigne - toujours par exemple - cette critique journalistique d'«un produit destiné à une consommation de masse et formaté en conséquence», réduit à une "mélasse de bons sentiments». (Moreault, 2016, p. Ao6). Dans un cas comme dans l'autre (trop ou pas assez), ce genre de valeurs joue indiscutablement sur « ce qui nous plaît ».

Évidemment, les livres et les films sont des discours « discourants » : ils emploient le langage et peuvent donc être jugés sur ce qu'ils expriment textuellement. Mais les valeurs ne sont pas moins présentes dans l'appréciation de discours artistiques beaucoup moins loquaces, comme des œuvres d'art moderne. Oscar Wilde avait beau défendre l'idée que "la sphère de l'art et la sphère de l'éthique sont absolument distinctes et séparées » (1891/2003, p. 1145), elles le sont souvent peu dans l'esprit du public. Examinant les commentaires négatifs portés sur les livres d'or d'expositions, Nathalie Heinich (1998) a pu établir une sorte de rhétorique de la désapprobation et constater que celle-ci se référait toujours implicitement ou explicitement à des valeurs : la morale religieuse ou sexuelle (dans le cas des œuvres provocantes), mais aussi le travail (l'œuvre n'a pas demandé assez d'effort à son créateur), l'utilité sociale (l'argent est mal dépensé), le respect du patrimoine environnant (pour des installations extérieures), etc.

Cependant (refrain) la typologie très spécifique de Heinich, comme celle démesurément large de Schwartz, ne peuvent rendre compte que d'une partie des valeurs influant sur les préférences discursives. Échappe par exemple à ce double coup de filet le critère de la validité référentielle ${ }^{21}$ des discours - la «vérité » de ce qu'ils avancent - dont Kant, entre autres, pensait beaucoup de bien et à laquelle les journalistes, entre autres, accordent un vif intérêt.

La fatigue gagne : arrêtons-nous ici. On ne reviendra de toute façon pas de ce vagabondage dans les jardins des préférences sociales

21 À dire vrai, Heinich entrevoit ce critère, mais décide de le négliger, considérant une telle considération comme «un malentendu sur l'objet du jugement», la valeur référentielle se trouvant "au plus loin de la sphère d'autonomie de l'art et du jugement artistique » (p. 209). 
muni d'un appareil analytique d'usage commode et d'application universelle. C'était d'autant plus prévisible que, si les variables de la pertinence sont relativement constantes, les critères de valorisation sociale varient non seulement selon les sociétés et les individus, mais aussi, pour chacun d'eux, selon les circonstances dans lesquelles ils sont invoqués. Visitant une exposition, on appréciera l'originalité, mais à son bureau, on préférera l'efficacité, dans sa famille la fidélité, etc., et ces valeurs peuvent s'étendre par analogie à des situations plus larges ("notre entreprise est une grande famille »), en particulier lorsqu'il s'agit de résoudre des désaccords (Boltanski et Thévenot, 1991).

Bref, il semblerait que ce qui nous plaît dépende, d'un côté de la pertinence cognitive de l'offre discursive et de l'autre de facteurs socialement déterminés au nombre desquels figurent les questions de légitimité et de distinction, mais aussi bien d'autres choses, dont on sait essentiellement qu'elles sont nombreuses et qu'elles varient selon les moments et les circonstances. Devra-t-on vraiment se contenter de ça? 
Page blanche conservée intentionnellement 


\section{(Précisions)}

$\mathrm{L}$

e choix d'une aimable promenade à travers quelques manifestations empiriques de la convenance et certains apports des recherches, plutôt que d'un inventaire méthodique et scrupuleux de tous les enseignements des sociologies culturelles ${ }^{1}$, ne vise pas seulement à éviter de ressembler à un manuel de premier cycle (on en trouverait facilement de plus complets et plus orthodoxes). Il reflète surtout l'idée, assez consensuelle, que les normes de l'induction sociologique ne permettent de toute façon pas - ou plus d'abstraire légitimement d'études spécifiques un système généralisable à l'ensemble des productions discursives dans toutes les situations.

Pourtant, un sociologue, au moins, a formulé explicitement ${ }^{2}$ la question inconvenante qui nous accompagne: "Why do we like what we like? »(Stewart, 2014). Existerait-il donc une façon purement sociologique d'y répondre? Le fait que les trente pages qu'elle introduit constituent un exposé très « premier cycle » de la théorie positionnelle de Bourdieu, précédant, au chapitre suivant, une longue revue des débats qu'elle a pu susciter (où Zygmunt Bauman, le penseur de la "société liquide», occupe une place insolite) confirmerait plutôt que, s'il existe des façons sociologiques d'y répondre utilement, il n'en existe pas d'y répondre complètement ${ }^{3}$. Et encore l'ouvrage ne traite-t-il, comme bien d'autres, que de l'offre «culturelle », catégorie intuitive dont - pour des raisons

1 S'agissant des distinguos possibles - et localement nécessaires - entre la sociologie de l'art, de la culture, de la consommation culturelle, des pratiques culturelles, etc, on se contentera de dire qu'ils existent.

2 C'est même le titre de son chapitre 3.

3 À tel point que Stewart en vient, comme souvent, à déboucher sur la vieille question de la perception esthétique. 
que l'on se soucie rarement d'expliquer - les chatons de YouTube et les articles de presse ne font apparemment pas partie.

Dans la problématique générale qui nous intéresse, les éclairages de la sociologie de l'art, de la culture, etc. sont principalement de deux natures. La plus évidente réside dans les éléments qu'elle apporte, tels qu'on les a évoqués au chapitre précédent. Mais la seconde réside dans la démonstration qu'elle fournit de la nécessité de considérer en parallèle les facteurs cognitifs. On avait remarqué plus haut que l'une des vertus de la théorie de la pertinence - originelle ou amendée - résidait paradoxalement dans sa capacité à désigner tout ce qu'elle laissait dans l'ombre. Or, le même effet de contraste, un peu moins net il est vrai, se distingue ici.

Ainsi, lorsque Peterson se demande "Pourquoi l'omnivorité s'accroît-elle?", question judicieuse s'il en est, sa première réponse met en cause « la concurrence des divertissements populaires » qui «se font la lutte pour attirer l'attention du public » (2004, p. 145-164). Mais évoquer la concurrence attentionnelle (ce dont on ne s'était pas privé au chapitre premier) ne peut conduire qu'à une nouvelle perplexité. Le fait que d'autres propositions discursives soient offertes sur le marché n'explique nullement que des individus de statut social élevé puissent régulièrement les préférer à l'offre légitime : même si l'étau de la légitimité s'est desserré, les connaissances et appétences acquises devraient plutôt favoriser les formes traditionnellement consommées. Et évoquer l'influence des réseaux affinitaires (j'aime ce que mes amis aiment) ne ferait que repousser le problème vers les amis en question. "De toute évidence, s'avise Peterson, il faut pousser plus à fond l'étude de l'importance de la concurrence des divertissements populaires. » De toute évidence... mais cette route-là conduit au-delà des frontières sociologiques.

Plus significatif encore est l'imposant tableau que Lahire dépeint de la culture des individus. Évidemment, les enquêtes quantitatives (et dans une moindre mesure qualitatives) ne peuvent capter la fréquence des pratiques les moins légitimes, comme cette abondante consommation de pornographie dont nul n'arrive à mesurer l'ampleur réelle4, mais même en ne considérant que les

4 Les estimations varient considérablement selon les méthodes - toutes imparfaites - mais elles s'accordent à dire que cette consommation, 
préférences déclarées, les observations qu'il rassemble ne cessent d'illustrer, aussi explicitement que possible, l'opposition entre pertinence cognitive et légitimité sociale. Dès les premiers témoignages rapportés fuse ainsi le rejet des films sans effet cognitif évident " Y a pas d'action, y a rien qui se passe » et de ceux réclamant trop d'effort «Moi, les films compliqués... » (2004, p. 43).

Lahire n'en réitère pas moins le verdict de Bourdieu selon lequel les penchants psychologiques ne sont « que du social incorporé » (p. 716). Une position un peu délicate, dans la mesure où les dissonances célèbres qu'il met en exergue - la coupable passion de Wittgenstein et de Sartre pour les romans policiers - constituent justement des attestations éclatantes de la dialectique des valeurs sociales et des inclinaisons hédoniques (quoi de plus pertinent qu'un meurtre ? quoi de plus délassant qu'un polar ?) On pourrait sans doute étendre cette liste, en y ajoutant, entre autres, un Lévi-Strauss se délectant de théâtre de boulevard à la télévision (l'émission Au théâtre ce soir), mais on sent bien qu'une accumulation de cygnes noirs n'ébranlera pas cette conception, d'autant qu'elle s'appuie - dans le cas particulier de Lahire - sur une réelle connaissance de ce qu'il combat.

La nécessité de garder les frontières de la sociologie face aux vénéneuses séductions des sciences cognitives, lesquelles, menaçantes depuis toujours (Tarde, 1895), détournent du droit chemin un nombre inquiétant de sociologues et d'ethnologues - et ils s'enhardissent ${ }^{5}$ - pourrait suffire à motiver la proscription de ce genre

extraordinairement élevée en tout état de cause, s'est beaucoup accrue.

5 Voir entre autres l'ouvrage de Karen Cerulo (2002) en faveur d'une sociologie cognitive de la culture et les positions de Gerald Bronner (par exemple Bronner, 2008) réclamant avec pugnacité une prise en compte des biais cognitifs dans l'appréhension sociologique des croyances collectives. Incidemment, la perspective défendue par cet auteur, de même que la réception contrastée qu'il rencontre, rappelle que la discussion épistémologique dans ce domaine associe non pas deux (comme on l'avait simplifié ci-dessus) mais au moins trois enjeux étroitement enchevêtrés : définitoire (l'identité disciplinaire), méthodologique (les façons légitimes de connaître) et axiologique (l'engagement ou la neutralité du chercheur). Ceci sans compter la question, toujours brûlante depuis un bon siècle (Gabriel Tarde, Max Weber...), du degré d'autonomie qu'il convient de prêter aux acteurs sociaux. On 
d'explication dans une perspective disciplinaire (laquelle, dans ce cas, ne me regarde en rien, les problèmes définitoires de ma propre famille dysfonctionnelle me semblant autrement plus tortueux $x^{6}$. Mais réduire ce rejet à ses seuls enjeux de police institutionnelle méconnaîtrait une justification épistémologique plus fondamentale : le fait que les motivations humaines soient multifactorielles n'implique pas que leur investigation empirique gagnerait forcément à l'être. Ainsi, l'impulsion qui, considérant que «tout est dans tout ", pousserait à vouloir psychologiser la recherche sociologique à la racine aurait autant de chances d'aboutir à une mélasse conceptuelle de composition incertaine et de texture bourbeuse qu'à un appareil analytiquement fécond (le genre de mélasse dont le boursouflement contemporain du terme «cognition» donne déjà une idée). On ne parle évidemment pas là des approches qui, à l'instar de la psychologie sociale, exploitent avec des méthodes définies un espace scientifique délimité (et encore moins de ce livre, qui n'appelle en rien à la confusion épistémologique). Cependant, l'alternative entre le déni du psychologique et la désintégration des sciences sociales est imaginaire. Indépendamment $\mathrm{du}$ fait que la psychologie scientifique moderne se soucie à vrai dire fort peu d'étendre son territoire à quelque question que ce soit où l'on ne peut tester des corrélations avec une valeur de $p$ inférieure à 0,05 , la tradition séculaire qui a gravé cette défiance au plus profond de l'habitus sociologique autorise une autre lecture.

De l'extérieur de ce débat, en effet, le manifeste par lequel Durkheim (1895) l'a arrachée à l'emprise de sa rivale pour l'instituer sur un pied d'égalité - «la sociologie n'est pas un corollaire de la psychologie » (p. 125) - ne paraît pas receler la moindre incitation à nier l'influence de cette dernière sur les motivations humaines. Certes, les attitudes et comportements collectifs résultent avant tout de facteurs sociaux (qui le contesterait sérieusement aujourd'hui ?),

comprend donc que ce débat interne, lourd de convictions sousjacentes et de dissensions historiques, puisse facilement devenir acrimonieux. "Les désaccords ne sont pas minces" note sobrement Jean-Hughes Déchaux (2010, p. 643), appelant néanmoins à la poursuite de cette discussion.

6 Pour un exemple de ces problèmes, celui du statut de l'expertise discursive en sciences de la communication, voir Labasse (2012b). 
mais on se méprendrait étrangement sur notre pensée, si, de ce qui précède, on tirait cette conclusion que la sociologie, suivant nous, doit ou même peut faire abstraction de l'homme et de ses facultés. Il est clair, au contraire, que les caractères généraux de la nature humaine entrent dans le travail d'élaboration d'où résulte la vie sociale. (p. 130)

Page après page, le texte fondateur martèle ainsi la nécessité de comprendre les phénomènes sociaux à l'échelle sociale où ils se manifestent et qui les explique, mais il ne le fait jamais sans signifier d'un mot ou d'une phrase que son propos ne porte que sur cette échelle?

Il n'est pas question, bien sûr, d'administrer un cours d'histoire de la sociologie aux sociologues (tous les toupets, même le mien, ont des limites), mais du moins de soutenir - ce qui ne sera déjà pas si mal en matière de toupet - que, dans la perspective multiscalaire requise par le problème spécifique de la valeur discursive, la nécessité empirique d'articuler, et non d'amalgamer, le cognitif et le social s'inscrit dans la plus pure conformité à la pensée durkheimienne :

Si la vie collective ne dérive pas de la vie individuelle, l'une et l'autre sont étroitement en rapports; si la seconde ne peut expliquer la première, elle peut, du moins, en faciliter l'explication. (p. 136)

Et réciproquement.

7 Soulignons quelques exemples : «Sans doute, il ne peut rien se produire de collectif si des consciences particulières ne sont pas données; mais cette condition nécessaire n'est pas suffisante » (p. 127); «Par conséquent, toutes les fois qu'un phénomène social est directement expliqué par un phénomène psychique, on peut être assuré que l'explication est fausse » (p. 128); «Les représentations, les émotions, les tendances collectives n'ont pas pour causes génératrices certains états de la conscience des particuliers » (p. 130); «La cause déterminante d'un fait social doit être cherchée parmi les faits sociaux antécédents, et non parmi les états de la conscience individuelle» (p. 135); «Les phénomènes psychiques ne peuvent avoir de conséquences sociales que quand ils sont si intimement unis à des phénomènes sociaux que l'action des uns et des autres est nécessairement confondue » (p. 137). 
Page blanche conservée intentionnellement 


\section{À la charnière des valeurs}

Nous sommes très riches en observations : c'est un fonds qui s'est grossi de jour en jour [...]. Mais ce fonds si riche, nous gêne plus qu'il ne nous sert [...] tout s'échappe ; parce qu'il y a un nombre infini de parties qui, n'étant nullement liées entr'elles, ne font qu'une masse informe, au lieu de faire un corps régulier. Toutes les Régles sont des branches qui tiennent à une même tige. Si on remontoit jusqu'à leur source, on y trouveroit un principe assez simple, pour être saisi sur le champ, \& assez étendu pour absorber toutes ces petites régles de détail.

Charles Bateux (1746, p. ii)

onsidérez l'appréciation des discours comme un phénomène
psychologique : l'évidence de sa détermination sociale vous
harcèlera sans répit. Considérez-la comme un phénomène social : sa
dimension psychologique vous sautera aux yeux (à moins, bien sûr,
que vous ne les fermiez vraiment très fort). Ce n'est pas un problème
propre à la valeur des discours. Il suffit, pour s'en assurer, de consi-
dérer à nouveau ces piétons téméraires qui traversent les rues aux
endroits ou aux moments les plus malavisés. L'effet attendu (se
retrouver de l'autre côté) étant constant, la « loi du moindre effort »
est apparemment le seul moteur de leur témérité. À ceci près que le
même bénéfice et la même paresse ne produisent pas forcément les
mêmes choix : observant des carrefours similairement dangereux à
Strasbourg et à Inuyama, une équipe a ainsi pu vérifier que 67 o des
piétons français traversaient au feu rouge, contre 6,9\% des piétons
japonais (Sueur, Class et al., 2013). Mieux, un prolongement de
l'étude (Pelé, Bellut et al., 2017) a permis de constater que la présence
d'autres piétons influençait significativement leurs décisions (dans 
un sens ou dans l'autre, selon que ceux-ci transgressent ou non l'interdiction).

En somme, un biophysicien qui couvrirait des piétons de capteurs pour mesurer exactement la quantité d'effort qu'ils s'épargnent n'aurait ni plus ni moins raison qu'un sociologue qui examinerait les valeurs et normes déambulatoires des Français et des Japonais. On pourrait étendre indéfiniment la liste des approches disciplinairement pertinentes (droit comparé, épidémiologie des accidents...) : assez curieusement, l'étude franco-japonaise a d'ailleurs été menée par des éthologues, lesquels n'ont pas manqué de rapprocher les comportements imitatifs des piétons de ceux des suricates et des primates qu'ils étudient d'habitude, plutôt que d'y voir des effets de légitimité (conformité ou transgression). Or, les choix de consommation discursive, surtout au sens large, sont autrement plus complexes que les préférences piétonnières. Voilà donc pourquoi le grand mystère se montrait si grand, persistant et mystérieux.

Lorsqu'il s'agit d'expliquer pourquoi ce qui nous plaît nous plaît, le fatras des déterminants sociaux obscurcit plus qu'il n'éclaire. Une façon empirique de l'appréhender pourrait être d'examiner, non pas l'approbation des discours, mais leur désapprobation (généralement plus véhémente, donc plus repérable) telle qu'elle se manifeste dans les médias et sur Internet. J'avais rassemblé dans cette perspective trois corpus exploratoires. Le premier, subjectif, s'est construit tout seul depuis des années par le prélèvement, chaque matin ou presque au petit-déjeuner, des articles faisant état d'une quelconque controverse discursive dans les nouvelles du jour (telles que les reflètent les agrégateurs d'actualité et les sites de quelques quotidiens). Le second, plus systématique en théorie, mais plus étroit en pratique, est constitué par une recherche intégrale sur la base de textes Eureka.cc des articles publiés à ce sujet durant un an dans la presse canadienne francophone $^{1}$. Le troisième, mettant à profit l'évaluation de professionnels

1 Le critère d'inclusion était la présence dans la même phrase d'une racine traduisant une forte réprobation (choqu* $\mid$ scandal $^{*} \mid$ offens ${ }^{*} \mid$ inaccep* | blessa*) et d'une racine associée à un objet discursif (texte $\mid$ articl $^{*}$ | propos I mots | parol* | déclara* | humo* | œuvre | livre | film I phrase I discou*). Un filtrage secondaire a permis d'inactiver des termes non pertinents (comme « offensive » ou «à propos »), conduisant à recueillir 649 articles publiés entre septembre 2015 et septembre 2016. Une 
particulièrement friands de chamailleries, a exploité l'indexation adoptée par deux magazines populaires : Gala (« Polémiques ») et Voici («Critiques »).

L'examen de la cacophonie ainsi rassemblée a d'abord confirmé ce qui était à craindre : en tirer des conclusions quantifiées, ou même des enseignements méthodologiquement robustes, exigerait un traitement d'une ampleur disproportionnée par rapport aux nécessités et à la pagination de cet ouvrage : trop d'effort et pas assez d'effet, pour ce qui ne constituerait ici qu'une petite pièce dans un grand puzzle. On se contentera donc jusqu'à nouvel ordre de considérer cette collection comme une banque de cas, c'est-à-dire un gisement d'exemples empiriques (dont certains, à dire vrai, ont déjà été exploités dans les chapitres qui précèdent), mais aussi comme une source indicative plus générale, fondée sur des typicités facilement identifiables qualitativement à défaut d'être probantes.

Si les deux prélèvements opérés sur la base de mots clefs ou d'indexations éditoriales présentent de ce fait un spectre de situations beaucoup moins large que celui réalisé manuellement - d'autant que celui-ci ne se limite pas à une tranche de douze mois - tous trois manifestent avant tout la prévalence écrasante d'un même critère : le respect des différences identitaires (pour ses promoteurs) ou la domination du "politiquement correct» (pour ses détracteurs). Ce n'est pas exactement une surprise, tant le resurgissement de la contrainte morale - imposée par le haut jusqu'à sa décomposition dans les années 1950 et 1960, puis reconstruite sur des bases plus ou moins inversées depuis quelques décennies - est intuitivement connu et publiquement débattu. Si approximative que soit cette étiquette, qui recouvre des positions assez différentes, elle suffira ici. Mais cette catégorie, même distendue, est loin d'enceindre le riche spectre des désapprobations. On y trouve aussi, par exemple, le rejet du langage «vulgaire » ou de gestes explicites, celui des modernisations orthographiques, la dénonciation d'une révélation journalistique (sur des

sous-sélection requérant en outre la présence des mots cibles dans l'introduction (lead) de l'article a par ailleurs cerné un «noyau dur » de 200 articles (précisons cependant que les quotidiens du groupe Québécor, dont Le Journal de Montréal, n'étaient pas compris dans la bases de textes utilisée, mais qu'un bon nombre de leurs articles a en revanche été recueilli dans le corpus manuel). 
opérations militaires en cours), celle du contresens historique proféré par un homme politique ou de l'erreur géographique commise par un manuel scolaire, les affabulations d'un nageur olympique, la retouche sous Photoshop de célébrités. Et, bien sûr, principalement, les dénonciations de campagnes publicitaires, de films à l'affiche et de propos de Donald Trump, mais ces trois thèmes, particulièrement fournis, relèvent en bonne part du registre du respect identitaire. Et encore ce brouhaha dans lequel «les réseaux», remarquablement inflammables, sont souvent le locuteur de référence, n'est-il que celui de l'indignation ostensible. La désapprobation commune, privée ou même publique, des productions discursives médiocres, ennuyeuses, douteuses, triviales, voire franchement mauvaises, échappe aux critères de prélèvement utilisés pour cette sélection, ce qui peut du reste expliquer pourquoi les effets de distinction culturelle y sont étonnamment peu perceptibles. Bref, même en ne se penchant que sur la fraction la plus outrée des seuls jugements de désapprobation, sa diversité défie toute typologie commode.

Les choses en étaient à peu près là, lorsqu'est arrivée une somme très attendue. Dix ans après avoir dénoncé à juste titre le fossé entre "l'omniprésence du thème des "valeurs" dans maints travaux se réclamant de la sociologie, et la pauvreté de ses conceptualisations, voire son absence comme objet de recherche à part entière » (Heinich, 2006), Nathalie Heinich s'essayait à combler le fossé en question. Le propos était d'autant plus considérable qu'il s'appuyait, entre autres, sur les recherches de terrain qu'elle avait consacrées depuis plus de deux décennies à l'appréciation des produits culturels (art contemporain, patrimoine architectural...). Mais il s'agissait maintenant (Heinich, 2017) d'étendre ces observations aux valeurs en général, telles qu'elles peuvent être mobilisées dans toutes les appréciations, quel que soit leur objet. Bigre.

Ayant détaillé ailleurs (Labasse, 2017a) tout le bien que l'on pouvait penser de cet ouvrage en général et les quelques réserves plus spécifiques qu'il pouvait susciter sur tel ou tel aspect (voir aussi les contributions réunies par Fleury et Walter, 2017), on se contentera d'en mentionner deux points. Le premier est l'arrivée on ne peut plus opportune sur le marché des idées d'une nomenclature explicite et réfléchie des "registres de valeurs ", c'est-à-dire des catégories générales regroupant les critères de jugement. L'inventaire en comporte 16, sans autre hiérarchie que celle de l'alphabet: 
Aesthésique (plaisir, gourmandise, sensorialité, sensualité...)

Affectif (attachement, émotion, amour, tendresse, sensibilité...)

Civique (responsabilité, patriotisme, souci de l'intérêt général...)

Domestique (proximité, présence, lien, entraide, protection...)

Économique (cherté, coût, juste prix...)

Épistémique (vérité, connaissance, savoir...)

Esthétique (beauté, art...)

Éthique (moralité, fraternité, charité, sollicitude, décence, respect...)

Fonctionnel (utilité, commodité, sécurité...)

Herméneutique (sens, significativité...)

Juridique (légalité, conformité aux règlements...)

Ludique (jeu, humour, distanciation, exploration, expérimentation...)

Mystique (spiritualité, foi, sacralité...)

Pur (pureté, authenticité, propreté, intégrité, sincérité...)

Réputationnel (renom, réputation, honneur, célébrité, visibilité...)

Technique (efficacité, performance, virtuosité.)

À ces registres évaluatifs, la chercheuse ajoute trois «valeurs de base ", surplombant les autres (la rationalité, la justice et l'ordre) et quatre valeurs «cardinales » (l'originalité, la rareté, la pérennité et l'universalité). Ces dernières, "constitutivement ambivalentes, ne suffisent pas à elles seules à créer de la valeur » (p. 259), mais ont le pouvoir d'amplifier ou d'affaiblir les autres selon le contexte. Elles ressemblent assez en cela aux « modulations », telles que la surprise et l'humour, que l'on avait évoquées plus haut à propos des facteurs d'effet cognitif.

Pour séduisant que soit ce panorama des valeurs tiré (dans une certaine mesure) des arguments exprimés par les acteurs, il ne se prétend pas exhaustif et il ne l'est pas. Même s'il s'est élargi à d'autres champs, les domaines de l'art et du patrimoine où plongent ses racines semblent, par exemple, peu concernés par une question comme celle de la correction orthographique et grammaticale, si obsédante dans 
d'autres champs (ou dans d'autres lieux de la francophonie). Plus fondamentalement, il ne peut transgresser les limites inscrites dans sa construction même. Comme toute "façon de voir ", a fortiori lorsqu'elle est ancrée dans une tradition disciplinaire ombrageuse, l'approche proposée par Heinich tend à aplanir sous sa perspective les objets qu'elle examine, quitte, de ce fait, à brider un peu sa capacité explicative. Cet inévitable effet de perspective conduit ainsi à rapprocher des ordres de valeur dont l'homologie ne va pas de soi, notamment le registre "éthique ", dont la portée tend aussitôt à déborder de la case qui lui est assignée (la chercheuse remarque du reste qu'il l'emporte généralement sur les autres considérations), le registre "affectif » (peut-on imaginer de critères plus individuels ${ }^{2}$ ?), mais aussi le registre «fonctionnel », qu'il semble difficile de ramener simplement à une famille de valeurs sociales. Apprécier un bon manuel de bricolage ou un livre de cuisine infaillible, c'est effectivement les créditer d'une valeur, mais le caractère social de cet attachement est aussi discutable que la prédilection que l'on peut éprouver pour un tournevis fidèle ou un épluche-légumes efficace. De même, et surtout, enrôler le registre du plaisir et de la gourmandise parmi les valeurs fait bon marché de la classique différence entre les jugements d'agrément (ceux qui, selon l'adage, ne se discutent pas) et les jugements... de valeur (argumentables). La nuance est effectivement fragile, surtout si l'on accepte de définir les valeurs comme étant n'importe quelle qualité à laquelle des acteurs disent attacher du prix. Et surtout à une époque où, sur les sites de vente en ligne, les jugements d'agrément spontanés (" $A$ moi, ça m’a pas plu»), censés jusque-là relever du domaine privé, entrent en collision avec les jugements de valeur ("C'est une œuvre médiocre parce que... »). Sans développer ce problème (que l'on abordera plus loin), force est de remarquer que réduire la dimension hédonique à une catégorie banalisée ne permet guère de percevoir les ressorts de telles divergences, et plus généralement de rendre compte des polarités qui distendent le marché discursif. $\mathrm{Ni}$ quantitativement (l'offre et la demande effectives de livres, disques, films, contenus Internet...) ni qualitativement (par exemple la profondeur de la tension, largement attestée, entre la critique cinématographique « culturelle » et la critique « consumériste »).

2 D'accord, on sait que les affinités électives sont en bonne partie déterminées socialement (endogamie du mariage, etc.), mais ça ne suffit pas à réduire le registre affectif à cette seule échelle d'explication. 
Tout cela ne met pas le moins du monde en cause l'importance ou la validité interne de cette nomenclature. Il serait aussi candide de la contester sur cette base qu'il serait symétriquement naïf de reprocher à la psychologie expérimentale la désinvolture avec laquelle elle rangeait les «dispositions personnelles » (dont les déterminants sociaux) parmi les facteurs de l'intérêt cognitif 3 .

Au bout du compte, le catalogue raisonné d'Heinich ressemble un peu à la devanture d'un marchand de glaces : son menu permet fort bien de répondre à la question "qu'est-ce qui nous plaît? », mais il ne propose nullement d'expliquer pourquoi ce qui nous plaît nous plaît (ce qui serait, du reste, en contradiction avec sa démarche4). À cet égard, le modèle bourdieusien de la distinction, pour insuffisant qu'il s'avère aujourd'hui, éclairait mieux la hiérarchie qui sépare l'humble glace à la vanille du distingué granité à l'eau de fleur d'oranger.

Constater une fois de plus que les déterminants sociaux de la valeur discursive ne se laissent pas facilement inventorier ne peut que contraindre, dans une perspective analytique globale, à se résoudre à les envisager ici selon leurs caractéristiques les plus élémentaires. Un peu frustes, sans doute, mais empiriquement robustes et largement admises.

Un de leurs caractères les plus manifestes réside dans l'ambivalence de leur action. Il n'est même pas nécessaire de s'interroger sur la nature des influences sociales pour noter que leurs effets se traduisent aussi bien en attirance (ou préférence, attrait...) qu'en répulsion (ou aversion, rejet...). Cette opposition directionnelle est si régulière que Heinich a pu associer de façon presque systématique des anti-valeurs aux valeurs qu'elle a collectées : ce qui sera apprécié comme minutieux dans un cas serait jugé laborieux dans un autre, la célébration de

3 Signaler, en somme, que la sociologie produit de la sociologie et que la psychologie élabore de la psychologie n’est pas précisément une révélation, mais on voit mal qui - à moins d'exiger pour sa discipline une préséance universelle - pourrait le prendre comme une critique.

4 Le courant méthodologique dont se réclame l'auteure (la sociologie « compréhensive ») se fonde sur les perspectives qu'adoptent les acteurs eux-mêmes, s'abstenant d'en soupeser les motivations ou de les expliquer par des mécanismes qui leur seraient dissimulés. 
l'intégrité s'opposera à la dénonciation de la rigidité, et ainsi de suite ${ }^{5}$ (exceptionnelles sont les valeurs qui, comme la justice, ne semblent pas posséder d'opposé direct). De même, dans la sociologie positionnelle de la distinction, l'invocation du « bon goût » appelait symétriquement celle du « mauvais goût ». De façon générale, produits culturels ou pas, il parait difficile de trouver une articulation sociale plus solide que la dialectique du bien et du mal, sur laquelle reposent la prescription et la proscription religieuse depuis quelques millénaires.

Or, rapportée au problème de la valeur discursive, cette antinomie évoque un schéma agréablement familier. On se souvient que la pertinence cognitive reposait sur l'opposition entre les facteurs dépréciatifs (l'effort de traitement) et les facteurs appréciatifs (l'effet cognitif).

Souligner, en regard de la pertinence cognitive, que la convenance sociale joue elle aussi de façon dépréciative et appréciative ne produit pas seulement un effet de symétrie esthétiquement plaisant. Cet angle de vue présente aussi l'intérêt de juxtaposer des propositions dont le statut empirique est équivalent, l'une comme l'autre étant assez fondamentale et assez évidente pour être assimilable à un axiome (si ce n'est à un truisme). De plus, leur confrontation permet de percevoir que les différentes familles de facteurs ne s'opposent pas en bloc : un critère appréciatif d'ordre social peut par exemple se combiner avec un facteur d'effet cognitif pour contrebalancer l'effort que requiert une œuvre. Ou, à l'inverse, une proscription sociale comme celle visant le mensonge s'accordera avec une destruction de l'effet cognitif (un énoncé fallacieux perd son attrait utilitaire ou stochastique) pour dévaloriser un discours.

En d'autres termes, l'appréciation de la valeur discursive ne se réduit pas à un face-à-face frontal entre «le » social et «le » cognitif (dichotomie pratique mais sommaire); bien que l'on puisse la plupart du temps s'en tenir à cette approximation, d'autres situations peuvent utilement être abordées à travers l'interaction croisée des ordres de facteurs relevant de l'un et de l'autre regroupement.

5 On trouvera dans Jourde (2002) une brillante et féroce illustration de cette ambivalence dans le cas des productions littéraires, où des valeurs comme l'authenticité et la sincérité peuvent aussi bien justifier le blâme pour leur carence («affectation») que pour leur ostentation (« excuse de la médiocrité »). 


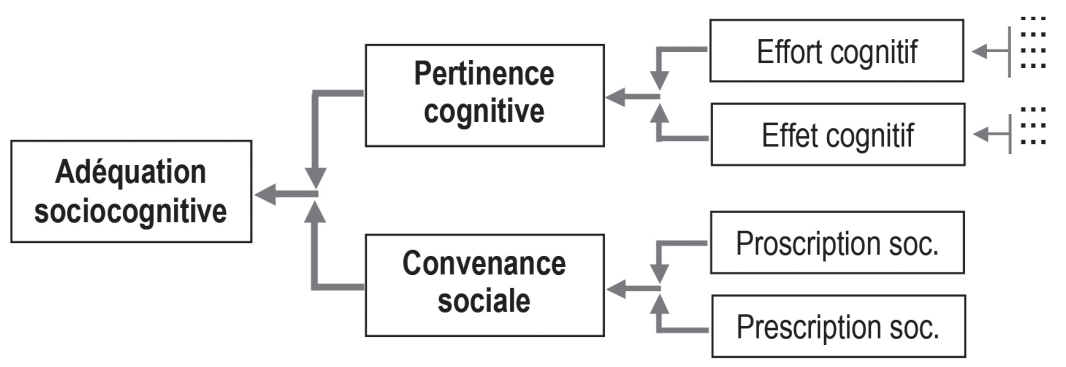

Figure 4 b. L'articulation (complétée) de l'adéquation discursive

Encore faudrait-il préciser dans la mesure du possible comment les variables sociales se manifestent. C'est ici que l'on doit considérer un second attribut élémentaire des influences sociales, lui aussi robuste et largement admis : celles-ci n'ont pas toutes la même force contraignante. De même que l'on avait, dans le cas de l'effort cognitif, glissé des processus aux tâches, on glisse ici des valeurs, décidément compliquées, à leurs conséquences : les normes sociales et leur sanction. Cette dimension est également compliquée - des générations de philosophes, sociologues, juristes, etc. y ont veillé-, mais on n'a besoin que de quelques repères pragmatiques.

La hiérarchie des normes est dans une certaine mesure associée à leur formalisation, de la plus explicite - lois, règlements, textes déontologiques, prescriptions, recommandations... - à la plus tacite, laquelle peut se réduire à une moue ou à un malaise fugace ${ }^{6}$. Cependant, cette formalisation est souvent décalée par rapport aux contraintes effectives. Les guides de "bonnes manières ", très formels, que s'arrachait jadis la moyenne bourgeoisie ont perdu une bonne partie de leur emprise (mais pas toute : l'appel de la distinction y veille) et les règles d'acceptabilité comme celles édictées par Apple pour sa boutique en ligne sont souvent dénoncées comme pudibondes. Même les textes de loi, en principe au sommet de l'échelle des contraintes, n'échappent pas à ce décalage. Ainsi le délit français d'offense à un chef d'État étranger était-il devenu désuet bien avant sa disparition du Code pénal. Les spécialistes du droit en sont venu à questionner la « force normative » des lois, dans laquelle la légitimité que leur attribuent ceux qui leur sont assujettis tient une

$6 \mathrm{Ou}$, de façon encore plus diffuse, à l'exemple offert à l'imitation par les comportements et préférences des pairs. 
bonne place (Thibièrge, 2009). En parallèle, d'autres ont, par la force des choses, pris en compte l'importance de la "soft law ", soit la masse « des recommandations, des déclarations, des activités d'information et de persuasion, des gentlemen's agrement, des chartes ou d'autres actes incitatifs ». Mais cet ensemble de prescriptions sans obligations est juridiquement perturbant, puisqu'« admettre une graduation du phénomène normatif conduit à admettre non seulement l'existence de degrés dans les actes non obligatoires, mais également dans les actes de hard law" (Flückiger, 2009, p. 74).

D'autre part, l'emprise effective des diverses normes, codifiées ou non, explicites ou non, varie non seulement selon les individus et les stratifications sociales, mais aussi selon l'échelle considérée (sphère personnelle, communauté de travail ou de loisirs, espace public) et le groupe qui les applique : le stéréotype du "bon élève », admirablement conforme aux normes de l'institution, mais pas à celles (non écrites, mais plus ou moins explicites) de ses camarades les moins studieux en est un bon exemple. Elles s'ajustent aussi au caractère autojuridictionnel des discours : les tribunaux admettent par exemple de bonne grâce que les normes d'acceptabilité s'appliquant à un texte satirique sont plus souples que celles s'imposant à un écrit présenté comme factuel.

Au niveau de généralité qui nous intéresse, on se bornera à prendre note de la gradation classique des contraintes sociales en fonction de leur niveau d'assertion, tant qualitatif (la hiérarchie des textes normatifs) que quantitatif (par exemple, l'abondance d'un blâme sur les réseaux sociaux). On retiendra cependant que cette gradation est modulée ${ }^{7}$ par l'évaluation de leur légitimité, de leur intensité injonctive ("doit», « devrait »...) et de leur applicabilité au type de discours considéré. Elle l'est d'autre part, voire surtout, par la perception de leurs conséquences personnelles, tant pratiques - par exemple pécuniaires - que symboliques, c'est-à-dire leurs enjeux en termes de disqualification ou de reconnaissance, y compris sur le plan de l'auto-appréciation. On aura deviné que cette dernière précision ne vise qu'à esquiver le vieux problème philosophique de l'origine du jugement moral, incidemment la question de sa sincérité, et

7 Plus que modulée, même, si l'on considère cette évaluation comme une condition originelle des normes et une clef de leur évaluation au fil du temps. 
plus généralement celui des convictions ou des croyances. Dans la mesure où ces variables nébuleuses jouent autant sur l'évaluation de la légitimité des normes et des discours qu'elles peuvent être affectées par leurs conséquences (voir les débats sur l'enseignement du créationnisme aux États-Unis), étiqueter la « morale » comme une modulation sociale d'ordre amont ou aval est un choix qui ne s'impose pas. Disons qu'elle est par là.

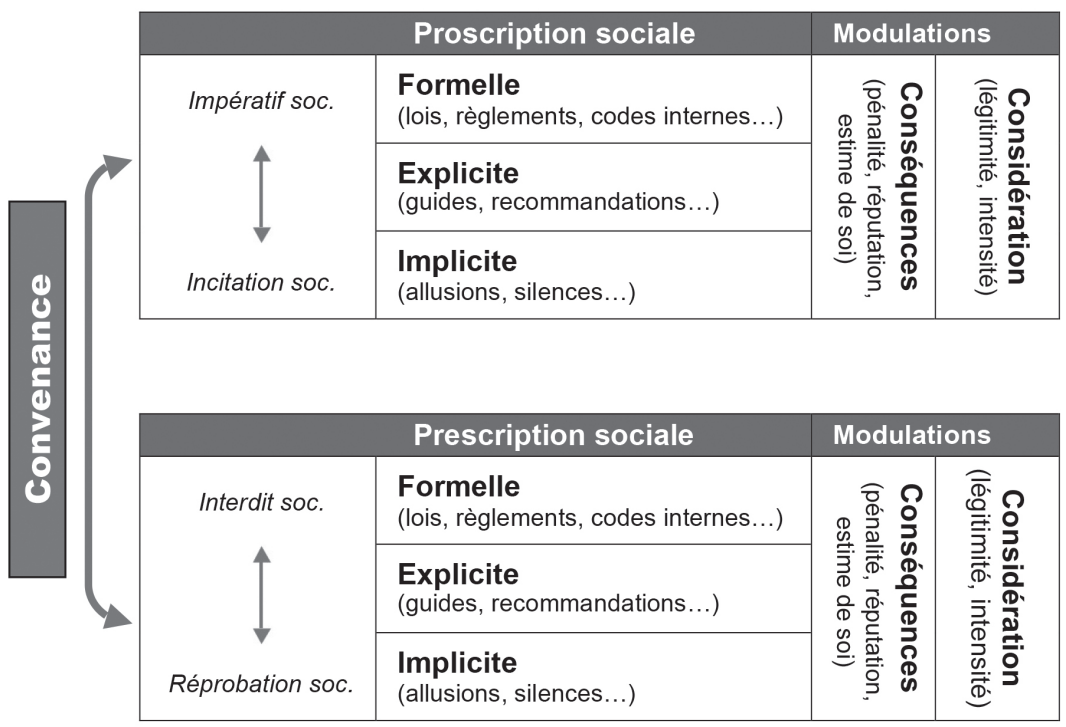

Figure 5. Une typologie oppositionnelle de la convenance sociale

À l'évidence, cette conceptualisation abstraite n'est pas le summum du raffinement théorique. Outre que les facteurs qu'elle schématise sont loin d'être aussi étanches que les cases qui les situent, le fait que ces facteurs (contrairement aux variables appréciatives et dépréciatives de la pertinence cognitive) soient manifestement identiques de part et d'autre de cette séparation jette un sérieux doute sur celle-ci. Il est scientifiquement plus courant, et généralement plus sensé, de rapprocher les objets semblables. Après tout, les valeurs et les anti-valeurs (Heinich), de même que l'ennoblissement et la stigmatisation (Bourdieu), ne diffèrent pas dans leur nature ${ }^{8}$.

8 Comme le remarque par ailleurs Carole Talon-Hugon, la pression moraliste des siècles passés et les provocations transgressives des créateurs 
Comme toujours, une question de perspective est à l'œuvre. D'un certain point de vue, les panneaux de sens interdit et de stationnement appartiennent à la même classe, celle de la signalisation routière. Mais en termes d'effets, cette similitude n'est pas forcément pertinente : s'il s'agit de comprendre la logique d'un trajet automobile, un sens interdit est presque identique à un tronc d'arbre en travers de la route, et tout à fait différent d'un panneau anodin. S'il s'agit de clarifier les logiques de l'adéquation discursive, le sens dans lequel s'exercent les influences sociales est plus important que leur nature spécifique, d'autant que leur symétrie n'est que partielle.

A priori, il existe aussi bien des normes formelles, perçues comme légitimes et impérieuses, qui obligent à prendre connaissance des discours, que des normes interdisant de consommer d'autres offres discursives. Ainsi, des règles imposent-elles expressément aux membres de certaines professions (médecins, avocats...) de suivre l'évolution des connaissances dans leurs domaines, que celle-ci les passionne ou non. Des exigences légèrement moins explicites font qu'un professeur ne peut pas, en tout cas pas durablement, se dispenser de lire des copies d'examen, pas plus que les étudiants ne peuvent sans risque s'abstenir de connaître le contenu du cours. En fait, dès lors que la plupart des emplois supérieurs peuvent être considérés comme des activités d'opérateur sur le marché discursif - ainsi qu'on le remarquait en introduction - la plupart de ces emplois imposent de consommer des discours indépendamment de leur pertinence cognitive. C'est même le critère introspectif qui permet de distinguer l'intérêt utilitaire (en tant que facteur d'effet cognitif) et la conséquence pratique (en tant que contrainte sociale) : le premier, contrairement à la seconde, est rarement associé à l'ennui'.

Cependant, l'intensité des règles appréciatives formelles est généralement moins prononcée que celle de leurs homologues dépréciatives,

contemporains sont d'une certaine façon similaires face à la conception autonomiste de l'art: "Né en effet du combat contre l'idée d'un art au service de la morale, [l'autonomisme] doit être logiquement tout aussi hostile à un art dont l'intention explicite serait de mettre à mal les valeurs éthiques » (2009, p. 145).

9 Ce qui n'empêche bien sûr pas l'une et l'autre dimensions de se mélanger parfois, puisque les catégories analytiques ne traduisent pas des délimitations «naturelles ». Mais il est assez rare que l'on juge un mode d'emploi « ennuyeux», contrairement, hélas, à un texte que l'on doit examiner. 
lesquelles sont souvent assorties de sanctions pénales. Les proscriptions explicites sont en outre moins liées au statut professionnel, qui peut en revanche les neutraliser (policiers, journalistes ou chercheurs en sont exonérés pour des enquêtes «légitimes »). C'est le cas des discours de nature sexuelle, qui sont proscrits en fonction de critères très variables selon les époques et les pays, mais impérieux dans tous les cas. La possession d'œuvres politiquement ou religieusement douteuses est également un motif traditionnel d'arrestation, toujours employé dans certains pays. De même, en France, la consultation de sites Internet faisant l'apologie d'actes terroristes est (brièvement) devenue sanctionnable de deux ans de prison.

Cette loi mérite que l'on s'y arrête, car, outre sa récence (2016) dans un pays démocratique, elle est particulièrement révélatrice des tensions internes de la régulation légale de l'adéquation discursive. $\mathrm{La}$ contradiction qui n'a échappé à personne - et surtout pas au Conseil constitutionnel qui a invalidé le texte à deux reprises - est l'opposition entre cette interdiction et un principe supérieur tel que la liberté de s'informer, d'où sa légitimité problématique.

Une autre contradiction, en revanche, a suscité moins d'attention. La loi posait comme conditions alternatives, soit que le site visité appelle à commettre des actes de terrorisme, soit qu'il « comporte des images ou représentations montrant la commission de tels actes consistant en des atteintes volontaires à la vie ${ }^{10} »$. Or, si le premier cas pouvait correspondre à l'objectif sécuritaire déclaré, la justification du second semblait beaucoup plus confuse (à l'image de sa formulation). Pourquoi viser spécifiquement des images d'assassinats, si ce n'est pour lutter contre leur effet cognitif extrême, trop élevé (insoutenable) pour une personne "normale", mais attirant pour certains? Auquel cas cette disposition relèverait de la morale publique, à l'instar de la prohibition des atteintes à la pudeur, plutôt que de la sécurité publique qui motivait le texte. On voit ici - et on le reverra que la question des déterminants de l'adéquation sociocognitive n'est pas aussi abstraite que ça : elle aide par exemple à percevoir la nuance possible entre la répression du terrorisme et la répression du voyeurisme. Mais en contrepartie, un tel cas d'espèce aide à percevoir un aspect paradoxal de cette adéquation. Si celle-ci résulte de façon générale de la confrontation entre la pertinence cognitive et la

10 Article 18 de la loi $n^{\circ}$ 2016-731 du 3 juin 2016. 
convenance sociale, ces modes d'appréciation ne paraissent pas toujours agir dans le sens attendu.

Les vidéos d'égorgement sur lesquelles repose en partie la communication des groupes les plus violents ne tendent pas seulement à «valoir» par la brutalité de leur effet cognitif de premier et second degré (l'atrocité pure et l'appréhension), mais tout autant par leur violation ostentatoire des principes élémentaires du comportement humain : au lieu d'étouffer la réception de ces discours, les normes sociales qu'elles bafouent leur servent d'amplificateur. C'est la version démente d'un effet bien connu dans d'autres domaines, la shock value, qu'ont exploité des pans entiers de la musique rock (punk, death metal...) et de la publicité (shockvertising), mais aussi de l'humour - celui qui "évoque les choses les plus horribles, les plus contraires à la morale en usage " (Bailly, 2008) - et, bien sûr, de l'art contemporain. C'est ainsi que la capacité à scandaliser de La Nona Ora de Maurizio Cattelan (représentant le pape Jean Paul II écrasé par une météorite) ayant déçu son propriétaire, celui-ci eu l'idée judicieuse de l'exposer à nouveau, mais cette fois en Pologne, pays où il était sûr d'obtenir plus qu'un haussement d'épaules. De fait, scandale il y eut enfin, de proportion homérique, et la cote de La Nona Ora s'envola sur le marché de l'art.

L'instrumentalisation de la transgression normative n'est ni limitée à quelques secteurs turbulents du marché discursif, ni particulièrement récente, quoiqu'elle le paraisse souvent à ceux qui s'en lamentent. Par exemple, Paul Acker en 1906 :

On avait annoncé cet hiver qu'un étranger allait installer à Paris une agence de notoriété : il se targuait d'assurer à tout écrivain pressé d'arriver une réputation plus ou moins brillante, plus ou moins durable, selon les honoraires qui lui seraient servis. Que cela est symptomatique de notre temps ! Aujourd'hui, en effet, il s'agit, non pas d'écrire un beau livre, mais de vendre bien le livre qu'on a écrit. Or, comment le vendre bien, s'il passe inaperçu ? Et comment empêcher qu'il passe inaperçu, sinon en tâchant qu'on en parle ? [...] Cependant aucun de ces jeunes écrivains n'a encore tenté ce que tenta dernièrement un journaliste américain. Il avait publié un roman, dont personne ne parlait. Afin d'attirer l'attention sur lui, il tua un Chinois. Le jour du jugement, il avoua, avec une grande aisance, qu'il avait tué ce Chinois afin qu'on connût 
le meurtrier et qu'on achetât son roman. Je ne sais si on acheta le roman, mais lui fut condamné à mort. Je voudrais qu'on l'eût condamné à mort encore moins pour avoir tué ce Chinois, que pour s'être formé de la réussite littéraire une idée si méprisable. (p. 161-162)

Que l'anecdote soit factuelle ou non, placer un tel meurtre en dessous de l'avilissement littéraire sur l'échelle des crimes n'est acceptable que dans le cadre autojuridictionnel d'une chronique. Mais si l'on se situe dans ce registre, on doit distinguer un motif d'inculpation beaucoup plus grave que l'offense à la littérature : l'offense à la théorie. Il est conceptuellement intolérable que la violation de l'acceptabilité sociale puisse être mise par quiconque au service de la valeur discursive.

Cependant, comme la plupart des paradoxes, celui-ci ne tient qu'à la variabilité des éléments qu'il oppose : la «convenance », qui fluctue un peu trop facilement des discours aux actes en général, mais surtout la " valeur ", que l'on s'était bien gardé de trop expliciter aussi longtemps que cela n'était pas indispensable.

Une conception possible, et très classique, de la valeur d'un discours est l'estime que l'on accorde à celui-ci. D'autres, assez proches mais plus spécifiques, sont le crédit qu'on lui accorde, l'approbation de ce qu'il énonce, voire, le cas échéant, l'adhésion aux opinions qu'il distille. D'autres encore sont le plaisir que l'on en retire, ou la satisfaction qu'il apporte (ce qui n'est pas du tout pareil : je suis satisfait d'être vacciné, mais je n'ai éprouvé aucun plaisir à l'être). Il n'est pas besoin d'allonger plus la liste, ce qui serait facile, pour constater qu'elle ne peut être plus que l'écho des métonymies conceptuelles que l'on s'efforce justement de dépasser : la partie pour le tout ou la cause pour la conséquence. Si l'on tentait de la ramener à ses polarités principales, elle reviendrait à l'opposition entre la "valeur» hédonique et la «valeur » symbolique, non sans l'alourdir d'une complication supplémentaire, la redoutable dichotomie entre la forme et le fond.

Ces considérations théoriques se transposent aisément en termes introspectifs moins abstraits. Je crédite par exemple de beaucoup de valeur les livres du Mahatma Gandhi ou de Nelson Mandela, mais je n'envisage aucunement de les lire - en tout cas entièrement - à moins de circonstances imprévues (la plus plausible impliquant un échouage sur une île déserte). Ce qui revient à admettre que malgré la 
qualité de leurs auteurs, mais aussi le grand intérêt historique ou politique de ces œuvres, je ne leur accorde pas réellement de valeur. Pas assez, sans doute, mais c'est ainsi. À l'inverse, je peux raffoler de certains auteurs (Borges, Vialatte...) sans forcément éprouver un grand enthousiasme pour leurs options idéologiques, ni attribuer à ces livres un intérêt historique ou documentaire particulier. Sans parler de ces romans policiers que - comme le rappelait Lahire - je suis loin d'être le seul à apprécier.

En fait, chacun n'a qu'à parcourir les étagères de sa propre bibliothèque ou l'historique de son navigateur Internet pour décaper la «valeur» discursive de tout vernis mystique. Parmi tous les sens dont on peut essayer de revêtir cette locution, ceux qui résistent le mieux - la valeur d'échange, la valeur concurrentielle et la valeur attentionnelle - sont un peu désabusés mais convergents, et au bout du compte extrêmement proches. Tous obligent du reste à prolonger d'une façon ou d'une autre la métaphore du « marché » discursif.

Le premier, cher aux économistes ${ }^{11}$, revient à considérer la valeur en fonction de la contrepartie que l'on est prêt à lui donner. La valeur, c'est ce que l'on paie, parfois avec de l'argent, mais surtout avec du temps dans le cas des discours. L'approbation n'entre pas nécessairement dans ce calcul : si, par exemple, paraissait un règlement interdisant les mauvais calembours dans les cours universitaires, il serait assuré de me compter toutes affaires cessantes parmi les lecteurs les plus passionnés de ses moindres clauses ou arguments (même s'il fallait le commander à grands frais). Que l'on puisse par ailleurs ne pas terminer, voire ne pas commencer, un livre emprunté ou acheté traduit clairement une réestimation de la contrepartie que l'on avait prévu de lui accorder.

La seconde approche considère la valeur de façon relative au sein de la surabondance de l'offre discursive. La valeur c'est ce qui fait qu'un discours est consommé plutôt qu'un autre. Cette perspective concurrentielle est à peu près similaire à l'«équation de sélection» qu'avait recherchée Wilbur Schramm. Elle peut aussi s'exprimer

I1 Sur cette question, d'autant plus sérieuse en économie que l'assymétrie d'information entre les acteurs se répercute sur le fonctionnement des marchés, voir notamment l'article classique de Joseph Stigler (1961) et celui, particulièrement accessible, de Sakalaki et Thépaut (2005). 
comme la somme de tout ce qui fait que l'on s'arrête devant un tableau particulier dans un musée qui en expose des milliers : les raisons peuvent être multiples, de la renommée de l'œuvre à l'effet qu'elle produit, mais le résultat est le même.

Le troisième sens, dans l'air du temps, considérerait la valeur comme un équivalent de l'attention. Bien en phase avec la «crise de l'attention » évoquée au début de ce livre, mais aussi avec les évolutions de la communication associées aux réseaux sociaux, il serait particulièrement approprié si la notion d'attention ne s'était singulièrement démonétisée. Pendant que des journalistes sont chargés de résumer à la radio les sujets qui « retiennent l'attention » des réseaux sociaux, d'autres consacrent des articles amers au fait que ces mêmes réseaux sociaux favorisent le partage instantané d'informations sans le moindre examen de celles-ci, si ce n'est de leur titre, voire d'un message s'y rapportant : quantitativement formidable, l'attention de tous ne requerrait l'attention de personne en particulier (elle n'aurait donc pas à se payer avec du temps, ne durant que le temps d'un clic). Ce genre d'attention inattentive n'est cependant pas l'apanage de Twitter. Comme dans toute polémique littéraire, Richard Millet a eu beau jeu de reprocher à la plupart de ses détracteurs de ne l'avoir pas lu. Quant au champ des sciences expérimentales, la tendance à recopier à partir d'autres publications l'analyse préalable des travaux antérieurs (la « revue de question ») y est si répandue qu'il est parfois impossible de retrouver la source d'une référence erronée. Bref, l'équivalence entre valeur discursive et attention accordée peut être très judicieuse, mais seulement dans le cas où il s'agit bien d'attention.

Comme on le voit, ces trois façons d'aborder la valeur discursive diffèrent plus par leur point de départ que par leur point d'arrivée. On peut ainsi considérer la valeur discursive, comme la préférence accordée par rapport à d'autres contenus (ou à des activités concurrentes), telle qu'elle se traduit par le temps et l'attention réelle dévolus à son objet. À titre d'illustration, on pourra évaluer la valeur discursive de la télévision à l'aune des études montrant qu'elle capte une part de temps considérable, mais s'avère modérément concurrentielle (elle permet des activités simultanées) et mobilise assez peu d'attention effective (voir supra, chapitre 2).

Au-delà de son pragmatisme, la principale vertu de cette façon de voir est de ne pas séparer a priori la valeur hédonique (jugement 
d'agrément) et la valeur symbolique (jugement axiologique) : elle s'applique aussi facilement à une soirée de gala à l'Opéra, même si l'on y a été traîné par son ou sa conjoint(e), qu'à la lecture d'un magazine lubrique, même si l'on est peu enclin à s'en vanter.

En revanche, dans le cas des discours visant à choquer, elle permet de distinguer la shock value de la valeur discursive, en remarquant que la première, malgré son impact en termes de visibilité publique, n'entraîne pas mécaniquement la seconde. La remarque d'Acker, «Je ne sais si on acheta le roman", est à cet égard tout à fait judicieuse. De même, on peut penser que la forte résonance des vidéos d'exécution des talibans se traduit modérément dans les choix de visionnage du grand public. Quant aux strates plus acceptables du marché de l'inacceptable, elles témoignent également de cette différence : plus d'un chanteur ou d'un groupe rock s'est avisé, au désespoir de ses fans originaux, qu'une posture moins radicale lui permettrait d'élargir son audience, et la capacité du shockvertising à susciter efficacement des intentions d'achat a soulevé de nombreux doutes chez les annonceurs. Il ne s'ensuit évidemment pas que la capacité à choquer est sans effet sur la consommation discursive, non seulement en conséquence de la visibilité qu'elle procure, mais aussi parce que, les normes sociales étant modulées différemment en fonction des groupes et des individus, un discours acceptable pour certains tout en révoltant le plus grand nombre n'en est que meilleur aux yeux des initiés. Mais il n'y a plus là de paradoxe.

Dès lors, il est temps de rapprocher les divers déterminants cognitifs et sociaux examinés en les figurant dans un modèle d'ensemble (figure 6), un peu rébarbatif de prime abord mais réellement très simple dans les relations qu'il schématise.

Il ne s'agit là que d'une façon de situer les grandes familles de variables dont les interactions peuvent expliquer les préférences discursives ressenties (ce qui nous convient). Toutefois, il est très facile d'en dériver les logiques d'autres modes d'appréciation, tout particulièrement les préférences discursives exprimées (ce que l'on dit apprécier). Communiquer un jugement de valeur discursive revient par définition à "prendre position", dans le sens commun du terme comme dans son sens sociologique. Les déterminants de la convenance y acquièrent donc une importance accrue par rapport à ceux de la pertinence et tendent plus ou moins à s'ajuster à ceux des interlocuteurs. 
Cependant, cette situation ne modifie en rien la nature et les interactions des éléments en jeu : elle n'en implique qu'une repondération en fonction des circonstances (souvent au détriment de l'effet cognitif). En fait, les variations du poids attribué aux différents critères permettent enfin de parcourir tout le spectre allant du contenu le plus «vulgaire» au plus « distingué » sans rencontrer la moindre ligne de démarcation.

On verra plus loin que cette structuration conceptuelle peut tout autant rendre compte des logiques et des stratégies de la production discursive dont elle est un miroir. Ce qui n'a, là non plus, rien d'étonnant puisque la communication est essentiellement déterminée par la réception, ou, pour reprendre la formule bourdieusienne, qu'elle est

un marché sur lequel le locuteur place ses produits et le produit qu'il a produit pour ce marché dépend de l'anticipation qu'il a des prix que vont recevoir ses produits. 


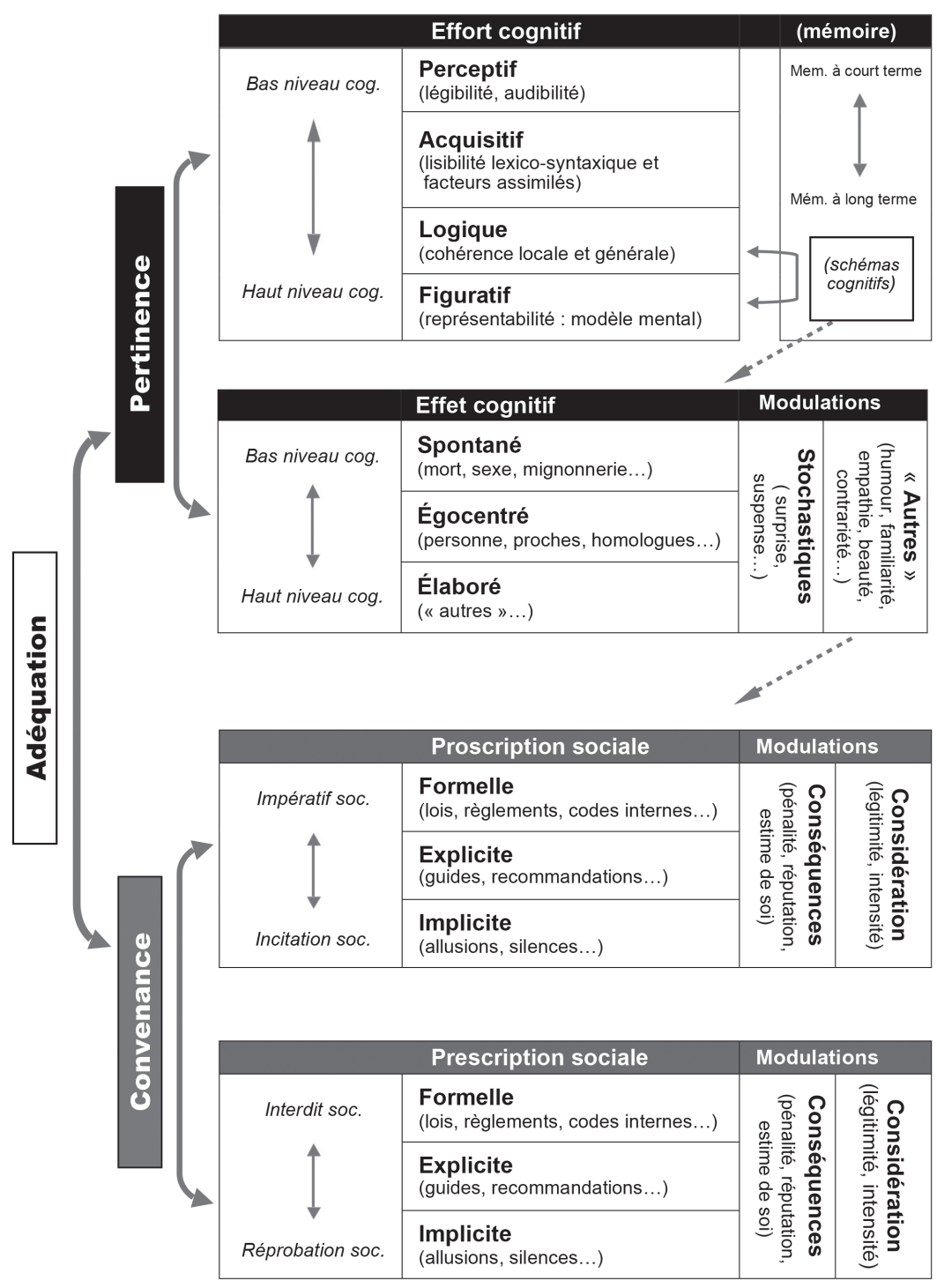

Figure 6. Le modèle général de l'adéquation sociocognitive 


\section{(Précisions)}

Darmi tous les raccourcis imposés par la stricte gestion de la 1 désinvolture qu'exige le vaste problème de la valeur discursive, certains sont plus embarrassants que d'autres. Autant il aurait été impensable de s'étendre sur les fondements de l'éthique (à supposer que j'en sois le moins du monde capable), autant il serait décidément dommage de ne pas évoquer brièvement quelques variables qui peuvent non seulement éclairer les rouages de l'adéquation discursive, mais aussi relativiser et affiner ce que l'on en a dit.

L'une des plus déprimantes, connue depuis Aristote, ne tient qu'à l'apparence physique de l'interlocuteur. Heinich l'évoquait comme l'exemple type d'une valeur "privée »: «valeur intensément utilisée par les gens pour classer et évaluer leurs contemporains (et surtout les femmes), elle ne peut guère toutefois se revendiquer publiquement comme un critère d'évaluation » (2017, p. 210-211). Elle est bien connue dans le cas des recrutements de salariés, pour ne pas parler des sites de rencontres, mais agit également sur l'appréciation des discours. Si illégitime soit-elle, son emprise a été soulignée à de multiples occasions. La plus notoire est le débat qui avait opposé à un Nixon patibulaire un Kennedy jeune et bronzé : ceux qui l'avaient écouté à la radio l'auraient perçu à l'avantage du premier, tandis que ceux qui le regardaient à la télévision l'auraient jugé favorable à son adversaire. Cependant, la qualité des données analysées a fait l'objet de sérieuses critiques. C'est dans des conditions artificielles (expérimentales) que cette variable est la plus facile à isoler. Ainsi, des étudiants $(n=60)$ invités à évaluer une copie assortie d'une photo lui ont attribué une note significativement meilleure lorsque le visage de la photo était plaisant (Landy et Sigall, 1974). D'autres sujets $(\mathrm{n}=432)$, à qui l'on soumettait une fausse publicité utilisant un témoignage de consommateur la jugeaient non seulement plus appréciable, mais aussi plus convaincante lorsque cet utilisateur était attrayant (Patzer, 1983). 
L'apparence peut être perçue comme un signe de conformité aux normes, voire un prédicteur de performance sociale, mais une explication souvent avancée implique par ailleurs une économie d'effort cognitif : l'apparence serait utilisée comme un indice pratique de la valeur du propos, simplifiant ainsi son évaluation. Que l'on y voie une résurgence de l'association platonicienne entre le beau et le bien ou le genre de raccourci commode qui nous fait soupçonner derrière une carrosserie rouillée un moteur à l'agonie, cet effet reste généralement assez modéré par rapport à d'autres. Il peut influer dans une certaine mesure sur la sélection des présentateurs de télévision, mais ne semble pas avoir beaucoup de prise sur le succès des cinéastes ou des romanciers. Le fait, justement, que le débat Nixon-Kennedy ne soit, en fin de compte, pas aussi concluant que ça malgré la nette différence de séduction des deux candidats va dans ce sens.

Tel n'est en revanche pas le cas d'un autre type de variables bien connu, et celui-là dominateur, les convictions dites "personnelles", quoique l'adjectif soit pour le moins discutable. Ces opinions, croyances, etc., sont d'autant plus liées à la réception des discours que, comme on l'avait remarqué, celles-ci et ceux-là peuvent récursivement s'influencer. Comme toujours, cette observation n'est pas d'une grande nouveauté : Francis Bacon, par exemple, soulignait que l'« œil de l'entendement humain n'est point un œil sec, mais au contraire un cil humecté par les passions [...] car plus l'homme souhaite qu'une opinion soit vraie, plus il la croit aisément » $(1620 / 1843, p .17)$. Cet effet a notamment été vérifié par une recherche classique, confrontant 48 personnes, favorables ou défavorables à la peine de mort, à deux argumentations factuelles, l'une favorable, l'autre défavorable à celle-ci (Lord et Lepper, 1979). Elle a permis d'observer que les opinions préalables modifiaient sensiblement le traitement même des discours : les thèses convergentes avec les opinions des lecteurs étaient approuvées sans grande exigence critique alors que la méthodologie de celles qui s'y opposaient était disséquée de façon pointilleuse. En outre, la confrontation à des argumentations contradictoires, loin de distiller le doute dans l'esprit des participants, tendait au contraire à rigidifier leurs positions initiales (polarisation). Une étude non moins classique (Festinger, Rieken et al., 1956), mais menée cette fois sur le terrain auprès d'un groupe apocalyptique avait également observé que l'absence, pour le coup indiscutable, de 
toute fin du monde à la date prévue n'avait pas sapé sa confiance envers sa fondatrice et avait en outre renforcé son prosélytisme.

Un bon demi-siècle de recherches en psychologie sociale n'a cessé de dilater le catalogue fascinant des multiples biais plus ou moins apparentés (de similarité, de conformisme, de confirmation...) qui pèsent sur la perception et le traitement des discours. Pour conjurer à nouveau le risque de se transformer en une improbable encyclopédie des sciences humaines et sociales, on se bornera à remarquer que ces recherches tendent souvent à troubler la limpide géométrie de l'adéquation discursive. Ainsi, l'observation selon laquelle les argumentations adverses bénéficient d'un surcroît de vigilance méthodologique paraît-elle s'opposer à l'idée, également démontrée empiriquement, selon laquelle les publics - notamment les utilisateurs des réseaux sociaux (Bakshy, Messing et al., 2015) et les téléspectateurs de Fox news (Iyengar et Hahn, 2009) - se détournent des discours adverses. Dans le premier cas, les arguments défavorables auraient donc plus de valeur, en termes de temps et d'attention alloués, et dans le second, moins. On pourrait facilement invoquer le spectre artefactuel qui hante les méthodes expérimentales : en situation de laboratoire, les sujets sont souvent obligés de lire le genre de textes qu'ils fuiraient dans la vie réelle, et les lisent de surcroît dans des conditions très différentes de celle-ci. Il serait cependant dommage d'écarter la possibilité qu'un discours contraire puisse être plus intéressant - donc avoir affectivement plus de valeur - qu'un discours convenu (d'autant que, d'un point de vue introspectif, la première catégorie m'intéresse personnellement plus).

De façon plus générale, la majorité des études sur la confrontation entre des éléments incompatibles est liée de près ou de loin à de la théorie de la dissonance cognitive proposée par Festinger en 1957. Selon celle-ci, débattue dans ses détails mais résistante sur l'essentiel, l'incompatibilité entre des "connaissances » (terme à prendre ici au sens large : idées, faits, croyances, valeurs), ou entre une "connaissance " et une action (par exemple une pratique culturelle peu légitime) provoque une tension psychologique suffisamment pénible pour pousser à réduire cette dissonance et retrouver une cohérence interne, que ce soit par une justification $a d$ hoc, par un changement ou par un rejet. Cette dissonance tendrait donc plutôt - en ce qui nous concerne ici - à réduire la valeur des informations non consonantes. D'un autre côté, l'exploitation de la 
dissonance est un des plus solides procédés de valorisation des discours. Bien connue en pédagogie (en particulier en didactique des sciences), la mise en jeu des croyances préalables par des éléments qui les contredisent est également précieuse dans le champ du journalisme, en particulier lorsqu'il porte sur des sujets complexes («Pourquoi ceci n'est pas ce qu'on pense» "Faut-il renoncer à cela [concept, pratique, position] ?», etc.), mais aussi dans les domaines les plus triviaux («La vraie personnalité de [vedette] »). À cet égard, les éléments dissonants paraissent agir comme les éléments choquants évoqués plus haut : une certaine dose donne beaucoup plus de goût aux discours, mais trop les rend impossibles à avaler.

Invoquer tant de recherches pour n'aboutir qu'à une banalité solidement ancrée dans le sens commun (trop de ceci tue ceci, le mieux est l'ennemi du bien, etc.) est en soi un assez piètre exploit conceptuel. Et pourtant, ce genre de considération est indispensable pour bien prendre la mesure des déterminants de la valeur discursive.

1. Ces déterminants sont souvent ambivalents, que ce soit parce que leur intensité peut inverser leur influence (on avait par exemple remarqué que trop d'effet cognitif pouvait devenir insoutenable), ou parce que cette influence s'exerce de façon différentiée, souvent liée au statut autojuridictionnel de chaque discours. Ainsi, l'incohérence, augmentant l'effort cognitif, se manifeste-t-elle essentiellement de façon dépréciative, mais elle peut aussi agir de façon atypique en contribuant à l'effet produit par des discours littéraires (cf. la terre «bleue comme une orange » d'Éluard...), humoristiques (le comique de l'absurde) ou encore de récits fondés sur le suspense (où ni le héros ni le lecteur ne comprennent initialement ce qui se passe). De même, le respect de la langue est avant tout affaire de conformité normative, mais il peut aussi influer sur la compréhension, donc sur l'effort cognitif, et parfois sur l'effet cognitif ${ }^{1}$. Les

1 Dans les cas où «il appartient à la définition légitime du producteur légitime de transgresser ces normes » (Bourdieu, 1977, p. 21). 
attentes liées aux "genres » textuels sont également d'ordre normatif, mais elles conduisent également à des allocations d'effort cognitif différentes selon le type de récit attendu (Zwaan, 1994), ce qui est normal si l'on considère que le "genre » n'est pas une propriété formelle mais bien une inscription autojuridictionnelle. Il existe cependant une nette disproportion entre le sens fondamental dans lequel s'exerce usuellement une variable et l'influence divergente ou même inverse qu'elle peut avoir incidemment, ce qui permet de la considérer en fonction de son mode dominant («normal »), le reste étant surtout affaire de repondération, parfois considérable, dans certaines circonstances.

2. Bien des aspects de l'adéquation sociocognitive pourraient sans nul doute s'enrichir des ressorts apparemment inépuisables qu'exposent empiriquement les recherches de terrain, de corpus ou expérimentales. Ainsi, les travaux sur l'« illusion de profondeur explicative » (Rozenblit et Keil, 2002) aident-ils à relativiser la puissance du désir de savoir évoqué parmi les facteurs d'effet cognitif de haut niveau, en montrant que le niveau de compréhension dont se satisfont les individus est en réalité beaucoup plus faible qu'ils ne le pensent. Ce n'est nullement un détail si l'on considère, comme on le verra plus loin, que cet aspect peut contribuer à éclairer la propension à l'extrémisme idéologique (Fernbach, Rogers et al., 2013), mais, comme on le suggérera aussi, le jeu de la pertinence cognitive et des contraintes sociales permettrait, dans l'autre sens, de mieux expliquer ces résultats expérimentaux. De même, la prévalence de l'«ignorance pluralistique» (Van Boven, 2000), biais conduisant à surévaluer l'approbation d'une norme par les autres membres d'un groupe, et donc à la perpétuer bien au-delà de l'adhésion dont elle bénéfice réellement, permettrait de nuancer le poids que l'on a attribué aux normes sociales dans l'appréciation individuelle d'un discours, ou, du moins, de préciser 
les modulations évoquées à leur propos. De façon générale, toutes les recherches exposant un «biais » ou quelque chose d'apparenté informent à un titre ou un autre sur l'appréciation de la valeur discursive. Il n'est cependant pas certain qu'un recours qui se voudrait systématique à tous ces résultats (appelons ça l'illusion encyclopédique) ne perdrait pas, en termes de complexité, plus que ce qu'il gagnerait en termes de précision. L'alternative, heureusement, n'est pas binaire : dès lors que de telles données ne paraissent pas incompatibles avec le schéma d'ensemble de la valeur discursive (ce qui imposerait évidemment d'en tenir compte plus attentivement), rien n'empêche de gagner en densité conceptuelle avec elles dans les situations où ceci serait analytiquement nécessaire et de se soulager de ces précisions lorsque la gamme de phénomènes analysés ne les justifie pas.

3. Plus généralement, il est très significatif que la plupart de ces éléments jalonnent l'une des frontières conceptuelles les plus problématiques qui soient, celle où se recouvrent les explications personnelles et collectives des attitudes. L'intitulé même du champ qui s'y intéresse, la psychologie sociale ${ }^{2}$, témoigne de cette dichotomie. Et du projet de la surmonter, bien sûr, mais seulement en fonction de l'approche et du niveau qui lui sont propres, dont on ne saurait penser que le plus grand bien (on vient de voir quelques-uns de ses apports considérables), mais qui lui interdisent en général de prendre en compte les phénomènes de plus grande et de plus petite échelle. Ainsi, le manuel francophone de référence traitant de la «psychologie de la communication " sous l'angle de la psychologie

2 Science "charnière ", comme la définit Maisonneuve, "son émergence et son développement croissant proviennent de l'incapacité de la seule sociologie ou de la seule psychologie à rendre compte de l'intégralité des conduites humaines concrètes » $(1989$, p. 8). On notera que cet auteur écarte à bon escient le distinguo que certains praticiens ont tenté d'établir entre «psychologie sociale » et «psychosociologie», désignations qui sont aussi employées indifféremment dans ce livre. 
sociale (Abric, 2011) peut-il ignorer simultanément les fondements de la psycholinguistique cognitive et la sociologie des pratiques ou industries culturelles : il n'y a bien sûr aucun jugement de valeur dans ce constat (à l'exception de celui qu'appellerait le titre abusif de l'ouvrage), l'auteur lui-même réduisant explicitement sa conception de la « communication» à l'étude empirique des interactions dans les groupes restreints. Malheureusement, la difficulté évidente qu'il y aurait à l'heure actuelle à superposer la psychologie sociale et l'approche psychologique et sociale (donc elle aussi psychosociologique) de l'adéquation discursive que l'on a esquissée ici pose plus de problèmes à la seconde qu'à la première. Si l'une est solidement campée au confluent de l'individuel et du social, l'autre est contrainte d'enjamber celui-ci. D'où le flou que l'on a préservé quant au périmètre des facteurs d'effet de haut niveau et, par exemple, l'incertitude évoquée (et préservée) sur le caractère "psychologique» ou "sociologique» d'un facteur comme la compassion.

4. Ces catégories abstraites étant poreuses, le situer ici ou là n'a pas beaucoup de conséquences sur les logiques d'appréciation de la valeur discursive : que l'on pivote un peu cette façon de voir et quelques éléments pourront aussitôt paraître changer de camp sans déstabiliser l'ensemble. Mais il n'en reste pas moins que les apports de la psychologie sociale soulignent utilement le caractère symbolique d'une telle délimitation, tout en confirmant de facto sa nécessité heuristique dès lors que les réalités des choix sur le marché discursif imposent de prendre en compte l'amplitude de variables d'échelles très différentes. Il est du reste frappant de remarquer à cet égard que certains travaux qui visent à synthétiser les diverses motivations des décisions individuelles, notamment ceux de Tversky et Kahneman (voir Kahneman, 2011 pour une présentation générale), s'ils puisent explicitement dans la longue tradition des explications 
hédoniques (y compris la séculaire « Loi du moindre effort $»)$, peinent à rapprocher vraiment celles-ci de la complexité des déterminants sociaux qui jouent sur ces mêmes décisions. En revanche, le fort classique ${ }^{3}$ modèle des «deux systèmes » de pensée développé et popularisé dans ces travaux (un mode de réflexion heuristique, rapide et économique, complété au besoin par un mode plus approfondi, mais mentalement plus coûteux) éclaire non seulement l'importance que revêt l'effort cognitif, mais aussi la distinction entre les facteurs d'effet de bas et de haut niveau cognitif (spontanés ou construits) jouant notamment sur le rejet ou la consommation d'œuvres « exigeantes».

3 "La distinction entre deux modes de pensée, l'un rapide et intuitif, l'autre lent et réflexif, est à la fois ancien dans ses origines et largement répandu dans la littérature philosophique et psychologique. Une telle distinction a été faite par de nombreux auteurs dans de nombreux domaines, souvent dans l'ignorance de ce que d'autres avaient écrit à ce propos» (Evans et Stanovich, 2013, p. 223). 


\section{Sur les étals du marché discursif}

Le processus éducatif n'est pas d'abord une affaire d'accès au savoir, mais bien plutôt une manière de poser la question, fondamentale, du désir de savoir.

Philippe Breton (1997, p. 145)

"P ourquoi les discours qui nous plaisent nous plaisent-ils?». Posée en termes aussi généraux, la question n’a pas vraiment de sens. Non seulement parce que dire, ou même se dire, que l'on adore le théâtre de Becket tout en regardant plutôt une émission de variétés à la télévision est un peu inconfortable, mais aussi parce que beaucoup de ce qui « nous plaît » nous est caché. Comme le remarquait François de Closets (1980), les millions de Français qui se délectaient des chansons de Tino Rossi ou de Luis Mariano ne pouvaient présager qu'ils aimeraient aussi celles, très différentes, de Jacques Brel ou de Georges Brassens (d'où la déficience prédictive bien connue des études de marché dans le domaine des médias et de la culture). Et surtout parce qu'il existe une multitude de raisons d'apprécier des discours, lesquels ne sont pas moins multiples dans leur nature et dans leur façon particulière de s'offrir au jugement : j'hésite à reprocher à Flaubert de ne pas beaucoup me faire rire, mais j'aurais été intransigeant sur ce point vis-à-vis de Groucho Marx. Le déterminisme social dont Asimov a tiré la trame de la saga Fondation est scientifiquement consternant, mais ça n'empêche pas ce cycle romanesque d'être captivant.

Tout devient plus clair lorsque l'on reformule la question dans le contexte de ce marché discursif pléthorique au sein duquel Flaubert, Groucho Marx et Asimov sont bel et bien en concurrence : "Pourquoi préférons-nous les discours auxquels nous accordons notre préférence? » Sous cet angle ne s'affrontent pas seulement toutes les offres discursives accessibles, mais aussi l'ensemble des raisons de les apprécier ou 
non, dont chacune est en lice à tout moment, parfois dominante, parfois écrasée, mais toujours présente (ainsi le déterminisme d'Asimov reste-t-il tout de même un petit peu gênant). Il est dès lors possible d'avancer une réponse d'ensemble :

Nous préférons ce qui nous semble le plus pertinent-c'est-àdire ce qui nous offre le plus d'effet pour l'effort demandé-en regard des contraintes sociales que nous intégrons.

Est-ce donc si simple? Oui et non.

Soulignons d'abord à nouveau que l'on n'a rien « démontré » du tout. Si l'on entend par là développer une argumentation contraignante (c'est-à-dire fondée sur la force de la preuve), on n'a même pas essayé de le faire. Et encore moins de paraître le faire. Par nature, l'échelle considérée ne pouvait permettre d'avancer qu'une proposition, une forme de compréhension du réel, ce qui, du reste, est à un degré ou un autre le sort de toutes les hypothèses ayant cours en sciences humaines et sociales (y compris les plus péremptoires) dès lors qu'elles ne se cantonnent pas à un objet très restreint. À dire vrai, même les postulats se limitant à l'interprétation de quelques données expérimentales n'y échappent pas ${ }^{1}$.

Au minimum, cette proposition livre un outil analytique, l'adéquation sociocognitive, qui, comme toute abstraction instrumentale $-\mathrm{y}$ compris les indices quantitatifs comme le PIB ou le QI - est très exactement et indiscutablement égale à ce qui la définit. Cependant, on pourrait tout aussi rigoureusement définir, par exemple, la cyanophobie comme étant la peur de la couleur bleue sans que ceci établisse sa validité empirique (traduit-elle un phénomène significatif?), sa

I On connaît assez les problèmes de puissance statistique (la représentativité des petits échantillons) et de validité écologique (le rapport entre les conditions de laboratoire et celles de la vie quotidienne) que doivent affronter les méthodes expérimentales, ainsi que, plus généralement, leurs limites inductives et la «crise de reproductibilité » qu'elles rencontrent pour ne pas s'attarder sur ces points. Du reste - faut-il le préciser ? - ceux-ci ne les invalident pas pour autant : le fait que la psychologie expérimentale se soit saisie avec vigueur et au grand jour des difficultés de reproduction des résultats (voir notamment Weir, 2015 et Bohannon, 2015) témoigne au contraire en sa faveur. 
cohérence théorique (les notions de peur et de bleu sont-elles fondées ${ }^{2}$ et appropriées ?) et plus généralement son utilité conceptuelle. Ces trois aspects mériteront donc un examen attentif.

Plus ambitieusement, mais toujours par analogie (donc pour clarifier et non pour démontrer), le modèle proposé peut être considéré comme une carte. À l'instar de toute synthèse cartographique depuis l'Antiquité, ce schéma est étroitement dépendant de l'état des connaissances disponibles : il comporte son lot de zones incertaines ou un peu distordues et de pondérations entre des revendications de frontières hétérogènes. Il a en outre supposé de négliger la précision superflue des cadastres locaux, mais aussi d'écarter (souvent sans même les mentionner) un certain nombre de figurations désuètes, fantaisistes ou mystiques. Son principal mérite est d'exister et de permettre ainsi d'embrasser d'un coup d'œil des régions différentes, et surtout d'interdire de les ignorer. Tant mieux si, au fil du temps, le dessin devait être précisé, rectifié ou complété : l'important est que les grandes masses soient bien là. Peu importe aussi que les besoins conceptuels de tel ou tel fief n'impliquent pas de s'inquiéter de régions lointaines : elles sont là tout de même, et leur influence peut s'exercer de façon localement insoupçonnée.

L'analogie avec une représentation cartographique est moins superficielle qu'il n'y paraît. Non seulement parce qu'une carte et une figuration théorique sont des outils cognitifs fonctionnellement équivalents (voir ci-dessus le rôle des schémas et des modèles mentaux), mais aussi parce qu'elles peuvent partager une même propriété : la fractalité. Benoît Mandelbrot (1995) remarquait que la longueur d'une côte rocheuse dépendait de l'échelle à laquelle on la considérait : plus l'échelle était petite et plus son tracé, lissé à grande échelle, devrait s'allonger pour tenir compte d'accidents de terrain de plus en plus petits, jusqu'au contour de chaque rocher puis, zoomant toujours, à la granularité du moindre caillou. Cet état de choses, contre lequel on n'a cessé de maugréer au fil des pages, s'avère une bénédiction au terme de ce parcours. En effet, le modèle qui en résulte tend de ce fait

2 Comme le signalait un linguiste, le « bleu » est une catégorie arbitraire du spectre lumineux, qui ne renvoie pas à un universel, même pour les Occidentaux : "en breton et en gallois un seul mot glas s'applique à une portion $d u$ spectre qui recouvre à peu près les zones françaises $d u$ bleu et du vert » (Martinet, 1967, p. 12). 
à s'adapter spontanément au niveau de précision dont on a besoin. Pour un praticien, par exemple un journaliste confronté à un choix discursif, percevoir au minimum la tension qu'exercent deux variables générales, la pertinence cognitive et les contraintes sociales, pourrait souvent suffire à mieux interpréter les arbitrages qu'il doit opérer. À l'inverse, des problématiques scientifiques ciblées impliqueraient une granularité locale beaucoup plus fine (on avait, par exemple, remarqué que ce que l'on mentionnait comme l'établissement de la cohérence lissait des nuances telles que la cohésion et la connexité), mais cette précision locale s'accommodera fort bien d'une perception plus vague (lissée) du reste des facteurs³.

Ainsi s'affranchit-on de l'alternative traditionnelle entre tout considérer avec la même attention - ce qui serait conceptuellement vain, en plus d'être disciplinairement suicidaire - et ignorer tout facteur non dicté par l'approche choisie, ce qui constitue une source inépuisable de fourvoiement pour des recherches fières de leur méthodologie, mais étonnamment vulnérables aux variables qu'elles ne songent pas à contrôler (les exemples ne manquent pas dans les pages qui précèdent). On pourrait même se demander si la rigidité que quelques écoles brandissent comme de la rigueur n'est pas une façon de conjurer cet extérieur mystérieux et confusément menaçant. Quoi qu'il en soit, une cartographie générale de la valeur discursive ne peut être empiriquement solide qu'en étant souple. Ce qui pose un problème d'une autre nature. Comme le signale en particulier Popper, une théorie qui serait assez souple pour s'adapter à tout et n'importe quoi s'annihilerait du même coup : pouvant toujours se contorsionner pour échapper à la contradiction, elle serait au bout du compte incapable d'affirmer scientifiquement quoi que ce soit. On reviendra sur cette question un peu aride (et qui promet d'être longue) dans les «Précisions » de ce chapitre, mais il faut remarquer qu'en ce qui concerne l'adéquation sociocognitive, cette souplesse est en réalité relative et surtout très variable : loin de relever d'une pusillanimité rhétorique ou diplomatique, elle calque fidèlement la « fractalité » de son objet.

3 Si certains systèmes théoriques semblent exiger une dévotion pointilleuse et exclusive à une œuvre fondatrice, comme le remarquait prophétiquement Bourdieu (mais à propos de la postérité de Marx, pas de la sienne), la façon de voir défendue ici est assez éloignée du type « tout ou rien ». 
L'approche sociocognitive de la valeur discursive, en effet, ne produit pas un bloc doctrinal uniforme, mais constitue un système cohérent d'assertions hiérarchiquement décroissantes, tel que chaque articulation est plus importante que ce qu'elle articule.

- L'hypothèse de premier niveau ${ }^{4}$ est que la préférence qui conduit à la sélection d'une offre sur le marché discursif peut s'analyser comme le rapport entre la satisfaction hédonique que cette offre propose (sa pertinence cognitive) et les contraintes normatives qui s'exercent sur son appréciation (sa convenance sociale). C'est la thèse fondamentale de ce livre. On pourrait la juger évidente : le fait qu'elle soit loin d'être reconnue comme telle par ceux qui ont traité jusqu'à présent de l'appréciation des discours montre qu'elle ne l'était pas. Les éléments que l'on a examinés ici montrent que c'est bien dommage. Quant à la petite zone de flottement qu'elle souligne à la frontière des déterminants psychologiques et sociaux, elle rappelle utilement que ces deux regroupements sont des nécessités conceptuelles et non des entités naturelles qui s'excluraient mutuellement (Durkheim disait qu'il fallait étudier les faits sociaux « comme des choses »; il n'a jamais conseillé de croire que c'était des choses).

- Les hypothèses de second niveau sont, d'une part, que la pertinence cognitive peut s'analyser comme le rapport entre l'effort et l'effet cognitif, d'autre part que l'emprise sociale peut - en ce qui concerne la convenance - s'analyser comme le rapport entre les contraintes appréciatives et dépréciatives ressenties. La réfutation, très improbable, de l'un ou l'autre de ces axiomes robustes ne détruirait pas la thèse fondamentale, mais lui ôterait une partie de sa fonctionnalité à moins de sérieux travaux de rénovation.

4 On pourrait sans doute énoncer une hypothèse de niveau zéro selon laquelle la probabilité de consommation effective d'un discours peut s'analyser comme le rapport entre la préférence dont il bénéficie (son adéquation sociocognitive) et les contingences extradiscursives qui la contrebalancent, telles que sa disponibilité et l'investissement concret qu'il requiert (temps, argent, etc.) mais ce n'est pas un aspect que l'on développera ici. 
- Les hypothèses de troisième rang, encore un peu plus souples, structurent comme on l'a vu les principaux facteurs contribuant respectivement à l'effort cognitif, à l'effet cognitif, à l'appréciation sociale et à la dépréciation sociale. En l'état, ces quatre articulations rendent apparemment très bien compte de leurs incidences empiriques, mais une contre-proposition visant l'une ou l'autre d'entre elles ne pourrait sans doute qu'enrichir l'ensembles. En particulier en ce qui concerne la prise en compte un peu fruste des inextricables déterminants sociaux.

- Enfin, chacun des facteurs mentionnés peut en soi être l'objet d'approfondissements considérables, et beaucoup d'entre eux le sont isolément, quoique souvent confusément : on signalait par exemple que la seule question de la lisibilité lexico-syntaxique avait été l'objet de plusieurs centaines de publications scientifiques, mais qu'une question comme celle-ci était pourtant loin d'être close. Une perspective d'ensemble pourrait justement aider à débrouiller ce genre de problèmes et, en retour, s'alimenter de leur avancement.

On aura à peu près fait le bilan de ce qui a effectivement été soutenu ici lorsqu'on aura en outre précisé ou rappelé trois corollaires.

- D'une part, contrairement à une carte classique ou à une nomenclature figée, cette schématisation est avant tout dynamique : elle vise moins à cataloguer des objets, ou même à leur affecter une position spatiale ou hiérarchique intangible, qu'à rendre visible le jeu global de leurs actions et interactions dans l'établissement de la valeur discursive.

- De ce fait, elle n'est pas seulement de plus en plus souple au fur et à mesure que l'on s'éloigne de son tronc vers le bout de ses branches. Elle est aussi moins rigide dans les rapports entre ses composants que ce qu'il est possible de représenter de façon raisonnablement claire. Les

5 Toute théorie étant par essence une contribution open source offerte au développement collaboratif. 
positions figurées sont les plus significatives (dominantes) et globalement les plus explicatives, mais elles n'excluent pas que dans tel ou tel cas des facteurs puissent court-circuiter ces rattachements dominants. On l'avait par exemple remarqué à propos de l'humour noir (dont l'effet cognitif est renforcé par la violation des convenances) ou de la vérité, dont les deux antonymes, erreur et mensonge, reflètent assez bien l'ambivalence utilitaire et normative ${ }^{6}$. Il serait possible de figurer tout cela en adoptant une perspective tridimensionnelle, ou simplement en multipliant les flèches de ce modèle, mais ça ne le rendrait qu'absurdement et inutilement compliqué. L'important résidant dans les types de déterminants et les interactions qu'il conduit à prendre en compte, il est facile de les moduler en fonction des situations considérées et du niveau de granularité requis.

- Enfin, comme on l'avait souligné dès le début de cette quête et rappelé de temps à autre, ce que l'on avance ici est une «façon de voir ». Une construction très résolue, tout à fait confiante dans sa capacité à mieux expliquer une très large gamme de phénomènes, mais pas au point de se prendre pour la réalité de ces phénomènes, ni même pour la seule façon utile de les considérer. D'abord parce que les méta-théories ne sont jamais des superthéories. Cette synthèse composite n'est pas plus « supérieure » aux divers apports scientifiques qu'elle relie dans une perspective élargie que la biologie n'est « supérieure » à la chimie ou que la chimie ne l'est à la physique (il n'y a pas de prééminence scientifique entre les cellules, les molécules et les atomes, ni entre ceux qui les étudient). D'autant que cette intégration n'est pas seulement moins précise que ses constituants : elle est aussi beaucoup plus focalisée : on ne s'intéresse pas ici à la cognition en général ou à la société en général, mais seulement à leurs effets sur l'appréciation discursive (ce que l'on décide de connaître ou d'ignorer n'est donc pas

6 Une assertion fausse est dépourvue d'effet positif pour la sphère personnelle (pertinence) mais elle peut aussi être moralement condamnable (convenance). 
moins strict, mais différent). Ensuite parce que son intérêt opératoire est évidemment variable.

Quoique l'approche proposée soit a priori applicable à n'importe quelle production discursive, sa contribution à l'analyse des discours non figuratifs est assez réduite7. Même si l'on s'en tient au cas des textes, ce qu'elle peut apporter à l'examen d'une lettre d'adieu ou d'un menu de restaurant ${ }^{8}$ est relativement modeste par rapport à d'autres considérations. Enfin et surtout parce qu'elle ne vise pas à saisir dans toute leur profondeur certaines des dimensions de la production et de la réception discursives (idéologiques, esthétiques...). On verra plus loin qu'elle peut leur apporter divers éclairages, mais aucun cadre théorique ne pourrait sérieusement se prétendre "suffisant " sans l'être au sens péjoratif du terme. Même la bonne vieille tripartition ethoslogos-pathos de la rhétorique antique peut s'avérer plus efficace pour appréhender certaines faces du débat public (quoiqu'elle puisse se combiner à merveille avec une approche sociocognitive). C'est bien pourquoi les allégations hiérarchiquement décroissantes exposées un peu plus haut soutenaient que chaque niveau pouvait s'analyser de la façon proposée, et non qu'il le devait.

Une telle nuance est heureusement moins rare dans la littérature scientifique que l'on ne pourrait le penser, et sa justification est généralement la même. On peut donc emprunter sans vergogne celle qu'un autre, en l'occurrence un linguiste, a pris la peine de formuler (et que Barthes avait déjà repris en connaisseur) :

Entre parenthèses - mais c'est important - je viens de dire « on jugerait ${ }^{9}$ commode d'opérer » avec cinq unités » et non pas « on doit». Il faut insister ici sur le fait que les descriptions linguistiques ne sont pour ainsi dire jamais monovalentes. Une description n'est pas « exacte » ou «fausse » en soi (elle peut être fausse, bien entendu, si elle n'est pas conforme aux faits!) : elle est

7 Mais pas à celle des conditions de leur réception, et encore moins à celle des débats qu'ils suscitent souvent.

8 Mais pas à celui des modes d'emploi, type de discours qui constitue au contraire l'une des meilleures illustrations possibles des difficultés de l'adéquation et de ses logiques en action.

9 La version française de cette publication comportait une coquille non reproduite ici («on le [sic] jugerait commode... »). 
meilleure ou pire, plus ou moins utile. [...] On cherche toujours la description la plus simple à condition qu'elle convienne aux faits observés. (Halliday, 1962, p. 12)

Il est significatif que son propos s'inscrive ouvertement dans une perspective impure, celle d'une connaissance "appliquée » et qui plus est - comme le souligne son titre ${ }^{10}$ - appliquée à l'enseignement. Sans égard pour la tradition très Ancien Régime d'une aristocratie de la pensée où des concepts éthérés ne vivraient que pour eux-mêmes et pour leurs clercs, le critère d'utilité qu'Halliday met en avant suggère à point nommé qu'une théorie ne vaut réellement que par le travail qu'elle est capable de fournir : ce que l'on peut faire avec (ou voir avec, comprendre avec...) Or, son utilité pédagogique, bien qu'elle n'en soit pas la condition, en est souvent un excellent révélateur. D’abord parce qu'enseigner sans mystifier met à nu toutes les carences et nébulosités que pourraient couvrir un manteau de jargon et une posture assurée. Ensuite, en particulier dans les nombreuses branches des sciences humaines consacrées à des activités professionnelles, en raison de l'écart inconfortable séparant « la théorie », qui est ce qui intéresse les profs, et « la pratique » qui est ce qui intéresse les étudiants, futurs profs mis à part (on pourra facilement y reconnaître une tension entre la légitimité et la pertinence du discours de l'enseignement supérieur). Dans le champ de la communication et des médias, cet écart rituellement dénoncé par les étudiants et employeurs, mais remarquablement peu débattu dans la littérature savante francophone (si ce n'est de temps à autre pour le réfuter ${ }^{11}$ ) est justement l'un des questionnements qui m'avaient à l'origine conduit à m'intéresser à la (non)structuration et à la portée opératoire des multiples interprétations disponibles sur le marché des idées. L'autre raison, plus noble, plus théorique, était une certaine insatisfaction face à la capacité explicative et prédictive globale de ce kaléidoscope. Mais l'une et l'autre de ces questions reviennent à celle de l'utilité :

La chose la plus fondamentale que l'on puisse sans doute dire de la mémoire humaine, au terme d'un siècle de recherches intensives,

10 «Linguistique générale et linguistique appliquée à l'enseignement des langues ».

11 Ce problème épistémologique et didactique complexe mériterait plus qu'une affirmation péremptoire, mais on pourra en trouver des analyses plus détaillées dans Labasse 2012 b et 2014 b. 
est qu'à moins qu'un détail ne soit placé dans un schéma structuré, il est rapidement oublié [...] Une bonne théorie n'est pas seulement le moyen de comprendre un phénomène sur le moment, mais aussi celui de s'en souvenir plus tard. (Bruner, 1960, p. 24-25)

Le lien entre l'utilité pédagogique et l'utilité scientifique est d'ailleurs plus profond qu'il n'y paraît, si l'on estime avec Kuhn que la maturité d'un domaine scientifique peut se reconnaître à la réduction de la «confusion bourdonnante et foisonnante ${ }^{12}$ » que tend à entretenir le «fatras » des observations et des hypothèses non intégrées.

L'intégration sociocognitive étant ainsi promise dès le départ à une vie de labeur, il n'est pas inutile d'examiner brièvement des exemples de sa «mise au travail » dans quelques cours de premier et de second cycle au Canada et en France (que l'on ne s'étonne pas d'y retrouver bien des points déjà évoqués dans les pages qui précèdent ou qui le seront plus loin). Malgré leur diversité croissante, ces cours-là avaient en commun d'être impurs, c'est-à-dire de viser la compréhension des logiques d'un domaine discursif en vue de permettre à la fois aux étudiants d'en acquérir une connaissance analytique et critique, et de développer une expertise discursive dans le domaine concerné.

Ainsi, dans un cours consacré à la vulgarisation scientifique, la notion d'adéquation a-t-elle notamment été appliquée à l'examen des recherches consacrées aux rapports entre scientifiques et vulgarisateurs, en s'arrêtant en particulier sur la prépondérance des débats visant la «sursimplification » et le «sensationnalisme», respectivement analysables comme un allègement de l'effort cognitif et un renforcement de l'effet cognitif (jugés l'un comme l'autre inadéquats en regard du système normatif des scientifiques). Elle a également aidé à observer certaines des tensions qui jalonnent l'histoire de la divulgation scientifique (on sait par exemple que les ennuis qu'a connus Galilée venaient moins de sa défense de la thèse copernicienne que de la pertinence cognitive qu'il lui avait conférée). Appliqué à la production discursive, le même cadre notionnel a guidé l'exploration des stratégies d'optimisation de l'adéquation discursive des sujets complexes, notamment en approfondissant le rôle de l'imagerie mentale dans la compréhension et en étudiant et expérimentant la vaste palette des

12 Kuhn transpose (1983, p. 160) à la connaissance scientifique la célèbre formule par laquelle William James résumait la perception d'un nouveau né. 
facteurs d'effet de haut niveau (problématisation, paradoxes...), tout en soupesant, bien sûr, les transgressions normatives qu'ils impliquaient par rapport au discours savant légitime.

Dans un cours portant sur la communication, ce référentiel a, toujours par exemple, aidé à situer les stratégies publicitaires de captation de l'attention en fonction des facteurs d'effet cognitif sollicités (primaires, utilitaires, surprenants, intrigants...) et aux contraintes, tantôt bravées (depuis la timide provocation jusqu'au "shockvertising » assumé) tantôt observées (euphémisation de promesses non légitimes). Mais il a aussi permis de comprendre les ambiguités et non-dits de la négociation du mandat entre commanditaires et concepteurs-rédacteurs, ou, dans une perspective purement technique, d'évaluer la portée réelle et les limites des formules de lisibilité. Il a encore été appliqué à l'examen des formes de l'injonction au sein des organisations ( « je veux que... » étant un énoncé plus pertinent que « je souhaiterais que... » tout en étant généralement moins adéquat) ou, à propos des controverses publiques, par exemple sur les risques sanitaires, à une réflexion sur les conditions d'une argumentation fondée en raison, laquelle réclamant plus de ressources cognitives, suppose de ce fait (à contraintes constantes) de veiller à ménager assez d'effet cognitif pour être entendu.

Dans un cours consacré à la critique culturelle, l'opposition entre succès commercial et succès légitime a pu être abordée comme l'expression d'un écart entre la pertinence cognitive d'une œuvre littéraire ou cinématographique (reposant généralement sur un effort cognitif réduit et des facteurs d'effet robustes, comme la violence ou l'érotisme, mais aussi l'humour) et les contraintes qui déterminent le jugement esthétique cultivé, lesquelles bannissent aussi bien l'effet immédiat que l'absence d'effort :

On pourrait montrer que tout le langage de l'esthétique est enfermé dans un refus principiel du facile, entendu dans tous les sens que l'éthique et l'esthétique bourgeoises donnent à ce mot; que le "goût pur», purement négatif dans son essence, a pour principe le dégoût $[\ldots]$ pour tout ce qui est «facile». (Bourdieu, 1979, p. 556)

Plus généralement, le concept d'adéquation a contribué à faire mieux percevoir le caractère autojuridictionnel des œuvres et les options 
critiques à son égard (situer ou non le jugement dans le cadre revendiqué), mais aussi à structurer à la fois des démarches introspectives ("Pourquoi les livres que j'ai aimés m'ont-il plu?»), évaluatives ("Que dire de cette cuvre? ?) et discursives ("Comment y intéresser des lecteurs?»).

Dans un cours consacré à l'histoire de l'édition et aux mutations des pratiques éditoriales, cette façon de voir a aidé à échapper à une chronologie purement techniciste des transformations successives du codex à l'imprimé, puis au numérique, en l'accompagnant d'une réflexion sur l'aménagement cognitif de la lecture que reflètent les évolutions de la typographie et de la mise en page depuis l'Antiquité, mais aussi sur la transformation quantitative et qualitative des modalités de sélection et de consommation de l'offre discursive au fil des siècles.

Dans plusieurs cours de journalisme, enfin, la même notion a notamment permis d'éclairer les processus de sélection de l'information (newsworthiness) ou d'autocensure que mettent en œuvre les professionnels, mais aussi de réexaminer sous un angle technique les préceptes et les moules canoniques de l'écriture de presse, dont la pertinence cognitive est souvent désastreuse (voir plus loin dans ce livre "Le mystère de la pyramide inversée »), et d'expérimenter de façon réfléchie diverses formes de traitement d'une même actualité. Elle a tout aussi concrètement aidé à remarquer les déterminants que reflètent les thèmes de couverture des magazines d'actualité, par exemple celles qui font appel aux facteurs d'effet de second niveau (l'interaction avec la sphère personnelle) comme " le salaire des cadres » ou « les prix de l'immobilier ». Et, bien sûr, elle a aidé à approfondir l'opposition machinale entre la dimension civique du journalisme ( l'intérêt public») et sa dimension marchande ("l'intérêt du public») en montrant les limites de cette dichotomie dans l'absolu et plus encore dans un contexte d'âpre concurrence attentionnelle.

On exagérerait cependant beaucoup en laissant penser que le modèle de l'adéquation sociocognitive - proposé comme une structuration analytique et non une vérité première - constituait l'essentiel de tels cours, qui associaient un bon nombre d'autres aspects techniques, créatifs, conceptuels et historiques. D'autant qu'il y était souvent accompagné de propositions théoriques concurrentes ou complémentaires (pas moins de quatre dans le cas de la critique culturelle) et qu'il était replié à ses articulations de premier et second 
niveau chaque fois que les thèmes abordés ne requéraient pas une granularité plus fine. Paradoxalement pourtant, il s'est avéré que les cours comportant une certaine dimension appliquée - les seuls évoqués ici - pouvaient réclamer plus de précision locale que des séminaires traitant de questions réputées plus élevées, mais de ce fait moins sensibles aux détails.

Quoi qu'il en soit, si, comme le suggéraient Halliday et bien d'autres («Rien n'est plus pratique qu'une bonne théorie» disait aussi Kurt Lewin, 1951), on peut juger une perspective théorique en la regardant travailler, y compris dans une salle de cours, celle-ci ne rechigne pas à la tâche. Évidemment, ses mains calleuses la condamnent si l'on est à l'inverse persuadé qu'une théorie assez simplifiable pour être claire en seconde ou troisième année n'est pas digne de ce nom ${ }^{13}$, surtout si elle a le mauvais goût d'avoir des implications pratiques, et $a$ fortiori de les revendiquer.

Du reste, sa propension à relier théorie(s) et pratique(s) n'est pas sa seule inconvenance. J'ai longtemps été fasciné et naïvement fier de constater que, contrairement aux usages admis, il était bel et bien possible d'envisager sous le même angle théorique des phénomènes de très petite échelle comme la sélection lexicale (pourquoi un écolier choisit-il d'écrire «monture » ou «cheval » ?) ou le jugement institutionnel (qu'est-ce qu'un style «trop journalistique»?) et de grande échelle, comme la stratégie d'une chaîne de télévision, voire de très grande échelle, comme la diffusion publique des connaissances complexes. Mais cette autosatisfaction était assez mal placée : en y réfléchissant mieux, le caractère multiscalaire de cette façon de voir ne devait rien à la perspicacité de son auteur et tout à son objet. Ce n'est pas le modèle qui est multiscalaire, c'est la communication ellemême. À moins de croire que la rédaction scolaire, la programmation télévisée ou la diffusion sociale des connaissances (ou encore la création culturelle, l'information journalistique...) ne sont pas des processus communicationnels, c'est-à-dire des productions discursives

13 Le «refus principiel du facile » que Bourdieu associait un peu plus haut à l'esthétique bourgeoise ne se limite pas au domaine de la consommation culturelle, pas plus que les logiques de distinction en général. Cependant, il y a également là des motivations épistémologiques plus défendables (il y a aussi, du reste, des arguments musicologiques tout à fait consistants pour préférer la musique classique à la variété). 
orientées par l'anticipation de leur réception ${ }^{14}$, il est normal et même banal de noter qu'ils relèvent de ce point de vue $e^{15}$ de logiques similaires. Ce qui revient à dire que la perspective multiscalaire de l'adéquation sociodiscursive est moins une conceptualisation linguistique, sociologique ou psychologique, qu'elle n'est, par essence, une théorie communicationnelle.

14 Ce n'est pas la seule définition possible, mais on peut soutenir que c'est la meilleure (voir Watzlawick pour un exemple - très curieux - de conception alternative).

15 On ne dit pas que l'éducation, la littérature ou le journalisme sont seulement des processus communicationnels, on affirme qu'ils le sont aussi. 


\section{(Précisions)}

L

e meilleur argument en faveur d'une approche sociocognitive de la valeur discursive est sa capacité globale à intégrer des déterminants traditionnellement séparés, mais susceptibles, une fois reliés, d'éclairer des phénomènes très variés dans leur nature, leur échelle et leur manifestation empirique. En d'autres termes, sa capacité à avancer une réponse heuristiquement satisfaisante à la question posée en exergue.

Cependant, l'analogie qui suivait presque immédiatement cette question initiale marquait la différence possible entre une réponse heuristiquement satisfaisante et une réponse scientifiquement fondée : on peut rassembler des éléments empiriques solides montrant qu'un dauphin est un poisson (son habitat, ses nageoires...) sans que cette réponse, acceptable en apparence, voire souvent en pratique, soit épistémologiquement valide. Bien sûr, il ne s'agit pas, à l'inverse, de tomber dans la naïveté scientiste selon laquelle le travail de la recherche consisterait à produire des relations d'identité entre ses objets et le réel (ceci est ainsi) plutôt que des relations de concordance (ceci s'interprète mieux ainsi). On a, en outre, assez discuté des raisons qui justifiaient chaque étape de cette intégration et des précautions et restrictions que requérait chacune d'elles pour pouvoir affirmer celle-ci possible, plausible et pratique. Mais à quel degré de validité épistémologique peut-elle vraiment prétendre dans l'absolu (s'il y a un absolu en la matière) ?

(Cette courte question appelant des considérations assez longues, on rappelle que le lecteur que cette discussion apologétique n'intéresserait pas peut toujours passer à la suite sans perdre le fil de ce livre).

Renonçons d'abord à la tentation de comparer la scientificité de cette théorie - notamment ses justifications empiriques - à un 
certain nombre d'autres, en commençant par quelques-unes des herméneutiques du discours, de l'esprit ou de la société sous lesquelles s'abritent sereinement tant de thèses de doctorat : le modèle de l'adéquation sociocognitive pourrait sortir grandi d'une série de duels épistémologiques bien choisis, mais certainement pas son auteur. Le critère de scientificité, en effet, est initiatique : une fois qu'une hypothèse a acquis droit de cité - celui d'être citée - il ne peut plus guère lui être opposé sans faute de goût. En sciences humaines et sociales au moins, les théories chenues peuvent finir leurs jours en paix, entourées de ceux qui les aiment toujours (quoique dans les sciences de la communication anglophones, les choses puissent se passer différemment ${ }^{1}$ ).

N'attendons rien non plus de la conception kuhnienne de la science légitime, perspective particulièrement féconde, mais qui n'éclaire vraiment qu'a posteriori. Non seulement parce qu'elle constate une réception plus qu'elle ne juge une validité, mais aussi parce qu'elle est de ces choses que l'on ne peut invoquer sans ridicule (un statut paradigmatique, si minime soit-il, ne se réclame pas plus qu'une médaille honorifique).

Écartons enfin l'idée que l'on pourrait directement étendre à une perspective intégrée la crédibilité durement acquise par les travaux scientifiques qui l'alimentent. Outre que les exemples de billevesées dérivées de recherches originellement scientifiques (par

1 Ainsi l'International Communication Association vient-elle de consacrer un séminaire puis un numéro de sa revue à la traque impitoyable des «concepts bons pour la retraite» (Annals of the International Communication Association, vol. 41, n 1, janvier 2017). La purge a commencé par des théories déjà dévaluées depuis longtemps sur le marché des idées (comme le modèle à deux niveaux de Lazarsfeld, que les phénomènes du Web 2.o raviveront peut-être un peu), avant de s'emballer, Klaus Krippendorff montrant notamment la porte à l'hypostasie du pouvoir par Foucault et d'autres, et même à l'analyse de cadrage de Goffman. Précisons à toutes fins utiles que je n'avais en tête aucune des théories visées dans ce numéro - il y en a de bien plus douteuses - et que je serais plutôt réservé sur l'exclusion d'un bon nombre d'entre elles : apparement, la distinction entre vérité et versimilitude a échappé à ce tribunal dont l'impartialité pourrait être discutée. Mais du moins témoigne-t-il dans la sphère anglophone d'une pensée disciplinaire dynamique et enviablement encline aux vivifiants débats d'idées. 
exemple de l'asymétrie cérébrale de Sperry) suffisent à dévaluer ce genre d'argument, le rapprochement d'apports psychologiques et sociologiques, même précautionneux, permet d'autant moins de fusionner leurs validités réciproques que leur suspicion mutuelle tendrait plutôt à le décourager. On pourra donc affirmer que les éclairages principaux sur lesquels il s'appuie sont intrinsèquement substantiels, mais non qu'ils le justifient et encore moins qu'ils le cautionnent. En outre, tandis que les reconnaissances intradisciplinaires de ces approches ne peuvent s'additionner, les limites inductives propres à chacune d'elles ne manquent pas, pour leur part, de se cumuler. En d'autres termes, la conception sociocognitive de la valeur discursive est d'autant moins "prouvable» que ses deux piliers ne le sont pas non plus. Il n'y aura pas d'expérience décisive.

On connaît la solution proposée par Popper (1973) au problème fondamental de l'induction (l'impossibilité de construire des connaissances certaines à partir d'observations empiriques). Outre que sa propre validité épistémologique a été débattue, étendre inconsidérément sa juridiction hors du champ des sciences de la nature pourrait conduire à un pyrrhonisme généralisé. Mais si cette conception réputée « scientiste » ne peut constituer un examen de scientificité, elle n'en invite pas moins à un exercice de pensée salutaire. D'abord parce qu'elle pousse à expliciter ce que l'on affirme, comme on l'a justement fait un peu plus haut. Ensuite parce qu'elle oblige à s'interroger sur ce qui peut le contredire (une théorie popperienne se définissant par ce qu'elle prétend impossible). En l'occurrence, on pourrait notamment placer dans la classe des réfutations potentielles tout ce qui démontrerait que les déterminants psychologiques et sociaux ne peuvent être mis en relation, soit parce que leur essence est si différente qu'elle les rend incommensurables, soit à l'inverse parce qu'ils sont la même chose ou, ce qui revient au même, que l'un absorbe l'autre.

L'invocation de tels dangers ressemblerait aux périls feints dont les bateleurs pimentent leurs exploits d'estrade si la dernière thèse n'avait bel et bien été soutenue, y compris par des membres éminents du Collège de France (professant que les comportements sociaux sont affaire de potentiels neuronaux ou, au contraire, que les représentations cognitives ne sont que du social incorporé). Mais dans la mesure où ces énonciations s'affranchissent elles-mêmes des critères popperiens de scientificité, elles prouvent surtout que la 
détermination sociocognitive de la valeur discursive n'est pas facile à improuver. Ce qui, selon les mêmes critères popperiens, est plutôt embarrassant pour elle : on peut préciser ce qui la réfuterait à tous les niveaux (ce qui, scientisme ou pas, est toujours un bon réflexe), mais pas garantir que cela soit possible.

Une façon moins abstraite de considérer la même question est de se demander si l'on peut trouver ou imaginer des cas ne s'accordant pas directement, ou difficilement, à ce que prévoit le modèle de l'adéquation discursive. Au terme de plusieurs années passées à en rechercher, la réponse est oui. Ils paraissent quantitativement négligeables par rapport à la grande masse des cas «normaux » (tant constatés au départ que prédits et observés par la suite), mais sont qualitativement déplaisants. L'adéquation suggère notamment que, toutes choses étant égales par ailleurs, une même offre discursive devrait en principe posséder une valeur à peu près équivalente pour des personnes à peu près comparables. Or, il s'est avéré que le personnage du commissaire San Antonio créé par Frédéric Dard ne manifestait pas le moindre égard pour ce principe. Ses aventures pouvaient être reçues de façon opposée par des lecteurs de même niveau social et culturel, moins en raison du libertinage du héros (une réticence à cet égard n'exprimerait que le jeu des facteurs normatifs) que du fait de la truculence particulière de leur écriture ${ }^{2}$. Les plus littéraires remarqueront que les exubérances de plume ne sont pas si particulières que ça, accompagnant la littérature depuis Rabelais au moins (ont-ils

2 «La grosse avait aussi ça de bath, en plus de son don culinaire, c'est qu'en clappant, elle ne bonnissait pas une broque. Fallait voir le turbin. A la baïonnette, qu'elle le chargeait, son cassoulet... De la manière qu'elle bâfrait, on avait envie de sonner la charge... La graisse lui dégoulinait aux commissures... Il en perlait dans ses bacchantes... Ça zigzaguait sur ses quatorze mentons et ça plongeait directo dans son décolleté du genre béant! A partir de là, on la perdait, comme ces rivières souterraines qu'il faut charger en colorants pour les retrouver... C'était le gouffre de Padirac : bonsoir tout le monde, rien ne va plus! Elle clappait à la soudard, en faisant claquer ses mandibules! Le régime dissocié, elle l'ignorait, Mame Dubois! Avec ses quarante-huit livres de nichons à entretenir, elle ne pouvait se permettre la carotte râpée-citron. Il lui fallait de la calorie : de la crème fraîche, de l'huile, $d u$ beurre et $d u$ carné aussi, du féculent, du qui-tient-au-corps! »San Antonio (Frédéric Dard), À tue... et à toi. Paris, Fleuve Noir, 1956. 
aussi remarqué que la transgression stylistique s'y joint généralement à la transgression morale, de Pétrone à Céline, ce qui semble témoigner de la cohérence de la dimension normative par delà la dichotomie de la forme et du fond ?). Cependant, les aventures de San Antonio avaient ceci de particulier que les critères selon lesquels elles s'offraient au jugement étaient essentiellement d'ordre hédonique : elles ne prétendaient pas bénéficier de la valorisation sociale qui favorise l'investissement dans la lecture dite « littéraire $^{3} »$. Mais si la pertinence cognitive de ces romans policiers était la principale motivation de leur lecture, comment se pouvait-il que l'effort cognitif imposé par leur style (malgré sa lisibilité lexico-syntaxique élevée) décourage des lecteurs et en ravisse d'autres au sein d'un même environnement social et culturel, en l'occurrence le mien ? Ce problème n'étant apparu que rétrospectivement, il est bien trop tard pour poser la question aux intéressé(e)s, ce qui violerait en outre le rapport au terrain assumé par ce travail, pour lequel personne, à aucun moment, n'a été interrogé directement ou soumis à un quelconque protocole expérimental ${ }^{4}$.

Par ailleurs, le cas de San Antonio fait écho à un phénomène introspectivement familier. Comment comprendre que l'on puisse parfois, en consommant à nouveau une offre discursive, se demander ce que l'on avait bien pu lui trouver la première fois?

3 La notion même de « lecture littéraire » méritant d'être discutée (sans forcément la rejeter), on ne l'emploie ici que pour simplifier. D'autant que l'on ne songe certainement pas à minorer la « littérarité » de San Antonio (quoi que ce terme puisse réellement signifier), pas plus que l'on ne méconnait l'anachronisme qui rangerait dès l'origine Rabelais dans la littérature canonique.

4 À dire vrai, il m'est arrivé à quelques occasions de poser des questions à des gens - 30 ooo dans un cas - afin d'éclairer un problème spécifique ou encore de vérifier selon un protocole minutieux qu'une affirmation publiée n'était pas reproductible (elle ne l'était pas), mais je ne les ai jamais rencontrés moi-même. Évidemment, les diverses expériences personnelles mentionnées dans ce livre ne comptent pas, faute de préparation méthodologique et de contrôle statistique (quant à mon exploitation plus coutumière de corpus petits et gros, de listes de fréquence, etc., elle peut certainement être qualifiée d'empirique et, j'espère, de méthodologiquement construite, mais ce n'est pas ce que l'on appellerait du terrain). 
Il y a plusieurs façons de relativiser ce genre de difficultés. D'abord en remarquant que la prémisse «toutes choses étant égales par ailleurs » n'est réellement soutenable que pour les atomes ou les molécules. Deux personnes socioculturellement équivalentes ne sont pour autant interchangeables ni cognitivement ni normativement (l'exemple de la perception différenciée de l'affaire Dreyfus au sein de familles jusqu'alors unies est resté proverbial), et nous ne sommes certainement pas semblables à nous-mêmes à quelques années d'écart. Ensuite en rappelant, s'il en en est besoin, que les hypothèses (quelle qu'en soit la source disciplinaire) n'informent que sur des tendances, des probabilités statistiques, des attitudes types, des logiques communes... : si l'induction des cas particuliers à une règle générale est fragile par nature, le retour déductif de celle-ci à la prédiction d'un comportement individuel est tout à fait aléatoire (probabiliste au mieux). Enfin, en soulignant à nouveau que les polarités que propose la conception sociocognitive de la valeur discursive sont explicitement compatibles avec la pondération individuelle des facteurs en jeu, de même qu'avec des interactions atypiques entre ces facteurs.

C'est précisément ici que l'intransigeance popperiene prend tout son intérêt. Non pas que son rasoir bien connu (si un cas comme celui-ci n'annihile pas la théorie, alors qu'est-ce qui le pourrait ?) ait beaucoup de portée en l'espèce. Compte tenu du nombre, de l'ambivalence et de la diversité des variables en jeu, il est difficile de se sentir pris la main dans le sac: on náa jamais revendiqué ce genre de scientificité là. Du reste, même des conceptions bâties sur un socle monodisciplinaire plus homogène lui échappent (on pourrait, surtout au $\mathrm{xxI}^{\mathrm{e}}$ siècle, réfuter le modèle de La Distinction par bien des objections empiriques : ça le relativise, mais ça ne l'invalide pas). Cependant, la méthode critique de Popper ne livre pas seulement un verdict. Elle offre aussi des diagnostics ou des alertes profitables.

Si une hypothèse ne satisfaisant pas aux stricts critères de la réfutabilité est toujours susceptible d'être requalifiée de "métaphysique », elle peut aussi trahir des vices plus fondamentaux. L'un est le recours trop complaisant à des «stratagèmes conventionnalistes ", stipulations ad hoc équivalant plus ou moins aux clauses en petits caractères des contrats commerciaux. Indiquer, en particulier, que les facteurs déterminant l'adéquation discursive peuvent parfois agir de façon atypique, ne reviendrait-il pas à professer, en 
somme, que "les choses sont ainsi... sauf quand elles ne le sont pas", et à se prémunir ainsi contre toute objection? Un autre risque, qui englobe le premier, est celui de la tautologie, énonciation circulaire (donc toujours vraie) se réduisant au bout du compte à une simple reformulation, impressionnante mais toujours descriptive, de ce que l'on prétend expliquer. On en a croisé dans les pages qui précèdent quelques exemples possibles chez certains auteurs, un médecin de Molière en fournit un autre, resté célèbre, en expliquant que « la cause et la raison » de l'assoupissement provoqué par l'opium réside dans sa virtus dormitiva (l'invocation de cette « vertu dormitive » revenant à dire qu'il fait dormir parce qu'il fait dormir). On voit que si la batterie complexe des tests popperiens est absurdement surdimensionnée par rapport à une proposition analytique en sciences humaines (Popper fluctue même dans les conclusions qu'il en tire sur... la théorie darwinienne de l'évolution), la vigilance qu'elle suggère reste salutaire pour n'importe quel énoncé. Elle est cependant paradoxale.

Penser que la conception sociocognitive de l'adéquation pourrait être tautologique reviendrait à l'honorer d'un degré de "vérité » bien supérieur à celui qu'elle revendique, puisque par définition une tautologie est toujours vraie. La question est probablement un peu plus compliquée que ça, le même argument pouvant s'appliquer au genre de théories pseudo-scientifiques ou para-scientifiques que Popper visait à démasquer. La façon la plus simple et la plus courte de la considérer ici est sans doute de se demander si la façon de voir, proposée, y compris les interactions atypiques qu'elle admet, n'équivaut épistémologiquement qu'à une reformulation tortueuse des phénomènes qu'elle décrit. En d'autres termes, dans quelle mesure ce qui sort de cet outil analytique est-il plus que ce que l'on y rentre ? L'ennui, c'est qu'en arrivant ainsi sur un terrain nettement favorable (selon moi...) on en revient à la question de l'utilité telle qu'on l'avait évoquée dans le chapitre qui précède, ce qui est assez éloigné des critères théoriques popperiens. Mais on pourrait continuer à tourner en rond sans tirer de ceux-ci plus que des éclaircissements conceptuels, ce qui n'est pas inutile mais devient vite lassant. Du reste, tout scrupule s'envole en constatant que ce à quoi on a abouti n'est autre que le mode d'évaluation que Popper applique après mûre réflexion à sa propre thèse : 


\begin{abstract}
Autant que je puisse en juger, il n'y a qu'une seule manière de présenter des arguments rationnels en faveur de mes propositions. C'est d'analyser leurs conséquences logiques : mettre en évidence leur fécondité, leur pouvoir d'élucider les problèmes [...] (1973, p. 34)
\end{abstract}

Incidemment, la pertinence de cette forme d'évaluation est solidement attestée en psychologie ${ }^{5}$ comme en sociologie ${ }^{6}$ (moins dans les prescriptions méthodologiques à l'usage des étudiants que dans les productions de leurs maîtres). Et puis, c'est après tout celle qui a guidé en permanence la prise en compte ou non des recherches sur lesquelles s'appuie ce livre. Mais suffit-elle pour autant?

Si l'appareil critique popperien était clairement surdimensionné, tel n'est pas le cas de celui qu'a proposé Bernard Miège. Il ne s'agit plus là de la scientificité dans l'absolu, ni de celle des méthodes expérimentales, mais très précisément du problème de la validité des théories communicationnelles. De surcroît, ce filtre n'est pas isolé : il couronne la défiance collective vis-à-vis de toute approche générale de la communication d'un champ qui pourrait, à l'instar du Méphistophélès de Goethe, se présenter comme «l'esprit qui toujours nie » (" et c'est avec justice : car tout ce qui existe est digne d'être détruit7 »). Il est vrai que ce domaine interdisciplinaire nécessite plus que

5 "Des affirmations abstraites [...] ne peuvent être jugées en se bornant à se demander si elle est "juste". On a meilleur compte à poser des questions pragmatiques, perspectivistes : "Quelle conséquence y aurait-il à être d'accord avec cette proposition ?" » (Bruner, 1991 p. 40).

6 "À quoi peut bien servir notre grammaire axiologique? La première réponse est qu'elle sert à savoir. [...] L'on peut toutefois aller plus loin, en affirmant que non contente de produire du savoir, la "sociologie axiologique" permet aussi de comprendre. Car, par-delà la description analytique des structures sous-jacentes à la production d'un jugement de valeur, elle donne des outils pour élucider [...]» (Heinich, 2017, p. 276).

7 Pièce d'autant plus recommandable qu'elle offre, dans le dialogue du directeur de théâtre, du bouffon et du poète, une admirable illustration du conflit entre la pertinence cognitive et la légitimité axiologique :

[Le directeur] « Offrez un univers aux spectateurs surpris [...] Sachez donc par l'EFFET conquérir leur estime, / Et vous serez pour eux un poëte sublime. » [Le poète] «Cherche un autre valet! tu méconnais en vain / Le devoir du poëte et son emploi divin! » (trad. Nerval, 1877, p. 31-32 ; «EFFET» en capitales dans l'original). 
d'autres une attention critique sans laquelle ses objets se donneraient souvent à voir sous un jour enchanté. Il est vrai aussi qu'une partie des théories perçues comme "communicationnelles", surtout à l'extérieur de ce champ (on en trouve de bons exemples dans l'offre de formation destinée aux cadres d'entreprises), sont à peine « dignes d'être détruites ». Mais il n'en est pas moins intéressant, en constatant que l'imposant territoire institutionnel conquis par les sciences de la communication est jonché de cadavres conceptuels (pour ne pas parler des théories zombies ou ectoplasmiques qui le hantent comme bien d'autres $\left.{ }^{8}\right)$, de se demander quelle sorte de malédiction épistémologique semble condamner par avance toute tentative d'intégration dans cette interdiscipline.

C'est la question à laquelle pourrait répondre l'analyse de Miège ${ }^{9}$, bien qu'il ne l'ait pas envisagée de cette façon. Compte tenu de l'importance de ses observations et de la netteté de leur énonciation, mieux vaut en reproduire un assez large extrait que les paraphraser :

[...] les parutions de «théories générales » se sont succédé à intervalles réguliers, sans que leurs auteurs prennent appui sur les théories précédentes, pour en réfuter l'argumentation ou même... en reprendre des éléments clés. Tout se passe comme si un coup de force théorique avait d'autant plus de chance de réussir que l'auteur (ou le courant) concerné, faisant table rase du passé scientifique, affirmait de ce fait avec plus de conviction la radicale nouveauté de son propos. [...]

À quelles limites se heurtent ces théories générales ? Sans nier les apports particuliers des unes ou des autres, surtout quand elles sont envisagées seulement comme des « problématiques

8 Plus d'un domaine scientifique est habité par des théories ayant apparemment livré leur dernier souffle depuis longtemps, mais titubant encore (zombies), ou si vagues dans ce qu'elles spécifient et diffuses dans leur portée (ectoplasmes) que l'on peut en faire l'usage que l'on veut tout en se réclamant d'un « cadre théorique » légitime. En termes d'utilité rhétorique, il est vrai, cette qualité est insurpassable, mais même d'un point de vue strictement analytique, ces façons de voir conservent souvent une certaine pertinence.

9 Miège (1995, p. 85-86). 
partielles », nous considérerons qu'elles sont marquées par les traits suivants :

- le réductionnisme (un aspect de la communication, par exemple l'informatisation, est censé représenter le tout, c'est-à-dire l'ensemble des phénomènes informationnels et communicationnels, et suffire pour tirer des conclusions valables hic et nunc) ;

- l'abstraction (les modèles abstraits évitent souvent l'approche de la complexité du social, ou se fondent pour l'essentiel sur les tendances émergentes ou les innovations);

- le primat accordé à un paradigme unique (ainsi, entre autres, la pragmatique ou certaines théories linguistiques n'envisagent-elles aucune autre approche de la communication que celle qu'elles proposent) ;

- la confusion des instances envisagées (cette critique recoupe et complète la précédente : ici, l'une des instances, celle de la communication langagière et interindividuelle, est confondue avec les autres instances de la communication dans la société, ou censée les représenter) ;

- la dérive futurologique (les théoriciens de la communication sont de moins en moins nombreux à nous promettre un "monde meilleur ", et un avenir heureusement régulé par les techniques infographiques ou télé-communicantes ; mais ils ont en commun avec les auteurs post-modernistes de nous projeter dans le futur, ou même dans un présent «futurisé ») ;

- l'absence ou l'insuffisance de procédures de vérification empirique (les démonstrations s'appuient au mieux sur des études de " cas », mais le plus souvent sur le relevé de phénomènes présentés comme « exemplaires » ou en devenir).

Il serait malvenu, après avoir convié l'un des meilleurs arbitres possibles, de contester les règles qu'il applique. Mais ça sera d'autant moins opportun que celles-ci, même présentées comme de simples constats, sont d'excellentes règles, quoique leur interprétation soit parfois délicate. 
Deux d'entre elles, déjà évoquées, sont même des piliers fondamentaux des sciences humaines et sociales. Des piliers jumeaux : la question de l'abstraction et celle des "procédures de vérification" (curieusement popperienne sous cette plume) questionnent toutes deux le rapport aux observations empiriques. Elles sont si proches que dans certaines disciplines, par exemple en psychologie, le recueil de données conditionne le droit de parler (ainsi des propositions théoriques intéressantes sont-elles parfois introduites par des expériences de laboratoire bien trop limitées pour les soutenir, mais montrant que l'on a suivi le rituel d'appartenance). Elles constituent en outre des instruments de qualification et de disqualification d'autant plus utiles qu'elles sont - comme on le remarquait plus haut - assez librement paramétrables pour ne pas se retourner instantanément contre ceux qui les empoignent. Mais elles n'en demeurent pas moins des critères indispensables à l'évaluation du discours scientifique, une abstraction dépourvue de fondement empirique relevant (dans le meilleur des cas) de la spéculation métaphysique, tandis qu'un constat sans portée théorique relève de la description ou de la comptabilité. En l'espèce, les précisions apportées par Miège, donc les paramètres qu'il adopte pour ces critères, suffisent pour toucher les cibles qu'il vise à très juste titre. Affirmer qu'ils n'affectent pas l'approche défendue ici supposerait non seulement de revenir sur la totalité des éléments empiriques évoqués dans les chapitres qui précèdent et de vérifier le recul critique avec lequel chacun de ces éléments hétérogènes est évalué, contextualisé et relié aux autres, mais aussi d'empiéter sur les prérogatives du lecteur qui est le mieux placé pour en décider (quitte à recourir au besoin à cette comparaison avec d'autres théories à laquelle on a renoncé plus haut). Disons seulement que je soutiens expressément les paramètres adoptés dans cette recherche (y compris le genre d'analogies clarificatrices auxquelles Popper n'était d'ailleurs pas le dernier à recourir lourdement ${ }^{10}$ ).

10 "La science ne repose pas sur une base rocheuse. La structure audacieuse de ses théories s'édifie en quelque sorte sur un marécage. Elle est comme une construction bâtie sur pilotis. Les pilotis sont enjoncés dans le marécage mais pas jusqu'à la rencontre de quelque base naturelle ou "donnée" et, lorsque nous cessons d'essayer de les enjoncer davantage, ce n'est pas parce que nous avons atteint un terrain ferme. Nous nous arrêtons, tout simplement parce que nous sommes convaincus qu'ils sont assez solides pour supporter l'édifice, du moins provisoirement » (1973, p. 111). 
Quant aux autres remarques, j'y souscris d'autant plus volontiers que, comme on a pu le remarquer, le réductionnisme technocentrique (ou autre), l'hégémonie revendiquée par une perspective scientifique exclusive et la confusion des instances (ou des niveaux, des déterminants, etc.) de la communication sont le genre de choses contre lesquelles on n'a cessé de ferrailler dans les chapitres qui précèdent. Sans compter que - toujours sans empiéter sur le jugement du lecteur - la façon de voir proposée ici est typiquement de celles à qui Miège accorde les circonstances atténuantes d'avoir été «envisagées seulement comme des "problématiques partielles" ». J'aime à penser qu'elle est même la plus explicite à cet égard, n'abordant par exemple pas (ou pas directement) des questions aussi classiques que celles des effets des médias ou de la nature de l'ineffable dans l'art et la littérature ${ }^{11}$, pas plus que celles - primordiales sous d'autres angles de l'influence des technologies, des idéologies ou de l'économie.

Pour être plus concret, revenons donc sur un exemple antérieur : le modèle de l'adéquation discursive est absolument incapable d'expliquer à lui seul pourquoi l'évolution typographique et éditoriale, qui depuis les premiers rouleaux de papyrus respectait admirablement les logiques attendues, a soudain bifurqué vers l'aberration cognitive des caractères gothiques. Il permet juste de remarquer et de caractériser (situer analytiquement) cette anomalie, ce qui n'est déjà pas si mal. Pour expliquer cette dégradation de la valeur discursive, il faut faire appel à d'autres dimensions de la communication, notamment la course à la rentabilité économique des industries culturelles ${ }^{12}$.

Mais même "partielle», comment se pourrait-il que l'approche générale proposée ici se soumette de si bonne grâce aux

11 Notamment la traque bimillénaire du «sublime » (un peu passée de mode il est vrai).

12 En l'occurrence, le fait que les caractères gothiques étaient plus faciles à produire de façon régulière (n'exigeant pas de tracé courbe mais seulement des applications successives de la plume) et que leur parallélisme permettait d'adopter une chasse (étroitisation) très réduite, et donc d'économiser le papier si onéreux. Ultérieurement, l'impression de certains ouvrages en caractère gothiques pouvait en partie s'expliquer par le désir d'imiter l'aspect des ouvrages manuscrits, plus « nobles » et plus chers (et, encore beaucoup plus tard, par le nationalisme allemand). 
critères évaluatifs développés par Miège, tout en constituant, du simple fait de son existence, une objection respectueuse mais résolue à son hypothèse selon laquelle toute approche théorique de grande échelle est dans ce domaine «improbable et même inconcevable»?

Il est bien sûr possible que ses critères aient été mésinterprétés ou dévoyés dans les lignes qui précèdent, mais ce n'est pas la seule explication envisageable. Outre le fait qu'une authentique « théorie du tout » serait effectivement plus qu'improbable (la prétention à la panacée trahit généralement le charlatanisme), une approche générale peut certainement sembler inconcevable puisqu'elle n'offre guère que deux voies également impraticables. La première, confortable, mais illusoire, est de tout considérer à partir d'un seul angle restreint, avec assez d'assurance pour écraser le reste. C'est la méthode monodisciplinaire habituelle. La seconde, moins confortable, est de tout considérer sous une perspective plus large en acquittant le coût épistémologique (simplification, etc.) qu'elle implique, et donc en s'exposant à des remontrances justifiées. Bref, on ne peut, de fait, choisir qu'entre la carence et l'approximation, quoique les exemples disséqués par Miège montrent que la première exclut rarement la seconde. Et puisque l'une et l'autre de ces voies sont également contestables $a$ priori, elles ne peuvent s'évaluer qu'a posteriori. Nous voici donc revenus au critère d'utilité, mais cette fois lestés d'un solide handicap : il est inutile de soutenir l'utilité analytique de l'articulation du social et du cognitif, car il est de toute façon interdit d'invoquer ce dernier, en tout cas en France. Comme le précise Miège, soudain normatif, "Pour les sciences de l'information et de la communication, cette nouvelle perspective [les sciences cognitives] ne saurait être admise » (p. 104). Elle ne saurait même pas être connue, si j'en crois mon expérience ${ }^{13}$. C'est d'ailleurs ce tabou disciplinaire qui m'avait

13 Illustrons-la juste par une autre de ces anecdotes qui ne prouvent rien. Pour passer le temps avant une intervention dans un prestigieux institut du Sud-Est de la France consacré à la communication, j’avais flâné dans son imposante bibliothèque. Quoique richement fournie, elle comprenait en tout et pour tout... deux ouvrages traitant sérieusement de psychologie. L'un portait sur une théorie zombie (la gestalt, qui a bien travaillé dans sa jeunesse et peut encore rendre de menus services, mais semble avoir épuisé l'essentiel de sa vitalité), l'autre 
incité il y a une vingtaine d'années à aller faire ma recherche doctorale dans un domaine où je pouvais bénéficier de compétences et de séminaires en sciences cognitives. Non pour braver un interdit, mais parce qu'il me semblait collectivement et personnellement délicat d'analyser et enseigner ce qui est au cœur de la communication, la production et la réception discursives, en acceptant d'en savoir si peu sur les processus cognitifs qu'elles mettent en jeu (ce n'est pas non plus par bravade que j'ai soutenu cette thèse devant un jury composé paritairement de professeurs de communication, mais pour qu'on ne me soupçonne pas d'importer en contrebande un acquis non soumis à mes pairs).

Quoi qu'il en soit, la question de la possibilité d'une théorie partielle mais générale de la communication est ainsi réglée : $I^{\circ}$ une théorie de ce genre ne peut réellement s'évaluer qu'a posteriori, donc en fonction de sa portée heuristique (ce qu'elle permet de comprendre) $; 2^{\circ}$ or, une théorie de ce genre ne peut (selon moi) être réellement explicative qu'en prenant enfin en compte les facteurs cognitifs ; $3^{\circ}$ mais de ce fait, une théorie de ce genre «ne saurait être admise » comme une théorie communicationnelle ; $4^{\circ}$ donc une théorie communicationnelle de ce genre est bel et bien impossible. Ce n'était pas si compliqué.

Avant de refermer le dossier, il convient toutefois de prendre note de la justification épistémologique qui suivait immédiatement cette proscription formelle :

[...] en effet, les technologies intellectuelles (et parmi elles les médias) étant supposées jouer un rôle capital dans les processus cognitifs, c'est pratiquement l'ensemble des activités communicationnelles qui sont ainsi réduites à des processus cognitifs, relevant donc des sciences cognitives.

Curieusement, cette opinion est exactement l'inverse de celle à laquelle aurait pu conduire un examen de ces savoirs interdits, s'il avait été assez soutenu pour traverser le brouillard des prétentions

était un excellent manuel de psycholinguistique de premier cycle (le Précis de Caron) mais il semblait peu feuilleté. Peut-être aurais-je pu aussi y trouver un ou deux livres de méditations abstraites sur la cognition au rayon philosophie, mais je ne faisais que passer. 
fantaisistes ou métaphysiques que des essayistes et des penseurs entretiennent aux contours de cette nébuleuse ${ }^{14}$. Ce qu'un tel examen pourrait d'abord montrer, c'est que la psychologie cognitive expérimentale - qui est pour le moment la plus pertinente et la plus avancée si l'on parle bien des « processus cognitifs »- ne serait aucunement en mesure d'avaler le domaine de la communication dans l'hypothèse où elle se soucierait le moins du monde d'aller se perdre dans cet entrelacs multiscalaire. À elle seule, la construction d'un modèle réellement consensuel et prédictif des processus de compréhension des énoncés textuels reste pour elle un défi imposant malgré les progrès considérables accomplis dans ce sens (et le peu que je crois savoir des autres disciplines associées à la cognition est qu'elles auraient encore beaucoup à envier à la première sur ce point).

Mais ce que cet examen pourrait du même coup montrer, c'est surtout que la dimension cognitive de la communication humaine, loin de menacer les sciences de la communication, est au contraire ce qui est le plus à même de justifier leur nature interdisciplinaire et leur nécessité scientifique (sans compter - mais ça compte rarement - leur attractivité pédagogique). Comme l'a illustré le cas particulier de la valeur discursive, plus l'on tente de cerner les aspects proprement cognitifs du traitement de l'information, plus ils exigent en regard la prise en compte des déterminants sociaux de ce traitement (et soulignent l'impossibilité de les mélanger indistinctement). Si la sociologie - ainsi qu'on l'avait remarqué - n'a pas grand-chose à craindre de la psychologie (et réciproquement ${ }^{15}$ ), chacune ne pourrait que profiter d'une interdiscipline (ou métadiscipline ?) vraiment capable de clarifier les liens entre de tels aspects (et d'autres, historiques, technologiques, idéologiques, etc. qui lui sont familiers) aux niveaux de complexité requis par les phénomènes abordés.

Pour prendre un exemple, l'approche psychologique de l'attention médiatique qui conduit Kahneman à l'attribuer à un biais bien connu des expérimentalistes (l'heuristique de disponibilité) est extraordinairement fragmentaire au regard de toutes les connaissances accumulées depuis l'époque où McCombs et Shaw

14 Pour ne pas parler des captations de budgets ou des intrigues institutionnelles dont bruissent les organismes de recherche.

15 Pas plus que l'une ou l'autre n'ont à redouter la linguistique (et réciproquement). 
(1972) mettaient en évidence les phénomènes d'«agenda setting» médiatiques. Mais il serait aussi farfelu de reprocher à Kahneman de méconnaître ce corpus que de ne pas enrichir ce dernier avec les résultats du premier. Ainsi, assumer réellement la nature et la culture censément interdisciplinaires des sciences de la communication - et le caractère composite de ses objets propres - n'implique ni de rivaliser avec des approches monodisciplinaires ni de les surplomber. Mais cela n'implique pas non plus de les solliciter toutes indistinctement pour toutes les problématiques : c'est justement la distance entre la noyade multifactorielle et la myopie monofactorielle qui décrit de facto - mieux que n'importe quelle autolégitimation abstraite - les territoires de tous les domaines de recherche définis par un objet plutôt que par une méthodologie.

Tout irénisme mis à part, une telle chose serait-elle... utile? Peut-être bien, si l'on considère justement deux des chantiers prioritaires que Miège inscrivait au programme de travail (Miège et Odin, 1993) d'une interdiscipline qu'il a, en France, largement contribué à édifier conceptuellement et à normer institutionnellement :

- l'articulation entre les dispositifs techniques de la communication et la production des messages et du sens ;

- la mise en place des "procédures » d'écriture des messages [...] et des conditions présidant à leur conception et à leur réalisation ;

Le sens? Les conditions de la production discursive ? On pouvait certainement essayer de les aborder sans s'embarrasser de psychologie à une époque où l'état de celle-ci laissait peu d'autre choix que de transposer spéculativement à ces problèmes la «façon de voir» de la linguistique structurale (avec des résultats initiaux brillants, puis de plus en plus tenus au fil du temps). Mais l'ignorance des processus cognitifs est aujourd'hui d'autant plus intenable que si le progrès des connaissances en la matière ne menace pas les disciplines qui les apprivoisent, il ne peut que saper la crédibilité et la pertinence de celles, de plus en plus rares (en reste-t-il d'autres, d'ailleurs ?) qui s'imposent de les ignorer ${ }^{16}$.

16 L'interdit, du reste, se fissure depuis belle lurette, non seulement dans la sphère francophone (voir par exemple l'Introduction aux théories de la 
Une sorte de fatalité homophage veut que les auteurs dont la pensée est la plus utile dans une recherche finissent souvent par être ceux qu'elle discute le plus (comme, plus haut, Sperber et Wilson et quelques autres). D'où mon embarras à être, en ce qui concerne la cognition, en net désaccord avec Bernard Miège, et généralement avec la position très majoritaire qu'il exprime. Mais ça ne m'empêche pas de partager, parmi bien d'autres choses, son jugement sur l'inculture communicationnelle de beaucoup d'auteurs prétendant éclairer ce domaine (voir, plus haut, le cas de Sperber et Wilson) et sa méfiance vis-à-vis des théories "faisant table rase du passé scientifique ». Avec, cependant, deux perplexités personnelles. La première est que la culture communicationnelle, complexe et hétérogène, excelle avant tout dans son rôle faustien (der Geist, der stets verneint) ou, comme le résume encore mieux Miège, dans sa capacité «à interroger, voire à mettre en question des pratiques et des points de vue actuellement dominants » (p. 105). Cependant, force est de remarquer que sa volonté de proposer en contrepartie des paradigmes explicatifs, voire sa capacité à en produire, n'est pas toujours discernable, en tout cas de l'extérieur ${ }^{17}$, ce qui pourrait peut-être contribuer à expliquer que tant d'auteurs (et d'étudiants) se dispensent d'accorder à son canon non canonique l'effort cognitif qu'il requiert.

La seconde perplexité, qui ramène directement aux tensions de ce travail, est l'ambivalence intrinsèque du devoir de connaître, critère aussi nécessaire que le rejet de l'abstraction, mais aussi malléable que lui. En effet, le rejet que justifie amplement «l'ignorance de pans entiers des connaissances produites antérieurement » n'exorcise la méconnaissance que pour pousser vers l'écueil, tout aussi dangereux, de l'éclectisme : d'un côté l'autarcie de la théorie batracienne

communication de Meunier et Peraya) mais même en France. Un spécialiste de psychologie sociale a été invité à contribuer à un numéro thématique de la revue Hermès consacré à la réception des médias, le directeur d'un département des Sciences de l'Information et la Communication a commis divers ouvrages consacrés à ce qu'il appelle la "psychologie de la communication" (en l'occurrence les approches interprétatives de la communication interpersonnelle), etc.

17 Que l'on considère sa «balance commerciale » (importations contre exportations) sur le marché des idées, ou la prépondérance des conceptualisations pseudo-scientifiques de la communication sur celui de la formation et du conseil. 
du langage échafaudée par Brisset, de l'autre le syncrétisme érudit mais chimérique de Pic de la Mirandole.

A priori, une approche explicitement biréférentielle, bien ancrée (au-delà d'à-côtés contextuels multiples, mais secondaires et surtout heuristiques) sur les deux cadres définis dont elle explore la complémentarité dans une perspective très classique - les théories de la réception - et selon un angle qui n'est pas moins classique $^{18}$, ne devrait pas avoir grand-chose à craindre de tels périls. D'autant qu'elle n'en conteste aucune autre, se bornant à revendiquer sa complémentarité. Pourquoi faut-il alors que la défense pathétique de Pic de la Mirandole continue à me hanter?

[...] selon eux, j'aurais accumulé des vétilles et des bagatelles pour produire un effet de nombre, comme s'il ne s'agissait pas de questions extrêmement incertaines et controversées opposant violemment les principales écoles; comme si je n'avais pas fourni, à ceux-là mêmes qui mettent mon travail en pièces en se prenant pour les princes des philosophes, un apport inédit et quantité d'idées jamais encore soumises à examen! Or, je me suis si bien gardé de cette faute que j'ai veillé à restreindre, dans toute la mesure du possible, le nombre des points en discussion. Si j'avais voulu, comme tant d'autres, marquer toutes les divisions et articulations, le nombre des thèses serait à coup sûr devenu incalculable. Et sans parler du reste, comment ne pas voir que j'aurais pu, sans me faire soupçonner le moins du monde de multiplication artificielle [... l'accroître...] par la simple énumération de tous les passages où les autres croient ces auteurs en désaccord et où j'estime, quant à moi, qu'ils concordent? Mais je

18 Sans parler du problème de la « sélection » posé par Schramm (et par divers auteurs extérieurs à ce champ), on pourrait, si l'on y tient, lui prêter une certaine affinité avec la mouvance débattue des usages et gratifications, bien moins pour ce qui a pu être dénoncé par certains (non sans forcer le trait) comme du fonctionnalisme que pour le renversement plus général de perspective qu'elle proposait contre l'hégémonie de la problématique de l'influence des médias : «même le plus puissant des médias ne peut normalement influencer un individu qui n'en a aucun "usage" dans le contexte social et psychologique où il se trouve " (Katz, 1959 p. 3, souligné par moi). 
l'affirmerai, bien que ce ne soit guère modeste ni conforme à mon tempérament [...] (1486/1993, p. 99-101)

Peut-être est-ce à cause du ridicule que la postérité a niaisement attaché au nom de cet humaniste imprudent. Peut-être aussi parce que son château de cartes branlant était bien trop ambitieux pour ne pas s'effondrer de lui-même. Mais sans doute surtout parce qu'il symbolise les deux problèmes qui sont inhérents au refus de "l'ignorance de pans entiers des connaissances » : celui de la vulnérabilité des détails et celui de la recevabilité de l'ensemble. On a assez parlé du premier pour ne pas y revenir une fois de plus, d'autant qu'il est en bonne partie réglé depuis 24 siècles (Sutor, ne supra crepidam). Quant au second, c'est-à-dire le fait que - toute considération épistémologique mise à part - la crédibilité scientifique attachée à un propos soit inversement proportionnelle à la diversité des aspects qu'il s'abstient d'ignorer, il a ceci de délicieusement ironique que l'on pourrait notamment l'expliquer par la conjonction de facteurs cognitifs et sociaux.

Sous le premier angle, il relève d'une propension largement documentée scientifiquement : celle consistant à économiser son effort cognitif en se fondant sur des indices de valeur ${ }^{19}$ qui facilitent ou épargnent un examen cognitivement coûteux et possiblement inutile des énoncés (" peut-il vraisemblablement savoir de quoi il parle? »). C'est le même type de biais qui, dans un colloque, me font percevoir spontanément, et parfois à tort, les gravités trop affectées comme des indices d'insécurité ou de vacuitée ${ }^{\circ}$. Bref, ce mode d'évaluation, relevant de ce que Kahneman désigne comme le mode « rapide » de la pensée, est certainement aléatoire, mais il n'en est pas moins nécessaire dans un marché de l'attention scientifique où, comme le signalait Franck, chaque producteur «doit employer sa propre attention de la façon la plus productive possible».

Sous le second angle, il relève tout autant des normes implicites ou même tout à fait explicites (voir Miège ci-dessus) d'un

19 Ce qui - doit-on encore s'étonner de telles correspondances ? - pourrait se rapprocher de ce qu'Heinich appelle des «prises » dans son étude sociologique de l'évaluation des produits culturels.

20 À l'inverse, c'est aussi lui qui m'avait fait refermer un ouvrage formellement insolite (Latour, 1993) dont je n'ai compris l'intérêt que bien plus tard. 
secteur ou d'un autre de la production intellectuelle, la doxa de chacun d'entre eux définissant ce qu'il est ou non légitime de prendre en compte et du niveau de précision requis pour garantir la recevabilité des discours. On pourrait même, dans une perspective positionnelle, considérer qu'il dénonce ces louches stratégies de multipositionnement que Bourdieu traquait avec une vigilance de garde-frontière. Mais qu'elle soit condamnable pour dilettantisme ou pour duplicité (la seconde inculpation étant d'ailleurs bien moins grave que la première) la valeur discursive d'un propos pluriréférentiel - indépendamment de sa validité éventuelle - ne relève pas seulement de déterminants cognitifs ou sociaux, mais bien des deux à la fois. On ne sortira pas de cette dualité.

Arrêtons ici la litanie des explications et considérations diverses, tout à fait nécessaires, mais qui finiraient par devenir gênantes si quelqu'un avait l'idée saugrenue d'évaluer la solidité d'une proposition théorique - ou d'ailleurs... celle d'une discipline - au temps qu'elle passe à disserter sur sa scientificité et sa légitimité.

Des divers filtres que l'on a envisagés plus haut, on retirera divers éclaircissements utiles, mais, bien sûr, aucune réponse contraignante à la question en jeu, celle de la validité épistémologique de l'approche sociocognitive de la valeur discursive. Ni approbation, ni improbation, mais du moins cette question aurat-elle été examinée avec plus d'exigence que de coutume en pareille matière. C'est le mieux que l'on pouvait faire. Et, pour autant que je puisse en juger, aucune de ces considérations n'interdit d'affirmer que l'approche proposée est aussi éminemment scientifique qu'il est possible de l'être en regard du problème qu'elle aborde : compte tenu de l'étendue des facteurs en jeu, prétendre rehausser sa scientificité par une recherche empirique ad hoc ou l'ennoblir par la dignité d'une écriture hermétique aurait été de la poudre aux yeux.

Quant au bref élargissement de cette question à celle, plus générale, des recherches en communication, il assume le fait que, par le choix de ce qu'il traite et de la façon selon laquelle il le traite (donc de ce qu'il présente comme un discours légitime) tout ouvrage de sciences sociales est de fait un manifeste épistémologique, qu'il le veuille ou non. Celui-ci ne fait pas exception à la règle, tant sur le fond que sur la forme. 


\title{
Des idées « libres » dans un poulailler libre
}

Les meilleurs manquent de toute conviction, pendant que les pires sont pleins d'intensité passionnée.

W.B. Yeats (1926, p. 346)

\begin{abstract}
u printemps 2017, dans un zoo perdu du nord des États-Unis, la 1 bien nommée girafe Avril donnait le jour à un girafon, sous l'œil attendri de ses gardiens et de plus d'un million d'internautes. En termes de valeur discursive, cet heureux évènement était effectivement un évènement : la chaîne en ligne qui permettait de suivre l'avancement des choses (que la mère avait eu la coquetterie de faire traîner pendant des semaines) était devenue, depuis son ouverture deux mois plus tôt, le second canal le plus regardé en direct de l'histoire de YouTube, totalisant, selon Associated Press, 232 millions de vues².

À peu près au même moment, le Fonds monétaire international exprimait de sérieuses craintes vis-à-vis de la déréglementation bancaire promise par le président des États-Unis et des risques, « dans les pays avancés [d'] une orientation protectionniste ${ }^{3} »$. On ignore le poids exact du FMI sur le marché discursif, mais du moins a-t-on une certaine idée de celui de sa sœur, la Banque mondiale : sur une période de cinq ans, seuls $13 \%$ de ses rapports sur les politiques économiques
\end{abstract}

1 Certains des points présentés dans ce chapitre avaient été examinés dans des textes antérieurs, notamment Une dynamique de l'insignifiance (Enssib, 2003) et L'offre éditoriale face à l'évolution des publics (FNPF, 2008).

2 The Associated Press, Live Video Feed That Showed Giraffe Birth to BeTaken Down, 18 avril 2017 à $22 \mathrm{~h} 54$ UTC.

3 Fonds Monétaire International (2017). Rapport sur la stabilité financière dans le monde-Résumé analytique. Washington : FMI (p. 1). 
ont été téléchargés plus de 250 fois, et près d'un tiers (31 \%) n'ont pas été téléchargés une seule fois ${ }^{4}$.

L'effet de contraste saisissant produit par ce genre de rapprochement serait propre à alimenter un essai cataclysmique sur la désintégration actuelle de la sphère du débat public, si l'on oubliait que de tels contrastes pourraient être relevés à toutes les époques : il y a plus d'un siècle, par exemple, la mort en Ontario de l'éléphant Jumbo (dont le nom est même devenu un adjectif) avait elle aussi capté une bonne partie cette attention que le public de 1885 aurait pu - toujours par exemple - consacrer au problème plus important, mais plus austère, des frontières roumaines.

Aucun rapprochement anecdotique ne permet de trancher sérieusement entre la vision discontinuiste ("tout est différent») et continuiste ("rien n'a changé ») du marché discursif contemporain. À supposer qu'il y ait lieu de trancher entre ces deux façons de voir, ce qui est très douteux. Mais puisque l'on avait commencé ce parcours par un survol du gonflement historique de la masse des discours et de la « crise de l'attention » qui semblait en découler, on se doit d'y revenir une fois explicitées certaines des forces à l'œuvre. Sans perdre de vue cependant que cette échelle d'analyse - la plus large de toutes est particulièrement problématique, comme en témoignent les multiples lectures qu'en ont proposées les auteurs les plus variés. La diversité de ces façons de voir s'explique non seulement par le fait que cette échelle cumule techniquement les complications de toutes les autres (d'où la "confusion bourdonnante et foisonnante » qui la caractérise), mais aussi par les enjeux qui lui sont propres.

Il est particulièrement difficile de faire totalement abstraction de ce que l'on pense être «bon » pour la vie publique, la culture ou la société en général, et, en tout cas dans ce chapitre conclusif, je n'ai pas la prétention d'échapper plus que n'importe qui à un certain biais normatif : si l'adéquation sociocognitive en tant qu'appareil analytique se veut indifférente aux valeurs, rien n'interdit d'appliquer dans un second temps cet outil aux préoccupations plus subjectives auxquelles s'abandonnent sans vergogne tant d'autres recherches.

4 Doerte Diemeland et James Treviono (2014). Which World Bank Reports Are Widely Read? (Policy Research Working Paper $n^{\circ}$ 6851). Washington : Banque mondiale. 
L'une des manifestations les plus saillantes des transformations, qualitatives sans doute, quantitatives sûrement, que connaît le marché discursif est celle qui ébranle les conceptualisations de la communication comme un instrument univoque d'oppression et d'uniformisation des consciences. Non que ces façons de voir aient pour autant perdu toute leur pertinence. Ainsi, la plus éminente d'entre elles, la théorie critique des industries culturelles, a beau être née pendant la guerre de l'incompatibilité entre le rêve d'une modernité guidée par la raison et l'évidence de la barbarie nazie, il est difficile, soixante-quinze ans plus tard, de ne pas reconnaître un peu partout le développement de la marchandisation de l'offre discursive qu'elle dénonçait (la désignation de plus en plus fréquente et sereine des étudiants comme des «clients » par les instances universitaires - et parfois par les intéressés - montre que ce phénomène a largement dépassé la sphère éditoriale). De même, la prédilection de la production culturelle de masse pour les contenus facilement consommables, normalisés et anodins est facile à constater.

Éclairant mais circonscrit, cet angle de vue engagé - dévoiler l’hégémonie de la trivialité comme instrument de contrôle idéologique et d'étouffement des dissidences - dans lequel on pourrait plus ou moins rapprocher (au mépris de toute nuance) des auteurs aussi différents qu'Adorno, Habermas, Bourdieu et Chomsky, était suffisamment déterminé pour résister aux divers contre-exemples empiriques et aux objections compréhensibles des producteurs discursifs visés 5 , tant que la concentration croissante des industries culturelles tendait à le corroborer. Mais il paraît de plus en plus difficile de considérer sous cette seule perspective la complexité contemporaine du marché discursif. Non seulement en raison de la fragmentation de l'offre et de l'abaissement des barrières d'entrée sur ce marché (faire connaître sa pensée ne coûte plus rien, à défaut de rapporter grand-chose), mais aussi parce qu'a disparu un point important de la critique classique, ce caractère unilatéral des médias de masse que dénonçaient aussi bien Habermas - pour qui ils captivent les spectateurs tout en leur retirant « la possibilité de prendre la parole et de contredire » (1978, p. 179) - qu'Horkheimer et Adorno, lesquels déploraient qu'« aucun mécanisme de réponse n'a été développé» (2002, p. 96).

5 Les premiers, quel qu'en soit le nombre, pouvant toujours être rejetés comme des exceptions tolérées ou entretenues par le système pour camoufler son emprise, les secondes comme la preuve de l'illusion dans lequel le même système maintiendrait ses serviteurs. 
Comment expliquer que les conglomérats des industries culturelles, tout en renforçant bel et bien leur mainmise économique sur les médias, puissent favoriser à une échelle sans précédent, non seulement le partage des contestations idéologiques les plus radicales, mais aussi la mise en cause des entreprises elles-mêmes (aujourd'hui obnubilées par les appréciations en ligne et la crainte du déchaînement des réseaux sociaux) et jusqu'à la concurrence par tout un chacun de leur propre production (et donc la dévalorisation de celle-ci)? Les marxistes ne s'étonneraient peut-être pas de ce genre de contradiction (selon la boutade attribuée à Lénine, «les capitalistes nous vendraient la corde pour les pendre »), mais elle n'en reste pas moins difficile à concilier avec l'hypothèse d'une domination orchestrée de façon stratifiée par « le pouvoir » économique et politique ${ }^{6}$.

Sans renoncer à évoquer la communication comme une technique de "gestion du social», la version moderne de la théorie des industries culturelles, moins incantatoire, moins monolithique et méthodologiquement beaucoup plus solide que sa ou ses devancières, s'accommode nettement mieux qu'elles de la coexistence de grandes masses industrielles et de multitudes de microacteurs individuels ou collectifs. Mais si elle décrit très bien les logiques à l'œuvre derrière la diversification et le renouvellement permanent du secteur de la communication, elle le fait sous un angle de vue et à une échelle (celle des structures, des flux, des mutations...) très différents de ceux des déterminants adéquationnels du marché discursif, celui-ci étant surtout envisagés par elle sous l'angle macrosocial et mésosocial des rapports de production et de consommation.

Si la communication se résume à un appareil de "gestion $d u$ social 7 ", les résultats que l'on a pu en observer suggèrent en tout cas

6 Bien sûr, on schématise ici des positions plus subtiles, et qui plus est non dépourvues d'exemples empiriques (comme, aux Etats-Unis, le rôle des frères Koch dans l'organisation d'un puissant réseau d'influence idéologique), mais la réification rituelle du "pouvoir», de même que le biais d'intentionnalité (ou d'agentivité) qui attribue classiquement aux organisations et professionnels des médias des visées sulfureuses n'en conservent pas moins un poids épistémologiquement insolite dans les débats sur ce thème.

7 Il serait particulièrement caricatural de réduire les recherches modernes sur les industries de la communication à la conception manipulatoire 
que ses gestionnaires, quels qu'ils puissent être, sont extraordinairement maladroits. L'élection d'un président discordant aux États-Unis, le retrait britannique de l'Europe et le niveau d'adhésion aux positions extrêmes lors de la présidentielle française montrent que le monopole explicatif accordé aux finalités manipulatoires et uniformisantes prêtées aux industries culturelles a fait son temps.

Peut-être même le temps est-il venu de cesser de mélanger la dimension normative et la dimension hédonique de l'offre communicationnelle, de même que les enjeux qui leur sont prioritairement attachés : idéologiques pour la première, marchands pour la seconde. Évidemment, l'une ne va pas sans l'autre : si l'on peut soutenir que la majeure partie des industries culturelles vise beaucoup moins à imposer une quelconque hégémonie qu'à vendre de la pertinence cognitive (en particulier les entreprises de la «nouvelle économie», qui n'ont même pas besoin de la fabriquer elles-mêmes), la réduction de la communication à la satisfaction hédonique n'en reviendrait pas moins à un choix de société dont Aldous Huxley livrait une image frappante dans Le Meilleur des mondes. Par ailleurs, la comparaison entre la production idéologique de Fox News et celle, mercantile, d'Endemol, ou a fortiori du groupe A\&E8, montre qu'il n'est pas forcément plus anodin de vendre du spectacle que de l'opinion. Mais en amont de toute conception polarisée de ce que les médias devraient être et dire, la question la plus fondamentalement émancipatrice est

que pourrait suggérer la persistance de cette désignation assez discutable (voire vaguement fonctionnaliste) dans son ambiguïté : les travaux scientifiques sur cette question ont justement tourné le dos depuis des décennies aux fantasmes d'un contrôle concerté de l'opinion pour leur substituer une image beaucoup plus complexe et informée des logiques et des instances de la communication. Comme le remarquait Miège, l'évolution du modèle de la «communication généralisée » va dans un sens "axé plus nettement [...] vers l'acquisition d'outils techniques individuels, et donc moins orienté vers la mise en auvre de techniques de gestion du social » (1997, p. 7).

8 Dont les chaînes, en particulier la fort mal nommée History Chanel, diffusent en Amérique du Nord un chapelet de pseudo-documentaires (consacrés par exemple à la réalité de créatures surnaturelles ou de complots extra-terrestres) invariablement présentés comme des enquêtes méticuleuses - «experts » à l'appui - ponctuées de perplexités feintes («se pourrait-il que...? ?). 
plus que jamais, au-delà des postures dénonciatrices, celle des conditions de pertinence d'un débat démocratique fondé en raison.

Celle-ci conduit par contraste à reconsidérer la question du "populisme ", lequel, comme toujours en matière de communication, est plus facile à dénoncer qu'à caractériser. Cette notion, dont le flou a très souvent été souligné (Charaudeau, 2011 ; Godin, 2012 ; Laclau, 2005 ; Taguieff 1997, etc.) est généralement envisagée sous une perspective plus thématique qu'analytique comme l'opposition outrancière à une élite politique ou socioculturelle, mais l'appréciation de ce qui est abusif ou non, et donc de ce qui est populiste ou non, entraîne vers un problème de valeurs inextricablement circulaire 9 . Dans quelle mesure est-il possible d'aborder ce phénomène en s'affranchissant du jugement idéologique qu'il réclame?

Si l'on considère que le populisme est fondamentalement un caractère lié au discours ${ }^{10}$, on remarque facilement que celui-ci repose sur une surexploitation des registres de pertinence de bas niveau cognitif. Il s'agit d'abord de réduire au strict minimum l'effort de traitement demandé, notamment en éliminant les nuances et même les justifications des assertions (dont la cohérence devient secondaire). La personnalisation des idées par un chef charismatique favorise également cette économie cognitive. Le discours populiste vise parallèlement à amplifier sur la forme et le fond les facteurs d'effet cognitif qui rendent ces assertions pertinentes, en mettant en particulier en jeu les émotions fondamentales (aversion, compassion...) et les interactions avec la sphère personnelle. Cette dernière est sollicitée non seulement par les craintes individuelles (de la violence, de la paupérisation, de

9 L'appel au peuple contre les élites ne peut être considéré comme du « populisme » que lorsqu'il est reconnu comme illégitime, d'où une circularité sans issue : le populisme est mal, mais seul ce qui est mal peut être populiste (on verra que Laclau et ses collègues ont résolu ce problème, mais d'une façon glaçante). Par ailleurs, comme le note Guillebaud, la dénonciation du populisme "fournit en même temps un alibi à un élitisme peu soucieux de démocratie » (1995, p. 109).

10 On peut être populiste discursivement sans vraiment l'être politiquement (c'est un peu ce que l'on avait reproché à Guy Mollet) alors que l'inverse est beaucoup plus improbable. Du reste, une politique qui serait « discrètement » populiste semble une contradiction dans les termes, une mesure populiste valant généralement par les profits communicationnels qui en sont retirés (à défaut desquels on parlera plutôt de « clientélisme »). 
l'exclusion...) et les aspirations qui leur correspondent, mais aussi par l'identification à un groupe, produisant un sentiment de proximité catégoriel (à une identité socioculturelle, à une classe sociale ou encore au "citoyen moyen ») en opposition à d'autres groupes : comme le spectacle du sport, celui de la politique ne semble pouvoir intéresser qu'en ralliant à une équipe. Et, bien sûr, le populisme repose largement sur l'activation de schémas cognitifs frustes, mais puissants et solidement ancrés (le bolchevique, le capitaliste, le travailleur, l'étranger...) dont les effets de typicité et la bivalence axiologique simpliste (gentil/méchant) font merveille en l'espèce. En somme, il prospère sur ce que Jean-Claude Guillebaud (1995) appelle joliment « une propension disputeuse, agrippée à quelques affrontements clairs et nets » qui permet de s'abandonner «à la chaleur rassurante du groupe, du clan, de l'école ou de la secte, à la douce saveur des "mots de la tribu" ».

On pourrait incidemment se demander si la pertinence cognitive est seulement un mode de diffusion de la «pensée » populiste ou si elle en est la source : l'économie d'effort qu'offrent la sursimplification du réel et le relâchement de la cohérence réflexive, de même que l'égocentrisme réduisant les enjeux à des implications personnelles ou proximales - ce qui n'exclut pas un altruisme à géométrie variablepeuvent aussi bien façonner la vision du monde des tribuns populistes que leurs stratégies de communication.

En tout état de cause, cet angle peut aider à percevoir la grande efficacité cognitive de la mécanique populiste classique, mais ne peut suffire à la caractériser. L'autre volet de celle-ci réside dans la transgression ostensible des déterminants sociaux de la convenance discursive : la correction langagière de l'expression politique légitime, le respect de l'altérité et, surtout, la concordance des assertions avec la factualité du réel ou avec sa complexité. Ainsi, la dénonciation des corps intermédiaires - journalistes, technostructure, intelligentsia, experts... - et de leur "pensée unique » (ou, dans la bouche d'un ancien président, de leur «conformisme intellectuel»; voir Mayaffre, 2013), connue pour être l'un des indices les plus visibles du populisme se traduit-elle par un double bénéfice cognitif et social : elle renforce, en termes d'effets, le sentiment de proximité et elle légitimise la violation des normes usuelles de véracité et de mesure, tout en renforçant la communauté interprétative au sein de laquelle ce discours est perçu comme le seul valide. 
Souligner l'importance de la posture conflictuelle constitue d'autant moins une révélation que la stratégie de la polarisation, «eux» contre «nous », est revendiquée sans détour par les théoriciens de la radicalité qui se réclament explicitement d'un populisme de gauche rénové et assumé, en particulier Ernesto Laclau et Chantal Mouffe (rejoints notamment par Judith Butler et certains responsables politiques en Espagne et en France). Prenant acte de l'épuisement du modèle de la lutte des classes, ce courant envisage «la construction discursive de la division sociale » (Laclau, 2005, p. 231) comme le moyen de rassembler des positions revendicatrices et contestataires hétéroclites dans un front se définissant moins par un programme politique que par son antagonisme fondamental aux partisans du consensus : "La catégorie centrale de la politique démocratique devrait être celle de l" adversaire" » (Mouffe, 2003, p. 149).

Conformément à sa logique, cette optique exige soit l'adhésion soit le dissentiment. Si l'on choisit avec une naïveté obstinée le « camp » de la démocratie délibérative et de l'éthique d'une communication fondée sur l'usage dialectique de la raison, l'impression de se retrancher dans une forteresse délabrée est saisissante, même si l'on s'y retrouve en très bonne compagnie. Certes, le mot d'ordre de Condorcet n'a rien perdu de sa grandeur :

Épuisez toutes les combinaisons possibles pour assurer la liberté ; si elles n'embrassent pas un moyen d'éclairer la masse des citoyens, tous vos efforts seront inutiles. (1971/1847, p. 389)

Mais cette formule peut-elle toujours résumer un projet émancipateur ou doit-on aujourd'hui la lire comme un diagnostic lugubre? Tout ce qu'on a vu jusqu'à présent, même en le nuançant, semble pencher vers la seconde hypothèse. La surabondance toujours croissante de l'offre discursive, la volatilité de l'attention qui en résulte, de même que l'affaissement économique et symbolique de ces instances intermédiaires que les populistes continuent pourtant à conspuer machinalement, paraissent livrer le marché discursif à ceux qui, prévenait Condorcet, s'entendent à "maintenir l'ignorance du peuple pour le maîtriser tantôt au nom des préjugés anciens tantôt en appelant à leur secours des erreurs nouvelles » (ibid.).

D'un point de vue expérimental, ce que l'on sait de la réception différentiée de l'information, c'est-à-dire la tendance à accueillir sans 
recul ce qui est conforme aux convictions et à rejeter tout ce qui peut les mettre en jeu, n'est pas plus encourageant. Dans le même sens, l'«illusion de profondeur explicative », qui donne à chacun le sentiment de comprendre assez bien une situation pour porter un jugement définitif à son égard, constitue un facteur important de polarisation. Contrairement à ce que l'on aimerait croire, ce biais ne touche pas seulement les adolescents ${ }^{11}$ (même s'ils s'y révèlent extraordinairement vulnérables).

$\mathrm{Vu}$, enfin, sous une perspective technocentrique, le paysage discursif ne paraît pas moins dévasté. Les réseaux sociaux, en particulier, s'accordent si bien aux tendances que l'on vient de rappeler (dissolution de l'attention, confinement de celle-ci aux idées qui flattent les croyances $^{12}$, limitation des développements explicatifs...) que l'on pourrait les croire soigneusement mis au point par des psychologues diaboliques. Ce qui serait aussi absurde que de croire que l'industrie pétrolière s'est développée dans l'intention de polluer la planète : outre qu'elle est en partie discutable, et en tout cas ambivalente ${ }^{13}$, la dégradation apparente de la sphère du débat public n'implique pas plus l'intention des nouveaux entrepreneurs des industries culturelles qu'elle n'impliquait auparavant celle de leurs prédécesseurs. Et ce n'est pas dans le but d'étrangler l'institution intermédiaire capitale constituée par les médias d'information que les entrepreneurs des nouvelles technologies ont exercé une prédation aussi implacable sur ses ressources publicitaires: "Ce n'est pas la faute de Google, c'est l'Internet qui est arrivé", répondait ainsi un vice-président de Google aux journalistes, « $\mathrm{Ce}$ sont des choses qui arrivent, et ça va probablement arriver de nouveau » (Codère, 2017, écran 13).

11 Une série d'expériences menées sur des adultes de plus de 30 ans en moyenne a montré une nette modération des positions extrémistes après que les participants aient été invités à expliquer les mécanismes du problème sur lequel ils s'étaient prononcés (par exemple l'aide sociale), puis à évaluer leur niveau de connaissance à son propos (Fernbach, Rogers et al., 2013).

12 Ceci, comme toujours, serait à nuancer (voir notamment Bakshy, Messing et al., 2015).

13 Il serait évidemment absurde de dénier aux réseaux sociaux ce qu'ils se montrent également capables d'apporter à l'expression des idées et au débat démocratique en général. 
D'un point de vue démocratique, les questions d'intention n'ont pas un grand intérêt. En tout état de cause, les efforts ostentatoires mais dérisoires que les géants du numérique, dans le rôle classique du pompier pyromane, disent consacrer dorénavant à la lutte contre les fausses nouvelles ou à la diversification de leurs algorithmes de présélection des contenus ne semblent pas exactement en mesure de protéger le rêve de Condorcet. Mais si l'on tient absolument à attribuer un blâme, la perspective sociocognitive de la valeur discursive rend attentif à une hypothèse dérangeante : les défenseurs des Lumières et de la politique délibérative pourraient avoir à endosser une bonne part de la responsabilité qu'il est si confortable d'attribuer aux nouvelles technologies ou aux nouvelles générations.

On ne peut s'effarer de l'élection de Donald Trump sans remarquer que celle-ci couronne une remarquable progression de présidents républicains dont, depuis quarante ans, chacun (ou presque) semble plus fruste, plus radical et plus dénué de scrupule discursif que le précédent. Or seul le dernier de la série a pu bénéficier des réseaux sociaux. Georges W. Bush est arrivé au pouvoir quatre ans avant que le public ne découvre Facebook ou Twitter. Quant à Ronald Reagan, il n'a même pas pu s'appuyer sur Fox News, pas plus que sur le Web, l'un et l'autre étant apparus plus de dix ans après son élection. Il paraît donc difficile d'interpréter sous un angle technocentrique le fait que tous sont parvenus à rassembler un électorat populaire autour de programmes fiscaux et sociaux assez manifestement défavorables à cet électorat (en tout cas en comparaison des avantages qu'ils promettaient à la fraction la plus aisée du corps électoral).

Condorcet, lui, a une réponse à proposer (les sciences politiques contemporaines en ont aussi, convergentes d'ailleurs, mais superfétatoires dans la perspective de ce chapitre affranchi des contraintes documentaires de l'énonciation scientifique). La rhétorique de tous ces candidats reposait lourdement sur la promesse d'une prospérité nouvelle associée à des schémas cognitifs immuables et inlassablement martelés : le technocrate fédéral liberticide et, surtout, le politicien démocrate dilapidateur. Elle reposait, en d'autres termes, sur une gestion habile de l'«ignorance du peuple ». On sait par exemple qu'au cours de ces quarante années, les démocrates ont invariablement été moins dépensiers que leurs adversaires : sous chacune de leurs présidences, le déficit budgétaire fédéral a été réduit par rapport à celui 
que leur avaient laissé les républicains ${ }^{14}$. Pourtant, quelques semestres avant l'élection de Georges W. Bush, une enquête approfondie avait montré que ce genre de «fait» n'avait pas de poids face à celui des croyances $^{15}$. Alors que le déficit budgétaire avait chuté de 290 à 109 milliards de dollars sous la présidence de Bill Clinton, seuls $10 \%$ des répondants pensaient qu'il s'était réduit au cours des dernières années, $69 \%$ étant au contraire persuadés qu'il s'était creusé. Or, la «mauvaise santé » de l'économie du pays (qui ne se portait pourtant pas si mal), était attribuée prioritairement à ce déficit (77\%), suivi par l'aide sociale $(70 \%)$ et l'aide au développement $\left(66 \%{ }^{16}\right)$. Compte tenu $\mathrm{du}$ résultat très serré des élections, tout porte à croire que dans un pays où le tiers au moins des répondants auraient eu une idée un peu moins fausse de la situation économique ${ }^{17}$, Georges W. Bush n'aurait jamais pu être élu. Pas plus que Donald Trump.

$\mathrm{Si}$, selon la célèbre formule de Woody Allen, "les méchants ont sans doute compris quelque chose que les bons ignorent» (1979, p. 47), cette chose-là est en l'espèce connue depuis des millénaires. Elle ne demande guère aux bons qu'à puiser dans leurs souvenirs scolaires. Les grands registres de l'argumentation, ce qu'Aristote appelle «les preuves inhérentes au discours » ont, comme on le sait, été ramenés par lui à trois dimensions fondamentales : l'ethos, soit les arguments fondés sur la personne de l'orateur, le logos, les arguments fondés en raison, et le pathos, les arguments fondés sur les émotions de l'auditoire. Il n'est pas indispensable de se plonger dans les finesses de la rhétorique classique ou moderne pour remarquer dans les joutes électorales la

14 En pourcentage du produit national brut. Une analyse beaucoup plus fine serait nécessaire pour tenir compte de l'incidence des majorités au Congrès, de celle des circonstances économiques, et du biais propre à l'indice utilisé. Peu importe.

15 Cette série de sondages détaillés était coorganisée par la fondation Kaiser, une équipe de l'Université Harvard (John F. Kennedy School of Government et Harvard Program on Public Opinion and Health/Social Policy) et le Washington Post. Ses résultats ont notamment été exposés dans Kaiser Fondation (1996) et Blendon, Benson et al. (1997).

16 Contrairement aux 250 économistes interrogés par ailleurs, qui étaient respectivement $32 \%, 11 \%$ et $1 \%$ à attribuer une responsabilité à ces trois facteurs.

17 On ne débattra pas ici de la validité intrinsèque des statistiques gouvernementales. 
surexploitation de l'ethos, que ce soit pour établir sa légitimité (je suis un homme nouveau, une femme, un ancien soldat, un fils du peuple, un businessman couronné de succès, un parangon de droiture, une victime, un expert) ou au contraire pour discréditer son adversaire (il est l'opposé). Il n'est pas plus difficile de constater l'instrumentalisation politique des émotions premières (la peur, la pitié, le ressentiment...). Lancienneté même de l'ethos et du pathos dit assez qu'ils ont servi à toutes les époques, pas seulement la nôtre. Et qu'ils ont servi bien des causes, pas seulement les plus exaltées. Mais face à la puissance que l'un et l'autre tirent de leur formidable séduction cognitive (l'effet qu'ils produisent et, surtout, l'effort dont ils dispensent), le registre du logos paraît de plus en plus désarmé : son effet, celui que procurent le doute et la nuance, ne paie pas l'effort cognitif bien supérieur qu'il réclame pour comprendre les tenants et les aboutissants des problèmes complexes des sociétés contemporaines. Quant aux normes discursives qui pouvaient le favoriser, elles sont étouffées non seulement par la désinvolture ingénue des certitudes militantes, mais aussi par le vieux drame de la raison : son incapacité intrinsèque à fonder des valeurs morales (mais non à les soupeser).

C'est n'est cependant là qu'une autre façon, assez banale au fond - et qui appellerait une analyse moins succincte des notions et facteurs en jeu - d'entonner l'air connu de la disculpation fataliste des élites discursives. Que l'on attribue la crise (... de la politique, de la culture, de la presse, etc.) aux nouvelles technologies de communication, à la futilité prêtée aux nouvelles générations ou aux implacables déterminants sociocognitifs de la valeur discursive, le refrain est toujours celui de l'impuissance navrée, celui que sifflotait plus haut un dirigeant d'Internet ("Ce sont des choses qui arrivent»), ou, bien pire, celui que grommellent des producteurs discursifs désabusés (« les citoyens ont les élus qu'ils méritent », «les lecteurs ont les journaux qu'ils méritent»). La faute du consommateur... Un argument de dealer de drogue. Si l'on analyse de façon plus précise l'offre discursive de la plupart des locuteurs « légitimes », la perspective s'inverse : ce sont bel et bien eux qui ont les électeurs et les lecteurs qu'ils méritent. On reviendra un peu plus loin sur les lecteurs. Voyons les électeurs, en s'arrêtant à un cas précis.

Les grandes secousses qui ont ébranlé les intelligentsias étatsunienne, britannique et française, culbutant les deux premières, avaient en commun la dénonciation des coordinations supranationales : les traités commerciaux internationaux pour Donald Trump, les institutions 
européennes dans les deux autres cas. On pourrait facilement remarquer que l'instrumentalisation de l'égocentrisme identitaire est le cheval de bataille du discours populiste, comme l'ont aussi illustré les extrémismes en Flandre, en Italie du Nord et en bien d'autres lieux. Cependant, outre qu'il serait dangereux de tirer de ce symptôme un diagnostic infaillible ${ }^{18}$, le condamner pour ce qu'il est revient à opposer le péremptoire des uns au péremptoire des autres, les valeurs du haut aux valeurs du bas. À combattre, en quelque sorte, le populisme de l'invective par l'infatuation du mépris.

Je n'ai aucune information privilégiée sur les élections étatsuniennes ou le référendum britannique, mais j'avais eu il y a quelques années en France l'occasion ${ }^{19}$ d'étudier de plus près un cas assez similaire, celui du passage à l'Euro. Bien décidés à «arrêter toutes les dispositions qui permettront à chaque citoyen français d'avoir une pleine et entière confiance dans l'euro ${ }^{20} "$, les pouvoirs publics français avaient développé un formidable arsenal de communication, étendu sur plusieurs années et fort de toutes les ressources de la publicité écrite et audiovisuelle (dont une première salve de 25 millions de brochures appuyée par une campagne massive à la radio et la télévision). Les progrès des consciences étaient en outre mesurés attentivement par des sondages semestriels. Cependant, ces derniers révélèrent quelque chose d'assez curieux pour qui ne connaît pas les recherches en communication et d'encore plus curieux pour qui les connaît un peu.

Sur la période statistiquement analysable (avril 1996 à octobre $1998^{21}$ ), les courbes montraient une montée très régulière des connaissances factuelles du public sur les modalités du passage à l'euro, mais pas la moindre progression en termes de confiance et d'adhésion à ce changement. Or, s'il est une chose qu'avaient jusqu'alors

18 En particulier hors du contexte démocratique occidental (à moins de contester que les luttes de l'après-guerre contre les dominations coloniales étaient bel en bien fondées).

19 Je reprends ici des observations financées à l'initiative de Jean-Loup Molin par la Mission prospective de la Courly et publiées dans Une dynamique de l'insignifiance (Presses, de l'Enssib, 2002).

20 Ministère de l'Économie et des Finances (1997). Dossier de presse. Paris : MINEFI.

21 Un changement des questions posées ne permet malheureusement pas de prolonger cette série après le sondage d'octobre 1998. 
enseignée les études, notamment celles menées sur la perception des risques et des choix technologiques, c'est que les jugements sur une question sont étroitement corrélés aux connaissances à son propos (et l'on a vu plus haut qu'un effet du même genre pouvait être observé expérimentalement). L'énorme campagne pour l'euro faisait mentir la règle, sans troubler le moins du monde l'autosatisfaction affichée par ses organisateurs. Cependant, l'étude des discours dont ils bombardaient l'opinion montrait que ceux-ci obtenaient très exactement l'effet que l'on pouvait en attendre. Ils se réduisaient essentiellement à deux types de messages. Le premier se concentrait sur les modalités pratiques du passage à l'euro (Quel sera son taux? Quand entrera-t-il en vigueur? Que faire de ses anciens billets ?). Le second, composé surtout de saynètes télévisées, était une variation inépuisable sur le thème Qu'est-ce que c'est bien l'euro! Qu'est-ce que ça va être amusant! dans lesquelles une gamine espiègle tenait le premier rôle. On peut toujours compter sur l'ethos de la mignonnerie : «Cette prime à la sympathie nous aida sans aucun doute à fédérer les convaincus et les indécis, à rassurer les inquiets, et à atténuer grandement les rejets » se félicite ainsi l'un des concepteurs de la campagne ${ }^{22}$.

À aucun moment, en revanche, ceux-ci ne paraissent avoir songé qu'il pouvait être dans leur propre intérêt de solliciter des facteurs d'effet de plus haut niveau cognitif en intéressant leurs concitoyens aux raisons qui pourraient bien justifier un tel bouleversement monétaire. Vu d'en haut, tout ce que ceux-ci étaient dignes de savoir et capables de comprendre était la marche à suivre et le caractère positif de ce changement. Quant à la montée indiscutable du niveau des connaissances pratiques (opératoires) sur le passage à l'euro, elle doit sans doute quelque chose aux dépenses publicitaires, mais sans doute aussi beaucoup au puissant effet de second niveau (que devrais-je faire de mon argent ?) dont elle aurait bénéficié de toute façon ${ }^{23}$.

Le plus troublant est qu'une telle conception de la communication avait déjà été observée en psychologie sociale (Rimé, 1984) :

22 L'euro, c'était plus facile ensemble ; analyse-témoignage de l'un des concepteurs de la campagne, Yves Deschamps, publié le 17 février 2012 sur le site viadeo.com.

23 Par contraste, les facteurs d'effet de haut niveau n'ont à aucun moment été sollicités, l'essentiel étant de maintenir l'effort de traitement à un niveau extraordinairement bas. 
Voilà un paradoxe qui donne à réfléchir : des individus s'adressent à des gens moins compétents qu'eux sur le plan verbointellectuel ; ils manifestent des efforts adaptatifs qui paraissent tendre à ce que ces gens puissent accéder à la compréhension ; mais dans le même temps, le contenu de ce qu'ils communiquent se restreint et paraît se limiter aux questions opératoires et normatives.

Ce qui donne surtout à réfléchir ici, c'est que cette observation qui paraît s'appliquer si bien à la communication gouvernementale en faveur de l'euro résume en réalité une étude menée sur les rapports entre infirmiers et patients en milieu psychiatrique. Elle synthétise, en somme, ce que veut trivialement dire « parler à quelqu'un comme à un taré ». Y compris lorsque ce quelqu'un est un peuple. Une illustration assez différente de ce point est du reste fournie par la phraséologie de Donald Trump, laquelle recoure lourdement aux énoncés opératoires (Il faut... On doit... Nous allons...) et aux assertions normatives : des contradicteurs sont des «personnes mauvaises » («bad people»), le charbon est «magnifique» (« beautiful »), etc.

Convenons que le cas du passage à l'euro, même s'il est loin d'être isolé, remonte aujourd'hui à deux décennies. Les bergers des consciences ont-ils depuis lors appris quelque chose? La question, très actuelle, de la justification des traités commerciaux internationaux permet d'en douter. Elle permet incidemment à chacun d'improviser sa propre expérience introspective sur l'«illusion de profondeur explicative » ou d'y soumettre son entourage : qui est donc capable d'expliquer pourquoi au juste ces accords sont généralement considérés comme une «bonne chose ${ }^{24} »$ ? Elle pourrait du même coup

24 En ce qui me concerne, j'ai été consterné de réaliser un beau jour que si je distinguais de plus en plus de raisons de s'y opposer (dont les insupportables clauses d'arbitrages et autres dispositions empoisonnées), je n'avais au fond aucune idée précise de ce qui pouvait bien justifier de tels accords, au-delà de la confiance que j'accordais depuis l'école à des affirmations vagues (ils favorisent la prospérité de tous) et à des locuteurs censément compétents. Il ne m'a fallu que quelques lectures pour refonder plus ou moins cette relative confiance - sans l'étendre en rien aux clauses susdites -, mais il me faudra plus de temps pour me prendre à nouveau pour un observateur éclairé et sceptique du débat public. Du moins en ai-je tiré un bon motif de réflexion pour mes étudiants en journalisme. 
permettre aux responsables et praticiens du débat public de mesurer le peu d'importance qu'ils accordent à la santé de celui-ci. Sans soutenir un instant que les dogmes économiques doivent constituer la source exclusive de toute raison politique (et encore moins méconnaître l'extraordinaire fragilité de ces dogmes), tout porte à croire que la capacité des électeurs à évaluer les propositions qui s'affrontent demeure une étonnante zone d'ombre de la pensée et de l'action politique.

En France, «phare de la démocratie » comme on ne perd jamais une occasion de le claironner, les dernières données sérieuses sur ce que les citoyens comprennent des discours économiques datent de près d'un demi-siècle : elles montraient par exemple (en 1971) qu'à peine $40 \%$ des personnes interrogées pouvaient donner le sens du terme " inflation » ou en indiquer un vague équivalent (" la vie chère ", «les prix qui montent»), et que moins de $20 \%$ appréhendaient, même approximativement, les notions de " charges sociales » et de « dévaluation» (C.E.R.C., 1971). Quelques sondages plus récents, quoique discutables dans leur méthode (choix entre trois réponses, soit une probabilité de $33 \%$ d'avoir raison au hasard) et inexploitables dans leur présentation, ne démentent pas ce constat.

Mais si la carence des enquêtes approfondies sur la compréhension du public en dit long sur l'importance accordée à cette question, le risque de ce genre de données serait à l'inverse de renforcer la vision de la société dont elles sont en partie la conséquence, celle d'une masse ignare, peuple enfant qu'il appartient aux grandes personnes de guider. Une telle perception avait déjà été mise en évidence il y a plusieurs décennies dans un autre secteur du marché discursif, celui de la culture scientifique, question dont l'importance civique n'a pas besoin d'être soulignée : comment, par exemple, débattre des OGM lorsque $35 \%$ à peine des répondants savent que les tomates ordinaires ont aussi des gènes? (INRA Europe, 2000).

S'inscrivant dans la tradition engagée et volontiers généralisante, mais souvent instructive, des études critiques, la longue liste des reproches adressés aux discours vulgarisateurs visait plusieurs points également dénoncés dans les discours économiques, en particulier leur tendance fréquente à réifier l'état des connaissances (en présentant des hypothèses comme des faits acquis) et l'idéologie dont ils seraient souvent les vecteurs (celle du progrès technoscientifique 
dans le cas des sciences dites exactes). Cependant, d'autres observations critiques du discours de vulgarisation pourraient tout autant éclairer le discours économique, et, au-delà de lui, le discours politique en général. C'est en particulier le cas de ce qu'on a appelé le modèle de déficit, en vertu duquel le public est perçu comme déficient par rapport à ce qu'il « devrait savoir », les connaissances en question étant précisément celles que l'évaluateur estime légitimes, voire requises pour une personne «normale» (ainsi les physiciens déploreront-ils la méconnaissance de la physique, les hellénistes la méconnaissance du grec ancien, etc.). Un problème proche, quoique distinct, est ce que l'on pourrait appeler le modèle des outres vides, c'est-à-dire la perception de la foule profane comme un ensemble de récipients qui ne demanderaient qu'à s'emplir de bons savoirs dès lors que l'on simplifierait suffisamment ceux-ci. Ces deux optiques « pédagogiques » ont accompagné au $\mathrm{xx}^{\mathrm{e}}$ siècle, la multiplication des instances de diffusion culturelle (centres de sciences, sites Internet...), dont on ne peut que se féliciter, mais qui tendent essentiellement à toucher les bons élèves du corps social, ceux, justement, qui fréquentent les musées et goûtent les contenus culturels à la télévision ou sur Internet. D'où, peut-être, un étrange retour de balancier, celui qui a conduit bien des musées à tout miser sur la découverte spontanée (si possible interactive) voire, dans la crainte d'ennuyer, à abolir les explications que fournissaient les cartels imprimés.

Or, ce que l'on a vu de l'évolution du marché discursif et de celle de la désagrégation de la consommation culturelle suggère de réévaluer ces deux présupposés.

1. Les discours réputés élevés (sciences, politique, culture...) peuvent de moins en moins compter sur les privilèges attentionnels que pouvait jadis leur valoir la noblesse attachée à leur objet. On peut le déplorer autant que l'on voudra, mais la prescription sociale qui assurait leur convenance normative (il est mal d'ignorer ceci ou cela) ne suffit plus à fonder leur valeur discursive. Et avant d'accuser la frivolité juvénile des internautes, on ferait bien de se souvenir qu'une ministre de la culture française n'avait éprouvé aucun embarras à dire qu'elle n'ouvrait pas un livre, ou qu'un récent Premier ministre canadien ne s'était pas non plus signalé par son amour de la culture 
littéraire (ni scientifique, du reste). Pour ne pas ajouter à ces repères anecdotiques la liste entière des présidents républicains évoqués plus haut. Entre autres.

2. Les citoyens ne sont pas des outres vides : leur esprit est au contraire aussi plein de connaissances qu'il est possible de l'être, notamment de connaissances spécialisées dont l'érudition légitime ignore tout. Celles du Premier ministre canadien étaient impressionnantes dans le domaine du hockey sur glace ${ }^{25}$, et même Donald Trump sait sûrement quelque chose à propos de quelque chose (au minimum les concours de mannequins et les vedettes de télé-réalité). Or, la tentation d'écarter ces « connaissances » concurrentes comme de la pacotille, quoique la plupart d'entre elles le soient en effet, renvoie à l'impasse précédente : celle d'une hiérarchisation unilatérale dont la légitimité est de moins en moins évidente.

Si l'on ne peut plus fonder la valeur des discours ci-devant nobles sur la convenance normative, peut-on au moins renforcer leur pertinence cognitive en réduisant l'effort qu'ils réclament (et en renforçant si possible leur caractère distrayant ou pratique) ? Sur ce point se rejoignent, entre autres, les stratèges en communication et un bon nombre de muséographes ${ }^{26}$. Cependant, une bonne vingtaine d'années passées à dépouiller les travaux sur la production et la réception de l'information m'oblige à signaler un élément qui pourrait surprendre une bonne partie de la noblesse discursive : les gens ne sont pas idiots. Son fondement est surtout négatif : je n'ai jamais croisé d'indice théorique ou empirique pouvant réellement accréditer l'idée d'une infériorité foncière de "l'homme de la rue » en termes de capacités intellectuelles. En revanche, j'ai croisé beaucoup de traces plus ou moins explicites de cette croyance. Dans les manuels techniques de communication, par exemple, où une expertise aux racines introuvables conduit rituellement à imaginer «le lecteur»

25 Il est, du reste, membre de la «Société internationale de recherche sur le hockey » et auteur d'un livre paraît-il érudit sur l'histoire de ce sport.

26 "Les longs cartels imprimés (la malédiction des expositions éducatives) ont été éliminés dans ces présentations, de même que tous les mots techniques. L'exposition interprétative devrait montrer plutôt que dire » (Kuh, 2001, p. 53). 
comme une créature volage et bornée. Son vocabulaire serait de 1500 mots affirment certains, 3 ooo mots avancent d'autres... Il est au moins dix fois plus élevé selon la littérature scientifique disponible (Labasse, 2001). Les jeunes, comme on s'en doute, ne sont pas plus estimés. Un des manuels de journalisme les plus diffusés rapporte ainsi les résultats affligeants d'« une expérience réalisée dans un lycée, avec des élèves sortant d'une classe de troisième ». En l'absence de référence bibliographique, j'avais tenté de reproduire ces résultats dans deux établissements différents (Labasse, 2006). Même en recourant au protocole le plus sévère que je puisse imaginer, la moyenne observable, sans être fameuse, était trois fois plus élevée que celle indiquée $(33,5$ contre 11,6).

Étant affligé de trois enfants, j'aurais a priori de bonnes raisons de me convaincre que les générations suivantes ne valent pas la mienne. Mais pas au point de les prendre, toujours par exemple, pour les machines à zapper que décrivent les consultants, selon qui l'attention de la génération $X, Y$, ou $Z$ (étiquettes mystérieuses) ne dépasserait pas quelques minutes. Dialoguant avec l'un de ces «spécialistes » à l'occasion d'une table ronde, j'avais ravalé in extremis un réflexe bien naturel d'arrogance universitaire ("qu'est-ce qui vous permet donc d'affirmer ceci ?») pour tenter de m'instruire auprès d'une source si assurée : pouvait-elle m'aider à comprendre pourquoi, dans ce cas, tant de jeunes pouvaient rester interminablement captivés par un jeu vidéo, un roman de sorciers ou une série télévisée? Elle ne pouvait pas.

On se gardera bien ici de passer de l'image, infondée, d'une masse obtuse à celle, exaltée, d'un peuple spontanément brillant et concentré : quelle que puisse être mon opinion (contrastée) sur la question, réhabiliter qui que ce soit ne fait pas partie de mon programme de travail, d'autant que bien d'autres et non des moindres (de Certeau, Hall, Hoggart...) l'ont fait depuis longtemps. En revanche, examiner les conséquences discursives d'une apparente sousévaluation des capacités de «l'homme de la rue » s'inscrit mieux dans celui-ci. Or, le schéma d'un destinataire irrémédiablement benêt entraîne effectivement de sérieux problèmes.

Le premier est l'abandon plus ou moins perceptible de l'ambition de signifier. On se souvient de la formulation nietzschéenne de la pertinence : "plus abstraite est la vérité que tu veux enseigner, plus tu dois 
en sa faveur séduire les sens » (en d'autres termes : "plus la vérité que tu veux enseigner réclame d'effort cognitif, plus tu dois en sa faveur produire d'effet cognitif »). Mais sa version infantilisante - celle, par exemple, de la campagne en faveur de l'euro - devient «plus abstraite est la vérité que tu veux enseigner, plus il te faut à la place séduire les sens ». Quoiqu'elle ressemble apparemment à la première, elle en est l'opposé : le moyen se substituant insensiblement à la finalité (si ce n'est pour des messages opératoires).

Le second est la tentation d'imposer par la coercition discursive ce qu'on sent incapable d'assurer par la pertinence argumentative. D'où, en France, l'interdiction de la consultation des sites terroristes, la large proscription des propos discriminatoires ou encore celle des discours négationnistes et même l'élaboration récente d'une loi contre les fausses informations. Au début des années 2000, un juriste proche du Syndicat national de l'édition évaluait déjà à près de 400 les textes légaux restreignant la liberté d'expression dans ce pays (Le Naire, 2002). De même en Espagne, à la suite d'une « loi du bâillon » (Ley mordaza) extraordinairement vague votée en 2015, "des dizaines d'utilisateurs ordinaires des réseaux sociaux ainsi que des musiciens, des journalistes et même des marionnettistes ont été poursuivis en justice pour des raisons liées à la sécurité nationale. Cela a eu pour effet de créer un climat de crainte, les gens hésitant de plus en plus à exprimer des opinions différentes ou à faire des plaisanteries prêtant à controverse » (Amnesty International, 2018).

Rappeler ce que le formatage législatif des déterminants normatifs de l'adéquation a d'artificiel n'est pas une tâche agréable. D’abord parce que ces prohibitions visent souvent des discours profondément répugnants, si douteuse que soit cette justification (la même logique qui proscrit en France la négation de la Shoah avait criminalisé en Pologne les rappels historiques de la participation polonaise à celle-ci, la répression de l'« apologie du terrorisme » sert en Turquie à emprisonner des journalistes démocrates, etc.). Ensuite parce qu'il est assez inconfortable de se retrouver simultanément favorable à la régulation du marché financier et à la liberté du marché discursif (non pas illimitée, mais telle que l'assure par exemple le premier amendement de la constitution états-unienne, fruit des Lumières s'il en est).

On se bornera donc à remarquer que - dans les pays démocratiques - cette propension à régenter le dicible et le recevable dénote 
en tout état de cause une très faible confiance vis-à-vis du débat public et du public lui-même ${ }^{27}$. Elle illustre à nouveau un biais bien connu, l'« effet de troisième personne » (Davison, 1983), en vertu duquel chacun croit que les autres sont beaucoup plus manipulables qu'il ne l'est lui-même. Mais si l'efficacité de telles mesures est délicate à évaluer dans l'absolu, du moins ont-elles une qualité indéniable, celle d'être simples à concevoir et peu coûteuses à mettre en œuvre, ce qui les qualifie admirablement pour le «syllogisme du politicien » (Lynn et Jay, 1989) : «Il faut faire quelque chose. Or, ceci est quelque chose. Donc il faut faire ceci $\gg$. On comprend que face à un tel attrait, celui de l'éthique communicationnelle d'Habermas - laquelle requiert «pour tous les participants la possibilité [...] de faire valoir leurs propres arguments à égalité de chance » (1986, p. 111) - soit de peu de poids. Il est vrai qu'elle demande sensiblement plus d'effort de réflexion et produit moins d'effet utilitaire.

Un troisième problème, moins évident mais tout aussi sérieux, réside dans la dégradation obstinée de la valeur discursive qu'entraîne la dévaluation des destinataires.

D'une part, comme on l'avait remarqué plus haut à propos de la faible séduction que les livres pour enfants exercent sur les adultes, les bénéfices que l'on peut retirer d'une réduction de l'effort cognitif ne sont pas linéaires : si celle-ci est capitale lorsqu'il s'agit d'éclaircir des contenus abscons, son profit est négligeable, voire négatif, à partir d'un certain degré d'intelligibilité. On pourrait le comprendre en ne considérant que le facteur cognitif le plus bas : la légibilité. Si un texte dont la typographie est minuscule est d'une lecture pénible ou impossible, le composer en caractères gigantesques serait tout aussi absurde. Or, il en va de même pour le reste des facteurs, depuis la lisibilité, dont le gain est nul en dessous d'une certaine charge syntaxique,

27 On simplifie (comme toujours), notamment en se réduisant à l'information des citoyens adultes, alors que celle des enfants peut facilement justifier un encadrement prononcé, au risque que l'accumulation des initiatives législatives et sectorielles provoque un empilement assez confus de normes, de cadres réglementaires et d'instances régulatrices. Le Canada fournit un bon exemple de cette complexité normative (détaillée par Caron et Cohen, 2013), d'autant qu'à la protection de l'enfance s'ajoute d'autres impératifs, comme celui d'y préserver la langue française, y compris dans la musique populaire (Grenier, 1993). 
jusqu'à la construction d'un modèle mental, que rend difficile une énonciation trop vague.

D'autre part, le processus de simplification opère généralement au détriment de la substance. Là encore, cette opposition n'est pas linéaire. Comme l'ont montré tant d'expériences, clarifier un discours embrouillé, verbeux ou abstrait renforce considérablement sa valeur. Mais prolonger ce traitement affecte de plus en plus sévèrement ce contenu, d'abord les précisions et les nuances, puis les idées ellesmêmes. Et il en vient du même coup à détruire sa pertinence, $l^{\prime}$ « homme de la rue » étant, pour autant qu'on sache, tout à fait capable de détecter le peu de profit cognitif que camoufle si mal la jovialité affectée ou le moralisme paternaliste d'un discours creux.

On rejoint au bout du compte par un chemin un peu plus technique le point évoqué plus haut (la renonciation à signifier), mais cet aspect technique, justement, aide à percevoir un autre point : la représentation du destinataire-enfant justifie opportunément l'incapacité d'une grande partie des communicateurs à produire de l'intérêt mettant en jeu les facteurs cognitifs de haut niveau. Ceux-ci sont aussi universels que les autres, mais leur stimulation suppose une réelle compétence discursive (le même genre de compétence qui permettrait d'évaluer où se situe le degré - variable - à partir duquel la simplification devient contreproductive) et un réel désir de signifier.

Pour tempérer ce qu'une telle généralisation a d'outrancier, on mentionnera un seul contre-exemple, à charge pour lui d'en représenter d'autres. Ce sera, disons, celui du héros anonyme de la SNCF qui, vers la fin des années 2000, persuada son institution de s'écarter un peu du style prescriptif-normatif avec lequel elle s'adressait traditionnellement à ses voyageurs-enfants. Alors que des intempéries hivernales prévisibles provoquaient des retards inouïs, les voyageurs eurent la surprise d'obtenir un dépliant qui se donnait la peine de leur expliquer, schémas à l'appui, pourquoi quelques centimètres de neige exigeaient bel et bien que l'on ramenât la vitesse des TGV à celle des trains à vapeur. Mais si ce document représentait un bond prodigieux par rapport à l'abstraite condescendance habituelle (« Pour votre confort et votre sécurité, votre train aura deux heures de retard»), il était techniquement facile à concevoir puisque sa valeur discursive était au préalable assurée par un vif désir d'explication : l'exploit était surtout de s'être avisé de celui-ci. 
Les choses sont en revanche un peu plus délicates lorsque cette envie de connaître doit être suscitée ou entretenue, mais les façons d'y parvenir sont multiples. Comme il n'est pas question de convertir tout à coup ce livre en traité de rédactologie, il suffira de souligner qu'elles ne se trouvent pas sur une liste de recettes, mais plutôt dans une approche raisonnée de l'interaction communicationnelle en contexte de vive concurrence attentionnelle. Comme le remarque Céline Beaudet (1999, p. 6),

le savoir-faire du rédacteur ne saurait se dissocier d'un savoir sur les conditions linguistiques d'émergence du sens dans le discours. Dans notre perspective, le rédacteur n'est un professionnel responsable de ses productions que s'il est conscient de la complexité des choix qui s'offrent à lui.

On aura fait la moitié du chemin vers le professionnalisme en substituant à l'image du destinataire indigent celle d'un destinataire apte à comprendre, mais résolument indifférent, tels que nous le sommes tous face à la majeure partie des «connaissances » hétéroclites que nous prodigue sans cesse le marché discursif (pour qui veut s'en assurer, le dispositif expérimental peut se réduire aux cartes de Trivial pursuit ou d'un jeu équivalent, pourvu qu'il soit récent ${ }^{28}$ ).

Que des connaissances soient supérieures à d'autres, j'en conviens volontiers (j'en suis même persuadé). Mais la responsabilité de leur valorisation incombe à ceux qui le pensent et à eux seuls. Et si c'est la raison publique que l'on veut défendre, cette raison commence par l'hypothèse que, contrairement à un arbitraire « devoir de savoir ", l'ignorance des discours insignifiants est parfaitement rationnelle.

Une hypothèse similaire a du reste été soutenue sous d'autres angles de vue par des spécialistes des choix économiques ou politiques (voir les "Précisions» infra). Il n'est cependant pas indispensable d'invoquer les théories des choix pour noter qu'il pourrait être plus rationnel de regarder un film d'action que de s'infliger - sous l'emprise de la distinction - certaines émissions d'actualité culturelle

28 La pléthore de noms propres inconnus et d'allusions incompréhensibles pour les plus de quarante ans fait mieux mesurer la fuite des années que les cheveux blanc dans le miroir. 
(celles qui, à l'instar du journalisme mondain de jadis, se réduisent à un chapelet soporatif de descriptions complaisantes et de ravissements candides).

Quoi qu'il en soit, la question est moins de savoir s'il est, pour le public, raisonnable ou non de s'intéresser à ceci ou cela que de savoir s'il est, pour les producteurs discursifs traditionnels, raisonnable de s'interroger sur les conditions de la valeur sociocognitive de leurs propres discours. Ceci changerait-il quelque chose à l'évolution du marché discursif ? Il n'existe aucune base empirique assez solide pour pouvoir l'affirmer (à moins d'être futurologue, auquel cas on n'en a pas besoin de toute façon), mais il existe des raisons de le penser. En particulier dans le cas le plus inquiétant de dégradation spontanée de la valeur discursive, celui qui affecte justement l'instance intermédiaire censée donner du sens et de l'intérêt au débat public : les médias d'information.

Attaqués de toute part, sur leurs ressources publicitaires par les prédateurs numériques, sur leur légitimité par les zélateurs d'une faction ou d'une autre, sur leur identité professionnelle par les prophètes des nouvelles technologies, sur leur part d'attention par les fournisseurs de distractions, et souvent sur leurs moyens éditoriaux par des actionnaires à courte vue, les professionnels des médias d'information n'ont pas besoin, en plus, qu'on leur attribue une part de responsabilité dans la crise qui les frappe. Et pourtant...

On ne parle pas ici de la responsabilité qui découlerait des déficiences, si souvent dénoncées, du processus journalistique (erreurs, complaisance ou agressivité, suivisme, etc.) : chercher à soupeser ce qu'il y a tantôt de fondé et tantôt d'outrancier dans ces accusations récurrentes dépasserait de beaucoup l'objet de ce livre et l'intérêt que j'accorde à mes propres opinions dans un débat dominé par les convictions. Notons cependant que la promesse autojuridictionnelle du journalisme - ce par quoi il s'engage à valoir - est particulièrement floue en France et remarquablement peu défendue par ceux qui en vivent ${ }^{29}$ : indépendamment de tout jugement moral, une

29 Le bidonnage, par exemple, y jouit encore d'une indulgence déconcertante pour des non latins, et la partialité demeure considérée comme une vertu journalistique par un certain nombre de praticiens de la vieille école et de novices ardents (les mêmes, souvent, pour qui l'idée de 
question en jeu est celle de la «valeur ajoutée » du journalisme professionnel, c'est-à-dire les raisons pour lesquelles son offre discursive pourrait se prétendre préférable à la nuée des discours excitants ou exaltés offerts sur Internet (sans compter qu'on y trouve également des textes remarquables). L'ennui n'est pas seulement qu'évoquer ainsi le journalisme « en général » amalgame des pratiques, des postures et des compétences professionnelles extrêmement différentes. L'ennui est surtout que celles-ci sont bel et bien amalgamées dans la dévaluation du discours journalistique par le public, l'impéritie des uns disqualifiant la production de tous. Or, l'impensé libertarien de la profession, entretenu par ceux, patrons ou militants, qui y trouvent leur intérêt, s'oppose à toute réflexion interne sur les critères génériques pouvant justifier une quelconque valeur propre à l'offre journalistique, et plus encore sur les moyens collectifs de les garantir aux consommateurs d'information. Il est vrai que cet engagement autojuridictionnel, à défaut d'être attaché à une masse discursive indéfinie, l'était du moins à un organe d'information à une époque où l'on savait plus ou moins ce que l'on achetait. D'où la perplexité que l'on peut éprouver devant l'acharnement avec lequel tant d'éditeurs, tenaillés par la crainte de rester en arrière sur la voie de la nouveauté, s'emploient à banaliser leur offre en la mélangeant indistinctement avec la copie d'agences disponible partout - ce qui est en soi une très mauvaise idée ${ }^{30}-$, mais aussi avec des blogues d'amateurs difficiles à distinguer graphiquement des articles de la rédaction (pour ne pas parler de la « publicité native » qui, en mimant l'apparence de ceux-ci, parachève le processus).

recouper une information reste une fantaisie exotique), d'où la sévérité un peu condescendante des spécialistes du journalisme comparé pour qui : « la professionnalisation du journalisme [en France] est assez faible, dans la mesure où le journalisme n'est pas différencié de façon particulièrement forte de l'activisme politique » (Hanitzch, 2009, p. 421).

30 Curieusement, l'une des études qui montrent l'effet de cette multiplication d'articles similaires sur la désaffection des jeunes internautes (Blinkoff, 2008) a justement été commanditée par une agence de presse, en l'occurrence Associated Press. On notera par contraste que certains journaux nord-américains veillent à préciser l'édition et la page à laquelle leurs articles en ligne ont été publiés, non pour plaire aux documentalistes, mais parce qu'il s'agit d'indices de valeur précieux, surtout à une époque où le moindre poulet en barquette est constellé de labels d'origine et de sceaux de qualification. 
Une telle indifférence s'explique peut-être par la croyance désabusée selon laquelle la qualité des articles est aujourd'hui sans effet sur leur valeur. Cette thèse a du reste été explicitement soutenue, à l'aide d'analogies frappantes (les compagnies aériennes low cost, la chute de Kodak...) et de beaucoup d'assurance, par un institut de presse nord-américain, lequel a appelé les éditeurs à se replier sur le "good enough", c'est-à-dire la production de l'information la moins onéreuse possible restant néanmoins consommable. Malheureusement, ou heureusement, une dizaine de recherches nettement plus approfondies menées en Amérique comme en Europe sont parvenues à des conclusions inverses par des méthodologies très variées $^{31}$ (mais dont, à dire vrai, aucune n'a songé à exploiter le pouvoir analytique des analogies managériales). Quelle que soit la façon selon laquelle on l'évalue - ce qui est un problème captivant dès que l'on se dégage des a priori moralisateurs - la qualité journalistique apparaît, indépendamment de ses enjeux civiques, comme un atout commercial majeur. Pour sa part, l'étude (évoquée plus haut) qui avait été commanditée par la Fédération nationale de la presse française n'abordait pas la qualité journalistique en tant que grandeur objective, mais en tant que valeur perçue par 30 ooo répondants à partir de huit critères d'appréciation (intérêt, fiabilité, vivacité, style...). Or, cette perception s'est avérée plus fortement corrélée avec l'évolution individuelle de la consommation d'information quotidienne que n'importe laquelle des nombreuses autres variables examinées - y compris les suspects habituels : âge, niveau d'étude et usage d'Internet - à la seule exception des habitudes parentales (Labasse, 200832).

Cette corrélation statistique ne montrant pas dans quelle mesure l'appréciation qualitative pouvait être la cause (ou la conséquence) des habitudes de lectures autodéclarées, une seconde analyse a comparé séparément la perception de la valeur de chaque quotidien national et régional français à l'évolution pendant cinq ans de sa diffusion payante, et ce, en distinguant l'appréciation de ses lecteurs et de ses non-lecteurs. Pour les uns comme pour les autres, la coïncidence entre ces appréciations et la santé commerciale des journaux concernés était éclatante. Mais moins étonnante que le peu d'intérêt manifesté par

31 Voir la synthèse publiée sous le titre La «qualité » est-elle l'avenir de la presse? par le CNDI (2003, p. 1-8).

32 On en trouvera l'essentiel condensé en 8 pages dans CNDI (2008). 
bien des responsables de journaux, notamment régionaux, pour les résultats qu'ils avaient financés. Il semblerait qu'une étude suggérant que la crise de la presse relevait moins d'une fatalité générationnelle ou technologique que de problèmes de routine éditoriale et de valeur ajoutée journalistique en contexte ultraconcurrentiel ne délivrait pas exactement le message attendu par les gestionnaires. De fait, d'autres études, plus restreintes mais plus techniques, menées au sein de la même structure (et vers la même époque) sur le contenu de quelques journaux ${ }^{33}$ n'ont pas permis d'y déceler de traces d'un effort concerté de consolidation de leur valeur discursive. Même le dogme le plus rudimentaire de l'écriture journalistique, celui de la lisibilité lexicosyntaxique (voir plus haut), témoignait de cette désinvolture. Non seulement parce que celle des journaux en question se situait en moyenne entre celles de Flaubert et de Chateaubriand (se rapprochant pour l'un d'eux de celle de Durkheim), mais aussi et surtout parce qu'elle variait prodigieusement d'un article à l'autre au sein de chaque titre, montrant que les cadres éditoriaux s'en remettaient aux compétences individuelles de leurs rédacteurs et à leur désir de signifier. Pour en rester au b.a.-ba de l'énonciation journalistique, un autre symptôme frappant (bien que des contraintes budgétaires n'aient pas permis de le quantifier) était la dissymétrie qui touchait les éléments traditionnels de $l^{\prime} \ll$ attaque récapitulative » (ou summary lead). Si les articles veillaient le plus souvent à indiquer qui a fait quoi, quand et où, les deux autres questions rituelles, pourquoi et comment, étaient en général peu ou pas traitées. Il est vrai que la capacité à interroger le réel (pourquoi ?) et à l'expliquer (comment?) a peu d'importance lorsque l'on compte sur la pertinence spontanée des nouvelles pour produire de l'intérêt. Mais elle pourrait en avoir beaucoup si l'on réalise qu'un nombre croissant de lecteurs ont mieux à faire que de savoir qui a fait quoi dans le monde politique, économique ou culturel.

D'un autre côté, ces observations montreraient, s'il en était besoin, que bien des journalistes nacceptent pas passivement cette indifférence : la ténacité d'une partie d'entre eux se devine par exemple aux grandes variations d'adéquation cognitive (lisibilité, problématisation...) remarquées plus haut. Comme le soulignait déjà Socrate, "un tempérament intuitif, intrépide et très doué pour les relations "

33 Lesquels, quoique tous consacrés à l'actualité générale, ne sont pas nécessairement représentatifs de l'ensemble. 
peut dans une certaine mesure compenser la défaillance de l'expertise communicationnelle (les textes de certains professionnels suggèrent même que ceux-ci «sentent» si bien les déterminants de la valeur discursive qu'ils n'auraient pas grand-chose à apprendre d'une modélisation analytique ${ }^{34}$ ).

Mais ce qui est vrai pour un individu, ou même pour un organe d'information (l'un de mes quotidiens préférés témoigne par exemple d'une réflexion éditoriale très poussée), ne l'est pas pour un secteur économique ou une profession dans leur ensemble. Or, face à la crise attentionnelle qu'ils rencontrent, leurs efforts présentent de curieuses similitudes avec ceux des responsables politiques et administratifs rencontrés plus haut.

La méconnaissance du public, d'abord, qui surprendrait sans doute ledit public, mais qui est l'un des secrets les moins gardés qui soient chez les chercheurs et même les professionnels dont l'étonnante mécompréhension de leurs destinataires a souvent été observée ${ }^{35}$.

34 De même, 40 ans de recherches expérimentales sur les processus cognitifs des rédacteurs experts et novices ont montré que les premiers font généralement d'instinct ce que les autres peinent à envisager. Pour n'en mentionner qu'un exemple, les premiers réorganisent profondément les éléments fournis dans une documentation source tandis que les seconds tendent plus ou moins à les reproduire dans l'ordre où ils les ont reçus. Outre les témoignages livresques de plusieurs journalistes et cadres éditoriaux, toutes les recherches menées au sein des entreprises de presse ont montré la même ignorance surprenante des attentes et aptitudes de ceux pour qui elles taillaient leurs discours, souvent réduits à une figure féminine archétypique (la «veuve de Romorantin » en France, une «petite fille de 12 ans » aux États-Unis, « Madame Koulibali » en Afrique). Ma dissection de 50 manuels de rédaction a constaté la même nébulosité chez les formateurs. Quant aux expériences menées par Valerie Crane (1992) auprès de professionnels de l'information et de spectateurs ordinaires à qui elle avait demandé d'évaluer en temps réel l'intérêt d'un montage de sujets télévisés, elles ont révélé des appréciations si différentes que la chercheuse dit avoir eu l'impression de ne pas leur montrer les mêmes séquences. De même, une étude dans dix pays coordonnée par Cohen et Shoemaker a mis en évidence « une déconnexion » entre ce que le public et les journalistes jugent mériter d'être rapporté : «dans certains pays, il y avait même une relation [statistique] négative » entre ces appréciations (Shoemaker, 2006). Or, en l'absence d'une réelle expertise dans ce domaine, les astuces utilisées pour 
La tentation normative, ensuite qui incite, à partir d'une position perçue comme supérieure, à dire à ce public ce qu'il doit penser du monde (ceci est bien, ceci est mal, eux sont les gentils, eux sont les méchants) plutôt qu'à lui fournir les moyens de le penser. Comme l'a vigoureusement souligné Marcela Iacub :

Les journalistes et les intellectuels qui s'expriment dans les journaux de gauche se sentent investis d'une fonction civilisatrice envers les masses. Ils croient que ces dernières ont besoin d'être guidées dans la vie sociale, politique et culturelle. [...] Et si les masses auxquelles ils s'adressent n'achètent plus cette presse-là, il faut dire que c'est la faute d'Internet, des tablettes, etc. Comme si le papier était le coupable et non pas ce qu'on y lit. [...] Ce faisant, outre devenir des piètres affaires pour les investisseurs, ces journaux ne sont pas capables de remplir leur rôle dans un régime démocratique : celui de rendre possible le débat d'idées, entier et sans retenue. (2014, p. 37)

Cependant, le renoncement au journalisme de chaire, qu'il soit progressiste ou conservateur, est non seulement inimaginable pour ceux des producteurs les plus convaincus de l'importance de leurs propres opinions, mais aussi - pour tous - nettement plus exigeant et plus onéreux en termes de temps et d'effort journalistique (le fait que certains s'y astreignent néanmoins témoigne à nouveau de l'opiniâtreté de l'ethos journalistique). Faut-il préciser que l'on ne parlait ici que des articles d'information et non des textes d'analyse ou de plume (éditoriaux, chroniques) qui contribuent également à la valeur discursive d'un journal ?

Enfin, la prédilection de beaucoup de hiérarchies pour les solutions facilement concevables et les contenus facilement recevables. Elle a même conduit une équipe canadienne (Charron et de Bonville, 1997) à avancer, il y a vingt ans ans déjà, l'hypothèse discontinuiste d'un basculement entre le "journalisme d'information » antérieur et un «journalisme de communication», qu'elle caractérisait notamment par un effacement de l'objectivité, une culture promotionnelle et

orienter la production des nouvelles (focus groups, statistiques de pages vues ou partagées, etc.) favorisent des erreurs d'interprétation d'autant plus dangereuses qu'elles ne se manifestent qu'à long terme et qu'elles correspondent exactement à ce que les responsables souhaitaient entendre. 
conversationnelle, une célébration du direct et de l'information immédiate et une prépondérance des sujets d'intérêt privé ou distractifs dans un contexte d'« hyperconcurrence " (Brin, Charron et al., 2004). Si les nombreux symptômes empiriques qu'elle décrivait n'ont guère été contestés, cette thèse importante a suscité un intéressant débat (Mathien, 2001 ; Gauthier, 2010), portant sur l'analyse théorique de ces phénomènes (question hors sujet ici), mais aussi sur la rupture symbolique qu'ils étaient supposés traduire, beaucoup d'observateurs - dont je suis - répugnant à envisager que l'information puisse ne plus être la visée définitoire du journalisme.

Mais, si attaché que je demeure à l'idée selon laquelle le journalisme est par essence différent de la communication, il est un peu perturbant de remarquer que les symptômes du "journalisme de communication » ressemblent fort à ce qui pourrait similairement être décrit dans le cas de la culture artistique ou scientifique comme une «muséologie de communication» ou, en politique, comme une « politique de communication ». Au sein d'un marché discursif chaotique, les mêmes causes - surabondance, délégitimation, réduction des coûts et délais de production, sous-évaluation des destinataires, hégémonie de la nouveauté technologique et, surtout, crise attentionnelle - paraissent produire les mêmes effets. Au bout du compte, il n'y a peut-être pas là de contradiction. Pas plus qu'il n'y en a forcément dans le fait que, à l'université, des étudiants en communication et en journalisme puissent apprendre des techniques de rédaction similaires, souvent confiées aux mêmes intervenants. C'est que d'un point de vue pratique, toutes les productions discursives relèvent plus ou moins des mêmes conditions de pertinence cognitive alors que leurs critères de convenance normative (que l'on espère enseignées dans des cours divergents) sont en bonne partie spécifiques à ces champs. En d'autres termes - même si je n'aime décidément pas beaucoup cette idée - le journalisme est bel et bien, et depuis toujours, une pratique communicationnelle, sans qu'il en résulte nécessairement qu'il soit assimilable à « la communication ».

Sous cette perspective, on remarque que certaines des évolutions prêtées au journalisme sont extradiscurisves (par exemple la réduction des coûts de production), souvent au détriment de sa valeur. À l'inverse, la plupart visent au maintien de sa pertinence, mais généralement moins pour renforcer l'effet produit par le traitementjournalistique que pour rechercher et livrer des sujets censés être spontanément 
pertinents - donc demander peu de traitement cognitif - comme la vie des célébrités ou les conseils pratiques. Mais cette stratégie opportuniste, outre qu'elle expose à la concurrence d'autres "producteurs de contenus ", fait subir une vive tension aux critères de convenance normative, lesquels définissent notamment ce qui est ou non une information légitime, ainsi que les règles de sa validation. Or, l'assouplissement de ces normes - quoi qu'il ne soit pas si nouveau - n'est pas sans effet sur la valeur discursive, d'où l'importance qu'il y aurait à le soupeser plus soigneusement.

Bref, s'il y a probablement dans l'évolution du journalisme d'information générale quelque chose d'inéluctable, et peut-être de civiquement souhaitable (à moins de considérer qu'il ne doive plus atteindre que les bons élèves du corps social), une perception moins embrouillée de l'interaction des facteurs en jeu et des marges de manœuvre qui en découlent ne pourrait que l'aider à reconquérir la maîtrise de ses choix et l'évaluation dépassionnée de leurs conséquences. Plutôt que de replonger ici dans les débats académiques, illustrons ce point par deux fictions.

La première est empruntée à un auteur dont l'institution littéraire peine à percevoir la grandeur ${ }^{36}$, Terry Pratchett, dans la fantaisie pseudo-médiévale où il imagine un inventeur précoce du journalisme face à l'autocrate d'une cité-état :

- Oh, je vois. Vous vouliez dire que vous devriez être libre d'imprimer ce qui vous plaît?

Il n'y avait pas d'échappatoire.

- Eh bien... en gros, oui, Monsieur.

- Parce que c'est dans, quel était l'autre terme intéressant? Ah, oui... l'intérêt public ? Lord Vetinari ramassa un caractère en plomb et l'examina attentivement.

- Je le crois, Monsieur.

36 Il n'est que justice que ce fléau des clichés et des mythes soit surtout apprécié en fonction des critères hédoniques selon lesquels il se soumettait lui même au jugement, critères assez éloignés de ceux de la littérarité légitime, mais ça n'enlève rien à la prodigieuse inventivité de cet écrivain (en ce qui me concerne, il n'y a aucun problème à ce que l'on considère Pratchett comme un auteur « rigolo » tant que l'on range Rabelais, Molière et Chevillard dans la même catégorie). 
- Ces histoires de poissons rouges mangeurs d'hommes et de maris qui disparaissent dans des grandes soucoupes argentées ? - Non, Monsieur. Ça, c'est ce qui intéresse le public. Nous faisons l'autre chose. [...]

- Donc... Nous avons ce qui intéresse les gens, et les sujets d'intérêt humain, qui sont ce qui intéresse les humains, et l'intérêt public, qui n'intéresse personne.

- Sauf le public, Monsieur, dit William, tentant de rester à flot.

- Qui n'est pas la même chose que les gens et les humains?

- Je crois que c'est plus compliqué que ça, Monsieur.

- Manifestement. Voulez-vous dire que le public est une chose différente que les gens qu'on voit marcher un peu partout? Le public réfléchit à des grandes questions, délicates et mesurées, pendant que les gens courent dans tous les sens en faisant des choses idiotes?

- Je le crois. Il faudrait peut-être que je creuse cette question, je l'admets ${ }^{37}$.

Il le faudrait, en effet. C'est aussi ce qu'illustre une fiction beaucoup plus perturbante. Celle qu'a camouflée en information la Radio Télévision belge francophone (RTBF), le jour de décembre 2006 où elle a interrompu ses programmes pour annoncer, sur fond de barricades et de milices, la brusque sécession de la Flandre. Face à la tempête qui a suivi cette violation effarante de la plus fondamentale des normes journalistiques ${ }^{38}$, l'un des coauteurs de la mystification s'est justifié par les nécessités de la pertinence cognitive : «Certes, j'ai poussé le bouchon un peu loin, mais il fallait créer un effet de surprise, imaginer une forme d'écriture particulière pour attirer l'attention du téléspectateur [...] afin de porter un débat fondamental sur la place publique ${ }^{39}$. " Le plus intéressant est toutefois la réflexion perplexe de la rédactrice en chef d'un quotidien de qualité devant cette transgression normative majeure :

Faut-il que l'information se transforme en fiction pour toucher le lecteur-téléspectateur et passer la barre de l'indifférence et de la banalisation? Non, évidemment. Se résoudre à cette équation

37 Terry Pratchett (2000/2008). The Truth. Londres ; Random House, p. 426-427.

38 L'engagement à ne pas mentir sciemment, énoncé dès 1632 par Théophraste Renaudot.

39 Rapporté in Quatremer, 2006, p. 7-8. 
serait suicidaire pour notre profession. Mais faut-il pour autant fermer les yeux sur une réalité qui frappe tous les médias qui ont fait de l'information, et non de la distraction, leur métier : comment capter et conserver l'intérêt des lecteurs sur des sujets ardus, répétitifs et pourtant capitaux ? Faire la une sur les enjeux communautaires, le développement durable ou le Darfour devrait être la priorité mais, souvent, ces sujets lassent les lecteurs-téléspectateurs : trop compliqués, trop ennuyeux, trop tristes. Pour les journalistes qui n'ont pas renoncé à traiter ces sujets qui comptent, il est devenu impératif d'explorer des voies nouvelles pour capter l'attention. (Delvaux, 2006, p. 24)

On ne saurait résumer de façon plus saisissante les enjeux contemporains du débat public. Ni exposer de façon plus saisissante le désarroi de ses animateurs traditionnels face à ce problème qui, s'il devient chaque jour plus crucial, n'est pas foncièrement nouveau. Comme le notait Robert E. Park en 1922 :

Une plus grande exactitude dans le journalisme dépend de l'avancement des sciences sociales. Mais il y a une seconde condition, à savoir que, pour être une information, un fait doit être intéressant. Comment rendre les faits qui sont importants intéressants est un problème auquel le journal est confronté tous les matins. L'une des raisons pour lesquelles les faits ne sont pas intéressants est qu'ils ne sont pas intelligibles. (p. 234)

Le propre des constantes étant leur constance d'un contexte à l'autre, Léon Trotsky pouvait à la même époque analyser ${ }^{40}$ cette question en des termes quasiment identiques :

[...] pour que les dépêches occupent la première place dans un journal soviétique, il faut qu'elles présentent des faits importants

40 On aimerait tout citer de ce texte où Trotsky, fort de son expérience de la presse, examine les problèmes d'effet cognitif des journaux soviétiques (leur intérêt) mais dissèque aussi l'effort qu'ils réclament, notamment en termes de légibilité («impossible de lire tant l'impression est défectueuse », «le lecteur, malgré tous ses efforts, n'en déchiffre pas la moitié » (p. 22) - mais aussi de cohérence - «aucune suite logique», "déroutent totalement la masse des lecteurs » (p. 23), etc. 
et intéressants sous une forme compréhensible à la masse des lecteurs. Cependant il n'en est rien. [...] Chaque dépêche ressemble à un éclat tombé là par hasard. Les explications qui s'y rapportent ont un caractère fortuit et pour la plupart irréfléchi. [...] Nous considérons souvent que les évènements et les faits sont en euxmêmes connus du lecteur, ou qu'il les comprend par une simple allusion, ou encore qu'ils n'ont aucune importance et que le but $\mathrm{du}$ journal est soi-disant, «à propos » de tel ou tel fait (que le lecteur ignore ou qu'il ne comprend pas) de raconter des tas de choses édifiantes dont on a depuis longtemps par-dessus la tête. [...] Un journal n'a pas le droit de ne pas s'intéresser à ce qui intéresse la masse, la foule ouvrière. Bien sûr, tout journal peut et doit donner son interprétation des faits, car il est appelé à éduquer, à développer, à élever le niveau culturel. Mais il n'atteindra ce but que dans le seul cas où il s'appuiera sur les faits, les pensées qui intéressent la masse des lecteurs. (1923/2010, p. 23)

Jusqu'où aller pour intéresser les masses ? Le révolutionnaire exhorte non seulement à clarifier et à expliquer, mais aussi à exploiter toute la gamme de l'effet cognitif et ne pas se «détourner de la curiosité et des instincts de l'homme en général » (p. 29). La hiérarchie normative est explicitement contrebalancée par les impératifs de pertinence : «Nous sommes un État révolutionnaire et non un ordre spirituel ni un monastère. Nos journaux doivent satisfaire non seulement la curiosité la plus noble, mais aussi la curiosité naturelle » (ibid.).

On retrouve là, encore, toujours, le problème crucial que Breton soulevait plus haut à propos des espoirs pédagogiques suscités par les réseaux numériques : "la question, fondamentale, du désir de savoir ». L'objection que, sous une autre forme, Quintilien assénait vingt siècles plus tôt à la noblesse discursive :

En effet, dans les [débats dialectiques], des hommes doctes et qui cherchent la vérité avec d'autres hommes doctes pèsent tout avec un scrupule minutieux, pour arriver à des notions claires et incontestables [...] Nous, c'est le goût des autres qui doit régler notre langage, et le plus souvent il nous faut parler devant des hommes sans culture aucune, et, dans tous les cas, ignorants de l'art dont je viens de parler; si nous ne savons pas les attirer par le plaisir, les entraîner par la force, et parfois les troubler par 
l'émotion, nous ne pourrons faire triompher même la justice et la vérité ${ }^{41}$.

Pourtant, Quintilien, en opposant (comme avant lui Aristote) la dialectique, censée se passer d'apprêt, et la rhétorique, seule condamnée à attirer l'attention, a justement contribué à enraciner la confusion qui paralyse aujourd'hui les promoteurs du débat public, de la culture et de toutes les choses de ce genre : l'assimilation bimillénaire de la communication en tant que condition de réception de n'importe quel discours à la communication en tant que manipulation de l'opinion.

Une confusion si peu questionnée par la suite que la seconde dimension (la communication comme instrument de pouvoir idéologique) est restée, comme le sexe pour les psychanalystes, une variable explicative hégémonique ${ }^{42}$. Et ce d'autant plus facilement que, de fait, toute communication est bel et bien idéologique ${ }^{43}$. Mes cours distillent insidieusement la croyance que l'approche universitaire du réel est supérieure, disons, à l'approche astrologique de celui-ci. Le récit médiatique d'un hold-up sous-entend, mine de rien, qu'il ne s'agit pas là d'une façon appropriée de se procurer de l'argent. Et les panneaux routiers canadiens ne cessent de célébrer sournoisement le système métrique au détriment des miles et des yards.

Bref, un truisme (celui des idéologies sous-jacentes) en a simplement écrasé un autre (celui de la pertinence requise), ce qui a eu l'avantage indiscutable de rendre attentif à des aspects politiques importants de la communication dans la société, ainsi que l'inconvénient indiscutable de rendre aveugle à des aspects opératoires tout aussi capitaux, mais beaucoup moins connus : si l'on croit que toute communication est avant tout manipulatoire, il faudrait être pervers ou mercenaire pour mener des recherches sérieuses pouvant renforcer son audience (sans compter que si l'on est absolument convaincu de la supériorité de ses propres valeurs morales, il serait malavisé d'aider le logos, registre du doute, à se faire entendre face à l'ethos et au pathos).

41 Institution Oratoire (trad. Bornecque, 1933, p. 275).

42 Précisons cependant qu'épistémologiquement parlant, les deux perspectives sont en principe incluses dans l'acception disciplinaire des sciences de la communication.

43 Comme insistait notamment Angenot, « Tout ce qui s'analyse comme signe, langage et discours est idéologique » (1988, p. 85). 
Bien sûr, puisque tout discours présente effectivement une dimension idéologique, séparer la communication en tant que condition de tout échange discursif et la communication en tant que stratégie d'influence ne revient qu'à adopter un angle de vue. Cette perspective permet de clarifier en quoi le journalisme est bien un processus communicationnel (au sens 1) sans forcément lui attribuer des intentions manipulatoires (la communication au sens 2). Mais ce distinguo est-il bien utile dans le champ de la communication politique ou commerciale, dont la visée est explicitement persuasive? La vieille tradition opinioncentrique suggère qu'il n'en est rien : puisque les relations publiques, la publicité, etc. mêlent intimement ces deux aspects, les démêler ne se justifie ni en théorie ni en pratique. Ce en quoi ladite tradition manifeste une fois de plus sa souveraine indifférence vis-à-vis des réalités opératoires ${ }^{44}$. En effet, les recherches qui, en Amérique du Nord, se sont penchées sur les rouages de la persuasion attestent justement du contraire.

C'est en particulier le cas du modèle ELM développé par Petty, Cacioppo et leurs collègues, lequel est aujourd'hui un cadre de référence non seulement pour un bon nombre de chercheurs, mais aussi pour les communicateurs qui essaient de comprendre ce qu'ils font (c'est particulièrement le cas lorsque sont en jeu des sujets complexes, comme la santé publique). Je n'ai aucune idée de l'efficacité de cette approche en pratique, mais elle démontre en théorie l'importance de l'adéquation sociocognitive des discours par le fait qu'elle se situe explicitement en aval de celle-ci. Comme ces spécialistes de la persuasion en sont venus bon gré mal gré à le souligner "l'ELM se concentre sur une nouvelle étape, après l'attention et la compréhension" (Petty, Wegener et al., 1993, p. 340). En d'autres termes, la communication au sens 2, celle qu'ils se proposent d'expliquer est préalablement conditionnée par la communication au sens 1 .

Peut-être l'auraient-ils concédé plus tôt s'ils s'étaient avisés (sans même remonter à l'ancienne rhétorique) que ce point avait largement été admis un demi-siècle plus tôt par leurs grands devanciers étatsuniens, depuis Lasswell - apologue s'il en est des techniques de manipulation de l'opinion - soulignant que,

44 Si elle est à l'opposé de la «cuisine » pratique que dénonçait Socrate, elle est aussi éloignée d'être la «technique » dont il attendait qu'elle puisse « rendre raison [...] du destinataire », de la "nature effective » et des « causes » de ce qu'elle prescrit. 
avant que quiconque soit en droit d'appeler n'importe quel recueil d'énoncés un «contenu », il doit être présumé ou démontré que l'attention des destinataire atteint un certain niveau minimal. [...] La très large somme de recherches qui ont été menées sur la compréhension montre que la majorité du discours public est portée par un langage qui passe « au dessus de la tête » du public de masse (1946, p. 95)

jusqu'à Katz, appelant les chercheurs à sortir de l'impasse à laquelle avait (déjà) conduit la quête exclusive des effets des médias :

Il y a plus en recherche en communication, en tout cas potentiellement, que l'étude des campagnes. Et il y a plus - bien plus dans les communications de masse que la persuasion. L'approche des « usages ", comme je l'appellerais, part de la supposition que même le message du plus puissant des médias ne peut pas normalement influencer un individu qui n'en a pas l'usage dans le contexte social et psychologique dans lequel il vit. L'approche des « usages » présume que les valeurs des individus, leurs intérêts, leurs associations, leurs rôles sociaux, sont prédominants [...] Je ne prétends pas que c'est une idée originale : les études classiques [...] pointent toutes dans cette direction. (1959, p. 2)

En somme, si l'on ne peut apparemment pas convaincre de cette « prédominance » des chercheurs qui ne veulent pas l'entendre, on ne peut en tout cas pas convaincre un public qui n'écoute pas. Et, comme le remarquait plus haut la rédactrice en chef du Soir, on ne peut non plus l'informer. Et, comme on le remarquait encore plus haut, on ne peut non plus le lui reprocher. Être écouté suppose d'en assumer les conditions d'intelligibilité et d'intérêt, dont les modalités les plus importantes ne sont pas forcément les plus évidentes. Ce qui implique non seulement de les discerner, mais aussi d'être capable d'évaluer clairement le coût normatif - parfois trop élevé - des transgressions qu'elles peuvent ou non réclamer.

En d'autres termes, les gentils auraient tout intérêt à mieux percevoir le genre de facteurs que les méchants, qui peuvent se contenter de leviers plus grossiers, n'ont jamais eu besoin de prendre en considération : la communication est peut-être manipulatoire, mais sa compréhension, elle, est émancipatrice, en particulier dans le contexte de 
la concurrence attentionnelle contemporaine. Elle l'est en tout cas plus que les discours moralisateurs, les prédictions enthousiastes ou lugubres, les mesures improvisées et les exhortations vaines ${ }^{45}$.

45 Comme toujours, il y a plusieurs façons de parvenir aux mêmes conclusions. Sans aucun recours aux sciences cognitives, un spécialiste de l'étude des mégadonnées analysant l'effet des discours du président Obama contre l'islamophobie sur les recherches en ligne des internautes a récemment constaté que les prêches du président sur la tolérance envers les musulmans américains ne faisaient que renforcer l'hostilité de ceux qu'il voulait convaincre, mais que les connaissances stimulantes qu'il s'est un beau jour avisé de mentionner (des gratte-ciels de Chicago ont été dessinés par un musulman américain, etc.) ont en revanche eu un impact important (Stephens-Davidowitz, 2017) : «Lorsque l'on sermonne des gens en colère, les données de recherche indiquent que leur rage peut s'accroître. Mais provoquer subtilement la curiosité des gens, leur apporter de nouvelles informations [...] peut orienter leurs pensées dans une direction différente et plus positive. » 


\section{(Précisions)}

Tl n'est pas surprenant que la griserie de la prédication normative 1 se paie après coup d'un peu de gêne à avoir de nouveau multiplié le genre de raccourcis péremptoires qui m'affligent chez tant d'autres. Mais, à dire vrai, elle se paie surtout de la crainte que l'on ne retienne de l'approche psychosociologique de l'adéquation discursive exposée auparavant que cette mise en perspective finale, plus conforme aux canons de la dissertation scolaire (conclure en élargissant le propos) qu'à ceux de la démonstration scientifique. Bien sûr, elle se situe au-delà du modèle proposé, et en partie au-delà de ce qu'il permet strictement de soutenir, mais d'un autre côté, s'en tenir au problème analytique un peu plus abstrait auquel ce livre est consacré aurait été discourtois, dès lors qu'il avait justement été introduit par un questionnement sur la société dite « de l'information ».

Passons donc sur les détails : personne, je l'espère, ne prendra de toute façon les remarques de ce dernier chapitre pour une réflexion de philosophie politique, domaine dont ma connaissance est assez mince pour me faire croire que Rawls et Habermas disent vaguement la même chose (celle que j'ai envie d'entendre). Et il va sans dire qu'Avril la girafe, la théorie des industries culturelles, le populisme, la publicité en faveur de l'euro, la culture scientifique, les horaires des trains et le management des entreprises de presse n'ont pas grand-chose à faire dans un même chapitre. Sauf d'un certain point de vue.

Sous le bon angle, en effet, les facettes de ce kaléidoscope posent, chacune à leur façon, mais chacune crûment, la question de la valeur des discours dans l'espace contemporain de la communication. Mon seul doute à ce propos vient de la puissance avec laquelle un angle de vue tend à tout ordonner dans sa perspective sans s'embarrasser le cas échéant de contradictions époustouflantes 
(Adorno condamnant les Lumières au nom des Lumières, Laclau dénonçant Rousseau au fil d'un discours dégoulinant de rousseauisme, d'autres attaquant les fondements du journalisme au nom de la démocratie, ou même la démocratie au nom d'ellemême, etc.). Je préfère donc laisser l'hypothétique lecteur qui serait parvenu jusqu'ici soupeser ce que l'angle de vue proposé peut générer d'aberration optique et me dessiller s'il y a lieu.

En attendant (pour poursuivre l'espèce de conversation que je m'imagine entretenir avec lui depuis le début), ces observations me semblent clairement indiquer que les conditions de possibilité de la communication restent l'impensé majeur des discours critiques concernant la communication et les médias. Pourquoi pas, après tout? Les critiques de la "société de l'automobile" devaient-ils savoir ce qu'est un moteur à explosion ou un crédit sur cinq ans avec option d'achat? Admettons que la compréhension opératoire des phénomènes, outre qu'elle est symboliquement peu gratifiante, n'est pas une nécessité épistémologique absolue. En revanche, dans le cas qui nous occupe, l'inattention de chacun à ces problèmes de garagiste engendre et perpétue, comme on l'a souligné, l'impuissance de tous. "L'information n'est pas une marchandise » répète-t-on dans les écoles de journalisme et les rédactions. Bien que j'en sois viscéralement persuadé, j'aimerais par moments que celle-ci soit considérée avec le même sérieux que les vrais industriels accordent à leurs produits.

La notion de « qualité », par exemple, telle qu'elle est envisagée dans la production discursive et dans la production industrielle signale que l'une et l'autre vivent non seulement dans des mondes différents, ce qui est heureux, mais aussi à des époques très éloignées, ce qui l'est moins ${ }^{1}$.

En hommage à Julien Benda ${ }^{2}$, signalons tout de même - comme on l'avait déjà fait en d'autres occasions - quelques points faibles dans ce qu'avançait ce chapitre.

1 Pour une comparaison (peu flatteuse) entre le rapport à la qualité des responsables d'entreprises de presse et ceux des industries manufacturières, voir CNDI, 2003.

2 «Il y a là un trait commun à toutes les doctrines - démocratique, monarchique, socialiste, communiste - en tant qu'elles s'adressent à des foules : prétendre avoir toutes les vertus et ne point admettre que, si elles ont celle-ci, 
Le plus flagrant (si l'on excepte, bien sûr, son mutisme sur les variables politiques et sociales qui dépassent son objet3) est le contraste, pratique mais candide, qu'il entretient entre l'explication et la manipulation, comme si un discours se présentant comme fondé en raison ne pouvait être suprêmement manipulateur : après tout, il est rare que les marchands de solutions miracles, qu'il s'agisse de régimes amaigrissants ou de politiques économiques, ne les appuient pas sur une argumentation quelconque. De surcroît, les registres "irrationnels" (ethos, pathos) pour lesquels j'éprouve si peu d'enthousiasme sont parfois les seules armes des faibles : parce qu'elles sont très puissantes et faciles à utiliser, elles tendent à égaliser un peu leurs capacités à faire valoir leurs vues (fondées ou non) face aux ressources communicationnelles des institutions. Même si, dans les faits, ce sont très rarement les plus faibles qui en profitent, voici typiquement le genre de problèmes, pas vraiment nouveaux, qui dépassent à la fois le cadre de ce livre et les compétences d'un simple garagiste du discours public (tenter d'invoquer confusément l'impératif catégorique, la dialectique éristique ou l'éthique habermassienne de la communication ne ferait que le souligner). Disons simplement que je demeure assez proche de la conclusion à laquelle est parvenu un examen plus minutieux des études menées sur le discours politique :

Les discours simplificateurs, ritualisés, métaphoriques et mythiques ont en commun le même potentiel démocratique et antidémocratique. [...] En fonction des études citées ici, il apparaît que ces stratégies sont plus souvent les adversaires

elles n'ont pas celle-là. Je cherche celle qui déclare: "Ici notre thèse a un point faible." (Je la cherche aussi dans l'ordre philosophique, du moins pour l'âge moderne). On m'assure qu'un tel aveu éloignerait toute une clientèle, laquelle ignore la distinction des idées et veut en effet tous les avantages, fussent-ils les plus contradictoires » (1975, p. 53).

3 Ce centrage sur les conditions discursives d'un débat fondé en raison n'implique pas pour autant que les conditions politiques sont favorables à un tel débat, ce qui reviendrait à tout réduire à des problèmes communicationnels (comme l'a par exemple montré l'élection de 2016 aux États-Unis, si l'élite discursive peine à se faire entendre, c'est peut-être aussi parce qu'elle a peu à dire à des citoyens confrontés notamment à l'enrichissement rapide d'une faible partie de la population et à la crainte de leur propre appauvrissement). 
que les amies du processus démocratique. Comme en ce qui concerne l'ambiguïté lexicale, nul ne voudrait s'engager dans un combat chevaleresque pour purger notre discours de ce genre de choses. Pour autant, ceux qui cherchent à démocratiser le discours politique ne devraient se sentir réconfortés, ni par la prévalence de ces formes d'argumentation, ni par la propension des auditeurs à les accepter. (Gastil, 1992, p. 490)

Ce qui conduit à un autre présupposé pratique mais candide : le rôle éminent accordé aux instances intermédiaires censées assurer l'intelligibilité, l'intérêt, mais aussi la sincérité et la fiabilité des arguments publics, et l'assignation (traditionnelle) de ce rôle aux médias d'information. Écarter le soupçon d'hérésie fonctionnaliste $^{4}$ ou de célébration béate $\mathrm{du}$ « quatrième pouvoir » impliquerait de nouveaux détours, possiblement longs : on s'en dispensera donc, comme on se dispensera de défendre l'importance intrinsèque d'une médiation pour renforcer la pertinence cognitive des informations, ainsi que la conformité des assertions aux normes usuelles de véracité. Mais, outre que cette médiation n’a jamais été exclusive aux journalistes ${ }^{5}$, en tout cas en théorie (en pratique elle l'était plus ou moins), la multiplication des discours se réclamant, sur Internet, d'une légitimité non moindre à délivrer une parole « citoyenne » sur l'actualité interroge évidemment l'importance conférée au journalisme professionnel. Une façon rapide d'aborder cette question serait de se livrer à une expérience de pensée très classique (voir Jefferson, Tocqueville...), mais renouvelée par le contexte contemporain : à quoi ressemblerait une société sans les médias d'information professionnels se réclamant des principes

4 Pour des raisons mystérieuses, ce qui était à l'origine une critique épistémologique (et politique) très justifiée est devenu au fil du temps un anathème scientifique si large, si vague et pourtant si grave qu'il serait très imprudent de paraître suggérer, même involontairement, que quelque chose puisse servir à quoi que ce soit ou à qui que ce soit (la carrière scientifique et la carrière religieuse passent heureusement toutes deux par des séminaires où l'on apprend à chasser les mauvaises pensées).

5 Les sondages rappellent par exemple que les citoyens disent faire plus confiance aux scientifiques, médecins, enseignants, associations de consommateurs, etc. 
journalistiques? D'un côté, on peut penser que le reste de la noblesse discursive, prompte à les critiquer, aurait toutes les raisons de les regretter (c'est en tout cas ce que suggèrent déjà les déplorations alarmées visant l'anarchie discursive des réseaux). De l'autre, il n'est pas totalement impossible que se constituent des formes non professionnelles de journalisme collaboratif qui finissent par acquérir une valeur discursive significative. Cependant, l'une et l'autre de ces spéculations ne sont pas nécessaires si l'on met un instant de côté la question de l'avenir des entreprises d'information - source inépuisable de prédictions fantaisistes - et même celle des acteurs, pour s'en tenir à l'essentiel : le journalisme lui-même. On peut en effet montrer (Labasse, 2015b) que celui-ci est non seulement caractérisable par sa gestion de la pertinence discursive (la réduction de l'effort et le renforcement de l'effet cognitif que l'on appelle parfois le "style journalistique »), mais aussi et surtout, sur le plan normatif, par une façon spécifique d'appréhender et évaluer le réel (une mésoépistémologie), laquelle s'avère de plus en plus explicitement formulée dans les chartes et codes déontologiques.

Or, en détachant l'identité journalistique de son féodalisme traditionnel - l'« appartenance » (sic) à un organe d'information pour l'attacher à une définition autojuridictionnelle du journalisme lui-même, cette évolution, dont on ne peut rediscuter les nuances ici (voir l'article en question), pourrait régler la question lancinante des amateurs et des professionnels. Dès lors qu'une pratique discursive se présente comme plus ou moins journalistique, elle s'offre à l'évaluation en fonction du degré selon lequel elle se soumet à ces critères de valeur. Or, comme ceux-ci correspondent à un marché solvable, il y aura toujours des structures et des professionnels pour assurer ce type de production, sans que cela exclue que des amateurs puissent proposer de leur côté des contenus s'offrant au jugement selon les mêmes normes (ou s'en éloignant, mais devenant proportionnellement « moins » journalistiques).

6 On ne parle pas ici des blogues individuels, qui sont essentiellement la transposition numérique $\mathrm{du}$ journalisme de chronique $\mathrm{du}$ $\mathrm{XIX}^{\mathrm{e}}$ siècle : si beaucoup présentent un grand intérêt, ils commentent l'actualité plus qu'ils ne la rapportent. Par ailleurs, 15 ans après son lancement, le projet Wikinews ("la source d'informations que vous pouvez écrire ») n'a pas vraiment fait ses preuves. 
Bref, contrairement à ce que simplifiait beaucoup le chapitre précédent, les professionnels du journalisme ne sont pas si indifférents que ça à la préservation de leur valeur discursive et même à sa conceptualisation?. On ne peut toutefois manquer de noter que cette évolution doit sans doute moins à un appétit spontané pour la réflexion abstraite qu'à la pression de la concurrence discursive sur les réseaux numériques. Il en va plausiblement de même pour des évolutions plus techniques comme la mise en valeur de la vérification factuelle (fact checking) qui, indépendamment de son utilité civique, constitue un enjeu majeur de différentiation du discours journalistique depuis le $\mathrm{XvII}^{\mathrm{e}}$ siècle.

Cependant, et c'est là un autre problème qu'il faut souligner, le fait qu'il existe un marché solvable et durable pour le journalisme, et le fait que la qualité que lui prêtent ses destinataires constitue très clairement - comme on l'a montré plus haut - un atout majeur dans ce domaine n'implique pas que d'un point de vue strictement commercial, investir dans la valeur discursive soit la seule stratégie rentable. Il paraît d'autant plus habile à court terme de se contenter de la feindre (comme le montrent les sites capteurs de clics mimant les codes discursifs de l'information) que la production journalistique de la valeur est relativement coûteuse : elle demande plus de compétence, de talent, de temps, d'effort, etc. Au bout du compte, le principe du «good enough» dont témoigne par exemple la vacuité de tant de sujets télévisés dits « de société » - traiter une information par un micro-trottoir (vox pop au Canada) ou un tournage vite expédié auprès d'une famille « ordinaire » - pourrait moins relever de l'inaptitude professionnelle que d'une certaine rationalité managériale. Ils ne coûtent pas grand-chose à produire et à consommer (d'autant moins que

7 Ainsi, un comité de l'Association canadienne des journalistes a-t-il avancé en 2012, après des hésitations dont il ne fait pas mystère, que "le travail journalistique apporte clairement la preuve d'une discipline consciente d'elle-même, élaborée pour fournir une description juste et équitable des faits, opinions et débats en jeu dans une situation donnée » en recourant à «certaines approches reconnaissables » (p. 4). La révision en 2011 de la charte française du Syndicat national des journalistes va dans le même sens en précisant pour la première fois depuis 1918 que «Le journalisme consiste à rechercher, vérifier, situer dans son contexte, hiérarchiser, mettre en forme, commenter et publier une information de qualité ». 
l'image audiovisuelle fournit un modèle mental sans réclamer au spectateur d'effort cognitif) et leur légitimité est confortée au besoin par des consultants prêts à affirmer contre toute vraisemblance scientifique que c'est « ce que les gens préfèrent ». La même logique et la même conviction pourraient alimenter la célébration du direct, qui tend à réduire la charge du travail d'enquête et de traitement, montage compris, à un processus technique de captation. Mais comme il faudrait par ailleurs fournir de nombreux contre-exemples de traitement télévisuel remarquable, et comme le problème aigu des croyances du management éditorial est déjà soulevé dans plusieurs livres de chercheurs et de journalistes (lesquels en sont les premières victimes, la seconde étant l'espace public de la communication), on s'en tiendra là.

D'autant que si l'on voulait poursuivre le petit jeu - assez journalistique - de la recherche des responsables, le monde académique se trouverait bientôt en position délicate. Comment déplorer les insuffisances de l'expertise de ce champ, ou dénoncer vertueusement des dérives qui n'en sont que la conséquence (Labasse, 2010), si l'on n'est pas en mesure de lui proposer des solutions crédibles en fonction des contraintes qui lui sont propres ? À dire vrai, la tour d'ivoire est de moins en moins ce qu'elle était : un nombre croissant de chercheurs accordent plus d'attention aux problèmes opératoires des journalistes (voir notamment Mercier, 2007) et même à ceux de l'ensemble des professionnels de la communication ${ }^{8}$. Mais il faudra d'autant plus de temps pour permettre l'essor d'une expertise à la mesure des défis actuels que la facilité de la pensée moralisatrice - normativement gratifiante et cognitivement peu coûteuse - la rend encore séduisante pour une bonne partie du monde scientifique et politique.

Au bout du compte, l'élévation morale qui permet de jauger depuis l'Olympe de la pensée les dérives des journalistes n'est guère différente de celle qui permet de jauger de façon tout aussi assurée la futilité du public. C'est en cela que le renversement de la

8 Comme en témoignent notamment l'essor que connaît depuis quelques années le Réseau international sur la professionnalisation des communicateurs (problématique à peine concevable il y a une ou deux décennies) et le développement des études en Rédactologie (expertise discursive). 
charge de la rationalité constitue, comme on l'a soutenu, un enjeu aussi important d'un point de vue instrumental que sur le plan démocratique, culturel ou pédagogique. Penser diffuser un message «important» sans évaluer attentivement le prix normatif (la dégradation épistémologique) que l'on est prêt à acquitter pour sa réception expose aussi bien les politiques que les journalistes, les scientifiques ou les enseignants aux risques opposés de l'inaudibilité et de la mièvrerie.

L'idée qu'il puisse être plus rationnel de regarder un film d'action que l'une de ces émissions «culturelles» insignifiantes évoquées plus haut n'en est pas moins déplaisante, y compris pour moi. Mais elle n'est guère plus qu'une banalité pour les tenants d'une froide analyse économique des choix informationnels et politiques, laquelle va beaucoup plus loin dans ce sens. En 1957, Antony Downs avait ainsi avancé que, dès lors que le coût de traitement de l'information politique dépasse largement le profit que l'on peut en retirer :

il est irrationnel pour la plupart des citoyens d'acquérir de l'information politique dans le but de voter. Dans la mesure où chaque personne considère que le comportement des autres est acquis, il est simplement non rentable pour elle d'acquérir assez d'information pour voter "correctement" [...] Ainsi, l'ignorance politique n'est pas le résultat d'une apathie incivique ; elle est plutôt une réponse hautement rationnelle aux réalités de la vie politique dans une grande démocratie. (p. 147)

Il n'est pas nécessaire d'adhérer au réductionnisme abstrait de ce raisonnement (qui écarte toute motivation ne relevant pas d'un strict calcul coût-bénéfice en vue d'un choix électoral) pour remarquer les rapports entre ce qu'il aborde sous un angle économique et ce que la théorie de la pertinence avançait sous un angle psychologique. On pourrait du reste en dire autant des multiples approches économiques contemporaines qui tempèrent le dogme du choix rationnel, comme la théorie de la "rationalité limitée » d'Herbert Simon ou celle de la "rationalité peu informée » (low-information rationality) de Samuel Popkin, et plus généralement du large champ ce que l'on appelle aujourd'hui l'«économie comportementale». Mais ce n'est pas ici que l'on débattra des vertus et limites de ces courants 
- ce dont se chargent des auteurs bien plus qualifiés que moi - si ce n'est pour remarquer que ces approches opèrent toutes, sous l'angle de vue qui leur est propre, un recentrage bienvenu des théories économiques ou politiques sur la question centrale de la valeur accordée aux informations.

Cependant, la valeur de l'information est plutôt perçue dans ce cadre comme une contingence que comme une problématique en soi, ce qui conduit à envisager avec un certain détachement les constituants de cette valeur. Puisque les questionnements sont centrés sur des choix politiques ou économiques, la question amont de la réception de l'information est facilement réduite à une conception de l'effort assez vague (assimilant ce qui est discursif et ce qui ne l'est pas), une conception de l'effet plutôt sommaire (restreinte à l'interaction utilitaire avec la sphère personnelle) et une certaine indifférence vis-à-vis de l'influence des déterminants sociaux : même lorsqu'elles célèbrent implicitement l'importance de la valeur discursive, les théories économiques demeurent évidemment des théories économiques.

De même, comme on l'a vu, que la théorie de la persuasion de Petty et Cacioppo, demeure pour sa part une théorie de la persuasion, laquelle se situe explicitement en aval des déterminants de l'adéquation cognitive.

De même que, comme on l'a vu également, les grandes théories de la réception littéraire sont profondément littéraires, ce qu'il serait tout aussi malvenu de leur reprocher (il n'est pas intrinsèquement «erroné » de restreindre son optique à un type particulier de lecteur, même si celui-ci est peu susceptible d'attribuer le prix du livre de Montréal à un ouvrage sur les recettes de lasagnes ou de ressentir un frisson esthétique en feuilletant un roman d'amour).

De même que les plus éminentes théories contemporaines de la démocratie délibérative, celles de Rawls et d'Harbermas, supposent toutes deux la collaboration active des citoyens dans l'évaluation des arguments en lice, d'où la nostalgie non dissimulée du second pour les débats d'idées des salons du XviII ${ }^{\mathrm{e}}$ siècle. Il est du reste frappant qu'Habermas, s'il opère une distinction précieuse entre le discours propagandiste ( $\mathrm{l}^{\prime}$ agir stratégique») et le discours 
délibératif ( $\mathrm{l}^{\prime}$ «agir communicationnel9 $)^{2}$ n'en opère pas vraiment entre ces deux grandes finalités et le socle des prérequis attentionnels qui leur est commun (la communication « au sens 1 »).

$\mathrm{Au}$ cas, donc, où ça ne serait pas absolument évident, il ne s'agissait aucunement ici de critiquer à la chaîne - ni pour ce qu'elles sont, ni même pour ce qu'elles ne sont pas - de si substantielles et fécondes théories. En revanche, envisagées dans la perspective de la valeur discursive, c'est-à-dire non seulement sous un certain angle, mais aussi d'assez loin, chacune d'elles et bien d'autres renforcent un soupçon initial : la plus belle ruse du Grand Mystère était de faire croire qu'il n'existait pas.

9 Je rangerais volontiers dans le premier la publicité et la « communication» (au sens 2), et dans le second le journalisme d'information, quoique tout me donne à penser qu'Habermas et d'autres y trouveraient à redire. 


\section{SECONDE PARTIE}

\section{OCCURRENCES}

ous l'emprise d'une pulsion de piété filiale trop rare de nos jours', j'avais, adolescent, entrepris de feuilleter quelques ouvrages publiés par mon géographe de père. L'effort requis et le peu d'effet cognitif que j'en retirais mavaient vite renvoyé à des contenus plus adéquats, mais j'en avais au moins retenu une fascinante citation de Robert Oppenheimer : "plus on est précis, moins on va profond». Malheureusement, son efficacité rhétorique s'est révélée décevante : j'avais beau l'essayer sur mes professeurs, ils voulaient toujours des détails et des dates. En outre, il s'est avéré plus tard à la lecture de certains auteurs qu'il ne suffisait pas forcément d'être imprécis pour être profond. Il n'en reste pas moins que toute problématique d'une certaine étendue interdit de s'arrêter à l'examen de chacune des situations qu'elle rencontre.

C'est pourquoi se trouve rassemblée dans cette partie, beaucoup plus courte, une brassée d'observations qui examinent de façon un peu plus détaillée (mais pas trop) quelques manifestations empiriques des déterminants de la production et de la réception discursive.

Loin de chercher à compenser l'hétérogénéité des éléments évoqués dans la partie précédente, ces études de cas l'accentuent au contraire sans le moindre remords. La cohérence, comme on s'en doute, est fournie par les logiques de même nature que l'on pourra reconnaître ${ }^{2}$ en examinant des traductions absurdes (Ce que les traductions

1 Message privé.

2 Pour pouvoir les reconnaître, le lecteur vraiment pressé qui serait parvenu ici en omettant tout ce qui précède pourrait parcourir le résumé qui en est 
trahissent), des images publicitaires (Rhétorique du chiot), des recettes journalistiques (Le mystère de la pyramide inversée), des polémiques littéraires et cinématographiques (Souffrances et amertumes de la critique), des manuels militaires (Vertus martiales des images salaces) ou des batailles de mots (L'art de l'agonistique lexicale).

Certains de ces textes sont adaptés de publications ou de communications orales antérieures, mais d'autres ne constituent encore à ce stade que des ébauches heuristiques.

présenté dans l'introduction de l'ouvrage et les schémas récapitulatifs qui figurent plus haut. 


\title{
Ce que les traductions trahissent ${ }^{1}$
}

\begin{abstract}
yoins de la croire transparente, la traduction constitue évidemment en soi une production discursive, soumise à ce titre à des déterminants cognitifs et sociaux qui lui sont propres. Or, ce système d'adéquations en cascade, ajoutant à celle du texte source celle de la traduction elle-même, pourrait contribuer à expliquer certaines spécificités de ce processus et des arbitrages sur lesquels il repose.
\end{abstract}

Si l'on suppose que la vocation de la traduction est, au-delà de l'alternative désuète entre la lettre et le sens, de transposer la valeur sociocognitive des discours, et que cette valeur résulte du rapport entre des facteurs souvent contradictoires ou en tout cas interdépendants, la démarche du traducteur apparaît moins comme un art spontané ou comme la conséquence d'un savoir abstrait que comme une expertise stratégique et technique, l'optimisation réflexive de variables capricieuses.

La lettre et le sens d'un discours sont par nature des constituants conjoints de son adéquation générale (sa valeur), au même titre d'ailleurs que les variables culturelles : dire par exemple qu'une approche "culturellement orientée a mis en cause la notion d'équivalence qui jusqu'alors dominait les débats sur la traduction " (Jeon et Brisset, 2006) revient en réalité à suggérer que l'on employait cette "notion" sans prêter beaucoup d'attention à ce qu'implique le terme même d'[équi]-[valence].

Par ailleurs, aucune traduction ne peut s'abstenir de constituer une intervention sur cette valeur, qu'elle le veuille ou non :

1 Ce texte, ainsi que son grand frère (Labasse, 2017b), trouvent leur origine dans une conférence invitée à l'École supérieure d'interprètes et de traducteurs (Université Paris 3) à l'occasion du colloque Traduire, écrire, réécrire dans un monde en mutation (Paris, $1^{\mathrm{er}}-2$ décembre 2016). 
- Elle part toujours de zéro ou presque, dans la mesure où le texte source est, en l'état, privé de valeur pour les lecteurs qui n'en maîtrisent pas la langue : à supposer que des déterminants sociaux (par exemple le prestige) ou l'espoir d'un effet utilitaire (par exemple celui d'un mode d'emploi) conduisent ces lecteurs à tenter d'en extraire le sens, dictionnaire en main, l'effort cognitif colossal que réclamerait l'accès lexical mot à mot et l'établissement des cohérences serait vite décourageant.

- Elle ne peut pas assurer l'homologie parfaite en valeur de la source et de la cible, non seulement parce que cette valeur n'est, au départ, pas universelle pour les lecteurs de la langue originelle, mais aussi parce que la force des déterminants de celle-ci n'est pas strictement identique d'une culture à l'autre (quoique leur nomenclature le soit). À l'instar de la liberté ou de l'objectivité, une telle équi-valence est de ces points de référence dont on peut se rapprocher, mais que l'on ne peut atteindre. D'où il résulte que l'excellence en la matière est celle du moindre mal (à l'instar, du reste, de la transposition initiale des pensées dans un texte).

- Elle est nécessairement guidée par une évaluation implicite (mais qui gagne à être explicitée) des déterminants de la valeur du texte source. Celui-ci «vaut » forcément - sinon pourquoi le traduire? - et la dominante de cette valeur, qu'elle se situe au plus bas niveau cognitif ou au summum de la distinction culturelle, est bien celle qui déterminera les délicats arbitrages du traducteur : le fait de privilégier, par exemple la précision d'un document juridique, la clarté d'un guide, la fluidité ou l'originalité d'un texte littéraire, voire son caractère dérangeant, n'est rien d'autre qu'une stratégie d'adéquation fondée sur les modalités d'adéquation du texte source ${ }^{2}$.

Si l'exploit discursif que constitue une traduction possède une valeur qui lui est propre - comme en témoigne le fait qu'elle puisse être

2 Et plus précisément son caractère autojuridictionnel, c'est-à-dire les critères d'appréciation implicites ou explicites selon lesquels tout discours (scientifique, journalistique, littéraire, distractifs) s'offre spécifiquement et génériquement à être évalué. 
récompensée par un prix - cette valeur paraît intimement liée à la transposition de celle de la source. Une voie pour examiner empiriquement cette hypothèse est fournie a contrario par les traductions critiquables, dont les errements apparemment hétéroclites paraissent remarquablement lisibles sous l'angle de l'adéquation sociocognitive.

\section{Le Club des cinq contre l'emprise du temps}

Les traductions hasardeuses, car purement littérales (lexicales), de marchandises exotiques forment la partie la plus visible des transpositions problématiques. Or, celles-ci, bien que similaires dans leur démarche, peuvent produire des résultats curieusement opposés. Très souvent, les termes n'activent aucun schéma cognitif qui permettrait d'établir leur cohérence au sein d'un quelconque modèle mental, à l'instar de cet emballage promettant mystérieusement une «Preuve de flaque - Ami De Sport - Polyvalente Tasse - Rupture résistante, coffre-fort de lave-vaisselle ${ }^{3}$. Ce genre de traduction peut même s'apparenter à une démarche créative - la recherche de l'incohérence cognitive parfaite à l'exemple de ce soutien-gorge affirmant contre toute évidence qu'il est en réalité une voiture décapotable4. Mais, à l'inverse, les aléas langagiers du commerce international peuvent activer un schéma très vif, quoiqu'inapproprié, et conduire à construire à partir de lui un modèle mental peu séduisant de l'objet proposé, comme ce "Service de vaisselle de 16 morceaux ${ }^{5}$ » dont on ne peut s'empêcher d'imaginer les seize fragments tintant joyeusement au fond de la boîte. Les bévues des emballages ou des notices affectent toujours le traitement cognitif (l'effort), mais on voit qu'elles peuvent aussi produire un effet cognitif inattendu, parfois au défi involontaire des contraintes sociales (c'est le cas de ces peluches d'oursons dont l'étiquette clame "Nous baisons quand nous sommes proches", ou de ce verre qui propose «une manière spéciale de jouir sur la glace $\left.{ }^{6} »\right)$. Ainsi, les énoncés mal traduits sont-ils susceptibles d'acquérir accidentellement une valeur discursive élevée : ils obtiennent de l'attention, même si ce n'est pas celle qu'escomptaient

3 «Spill-proof-Sport Pal-All Purpose Cup-Break-resistant, dishwasher-safe ». On notera cependant que l'illustration fournissait directement le modèle mental approprié.

4 «I'm Convertible! » - «e suis Cabriolet!»

5 «16 piece dinnerware».

6 «We kiss as we get close! » - « Truly a new way to enjoy on the rocks ». 
leurs auteurs. La valeur discursive est décidément capricieuse (il n'est, par exemple, pas impossible que ce paragraphe ait procuré au lecteur plus de satisfaction que des volets plus essentiels mais plus austères du même texte, ce qui témoignerait de l'ubiquité des ressorts fondamentaux de l'adéquation sociocognitive).

Les traductions perçues comme critiquables ne sont pas nécessairement le fruit de la maladresse, mais elles se caractérisent toujours par un écart entre les modalités de valorisation du texte source et celles du texte traduit. Il en est ainsi de la tentation, si fréquente et si naturelle pour un professionnel de l'écrit, qu'il soit écrivain ou traducteur de métier, d'« améliorer » le discours originel, comme on l'a notamment reproché à Vialatte traducteur de Kafka, ou à Giono traducteur de Melville. Mais les transformations peuvent aussi avoir l'objectif inverse de réduire sciemment la valeur du texte pour son lecteur, exposant ainsi de façon remarquablement claire et brutale la tension fondamentale entre la pertinence cognitive et les contraintes sociales. C'est le cas, emblématique, de la traduction des vigoureuses épigrammes de Catulle, par exemple son apostrophe à Memmius?, dont les ouvrages classiques proposent des versions plutôt discordantes : "Ô Memmius, comme tu t'es joué de moi, comme tu m'as fait à loisir la victime de ton avarice! »; "Ô Memmius! assez longtemps tu m'as tenu le bec ouvert. »; "Ô Memmius, comme tu m'as longtemps tenu à ta discrétion!", etc.

Le motif de l'insatisfaction de Catulle, si c'en est une, se précisera graduellement à la fin du $x^{\mathrm{e}}$ siècle : « $\hat{O}$, Memmius, lentement et à ta guise, n'ai-je pas supporté ta poutre rouge? " (Markowicz, 1985, p. 35), et enfin «Ô Memmius, comme tu me l'as bien fourrée à la renverse, toute ta trique! » (Hecht 2011, p. 117).

À l'évidence, le puissant effet cognitif de premier niveau dont jouait le poète n'était pas acceptable pour le grand public du xix siècle, et encore moins pour la jeunesse des écoles, que l'on s'ingénie souvent à priver de pertinence cognitive. Les notes de certaines éditions ne font pas mystère de cette inadéquation sociale ${ }^{8}$. Mais, contrairement

7 O Memmi, bene me, ac diu supinum // Tota ista trabe lentus inrumasti (Catulle, XXVIII, Ad Veranium et Fabulum).

8 « - Bene ac diu, à ton aise et longtemps. - Supinum. L'image employée ici par Catulle est obscène. [...] - Irrumasti. Allusion aux mœurs débauchées de 
à d'autres énoncés de Catulle, riches en termes crus (par exemple « cacatus » qui... se passe de traduction), l'expurgation ne saurait ici se limiter à la superficie lexicale. L'épigramme ne peut être rendue acceptable qu'en en détruisant le sens, c'est-à-dire, la possibilité que des non-initiés en construisent un modèle mental, d'où des allusions aussi vagues que «tu m'as tenu à ta discrétion ». Ainsi se précise le sens figuré d'« édulcorer » (v. trans. - Au fig. Réduire l'effet cognitif d'un énoncé en atténuant sa figurabilité pour satisfaire des contraintes sociales).

Cependant, les complexes arbitrages par lesquels les traducteurs avertis gèrent la transposition de la valeur discursive se réduisent rarement à une intervention sur une seule variable, et peuvent en outre refléter des enchevêtrements de contraintes sociales beaucoup moins univoques. C'est ce qu'illustre à merveille la polémique qu'a suscitée la retraduction du Club des cing d'Enid Blyton, saluée sur Internet par des titres comme Le Club des 5 : la nouvelle traduction qui laisse sans voix, Coupeurs de langue, réducteurs de têtes, Le Club des 5 et la baisse du niveau et même (Traduttore, traditore !) Le Club des Cinq et les traîtres immondes.

Contrairement aux traductions ad usum Delphini, comme celles de Catulle au XIx ${ }^{\mathrm{e}}$ siècle, celles de l'édition commerciale moderne, surtout pour ce genre d'ouvrage, placent la valeur discursive (assimilable ici à la valeur économique) aux premiers rangs de leur cahier des charges. Malheureusement, celle-ci s'établit en l'occurrence sur un double marché et donc selon une double échelle : si la première est celle des jeunes lecteurs, la seconde est celle des acquéreurs, souvent les parents, qui l'établissent en vertu d'un statut symbolique très différent et beaucoup plus embrouillé, patrimonial dans tous les cas, mais conservateur ou progressiste selon les lectures9. Sa retraduction apparaît donc une mission délicate, pour ne pas dire désespérée, ne devant pas servir moins de quatre maîtres, le texte lui-même (malgré sa pauvreté stylistique bien connue), l'intérêt des nouvelles générations, la

Memmius. » (Louis Eugène Benoist, Commentaire, in E. Rostand, trad. Les poésies de Catulle, Hachette, 1879, p. 437).

9 Si le rôle attribué notamment aux personnages féminins reflète ostensiblement l'époque de sa rédaction (voire les conceptions assez conservatrices de son auteure), le refus par Claudine/Claude d'accepter son identité sexuelle biologique en a fait, pour d'autres lecteurs, une héroïne transgenre avant la lettre. 
nostalgie des adultes et leurs sensibilités normatives actuelles. En revanche, les «traîtres immondes » avaient à leur disposition une large gamme de leviers permettant d'ajuster les différents facteurs de l'adéquation discursive. Certains des réglages qui seraient a priori possibles relèvent plutôt du remodelage éditorial, en ce sens qu'ils modifient la diégèse elle-même, que ce soit pour accroître l'effet cognitif du récit (c'est le cas des scènes de sexe, ou même d'attaques de zombies, insérées dans des romans classiques ${ }^{10}$ ) ou au contraire pour l'expurger de passages devenus socialement perturbants. Épargné par la première stratégie, le Club des cinq l'est un peu moins par la seconde, qui conduit notamment à retirer aux " gitans » et "romanichels » leurs propensions les plus suspectes et à les nettoyer de la «crasse » censée les caractériser. On restreindra cependant ici l'examen aux décisions relevant plus directement du spectre traductologique (c'est-à-dire, de façon un peu arbitraire, aux choix ne modifiant pas substantiellement la diégèse), en se concentrant, pour des raisons de place et de temps, sur les chapitres 1 et 2 du premier roman de la série (Five on a Treasure Island), publié en 1942, puis traduit en 1962 (Claude Voilier) et en 2006 (AnneLaure Estève).

Parler, comme l'éditeur, de traduction « revue » est peut-être un peu modeste : les modifications entre les deux éditions françaises touchent environ les deux tiers de leur contenu, soit 3152 mots altérés, substitués ou supprimés sur 4919. Une partie de ces transformations vise clairement à ménager les sensibilités les plus aiguës, notamment par l'ablation des interjections d'origine religieuse ("Ma foi...»; "Dieu sait... ») et l'effacement de toute brutalité, qu'elle soit physique (1942: «She wants spanking » $\rightarrow 1962:$ "Elle a besoin d'une bonne fessée » $\rightarrow 2006$ : «Elle a besoin d'une bonne punition ») ou même verbale ("C'est une vilaine petite fille... » $\rightarrow$ " Je suis contrariée... »; "Quelle sotte tu fais » $\rightarrow$ [supprimé] ; «Deux cousins tout aussi stupides » $\rightarrow$ [supprimé]), la perte d'effet cognitif étant supposée compensée par le gain d'acceptabilité sociale.

L'aménagement vise aussi l'effort cognitif, par la suppression de la plupart des descriptions n'offrant pas de contrepartie en termes

10 Par exemple Pride and Prejudice and Zombies (Jane Austen et Seth Graham Smith, Philadelphie, Quirk books, 2009), ou, pour s'en tenir à ce roman, sa version sadomasochiste publiée sous le titre original et la signature de Jane Austen et Amy Amstrong par Clandestine Classics (2012). 
d'effet, mais aussi par l'amélioration de la lisibilité lexico-syntaxique. Sur ce point, il s'agit bien d'une rectification de la traduction initiale. Le gonflement de la charge lexicale ( $+15 \%)$ et syntaxique (+15\% également) entre le texte source et sa première version française était en effet anormalement élevé ${ }^{11}$, trahissant un goût marqué pour la fluidité du style et la richesse des détails (1942: "They are quite full up this year.» $\rightarrow 1962:$ "Les hôtels sont déjà complets et les touristes ne cessent d'affluer de partout. » $\rightarrow 2006:$ : Tout est déjà complet. ») En compensation de l'effort supplémentaire qu'elle réclamait, la première traduction dispensait très généreusement les points d'exclamation, saupoudrage d'effet artificiel que la nouvelle édition a ramené au niveau de l'original ( 51 occurrences en 1942, 75 en 1962 et 55 en 2006).

En somme, si l'on met à part l'abrasion de la moindre aspérité pouvant blesser une conscience sociale pointilleuse, les «coupeurs de langue, réducteurs de têtes » ne manifestent pas vraiment, dans l'échantillon examiné, le vandalisme que leur prêtent leurs critiques. D'un strict point de vue psycholinguistique, on pourrait même dire qu'ils ont parfois manqué de zèle, négligeant de scinder certaines phrases - qui l'étaient du reste dans l'original - voire de s'interroger sur la figurabilité de quelques termes («mansardée») pour leurs lecteurs d'aujourd'hui.

Restent cependant l'abandon du passé simple au profit du présent et l'indiscutable dégradation syntaxique des dialogues (1962: «Es-tu habillée, Annie ? [...] Nous vous attendons toutes les deux. »; 2006 : "Tu es habillée, Annie? [...] On vous attend!»). Ces décisions peuvent sembler malavisées, puisqu'elles dévalorisent forcément le texte aux yeux d'une bonne partie des acquéreurs potentiels alors que - contrairement à ce que suggéraient certains commentaires - elles ne " simplifient» la lecture que de façon négligeable (l'effort cognitif lié à l'inversion pronom-verbe est surtout à la charge de celui qui formule l'interrogative). Pourtant, de tels choix étaient inévitables. Si l'on se souvient qu'éditeur et traductrice étaient dépourvus de toute possibilité d'injecter de l'effet cognitif dans le texte pour le porter aux

11 Les différences des longueurs lexicales et syntaxiques moyennes entre les versions anglaise et française du Club des cinq sont les plus importantes qui aient été relevées dans le cadre d'une recherche en cours portant sur 25 romans pour enfants, jeunes et adultes (de Lewis Caroll à Flaubert et Proust, en passant notamment par Rowling et Verne). 
standards actuels, il leur fallait du moins préserver à tout prix le ressort essentiel de la littérature enfantine, l'identification aux héros, dont il bénéficiait à l'origine. De même que les Cinq devaient porter des jeans plutôt que des shorts - à l'instar de leurs homologues anglais depuis 1997 - un niveau d'expression qui aurait constamment rappelé leur distance par rapport à leurs lecteurs aurait été un handicap trop lourd pour des textes déjà affadis, puisqu'il aurait contrecarré le puissant jeu du second ordre d'effet cognitif : l'interaction avec la sphère personnelle (réelle ou imaginaire). Ce piétinement de la syntaxe relève donc du même registre d'effet cognitif que les couvertures d'hebdomadaires consacrées au salaire des cadres ou aux prix de l'immobilier. Et il relève de la même règle du jeu impérieuse : servir la valeur discursive en optimisant la pertinence en regard des contraintes. 


\section{Rhétorique du $\operatorname{chiot}^{1}$}

T 'analyse d'images n'est pas toujours perçue comme la captivante Lépreuve épistémologique qu'elle constitue. Tout en mettant l'observateur au défi de démontrer sa perspicacité - que pouvez-vous donc nous révéler ? - l'évidence de la représentation l'entraîne sournoisement vers les deux périls qu'illustrent si vaillamment les experts des plateaux de télévision. D'un côté, la spéculation acrobatique («sa cravate à rayures symbolise... ») et l'introspection hardie ("on ressent...»), de l'autre, la paraphrase pénétrée («il fronce les sourcils, ce qui signifie qu'il n'est pas content »).

Il ne s'ensuit évidemment pas que tout projet herméneutique ou sémiologique est dépourvu de vertu. Au contraire, la célèbre analyse de la publicité Panzani par Roland Barthes (ou, pour s'en tenir à elles, les contributions du numéro que la même revue consacrait en 1970 à l'analyse des images) démontre tout ce qu'un regard habile à déceler et interpréter des signes peut effectivement exposer, structurer et, dans une moindre mesure, expliquer. Mais l'épreuve épistémologique de l'image - et en particulier de l'image publicitaire - n'en reste pas moins redoutable: le double piège de la spéculation et de la paraphrase, s'il peut être déjoué, ne peut être désamorcé. D'où l'intérêt d'essayer sur le même type de discours des outils complémentaires².

Voici une publicité. Ainsi que Barthes avait relevé dans la photo d'un assortiment de produits Panzani la résurgence des natures mortes de la peinture classique, on pourrait facilement y voir, pour sacrifier à la vulgate interdiscursive, l'écho des dessins animés de

I Ce texte développe une étude de cas présentée lors d'une conférence invitée (Le problème de l'ajustement discursif; Repères scientifiques et perspectives, Université du Québec en Outaouais, 27 mars 2015).

2 Comme l'avait notamment esquissé le numéro de Recherches en Communication dirigé en 1998 par Daniel Peraya (voir Peraya, 1998). 


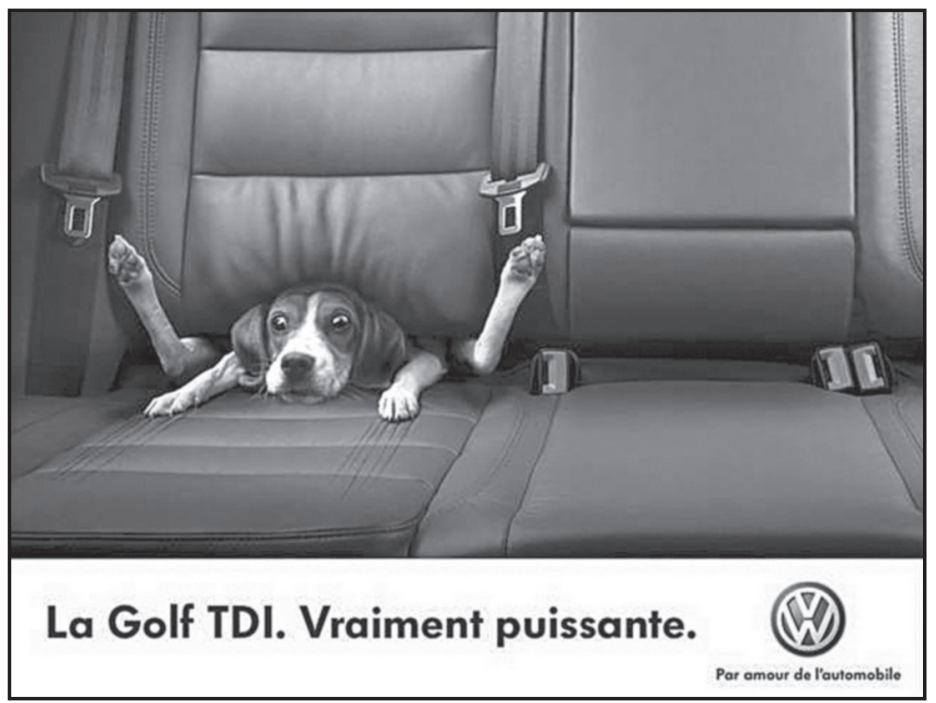

Tex Avery dans lesquels des animaux se retrouvent dans les positions les plus curieuses et les plus inconfortables.

Quant à sa logique sociocognitive, elle est aussi manifeste que son efficacité. Le bénéfice de toute image réside en partie dans l'économie d'effort cognitif qu'elle permet en dispensant du traitement lexical et syntaxique que réclamerait la lecture d'un texte descriptif, mais aussi en fournissant d'un coup le modèle mental que cette lecture conduirait plus péniblement à construire. Pour autant, toutes les images n'épargnent pas la tâche de mettre en cohérence les éléments qu'elles comportent et d'en inférer le sens : les plus subtiles, dans l'art comme dans la publicité, sont souvent celles qui prennent justement le risque de solliciter un tel effort. Dans le cas de celle-ci, on percevra la nature de ce travail en songeant qu'une accroche différente (par exemple : « La Golf TDI : vraiment confortable ») le rendrait vain et qu'une absence d'accroche le compliquerait sensiblement.

La pertinence des images, bien entendu, ne réside pas seulement dans l'effort cognitif qu'elles épargnent, mais plus encore dans l'effet cognitif qu'elles produisent et par lequel elles le justifient. Celle-ci est fort généreuse en la matière, l'effet de premier niveau produit par la «mignonnerie » (cuteness) du jeune beagle étant multiplié par l'imprévu de la situation et la drôlerie de la scène (voir 
chapitre 4), qu'accentuent son regard effaré et les traces de ses griffes. Sans oublier, à un plus haut niveau d'effet cognitif, la satisfaction d'avoir résolu l'énigme que proposait l'image, sensation familière aux amateurs de mots croisés et de romans d'Agatha Christie mais plus fugace ici.

En somme, le bref effort d'inférence qu'elle réclame pour être comprise est très largement compensé par le bénéfice qui en résulte (exemple type d'un effet cognitif « construit » plutôt que donné). À tel point qu'une publicité quasiment similaire sur le fond, mais moins pertinente en pratique, l'était encore assez pour obtenir, elle aussi, l'hommage de nombreuses reprises sur Internet. Créée pour la marque Hyundai, elle figurait dans un jardin, sans nul signe de l'univers automobile, un chien plus impressionnant (du genre que l'on aime voir porter une muselière), langue étirée au point d'entourer sa tête. Seule l'accompagnait, en caractères à peine légibles, une sobre mention : «o à $100 \mathrm{~km} / \mathrm{h}$ en $8,4 \mathrm{~s}$ » : avec moins d'effet spontané (mignonnerie) et plus d'effort inférentiel, le concept opérait encore.

On peut du reste aller très loin avec les inférences demandées, tant qu'on les récompense amplement par l'effet obtenu. Volkswagen, reconnue pour sa créativité depuis les célèbres annonces de Bill Bernbach dans les années 1960, l'avait testé avec succès en exhibant un dromadaire figuré dans un galop démentiel, sans fournir le moindre indice textuel à part les lettres «TDi» qui figuraient en bas de l'affiche. Il revenait au public de relier cette rapidité insolite à la propriété schématique saillante associée à l'animal en question (sa faible consommation). De nombreux prix publicitaires ont récompensé une telle audace, inusitée en $1976^{3}$.

Il ne s'ensuit cependant pas que l'attente de cohérence s'accommode de toutes les désinvoltures. C'est ce dont témoigne une version britannique de la publicité francophone, elle aussi largement partagée en ligne. On y voit le même chiot, en proie au même tourment, mais

3 Les publicités Volkswagen (chiot et dromadaire) ont toutes deux été conçues par l'agence DDB Bruxelles pour la société D'Ieteren qui représente la marque en Belgique. Je remercie à ce propos Jean-Marc Ponteville et André Lequime pour leur empressement à tenter d'en retrouver les fichiers images si longtemps après leur publication, ainsi que pour les utiles précisions qu'ils m'ont prodiguées en sus (quoique, bien sûr, mon analyse ne les engage en rien, pas plus que quiconque d'autre). 
accompagné cette fois de l'accroche "BMW M5. Ne sous-estimez jamais l'influence de la force centrifuge » et du logo de la marque bavaroise.

Une norme discursive d'un ordre très différent se manifeste alors : si pertinente que soit une création visuelle, il est hautement improbable qu'elle puisse être utilisée par des constructeurs différents (même les plagiats explicites qu'autorisent les normes autojuridictionnelles de l'ironie publicitaire se soumettent aux règles impérieuses de la propriété intellectuelle). Cette discordance trahit évidemment un détournement anonyme, rançon du succès des visuels forts, mais l'autre discordance est plus curieuse. Il n'y a pas besoin de théorie discursive pour s'aviser que le beagle n'est aucunement victime de la force centrifuge (auquel cas il se serait retrouvé dans le vide-poches), mais l'adéquation sociocognitive aide à remarquer à quel point il est anormal qu'un message dévalue ainsi sa pertinence discursive sans aucun profit apparent.

Une telle violation conduit dès lors à soupçonner l'influence de l'autre grand volet de l'adéquation : le jeu des contraintes socialement déterminées. Le fait que son auteur ait de lui-même entrepris d'en dégrader la valeur par l'incohérence remarquée plus haut pourrait ${ }^{4}$ en effet refléter la proscription des arguments liés à la vitesse sur le marché discursif britannique : s'affranchir de cette norme aurait dévoilé la supercherie.

De fait, on peut trouver une norme d'assez haut niveau (c'est-àdire explicite, non contestée et - relativement - coercitive) au chapitre 19 du code britannique de la publicité, lequel proscrit notamment le fait de présenter la vitesse automobile sous un jour séduisant, et même de faire de la vitesse ou de l'accélération le «principal message » d'une annonce ${ }^{5}$. La référence à la force centrifuge - qui pourrait à la rigueur être défendue comme une allusion à la tenue de route plutôt qu'à la vitesse - rend donc l'annonce détournée plus plausible en Grande-Bretagne : elle ne représenterait qu'une optimisation habile de l'adéquation du message par un annonceur préservant autant que possible sa pertinence en fonction des normes qui l'encadrent ${ }^{6}$.

$4 \quad$ Faut-il insister sur le fait que toute interprétation est une spéculation?

5 Committee of advertising practice (2014, p. 87).

6 Et ce, d'autant plus que la fraction du public la plus susceptible d'acquérir une BMW de 500 chevaux est probablement la moins susceptible 
N'était-ce là qu'une spéculation de plus ? Sans aucun doute - seul le détourneur anonyme connaît ses motivations, à supposer qu'il les connaisse - mais outre qu'elle recourt assez peu à l'abstraction, elle s'offre à un minimum de contextualisation empirique. On sait en effet que si les ressorts essentiels de la pertinence cognitive sont relativement constants (dans leur nature plus que dans leur intensité), ceux de la convenance sociale sont, en comparaison, très variables dans le temps, mais aussi dans l'espace.

Ainsi, le rapprochement de la version anglaise et des versions continentales pourrait donner une bonne idée des variations géographiques de l'emprise des contraintes discursives. On trouve, par exemple, dans la dernière édition du Code de l'autorité française de régulation de la publicité, et sans aucun doute dans les précédentes, une norme aussi explicite que celle de son homologue britannique :

La publicité ne doit pas argumenter sur la vitesse, non plus qu'exploiter l'attrait que celle-ci pourrait représenter, tant dans l'expression visuelle, sonore, qu'écrite dans les messages; $[. .$. Elle ne doit pas évoquer la réserve de puissance des moteurs ou les possibilités de freinage à des fins d'encouragement à la vitesse, mais les présenter comme des éléments de sécurité7 [...]

En Belgique, le code édicté par l'industrie automobile (Febiac) indique pareillement :

La publicité ne peut argumenter sur la vitesse, en particulier la vitesse de pointe ou suggérer son attrait. Il en est de même pour la puissance, l'accélération, la force de freinage ou toute autre caractéristique d'un véhicule si celles-ci font référence à la vitesse ${ }^{8}$.

Cependant, comme on l'avait souligné au chapitre 8, le degré de formalisation d'une proscription discursive (son caractère écrit et officiel) est toujours modulé par son intensité expressive (il ne s'agit en

d'adhérer à la proscription sociale de la vitesse (en paroles ou en actes) et que le pouvoir inférentiel des schémas cognitifs garantit en tout état de cause que les lecteurs comprennent le sens réel de l'annonce.

7 Autorité de régulation professionnelle de la publicité (2017, p. 158).

8 Fédération belge et luxembourgeoise de l'automobile et du cycle (2014, p. 1). 
France que d'une «recommandation » et en Belgique de règles d'autodiscipline). Il l'est surtout par la rigidité de son interprétation effective et par les conséquences qu'évalue le locuteur. Si l'autorité britannique de la publicité joue son rôle avec un zèle extrême et condamne de nombreuses campagnes - dont, récemment, un film où une BMW, quoique conduite à une allure légale, pouvait donner une impression $\mathrm{d}^{\prime}$ « excitation et de plaisir » et donc encourager à ses yeux un comportement «dangereux et irresponsable ${ }^{9}$ » - ses homologues continentales sont en général moins intransigeantes. Dans le cas d'une publicité automobile qui, pour le coup, était indiscutablement critiquable, l'organisme français a rendu son avis trois mois plus tard en évitant même de nommer l'annonceur coupable.

En revanche, les concepteurs initiaux de l'image ont, peut-être sans le réaliser, contrevenu à une autre norme, celle-là dépourvue de toute formalisation, mais nettement plus sévère elle aussi dans le monde anglophone que dans la sphère francophone. C'est celle dont s'est avisé trop tard un fabricant d'articles de sport après avoir représenté, sur un chemin de montagne désert, un athlète tentant un massage cardiaque pour réanimer le chien qu'il avait épuisé par sa propre endurance («Run longer» promettait l'annonce). Là encore, l'effet comique produit par cette brillante discordance avec les propriétés normales des schémas cognitifs en jeu attirait vigoureusement l'attention, mais « les réseaux » ne l'ont pas trouvée drôle : on ne plaisante pas avec la vie ou la santé d'un chien. L'entreprise a dû se confondre en excuses sur Facebook (29.08.2013) :

Comme la plupart d'entre nous, ici à Pearl Izumi possède des chiens, nous voulons que vous sachiez que nous ne ferions jamais, absolument jamais, quoi que ce soit qui pourrait nuire à un animal. S'il vous plaît, sachez que nous avons supprimé l'affiche et que nous l'avons retirée de toute publication ultérieure.

De chien en chien, le jeu de la pertinence cognitive et de la convenance sociale permet ainsi de percevoir derrière les images publicitaires des logiques de production et de réception assez similaires entre elles, mais aussi assez différentes de ce que pourraient en

9 ASA Ruling on BMW (UK) ltd. (Advertising Standards Authority, 9 mai 2018). 
révéler des approches fondées sur le code ou les signes. L'inverse, bien sûr, est également vrai (opposer des outils analytiques n'est pas moins vain que de prétendre un tournevis supérieur ou inférieur à une clef à molette) : si l'adéquation sociocognitive vise surtout à expliquer ${ }^{10}$, elle ne brille pas dans une perspective plus descriptive. Ainsi, le beagle de Volkswagen a-t-il été abordé par d'autres ${ }^{11}$ avec l'appareillage - descriptif s'il en est - de la rhétorique des tropes : on y a notamment repéré à juste titre une métonymie publicitaire (la cause figurée par l'effet) conjuguée à une hyperbole. L'espace pédagogique de l'Académie de Nantes incite pour sa part à y distinguer la dénotation et la connotation. Un regard plus artistique aurait pu apprécier le cadrage de cette image, le décentrement de son sujet ou la symétrie des pattes et des ceintures de sécurité, voire, en s'appuyant sur Erwin Panofsky, approfondir quelques aspects iconographiques et iconologiques non relevés ici.

Constatons en outre que l'approche adéquationnelle des discours brille encore moins dans le dévoilement de sens cachés. Laspiration du chiot trahirait-elle un désir inconscient de retour à la vie intra-utérine? Est-elle la résurgence inopinée d'un mythe antique $^{12}$ ? Le choix d'un beagle sous-entendrait-il que la voiture qui l'emporte constitue le sommet de l'évolution automobile ${ }^{13}$ ? Y a-t-il un sens social derrière l'arrogance avec laquelle la banquette luxueuse dévore les victimes de la société de consommation ? Dans cet «espace infini d'interprétations possibles » (Eco, 1992), l'outil sociocognitif s'avère décidément bien pataud. Tant pis.

10 Et encore... Elle n'explique pas la capacité ou non de ces publicités à faire vendre des voitures ou des articles de sport, tout au plus certaines des conditions essentielles qui leur permettent ou non de le faire.

11 Il est possible de trouver en ligne plusieurs exemples de pages didactiques se livrant à cet exercice.

12 Peut-être Chronos (chronomètre ?) dévorant ses enfants.

13 On se souvient que le H.M.S. Beagle était le navire à bord duquel Charles Darwin avait accumulé ses observations, dont le compte-rendu est connu sous le titre Le voyage du Beagle. 
Page blanche conservée intentionnellement 


\section{Le mystère de la pyramide inversée ${ }^{1}$}

T a construction dite en «pyramide inversée » est l'un des monu$\amalg$ ments les plus classiques de l'écriture de presse. C'est aussi l'un des plus étranges. Douteux dans ses origines, fragile dans ses fondements, ce modèle gagne d'autant plus à être revisité que l'âpre concurrence attentionnelle du marché discursif contemporain soumet l'expression de l'actualité à un impératif de pertinence croissant. En outre, la structuration rétropyramidale et son corollaire, l'attaque récapitulative (summary lead) se sont répandus dans la plupart des champs de production discursive où ils sont devenus une norme de référence censée accroître l'efficacité des textes. Or celle-ci traduit, au-delà de son aspect technique (mais il ne faut jamais négliger les aspects techniques), une façon très significative d'envisager les interactions communicationnelles.

Rappelons-en la substance. L'attaque récapitulative (ou summary lead, aussi appelée ${ }_{5} \mathrm{~W}+\mathrm{H}$, ou pire QQQOPC) consiste à commencer un texte par son information essentielle, laquelle doit autant que possible répondre à cinq questions : Qui ? (a fait) Quoi ? Quand? Où ? Pourquoi ? Comment ${ }^{2}$ ? La structuration en pyramide inversée repose sur le même principe de préséance de l'essentiel - dire l'important d'abord - prolongé cette fois à la totalité du texte : celui-ci comporte d'abord l'information principale, telle que définie précédemment, puis les éléments d'information accessoires, présentés par ordre décroissant d'importance.

Si ces deux outils ne sont pas toujours associés dans les manuels ou les cours, ils ont connu, ensemble ou séparément, un succès qui ne

I Ce texte est en partie repris de Structures narratives et congruence cognitive : cas du summary lead et de la pyramide inversée (Labasse, 2012c). Il incorpore en outre des éléments de Le journalisme au miroir de la littérature (à moins que ça ne soit l'inverse) (Labasse, 2014c).

2 Soit, en anglais : Who? (did) What? When ? Where? Why? How ? 
se dément pas. Ils sont fondamentalement attachés à l'écriture de presse : "Qui a fait Quoi, Où, Quand et Pourquoi ? Ce sont les 5 W que l'on enseigne depuis la nuit des temps (modernes) dans toutes les écoles de journalisme du monde... » (Antoine et al., 1995, p. 16). D'un auteur à l'autre, les différences portent surtout sur leur caractère impératif ou non3. Plusieurs suggèrent, implicitement ou explicitement, que ce moule est incontournable :

Vous rédigez d'abord l'essentiel des faits : Qui ? Où ? Quand ? Pourquoi ? Comment ? [...] Cette expérience vous enseignerait que tout peut se condenser, elle vous apprendrait aussi comment se bâtit un article : d'abord une phrase qui résume l'essentiel, puis des développements successifs, par ordre d'intérêt décroissant. (Florio, 1984, p. 72-734)

Lécriture journalistique ne peut connaître qu'un seul plan, celui qui valorise l'information centrale. [...] Le premier paragraphe de tout article doit dire le cœur de l'affaire. La suite les développe par ordre d'importance décroissante. Sans doute, les modalités de cette écriture-là sont très variées [...] Mais la construction est invariable. C'est le plan réputé «en pyramide inversée.» (Mouriquand, 1997, p. 485)

Comme pour mieux exprimer le caractère sacré des $5 \mathrm{~W}+\mathrm{H}$, Hartman (1968, p. 91) les figure sous la forme d'un temple grec, dont le fronton porte l'inscription «ELEMENTS OF LEAD» et dont chacune des six colonnes représente l'une des questions.

Mais d'autres voient plutôt dans ces règles une norme par défaut, qui peut admettre des exceptions, essentiellement pour certains articles longs de type magazine (feature) : «just as Five $W$ leads are inadequate for depth stories, so is the inverted pyramid" (Lorenz \& Vivian, 1996, p. 98). Pour ne pas y renoncer trop vite,

3 Bien que certains auteurs les réfutent globalement.

4 L'omission assez étrange de la question centrale «Quoi ? ", sans laquelle les autres sont un peu surréalistes, est probablement une coquille, l'auteur étant un enseignant et un praticien chevronné.

5 En une contradiction fascinante, mais significative, le même auteur affirmait peu avant que «La construction des écrits journalistiques, à l'inverse d'autres, ignore les moules » (p. 32). 
même lorsque le sujet doit aborder plusieurs thèmes, Ross (1994) a proposé une solution sophistiquée : «On pourrait parler de pyramide multiple: quelques petites pyramides (schématisant des divisions $d u$ texte) s'emboîtent pour en former une grande (schématisant l'ensemble de la structure du texte) » (p. 62).

Il convient cependant de noter que, tandis que ces préceptes maintiennent leur domination dans les manuels et les enseignements, il peut arriver qu'ils soient remis en question avec vigueur par des praticiens (ou praticiens-enseignants) expérimentés :

Dans les programmes de journalisme, les étudiants travaillent principalement les formes d'écriture les plus simples et les plus figées, des formes que la plupart de leurs professeurs n'ont pas utilisées depuis longtemps, et, pour dire la vérité, n'ont aucun désir de réutiliser. (Stephens, 2010, p. 40)

Comme on l'a dit, ces outils se sont développés bien au-delà de la rédaction journalistique pour se disséminer dans les différents domaines de la communication écrite. Cette influence doit probablement beaucoup à Gunning, l'un des auteurs dominants en la matière : "I have run across men in business who thought this was the way to write report: Feed out details gradually [...] But this is a poor way to organize a piece of writing that is chiefly information. [...] when your chief aim is to hand on information, the inverted pyramid form is best $\gg(1968$, p. 122). De fait, le principe de préséance de l'essentiel occupe aujourd'hui une place importante dans la rédaction en milieu de travail : «Pour un écrit professionnel, une lettre officielle, une note de service, répondre strictement à des questions simples : Qui ? Quoi ? Quand? Où ? Comment? Pourquoi ? Pour qui ?» (Peyroutet, 1997, p. 68) ; "Ricordiamo la regola giornalistica delle cinque Wh- : chi, che cosa, quando, dove, perché (dall'iniziale delle parole inglesi correspondenti : who 'chi ?', what 'che cosa?', when 'quando ?' where 'dove ?', why 'perché ?') (Bruni \& Alfieri, 1997, p. 37). Même la rédaction scientifique, au-delà de la sempiternelle description du plan IMRAD ${ }^{6}$, s'est laissée gagner par ce modèle : "L'introduction doit être courte [...] L'essentiel doit être dit dès les premières phrases. La fameuse accroche [sic] des journalistes et des publicitaires est, en effet, capitale pour appeler et retenir le lecteur » (Bénichoux, p. 62).

6 Introduction, Matériel et méthodes, Résultats et (and) Discussion. 
On peut ainsi retrouver ces idées dans les conseils donnés aux agronomes tropicaux par la revue d'un organisme de développement international ("Les modules de formation en communication écrite citent invariablement ces règles inspirées des méthodes journalistiques : [...] hiérarchiser l'information et respecter la règle des QQQOPC. Un message concis répond en effet à six questions [...] » CTA, 2000, p. 5) de même que dans les suggestions que prodigue aux ingénieurs la revue d'une agence nationale de veille technologique («La pyramide inversée : hiérarchiser pour structurer » ADIT, 1999). Enfin, la méthode connaît un succès considérable chez les concepteurs de sites Internet : « Une page Web n'est pas un document papier! La lecture écran est $25 \%$ plus lente que la lecture papier: on ne lit pas à l'écran, on scanne. D'où l'importance à accorder [...] à l'efficacité d'une écriture en pyramide inversée : donner en premier lieu l'idée principale [...] et ne détailler qu'ensuite » (Carrière et Schreiber, 2000, p. 2).

Toutefois, si la pyramide inversée et les $5 \mathrm{~W}$ méritent examen, c'est moins pour leur poids global dans le champ de la communication que pour ce qu'ils révèlent de celui-ci. À travers cet exemple, on constate d'abord que le journalisme exerce une ascendance inattendue - une expertise - sur l'ensemble du champ de la rédaction appliquée. Ce magistère, est, du reste, très souvent invoqué: « Les journalistes peuvent-ils constituer de bons exemples pour nous, écrivants d'entreprise? Mais bien sûr " (Berrou, 1991, p. 121). "My general advice for curing many of the problems that tend to recur in science writing is really quite simple: think of yourself as a reporter, as well as a scientist. You can learn something from journalists when you write the formal science report " (Porush, 1995 p. 19). "Les journalistes ont beaucoup à nous apprendre à ce niveau " (ADIT, op. cit.).

Sans débattre ici de l'ensemble des apports conceptuels du journalisme, il convient donc d'examiner avec attention les facteurs qui ont érigé la pyramide et des $5 \mathrm{~W}$ en « savoirs de référence ».

\section{Une genèse discutable}

Symboliquement, ces notions semblent représenter la transition entre une forme d'écriture ancienne, alambiquée et littéraire, et celle des "temps modernes", où l'on sait aller à l'essentiel (voir supra l'extrait d'Antoine et al.). Il n'est pas indifférent de noter qu'elles sont attachées aux États-Unis, pays dont l'image est traditionnellement 
associée à celle d'efficacité productive. Esquissant les contours du premier vrai programme d'enseignement du journalisme d'Espagne, Graña-Gonzáles (1930) note ainsi : "Los yanquis han llegado también a un grado de perfección que debemos imitar. El lead [...] es un artificio técnico que prontó serà adoptado por los buenos periódicos européos" (p. 157). De fait, contrairement à d'autres notions, qui semblent flotter dans ce domaine, venues d'on ne sait où et d'on ne sait qui, elles ont une origine claire et on ne peut plus "moderne", puisqu'elles coïncideraient avec l'essor de la civilisation industrielle et celui des « nouvelles technologies de l'information », en l'occurrence le télégraphe. L'histoire est connue :

Ce sont les défectuosités du télégraphe, en même temps que le coût de son utilisation, qui apporteront ses premières règles au journalisme américain. Ce sont elles qui imposent aux correspondants de guerre la grande concision de leur expression. [...] Ainsi, les premières recommandations aux journalistes américains sont nées des hasards de l'histoire ou des nécessités de la technique : le procédé de la pyramide inversée, qui impose la synthèse avant la présentation des détails; le respect des cinq interrogations de l'amorce, les 5 «W » Who, What, When, Where, Why - (Qui ? Quoi ? Quand ? Où ? et pourquoi ?) enfin la recommandation d'un style à la fois dépouillé et impersonnel. (Balle, 1987, p. 24-25)

Cette généalogie est même reprise par l'un des plus farouches contempteurs de ces procédés, Flesch, qui souligne que «the typical news lead is a Civil War relic. In those days, the telegraph wasn't too reliable and war correspondent invented [...]» (p. 63). L'histoire de cette «invention » est séduisante, mais elle est un peu courte : l'idée générale d'aller au fait, sans fioritures, remonterait au moins à Homère (Iliade ${ }^{8}$ ) et

7 Le terme «lead » est en italique dans l'original, avec une note de renvoi : «Pronúnciese liid. »

8 Ainsi que le souligne plus tard Horace qui, dans un vers célèbre de son Art poétique (ou Épitre aux Pisons), rappelle qu'Homère plonge son lecteur « in media res » (au milieu des choses). Pour contribuer, même très approximativement, à l'histoire des techniques rédactionnelles, aujourd'hui si lacunaire, on précisera qu'Horace est, de facto, l'un des principaux théoriciens de ce champ. Son Art poétique a directement inspiré celui de 
le «questionnement standard» à Aristote (Éthique 3:1). Si la préséance de l'essentiel est approuvée dans la rhétorique à Hérennius', le principe décroissant de la pyramide est notamment discuté par Quintilien, qui le rejette ${ }^{10}:$ "Les arguments les plus puissants doivent-ils être placés au commencement, pour s'emparer des esprits, ou à la fin, pour laisser les auditeurs sous cette impression, ou partie au commencement et partie à la fin [...] On suivra l'ordre qu'exige la cause, à une exception près : il ne faut pas une progression descendante du fort au faible ${ }^{11}$. » Le même rappelle par ailleurs les circonstances à traiter dans la narration: "personne, cause, lieu, temps, instrument, occasion» (id., p. 61). De saint Thomas d'Aquin (Somme théologique, 1266, Ia IIa, Qu. 7) jusqu'à Goscinny \& Uderzo (Astérix ${ }^{12}$ ) en passant, dans des optiques et registres bien différents, par Kipling $\left(1902^{13}\right)$ ou Lasswell $\left(1948^{14}\right)$, le

Boileau (qui l'a traduit) et ce dernier est l'un des deux auteurs les plus cités par les manuels de rédaction. Sur 50 ouvrages (12 anglophones et 38 francophones), il est cité 14 fois, soit plus que Flesch ou Gunning (seul Richaudeau parvient à le dépasser). Bref, une bonne partie des préceptes "modernes", tels que l'importance de la méta-cognition (que tendent aujourd'hui à confirmer les recherches en sciences cognitives) nous viennent de Boileau (1636/1711) transposant Horace (-65/-4), lequel invoque à son tour Homère (vers -80o).

9 «Nous pourrons captiver son intérêt si nous savons exposer rapidement le fond de la cause » $(1875$, p. 4$)$.

10 Perelman (1997, p. 163) rappelle cependant que les ordres croissant et décroissant étaient également envisagés dans la rhétorique antique, en fonction de la cause, même si l'ordre nestorien (le plus important au début et la fin, le reste au centre) était généralement préconisé.

11 Institution oratoire, Livre V (trad. Bornecque, 1933, p. 231).

12 «Quis, Quid, Ubi, Quibus auxiliis, Cur, Quomodo, Quando? " demande un centurion, effaré par l'aveu que vient de faire son supérieur (La serpe d'or, 1962, p. 43).

13 À la fin de son conte « The Elephant's Child» (in Just So Stories, 1902), consacré aux dangers de la curiosité, se trouve ce quatrain : "I keep six honest serving-men // (They taught me all I knew) // Their names are What and Why and When // And How and Where and Who."

14 C'est pour synthétiser les approches usuelles des processus de communication de masse que Lasswell, dans un article fameux, les résumera par la formule « Who // Says What // In Which Channel // To Whom // With What Effect? » (capitales selon l'original ; les doubles fractions correspondent à un retour à la ligne), son propos étant de regretter que les recherches se focalisent sur l'un ou l'autre de ces points, auxquels 
recours au célèbre questionnement de la tradition rhétorique - Quis, Quid, Ubi, Quibus auxiliis, Cur, Quomodo, Quando? - est décidément trop constant au fil du temps pour être attribué de but en blanc au télégraphe. Mais seul un très petit nombre d'auteurs paraît s'en aviser.

Quoi qu'il en soit, c'est surtout la question de son application effective qui importe. Et c'est surtout là que l'histoire collective de ces notions semble lacunaire : l'examen d'une vingtaine de quotidiens américains entre 1860 et 1910 (Errico et al., 1997) montre en effet que le taux d'articles utilisant vraiment le lead en $5 \mathrm{~W}$ reste inférieur à $1 \%$ pendant presque tout le $\mathrm{XIX}^{\mathrm{e}}$ siècle, n'atteignant $1,14 \%$ qu'en 1895 (trente ans après la guerre civile) : les messages de ce type étaient sans doute destinés à être réécrits, et non imprimés en l'état.

Un autre auteur qui a travaillé sur cette période (Mindich, 1998) suggère même que ce modèle pourrait avoir été développé, non par les journaux eux-mêmes mais, horresco referens, par les censeurs du ministère de la guerre. Quoi qu'il en soit, l'histoire des $5 \mathrm{~W}$ nous paraît pouvoir s'interpréter non comme une trouvaille soudaine, mais comme celle d'une confusion progressive, qui a insensiblement transformé ce qui est fondamentalement une technique de synthèse préalable de l'information - à l'origine pour structurer ses idées et plus tard pour transmettre des données essentielles - en une technique d'exposition directe de cette information.

\section{Des lectures confuses}

Un autre aspect essentiel de ces notions, comme on l'a dit, est leur valeur déclarative, donc didactique. Parce qu'elles sont désignées par des termes spécifiques, et qu'elles sont collectivement formalisées (même si l'on peut rencontrer à leur propos de nombreuses variations au hasard des auteurs) elles constituent quelque chose qui peut s'écrire ou se dire, et non de simples habiletés à conférer par la pratique. Mais ces « connaissances » enseignables sont-elles didactiquement pertinentes?

il oppose une approche plus globale. Toutefois, Lasswell ne se référant pas au questionnement classique, on ne peut dire dans quelle mesure ce dernier l'a inspiré ou non. 
Un premier doute peut provenir de l'apport cognitif des $5 \mathrm{~W}$ pour le rédacteur aux prises avec le matériel qu'il a à traiter. Sans s'attarder sur le fait, évident, que réduire le réel à une trame où quelqu'un a immanquablement fait quelque chose n'est souvent possible qu'au prix d'un élargissement considérable des notions d'agent et d'action (par ex., "l'inflation a augmenté... ») et paraît fort difficile à appliquer dans bien des cas, on notera que la détermination réciproque de l'agent et de l'action est généralement très délicate. Ainsi une forme telle que [Qui ? :] Le laboratoire... [Quoi ? :]... a connu des incidents, paraît-elle informativement équivalente à [Qui ? :] Des incidents... [Quoi ? :]... ont affecté le laboratoire. Il ne s'agit évidemment là que de permutations assez vaines (Belle marquise vos yeux... Vos yeux, belle marquise... etc.), mais c'est justement à ce type d'impasse que peut conduire le questionnement $5 \mathrm{~W}$ s'il est imprudemment érigé en dogme. Ainsi peut-on se demander si, au lieu de réduire la charge cognitive du rédacteur novice, cet «élément d'expertise » ne contribue pas à l'accroître en l'orientant vers un dédale réflexif (qui devient inextricable lorsqu'un sujet concerne de nombreux agents et actions). Paradoxalement, dans bien des cas, le désir de satisfaire à ce questionnement pourra même conduire à focaliser le sujet sur un élément qui s'y prête, mais qui n'est pas l'information essentielle : la lettre de la règle se retourne dans ce cas contre l'esprit de la règle.

La construction en pyramide inversée n'est pas en reste. Numérotant les dix éléments d'information que comprenait une dépêche assez typique (le déraillement d'un train de marchandises au Canada), j'avais demandé aux étudiants d'un cours de journalisme de les classer par ordre d'importance. Leurs classements étaient si différents que j'ai répété l'expérience d'année en année, mais sans jamais rencontrer de concordance entre les évaluations individuelles de l'importance de ces divers éléments.

La pyramide inversée pose par ailleurs un problème didactique d'un autre genre. L'utilisation d'un terme géométrique ou architectural puissamment évocateur (pyramide) constitue une façon presque métaphorique de concevoir le texte. Dès lors, la question est évidemment de savoir si le modèle mental qu'elle propose correspond à l'idée qu'elle est censée supporter. À quoi peut donc bien ressembler un texte conçu comme une pyramide inversée? La confrontation des idées que peuvent s'en faire divers auteurs est révélatrice des limites de cette métaphorisation. 


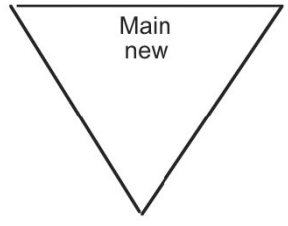

LORENZ et al. 1996 p.95
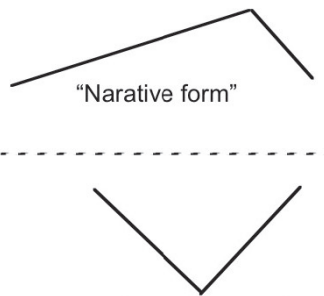

"Inverted pyramid form"

GUNNING

1968 pp.121-122

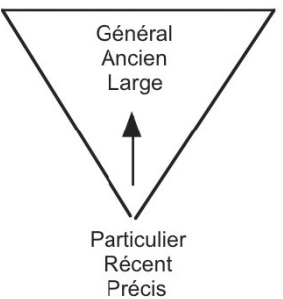

FAURE 1998 p.109

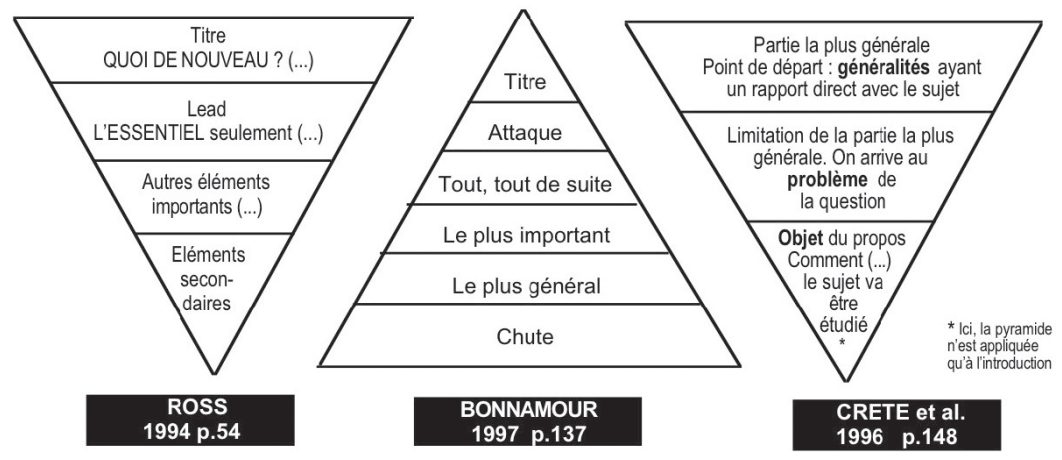

Figure 7. Quelques représentations du modèle pyramidal

On constate sans peine que ces exemples ne sont pas compatibles entre eux, et sont même opposés sur un point majeur : le « haut » de la pyramide, donc le début du texte, correspond-il à l'information principale de ce texte, ou, au contraire, à des généralités préliminaires ? En principe, la réponse est sans équivoque : le début du texte est bien censé être la partie la plus importante de celui-ci, comme l'indiquent Gunning, Lorenz \& Vivian et Ross. Mais les inversions (variations ou contresens) que l'on remarque chez les autres illustrent clairement l'inadéquation cognitive de ce modèle : la métaphore géométrique suggère un critère dimensionnel (le sommet doit être plus « large » que la base) alors qu'elle ne porte que sur un critère qualitatif (le sommet doit être plus « important » que la base). Ce point est d'autant plus notable que la pyramide inversée est le seul schéma qui soit largement répandu dans le champ de la rédactologie, où les discours sont essentiellement textuels. On mesure cette difficulté en constatant que Gunning rapproche deux graphismes qui ne présentent en réalité aucune cohérence. Dans ces deux figures, le critère est bien qualitatif 
(importance), mais le premier, qui représente les textes classiques, progressant jusqu'à un paroxysme avant de retomber vers leur conclusion, doit se lire de la gauche vers la droite (la hauteur correspondant à l'« importance » croissante des éléments), tandis que le second, qui est le modèle normal de la pyramide inversée, se lit du haut (début de l'article) vers le bas (fin), la largeur correspondant à l'« importance » des éléments. Comme le remarque justement Ross (seule à paraître s'en inquiéter) "Malgré son utilité [...] l'image de la pyramide risque de prêter à confusion" (op. cit., p. 55). En l'occurrence, le «risque» paraît une certitude : le fait que les auteurs eux-mêmes puissent s'y perdre montre que le seul schéma à vocation heuristique de ce champ est non seulement peu adéquat, mais même dommageable puisque, loin de faciliter la construction d'une représentation correcte, il tend à favoriser une mauvaise compréhension (l'un des gourous les plus influents de l'optimisation des sites Internet est même persuadé que la technique de la pyramide inversée - qu'il prescrit - consiste à commencer un texte... par sa conclusion $\left.{ }^{15}\right)$.

\section{Une efficacité très variable}

D'un point de vue opératoire, la question la plus importante que posent les $5 \mathrm{~W}$ et la pyramide inversée demeure celle de leur efficacité. S'il s'agit bien, toute considération historique mise à part, de notions résolument modernes, c'est parce qu'elles ne cherchent pas leur justification dans des considérations esthétiques, des normes institutionnalisées ou dans une meilleure adaptation au sujet traité, mais dans une volonté de rendement, d'efficacité informative. Celle-ci est d'autant plus marquée qu'elle concerne simultanément les trois degrés de l'interaction textuelle :

Le rédacteur. Le questionnement permet de rassembler ses idées, de vérifier que l'on dispose de tous les éléments nécessaires, d'éliminer l'inutile, voire de stimuler son inspiration. Beaucoup d'auteurs s'en tiennent, du reste, à cette utilisation : "Posez-vous ces questions. Elles servent d'ouvre-boite mental » (Barras, 1978, p. 38). C'est à ce titre qu'il

15 «En conséquence, les pages web doivent employer des textes scannables, en utilisant [...] le style en pyramide inversée, qui commence par la conclusion ». Jakob Nielsen, How Users Read on the Web (1997) en ligne à : www.nngroup.com/ articles/how-users-read-on-the-web. 
était un élément traditionnel de l'investigation rhétorique (l'inventio) et des manuels des confesseurs.

Le texte lui-même. Le document ainsi rationalisé devient aisément synthétisable, et surtout redimensionnable en fonction des besoins et de la place disponible, puisqu'il suffit d'en couper la fin pour conserver l'essentiel. C'est la raison pour laquelle cette structuration constitue à bon escient une norme dans toutes les agences de presse télégraphiques : "sur la dépêche de zoo lignes [...] un journal pourra, selon ses choix propres, offrir la totalité à ses lecteurs, se contenter de 200 lignes, des 80 premières ou du seul premier paragraphe [...]» (Hervouet, 1979, p. 73). Le même principe vaut pour des documents d'autres sources, tels que les communiqués de presse (qui visent à être partiellement ou totalement reproduits à l'identique) ou certains textes techniques particuliers.

Le lecteur. La raison la plus fréquemment invoquée en faveur du principe même de la préséance de l'essentiel (en général) est qu'il est le plus à même de répondre aux attentes du destinataire : « Le lecteur est une personne pressée » (Boclé \& Patriot, 1997, p. 60), qui, ainsi, "entre directement dans le vif du sujet» (Collectif, 1987, p. 150). En outre, cette préséance, quand elle s'étend à toute la structure du texte (pyramide inversée) favorise le redimensionnement de celui-ci par son destinataire lui-même puisqu'elle "permet à l'auditeur ou au lecteur de s'arrêter lorsqu'il a toute l'information qu'il désire » (Gunning, 1968, p. 189). Il s'agit donc d'un «plan psychologique» (MartinLagardette, 1994, p. 69). En termes strictement matériels (longueur du texte) l'efficacité de la construction rétropyramidale ne fait aucun doute : on voit mal, en particulier, comment les agences de presse pourraient remplir leur fonction sans y recourir. Mais ce type de nécessité reste très spécifique ; dans leur grande majorité, les textes n'ont pas pour vocation essentielle d'être retaillés à volonté. Or, l'argument dominant - celui des goûts des lecteurs - repose sur une vision assez partielle de leurs attentes.

Deux points sont en sa faveur. D'une part, nul ne prend plaisir à parcourir un délayage interminable, d'autre part, de nombreuses études ont montré que les lecteurs n'ont pas le temps matériel de parcourir toutes les informations qui s'offrent à eux. Mais d'un autre côté, supposer que le seul contenu de l'information est ce qui en fait la valeur (d'où l'idée de l'exposer au plus vite) est l'une des plus 
grandes erreurs que puisse commettre un rédacteur. Elle présuppose en effet de ses destinataires un appétit de savoir, spontané et plus ou moins universel, qu'il ne s'agirait que de satisfaire en fournissant les données correspondantes. Or, la très grande majorité des informations sont loin de bénéficier d'un tel désir. La surabondance d'informations, qui a instinctivement induit la question (passive) "Comment permettre au lecteur d'accéder vite à ce qui l'intéresse?", amène également une question très différente (active) "Comment amener le lecteur à s'intéresser à mon information?». Dans le monde entier, les sondages disponibles sur les connaissances et intérêts du public confirment que ceux-ci sont largement inférieurs à ce que pourraient escompter les professionnels. Ainsi, aux États-Unis, «An analysis of public attentiveness to more than 500 news stories over the last ten years confirms that the American public pays relatively little attention to many of the serious news stories of the day " (Parker \& Deane, 1997). Le problème qui se pose à la presse n'est pas foncièrement différent de celui que rencontre le rédacteur tentant, au sein d'une entreprise ou d'une institution, de donner à son texte une chance d'être lu : informer n'est pas seulement fournir, c'est aussi attirer, montrer d'abord que la question mérite que l'on s'y intéresse de préférence à une autre.

Le principe de préséance de l'essentiel méconnaît ainsi une distinction fondamentale : soit l'intérêt "va de soi ", c'est-à-dire qu'il repose sur une relation directe entre l'information et le lecteur - typiquement un effet cognitif de premier niveau (spontané) ou de second niveau (égocentré, utilitaire) -, soit il ne va pas de soi, et la relation doit alors être construite. L'un des éléments essentiels de cette construction est la «problématisation » : le fait de susciter un déséquilibre cognitif, une attente. Ce point est surtout familier aux didacticiens; "l'art de poser des questions provocatrices est au moins aussi important que celui qui consiste à donner des réponses claires" (Bruner, 1996, p. 158), mais ses implications sont beaucoup plus générales (voir Ram, 1990). En fait, elles sont même plus importantes dans la vie en général que dans la salle de classe, où de toute façon, l'attention est socialement contrainte (par l'obligation de présence, la perspective des examens et le maître lui-même). Du reste, le principe même de problème est au cœur de la rhétorique. Pour Meyer (1999, p. 242) c'est-là une de ses caractéristiques fondamentales : «elle traite de questions, de la problématicité, de l'énigme. " S'il n'y a pas 
de question à résoudre, il ne peut y avoir de débat, et le discours est inutile en soi. Cet auteur étend même le raisonnement au langage en général :

Parler, c'est soulever une question. Ecrire aussi. C'est pourquoi on ne parle pas de ce qui va de soi [...] le langage est utilisé pour attirer l'attention sur une question - c'est ce qu'on appelle la communication ${ }^{*}$ - à propos de laquelle on pourrait être en désaccord, ou tout simplement, à côté de laquelle on pourrait passer. (id., 1999, p. 73-74; * en ital. dans l'original)

Or l'art du lead en ${ }_{5} \mathrm{~W}$, comme l'art de la phrase courte et des mots simples, est précisément celui de fournir des réponses claires à des questions (Qui ? Quoi ?, etc.) sans se demander si le lecteur se les pose. Fondamentalement, il constitue plus une technique d'expression des faits qu'une technique de communication des faits. Il n'est donc pas bon ou mauvais en soi, et son utilité ne dépend pas nécessairement de la longueur du texte à produire. Elle dépend essentiellement du caractère «naturel » ou «à construire » de l'intérêt du sujet. Dans le premier cas - par exemple une catastrophe provoquant des centaines de morts - il va sans dire que la présentation des faits se suffit à ellemême et demande peu de compétence rédactionnelle. C'est le type même de textes que parviennent déjà à produire de façon relativement convaincante des prototypes de systèmes rédactionnels automatiques - des journalistes-robots, en quelque sorte - tels que Newsblaster développé par une équipe de l'Université de Columbia. Mais pour la majorité des thèmes importants de l'actualité - politiques, économiques, scientifiques, etc. - un tel traitement, loin d'être " efficace", est au contraire remarquablement inadéquat, parce que les « faits » à présenter ne répondent à aucune attente cognitive et se trouvent ainsi abandonnés à eux-mêmes au sein de la brutale concurrence du marché discursif.

On perçoit ce handicap en comparant par exemple l'attractivité de deux attaques portant sur le même sujet, mais dont l'une, passive, est centrée sur les faits [A], et l'autre, construite, est centrée sur le problème $[\mathrm{B}]$ :

[A] Une équipe de l'Institut de physique nucléaire de Lyon va installer cet hiver près de Modane (Savoie), un instrument 
destiné à étudier les particules massives à faible interaction (WIMPS). L'appareil, un détecteur à très basse température, sera placé dans une cavité communiquant avec le tunnel du Fréjus. (Information brute, non publiée)

[B] L'Univers nous cache quelque chose. Quelque chose d'énorme, dont nous ne savons strictement rien, si ce n'est, précisément, que ça nous est caché. Les physiciens ont en effet l'irritante conviction que toute la matière connue ne représente qu'une faible fraction de la masse du cosmos, et que la nature du reste leur échappe totalement. (Isotopes $n^{\circ}$ 6, septembre 1994, p. 8)

D'autre part, l'attaque récapitulative et le plan rétropyramidal ne sont pas seulement inertes en termes d'effet cognitif (lorsque l'information ne le produit pas d'elle-même), ils sont de surcroît très coûteux en effort cognitif dans la mesure où ils ne favorisent pas l'établissement de la cohérence discursive. Outre qu'ils impliquent de juxtaposer des énoncés successifs en fonction de leur importance plutôt que de leurs liens logiques, ils peuvent conduire à produire, ajout après ajout, des textes étonnamment redondants, comme dans cet extrait d'une dépêche d'agence de presse (soulignée par nous) :

L'A320 de Gulf Air a effectué une approche à une "vitesse excessive »

30 août $2000-20$ h 48

MANAMA (AFP) - L'Airbus A320 de Gulf Air, qui s'est abîmé le 23 août en mer à son atterrissage à Bahrein, a effectué une approche finale à une "vitesse excessive" ne pouvant pas lui permettre de se poser, a annoncé mercredi un responsable de l'Aviation civile de Bahrein.

« La vitesse de l'Airbus de Gulf Air au moment de son approche finale vers l'aéroport de Bahrein était excessive", a déclaré le sous-secrétaire du ministère des Transports pour les affaires de l'Aviation civile Ibrahim Al-Hamer, lors d'une conférence de presse à Manama.

"Cette vitesse est excessive pour un avion de ce type en phase d'approche finale avant de se poser [...] c'est pour cela que la tour de contrôle (ATC) a conseillé au pilote de remonter de 600 pieds 
à 2500 pieds d'altitude ( 180 à près de 800 mètres) pour tenter une deuxième approche », a poursuivi M. Hamar.

Il a toutefois souligné que «les raisons du crash ne sont pas encore connues, mais d'après les résultats préliminaires de l'enquête, la vitesse de l'approche finale de l'A320 était de 270 nœuds (environ $490 \mathrm{~km} / \mathrm{h}$ ), donc plus élevée que la vitesse maximale pour une approche finale ». [...]

Il n'est donc pas surprenant que de multiples recherches, dont deux de grande ampleur (Denton, 1993 ; Readership institute, 2001) menées auprès de lecteurs de presse et deux autres expériences menées auprès d'étudiants (Kelly, Knight et al., 2003 ; Zerba, 2008) aient clairement établi que les textes employant ce procédé étaient globalement moins bien reçus et retenus que les autres formes d'exposition testées (narrative, explicative, etc.).

Ce qui est surprenant est que la plupart des enseignants en rédaction professionnelle, auteurs de manuels et responsables éditoriaux ne s'en soient pas avisés de longue date. La méconnaissance scientifique, technique et professionnelle dont s'accommodent les intervenants du marché discursif, en particulier les éditeurs de presse, est d'autant plus frappante que ceux-ci sont les premiers à déplorer la désaffection qui frappe les contenus qu'ils proposent.

La logique qui sous-tend les $5 \mathrm{~W}$ et la pyramide, si elle est intégrée sans une claire conscience de ses limites, conduit à attribuer systématiquement au lecteur une responsabilité qui n'est pas seulement la sienne, mais avant tout celle du producteur discursif. La responsabilité de percevoir la valeur de l'information et même celle d'évaluer la longueur que celle-ci mérite (en interrompant sa lecture lorsqu'il estime avoir « suffisamment » d'information ${ }^{16}$ ).

Le lead en $5 \mathrm{~W}$ et la pyramide inversée possèdent clairement une justification dans divers cas, liés soit au fond (informations factuelles simples et répondant d'elles-mêmes à une attente spontanée) ou à la forme (nouvelles brèves, dépêches d'agence). Par ailleurs, d'autres avantages, qui relèvent plutôt des normes discursives, mais qui

16 Hormis les cas particuliers, doit-on considérer qu'un texte qu'un lecteur abandonne au milieu est un bon texte (puisqu'il est construit pour cela) ou un mauvais texte (puisque le lecteur ne va pas jusqu'au bout)? 
exigeraient des développements trop longs ici, pourraient dans une certaine mesure leur être reconnus ${ }^{17}$. En somme, ils peuvent être d'excellents outils, au même titre qu'un tournevis cruciforme ou un spectromètre de masse, mais, comme eux, ils ne sont utiles qu'à celui Qui sait à Quoi, Quand, Où, Pourquoi et Comment les utiliser.

\section{Des vertus littéraires insoupçonnées ?}

Curieusement, l'une de leurs vertus les plus fascinantes, mais aussi celle qui leur est le moins reconnue, pourrait être d'ordre... littéraire. Si les spécialistes de la littérature (surmontant un mépris constant de Brunetière à Gide) accordent aujourd'hui à certains textes journalistiques une réelle valeur créative, c'est pour réserver celle-ci aux formes les plus nobles et les plus travaillées du grand reportage et de la chronique. Même Alain Vaillant, promoteur du texte journalistique en "objet littéraire à part entière, au même titre que le drame romantique ou que tel roman de Balzac» (2001, p. 7) refuse cette élévation à l'article ordinaire d'actualité qui "n'est destiné qu'à une lecture immédiate et ponctuelle » (p. 26). De même, l'ajout d'écrits d'actualité aux textes légitimes étudiés à l'école suscite des réticences éloquentes (Paveau, 2001).

Il est vrai que le statut autojuridictionnel d'une information brute en pyramide inversée ne la soumet en rien aux critères de la délectation esthétique, les professionnels eux-mêmes n'étant pas les derniers à l'écarter : "Le journalisme n'est pas de la littérature » insiste ainsi un manuel (Voirol, 1992, p. 15). Mais si on la regarde bien, cette forme éculée dans le champ journalistique est aussi audacieuse et perturbante qu'il est possible de l'être dans le champ littéraire. Pour les étudiants en journalisme et les plus traditionnels de leurs professeurs, il s'agit de la façon «normale » de décrire et raconter. Il suffit pourtant de soumettre des étudiants en lettres au même exercice pour vérifier à l'aune de leur désarroi combien cette construction «normale » est inouïe : dans leurs recherches de nouvelles modalités narratives, ni l'Oulipo ni le Nouveau Roman n'avaient songé à un tel dispositif. Et il ne s'agit pas là d'une exploration expérimentale ou

17 Notamment le fait qu'une étude au moins suggère que la forme rétropyramidale pourrait être perçue par les lecteurs anglophones comme plus crédible que d'autres modalités d'exposition (peut-être parce qu'elle semble plus « objective » et correspond mieux au discours journalistique habituel), ce qui influence évidemment la valeur accordée au discours. 
d'un cauchemar d'écrivain, mais de la forme ordinaire des nouvelles : tenter d'imaginer un roman commençant par l'essentiel puis exposant le reste par ordre d'importance décroissant suffit pour percevoir à quel point cette structure pourrait être novatrice (et rébarbative, bien sûr, mais ça n'a jamais arrêté les pionniers du Nouveau roman).

Il n'en reste pas moins que, dans leur champ originel, l'examen des « savoirs » communicationnels de base que sont l'attaque récapitulative et la pyramide inversée conduit à relever de multiples problèmes, que l'on peut résumer par autant d'antinomies :

- entre l'origine historique qu'on leur prête et leur genèse réelle ;

- entre la simplification (structuration des idées) que les ${ }_{5} \mathrm{~W}$ apporteraient au travail du rédacteur et la surcharge cognitive qu'ils peuvent provoquer pour celui-ci ;

- entre le «message essentiel » qu'ils visent à cerner et le « scénario circonstanciel » qu'ils tendent à forcer ;

- entre le sens attaché au terme «pyramide » et la représentation mentale qu'il suscite ;

- entre l'image de l'une et l'autre de ces notions hors du champ du journalisme et les distances que prennent avec elles beaucoup de professionnels des médias ;

- entre leur justification par le contexte informationnel (surabondance des informations) et leur efficacité concurrentielle dans ce contexte (susciter et maintenir l'attention) ;

- entre leurs justifications "psychologiques» (accessibilité) et leurs limites fondamentales (cohérence, intérêt) selon ce même critère ;

- et plus globalement, entre deux prescriptions discursives dominantes : «soyez intéressant $t^{18}$ » et «allez droit aux faits ».

Ces contradictions donnent une idée de la distance qu'il reste à parcourir aux producteurs discursifs traditionnels pour construire une

18 Parfois exprimé sous une forme plus impérieuse (attribuée à Françoise Giroud) : "Si votre première phrase ne fait pas mouche, inutile de chercher la seconde." 
expertise épistémologiquement et techniquement digne de ce nom. Elles permettent également de percevoir concrètement des liens qui peuvent exister entre des questions ou des niveaux qui sont rarement reliés entre eux : une dimension sociale (les citoyens connaissent mal l'économie, le droit, les sciences, etc.) une dimension économique (les journaux perdent des lecteurs) et une dimension purement technique (la méthodologie de la communication écrite). Cette dernière ne saurait évidemment résoudre à elle seule les autres, mais son incidence n'en est pas moins évidente.

\section{Matériel}

On trouvera ci-dessous les références des guides de rédaction mentionnés supra (qu'il s'agisse de manuels du corpus ou d'autres documents prescriptifs)*.

ADIT (1999). « Rédiger avec pertinence ». Technologies internationales, vol. 52, p. 5-21.

Antorne, F., et Dumont, J. F. (1995). Écrire au quotidien; Pratiques du journalisme. Lyon : Chronique sociale.

Barrass, R. (1978). Scientists must write. Londres: Chapman \& Hall. Berrou, J.-P. (1991). Bien écrire en affaires. Paris : Dunod.

BÉNICHoux, R. (Dir). (1985). Guide pratique de la communication scientifique. Paris : Ed. G. Lachurié.

Boclé, C., et Pariot, C. (1997). La puissance de l'écrit. Saâcy : La Bohème.

Bonnamour, J.-Y. (1997). Guide pratique de l'écrit. Lyon : Chronique sociale.

Bruni, F. et Alfieri, G. (1997). Manuale di scrittura professionale. Bologne : Zanichelli.

Carrière, C. et Schreiber, C. (200o). Création de pages web (Support $d u$ cours "Utilisation pédagogique du multimédia». Grenoble : IUFM.

Collectif. (1992). Bien écrire et parler juste. Paris : Sélection du Reader's Digest.

CrÊte, J., et Iмвот, L. (1996). Comprendre et communiquer la science. Bruxelles : De Boeck.

CTA (2000). «Communication et développement : L'art de se faire comprendre ». Spore, vol. 86, p. 4-5.

FLESCH, R. (1949). The art of readable writing. New York: Macmillan. 
FLORIO, René (1984). L'écriture de presse. Lille : Trimédia /ESJ.

Gunning, R. (1968). The technique of clear writing. New York : MacGraw Hill.

Hartman, W. (1968). Journalism. River Forest (IL) : Laidlaw Brothers.

Hervouet, L (1979). Écrire pour son lecteur. Lille : Trimédia /ESJ.

Lorenz, A. L., et Vivian, J. (1996). News : Reporting and Writing. New York : Allyn \& Bacon.

Martin-Lagardette, J.-L. (1994). Écrire, informer, convaincre; les secrets de l'écriture journalistique. Paris : Syros.

Mouriquand, J. (1997). L'écriture journalistique. Paris : Presses universitaires de France.

Peyroutet, C. (1997). La pratique de l'expression écrite. Paris: Nathan Porush, D. (1995). A Short Guide to Writing About Science. New York: HarperCollins.

Ross, Line. (1994). L'écriture de presse : l'art d'informer. Québec : Gaëtan Morin.

Voirol, M. (1992). Guide de la rédaction. Paris : Eds du CFPJ.

* Les références des autres textes cités se trouvent dans la bibliographie générale en fin d'ouvrage.w 
Page blanche conservée intentionnellement 


\section{L'art de l'agonistique lexicale}

T a sphère du débat public est, par définition, un espace de confron$\amalg$ tation où tous les outils de l'argumentation sont susceptibles d'être mobilisés pour défendre une position ou en attaquer une autre. On a dressé au fil du temps d'imposants inventaires de ces armes, des plus classiques (comme les inusables arguments ad hominem et ad personam visant l'adversaire) aux plus récentes, comme l'argument ad Hitlerum $^{1}$ évoqué par Léo Strauss dans les années 1950. Pourtant, l'un des plus redoutables de ces procédés, celui qui vise les mots euxmêmes (on pourrait l'appeler "ad vocabulum ») ne figure pas dans les catalogues. Il est vrai qu'il ne se réduit pas à une tactique assurant un gain immédiat - dénoncer les termes « inacceptables » d'un adversaire imprudent -, mais tend, d'ailleurs comme d'autres², à s'inscrire dans une stratégie de bien plus large portée.

Celle-ci est connue et exploitée de longue date. Thucydide rapportait ainsi, quatre siècles avant notre ère, sa remarquable efficacité dans l'orchestration des massacres de Corcyre (aujourd'hui Corfou) :

1 La spécificité de la notion de «Reductio ad Hitlerum», qui désigne le fait de rapprocher un point de vue quelconque d'une position attribuée au régime nazi, est discutable, mais la prévalence de ce procédé dans les débats contemporains tend à justifier son succès, comme celui de la proposition de Mike Goodwin selon laquelle plus une discussion en ligne se prolonge, plus s'accroît la probabilité qu'y survienne une comparaison avec l'idéologie nazie.

2 L'une des limites bien connues des nomenclatures argumentatives usuelles est qu'elles n'envisagent que sous l'angle des tactiques discursives ce qui peut aussi relever de stratégies à plus long terme. Ainsi un argument ad personam d'utilité immédiate («Vous êtes mal placé pour dire ça!») relève-t-il de l'argumentation, mais les efforts que l'on mènera parfois pendant des mois pour saper l'image et la crédibilité d'un adversaire, y compris par la recherche de faits embarrassants, sont censés ne pas en relever. 
En voulant justifier des actes considérés jusque-là comme blâmables, on changea le sens ordinaire des mots. L'audace irréfléchie passa pour un courageux dévouement à l'hétairie; la précaution prudente pour une lâcheté qui se couvre de beaux dehors. Le bon sens n'était plus que le prétexte de la mollesse ; une grande intelligence qu'une grande inertie. La violence poussée jusqu'à la frénésie était considérée comme le partage d'une âme vraiment virile ; les précautions contre les projets de l'adversaire n'étaient qu'un honnête prétexte contre le danger. [...] Quiconque s'ingéniait à ne pas employer ces moyens était réputé trahir le parti et redouter ses adversaires33.

Souterrains ou ostensibles, les combats menés sur le champ lexical sont d'autant plus confus qu'ils sont à la fois rhétoriques et épirhétoriques : le contrôle du sens des mots (et de leur légitimité) est d'abord un enjeu de lutte, ensuite un outil de lutte. Mais ses conséquences, elles, sont tout à fait concrètes. Ainsi, Bruce J. Dickson remarque-t-il que, si une nette majorité de Chinois estiment que le niveau de démocratie de leur pays est "élevé » ou "très élevé », la définition qu'ils prêtent à ce terme est plutôt différente de celle qui a cours en Occident : seuls $3 \%$ d'entre eux associeraient la démocratie à des élections libres.

Instrument d'assujettissement dont Orwell imaginait l'ultime développement dans 1984, le lexique est symétriquement un outil d'émancipation (voire de subversion) dont Brecht rappelle l'ancienneté dans ce même pays :

Confucius falsifia un vieil almanach patriotique. Il se contentait de changer des mots. Là où il y avait : « Le seigneur de Kun fit mettre à mort le philosophe Wan parce qu'il avait dit ceci et cela...", il remplaçait "mettre à mort» par «assassiner». Disait-on que le tyran Untel avait été victime d'un attentat, il mettait : «avait été exécuté ». Ce faisant, Confucius ouvrit la voie à une vue nouvelle de l'Histoire. À notre époque, mettre au lieu de «peuple» la «population» et au lieu de «sol» «propriété terrienne ", c'est déjà retirer son soutien à bien des mensonges. C'est retirer aux mots leur auréole mystique et frelatée. (1970, p. 23-24)

3 Histoire de la guerre du Péloponnèse (trad. Jean Voilquin, 1937, p. 223-224). 
Depuis la théorie du Zhengming (rectification du langage) de Confucius et Xun Zi, les manipulations lexicales ont, au fil des siècles, donné lieu à suffisamment de dénonciations, sarcastiques ${ }^{4}$, alarmées ${ }^{5}$, emphatiques $^{6}$, sévères ${ }^{7}$ et au besoin apocryphes ${ }^{8}$, pour en décourager toute revue. Il en va évidemment de même pour les recherches plus contemporaines ayant abordé directement ou indirectement cette question d'un point de vue anthropologique, littéraire, philosophique, politique, psychologique, sociologique, et, bien sûr, linguistique.

La densité de ces observations reflète dans une certaine mesure le pouvoir que l'on prête au langage en général et aux désignations en particulier. Séduisante comme toute théorie déterministe, l'hypothèse (associée en particulier aux travaux d'Edward Sapir et de Benjamin Lee Whorf) selon laquelle le lexique façonne la conception individuelle et collective de la réalité a été largement nuancée ${ }^{9}$ : à

4 "J'y consens, leur dis-je; car je ne dispute jamais du nom, pourvu qu'on m'avertisse du sens qu'on lui donne. [...] c'est se jouer des paroles de dire que vous êtes d'accord à cause des termes communs dont vous usez, quand vous êtes contraires dans le sens » Blaise Pascal (1657, p. 6-7).

5 "... chacun a le droit de "définir ses termes". Mais ce droit bizarre, que l'on en est venu à s'accorder sitôt que l'on s'occupe de choses d'importance - comme s'il était vraiment identique au droit d'avoir son opinion - n'indique-t-il pas déjà que des termes comme "tyrannie", "autorité", "totalitarisme" ont tout simplement perdu leur signification commune, ou que nous avons cessé de vivre dans un monde commun » Hannah Arendt (1972, p. 126-127).

6 "Votre loi est une loi qui a un masque. Elle dit une chose et elle en ferait une autre. [...] C'est une confiscation intitulée donation. Je n'en veux pas. C'est votre habitude. Quand vous forgez une chaîne, vous dites : Voici une liberté! Quand vous faites une proscription, vous criez : Voilà une amnistie!». Victor Hugo (1875, p. 239).

7 «Mal nommer un objet, c'est ajouter au malheur de ce monde. [...] la grande tâche de l'homme est de ne pas servir le mensonge. » Albert Camus (1944/2006, p. 908).

8 Comme la belle citation de Platon, « La perversion de la cité commence par la fraude des mots ", abondamment sollicitée aux franges droite et gauche du spectre politique, mais dont la source semble introuvable.

9 Y compris par Sapir lui-même, soulignant à quel point « il serait naïf d'imaginer que toute analyse de l'expérience vécue est dépendante de modèles exprimés dans le langage. N'importe quel concept, qu'il fasse partie ou non du système des catégories grammaticales, peut-être transmis dans n'importe quelle langue » (Sapir et Swadesh 1946, p. 111). 
l'instar des effets des médias, les effets du vocabulaire sur la pensée sont loin d'être aussi puissants et directs que l'on serait tenté de le croire : le fait, par exemple, que le terme "démocratie » paraisse ne pas avoir le même sens pour les Occidentaux et les Chinois n'interdit nullement à ces derniers de concevoir la liberté électorale (ni, pour une partie d'entre eux, de la souhaiter). S'il ne corsète pas le pensable et l'impensable, le maniement des désignations influence non seulement le dicible et l'indicible, mais aussi, au-delà des mots, la perception des phénomènes et des individus en les catégorisant : « Nommer, on le sait, c'est faire voir, c'est créer, porter à l'existence. Et les mots peuvent faire des ravages » (Bourdieu, 1996, p. 19). D'où l'intérêt tout particulier que leur accordent les groupes militants, et notamment l'émergence de la notion diffuse de "politiquement correct», dont Geoffrey Hughes (2010) situe l'émergence dans la doctrine maoïste des années 1930, mais l'essor occidental un demi-siècle plus tard.

Ce cadre a accéléré selon Steven Pinker (2002), un processus d'euphémisation cyclique («euphemism treadmill») en vertu duquel une désignation originellement appropriée ( 1 : orthophémisme) se voit attribuer une connotation malséante $(2:$ dysphémisme) qui conduit à lui substituer un terme ou une locution moins expressive $(3$ : euphémisme), laquelle devient à son tour l'appellation appropriée (1) avant d'être perçue comme malséante (2) et d'être remplacée (3). Le moteur de ce processus est attribué par Allan et Burridge (2006) à une «loi de changement sémantique » (inspirée de la loi monétaire de Gresham) : «les mauvaises connotations chassent les bonnes » (p. 243). Il existerait, en d'autres termes, "une tendance générale de toute dénotation choquante ou défavorable au sein d'une expression langagière à dominer l'interprétation de son contexte immédiat » (p. 42).

Cette dynamique générale rend compte d'une très grande partie des transformations sémantiques "délibérées ${ }^{10}$ ", mais des motivations voisines peuvent aussi jouer en l'absence de toute malséance, comme celles tendant à obscurcir le propos (notamment la «langue de bois " politique ou managériale) ou à ennoblir son expression (en

10 Les seules auxquelles on s'intéresse ici, ce qui ne doit pas faire oublier que le sens des mots de toute langue évolue par ailleurs de façon plus ou moins «naturelle» dans le temps (effleurer signifiait retirer les fleurs d'une plante, naguère voulait dire récemment, etc.) et dans l'espace (fin signifie subtil en France et gentil au Canada). 
privilégiant des termes plus génériques et plus abstraits). Par ailleurs, l'euphémisation n'est pas le seul processus à l'œuvre : les captations sémantiques délibérées peuvent par exemple viser à valoriser l'objet désigné (le référent) en détournant un terme plus prestigieu ${ }^{11}$ ou plus frappant, quitte à dévaluer le sens de la désignation empruntée.

Ces opérations sur ce que Marie-Anne Paveau (2006) appelle les prédiscours (ou déterminations prélinguistiques) conditionnent la production et la réception de l'offre discursive. Elles présentent à ce titre un intérêt particulier dans la perspective analytique de l'adéquation communicationnelle en opposant nettement les contraintes sociales et la pertinence cognitive des discours.

D'un côté, en effet, les interventions sémantiques (modifier le sens d'un terme) ou lexicales (substituer un terme à un autre) sont clairement d'ordre normatif : elles visent à régenter ce qu'il faut ou qu'il ne faut pas dire (voir notamment Pinker, 2011). La locution même de politiquement " correct», quoi qu'elle soit beaucoup plus employée par les détracteurs de cette mouvance que par ses promoteurs, souligne explicitement ce caractère contraignant.

De ce point de vue, on remarque non seulement que les rectifications lexicales empruntent tout le spectre des intensités normatives, de la plus informelle à la plus catégorique (voir chapitre 7), mais aussi que le sens dans lequel elles l'empruntent paraît globalement lié au caractère plus ou moins spontané de ces évolutions. Alors que les transformations « naturelles » vont le plus généralement dans le sens du relâchement ${ }^{12}$ (proscription de moins en moins formelle), les aménagements délibérés tendent à parcourir le chemin inverse, pouvant même accéder au statut de contrainte réglementaire, voire pénale.

Évidemment, beaucoup de luttes normatives ne connaissent pas un tel succès. Ainsi, les bouchers-charcutiers qui s'étaient, en 1997, insurgés contre l'emploi du terme «boucherie » pour désigner des

11 «Depuis que les portiers s'intitulent concierges, les agents d'affaires se désignent sous le nom de jurisconsultes. Toute la vie est là, savoir se donner un nom convenable. Si vous êtes domestique, appelez-vous intendant. Si vous êtes coiffeur, coiffez-vous du nom d'artiste » (Scholl, 1885, p. 213).

12 Comme le notent Blanco et Bogacki (2014, p. 51), "Souvent, on élimine un mot ayant une nuance littéraire qui est remplacé par des termes expressifs, techniques, argotiques ou vulgaires [...]». 
attaques sanguinaires visant des civils ${ }^{13}$ ne rencontrèrent-ils qu'une indifférence ironique. Des sarcasmes similaires ont salué le code de langage très détaillé défendu par les responsables d'une revue :

Inévitablement, notre langage actuel à propos des animaux est un langage du passé bien que, crucialement, ce passé soit jonché de terminologies dépréciatives : "brutes", "bêtes", "bestial" [...] Nous ne pourrons penser clairement si nous ne nous disciplinons pas nous-mêmes pour employer des noms et des adjectifs plus impartiaux. [...] Nous voulons dire adieu aux nombreuses métaphores et locutions assimilées par lesquelles nous rabaissons nos semblables animaux ou humains : "sournois comme un renard", "manger comme un cochon" [...]"têtu comme une mule", et beaucoup d'autres. [...] Également, le terme "propriétaire", quoique techniquement correct en droit, renvoie à un temps où les animaux n'étaient considérés qu'ainsi : des propriétés, des machines ou des choses pouvant être utilisées sans contraintes morales. De même, "he" or "she" devrait être utilisé pour désigner un animal particulier plutôt que "it $\mathrm{it}^{14 "}$.

Toutefois, l'issue de ce combat normatif n'est pas nécessairement fixée : bénéficiant, contrairement aux bouchers, d'une dynamique favorable (dont témoignent la diffusion du terme «spécisme » et la vigueur des mouvements "pour l'égalité animale» ou «pour les droits des animaux $»)$, ces proscriptions perçues à l'origine comme une ridicule poussée de sensiblerie par nombre de commentateurs ${ }^{15}$ conservent de bonnes chances de paraître «normales » dans l'avenir.

Pour autant le destin des interventions lexicales ne peut jamais se réduire à des questions de justifications morales et de convenance sociale. Tendant par essence à modifier les conditions de l'adéquation communicationnelle, les changements de désignations mettent tout autant en jeu l'autre volet de cette adéquation : la pertinence cognitive. Ils peuvent tendre à rendre les énoncés moins pertinents

13 Voir le communiqué de la confédération française de la boucherie (C.F.B.C.T., 1997) « Bouchers, oui, mais ni bouchés ni... bouchers ».

14 «From the Editors. » Journal of Animal Ethics (2011, p. vii-ix).

15 Voir par exemple les échos de ces réactions dans les articles que le quotidien canadien (conservateur) National Post leur a consacré entre le 1o et le 14 mai 2011. 
(euphémisation) ou plus pertinents (emphase), mais ils ne sauraient être neutres. Non seulement parce qu'une transformation qui n'affecterait pas l'effet cognitif dans un sens ou dans l'autre ne serait en général d'aucun profit pour ses défenseurs, mais aussi parce que toute substitution d'une désignation nouvelle à une désignation courante implique un certain surcroît d'effort de traitement cognitif.

Celui-ci est particulièrement marqué dans le cas le plus courant, celui de l'euphémisation, puisque l'appellation promue réduit généralement la lisibilité lexico-syntaxique en allongeant les mots ou les locutions (« infirme » / « handicapé » / « personne à mobilité réduite ») et surtout, affecte la cohérence des énoncés et la construction d'un modèle mental de ce qu'ils signifient. D'où les protestations contre l'irruption de formules "creuses", "abstraites", "qui ne veulent rien dire ", etc. Mais il ne saurait en être autrement puisque ces opérations visent précisément à dire moins, ou de façon moins vive, ou plus englobante (hyperonymie) que les expressions ayant cours auparavant, ou encore, justement, à exiger du destinataire un effort de réflexion (non compensét ${ }^{16}$ à propos des réalités désignées.

Évidemment, l'agonistique lexicale ne vise pas les mots pour eux-mêmes : son objet réside dans les schémas cognitifs que les appellations activent ou non (voir ci-dessus, chapitre 5). C'est, du reste, suffisamment manifeste pour avoir été fréquemment remarqué, quoiqu'en recourant à des notions variées (connotations, représentations, métaphores, catégorisation, stéréotypes...), par ceux qui s'y sont intéressés sous un angle ou sous un autre : privilégier un vocable, c'est sélectionner les propriétés typiques qui lui sont associées. C'est ainsi que bien des termes s'étant au fil du temps chargés de caractères typiques très clairs, mais réducteurs ou discriminants ont été dévalués. Mais puisque la logique même de la schématisation cognitive est de simplifier les constituants du réel en les réduisant aux propriétés les plus saillantes (mais pas forcément les plus justes) qui leur sont associées, les effets de ce nettoyage langagier sont normalement peu durables $^{17}$. Si, comme le soulignait Pinker, le processus général d'euphémisation s'apparente à un «tapis roulant » continuel, le moteur

16 Puisqu'il n'offre pas nécessairement de contrepartie en termes d'effet cognitif.

17 Un militant herpétophile qui parviendrait à faire proscrire le terme «serpent » et à le remplacer par « ophidien » verrait rapidement cette 
de ce mouvement perpétuel réside justement dans l'économie cognitive qui conduit à typiciser (schématiser) le réel.

Cependant, les altérations lexicales peuvent parfois avoir des effets plus durables, notamment lorsqu'elles accompagnent une évolution plus profonde des schémas activés (au prix d'un travail d'explication et de justification lui-même plus profond ${ }^{18}$ ), mais aussi lorsque la substitution vise à réparer une désignation malavisée au départ. Ainsi, dans les hôpitaux, la Résonance magnétique nucléaire (R.M.N.) a-t-elle judicieusement été rebaptisée Imagerie par résonance magnétique (I.R.M.) : en supprimant ce qu'activait - d'ailleurs improprement $^{19}$ - l'adjectif nucléaire, la désignation a perdu son caractère inquiétant sans que les patients songent une seconde à regimber contre le flou qui en découlait (que fait-on résonner au juste ?).

Par ailleurs, les stratégies lexicales motivées, non par le besoin d'estomper les propriétés typiques d'un schéma, mais au contraire par le désir de s'approprier les propriétés associées à un autre schéma (comme « thérapeute » ou « architecte » pour les titres professionnels, « violence ", « haine » ou « terrorisme » pour les comportements, etc.) ne relèvent pas du cycle orthophémisme-dysphémisme-euphémisme: leurs principaux écueils résident à court terme dans les protestations que peuvent souligner des dénominations perçues comme abusives et à moyen terme dans l'affadissement ${ }^{20}$ qu'entraîne fatalement cette imposture sémantique.

Mais dans tous les cas, les luttes pour l'établissement des désignations «correctes », alors même qu'elles se situent en amont de la production des discours, mettent en jeu l'ensemble des déterminants cognitifs et sociaux de la valeur discursive.

désignation neutre endosser toutes les propriétés déplaisantes auparavant associées à « serpent ».

18 Lequel, contrairement à l'improbation unilatérale, implique en outre d'optimiser la valeur cognitive des arguments proposés.

19 Les noyaux en question sont les noyaux cellulaires et non les noyaux atomiques.

20 Ainsi la locution «violence routière » promue par une association pour désigner le non respect du Code de la route, même en l'absence de toute brutalité volontaire ou non, sans se soucier de déposséder les victimes de violences effectives (notamment domestiques) de la désignation de leur calvaire. 


\section{Souffrances et amertumes de la critique ${ }^{1}$}

L a critique des productions culturelles fait partie de ces activités discursives dont on annonce si routinièrement la crise que la locution « crise de la critique » tient plus du pléonasme que de la nouvelle. Depuis le bon vieux temps où les Grecs lapidaient Zoïle, féroce critique d'Homère ${ }^{2}$, l'évaluation publique des œuvres de l'esprit s'est toujours trouvée mise en cause dans ses fondements, ses ambitions, sa légitimité et, bien sûr, ses errances. Cependant, l'évolution du marché discursif - dont elle constitue l'un des témoins les plus fascinants - la soumet aujourd'hui à des tensions sans précédent, sinon dans leur logique, du moins dans leur intensité.

En se distendant, l'«art de juger les qualités et défauts des œuvres de

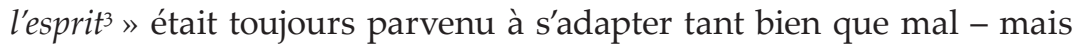
non sans récriminations - au gonflement continu de l'offre culturelle et à ses concomitances : la fragmentation des contenus et des publics, l'ébranlement des hiérarchies consacrées et le durcissement de la concurrence attentionnelle (voir chapitre 1). Ses lignes de fracture reflètent encore, en les accentuant, celle qui se dessinait au XvII ${ }^{\mathrm{e}}$ siècle entre la critique érudite et la critique mondaine, mais aussi celle, plus

1 Ce texte s'inspire d'une présentation orale intitulée L'art ou le mouchoir? Les facteurs sociocognitifs dans l'appréciation culturelle (conférence L'expérience esthétique, Ottawa, 19-20 février 2014).

2 À moins qu'il n'ait été crucifié, ou précipité d'une falaise. Les versions diffèrent mais, approuvait Vitruve, «quel qu'ait été le genre de son châtiment, il est certain qu'il le mérita: c'est ainsi que doit être traité celui qui s'avise d'attaquer un écrivain qui ne peut être appelé à se présenter pour défendre les pensées qu'il a répandues dans ses écrits " (De Architectura, trad. Ch.-L. Maufras, 1848, p. 111).

3 Définition instituée en 1580 par J. Scaliger (cit. in Thumerel, 2004, p. 9). 
ou moins parallèle, qui se creusait entre le raffinement et le divertissement. Ainsi, le versatile Donneau de Vizé, opposant les «bouffons » (comprendre : Molière) et les "grands hommes» (comprendre : Corneille) : "Les uns n'ont rien que de ridicule dans leurs ouvrages et ne travaillent que pour la rate, et les autres n'ont rien que de solide et ne travaillent que pour l'esprit » $(1665$, p. 87-884). Et, déjà, les arguments qui s'échangeaient entre les préférences du public et le respect de ce même public : "Il [Molière] veut encore nous persuader, pour rendre sa cause bonne, que les Français n'aiment qu'à rire; mais il faut voir par là qu'il les estime peu, puisqu'il ne les croit pas capables de goûter les belles choses" (p. 95). Cependant, l'opposition entre la noblesse de l'esprit et la basse soumission aux goûts du public n'était simple que pour ceux qui en tiraient argument. On se souvient en effet (supra, chap. 7) que Corneille, érigé par Donneau de Visé en modèle de pureté créatrice, n'était pas le moins du monde indifférent à la satisfaction des spectateurs :

[...] notre premier but doit être de plaire à la cour et au peuple, et d'attirer un grand monde à leurs représentations. Il faut, s'il se peut, y ajouter les règles, afin de ne déplaire pas aux savants, et recevoir un applaudissement universel; mais surtout gagnons la voix publique; autrement, notre pièce aura beau être régulière, si elle est sifflée au théâtre, les savants n'oseront se déclarer en notre faveur [...] (1637/1862, p. 119)

Ainsi, le «grand homme» s'accordait-il tout à fait avec le «bouffon», Molière recourant pour sa part à un argument similaire :

Je voudrais bien savoir si la grande règle de toutes les règles n'est pas de plaire, et si une pièce de théâtre qui a attrapé son but n'a pas suivi un bon chemin. Veut-on que tout un public s'abuse sur ces sortes de choses et que chacun n'y soit pas juge du plaisir qu'il y prend ? (1663, p. 91)

Tragédien ou comédien, l'un et l'autre, en somme, envisagent explicitement la valeur discursive comme le rapport entre le plaisir (hédonique) que procure leur production et les normes (sociales) auxquelles

4 Orthographe modernisée. Sauf précision contraire, l'orthographe de toutes les citations antérieures au $\mathrm{XvIII}^{\mathrm{e}}$ siècle rapportées ici a été modernisée. 
elle est soumise, dont, bien sûr, les règles impérieuses de la dramaturgie classique. Pour autant, le point où chacun situe l'équilibre optimal entre agrément et légitimité varie très logiquement en fonction de leur offre, Molière revendiquant la transgression la plus résolue : «ne consultons dans une comédie que l'effet qu'elle fait sur nous. Laissons-nous aller de bonne foi aux choses qui nous prennent par les entrailles » (id., p. 93).

\section{Bouleversements et invariances du positionnement critique}

Calquant la spécialisation des "gens de lettres " au XIx ${ }^{\mathrm{e}}$ siècle, le champ de la critique n'a pas manqué de reproduire la division du travail discursif qui a séparé les créateurs, les journalistes et les chercheurs, d'où les divergences de signification que l'on sait, en particulier entre la «critique » universitaire (dont aucune variante ne se propose plus de juger - «la chose est même carrément prohibée 5 »-, mais qui n'a pu se résoudre à rénover sa désignation) et la critique tout court, «la fonction critique au sens propre du terme, qui consiste à juger et apprécier les œuvres récentes pour éclairer les choix du public (fonction liée à l'institution journalistique) »(Genette, 1966, p. 146). On pourrait donc penser qu'assise sur une si solide expérience des bifurcations conceptuelles et des buissonnements de perspectives, la critique était solidement armée pour s'accommoder des nouvelles formes et des nouveaux locuteurs que multiplient les technologies de la communication. Ce n'est cependant pas si sûr. S'il ne s'agissait que d'évaluer une gamme de plus en plus large d'œuvres pour des publics de plus en plus variés, ce ne serait qu'une affaire de différentiation sur le marché discursif, chacun trouvant sa place à un étage ou un autre de la hiérarchie des choses de l'esprit. Mais on sait que les lignes de démarcation culturelles séparent de moins en moins les classes sociales, les lectorats et même les individus : les recherches des dernières décennies (voir ci-dessus, chapitre 7 ) témoignent surabondamment du panachage hétéroclite d'œuvres légitimes et triviales dont chacun - et en particulier les plus éduqués - compose son propre menu culturel.

En outre, la désacralisation touche la parole critique elle-même, fissurant la distinction traditionnelle entre les jugements de goût (j'aime / j'aime pas) et les jugements de valeur (c'est bon / c'est mauvais).

5 Hans Robert Jauss (1978, p. 26). 
Tandis que les premiers, intimes et non sujets à argumentation ${ }^{6}$, étaient censés rester confinés à la sphère personnelle, les seconds seuls étaient supposément dignes d'être publiquement communiqués et débattus. Ainsi, Nathalie Heinich (1999) a-t-elle observé, à propos des réactions suscitées par l'art contemporain, que «les arguments étaient investis d'un minimum d'objectivité ou de généralité pour pouvoir faire l'objet d'une argumentation dans l'espace public. Les gens ne vont pas écrire au ministre pour dire "moi ça ne me plaît pas" » (p. 159). En revanche, les commentaires des consommateurs de produits culturels, notamment sur les sites de vente en ligne, exposent volontiers les ressentis spontanés d'expériences personnelles, lesquels s'y mêlent indistinctement à des avis plus construits et généralement mieux orthographiés.

Évidemment, la distinction entre jugements de goûts et de valeur, quoique très pratique, est assez arbitraire. Tel internaute qui, sur Amazon, décrète Proust «ennuyant, répétitif et très $l o n g 7$ » rejoint non seulement les avis (à usage privé) des divers connaisseurs qui avaient refusé de le publier - Gide, Humblot, Madeleine... -, mais aussi l'aveu tout à fait public d'une critique célèbre, Rachilde, dans les pages du Mercure de France (1914, p. 362) : "j'ai fini par le laisser tomber avec effroi, comme on refuserait de boire un soporifique ${ }^{8}$. » Cependant, ce témoignage à la première personne était appuyé par la légitimité acquise par l'auteure, qui conférait à son opinion une valeur dépassant sa personne. C'est plus rarement le cas lorsque les goûts et dégoûts sont exprimés par des internautes dont les pseudonymes comportent des chiffres.

La libération du jugement critique ouvre en outre à tous un territoire privé de repères. Au fil des siècles, l'usure du temps et l'ardeur des avant-gardes ont eu raison, entre autres, des canons du théâtre classique, de la versification en poésie, de la ressemblance, puis de la figuration en peinture, et de la bienséance morale dans tous les cas. La nébulosité des références transcendantes, comme jadis le "sublime » et, au xx $x^{e}$ siècle, la « littérarité », a fini par décourager d'y recourir avec trop de confiance. Quant au critère de la «beauté», associé par les

6 Ce sont ceux dont, comme les couleurs, un adage latin rappelle qu'« on ne discute pas ».

7 Commentaire daté du 6 mars 2013 sur Amazon.ca.

8 Notons cependant que Rachilde - de même d'ailleurs que le consommateur d'Amazon - trouvait également de grandes qualités au roman. 
connaisseurs à une naïveté embarrassante, il peut même être rédhibitoire : le sec commentaire «C'est très beau, c'est joli. On n'en parle pas!" a ainsi suffi à éliminer une œuvre soumise à un jury d'art contemporain (Heinich, 2017, p. 94). Même la partialité doctrinale, si l'on en croit Maurice Nadeau, ne serait plus ce qu'elle était :

Aujourd'hui qu'a disparu, en littérature, l'engagement politique, sinon idéologique, sauf pour une certaine droite qui, littérairement, fait figure d'exception, aujourd'hui que sur la littérature moyen d'expression, création, art, prévalent toutes les formes de communication de l'audiovisuel, aujourd'hui que la critique littéraire, non seulement ne sait plus à quelles règles intellectuelles ou morales elle peut se fier, mais se voit contrainte d'accompagner les tendances du marché, de suivre les courants de la mode, fût-ce en s'en gaussant, fût-ce en trépignant [...] il devient difficile d'exercer, dans le respect de soi et le respect du lecteur, un métier qui paraît obsolète. (2002, p. 11-12)

À l'évidence, la confusion des appréciations, postures et critères de la critique contemporaine n'est pas de celles que l'on peut démêler en quelques pages. Mais du moins permet-elle de reconnaître quelques lignes de force (toujours les mêmes...) qui pourraient dans une certaine mesure contribuer à clarifier un peu les contraintes qui s'exercent sur une critique « peu à peu emportée par la mer des avis et des opinions » (Maggiori, 2011, p. 117).

\section{Valoir ou faire valoir?}

La caractéristique la plus fondamentale - et la plus manifeste - de la critique est qu'elle constitue une offre discursive (qui, donc, vise à valoir) à propos d'une autre offre discursive, laquelle vise elle aussi à valoir. Ce statut métadiscursif qui enchevêtre deux procès de valorisation ne lui est pas spécifique : la traduction (voir plus haut) ou la vulgarisation scientifique n'existent également qu'en rapport à des discours sources. Toutefois, la vocation évaluative de la critique rend nécessairement problématique la relation entre sa propre adéquation sociocognitive et celle de l'œuvre dont elle rend compte. Dans le cas de la critique littéraire, la chose se complique du fait que, comme le notait Genette, elle utilise le même matériau - l'écriture - que celui qu'elle examine. 
Cette tension est particulièrement apparente dans la mise en cause la plus traditionnelle de la critique celle qui - depuis Zoïle vise sa supposée malveillance :

Mais autant que la critique est légitime et utile, autant la satire est-elle injuste et pernicieuse : elle est injuste en ce qu'elle essaye de tourner les auteurs mêmes en ridicule, ce qui ne saurait être le droit de personne ; et elle est pernicieuse en ce qu'elle songe beaucoup plus à réjouir qu'à éclairer. Elle ne porte que des jugements vagues et malins, d'autant plus contagieux que leur généralité accommode notre paresse et que leur malice ne flatte que trop notre penchant à mépriser les autres. (de la Motte, 1715, p. 33-35)

Des titres de presse comme « Les dents de la critique » (La Presse, 1996) ou «Critiques : mais pourquoi sont-ils si méchants?» (Libération, 1992) rappellent de temps à autre la persistance de ce débat. La férocité critique a certes ses défenseurs, et non des moindres, au nom d'une mission purificatrice régulièrement mise en avant :

Les journaux littéraires devraient être la digue opposée au gribouillage sans conscience de notre temps et au déluge de plus en plus envahissant des livres inutiles et mauvais. Grâce à un jugement incorruptible, juste et sévère, ils flagelleraient sans pitié chaque bousillage d'un intrus, chaque griffonnage [...] On a absolument tort de vouloir transporter également à la littérature la tolérance qu'on doit nécessairement exercer dans la société, où partout ils grouillent, à l'égard des êtres stupides et sans cervelle. En littérature, ils sont d'éhontés intrus, et y rabaisser le mauvais, c'est un devoir envers le bon; car celui qui ne trouve rien mauvais, ne trouve non plus rien bon. D'une façon générale, la politesse, qui est la conséquence des rapports sociaux, est, en littérature, un élément étranger, souvent très nuisible. (Schopenhauer, 1851/1905, p. 37-39)

Cependant, ce type de justification, pour recevable qu'il soit, n'éclaire que le volet normatif de l'adéquation discursive : il oppose, en somme, une valeur sociale - l'intransigeance - à une autre valeur sociale - la courtoisie - pour faire prévaloir la première sur la seconde. Il fait du même coup abstraction de l'autre versant de l'adéquation, celle de la pertinence cognitive, d'autant plus cruciale pour le discours critique 
que la place qui lui est accordée par les médias ne cesse de fondre. Or, le spectacle de la férocité, garant d'un effet cognitif spontané (de même qu'un combat de catch est spontanément plus intéressant qu'un débat de philatélistes) est l'un des moyens les plus efficaces d'assurer cette pertinence, d'autant qu'il favorise le recours à de puissants modulateurs de l'effet cognitif comme l'humour et la surprise (que génèrent notamment les rapprochements insolites, figures typiques du genre9) et ne réclame pas trop d'effort cognitif. Ainsi, de la Motte, qui dénonçait plus haut la capacité de la virulence critique «à réjouir » (effet) et à "accomode[r] notre paresse » (effort) visait-il juste, sans que ceci ne soit le moins du monde incompatible avec la sincérité éventuelle des éreintements et la pureté de leurs motifs. Ou d'une partie de ces motifs, si l'on reconnaît par exemple dans le titre du recueil d'un chroniqueur - Les ais-je bien descendus ? (Besson, 1991) - la reconnaissance sans fard de la valeur cognitive d'une attaque vigoureuse. Plus explicite encore est l'hommage posthume inattendu rendu par un confrère (qui ne partageait rien de ses vues) au très conservateur Brian Sewell, dont les attaques contre les artistes contemporains se signalaient par une méchanceté hors de l'ordinaire, même selon les critères de ce domaine :

Le critique d'art controversé a vilipendé tant de choses parce qu'il savait que les journaux et les critiques devaient être un grand divertissement populaire. Les journaux - et les critiques adorent prétendre le contraire, parfois même en qualifiant de «verdicts» les opinions de leurs commentateurs artistiques, comme si nous étions des juges de la haute cour, mais en réalité, une recension à son meilleur est une sacrée bonne lecture. C'est une explosion stimulante, provocante ou agaçante d'adrénaline verbale $[\ldots]$ (Jones, 2015)

\section{L'introuvable légitimité des aficionados}

En tout état de cause, la vieille question de la méchanceté perturbe plus les créateurs que les professionnels du jugement culturel : si l'abattement gagne les rangs de ces derniers, il ne résulte apparemment pas

9 «La France avait Marceline Desbordes-Valmore, alias "Notre-Dame-DesPleurs", l'Angleterre nous offre J.K. Rowling, une desperate housewife qui se prend pour Dickens. » Jérôme Garcin (2012). « J.K. Rowling, quel pudding ", Le Nouvel observateur, 11 octobre 2012. 
d'une quelconque flambée collective de férocité. Il en va tout autrement de la désacralisation de la parole critique, non seulement face à la multiplication des avis d'amateurs en ligne, encouragée parfois par les quotidiens eux-mêmes ${ }^{10}$, mais aussi, voire surtout, à l'essor - dans les médias professionnels - d'une critique de surface délassante et consumériste, dont Catherine Voyer-Léger (2014) dresse un inventaire éloquent : "le club de lecture», "le règne des étoiles", "le conseil de consommation $» .$.

Cette évolution est communément associée à un abaissement du seuil des compétences et connaissances requis pour l'exercice du jugement critique, et, comme on le dénonce depuis le xix $x^{e}$ siècle (Sainte Beuve), à la marchandisation de la production culturelle. Mais, si fondés que puissent être les constats sur lesquels elles s'appuient, les dénonciations de la "critique au rabais ${ }^{11}$ " peuvent facilement conduire à un raisonnement circulaire (ce qu'on pense qu'il faut dire d'une œuvre découlant de ce qu'on pense que doit être une œuvre), voire à une pétition de principe : en se cantonnant au registre normatif de la légitimité, le débat en fait le seul mode de valeur du discours critique, ce qui reste à démontrer.

On retrouve un peu ici, toute différence mise à part, un problème courant des études universitaires de la réception médiatique, tel que l'a dénoncé Pierre Verdrager (2001) : construits sur un présupposé de grandeur - celle des œuvres dont elles examinent les recensions par la presse - ces travaux tendent spontanément à partager les critiques (clairvoyantes/ignorantes) en fonction de leur propre perspective.

Ainsi, l'étude de la réception de M. Yourcenar est assumée par les yourcenariens, celle de G. Bernanos par les bernanossiens, etc. En conséquence, la fiabilité de ces observations est entachée, tant il est vrai qu'il est difficile d'accorder sa confiance aux observateurs

10 Ainsi le quotidien Ouest-France a-t-il ouvert en 2017 une section en ligne «Critiques culturelles» entièrement sous-traitée à une association de « contributeurs bénévoles », suscitant l'indignation impuissante des journalistes (SNJ, 2018).

11 L'expression est déjà utilisée en 1865 dans un article de L.M. Ravary, « $L a$ critique et les critiques» (La France Littéraire, Artistique et Scientifique, 31 octobre 1865, p. 15-22). 
qui sont à la fois juge et partie. De ce biais perspectif découle, de manière récurrente, une analyse asymétrique qui gratifie ceux qui ont « eu raison » et stigmatise ceux qui ont « eu tort ». (p. 5)

Cependant, si l'on s'avisait d'appliquer aux problèmes généraux de la critique culturelle la neutralité axiologique que Verdrager réclame pour les études de réception, perspective qui «exige aussi bien l'abandon de la posture de dénonciation que celle d'admiration » (ibid.), il deviendrait délicat de répudier les évaluations ne visant qu'à optimiser un choix de consommation hédonique. À l'instar des conseils boursiers ou culinaires, ce type d'offre discursive répond en effet à un registre d'effet cognitif essentiel, l'interaction avec la sphère personnelle, qui pour être utilitaire n'est pas dépourvu de légitimité. Et, qui, après tout, est l'une des raisons d'être les plus traditionnelles de la critique, si l'on estime que, indépendamment des motifs parfois ambigus de sa production $^{12}$, sa consultation relève essentiellement - en termes d'usages et gratifications - de trois familles de motivation :

- pour soi-même (orienter ses prélèvements sur le marché culturel) ;

- pour les interactions avec d'autres (se faire une idée des œuvres dont on parle) ;

- pour le discours lui-même (lorsque des chroniques produisent une valeur indépendante de leur objet, survivant parfois à celui-ci $\left.{ }^{13}\right)$.

Or, dans le premier cas, et dans une certaine mesure dans le second, il est difficile d'imaginer plus pertinent qu'une note ou des étoiles, puisque cette condensation extrême du jugement réduit au minimum possible l'effort cognitif réclamé. On perçoit assez ce qu'une telle réduction de la critique culturelle à sa plus simple expression peut

12 Le cas, par exemple, des critiques mises au service d'intérêts souterrains - vénalité, servilité, réciprocité... - ne nous intéresse pas directement ici, dès lors que ces déterminants, illégitimes par définition, sont étrangers aux interactions « ordinaires » entre la production et la réception discursives (en d'autres termes, le public qu'elle servent n'est pas celui auquel elles affectent de s'adresser).

13 On peut les reconnaître à ce qu'elles finissent généralement dans des recueils (Rinaldi, Vialatte...) 
avoir de contestable (voire de contradictoire avec l'idée même de culture) pour qu'il ne soit pas utile d'y revenir. Pas plus qu'il n'est, à sa défense, utile de s'étendre sur le fait que les notes et étoiles sont généralement accompagnées d'un commentaire plus ou moins développé, dès lors que chiffres et symboles permettent de se dispenser de sa lecture (sinon, à quoi bon?).

On ne saurait pour autant lier l'essor des évaluations quantitatives - en tant que forme condensée (cognitivement pertinente) du discours critique - à celui des préférences qu'elles expriment quant aux œuvres elles-mêmes. On ne peut, en d'autres termes, confondre la multiplication de ce type d'évaluations avec la prépondérance dont jouiraient par ailleurs les produits culturels de grande consommation. Outre que l'usage des notes et des symboles a été adopté par des périodiques respectés, les plus grandes œuvres du patrimoine culturel pourraient tout autant se voir affublées d'un nombre variable d'étoiles (la chose se pratique d'ailleurs, entre autres, dans les guides touristiques). Or, si la forme quantitative des évaluations ne prédit ni leur objet ni leur verdict, ce sont bien ces derniers points - ce dont on choisit de parler et ce qu'on en dit - qui forment le cœur du débat critique, comme le rappellent périodiquement les titres annonçant un « divorce » entre les critiques et le public ${ }^{14}$.

Cette idée de rupture suggère cependant un phénomène circonscrit ou du moins délimité par un avant et un après, ce qui n’a rien d'évident, pas plus qu'il n'est évident que cette divergence soit propre à la critique médiatique. Il suffit, par exemple, de comparer la liste des 20 auteurs du $\mathrm{xx}^{\mathrm{e}}$ siècle les plus traduits dans le monde ${ }^{15}$ et la liste de tous ceux qui ont reçu le prix Nobel de Littérature au cours du même siècle, pour remarquer qu'aucun nom n'apparaît à la fois dans ces deux répertoires. Dans le premier figurent en revanche trois auteurs de romans sentimentaux, six de romans policiers ou de suspense, et sept de romans d'aventure ou de bandes dessinées ${ }^{16}$ :

14 Par exemple, l'Express (01.02.1996), Marianne (26.07.1999), Allo Ciné (15.02.2014).

15 Tels que recensés par le registre international (Index Translationum) tenu par l'UNESCO.

16 Ce relevé n'exclut en rien que divers auteurs des deux dernières catégories, par exemple Jack London ou Jules Verne, puissent être crédités d'une valeur littéraire. 
quelle que soit la supériorité symbolique des œuvres "élevées", et quelle que soit leur consécration par la critique, elle est de peu de poids face à l'attrait de la pertinence cognitive, qu'il s'agisse d'effet ou d'effort. Comme on l'a signalé plus haut, la lisibilité des romans a spectaculairement évolué entre le milieu du $\mathrm{XvIII}^{\mathrm{e}}$ siècle et celui du $\mathrm{xx}^{\mathrm{e}}$ siècle : la longueur de leurs phrases a décru de 41,5 mots à 15,2 mots en moyenne tandis que leur taux de mots de plus de 9 lettres a plongé de 47 à 28 (Danielson et Lasorsa, 1989). Ce genre de phénomène est encore mieux documenté dans le domaine du cinéma, où l'on sait par exemple que la durée moyenne des plans n'a cessé de diminuer depuis les années 1930 (de plus de 10 secondes à 4 secondes environ), tandis que l'indice d'activité à l'intérieur de ces plans était multiplié par trois (Cutting, Brunick et al., 2011). Pour autant, le cinéma dit «d'auteur » a fleuri au cours de la même période et l'on produit plus d'œuvres littéraires, artistiques ou musicales « exigeantes " qu'au XviII ${ }^{\mathrm{e}}$ siècle (où cette notion même aurait eu peu de sens $^{17}$ ). Ainsi semble-t-il beaucoup plus plausible de considérer que le gonflement numérique actuel de la sphère du débat public, plutôt que de causer la « crise ", ne fait que rendre plus visible et plus inconfortable le dilemme fondamental sur lequel s'est construit le jugement critique. Soit le critique est, pour reprendre un cliché du journalisme anglo-saxon, l'« œil du public », et il est déontologiquement irréprochable que son évaluation de la valeur discursive soit aussi proche que possible de celle de ses lecteurs (dont les palmarès de ventes donnent une idée), soit il vise à faire prévaloir sur la pertinence cognitive et la morale commune des critères plus élevés et - pour reprendre un cliché de la critique - «plus dérangeants » (l'art pour l'art), auquel cas sa propre légitimité, n'étant plus assurée par l'ancienne autorité de la hiérarchisation culturelle, ne peut plus faire l'économie d'une démonstration.

De ce point de vue, le territoire de la critique est traversé, non par une, mais par deux divergences classiques entre le profane et le transcendant : à l'opposition, propre à la production culturelle, entre la « voix publique » et celle des « savants » (selon les termes de Corneille), il ajoute celle, propre au journalisme, entre $l^{\prime} \ll$ information» et $l^{\prime} \ll$ opinion»

17 De même, note Olivier Bessard-Banquy (2012), l'édition contemporaine, réputée homogénéisée par l'obsession des best-sellers, fait également preuve d'une diversité créatrice foisonnante. 
(laquelle prétend également guider plutôt que servir son audience). Quoiqu'assez proches dans leur logique, ces divergences ne se confondent pas dans leurs modalités. La valorisation pour lui-même de l'effort cognitif que réclament les discours « exigeants » n'est pas un argument journalistique alors qu'en matière culturelle, suggérait Bourdieu (voir supra, chapitre 9) :

On pourrait montrer que tout le langage de l'esthétique est enfermé dans un refus principiel du facile, entendu dans tous les sens que l'éthique et l'esthétique bourgeoises donnent à ce mot ; que le "goût pur», purement négatif dans son essence, a pour principe le dégoût $[. .$.$] pour tout ce qui est « facile ». (1979, p. 556)$

Ainsi a-t-on, par exemple, vu des spécialistes juger "faible », voire «affligeant », le $3^{\mathrm{e}}$ mouvement (« Tuba Mirum ») du Requiem de Mozart où l'entrée du trombone produit un effet saisissant, mais facile. De même - toujours par exemple - a-t-on pu entendre un chef baroque renommé "gommer» un effet un peu voyant dans la Messe en si mineur de Bach.

La facilité paraît même se situer, en compagnie du cliché, au premier rang des tares condamnant une œuvre aux yeux des connaisseurs, alors que les consommateurs moins distingués ne semblent bouder ni l'une ni l'autre. Mais pour les critiques, dont les discours cherchent une voie médiane entre initiés et béotiens, il est plus délicat de condamner la facilité, gage de pertinence cognitive, que le cliché qui a peu de défenseurs.

\section{Le « spectacle » comme ligne de front}

Les affrontements ayant pour enjeu l'établissement de la valeur discursive se déroulent sur tous les étals du marché culturel, y compris ceux où le " grand public » est le moins à même de l'évaluer, comme les arts plastiques et la musique contemporaine. Ainsi la question de la pertinence cognitive (en particulier la cohérence et l'effet) de la musique atonale avait-elle suscité, en 2013, une vigoureuse empoignade entre compositeurs, où l'on a vu l'argument de l'inaccessibilité repoussé explicitement par les défenseurs de cette dernière (non sans se référer à une dimension behavioriste, certes obsolète, mais néanmoins psychologique). 
Mais la tâche du créateur consiste-t-elle à jouer sur des réflexes « quasi-pavloviens » du plus grand nombre de façon à s'en faire comprendre, ou, au contraire, à proposer des mondes alternatifs qui peuvent se révéler infiniment plus riches que celui de notre quotidienneté sonore? [...] La reconnaissance est-elle l'unique critère qui nous attache à elles? Il est plus que probable, comme Ducros le dit, que la musique atonale ne sera jamais aussi populaire que la musique tonale. Est-ce là un critère pertinent pour juger de la valeur des œuvres ? (Manoury, 2013, s.p.)

Que ce soit ou non le cas, le fait même qu'un tel affrontement n'oppose que des spécialistes dit assez que ces secteurs, dont la monétarisation dépend pour l'essentiel d'une très petite sphère de financeurs publics (combinée, dans le cas de l'art, à celle de la spéculation privée), se trouvent enviablement à l'abri des tensions qui s'exercent sur les produits culturels de grande consommation. Il serait donc incongru d'y parler de " divorce » avec le très grand public, lequel s'en soucie généralement bien peu (mais pas toujours, comme l'a observé Heinich).

Même le conflit entre les interprétations puristes et progressistes de la musique baroque et classique - qui oppose à nouveau une appréciation normative (l'authenticité) et une appréciation hédonique (l'agrément d'écoute) - n'est pas un thème majeur du débat public, bien que leur financement relève en bonne part des ventes de billets et d'enregistrements auprès d'une clientèle moins exiguë que celle des commanditaires de créations contemporaines.

Plus on s'éloigne d'une sphère de consommation restreinte, plus la rivalité entre deux systèmes d'appréciation discursive tend à s'accentuer, les deux polarités de la posture critique - être l'œil du public ou le gardien de l'ineffable - paraissant s'écarter inéluctablement.

Il est donc prévisible que ce soit dans le cas du cinéma que cette dissension se donne à voir de la façon la plus nette. Non seulement parce que la légitimation culturelle de celui-ci est encore récente - « Les films sont traités comme un véritable art par un conférencier au Metropolitan » s'étonnait un grand quotidien en $1936^{18}-$, mais aussi et surtout par l'« impureté » intrinsèque que soulignait Hervé Bazin (1987) :

18 New York Herald Tribune (16 novembre 1936), "Films are treated as real by lecturer at Metropolitan ", cit. in Thomas Y. Levin (1996, p. 27). 
Encore une fois, ne nous laissons pas tromper ici par l'analogie avec les autres arts, surtout ceux que leur évolution vers un usage individualiste a rendus presque indépendants du consommateur. Lautréamont et Van Gogh ont pu créer, incompris ou ignorés de leur époque. Le cinéma ne peut exister sans un minimum (et ce minimum est immense) d'audience immédiate. (p. 201)

La tension interne du cinéma ne peut, dès lors, que renforcer celle de la critique, voire s'y superposer. Toutefois, si la question de la valeur des films, comme auparavant celle des pièces de théâtre, bénéficie d'une visibilité dépassant nettement celle des autres champs de la création culturelle, son "impureté » économique n'explique pas tout. Certes, étant spectacle - à l'instar du théâtre - elle implique un coût de production et un seuil de rentabilité bien supérieurs à ceux d'un créateur isolé, mais on pourrait en dire autant du ballet ou de l'opéra, dont l'évaluation critique ne passionne pas les foules ${ }^{19}$. En revanche, son « impureté » cognitive est tout aussi essentielle : qu'il le veuille ou non, le cinéma - toujours comme le théâtre - suscite par nature une attente de pertinence plus impérieuse que d'autres domaines, non seulement parce qu'il annonce un récit (donc une cohérence raisonnablement facile à suivre), mais aussi parce qu'il montre directement ce qu'il raconte, épargnant ainsi l'effort considérable qu'implique la construction d'un modèle mental. Même lorsqu'elle n'est pas explicite, cette présomption de pertinence forme l'arrière-plan de tout débat sur la valeur d'un film.

Cependant, les facteurs de la pertinence, s'ils sont universels dans leurs modalités, ne le sont pas dans leurs proportions. C'est vrai de l'effort, qui varie avec l'expérience, c'est-à-dire avec l'entraînement. On sait par exemple que les lecteurs réguliers lisent non seulement plus vite, mais aussi avec beaucoup moins de peine que les lecteurs occasionnels, et que cette aisance se traduit à tous les niveaux de traitement (moins de fixations oculaires, plus d'inférences, etc.) C'est encore plus vrai de l'effet, pour lequel les facteurs de haut niveau, souvent abstraits, peuvent, également avec l'entraînement, supplanter

19 En tout cas aujourd'hui. Il est par exemple vrai que, de Rameau à Stravinsky, la juste appréciation des nouveautés musicales a soulevé son lot de polémiques, mais celles-ci n'atteignent pas l'ampleur publique des querelles théâtrales qui se sont égrenées du XvII ${ }^{\mathrm{e}}$ au XIX ${ }^{\mathrm{e}}$ siècles. 
les facteurs de bas niveau, leur réceptivité étant autocumulative (et autoprédictive). Il va sans dire que cet entraînement, loin d'être spontané, est déterminé et entretenu par sa valorisation sociale (tandis que la réprobation sociale tempère, mais beaucoup moins aujourd'hui qu'hier, les « vulgaires » séductions de bas niveau cognitif).

Comme le rappelle Roger Odin (2000),

Il existe donc non pas un mais des publics en fonction des contraintes partagées. [...]

Les ennuis commencent lorsque l'on se fixe comme programme de rendre compte des déterminations qui fondent ces publics. Comment maîtriser leur diversité, leur nombre, leur hétérogénéité ? (p. 55)

Imaginer un discours critique pouvant satisfaire ce large spectre d'attentes constitue à l'évidence un défi de taille. Il l'est d'autant plus dans le cas de la critique cinématographique qu'il ne lui est guère possible de se spécialiser : si un spécialiste de musique classique est rarement condamné à évaluer un enregistrement de black metal ou de country, un critique de cinéma est a priori confronté à la totalité des produits offerts sur grand écran, sans même maîtriser le calendrier impérieux des sorties hebdomadaires.

La perspective sémio-pragmatique d'Odin le conduit à proposer neuf modes de consommation du discours cinématographique :

1. Mode spectaculaire : voir un film comme un spectacle

2. Mode fictionnalisant : voir un film pour vibrer au rythme des évènements fictifs racontés

3. Mode fabulisant : voir un film pour tirer une leçon du récit qu'il propose

4. Mode documentaire : voir un film pour s'informer sur la réalité des choses du monde

5. Mode argumentatif/persuasif : voir un film pour en tirer un discours

6. Mode artistique : voir un film comme la production d'un auteur 
7. Mode esthétique : voir un film en s'intéressant au travail des images et des sons

8. Mode énergétique : voir un film pour vibrer au rythme des images et des sons

9. Mode privé : voir un film en faisant retour sur son vécu et/ou sur celui du groupe auquel on appartient

Mais, si judicieuse que puisse être cette nomenclature, dériver de ces modes une typologie des publics ${ }^{20}$ (même avec les nuances et précautions de cet auteur) paraît délicat en théorie - puisque la déstructuration contemporaine des préférences culturelles conduit chacun à panacher ses propres choix de consommation - et, de toute façon, inopérant en pratique - puisque le critique ne peut réserver son discours à l'un de ces « publics » virtuels.

La double perspective psycho-sociologique défendue ici - sans s'opposer le moins du monde à la précédente ni à tout autre angle (on ne le répétera jamais assez) - permet assez aisément de distinguer derrière ces types de consommation le poids variable des facteurs d'effet, parfois presque «purs » (spontanés : 1, 2, 8 ; égocentrés : 9 ; élaborés : 6,7$)$, parfois plus composites $(3,4,5)$, mais toujours régulés positivement $(3,4,5,6,7,9)$ ou négativement $(1,2,8)$ par la légitimité sociale, et bien sûr combinables entre eux.

Cette perspective réductrice permet-elle pour autant de soulager les tourments des critiques, tels que l'on vient de les survoler, ou mieux - comme le propose avec une feinte naïveté Laurent Juillier (2000) - de répondre à la question "Qu'est-ce qu'un bon film? » (il avance quatre critères, dont trois, selon lui, "relèvent $d u$ cognitif $»)$ ?

On ne nourrira évidemment pas de si hautes ambitions. Cependant, aborder la critique comme un discours évaluatif (visant à valoir) à propos de discours créatifs (visant également à valoir) peut aider à souligner quelques points utiles.

20 «Pour la sémio-pragmatique, un public est avant tout une communauté de faire: j'appelle public, un ensemble d'individus réunis par la mise en œuvre d'un système de modes de production de sens (c'est-à-dire d'un programme de production textuelle) »(Odin, op. cit., p. 6o). 
Le premier est que, sous cet angle, la "crise de la critique" n'existe pas, si l'on entend par là un phénomène singulier affectant spécifiquement la critique contemporaine. D'une part parce que la rivalité entre la pertinence cognitive et les valeurs est intrinsèque à la création culturelle et tout autant à l'exercice public du jugement sur celle-ci, qu'elle accompagne depuis toujours (voir plus haut Corneille et Molière). D'autre part parce que l'affaiblissement des hiérarchies symboliques et la multiplication des locuteurs (dont s'affligeait déjà Sainte Beuve au XIx ${ }^{\mathrm{e}}$ siècle) affectent indistinctement la totalité du marché discursif : la dilution de l'autorité discursive des critiques accompagne celle des journalistes généralistes, mais aussi des enseignants, des prêtres, des responsables politiques ou syndicaux, etc.

Dès lors qu'elle peut de moins en moins se jucher sur des normes transcendantes (ou sur l'«idéologie du goût naturel » observée par Bourdieu, 1979) pour statuer sur la grandeur ou l'indignité des productions et des consommations culturelles, la critique est-elle condamnée à agoniser en maudissant la baisse des exigences et des compétences ou, à l'inverse, à se soumettre sans vergogne aux «bas instincts » d'une foule frivole?

Ces deux positions, aussi caricaturale l'une que l'autre, ne sont que des transpositions des vieux archétypes (le gardien de l'ineffable et l'œil du public), lesquelles étaient utiles en première analyse, mais peuvent faire passer la délicate dialectique de la valeur discursive pour une stricte alternative entre des voies également impraticables. Si l'on ne peut (sauf au profit d'une poignée d'adeptes) s'ériger en gardien d'une légitimité évanescente, on ne peut pas plus être l'œil $\mathrm{d}^{\prime}$ « un » public dont on rappelait plus haut la grande variabilité des attentes, des modes de consommation et des facteurs d'effet auxquels ils s'apparentent. Ce qui n'empêche pas que le renforcement des attentes de pertinence cognitive soit suffisamment général et constant pour concerner la grande majorité des consommateurs culturels.

Au bout du compte, comme toutes les instances plongées dans la crise de l'attention, la critique pourrait être simplement condamnée à intéresser - ce dont il semble difficile de se lamenter - et cette nécessité croissante est d'autant plus impérieuse lorsque les œuvres qu'elle aborde ne sont pas spontanément pertinentes par elles-mêmes.

C'est là un défi familier à d'autres professionnels du métadiscours, tout aussi convaincus de l'élévation de leur objet, mais peu 
enclins à se reposer sur sa légitimité «naturelle »: ceux qui traitent de l'actualité scientifique, c'est-à-dire de discours fort compliqués et générant souvent peu d'excitation spontanée (ce qui a notamment amené ces professionnels à un certain recul, mais aussi à une réelle virtuosité dans les procédés sollicitant les facteurs d'effet de haut niveau, comme la problématisation, etc.)

Il ne s'agit pas de plaquer mécaniquement sur la culture littéraire et artistique ce qui vaut pour la culture scientifique. Mais à lui seul, le sentiment d'incongruité que peut susciter ce rapprochement entre la critique culturelle et la "vulgarisation » (dont le nom même sonne comme une condamnation ${ }^{21}$ ) pourrait en dire autant sur la « crise de la critique » que toutes les pages qui précèdent : les créations littéraires et artistiques sont-elles vraiment " dignes d'intérêt » - ce qui vaudrait mieux pour elles dans l'évolution actuelle du marché discursif - ou sont-elles « dignes » tout court?

21 On notera qu'à l'instar du savoir scientifique acquis, la vulgarisation des oeuvres consacrées (un opéra de Mozart, une sculpture de Rodin, une toile de Picasso...), telle que s'y efforcent notamment des émissions télévisées, ne perturbe personne. Les choses se gâtent lorsqu'il s'agit de traiter l'actualité de la production culturelle. 


\title{
Sur les pouvoirs du récit
}

\begin{abstract}
A vec l'essor des "fake news", le développement du «storytelling " 1 fait partie de ces phénomènes qui, une fois revêtus d'une appellation au goût du jour, surgissent comme des menaces nouvelles pour le débat public à l'heure des réseaux. Évidemment, dans les deux cas, les procédés visés sont aussi anciens que la communication humaine - en fait, la distinction entre fiction et réalité est nettement plus récente $^{1}-$, mais dans les deux cas, la multiplication des alarmes pourrait effectivement correspondre, non seulement à un développement quantitatif, mais aussi à une certaine progression de la technicisation et de la systématisation de ces pratiques discursives.
\end{abstract}

Elles sont du reste apparentées : la combinaison de la fiction (fond) et du récit (forme) a donné naissance à rien de moins que la littérature, après avoir engendré les mythes par lesquelles chaque civilisation expliquait son existence et justifiait ses règles. Autant dire qu'il n'est pas une seconde question ici de détailler - et a fortiori de concurrencer - tous les travaux qu'ont suscité la fiction ou le récit dans une perspective historique, ethnologique, philosophique, sémiologique et, bien sûr, littéraire. Entre autres.

En revanche, il pourrait être intéressant de s'interroger sur un détail technique : puisque le récit paraît si prégnant que certains vont jusqu'à lui attribuer l'essor initial de l'Homo sapiens (Harari, 2015) et d'autres le prescrivent comme l'ultime secret d'une communication efficace-«Storytelling:Réenchantez votrecommunication »(Durand, 2011),

1 On sait ce que la pensée occidentale doit, entre autres, à Hérodote et Thucydide dans la lente séparation des mythes et des faits. Cependant, les lecteurs du Moyen Âge se souciaient encore peu d'attribuer un statut soit « fictionnel » soit « véridique » au roi Arthur et à ses chevaliers et l'on ne s'est guère préoccupé avant le XIX $x^{e}$ siècle de l'historicité (très douteuse) de la Chanson de Roland. 
«Boostez vos présentations avec le storytelling " (Gabison, 2012), etc. - ou encore le dénoncent comme une terrifiante arme de domination mentale (Salmon, 2007), où se niche donc la source de cette mystérieuse puissance?

Le rapport au récit pouvant constituer un bon témoin de la transition entre la candeur de l'enfance («Raconte-moi une histoire!») et l'esprit critique du citoyen éclairé ("Ne me racontez pas d'histoire!»), l'intact appétit des adultes pour les fables - au-delà, bien sûr, de la consommation d'agrément - est certainement préoccupant d'un point de vue politique. Mais d'un point de vue cognitif, il n'a rien de surprenant. Si l'on envisage la pertinence d'un énoncé comme le rapport entre l'effort qu'il réclame et l'effet qu'il procure, on remarque aussitôt que le récit est par nature l'une des formes discursives les plus pertinentes qu'il soit possible d'imaginer².

En terme d'effet, même en mettant de côté les facteurs de bas niveau cognitif comme la violence et le sexe (quoique, l'incorporation de ces composantes de base - une personne en combat une autre en vue de se reproduire avec une troisième - offre une trame inusable depuis les contes les plus anciens jusqu'aux romans modernes, en passant par le cinéma et, bien sûr, l'opéra³), le récit a évidemment pour lui toute la force de l'identification, qui imbrique la sphère personnelle du lecteur et celle du personnage, mais aussi le puissant modulateur que constitue l'intrigue (que va-t-il se passer ensuite ?).

Cependant, même un récit où ces atouts ne joueraient guère - peu d'identification ou de curiosité pour la suite - resterait étonnamment pertinent. C'est que, avant tout, la narration est remarquablement peu coûteuse en termes d'effort cognitif : son caractère (plus ou moins) linéaire canalise une forte cohérence énonciative par laquelle le lecteur n'a qu'à se laisser porter ${ }^{4}$ et ses objets facilement

2 Des formes discursives non textuelles, par exemple une " photo-choc ", peuvent l'être encore plus, mais c'est... une autre histoire.

3 Dont tout le répertoire, selon une boutade (douteusement) attribuée à George Bernard Shaw, se résumerait à l'histoire d'un ténor voulant coucher avec une soprano en dépit d'un baryton importun.

4 On ne parle évidemment pas ici du «nouveau roman » des années 1950 ou d'autres projets de littérature expérimentale, qui, justement refusent ces avantages et s'enorgueillissent de la faible pertinence cognitive qui en résulte. 
figurables («concrets ») permettent de construire aisément un modèle mental de l'état du monde qu'elle décrit, puis de le faire évoluer au fil de la narration. On se souvient, du reste, que c'est en travaillant sur la compréhension des récits que Bartlett (1930) avait donné un nouveau départ aux recherches modernes sur la psychologie de la lecture.

Pour mieux prendre en compte toute la complexité de la dimension cognitive du récit, il faudrait se reporter aux nombreux travaux qui ont ultérieurement éclairé son versant fictionnel, depuis l'ouvrage pionnier de Michel Fayol (1985) jusqu'au numéro que les Cahiers de narratologie ont plus récemment consacré à cette question (Sevilla, 2015), sans oublier (toujours pour s'en tenir aux francophones) les propositions de Jean-Marie Schaeffer (1999) et Raphaël Baroni (2007). Cependant, les quelques facteurs simples évoqués plus haut, pour schématiques qu'ils soient, suffisent amplement à percevoir l'extraordinaire séduction qu'exerce le récit, depuis l'aube des civilisations jusqu'aux publicités actuelles, en passant par les chefs-d'œuvre littéraires 5 .

Ils aident aussi à situer ses enjeux rhétoriques, souvent soulignés, quoique difficilement distingués de ceux de la fiction (voir Booth, 1961; Schaeffer, 1999). L'incorporation du point de vue du héros à la sphère personnelle du destinataire, conduit à embrasser même temporairement et partiellement, les valeurs sociales et les aspirations de ce personnage (voir Jouve, 2001), si discutables que paraîtraient celles-ci avec du recul. Plus généralement, le récit tend à affranchir des normes d'acceptabilité discursive en estompant la présence de l'énonciateur (le personnage peut exprimer ou signifier ce que l'auteur ne saurait proférer) et en le dispensant de présenter une argumentation construite (l'exemple vécu valant démonstration par analogie). D'autant que les interrogations que pourrait soulever un exposé méthodique deviennent oiseuses dans l'enchaînement forcé du récit, qui masque facilement ses propres incohérences ${ }^{6}$. Et, bien

5 Comme le signalait Barthes (1966, p. 1) «Le récit se moque de la bonne et de la mauvaise littérature : international, transhistorique, transculturel, le récit est là, comme la vie. ».

6 Quel était le statut social ou le métier de Barbe-bleue ? Pourquoi sa barbe avait-elle cette couleur? Pourquoi était-ce jugé si repoussant? Que ne la teignait-il ou ne la coupait-il pas ? Pourquoi cet homme (plutôt charmant selon ce qu'en dit Perrault) s'est-il mit à trucider ses épouses ? Pourquoi 
sûr, il est plus attirant : comme le résumait La Fontaine en connaisseur, une "morale nue apporte de l'ennui; le conte fait passer le précepte avec $l u i^{7} »$.

En termes plus généraux et plus littéraires, le récit excelle à gommer l'« opposition entre [...] monde imaginaire et monde réel » (Jauss, 1978, p. 57), teintant toute histoire de cette «suspension consentie de l'incrédulité » qui selon Coleridge (1889, p. 145) « constitue la foi poétique ».

On comprend que des esprits parmi les plus attachés aux débats fondés en raison n'aient pas répugné à user d'expédients narratifs, par exemple les Lumières et leurs contes philosophiques ou l'ébouriffant Platon qui, après avoir sèchement condamné les récits fictionnels (dans la République) s'empresse d'exploiter - voire de forger ${ }^{8}-$ l'his- $^{\prime}$ toire de la déconfiture de Theuth (dans Phèdre).

Pour autant, réduire la question du récit à la domination de la raison par le mythe serait un peu trop simple. Sur le marché discursif, la narration n'est pas l'apanage des produits légitimement fictionnels (littérature) ou sournoisement mensongers (manipulation). En tant que structuration discursive - donc indépendamment de sa dimension fictionnelle ou non - elle est aussi la forme traditionnelle des comptes rendus d'assemblée et des rapports d'enquête, et même celle des discours les plus rationalistes qui soient : les publications de recherche en sciences expérimentales. La rigide trame requise en la matière (introduction, matériel et méthode, résultats et discussion) n'est rien d'autre que le récit standardisé d'une aventure scientifique : «ce problème se posait; des explorations antérieures en avaient révélé ceci;

a-t-il confié à la dernière d'entre-elles la clef qui le trahissait ? Pourquoi cette épouse - anonyme contrairement à sa sœur Anne - ne l'a-t-elle dénoncé aux amis qu'elle avait invités ou ne s'est-elle enfuie avant son retour? (Voir aussi la plaisante parodie pseudo-historique qu'AnatoleFrance, 1921, a donné de ce conte).

7 Le pâtre et le lion.

8 Bien sûr, Thot appartenait de longue date au panthéon égyptien, mais l'épisode étonnamment détaillé que raconte Socrate et le flou de son attestation (" une tradition des anciens... »; "J'ai donc oui dire qu'il existait près de Naucratis... », trad. Meunier, 1922, p. 274) peuvent laisser planer le doute, y compris, d'ailleurs, chez son interlocuteur («... ce que tu prétends avoir entendu raconter... "; "Il t'en coûte peu, Socrate, de proférer des discours égyptiens ; tu en ferais, si tu voulais, de n'importe quel pays que ce soit. ».) 
nous avons procédé comme cela, et avons ainsi recueilli tel et tel résultats, lesquels nous ont conduits (épilogue!) aux conclusions suivantes. » Si l'on oppose plus généralement l'ordonnancement chronologique aux autres dispositions textuelles, force est même de ranger dans le premier camp les modes d'emploi et les recettes culinaires (rappelons à tout hasard que l'analyse générale du marché discursif interdit d'opérer a priori des distinctions fondées sur l'élévation ou la trivialité des productions hétéroclites offertes sur ce marché).

Pourquoi, dès lors, le récit n'a-t-il pas totalement supplanté les autres formations discursives, porté par l'avantage que lui donne sa cohérence naturelle ? C'est qu'aucune des variables qui concourent à l'établissement de la valeur des discours ne joue isolément des autres, que toutes peuvent s'avérer tantôt favorables tantôt défavorables, et qu'aucune ne peut l'emporter sur toutes les autres dans tous les cas. Méconnaître l'interaction des facteurs en jeu serait refaire l'erreur commise par certains promoteurs de la « lisibilité » (la réduction de la longueur des phrases et des mots) en pensant que l'abaissement de la charge lexico-syntaxique des textes était le secret de leur efficacité.

Or, de même que les textes les plus lisibles (effort de deuxième classe) ne sont pas forcément les plus "efficaces", les textes hautement cohérents (effort de troisième classe) ne le sont pas nécessairement. La cohérence contraignante qui égrène les informations entre le début et la fin d'un récit, outre qu'elle est socialement proscrite lorsqu'une argumentation structurée est attendue, devient un sérieux obstacle lorsque la consommation discursive est motivée par l'attente d'une connaissance ou d'un argument précis, notamment dans un but utilitaire (c'est-à-dire lié à la sphère personnelle). C'est par exemple la raison pour laquelle les comptes rendus de réunions chronologiques ont été concurrencés par les comptes rendus analytiques qui regroupent par thème les décisions prises ${ }^{9}$. Mais le récit peut aussi être rejeté dans des contextes de haut, voire de très haut niveau, comme en témoigne la rébellion du Nouveau roman contre l'intrigue romanesque, ou, à mille lieues de la fiction, l'assaut donné à l'histoire chronologique (celle des rois et des batailles) par le courant de l'École des Annales et de la Nouvelle histoire qui

9 C'est aussi la raison pour laquelle les interminables «tutoriaux » informatiques pas à pas qui abondent sur YouTube seront fuis par ceux qui ne recherchent qu'une indication technique particulière. 
rejetait en effet sur les marges l'événementiel, répugnait au récit, s'attachait au contraire à poser, à résoudre des problèmes et, négligeant les trépidations de surface, entendait observer dans la longue et la moyenne durée, l'évolution de l'économie, de la société, de la civilisation. (Duby, 1973/2005, p. 8)

Évidemment, pour les élèves dont les programmes scolaires ont été chamboulés par cette réorientation de la pensée savante, l'évolution du prix du blé ou des représentations sociales au XvIII ${ }^{\mathrm{e}}$ siècle est nettement moins pertinente qu'une succession de péripéties habilement contées. Mais, comme on le signalait plus haut, l'enseignement scolaire peut toujours compenser le déficit de pertinence cognitive de ses contenus par une contrainte sociale suffisamment robuste pour forcer ses jeunes destinataires à en prendre connaissance.

C'est donc sur le marché discursif « libre», et particulièrement celui de l'actualité, que se sont produites les évolutions les plus révélatrices. Ayant vocation à raconter «ce qui s'est passé », la presse d'information a, des siècles durant, spontanément privilégié la forme du récit (les anglo-saxons parlent toujours de story pour désigner leurs sujets). Ce n'est qu'au cours du XIX ${ }^{e}$ siècle que les agences de presse ont commencé à penser qu'une histoire n'était pas un procédé si efficace que ça pour transmettre beaucoup d'information en peu de temps, et que - comme plus tard les comptes rendus de réunion « analytiques » - est apparue la façon la plus « rationnelle» d'empaqueter et livrer celles-ci : l'exposition des faits par ordre d'importance décroissante, en commençant par l'essentiel de la nouvelle (qui a fait quoi, quand, où, pourquoi et comment?). Sans trop creuser cette structuration dite en " pyramide inversée », que l'on a déjà examinée plus haut ${ }^{10}$, on rappellera que son incohérence radicale (imaginons donc un roman dont les paragraphes se succéderaient par ordre d'importance...) était en principe compensée par son caractère résolument fonctionnel : l'éditeur pouvait à tout moment couper la suite du texte, ou le consommateur interrompre sa lecture, en restant néanmoins assuré d'en avoir prélevé l'essentiel.

Or, dans la seconde partie du $\mathrm{xx}^{\mathrm{e}}$ siècle, le durcissement de la concurrence discursive a cruellement souligné le problème d'attractivité de cette façon de conditionner et livrer l'actualité : il ne suffit pas

10 Voir Le mystère de la pyramide inversée (supra). 
d'être pratique à consulter pour obtenir une part d'attention. Le journalisme narratif a donc refranchi les frontières du grand reportage (où il était resté inexpugnable) en se glissant d'abord dans les sections Loisirs des quotidiens états-uniens pour revenir - non sans résistances - dans les autres pages des journaux, y compris francophones (voir Lits, 2008, et Vanoost, 2013, pour une approche narratologique de ce phénomène).

Les recherches menées vers la fin du siècle, en particulier l'expérience en conditions réelles conduite pour l'association américaine des rédacteurs en chef (ASNE), ont cependant suggéré que le récit, pour séduisant qu'il soit, n'était pas la panacée. Si les articles narratifs étaient clairement préférés aux textes strictement informatifs, cet agrément semblait se payer, en particulier auprès des lecteurs éduqués, d'une confiance moindre vis-à-vis de la véracité et de l'impartialité de l'information proposée. Il est possible qu'une telle perception - qui reste à établir définitivement ${ }^{11}$ - ait été conditionnée par l'apprentissage des formes discursives, le récit étant associé dès l'enfance avec la fiction alors que la structuration en pyramide inversée est associée depuis des générations (en particulier aux États-Unis) au journalisme factuel et impartial. Comme il est possible - mais non contradictoire - que le traitement des récits factuels active des zones cérébrales différentes de celles qui sont stimulées dans le traitement de fictions (Altman, Bohrn et al., 2014). Ce bond vigoureux d'un extrême à l'autre du spectre scientifique rappelle opportunément que l'étendue du champ d'explications qui s'étend entre ces deux pôles et des échelles qu'il implique (du neuronal au social), aurait pu suffire à décourager l'espoir ou la crainte de découvrir une recette monofactorielle et universelle de communication « efficace», a fortiori si on la voudrait «scientifique ${ }^{12}$ ». Du reste, les résultats des autres expériences ayant ultérieurement tenté de vérifier l'efficacité du récit journalistique par rapport à l'exposition décroissante des faits pourraient être résumés par "plutôt oui... mais ça dépend ». Même à la télévision, où la simplicité conceptuelle de ce produit discursif - dans sa version

11 L'effet suggéré n'est pas considérable et d'autres expériences (sur des échantillons beaucoup plus minces) n'ont pu le mettre en évidence.

12 D'un point de vue expérimental, le contrôle une à une des innombrables variables en jeu dans différentes situations supposerait un effort inimaginable et plausiblement vain. 
de base - n'a pas manqué de séduire les producteurs et les responsables, son poids est moins écrasant qu'on ne tendrait à le penser (Ytreberg, 2001). Quant à sa version moins triviale mais plus coûteuse, telle que la défend notamment Érik Neveu (2014), elle ne représente encore qu'une modeste portion de l'offre quotidienne d'information.

Ce que montre surtout l'exemple du journalisme narratif, adopté, puis délaissé, et enfin redécouvert, mais accompagnant dorénavant d'autres formes qu'il semble peu susceptible d'évincer (Johnston et Graham, 2012), c'est que, même dans un cadre ne requérant normalement pas d'argumentation, l'avantage cognitif considérable que confère au récit sa facilité de traitement et son attrait n'est pas un avantage absolu ${ }^{13}$.

13 L'étude ASNE ne disait rien d'autre en concluant, au terme d'une recherche particulièrement poussée, que la meilleure façon d'écrire un journal... était de varier les formes. 


\section{Vertus martiales des images salaces}

ـ métier des armes était traditionnellement fameux pour le remaradministrative requise au quartier général et les rudesses du terrain, où (jusqu'à récemment) quelques obscénités vigoureuses n'étaient pas malvenues pour faire valoir un ordre. Pourtant, c'est bien dans des bureaux d'état-major qu'ont été approuvés certains des documents les plus incongrus qui aient jamais été produits et diffusés par des services gouvernementaux.

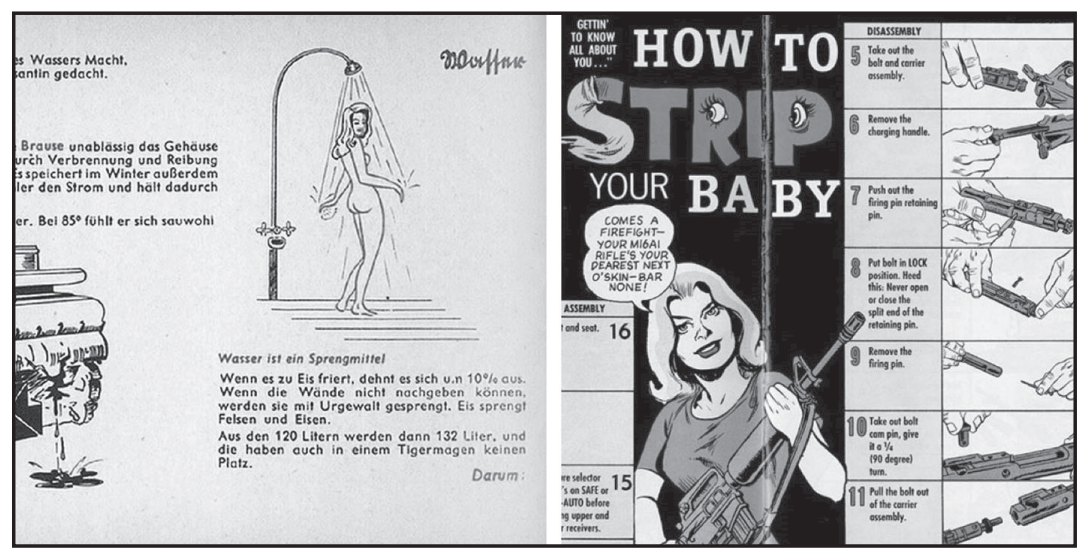

Ces manuels militaires consacrés à l'entretien de deux armes redoutables, le char Panzer VI Tiger (à gauche ${ }^{1}$ ) et le fusil d'assaut Mi6 (à droite ${ }^{2}$ ) sont, à l'évidence, assez éloignés de l'austère littérature

1 Generalinspekter der panzertruppen (1943). Tigerfibel [D656/27]. Berlin : Panzerwaffe, p. 18 (détail).

2 Departement of the Army (1969). The M16A1 Rifle-Operation and preventive maintenance (DA-pam 750-30). Washington, US Government, p. 2-3 (détail). 
technique ou bureaucratique habituelle. Le contraste entre ces publications égrillardes et les circonstances dans lesquelles ces armes allaient jouer un grand rôle ${ }^{3}$ est très perturbant a posteriori. Toutefois, si l'on se replace dans le contexte discursif de leur diffusion, une autre opposition se remarque, moins sinistre mais plus curieuse.

La logique qui a déterminé leur production est peu surprenante en soi : le combat à mener par leurs concepteurs était avant tout une lutte acharnée pour conquérir et conserver l'attention des soldats. Dans les deux cas, son enjeu était d'autant plus stratégique que chacune de ces armes payait sa supériorité technique d'une dangereuse fragilité : sans un entretien constant, le char Tigre s'arrêtait et le Mi6 s'enrayait quand les équipements plus frustes des adversaires restaient utilisables. Un soin acharné a donc été mis à réduire l'effort cognitif requis par des instructions techniques rébarbatives, en renforçant par tous les moyens la cohérence et la figurabilité de ces instructions (ainsi chaque page du manuel allemand comprend-elle une ou plusieurs comparaisons très didactiques avec la vie courante). Mais l'extraordinaire réside évidemment dans l'incorporation systématique d'images émoustillantes à des documents officiels dont l'objet était des plus sérieux. En effet, ceux-ci bénéficiaient d'un contexte qui semblerait largement suffire à assurer leur pertinence cognitive : le thème "si vous ne lisez pas ça, vous allez mourir »-d'ailleurs exploité par les deux brochures ${ }^{4}$ - garantissant une interaction plutôt solide avec la sphère personnelle des lecteurs. Mais malgré l'avantage vital - au sens propre - que ceux-ci trouveraient à cette lecture, les auteurs ont dans les deux cas estimé plus sûr de miser sur un inusable facteur d'effet de bas niveau cognitif, jugeant insuffisante la perspective de se retrouver désarmé face à un ennemi qui ne l'est pas. Une bonne dose d'humour (pas le plus léger...) a parachevé - dans les deux cas aussi ce renforcement obsessionnel de la pertinence discursive.

Toutefois, une telle lutte implique évidemment un conflit plus large, cette fois à l'échelle globale de la valeur discursive, entre la

3 Dont le massacre de civils d'Oradour-Sur-Glane en 1944 et celui de My Lai au Vietnam.

4 «Noubliez pas : étudiez bien votre guide où votre [char] Tigre partira en enfer! » souligne l'un (selon la traduction en anglais de Bob Carruthers), «le nettoyage est une nécessité et il peut vous sauver la vie », "[...] parce que vous jouez votre vie là-dessus ", insiste l'autre. 
pertinence cognitive et les normes d'acceptabilité de l'institution militaire. Celles-ci, qui se mesurent par exemple à la rigidité avec laquelle l'armée codifie ses règles de salutation et de parole, s'accommodent en principe mal de ce genre de badinage. Passe encore que, sous le feu ennemi, un sous-officier s'autorise une expression qui «offense grossièrement la modestie, la décence ou la bienséance ", voire "choque le sens moral » ou pire, "suscite des pensées lubriques 5 ", mais de là à publier au nom de la haute hiérarchie un document convoyant précisément de telles pensées, le pas était considérable. Il était même inconcevable si, comme Morroe Berger, on estime que les deux situations discursives constituent précisément des opposés :

Il y a différentes sortes de phraséologies militaires, cependant, qui descendent l'échelle du formalisme depuis le langage employé dans les manuels officiels jusqu'au ton beaucoup moins distingué des baraquements et du terrain. (1945, p. 258)

Mais même stratifiées par des siècles de tradition militaire, les contraintes sociales de la convenance pèsent moins que les impératifs de la pertinence cognitive lorsque les enjeux sont réellement élevés. On ne parle pas ici d'une sorte d'arbitrage spontané, voire impensé, mais d'une gestion attentive et raisonnée de la valeur discursive. C'est particulièrement vrai dans le cas des États-Unis, où l'on s'était avisé avant même la Seconde Guerre mondiale que la communication, entrant dans l'éventail des problèmes militaires, devait relever du type d'approche rationaliste que l'armée applique à ses dispositifs de combat (les chercheurs alors recrutés dans ce but deviendront aprèsguerre les moteurs du développement universitaire de cette discipline). Le dessinateur de la brochure du Mi6, Will Eisner (célèbre dans le civil comme créateur du super héros The Spirit) n'avait pas échappé à cette logique évaluative. Conscrit en 1942, il avait rapidement taillé son chemin à coups de crayon depuis le modeste journal de son régiment jusqu'à un périodique technique largement diffusé. Mais, raconte son biographe,

5 Selon l'article 134-89 (« Langage indécent») d'un autre manuel... celui de la justice militaire états-unienne (Manual for Courts-Martial, édition 2016). Notons cependant que ces précisions, de plus en plus détaillées au fil du temps, ne figuraient pas encore dans l'édition en vigueur au moment de la guerre du Vietnam. 
Il y avait naturellement des gens au-dessus de lui qui n'étaient pas enthousiasmés par son approche. Le service d'état-major chargé de la production des manuels techniques s'efforça de tuer l'utilisation des dessins d'Eisner pour des dossiers techniques sérieux. Les comics n'étaient jugés acceptables que pour la propagande. Le seul moyen par lequel le responsable administratif pensa stopper Eisner était une comparaison d'efficacité. Il obtint de l’Université de Chicago qu'elle mène un test entre un manuel technique standard et une brochure graphique d'Eisner sur le même sujet. Eisner l'emporta, car la lisibilité et le degré de rétention de son matériel étaient supérieurs à ceux des précis, mais secs et pédants manuels techniques. (Andelman, 2015, p. 5)

On imagine que cette étude, couronnant d'une légitimité savante les retours enthousiastes des soldats et sous-officiers, a définitivement aplani les objections qu'auraient ultérieurement pu soulever les comics militaires d'Eisner, devenu contractant civil lors de la guerre du Viêt Nam et ceux (encore plus suggestifs) de ses successeurs. De parution en parution, les tenues de la blonde conseillère technique sont en effet devenues de plus en plus «intéressantes », jusqu'à ce qu'elle apparaisse franchement nue, l'emplacement des traditionnelles feuilles de vignes se voyant occupé par deux panneaux : "L'entretien préventif des véhicules... " économisera votre carburant et vos équipements " «... et c'est la vérité toute nue, les gars !! », susurrait l'égérie des GI).

Quant au manuel du char Tigre, qui exploite tout aussi gaillardement les silhouettes féminines, rien ne permet de penser qu'il ait bénéficié d'expériences très poussées. En revanche, tout suggère que le pour et le contre de sa violation des normes d'acceptabilité discursive a été mûrement soupesée : la rigide culture prussienne de la Wehrmacht, mais aussi la caution explicite de l'Inspecteur général des troupes blindées (Heinz Guderian) et plus encore la mention un peu embarrassée qui la précède : "Même les moralistes, pour leurs prêches, sont parfois immoraux».

Dans les deux cas, cette transgression ne permettait pas seulement de jouer sans vergogne sur les facteurs d'effet et donc la pertinence. Elle permettait en outre, en renonçant à faire prévaloir les jugements de valeur de la hiérarchie, de se fondre à ceux prêtés aux publics visés. Alors que s'absorber dans un document technique aurait signalé aux autres membres du peloton un genre de «bon 
élève » rarement populaire, feuilleter une brochure effrontée était plausiblement beaucoup plus légitime. On peut supposer que la même logique de valorisation discursive - optimisant non seulement la pertinence cognitive, mais aussi la légitimité sociale du discours pour ses destinataires - explique une autre curiosité, le fait qu'aucun des deux écrits ne comporte ni ne représente le moindre marquage officiel : les drapeaux et logotypes dont les forces armées constellent leurs uniformes et équipements auraient été malvenus ici.

Peut-on mieux mesurer la pression de l'adéquation sociocognitive qu'en songeant qu'elle est capable de contraindre des armées à censurer leurs propres insignes (même la croix gammée) sur des imprimés visant leurs propres soldats?

Il est, du reste, symptomatique que ces documents aient été produits pour des troupes de conscrits, les appelés du contingent étant $a$ priori moins susceptibles de comprendre les équipements, moins motivés à les connaître, mais aussi plus rétifs aux normes et valeurs militaires que les soldats de métier.

Les mêmes contraintes devant conduire à des solutions assez similaires, on pouvait par exemple trouver en Suède (alors pays de conscription) un opuscule d'apparence familière. Mais ses circonstances de production et de réception discursive étant infiniment moins tendues, son contenu l'est aussi. En l'absence de guerre en cours ou imminente, les facteurs d'effets cognitifs y sont exploités avec modération : les enjeux pour la sphère personnelle du lecteur se limitent au risque d'une écoutille mal verrouillée et une seule silhouette féminine, celle de la jeune femme ci-dessous, sollicite un peu la libido du public visé. Cette pertinence modérée réduit en conséquence la nécessité de transgresser les normes du discours hiérarchiquement légitime, et ce d'autant moins que l'amertume du combat n'est pas là pour creuser un écart insurmontable entre les valeurs des soldats et celles de l'état-major (on chercherait néanmoins en vain le drapeau suédois ou le logotype des forces armées de ce pays).

Si lénifiant soit-il, cet opuscule est peut-être l'un des derniers vestiges d'une configuration discursive révolue en Occident. Non seulement parce que la professionnalisation des armées et leur technicisation transforment la compétence (en termes d'effort) et la motivation (en termes d'effet) que les producteurs des brochures 

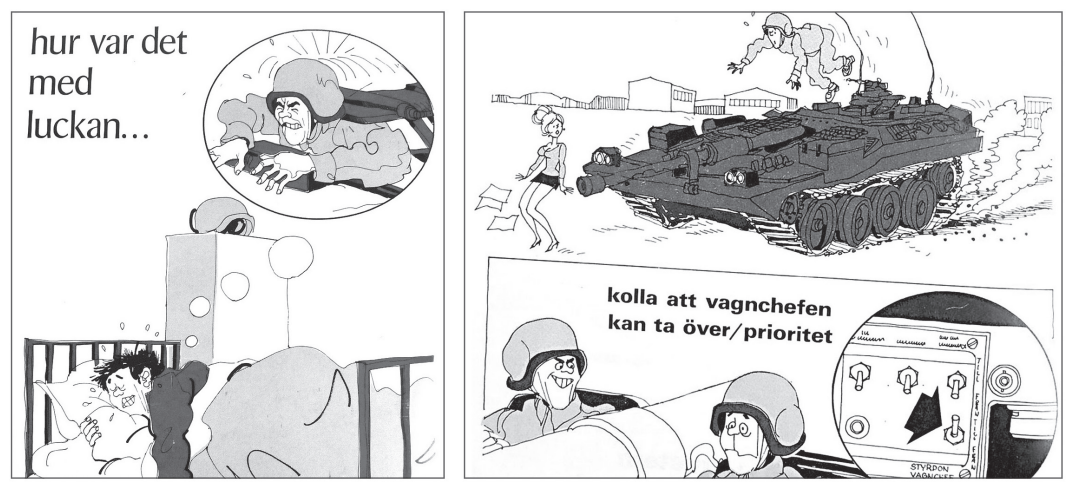

Försvarets materielverk (1970). En dag med STRV 103 (Une journée avec le STRV 103). Stockholm, FMV-A, p. 34 et 6 (détails).

techniques militaires peuvent espérer de leurs lecteurs, tout en rapprochant l'évaluation que ces derniers et leur hiérarchie font de la légitimité discursive. Mais aussi parce que les rôles qu'y jouent les personnages féminins et masculins (seuls à porter l'uniforme) sont factuellement et normativement en profond décalage avec l'époque actuelle.

Ainsi la paillardise des bulletins techniques militaires a-t-elle à la fois perdu sa nécessité cognitive et sa légitimité sociale. Mais cette truculence insolite reste révélatrice en ce qu'elle marque l'intersection de deux grands problèmes de valeur discursive.

Le premier, bien connu mais poussé ici à l'extrême, est celui de tous les modes d'emploi du monde. Il rappelle utilement que l'effet cognitif induit par l'utilité personnelle d'une information est moins puissant qu'on ne tend à le croire : si le risque d'une mort rapide n'est pas suffisant pour garantir qu'un public captif (et, à l'époque, peu sollicité par la concurrence des contenus en ligne) s'inflige l'effort de parcourir une série d'instructions techniques, même clarifiées avec soin, comment s'étonner que les consommateurs ignorent obstinément les modes d'emploi ou d'assemblage des produits qu'ils viennent d'acquérir?

L'autre, également connu, mais également paroxystique ici, est la chaotique ambiguïté des normes de l'acceptabilité discursive, généralement perçues comme intangibles et moralement justifiées, mais susceptibles en cas de besoin d'être aménagées au même titre 
que n'importe quelle autre variable communicationnelle. Or, les forces armées sont l'une des institutions les plus abruptement confrontées aux enjeux pratiques et symboliques de cette variabilité. Singulièrement celles des États-Unis, sujettes depuis l'origine à de remarquables tensions normatives. Ainsi, Georges Washington s'exaspérait-il déjà du fait que malgré "des ordres nombreux et précis [...] émis contre la coutume insensée et abominable de jurer [...] celle-ci prévaut, si c'est possible, plus que jamais », enjoignant aux officiers de tous les rangs de réprimer chez leurs hommes « un vice qui est aussi improfitable qu'il est abject et honteux » (1779/1973, p. 99-100). Qu'il soit moralement «honteux» est une question de conviction, mais qu'il soit «improfitable» ne va pas de soi, y compris chez les officiers eux-mêmes. Outre qu'un langage vigoureux a le mérite de la clarté - certains attribuent l'issue de la bataille de Gettysburg aux différences entre les injonctions du rude Stonewall Jackson et celles du (trop ?) distingué général Lee - l'élévation morale n'est pas forcément propre à élever le moral des troupes. Châtier le discours militaire (et ceux qui le profanent) présente un coût que le général Patton, théorisant l'obscénité en plus d'en être l'un des plus brillants praticiens, n'était pas prêt à payer :

Quand je veux que mes hommes se souviennent de quelque chose d'important [...], je leur donne ça très salement. Ça ne sonnerait peut-être pas bien pour quelques groupes de vieilles dames à un thé d'après midi, mais ça aide mes soldats à s'en souvenir. Vous ne pouvez pas diriger une armée sans grossièreté, et il faut que ça soit une grossièreté éloquente. Une armée sans grossièreté n'arriverait pas à se battre assez pour s'échapper d'un sac en papier trempé de pisse. (cit. in Brighton, 2009, p. 261).

De son aveu même, la rusticité radicale affectée par Patton ne devait donc rien à la spontanéité d'une âme simple et tout au calcul d'un stratège soucieux d'optimiser la portée de son discours. Le défenseur des expressions relâchées ne supportait d'ailleurs pas les uniformes débraillés. Et en fait de liberté de ton, Patton fera tout son possible pour censurer le plus fameux cartoonist du front - le reporterdessinateur Bill Mauldin de Stars and Stripes - coupable à ses yeux de représenter les GI crasseux, mal rasés et perpétuellement désabusés : « les boches devraient vous épingler une médaille pour les aider à bousiller la discipline chez nous » (Mauldin, 1971, 53). 
Du statut des jurons à celui des pin-up (jadis bienvenues sur les fuselages d'avions, aujourd'hui pourchassées avec acharnement ${ }^{6}$ ), le monde militaire, tantôt rigide tantôt pragmatique, constitue décidément un admirable champ pour apprécier les contorsions de la légitimité discursive entre ce qui est socialement acceptable et ce qui s'avère opérationnellement opportun.

6 Selon la revue militaire Stars and Stripes (18.01.2013), l'U.S. Air Force a entrepris une fouille systématique de ses bases, débusquant, entre autres contenus «inappropriés", plus de 200 photos représentant des bombardiers de la seconde guerre mondiale décorés de façon inconvenante. 


\section{Terminaison}

$\mathrm{O}$ ù interrompre cette promenade au fil des allées d'un marché discursif dont les étals s'étendent à l'infini ? On aurait pu visiter le quartier des pédagogues, où les auteurs de manuels scolaires tentent vaillamment de concilier les attentes de l'institution et celles des élèves. Peut-être s'aventurer dans le secteur des consultants en communication pour apprécier la faconde avec laquelle on y vend d'étonnants bricolages conceptuels. Repasser vers les étals de la presse, pour saluer l'exploit sociocognitif que peut constituer la rédaction d'un titre d'article, nécessairement bref, sous la double contrainte de la pertinence et de l'acceptabilité professionnelle et sociale. Ou encore traverser la zone des prédicateurs et compatir à ce que Daniel Bougnoux (2001) appelait « le dilemme du missionnaire » :

Le missionnaire, je le rappelle, veut étendre ou transmettre son message mais il ne peut le faire qu'au prix d'une certaine altération de l'inappréciable objet de sa transmission. Le Père de La Brosse s'interrogeait donc sur ce qu'il faut de communication dans la transmission. Ce que nous appelons ailleurs la pragmatique de l'énonciation, mais aussi de la réception, impose au missionnaire de ménager le monde propre des futurs fidèles, rendant du même coup l'objet de sa transmission essentiellement incertain : comment maintenir sa pureté tout en le diffusant? (p. 344)

Un nouvel arrêt ici aurait été doublement justifié. Non seulement parce que l'art du sermon (dont la dimension normative n'a pas besoin d'être soulignée) est de très longue date ${ }^{1}$ confronté au problème de la

1 Ainsi l'abbé Henry pouvait-il souligner, en défense des espiègles sermonnaires du $\mathrm{xv}^{\mathrm{e}}$ siècle, que "si cette concession au besoin d'intéresser l'auditoire et de réveiller son attention, ne les détourne pas du but moral [...] 
pertinence cognitive, alors même qu'il vise une assemblée présumée volontaire et temporairement isolée des distractions de ce monde. Mais aussi parce que cette tension entre la légitimité des énoncés et leur pertinence reproduit assez curieusement celle que rencontrent les locuteurs les plus rationalistes qui soient : les vulgarisateurs scientifiques.

Cependant, la fatigue gagne à nouveau, d'autant qu'à l'inverse du marché discursif, la pagination d'un livre n'est pas illimitée. Et puisqu'il faut tout de même marcher d'un bon pas, remarquer au passage ce que toutes ces boutiques disparates ont invariablement de commun ne permet décidément pas de trop s'attarder sur ce que chacune présente par ailleurs de particulier. Ce que rappellerait au besoin le fait que les reproches de sursimplification (réduction de l'effort cognitif) et de sensationnalisme (renforcement de l'effet cognitif) qui visent souvent les vulgarisateurs ${ }^{2}$ épargnent les prêcheurs : selon qu'ils se fondent sur des risques scientifiques ou sur les Écritures, les récits apocalyptiques ne relèvent pas des mêmes pressions normatives.

Mais où que l'on s'arrête, on ne peut à proprement parler écrire de « conclusion » à la quête des déterminants de la valeur discursive. Non seulement parce que les situations empiriques (les terrains) sont inépuisables, mais aussi parce que leurs angles et modes d'explication (les théories) le sont aussi. La question, après tout, est sur la table depuis plus de 2000 ans et la multiplication des approches et niveaux d'analyse ne cessera pas de sitôt d'en préciser de nouvelles facettes, au prix d'un enchevêtrement toujours plus dense des connaissances en jeu.

Le pari de ce livre était justement de considérer l'imprécision - appelons plutôt ça la distance analytique! - non comme une faute mais comme une méthode. Et même comme la meilleure méthode pour

ne serons-nous pas forcés de voir dans ce procédé un heureux artifice oratoire [...] Le premier mérite d'un homme qui parle n'est-il pas de se faire écouter et d'arriver à l'intelligence et au cœur de ceux qui l'écoutent? » (1876, p. 3). Et le même d'admirer en Bridaine « l'art merveilleux de se concilier, de soutenir et de ranimer l'attention de la multitude pendant toute la durée de ses plus longs sermons » (p. 110).

2 On pourra trouver dans Labasse 2012(a) un examen et une étude de cas consacrés à cette accusation plus paradoxale qu'il n'y paraît. 
tenter d'apercevoir, derrière l'imbroglio apparent de la production et de la consommation discursive, des articulations assez essentielles pour éclairer certaines de leurs logiques fondamentales. Une telle façon de voir méconnaissait d'autant moins la complexité de chacun des phénomènes en jeu que c'est précisément cette complexité qui la rendait nécessaire ${ }^{3}$ : elle visait avant tout, sous l'angle qui est le sien, à situer les principaux niveaux d'explication par rapport aux autres et à examiner leurs relations essentielles, sans pour autant prétendre les subsumer ni contester leur supériorité locale. Une carte géographique n'est ni moins ni plus juste que le plan d'une ville ou celui d'un bâtiment : elle ne répond pas aux mêmes besoins. Ainsi, chercher à examiner de façon encore plus détaillée les multiples courants théoriques - historiques ou actuels - dont la question posée traverse les territoires n'aurait pu qu'allonger outrancièrement, et en vain, la liste déjà fort dilatée et passablement hétéroclite des travaux cités.

Cette question, on s'en souvient, n'était pas le problème traditionnel de la persuasion (qu'est-ce qui influence les gens?), mais en amont celui, tout aussi crucial, qui constitue son préalable : qu'est-ce qui obtient l'attention des gens?

Elle a conduit à construire, tester et enfin soutenir une hypothèse dont les grandes lignes s'avèrent, au bout du compte, remarquablement banales : la valeur d'un discours pour un individu peut s'envisager comme le rapport entre sa pertinence cognitive (soit l'effort qu'il réclame contre l'effet qu'il produit) et sa convenance sociale (soit sa prescription contre sa proscription). En gros, tout le monde pouvait deviner ça et tout le monde s'en doute probablement plus ou moins. En tout cas, toute personne qui a déjà cédé à la coupable tentation d'un contenu frivole au détriment des productions plus légitimes qui s'offraient à elle. Donc tout le monde.

Pourquoi, alors, les phénomènes de production et de consommation discursives sont-ils aussi difficilement considérés sous cette perspective double par les praticiens ou les spécialistes?

3 L'ouvrage a même failli être sous-titré Une théorie approximative de la communication. Mais outre que quelques collègues auraient risqué de n'y voir qu'une vaine bravade (les usages du discours scientifique légitime sont plus férocement gardés que ceux du savoir-vivre mondain), un tel intitulé n'aurait pas rendu compte de sa vraie ambition, celui d'esquisser Une tout à fait rigoureuse théorie approximative de la communication. 
Peut-être, dans le cas des premiers, parce qu'elle est plus déstabilisante qu'il n'y paraît. Comparativement, la vague perception condescendante d'une populace frivole et erratique est d'autant plus attirante qu'elle permet aux élites discursives de ne pas examiner de trop près l'intérêt et le sens de ce qu'elles lui dispensent. En outre, l'idée qu'un prêtre ou un scientifique puissent rencontrer des problèmes discursifs apparentés, qu'un journaliste ou un écrivain soient confrontés à des logiques de réception voisines, qu'un enseignant ou un militaire aient à gérer des variables de même nature, n'est pas faite pour séduire l'un ou l'autre d'entre eux. Beaucoup de producteurs discursifs attachent à juste titre une noblesse intrinsèque, ou en tout cas une forte spécificité à leur tâche. Mais croire que cette particularité les exempte du même coup des logiques communes de la communication - de n'importe quelle communication - serait une coûteuse illusion : la spécificité de chaque secteur du marché discursif réside au contraire en bonne part (mais pas seulement !) dans la façon selon laquelle on y pondère différemment les multiples variables de l'adéquation sociocognitive. Et encore... Pour qui croirait vraiment que ces pondérations définissent des territoires discursifs autonomes, isolés de la concurrence des autres, il pourrait être instructif de se promener au fond d'un amphithéâtre, histoire de vérifier sur les écrans des ordinateurs et des téléphones des étudiants dans quelle mesure la légitimité institutionnelle du discours universitaire contrebalance la pertinence cognitive des distractions en ligne.

Comme on l'a vu plus haut, envisager la communication comme l'articulation de facteurs cognitifs et sociaux ne semble pas beaucoup plus séduisant ${ }^{4}$ pour certains des chercheurs, et non des moindres, qui se consacrent à un volet ou à un autre de ces variables sous un angle ou un autre. On sait que les nécessités tant épistémologiques qu'administratives de la division des tâches scientifiques conduisent souvent à négliger, voire à conjurer, ce qui ne s'inscrit pas dans une optique disciplinaire délimitée : si toute approche générale (et a fortiori celle-ci) se doit d'être réductrice, toute approche spécialisée le doit tout autant, mais dans l'autre sens, et également pour de bonnes raisons scientifiques et pratiques. Vaste ou exiguë, chacune devrait

4 Comment, sinon, la dialectique élémentaire de la satisfaction hédonique et des déterminants sociaux aurait-elle pu échapper depuis si longtemps à tant de manuels traitant de la production et de la réception discursive? 
simplement rappeler quelque part l'avertissement de Maslow : pour qui n'a qu'un marteau, tout finit par ressembler à un clou.

Pour ne rien arranger, il n'y avait pas, en l'espèce, de «preuve » à espérer, d'où les nuances et restrictions un peu fastidieuses qu'il a fallu multiplier. On a observé à plus d'une reprise tout ce qu'un modèle schématisant avec circonspection l'articulation des principaux facteurs qu'ont exposés les travaux de plusieurs disciplines complémentaires pouvait aider à remarquer ou à reconsidérer, y compris quelques problèmes définitoires ou opératoires figés depuis des lustres. Mais parce qu'elle imbrique dans des situations disparates des variables de nature et d'échelle extraordinairement variées, la communication humaine n'est pas un domaine où l'on pourrait brandir le genre de résultats décisifs quapprécient les physiciens (et encore... ces derniers se sont chamaillés pendant des siècles pour savoir si la lumière était faite d'ondes ou de particules avant de convenir qu'elle devait bel et bien être considérée comme les deux à la fois).

D'un autre côté... il n'y a pas forcément besoin d'expériences approfondies pour se demander si les choix de consommation discursifs sont d'ordre psychologique ou social, ou encore pour estimer que cette alternative est absurde. Dès lors que l'on baigne depuis l'enfance dans une dense soupe communicationnelle, la masse des observations que l'on en retire chaque jour pourrait plus ou moins compenser leur fragilité méthodologique individuelle. On a croisé au fil des pages un grand nombre de cas détaillés et d'exemples anecdotiques : chacun pourra les compléter par ceux qui lui viennent à l'esprit. Ou, mieux, par ceux auxquels il pourra être confronté comme analyste, enseignant ou praticien, et bien sûr comme citoyen, dans l'apparent chaos de la communication contemporaine.

Encore un mot, et je te laisse. Aie toujours présent à l'esprit [...] qu'un homme n'est pas une machine ; qu'une hypothèse n'est pas un fait : et sois assuré que tu ne m'auras point compris, partout où tu croiras apercevoir quelque chose de contraire à ces principes5.

5 Denis Diderot, 1754 (1821, p. 137). 
Page blanche conservée intentionnellement 


\section{Références}

Abric, J.C. (2003). « La recherche du noyau central et de la zone muette des représentations sociales ", dans Abric, J.C. (dir.), Méthodes d'études des représentations sociales, Saint-Agne, Érès, p. 59-80.

Abric, J.C. (2011). Psychologie de la communication: Théories et méthodes, Paris, Armand Colin.

Acker, P. (1906). "Le moyen de parvenir en littérature », Le Correspondant, 10 juillet 1906, p. 159-168.

Adams, M.J. et Collins, A. (1977). A Schema-Theoretic View of Reading (Technical Report 32), Urbana, Center for the study of reading.

Al-IssA, A. (2011). "Schema Theory and L2 Reading Comprehension, Implications for Teaching ", Journal of College Teaching \& Learning, vol. 3, $\mathrm{n}^{\circ} 7$, p. 41-47.

Alba, J.W. et Hasher, L. (1983). «Is Memory Schematic?» Psychological Bulletin, vol. 93, n 2, p. 203-231.

Albert, P. (1998). La presse française, Paris, La Documentation française.

Allan, K. et Burridge, K. (2006). Forbidden Words : Taboo and the censoring of language, Cambridge, Cambridge University Press.

Allen, W. (1979). Opus 1 et 2, Paris, Solar.

Altmann, U., Bohrn, I.C., Lubrich, O. et al. (2014). " Fact vs fiction - how paratextual information shapes our reading processes », Social cognitive and affective neuroscience, vol. $9, \mathrm{n}^{\circ} 1, \mathrm{p} .22-29$.

Amnesty InTERnATIONAL. (2018). Une loi antiterroriste est utilisée pour réprimer la satire et la créativité en ligne, Londres, Amnesty International.

Amossy, R. (1989). «La notion de stéréotype dans la réflexion contemporaine $»$ Littérature, $\mathrm{n}^{\circ} 73$, p. 29-46.

Amossy, R. (1991). Les idées reçues : sémiologie du stéréotype, Paris, Nathan.

Andelman, B. (2015). Will Eisner : A Spirited Life, Raleigh, TwoMorrows Publishing, p. 51.

Anderson, R.C., Spiro, R.J. et Anderson, M.C. (1978). « Schemata as Scaffolding for the Representation of Information in Connected Discourse», American Educational Research Journal, $\mathrm{n}^{\circ}$ 15, p. 433-443. 
Anderson, R.C. et Pearson, P.D. (1984). «A Schema-Theoretic View of Basic Processes in Reading Comprehension », dans Pearson, P.D., Barr, R., Kamil, M.L. et Mosenthal, P. (dir.). Handbook of Research on Reading, New York, Longman, p. 255-291.

Anderson, R.C., Reynolds, R.E., Schallert, D.L. et Goetz, E.T. (1977). «Frameworks for Comprehending Discourse », American Educational Research Journal, vol. 14, $\mathrm{n}^{\circ}$ 4, p. 367-381.

Ang, I. (1991). Desperately Seeking the Audience, Londres, Routledge.

Angenot, M. (1988). «Pour une théorie du discours social : problématique d'une recherche en cours ", Littérature, $\mathrm{n}^{\circ} 70$, p. 82-98.

Angenot, M. (1989). 1889, un état du discours social, Longueuil, Le Préambule. Arendt, H. (1972). La crise de la culture, Paris, Gallimard.

Aristote (1838). Métaphysique, L.I. (trad. V. Cousin), Paris, Ladrange.

Aristote (1883). Poétique et Rhétorique (trad. C.É. Ruelle), Paris, Garnier.

Autorité de régulation professionnelle de la publicité (2017). Le code de l'ARPP, Paris, ARPP.

AwAD, G. (2016). La pratique professionnelle de communication : organisation et parole vive, Paris, L'Harmattan.

Bachelard, G. (1938). La formation de l'esprit scientifique, Paris, Vrin.

BAcon F. (1843). Novum Organum (trad. F. Riaux, original : 1620), Paris, Charpentier.

Baddeley, A. (1992). La mémoire humaine: Théorie et pratique, Grenoble, Presses universitaires de Grenoble.

BaIlly, S. (2008). Anthologie de l'humour noir, Paris, Fayard.

Bakshy, E., Messing, S. et Adamic, L.A. (2015). «Exposure to ideologically diverse news and opinion on Facebook», Science, vol. 348, n ${ }^{\circ}$ 6239, p. 1130-1132.

Balle, F. (1987). Et si la presse n'existait pas. Paris, Lattès.

Baroni, R. (2007). La tension narrative: Suspense, curiosité, surprise, Paris, Seuil. Barsalou, L.W. (1999). "Perceptual symbol systems", Behavioral and Brain Sciences, $\mathrm{n}^{\mathrm{0}} 22$, p. 577-66o.

Barthes, R. (1964). «Rhétorique de l'image », Communications, $\mathrm{n}^{\circ}$ 4, p. 40-51.

BARthes, R. (1966). "Introduction à l'analyse structurale des récits », Communications, $\mathrm{n}^{\circ} 8$, p. 1-27.

Bartlett, F.C. (1932). Remembering, Cambridge, Cambridge University Press. Bateux, C. (1746). Les beaux-arts réduits à un même principe, Paris, Durand.

Bayle, P. (1820). Dictionnaire historique et critique, T. XVI (original : 1647), Paris, Desoer.

Bazın, H. (1987). Qu'est-ce que le cinéma? Paris, Éditions du Cerf. 
Beaudet, C. (1999). "Les compétences linguistiques et discursives du rédacteur professionnel : un ensemble à circonscrire», dans Z. Guével et I. Clerc (dir.), Les professions langagières à l'aube de l'an 2000, Québec, Ciral, p. 3-18.

Benda, J. (1975). La trahison des clercs, préface à l'édition de 1946, Paris, Grasset et Fasquelle.

Bentham, J. (1879). An introduction to the principles of morals and legislation (original : 1780), Oxford, Clarendon Press.

Berger, M. (1945). "Army langage », American Speech, vol. 20, n 4, p. 258-264.

Berger, P.L. et Luckmann, T. (1966). The social construction of reality : A treatise in the sociology of knowledge, Garden City, Doubleday Anchor.

Berlyne, D.E. (1949). " "Interest" as a psychological concept ", British Journal of Psychology, vol. 39, $\mathrm{n}^{\circ}$ 4, p. 184-195.

Bessard-Banquy, O. (2012). «Fin de la littérature ou crise de la lecture?», dans D. Viart et L. Demanze, Fins de la littérature, Paris, Armand Colin, p. $171-182$.

Bessard-Banquy, O. (2017). «Du best-seller aujourd'hui », Fixxion, n ${ }^{\circ}$ 15, p. 79-86.

Besson, P. (1991). Les ai-je bien descendus ?, Paris, Messidor.

BÉGUIN, A. (1996). « La métaphore absente : une particularité des manuels scolaires ", Communication et langages, $\mathrm{n}^{\circ}$ 110, p. 74-94.

Bitgood, S., Dukes, S. et Авву, L. (2006). «Interest and effort as predictors of reading : A test of the general value principle», Current Trends in Audience Research, n ${ }^{\circ}$ 19/20, p. 5-10.

Blair, A. (2010). Too Much To Know : Managing Scholarly Information Before the Modern Age, New Haven : Yale University Press.

Blanco, X. et Bogacki, K. (2014). Introduction à l'histoire de la langue française, Barcelone, Publicacions de la Universitat Autònoma de Barcelona.

Blendon, R.J., Benson, J.M., Brodie, M. et al. (1997). « Bridging the Gap Between the Public's and Economists' Views of the Economy ", The Journal of Economic Perspectives, vol. 11, n 3 , p. 105-118.

Blinkoff, R. (2008). A New Model for News - Studying the Deep Structure of Young-Adult News Consumption, New York: Associated Press.

Blumler, J.G. (1979). " The Role of Theory in Uses and Gratifications Studies ", Communication Research, vol. 6, $\mathrm{n}^{\circ}$ 1, p. 9-36.

Blyton, E. (1967). Oui-oui et son cerf-volant, Paris, Hachette.

Bohannon, J. (2015). " Many psychology papers fail replication test », Science, vol. 349, no 6251, p. 910-911.

Boltanski, L. et Thévenot, L. (1991). De la justification : Les économies de la grandeur, Paris, Gallimard, 
Воотн, W.C. (1961). The Rhetoric of Fiction, Chicago, University of Chicago Press.

Bougnoux, D. (2001). «Transmuniquer / Commettre », Les cahiers de médiologie, $\mathrm{n}^{\circ}$ 11, p. 343-349.

Bourdieu, P. (1977). "L'économie des échanges linguistiques ", Langue française, $\mathrm{n}^{\circ} 34$, p. 17-34.

Bourdieu, P. (1979). La distinction : critique sociale du jugement, Paris, Éditions de Minuit.

Bourdieu, P. (1980). Questions de sociologie, Paris, Éditions de Minuit.

Bourdieu, P. (1982). Ce que parler veut dire: L'économie des échanges linguistiques, Paris, Fayard.

Bourdieu, P. (1984). Homo academicus, Paris, Éditions de minuit.

Bourdieu, P. (1992). Les Règles de l'art: Genèse et structure du champ littéraire, Paris, Seuil.

Bourdieu, P. (1996). Sur la télévision, suivi de L'Emprise du journalisme, Paris, Liber.

Bourdieu, P. et Darbel, A. (1966). L'Amour de l'Art : les musées et leur public, Paris, Éditions de Minuit.

Bransford, J.D. et Johnson, M.K. (1972). "Contextual Prerequisites for Understanding : Some Investigations of Comprehension and Recall », Journal of Verbal Learning and Verbal Behavior, $\mathrm{n}^{\circ}$ 11, p. 717-726.

Brassart, D.G. (1991). "Connecteurs, organisateurs textuels et connexité dans les textes argumentatifs écrits », Recherches, $n^{\circ} 15$, p. 69-86.

Braun, T. et Zsindely, S. (1985). "Growth of scientific literature and the Barnaby Rich effect ", Scientometrics, vol. 7, nos 3-6, p. 529-530.

Brecht, B. (1970). Sur le réalisme, Paris, L'Arche.

Breton, P. (1997). L'utopie de la communication, Paris, La Découverte.

Breton, P. et Proulx, S. (1989). L'explosion de la communication : La naissance d'une nouvelle idéologie, Paris, La Découverte.

Brewer, W.F. et Nakamura, G.V. (1984). The Nature and Functions of Schemas (Technical Report 325), Urbana, Center for the study of reading.

Brighton, T. (2009). Patton, Montgomery, Rommel : Masters of War, New York, Crown publishing.

Brin, C., Charron, J. et de Bonville, J. (2004). Nature et transformation du journalisme : théorie et recherches empiriques, Québec, Presses de l'Université Laval.

Bronner, G. (2008). «L'apport d'une épistémologie cognitive à la démarche sociologique", Revue européenne des sciences sociales, vol. XLVI, nº 140, p. $155-174$. 
Bronner, G. (2013). La démocratie des crédules, Paris, Presses universitaires de France.

Bruner, J. (1960). The Process of Education, Cambridge, Harvard University Press, p. 24-25.

Buringh, E. et Van Zanden, J.L. (2009). "Charting the "Rise of the West" : Manuscripts and Printed Books in Europe, A Long-Term Perspective from the Sixth through Eighteenth Centuries », The Journal of Economic History, vol. 69, n² 2, p. 409-445.

Butcher, K.R. et Kintsch, W. (2003). "Text Comprehension and Discourse Processing », dans Healy, F. \& Proctor, R.W. (dir.), Handbook of psycho$\log y$ : Experimental psychology, New York, Wiley, p. 575-595.

Camus, A. (2006). CEuvres complètes, tome I, Paris, Gallimard.

CAReY, J.W. (1998). « McLuhan : généalogie et descendance d'un paradigme », Quaderni, vol. 37, $\mathrm{n}^{\circ} 1, \mathrm{p}$. 111-131.

Caron, A.H. et Caronia, L. (2005). Culture mobile : les nouvelles pratiques de communication, Montréal, Presses universitaires de Montréal.

Caron, A.H. et Cohen, R.I. (2013). Regulating Screens : Issues in Broadcasting and Internet Governance for Children, Kingston, McGill-Queen's University Press.

CARr, N. (2011). The Shallows: What the Internet Is Doing to Our Brains, New York, W.W. Norton \& Company.

Catherine, R. (1996). Le style administratif, Paris, Albin Michel.

Cavallo, G. et Chartier, R. (2001). Histoire de la lecture dans le monde occidental, Paris, Seuil.

Cefaï, D. et Pasquier, D. (1993). Les sens du public : Publics politiques, publics médiatiques, Paris, Presses Universitaires de France.

Centre d'étude des revenus et des coûts. (1971). Les Français et le vocabulaire économique (Documents du CERC $\mathrm{n}^{\circ}$ 9), Paris, La Documentation française.

Cerulo, K.A. (2002). Culture in mind : Toward a sociology of culture and cognition, New York, Routledge.

Chaffee, S.H. et Schleuder, J. (1986). « Measurement and Effects of Attention to Media News », Human Communication Research, vol. 13, nº 1, p. 76-107.

Changeux, J.P. (2008). Du vrai, du beau, du bien: une nouvelle approche neuronale, Paris, Odile Jacob.

Charaudeau, P. (2005). Les médias et l'information : L'impossible transparence $d u$ discours, Bruxelles, De Boeck.

Charaudeau, P. (2011). "Réflexions pour l'analyse du discours populiste », Mots - Les langages du politique, $\mathrm{n}^{\circ}$ 97, p. 101-116. 
Charron, J. et de Bonville, J. (1997). «Le paradigme du journalisme de communication : essai de définition», Communication, vol. 17, $\mathrm{n}^{\circ} 2$, p. 51-98.

Chesterton, G.K. (1914). The Wisdom of Father Brown, Londres, Cassell \& Company.

Chung, N. et Lee, K.C. (2011). «Empirical Analysis of Decision Maker's Schema and Cognitive Fit on Decision Performance», Asia Pacific Journal of Information Systems, vol. 21, $\mathrm{n}^{\circ}$ 2, p. 19-42.

Cicéron. (1848). De la nature des Dieux, dans D. Nisard (dir.), CEuvres complètes de Cicéron, t. IV, Paris, J. J. Dubochet.

Cicéron (attribué à). (1875). Rhétorique, à C. Hérennius, dans D. Nisard (dir.), CEuvres complètes de Cicéron, t. 1, Paris, Firmin Didot, p. 1-87.

Citton, Y. (dir.). (2014a). L'économie de l'attention, nouvel horizon du capitalisme? Paris, La Découverte.

Citton, Y. (dir.). (2014b). Pour une écologie de l'attention, Paris, Seuil.

Clerc, I. et Kavanagh, E. (2005). De la lettre à la page web, Québec, Gouvernement du Québec.

CNDI. (2003). «La "qualité" est-elle l'avenir de la presse ? "Études de presse, vol. $1, n^{\circ} 2$, p. $1-8$.

CNDI. (2008). «Crise et avenir de la presse : l'offre éditoriale face à l'évolution des publics ", Études de presse, vol. 1, n 5 , p. 1-8.

Codène, D. (2017). "Crise des médias : “Ce n'est pas la faute de Google”, dit son VP », La Presse+, 14 avril 2017, écran 13.

Coleridge, S.T. (1889). Biographia literaria (original : 1817), Londres, George Bell \& sons.

Committee of advertising practice. (2014). The UK Code of Non-broadcast Advertising and Direct $\mathcal{E}$ Promotional Marketing, Londres, Advertising Standards Authority.

Condorcet, N. de. (1847). CEuvres de Condorcet, t. 7 (original : 1791), Paris, Didot frères.

CONFÉDÉRATION FRANÇAISE DE LA BOUCHERIE, BOUCHERIE-CHARCUTERIE, TRAITEUR. (1997). Bouchers, oui, mais ni bouchés ni... bouchers [Communiqué de presse], Paris, C.F.B.C.T.

Confucius. (1987). Les Entretiens de Confucius (trad. P. Ryckmans), Paris, Gallimard.

Corneille, P. (1862). Préface à « La Suivante » (original : 1637), dans CEuvres de P. Corneille, Paris, Hachette.

Compagnon, A. (1998). Le démon de la théorie : Littérature et sens commun, Paris, Seuil. 
Coulangeon, P. (2004). "Classes sociales, pratiques culturelles et styles de vie : le modèle de la distinction est-il (vraiment) obsolète? » Sociologie et sociétés, vol. 36, $\mathrm{n}^{\circ} 1, \mathrm{p} .59-85$.

Coulangeon, P. (2006). Sans titre [Intervention lors de l'audition de Patrick Le Lay le 24 octobre 2006], dans Haut Conseil de l'Éducation artistique et culturelle, Rapport annuel 2006, Paris, HCEAC, p. 144-145.

Craig, R.T. (1999). "Communication Theory as a Field", Communication Theory, vol. 9, n², p. 119-161.

Crane, V. (1992). "Listening to the audience", dans B. Lewenstein (dir.), When science meets the public, Washington, American Association for the Advancement of Science, p. 21-32.

Cutting, J.E., Brunick, K.L., Delong, J.E. et al. (2011). «Quicker, faster, darker : Changes in Hollywood film over 75 years ", i-Perception, vol. 2, $\mathrm{n}^{\circ} 6$, p. 569-576.

Danielson, W.A. et Lasorsa, D.L. (1989). «A New Readability Formula Based on the Stylistic Age of Novels », Journal of Reading, vol. 33, n 3, p. 194-197. DAvenport, T.H. et Beck, J.C. (2000). "Getting the attention you need, " Harvard Business Review, vol. 78, n 5, p. 118-126.

Davis, H. et McLeod, S. L. (2003). «Why humans value sensational news : An evolutionary perspective », Evolution and Human Behavior, vol. 24, $\mathrm{n}^{\circ} 3$, p. 208-216.

Davison, W.P. (1983). "The Third-Person Effect in Communication ", Public Opinion Quarterly, vol. 47, n 1, p. 1-15.

de Certeau, M. (1980). L'Invention du quotidien, t. I, Arts de faire, Paris, Gallimard. De Solla Price, D.J. (1986). Little science, big science and beyond, New York, Columbia University Press.

De Closets, F. (1980). Le système E.P.M., Paris, Grasset.

DE LA Motte, A. (1715). Réflexions sur la critique, Paris, Du Puis.

DE WAAL, F. (1997). Le Bon Singe: Les bases naturelles de la morale, Paris, Bayard. Debord, G. (1967). La Société du spectacle, Paris, Gallimard.

Déchaux, J.-H. (2010). «Un "tournant cognitiviste" en sociologie? » Revue française de sociologie, vol. 51, n ${ }^{\circ}$, p. 641-644.

Delvaux, B. (2006). "Audace, oui ; crédibilité, oui ; esprit critique, oui », Le Soir, 15 décembre 2006, p. 24.

Demers, F. (2012). « Le défi de la construction d'un public pour le journalisme au temps de Google Analytics ", TicEsociété, vol. 6, n 1, p. 7-30.

Denton, F. (1993). (dir.) Ways with Words, Reston, VA, American Society of Newspaper Editors.

Dewey, J. (1913). Interest and effort in education, New York, Houghton Mifflin. 
Diderot, D. (1821). "Pensées sur l'interprétation de la nature », dans CEuvres de Denis Diderot, t. II (original : 1754), Paris, Brière, p. 132-226.

Dickson, B.J. (2016). The Dictator's Dilemma : The Chinese Communist Party's Strategy for Survival, New York, Oxford University Press.

DiMaggio, P. (1997). "Culture and Cognition », Annual Review of Sociology, $\mathrm{n}^{\circ} 23$, p. $263-288$.

DiMaggio, P. et Useem, M. (1978). «Social Class and Arts Consumption : The Origins and Consequences of Class Differences in Exposure to the Arts in America, » Theory and Society, vol. 5, n², p. 141-161.

Donneau De Vizé, J. (1665). «Lettre sur les affaires du théâtre », dans Les Diversitez galantes, Paris, Jean Ribou.

DouëL, J. (1981). Le journal tel qu'il est lu, Paris, Éditions du CFPJ.

Downs, A. (1957). " An Economic Theory of Political Action in a Democracy », Journal of Political Economy, vol. 65, n², p. 135-150.

Du Marsais, C.C. (1730). Des Tropes ou Des diferens sens dans lesquels on peut prendre un mème mot dans une mème langue, Paris, Veuve J.-B. Brocas.

Dubos, J.B. (1719). Réflexions critiques sur la poésie et sur la peinture, t. 1, Paris, J. Mariette.

Duby, G. (2005). Le dimanche de Bouvines : 27 juillet 1214 (original : 1973), Paris, Gallimard.

Durand, S. (2011). Storytelling : Réenchantez votre communication, Paris, Dunod.

Durkheim, É. (1895). Les Règles de la méthode sociologique, Paris, Alcan.

Eco, U. (1992). Les limites de l'interprétation, Paris, Grasset.

Eco, U. (1996). Six promenades dans les bois du roman et d'ailleurs, Paris, Grasset.

Eppler, M.J. et Mengis, J. (2004). «The Concept of Information Overload : A Review of Literature from Organization Science, Accounting, Marketing, MIS, and Related Disciplines", The Information Society, $\mathrm{n}^{\mathrm{o}} 20, \mathrm{p} \cdot 325-344$.

Ericsson, K.A. et Kintsch, W. (1995). «Long-term Working Memory », Psychological Review, $\mathrm{n}^{\circ}$ 102, p. 211-245.

Ernaux, A. (2012). « Le pamphlet de Richard Millet déshonore la littérature », Le Monde, $\mathrm{n}^{\circ}$ 21039, 11 septembre 2012, p. 20.

Errico, M. (1997). « The evolution of the summary news lead », Media History Monographs, vol. 1, $\mathrm{n}^{\circ}$ 1, Athens (OH), Ohio University.

Escarpit, R. (1966). « De la littérature comparée aux problèmes de la littérature de masse ", Études françaises, vol. 2, n 3, p. 349-358.

Escarpit, R. (1976). Théorie générale de l'information et de la communication, Paris, Hachette.

Estivals, R. (dir.). (1993). Les sciences de l'écrit, Paris, Retz. 
Evans, J.S.B.T. et Stanovich, K.E. (2013). "Dual-Process Theories of Higher Cognition: Advancing the Debate ", Perspectives on Psychological Science, vol. $8, \mathrm{n}^{\circ}$ 3, p. 223-241.

Fawcett T.W. et Higginson, A.D. (2012). « Heavy use of equations impedes communication among biologists ", Proceedings of the National Academy of Sciences, vol. 109, $\mathrm{n}^{\circ} 29, \mathrm{p}$. 11735-11739.

FAYOL, M. (1985). Le Récit et sa construction: Une approche de psychologie cognitive, Neuchâtel, Delachaux-Niestlé.

Fernbach, P.M., Rogers, T., Fox, C.R. et Sloman, S.A. (2013). «Political Extremism Is Supported by an Illusion of Understanding ", Psychological Science, vol. 24, nº 6, p. 939-946.

Ferrero, G. (1894). "L'inertie mentale et la loi du moindre effort", Revue Philosophique de la France et de l'Étranger, $\mathrm{n}^{\circ}$ 37, p. 169-182.

Festinger, L. (1957). A theory of cognitive dissonance, Stanford, CA : Stanford University Press.

Festinger, L., Riecken, H.W. et Schachter, S. (1956). When Prophecy Fails : A Social and Psychological Study of a Modern Group That Predicted the Destruction of the World, Minneapolis, University of Minnesota Press.

Findahl, O. et Hoijer, B. (1985). "Some Characteristics of News Memory and Comprehension », Journal of Broadcasting \& Electronic Media, vol. 29, n 4 , p. 379-396.

Fiske, S.T. et Linville, P.W. (1980). «What does the Schema Concept Buy us ? » Personality and Social Psychology Bulletin, $\mathrm{n}^{\circ}$ 6, p. 543-557.

FLeSCH, R. (1948). "A new readability yardstick », Journal of applied psychology, vol. $32, \mathrm{n}^{\circ} 3$, p. $221-233$.

Fleury, B. et Walter, J. (2017). «Les valeurs : quelles théories, quelles méthodes ? »Questions de communication, $\mathrm{n}^{\circ}$ 32, p. 153-166.

FLÜCKIger, A. (2009). "Pourquoi respectons-nous la "Soft Law" ? Le rôle des émotions et des techniques de manipulation ", Revue européenne des sciences sociales, vol. 47, $\mathrm{n}^{\circ}$ 144, p. 73-103.

Foucault, M. (1971). L'ordre du discours, Paris, Gallimard.

France, A. (1921). Les Sept Femmes de la Barbe-Bleue et autres contes merveilleux, Paris, Calmann-Lévy.

FrancK, G. (1999). «Scientific Communication - À Vanity Fair ? Science, vol. 286, $\mathrm{n}^{\circ}$ 5437, p. 53-55.

Frazier, L. et Fodor, J.D. (1978). «The sausage machine : A new two-stage parsing model », Cognition, no 6, p. 291-325.

From the Editors. (2011). Journal of Animal Ethics, vol. 1, no 1, p. vii-ix.

Gabison, Y. (2012). Boostez vos présentations avec le storytelling, Paris, Eyrolles. 
Galak J. et Nelson L.D. (2011). "The Virtues of Opaque Prose : How Lay Beliefs About Fluency Influence Perceptions of Quality », Journal of Experimental Social Psychology, vol. 47, $\mathrm{n}^{\circ}$ 1, p. 250-253.

Galtung, J. et Ruge, M.H. (1965). « The Structure of Foreign News », Journal of Peace Research, vol. 2, nº 1, p. 64-91.

Gandon, F. (1980). « La connotation parC. Kerbrat-Orecchioni », Communication et langages, $\mathrm{n}^{\circ} 47, \mathrm{p}$. 121-122.

Gans, H.J. (1992). "Sociological Amnesia : The Noncumulation of Normal Social Science ", Sociological Forum, vol. 7, nº 4, p. 701-710.

Gans, H.J. (1979). Deciding What's News : A Study of CBS Evening News, NBC Nightly News, Newsweek, and Time, Evanston, Northwestern University Press.

Gastil, J. (1992). «Undemocratic discourse : a review of theory and research on political discourse ", Discourses and Society, vol. 3, n 4, p. 469-500.

Gauthier, G. (2010). "Le journalisme de communication : expression de conviction et moralisme », Les Cahiers du journalisme, $\mathrm{n}^{\circ} 21$, p. 254-273.

Gaymard, H. (2009). Situation du livre : Évaluation de la loi relative au prix du livre et Questions prospectives, Paris, Ministère de la Culture et de la Communication.

Genette, G. (1966). Figures I, Paris, Seuil.

Genette, G. (1991). Fiction et diction, Paris, Seuil.

Ghosh, V.E. et GilboA, A. (2014). «What is a Memory Schema? A Historical Perspective on Current Neuroscience Literature», Neuropsychologia, $\mathrm{n}^{\circ}$ 53, p. 104-114.

Glocker, M.L., Langleben, D.D., Ruparel, K. et al. (2009). «Baby schema in infant faces induces cuteness perception and motivation for caretaking in adults ", Ethology, vol. 115, n 3, p. 257-263.

Godin, C. (2012). «Qu'est-ce que le populisme? »Cités, no 49, p. 11-25.

Goethe, J.W. (1877). Faust (trad. G. de Nerval), Paris, Garnier frères.

Goffman, E. (1991). Les cadres de l'expérience, Paris, Éditions de Minuit.

Goldhaber, M.H. (1997). « The attention economy and the net », First Monday, vol. $2, \mathrm{n}^{\circ} 4$.

Goodman, K.S. (1967). « Reading : A psycholinguistic gussing game », Journal of the Reading Specialist, vol. 6, n 1, p. 126-135.

Goscinny, René et Uderzo, Albert, (1962). La serpe d'or, Paris, Dargaud.

Graber, D. (1984). Processing the News, New York, Longman.

GracQ, J. (1950). La littérature à l'estomac, Paris, Éditions José Corti.

Graesser, A.C., Millis, K K. et ZwaAn, R.A. (1997). « Discourse comprehension ", Annual Review of Psychology, vol. 48, n 1, p. 163-189.

GrañA-GonzÁles, M. (1930). La Escuela de periodismo, Madrid, C.I.A.P. 
Gray, W.S. et Munroe, R. (1929). The Reading Interests and Habits of Adults : A Preliminary Report, New York, Macmillan.

Grenier, L. (1986). "La recherche fait la sourde oreille à la musique populaire », Communication, vol. 8, $\mathrm{n}^{\circ}$ 2, p. 82-110.

Grenier, L. (1993). "Policing French-Language Music on Canadian Radio », dans T. Bennett, S. Frith, L. Grossberg et al. (dir.), Rock and Popular Music ; Politics, Policies, Institutions, Londres, Routledge, p. 119-140.

Grice, H.P. (1975). "Logic and conversation », dans P. Cole et J.L. Morgan (dir.), Syntax and semantics, vol. 3 : Speech acts, New York, Academic Press, p. 41-58.

Grize, J.B. (1996). Logique naturelle et conversation, Paris, Presses Universitaires de France.

Guillebaud, J.C. (1995). La Trahison des Lumières, Paris, Seuil.

Habermas, J. (1978). L'Espace public, Paris, Payot.

Habermas, J. (1986). Morale et communication, Paris, Flammarion.

Halley, A. (1996). "Les dents de la critique», La Presse, 21 janvier 1996, p. B1. HallidaY, M.A.K. (1962). « Linguistique générale et linguistique appliquée à l'enseignement des langues", Études de Linguistique Appliquée, $\mathrm{n}^{\circ}$ 1, p. 5-42

Hanitzsch, T. (2009). "Comparative Journalism Studies », dans K. WahlJorgensen et T. Hanitzsch (dir.), The handbook of journalism studies, New York, Routledge, p. 413-427.

Harari, Y.N. (2015). Sapiens : Une brève histoire de l'humanité, Paris, Albin Michel.

Harcup, T. et O'Neill, D. (2001). «What Is News? Galtung and Ruge revisited ", Journalism Studies, vol. 2, n ${ }^{\circ}$ 2, p. 261-280.

Harcup, T. et O'Neill, D. (2017). "What is news? News values revisited (again) », Journalism Studies, vol. 18, $\mathrm{n}^{\circ}$ 12, p. 1470-1488.

Неснт, E. (2011). « Historique et haut de gamme », L'Express, $n^{\circ} 3118,16$ avril 2011, p. 117.

Heinich, N. (1999). « Les rejets de l'art contemporain », Publics et Musées, nº 16, p. $151-162$.

Heinich, N. (2006). « La sociologie à l'épreuve des valeurs », Cahiers internationaux de Sociologie, $\mathrm{n}^{\circ}$ 121, p. 287-315.

HeInich, N. (2012). De la visibilité : Excellence et singularité en régime médiatique, Paris, Gallimard.

Heinich, N. (2017). Des valeurs : Une approche sociologique, Paris, Gallimard.

Hennion, A. (2004). "Une sociologie des attachements : D'une sociologie de la culture à une pragmatique de l'amateur », Sociétés, no 85, p. 9-24. 
Henry, A. (1876). Histoire de l'éloquence: Avec des jugements critiques sur les plus célèbres orateurs, t. III, Paris, Librairie Adrien Le Clere.

Hidi, S. et BAIRD, W. (1986). "Interestingness - a neglected variable in discourse processing », Cognitive Science, vol. 10, n² 2, p. 179-194.

Hispalis, G. (2005). « Pourquoi tant de loi(s) ? » Pouvoirs, nº 114, p. 101-115.

Hoggart, R. (1970). La culture du pauvre, Paris, Éditions de minuit.

Hoey, J. (1997). Employer Satisfaction with Alumni Professional Preparation

- Alumni Satisfaction with Professional Preparation, Raleigh, North Carolina State University, University Planning and Analysis.

Honneth, A. (2004). «Visibilité et invisibilité : Sur l'épistémologie de la "reconnaissance" ", Revue du MAUSS, n²3, p. 137-151.

Horkheimer M. et Adorno T. W. (2002). Dialectic of Enlightenment-Philosophical Fragments, Stanford, Stanford University press.

Houdebine, A.M. (2015). "De l'imaginaire linguistique à l'imaginaire culturel », La linguistique, vol. 51, n 1 , p. 3-40.

HöIJer, B. (1992). "Socio-cognitive Structures and Television Reception", Media, Culture E Society, vol. 14, no 4, p. 583-603.

Hughes, G. (2010). Political correctness : a history of semantics and culture, Chichester, Wiley-Blackwell.

Hugo, V. (1875). «La liberté de l'enseignement », dans Actes et paroles - Avant l'exil, Paris, Michel Lévy, p. 231-249.

Hugo, V. (1893). Napoléon le petit, Paris, Émile Testard.

Hume, D. (1888). A Treatise of Human Nature, being an attempt to introduce the experimental method of reasoning into moral subjects, livre III (original : 1740), Oxford, Clarendon Press.

Iacub, M. (2014). « Presse de gauche, la débandade! » Libération, 12 avril 2014, p. 37.

Innis, H. (1951). The Bias of Communication, Toronto, University of Toronto Press.

InNis, H. (1952). The Strategy of Culture, Toronto, University of Toronto Press.

INRA Europe. (2000). Eurobaromètre 52.1 : Les Européens et la biotechnologie, Bruxelles, Commission européenne.

IsER, W. (1985). L'acte de lecture : théorie de l'effet esthétique, Bruxelles, Mardaga.

IsER, W. (2012). L'appel du texte : l'indétermination comme condition d'effet esthétique de la prose littéraire, Paris, Allia.

IyengAR, S. et HAHN, K.S. (2009). « Red media, blue media : Evidence of ideological selectivity in media use ", Journal of Communication, vol. 59, $\mathrm{n}^{\circ} 1$, p. 19-39.

Jackson, M. (2008). Distracted: The Erosion of Attention and the Coming Dark Age, Ammherst, Prometheus Books. 
Jaсові, D. (1986). Diffusion et vulgarisation : itinéraires du texte scientifique, Besançon, Presses universitaires Franche-Comté.

Jahangard, A., Moinzadeh, A. et Karimi, A. (2011). « The Effect of Grammar vs. Vocabulary Pre-teaching on EFL Learners'Reading Comprehension : A Schema-Theoretic View of Reading ", Journal of English Language Teaching and Learning, $\mathrm{n}^{\circ}$ 8, p. 91-113.

Jauss, H.R. (1978). Pour une esthétique de la réception, Paris, Gallimard.

JeAnneret, Y. (1992). «Le choc des mots : pensée métaphorique et vulgarisation scientifique ", Communication et langages, $\mathrm{n}^{\circ}$ 93, p. 99-113.

Jeanneret, Y. (1994). Écrire la science : Formes et enjeux de la vulgarisation, Paris, Presses Universitaires de France.

Jeanneret, Y. et Patrin-Leclère, V. (2004). "La métaphore du contrat», Hermès, $\mathrm{n}^{\circ}$ 38, p. 133-140.

Jodelet, D. et Ohana, J. (1989). «Bibliographie générale sur les représentations sociales », dans D. Jodelet (dir.), Les représentations sociales, Paris, Presses Universitaires de France, p. 9-43.

Johnson-Laird, P.N. (1983). Mental Models, Cambridge, Cambridge University Press.

Johnston J. et Graham, C. (2012). «The new, old journalism», Journalism Studies, vol. 13, $\mathrm{n}^{\circ} 4$, p. 517-533.

Jones, J. (2015). «Brian Sewell's pungent views got people arguing - that's what matters », The Guardian, 28 septembre 2015, p. 16.

Jourde, P. (2002). La littérature sans estomac, Paris, L'Esprit des péninsules.

Jouve, V. (1993). La Lecture, Paris, Hachette.

Jouve, V. (2001). Poétique des valeurs, Paris, Presses Universitaires de France.

Jouve, V. (2010). Poétique du roman, Paris, Armand Colin.

Jullier, L. (2000). «Qu'est-ce qu'un bon film? » Esprit, n² 268, p. 10-20.

Kahneman, D. (1973). Attention and effort, Englewood Cliffs, Prentice-Hall.

Kahneman, D. (2011). Thinking, Fast and Slow, New York, Farrar, Straus and Giroux.

Kahneman, D., Diener, E. et Schwarz N. (1999). Well-Being : Foundations of Hedonic Psychology, New York, Russell Sage.

Kaiser Fondation. (1996). Why Don't Americans Trust The Government? - The Washington Post/ Kaiser Family Foundation/ Harvard University Survey Project, Menlo Park, Californie, Henry J. Kaiser Family Foundation.

Kant, E. (1869). Critique de la raison pure (trad. J. Barni), Paris, GermerBaillère.

KATZ, E. (1959). "Mass communications research and the study of popular culture : An editorial note on a possible future for this journal », Studies in Public Communication, $\mathrm{n}^{\circ}$ 2, p. 1-6. 
Katz, E., Blumler, J.G. et Gurevitch, M. (1973). «Uses and Gratifications Research », Public Opinion Quarterly, vol. 37, n 4, p. 509-523.

Kavanagh, É., Roberge, J. et Sperano, I. (2016). «Typologie exploratoire des affordances textuelles », Pratiques, p. 171-172.

Kellogg, R.T. (1999). The Psychology of Writing, Oxford, Oxford University Press.

Kelly, J., Knight, J., Peck, L.A. et al. (2003). «Straight/Narrative ? Writing Style Changes Readers' Perceptions of Story Quality ", Newspaper Research Journal, vol. 24, n ${ }^{\circ}$, p. 118-122.

Kessous, E. (2012). L'attention au monde, Paris, Armand Colin.

Kintsch, W. (1988). « The Role of Knowledge in Discourse Comprehension : a Construction-Integration Model », Psychological Review, vol. 95, n², p. $163-182$.

Kintsch, W. (2005). «An Overview of Top-Down and Bottom-Up Effects in Comprehension : The CI Perspective", Discourse Processes, vol. 39, $\mathrm{n}^{\mathrm{os}} 2-3$, p. $125-128$.

Kintsch, W. et van Dijk, T.A. (1978). « Toward a Model of Text Comprehension and Production ", Psychological Review, vol. 85, n ${ }^{\circ}$, p. 363-394.

Kintsch, W. (1980). «Learning from text, levels of comprehension, or : Why anyone would read a story anyway? »Poetics, n ${ }^{\circ}$ 9, p. 87-98.

Kintsch, W. (1998). Comprehension : a paradigm for cognition, Cambridge, Cambridge University Press.

KuCER, S.L. (1985). « The Making of Meaning Reading and Writing as Parallel Processes », Written Communication, vol. 2, nº 3, p. 317-336.

Kun, K. (2001). "Explaining art visually », Museum International, vol. 53, n 4, p. 52-55.

Kunn, T.S. (1983). La structure des révolutions scientifiques ( $2^{\mathrm{e}}$ éd.), Paris, Flammarion.

La Bruyère, J. de. (1818). CEuvres, Paris, Belin.

LABASSE, B. (2001). «From linguistics to communication's didactics : the case of lexicology", International Review of Applied Linguistics, vol. 33, nº 9, p. $217-243$.

LABAsse, B. (2003). Une dynamique de l'insignifiance: Les médias, les citoyens et la chose publique dans la "société de l'information", Villeurbanne, Presses de l'École nationale supérieure des sciences de l'information et des bibliothèques.

LABAsse, B. (2004). «Quand le cadre fait le tableau : référentiels cognitifs et perception de l'actualité ", Les Cahiers du journalisme, $\mathrm{n}^{\circ}$ 13, p. 80-107.

LABAsse, B. (2006). La communication écrite : Une matière en quête de substance, Lyon, Éditions Colbert. 
Labasse, B. (2008). L'offre éditoriale face à l'évolution des publics : Étude quantitative sur les lecteurs et non lecteurs de la presse quotidienne, Paris, Fédération nationale de la presse française.

Labasse, B. (2009). " L'écrit professionnel : ambiguïtés et identités d'un objet académique indistinct ", Pratiques, $\mathrm{n}^{\circ}$ 143, p. 233-248.

LABAsse, B. (2010). «Éléments pour une éthique amorale du journalisme», dans M.F. Bernier (dir.), Actes du colloque «Les journalismes : réalités plurielles, éthique commune? »Ottawa, Université d'Ottawa.

LABAsse, B. (2012a). "Sexe, sang et physique des particules : le « sensationnalisme » est-il partout... ou nulle part ? » Les Cahiers du Journalisme, $\mathrm{n}^{\circ} 24$, p. 114-149.

LABAsse, B. (2012b). «Un trou noir dans la galaxie : la compétence opératoire dans les recherches en communication ", Canadian Journal of Media Studies, vol. 10, $\mathrm{n}^{\circ} 2$, p. 176-214.

LABAsse, B. (2012C). «Structures narratives et congruence cognitive : cas du summary lead et de la pyramide inversée », Rédactologie / Canadian Journal for Studies in Discourse and Writing, vol. 24, $\mathrm{n}^{\circ}$ 1, p. 65-83.

Labasse, B. (2014a). L'art ou le mouchoir ? Les facteurs sociocognitifs dans l'appréciation culturelle. Communication à la conférence «L'expérience esthétique », Ottawa, 19-20 février 2014.

LABAsse, B. (2014b). «De la fragmentation procédurale à l'émiettement épistémologique : quelles articulations pour la production discursive ? " Les Cahiers du RESIPROC, $\mathrm{n}^{\circ}$ 2, p. 75-101.

LABAsse, B. (2014C). «Le journalisme au miroir de la littérature (à moins que ça ne soit l'inverse) ", Analyses, vol. 9, n 3, p. 318-361.

LABAsse, B. (2015a). «Les déterminants cognitifs et sociaux de l'adéquation communicationnelle », dans Rey-Lafay, V. et Beaudet, C. (dir.), Écritures expertes en question, Aix en Provence, Presses de l'Université de Provence, p. 39-71.

Labasse, B. (2015b). "Du journalisme comme une mésoépistémologie», Communication, vol. 33, $\mathrm{n}^{\circ}$ 1, p. 1-29.

Labasse, B. (2016). Recevabilité des énoncés dans l'espace public : le cas de l'information nutritionnelle, Communication au colloque «Communication et innovation en santé : acteurs, enjeux, pratiques, savoirs », Montréal, 11-12 mai 2016.

Labasse, B. (2017a). «Nathalie Heinich : Des valeurs, une approche sociologique ", Communication, vol. 34, $\mathrm{n}^{\circ} 2$.

LAbAsse, B. (2017b). «La transposition de la valeur discursive : variables sociocognitives et enjeux traductologiques », Forum - Revue internationale d'interprétation et de traduction, vol. 15, $\mathrm{n}^{\circ} 2$, p. 178-211. 
Labasse, B. et Thoiron, P. (2002). "Characterization of the relation between word length and cumulated frequency in heterogeneous corpora", Langues et linguistique, $\mathrm{n}^{\circ}$ 28, p. 95-102.

Labov, W. The Social Stratification of English in New York City, Washington, Center for Applied Linguistics, 1966.

LAвоv, W. (2016). "Afterword : Where are we now? ", Journal of sociolinguistics, vol. 20, $\mathrm{n}^{\circ}$ 4, p. 581-602.

Laclau, E. (2005). On populist reason, Londres, Verso.

Lacroix, J.-G., Miège, B., Moeglin, P. et al. (1993). « La convergence des télécommunications et de l'audiovisuel : un renouvellement de perspective s'impose ", Technologies de l'Information et Société, vol. 5, nº 1, p. 81-105.

LAhire, B. (2004). La culture des individus : Dissonances culturelles et distinction de soi, Paris, La Découverte.

Lahire, B. (2008). «La Nature du cognitif en question », dans Bernard Lahire et Claude Rosental (dir.), La cognition au prisme des sciences sociales, Paris, Éditions des archives contemporaines, p. 56-105.

Lakoff, G. (1987). Women, Fire and Dangerous Things, Chicago, Chicago University press.

Lakoff, G. et Johnson, M. (1980). Metaphors we live by, Chicago, Chicago University press.

LANDy, D. et SigALL, H. (1974). «Beauty is talent: Task evaluation as a function of the performer's physical attractiveness », Journal of Personality and Social Psychology, vol. 29, nº 3, p. 299-304.

LARSEN, P.O. et Von INS, M. (2010). " The rate of growth in scientific publication and the decline in coverage provided by Science Citation Index ", Scientometrics, vol. $84, \mathrm{n}^{\circ} 3$, p. 575-603.

Lasswell, H.D. (1946). "Describing the effects of communications ", dans Bruce Lannes Smith, Harold D. Lasswell et Ralph D. Casey (dir.), Propaganda, Communication and Public Opinion, Princeton, Princeton University Press, p. 95-118.

Latour, B. (1993). Aramis ou l'Amour des techniques, Paris, La Découverte.

LeE, A.M. et Chyi, H.I. (2014). «When Newsworthy is Not Noteworthy : Examining the value of news from the audience's perspective", Journalism Studies, vol. 15, n 6, p. 807-820.

Lemaire, S. (2007). "Une défense de l'hédonisme psychologique », dans R. Lefebvre (dir.), Le Plaisir, Réflexions antiques, approches modernes, Mont Saint-Aignan, Publications des Universités de Rouen et du Havre, p. 199-214.

Le Naire, O. (2002). «Qui veut baillonner l'édition? » L'Express, n ${ }^{\circ}$ 2649, 11 avril 2002, p. 78 . 
Les AssociÉs D'EIM. (2004). Les dirigeants face au changement : baromètre 2004, Paris, Éditions du huitième jour.

Levin, T.Y. (1996). "Iconology at the Movies : Panofsky's Film Theory », The Yale Journal of Criticism, vol. 9, $\mathrm{n}^{\circ}$ 1, p. 27-55.

Levine, L.W. (1988). Highbrow/Lowbrow: The Emergence of Cultural Hierarchy in America, Cambridge, Harvard University Press.

Lewin, K. (1951). "Problems of research in social psychology", dans D. Cartwright (dir.), Field theory in social science: Selected theoretical papers, New York, Harper \& Row, p. 155-169.

Lits, M. (2008). Du récit au récit médiatique, Bruxelles, De Boeck.

Lopes, H. (2005). « Utilité, normes et sentiments moraux : De leur incommensurabilité ", Revue du MAUSS, n² 25, p. 404-427.

Lord, C.G., Ross, L. et Lepper, M.R. (1979). «Biased assimilation and attitude polarization : The effects of prior theories on subsequently considered evidence", Journal of Personality and Social Psychology, vol. 37, $\mathrm{n}^{\circ} \mathrm{11}$, p. 2098-2109.

LoRET, É. (1992). «Critiques : pourquoi sont-ils si méchants ? Libération [en ligne], 24 mai 1992.

Lynn, J. et Jay, A. (1989). The Complete Yes Prime Minister - The Diaries of the Right Hon. James Hacker, Londres, BBC Books.

Maggiori R. (2011). Le Métier de critique : Journalisme et philosophie, Paris, Seuil.

Maingueneau, D. (2004). Le discours littéraire : Paratopie et scène d'énonciation, Paris, Armand Colin.

Maisonneuve, J. (1989). Introduction à la psychosociologie, Paris, Presses Universitaires de France.

Malebranche, N. (1678). De la recherche de la vérité, Paris, Pralard.

Mandelbrot, B. (1995). Les objets fractals : forme, hasard et dimension, Paris, Flammarion.

Mandler, J.M. et Johnson, N.S. (1977). « Rememberance of Things Parsed : Story Structure and Recall », Cognitive Psychology, nº 9, p. 111-151.

Manoury, P. (2013). Mais de quoi donc ce "néo » veut-il nous parler? Texte en ligne [www.philippemanoury.com] daté du 24 avril 2013.

Martinet, A. (1967). Éléments de linguistique générale, Paris, Armand Colin.

Martino, L.C. (2003). «Épistémologie de la communication : scepticisme et intelligibilité du savoir communicationnel », Les Enjeux de l'information et de la communication, $\mathrm{n}^{\circ}$ 1, p. 52- 62.

Mathien, M. (2001). « Le journalisme de communication : critique d'un paradigme spéculatif de la représentation du journalisme professionnel », Quaderni, vol. 45, n 1, 105-135. 
Mathieu, D. (2009). "Cultural Knowledge in News Comprehension", Participations - Journal of Audience and Reception Studies, vol. 6, $\mathrm{n}^{\circ}$ 1, p. 77-117.

Mauldin, B. (1971). « My confrontation with Patton », Life, 6 août 1971, p. 51-54. Mayaffre, D. (2013). «Sarkozysme et populisme: Approche logométrique du discours de Nicolas Sarkozy (2007-2012) », Mots. Les langages du politique, $\mathrm{n}^{\circ} 103, \mathrm{p} \cdot 73-87$.

MсСомвs, M.E. et SHaw, D.L. (1972). «The agenda-setting function of the mass media », Public Opinion Quarterly, vol. 36, $\mathrm{n}^{\circ}$ 2, p. 176-187.

McQuall, D. (2010). Mass Communication Theory, New York, Sage Publications.

McVee, M., Dunsmore, K. et Gavelek, J. (2005). «Schema Theory Revisited», Review of Educational Research, vol. 75, n 4, p. 531-566.

Mercier, A. (2007). "Sciences de la communication et journalisme : de la compréhension des dérives à l'amélioration des pratiques », Studies in Communication Sciences, vol. 7, $\mathrm{n}^{\circ}$ 1, p. 53-74.

Meunier, J.P. et Peraya, D. (2010). Introduction aux théories de la communication, Bruxelles, de Boeck.

Meyer, M. (1993). Questions de rhétorique : Langage, raison et séduction, Paris, Le livre de poche.

Mi-YeOn, J. et Brisset, A. (2006). « La notion de culture dans les manuels de traduction : domaines allemand, anglais, coréen et français ", Meta : journal des traducteurs, vol. 51, n 2, p. 389-409.

MiÈGE, B. (1995). La pensée communicationnelle, Grenoble, Presses universitaires de Grenoble.

MiÈGE, B. (1997). La société conquise par la communication, t.2. Grenoble, Presses universitaires de Grenoble.

Miège, B. et Odin R. (1993). Les domaines de compétence de la $71^{e}$ section, Paris, Conseil National des Universités ( $71^{\mathrm{e}}$ section).

Millet, R. (2012). Langue fantôme, suivi de Éloge littéraire d'Anders Breivik, Paris, Pierre-Guillaume de Roux.

Mindich, D. (1998). Just the Facts : How «Objectivity "Came to Define American Journalism, New York, New York University Press.

Ministère de la Culture. (2018). Le secteur du livre : chiffres-clés 2016-2017, Paris, Ministère de la Culture.

Ministère de la Culture et de la Communication. (2001). Statistiques de la culture : chiffres clés 2000, Paris, La Documentation française.

Minsky, M. (1975). "A Framework for Representing Knowledge », dans P. H. Winston, (dir.), The Psychology of Computer Vision, New York, McGraw-Hill, p. 211-277. 
Moeschler, J. et Reboul, A. (1994). Dictionnaire encyclopédique de pragmatique, Paris, Éditions du Seuil.

Moles, A. (1967). Sociodynamique de la culture, Paris, Mouton et Cie.

Molière (1663). La critique de l'École des femmes, Paris, Gabriel Quinet.

Montaigne (2001). Essais (original : 1588), Paris, Livre de poche.

Montesquieu, C.L. de, (1765). Lettres persanes, Paris, Arkstée et Merkus.

Moreau, F. et Peltier, S. (2015). Fondamentaux et mutations du secteur de l'édition : les ressorts de l'économie de la création, Paris, Syndicat national de l'édition.

Moreault, É. (2016). « Sparks et sa recette classique », Le Droit, $\mathrm{n}^{\circ}$ 260, 6 février 2016, p. Ao6.

Morineau T. (2001). "Éléments pour une modélisation du concept d'affordance ", Actes du Colloque EPIQUE, Nantes, IRCCyN, p. 71-82.

Moufre, C. (2003). «Le politique et la dynamique des passions », Politique et Sociétés, $\mathrm{n}^{\circ}$ 223, p. 143-154.

Nadeau, M. (2002). Serviteur, Paris, Albin Michel.

NAssajI, H. (2007). "Schema Theory and Knowledge-Based Processes in Second Language Reading Comprehension : A Need for Alternative Perspectives ", Language Learning, vol. 57. $\mathrm{n}^{\circ}$ 1, p. 79-113.

Neveu, E. (2014). « Revisiting Narrative Journalism as One of The Futures of Journalism », Journalism Studies, vol. 15, nº 5, p. 533-542.

Nietzsche, F. (1990). CEuvres, t. II, Paris, Robert Laffon.

Odin, R. (2000). "La question du public : Approche sémio-pragmatique", Réseaux, vol. 18, n 99, p. 49-72.

Olivier, S. (2007). "Lecture d'un "mauvais genre" : le "roman sentimental" ", dans L. Rosier et M.C. Pollet (dir.), Les mauvais genres en classe de français ? : retour sur la question, Namur, Presses universitaires de Namur, p. 55-68.

Oppenheimer, D.M. (2006). «Consequences of Erudite Vernacular Utilized Irrespective of Necessity : Problems with Using Long Words Needlessly ", Applied Cognitive Psychology, vol. 20, n² 2, p. 139-156.

PAivio, A. (1991). "Dual coding theory : Retrospect and current status", Canadian Journal of Psychology, $\mathrm{n}^{\circ}$ 45, p. 255-287.

Paivio, A., Yuille, J.C. et Madigan, S.A. (1968). "Concreteness, imagery, and meaningfulness values for 925 nouns», Journal of Experimental Psychology, vol. 76, n 1 , p. 1-25.

Panksepr, J. (1998). Affective neuroscience: The foundations of human and animal emotions, New York, Oxford University Press.

PARK, R.E. (1922). "Recension de "Public Opinion" ", American Journal of Sociology, $\mathrm{n}^{\circ} 28$, p. 232-234. 
Parker, K. et Deane, C. (1997). Ten Years of the Pew News Interest Index, Washington, The Pew Research Center for the People and the Press.

Parmentier, M. (2017). "Lectures réelles et théorie littéraire », Poétiques, $\mathrm{n}^{\circ} 181$, p. 125-141.

Pascal, B. (1657). Les provinciales, Cologne, Pierre de la Vallée.

PAscal, B. (1954). CEuvres complètes, Paris, Gallimard, Bibliothèque de la Pléiade.

Patzer, G.L. (1983). "Source Credibility as a Function of Communicator Physical Attractiveness ", Journal of Business Research, n 11, p. 229-241.

Paveau, M.A. (2001). « Euvre littéraire et textes journalistiques : la querelle des implicites ", Le français aujourd'hui, vol. 134, n 3, p. 13-31.

Paveau, M.A. (2006). Les prédiscours : Sens, mémoire, cognition, Paris, Presses Sorbonne nouvelle, 2006.

Peillon, V. (2014). «Arrêtons d'opposer plaisir et effort à l'école » (Propos recueillis par Maryline Baumard et Mattea Battaglia), Le Monde, 8 février 2014, p. 10.

Pelé, M., Bellut, C., Debergue, É. et al. (2017). «Cultural influence of social information use in pedestrian road-crossing behaviours ", Royal Society Open Science, $\mathrm{n}^{\circ}$ 4, p. 160739 .

Peraya, D. (1998). «Image(s) et cognition : présentation du dossier », Recherches en communication, $\mathrm{n}^{\circ}$ 10, p. 7-19.

Perelman, C. (1997). L'empire rhétorique ; rhétorique et argumentation, Paris, Vrin.

Peterson, R.A. (2004). « Le passage à des goûts omnivores : notions, faits et perspectives », Sociologie et sociétés, no 361, p. 145-164.

Peterson, R.A. et Kern, R.M. "Changing Highbrow Taste : From Snob to Omnivore ", American Sociological Review, vol. 61, n 5, 1996, p. 900-907.

Peterson, R.A. (1992). «Understanding audience segmentation : From elite and mass to omnivore and univore ", Poetics, $\mathrm{n}^{\circ} 21$, p. 243-258

Petty, R.E., Wegener, D.T., Fabrigar, L.R., Priester, J.R. et Caciopro, J.T. (1993). «Conceptual and methodological issues in the elaboration likelihood model of persuasion ", Communication Theory, vol. 3, n 4, p. 336-362.

Pew Research Center. (2000). Journalists avoiding the news - Self censorship : How often and why, Washington, DC, Pew Research Center.

Piaget, J. (1923). Le Langage et la pensée chez l'enfant, Paris, Delachaux et Niestlé.

Pichert, J.W. et Anderson, R.C. (1977). «Taking Different Perspectives on a Story ", Journal of Educational Psychology, vol. 69, nº 4, p. 309-315.

Pico della Mirandola, G. (1993). De la dignité de l'homme, Paris, Éditions de l'éclat.

Picot, G. (1989). « Le Journal des débats sous la monarchie de juillet », dans Centenaire du Journal des Débats 1789-1889, Paris, Pion, p. 158-191. 
Pillet, F. (2011). «Que reste-t-il de l'École de Constance ? »Études Germaniques, vol. $263, \mathrm{n}^{\circ} 3$, p. $763-781$.

Pinker, S. (2002). The Blank State - The Modern Denial of Human Nature, New York, Penguin Group.

Pinker, S. (2016). "Block that metaphor», The New Republic, vol. 235, n 15 , 8 octobre 2016, p. 24-29.

Plant, K.L. et Stanton, N.A. (2012). «Why Did the Pilots Shut Down the Wrong Engine? Explaining Errors in Context Using Schema Theory and the Perceptual Cycle Model ", Safety Science, vol. 50, nº 2, p. 300-315.

Platon. (1922). Phèdre ou De la beauté des âmes (trad. Mario Meunier), Paris, Payot.

Platon. (1996). Gorgias (trad. Jacques Cazeaux), Paris, Librairie générale française.

Popper, K. (1973). La Logique de la découverte scientifique, Paris, Payot.

Porat, M.U. (1977). The Information Economy : Definition and Mesurement, Washington, U.S. Department of Commerce.

Postman, N. (1986). Amusing ourselves to death, New York, Penguin.

Pottier, B. (2000). Représentations mentales et catégorisations linguistiques, Louvain, Peeters Publishers.

Prairat, E. (2012). «L'autorité malmenée », Enfances \& Psy, nº 54, p. 109-117.

Pratchett, T. (2008). The Truth, Londres, Random House, p. 426-427.

Quatremer, J. (2006). "Les Belges y ont cru car notre scénario est crédible », Libération, 18 décembre 2006, p. 7-8.

Queneau, R. (1988). «La littérature définitionnelle », dans La littérature potentielle: Créations, re-créations, récréations, Paris, Gallimard, p. 115-118.

Quintilien. (1933). Institution Oratoire, livre V (trad. H. Bornecque), Paris, Garnier.

Quintilien. (1933). Institution oratoire, livres IV-VI. (trad. H. Bornecque), Paris, Garnier.

RACHILDE. (1914). «Les romans ", Le Mercure du France, 16 janvier 1914, p. 362.

Radvansky, G.A., ZwaAn, R.A., Curiel, J.M. et Copeland D.E. (2001). « Situation models and aging ", Psychology and Aging, vol. 16, n 1, p. 145-16o.

RAM, A. (1990). "Knowledge goals : A theory of interestingness ", dans Proceedings of the Twelvth Annual Conference of the Cognitive Science Society, Cambridge, MA, Cognitive Science Society, p. 206-214.

RAvary, L.M. (1865). " La critique et les critiques », La France Littéraire, Artistique et Scientifique, 31 octobre 1865, p. 15-22.

Read, D. (1999). The Power of News : The History of Reuters 1849-1989, Oxford, Oxford University Press. 
Readership Institute. (2001). The Value of Feature-style Writing, Evanston, IL, Readership Institute.

Renaudot, T. (1632). Préface au Recueil des Gazettes de l'année 1631, Paris, Gazette.

RichARD, J.F. (1990). Les activités mentales : comprendre, raisonner, trouver des solutions, Paris, A. Colin.

Rieffel, R. (2005). Que sont les médias? Paris, Gallimard.

Rieffel, R. (2014). Révolution numérique, révolution culturelle? Paris, Gallimard.

Rimé, B. (1984). "Langage et communication», dans S. Moscovici (dir.), Psychologie sociale, Paris, Presses Universitaires de France, p. 415-446.

Rozenblit, L. et KeIL, F. (2002). « The Misunderstood Limits of Folk Science : An Illusion of Explanatory Depth», Cognitive science, vol. 26, $\mathrm{n}^{\circ} 5$, p. 521-562.

Rumelhart, D.E. (1980). "Schemata : the Building Blocks of Cognition », dans Spiro, R.J., Bruce, B.C. et Brewer, W.F. (dir.), Theoretical Issues in Reading Comprehension, Hillsdale, Lawrence Erlbaum, p. 33-58.

Rumelhart, D.E. et Ortony, A. (1977). « The Representation of Knowledge in Memory », dans Anderson, R.C., Spiro, R.J. et Montague, W.E. (dir.), Schooling and the Acquisition of Knowledge, Hillsdale, Lawrence Erlbaum, p. 99-135.

SAdoski, M. (2018). « Reading Comprehension is Embodied : Theoretical and Practical Considerations ", Educational Psychology Review, vol. 30, n², p. 331-349.

Sadoski, M. et Paivio, A. (2007). "Toward a unified theory of reading ", Scientific studies of reading, vol. 11, $\mathrm{n}^{\circ}$, p. 337-356.

SAdoski, M. et Paivio, A. (2012). Imagery and Text : A Dual Coding Theory of Reading and Writing, New York, Routledge.

SAdoski, M., Paivio, A. et Goetz, E.T. (1991). « A Critique of Schema Theory in Reading and a Dual Coding Alternative », Reading Research Quarterly, vol. 26, n' 2, p. 463-484.

Sainte-Beuve, C.A. (1839). "De la littérature industrielle», Revue des Deux

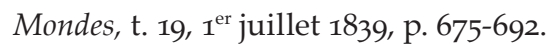

SAKAlaKi, M. et ThéPAut, Y. (2005). « La valeur de l'information », Questions de communication, $\mathrm{n}^{\circ} 8$, p. 355-365.

SALmon, C. (2007). Storytelling, la machine à fabriquer des histoires et à formater les esprits, Paris, Éditions La Découverte.

Salomon, G. (1984). "Television is "Easy" and Print is "Tough" : The Differential Investment of Mental Effort in Learning as a Function of Perceptions and Attributions ", Journal of Educational Psychology, n ${ }^{\circ}$ 76, p. $647-658$. 
SAPIR, E. et Swadesh, M. (1946). "American Indian Grammatical Categories ", Word, vol. 2, no 2, p. 103-112

Schaeffer, J.M. (1999). Pourquoi la fiction? Paris, Seuil.

Schaeffer, J.M. (2015). L'expérience esthétique, Paris, Gallimard.

Schank, R.C. (1979). "Interestingness : controlling inferences", Artificial Intelligence, $\mathrm{n}^{\circ} 12$, p. 273-297.

Schement, J.R. (1990). « Porat, Bell, and the information society reconsidered: The growth of information work in the early twentieth century ", Information Processing \& Management, vol. 26, $\mathrm{n}^{\circ}$ 4, p. 449-465.

Schickel, R. (1985). Intimate Strangers : The Culture of Celebrity, New York, Doubleday.

Scholl, A. (1885). Fruits défendus, III, Paris, Victor-Havard.

Schopenhauer, A. (1905). Parerga et Paralipomena (trad. A. Dietrich), Paris, Félix Alcan.

Schramm, W. (1954). " How communication works », dans W. Schramm (dir.), The process and effects of mass communication, Urbana, University of Illinois Press, p. 3-34.

Schramm, W. (1971). "The Nature of communication between humans", dans W. Schramm (dir.), The process and effects of mass communication, Urbana, University of Illinois Press, p. 1-53.

Schutz, A. (1962). Collected papers I : The problem of social reality, La Hague, Martinus Nijhoff.

Schutz, A. (2011). Collected papers V. Phenomenology and the social sciences, New York, Springer.

SchwARTz, S. H. (2006). « Les valeurs de base de la personne : théorie, mesures et applications ", Revue française de sociologie, n 47, p. 929-968.

SÉNÈQue. (1914). Lettres à Lucilius (trad. J. Baillard), Paris, Hachette.

Sevilla, G. (2015). «Introduction : Un pont narratif entre les deux cultures ? » dans S. Milan et G. Sevilla (dir.), « Le récit comme acte cognitif », Cahiers de Narratologie, $\mathrm{n}^{\circ}$ 28, s.p.

Shank, R. et Abelson, R. (1977). Scripts, Plans, Goals and Understanding, Hillsdale, Lawrence Erlbaum.

Shoemaker, P.J. (2006). "News and newsworthiness : A commentary", Communications, $\mathrm{n}^{\circ}$ 31, p. 105-111.

Silvia, P.J. (2006). Exploring the psychologie of interest, Oxford, Oxford University Press.

Simon, H.A. (1971). « Designing organizations for an information-rich world », dans M. Greenberger (dir.), Computers, communications, and the public interest, Baltimore, Johns Hopkins University Press, p. 40-41. 
Simonnot, B. (2008). «La pertinence en sciences de l'information : des modèles, une théorie? » dans F. Papy (dir.), Problématiques émergentes dans les Sciences de l'Information, Paris, Hermes-Lavoisier, p. 161-182.

Slama-Cazacu, T. (1993). "La "langue de bois" et quelques problèmes de communication $» \operatorname{Lin} x$, vol. 29, $\mathrm{n}^{\circ} 2$, p. 85-97.

SNyder, M. et Uranowitz, S.W. (1978). «Reconstructing the Past : Some Cognitive Consequences of Person Perception ", Journal of Personality and Social Psychology, vol. 36, n ${ }^{\circ}$ 9, p. 941-950.

Spencer, H. (1959). Philosophy of style (original : 1852), New York, Pageant Press.

Sperber, D. (1996). La Contagion des idées : théorie naturaliste de la culture, Paris, Odile Jacob.

Sperber, D. et Wilson, D. (1989). La pertinence : Communication et cognition, Paris, Éditions de Minuit.

Sperber, D. et Wilson, D. (1992). "Ressemblance et communication ", dans D. Andler (dir.), Introduction aux sciences cognitives, Paris, Seuil, p. 219-238.

Sperber, D. et Wilson, D. (1997). « Remarks on relevance theory and the social sciences ", Multilingua, vol. 16, nos 2-3, p. 145-151.

Spink, A., Wolfram, D., Jansen, M.B. et Saracevic, T. (2001). «Searching the web : The public and their queries ", Journal of the American Society for Information Science and Technology, vol. 52, $\mathrm{n}^{\circ}$ 3, p. 226-234.

Steel, N.M. (2012). "Schema(s)", dans Seel, N.M. (dir.), Encyclopedia of the Sciences of Learning, New York, Springer, p. 2933-2936.

Stephens, M. (2010). "Un manifeste pour l'enseignement du journalisme », Les Cahiers du journalisme, $\mathrm{n}^{\circ} 21, \mathrm{p} .38-45$.

Stephens-Davidowitz, S. (2017). Everybody Lies: What the Internet Can Tell Us About Who We Really Are, New York, Bloomsbury Publishing.

Stewart, S. (2014). A Sociology of Culture, Taste and Value, Londres, Palgrave-Mcmillan.

Stigler, G.J. (1961). "The economics of information», Journal of Political Economy, vol. 69, n 3 , p. 213-225.

Stout, G.F. (1896). Analytic psychology, New York, MacMillan.

Sueur, C., Class, B., Намm, C. et al. (2013). «Different risk thresholds in pedestrian road crossing behaviour : A comparison of French and Japanese approaches ", Accident Analysis and Prevention, no 58, p. 59-63.

Super, D. (1964). La psychologie des intérêts, Paris, Presses Universitaires de France.

Syndicat National de L'Édition. (2015). Les chiffres clés de l'édition 2015 - Données 2014, Paris, SNE. 
Syndicat nAtional des journalistes. (2018). " À Ouest-France, des clics et des claques ", Le Journaliste, $\mathrm{n}^{\circ} 327$, janvier 2018, p. 4 .

Tafani, É. et Bellon, S. (2003). «Études expérimentales de la dynamique des représentations sociales », dans Abric, J.C. (dir.), Méthodes d'études des représentations sociales, Saint-Agne, Érès, p. 255-292.

TAguiefF, P.A. (1997). « Le populisme et la science politique, du mirage conceptuel aux vrais problèmes ", Vingtième Siècle, no 56, p. 4-33.

Talon-Hugon, C. (2009). Morales de l'art, Paris, Presses Universitaires de France.

TARde, G. (1895). La Logique sociale, Paris, Alcan.

Teтьоск, P. (2005). Expert political judgment: How good is it? How can we know ? Princeton, Princeton University Press.

Thay, D. (2014). "Lakoff and the Theory of Conceptual Metaphor", dans J. Taylor et J. Littlemore (dir.), Bloomsbury Companion to Cognitive Linguistics, Londres, Bloomsbury Publishing, p. 49-6o.

The Rolling Stones. (1974). It's Only Rock'n Roll (But I like it), Amsterdam, Promotone.

Thibaudet, A. (1923). « Lettres et journaux », La Nouvelle Revue Française, $\mathrm{n}^{\circ}$ 117, p. $930-938$.

Thibièrge, C. (dir.). (2009). La force normative : Naissance d'un concept, Bruxelles, Paris, L.G.D.J.

Thoreau, H.D. (1854). Walden, or Life in the Woods, Boston, Ticknor and Fields.

Thorndyke, P.W. et Yekovich, F.R. (1980). «A Critique of Schema-Based Theories of Human Story Memory ", Poetics, n 9, p. 23-49.

Thucydide. (1937). Histoire de la guerre du Péloponnèse (trad. Jean Voilquin), Paris, Garnier.

Thumerel, F. (2004). La critique littéraire, Paris, Armand Colin.

Tichenor, P.J., Donohue, G.A. et Olien, C.N. (1970). « Mass media flow and differential growth in knowledge », Public Opinion Quartely, $\mathrm{n}^{\circ} 34$, p. $159-170$.

Tinker, M.A. (1963). Legibility of print, Ames, Iowa State University Press.

Твотsку, L. (2010). Le journal et son lecteur, dans J. Aubert-Yong (trad.), Les questions du mode de vie (p. 21-30), Chicoutimi, Les Classiques des sciences sociales (original 1923).

United States Government Accountability Office. (2012). Actions Needed to Evaluate the Impact of Efforts to Estimate Costs of Reports and Studies, Washington, GAO.

Vaillant, A. (2003). "Pour une histoire de la communication littéraire", Revue d'histoire littéraire de la France, no 103, p. 549-562. 
Vaillant, A. (2011). "De la littérature médiatique », Interférences littéraires, $\mathrm{n}^{\circ} 6$, p. 21-33.

VAlÉRY, P. (1941). «Littérature » (1929), dans Tel Quel, t. 1, Paris, Pléiade.

VAn Boven, L. (2000). «Pluralistic Ignorance and Political Correctness : The Case of Affirmative Action », Political Psychology, vol. 21, n², p.267-276. VAN DEN BroeK, P. et Helder, A. (2017). «Cognitive processes in discourse comprehension : passive processes, reader-initiated processes, and evolving mental representations ", Discourse Processes, vol. 54, $\mathrm{n}^{\mathrm{os}} 5-6$, p. 360-372.

Van Dijк, T.A. et Kintsch, W. (1983). Strategies of Discourse Comprehension, New York, Academic Press.

VANOOST, M. (2013). «Journalisme narratif : proposition de définition, entre narratologie et éthique », Les Cahiers du journalisme, $\mathrm{n}^{\circ}$ 25, p. 140-161.

Vedrager, P. (2001). Le sens critique : La réception de Nathalie Saraute par la presse, Paris, L'Harmattan.

Vialatte, A. (200o). Chronique de La Montagne, t. 1, Paris, Robert Laffont.

Vian, B. (2007). L'Écume des jours, Paris, Fayard.

Virilio, P. (2009). «L'instant contre la démocratie », Le Journal du dimanche, 11 juillet 2009.

Vitruve. (1848). De l'Architecture (trad. Ch.-L. Maufras), Paris, Pankouke.

Vorrol, O. (2005). "Visibilité et invisibilité : une introduction », Réseaux, $\mathrm{n}^{\mathrm{o}} 129$, p. 9-36.

Voyer-LÉGer, C. (2014). Métier critique, Québec, Septentrion.

Wagner, F. (2012). "Des coups de canif dans le contrat de lecture », Poétique, $\mathrm{n}^{\mathrm{o}} 172, \mathrm{p} \cdot 387-407$.

Washington, G. (1973). « General orders (20 juillet 1779)», dans General orders of Georges Washington, New York, Harbor Hill Books, p. 99-100.

WeIr, K. (2015). «A reproductibility crisis? » Monitor on psychology, vol. 46, $\mathrm{n}^{\circ} 9$, p. 5 .

Wernick, I.K., Herman, R., Govind, S. et Jesse, A. (1996). « Materialization and Dematerialization : Measures and Trends", Daedalus, vol. 125, $\mathrm{n}^{\circ}$ 3, p. 171-198.

Whitney, P., Budd, D., Bramucci, R.S. et Crane R.S. (1995). «On Babies, Bath Water, and Schemata : A Reconsideration of Top-Down Processes in Comprehension », Discourse Processes, vol. 20, n², p. 135-166.

Wilde, O. (2003). Complete Works of Oscar Wilde, New York, Harper Collins.

Wirth, J., Engammare, I. et al. (2008). Les marges à drôleries des manuscrits gothiques, Paris : Droz.

YeAts, W.B. (1926). Later Poems, Londres, MacMillan. 
Ytreberg, E. (2001). «Moving out of the Inverted Pyramid : narratives and descriptions in television news ", Journalism Studies, vol. 2, $\mathrm{n}^{\circ}$ 3, p. 357-371.

Zerba, A. (2008). "Narrative storytelling : Putting the story back in hard news to engage young audiences ", Newspaper Research Journal, vol. 29, $\mathrm{n}^{\mathrm{o}}$ 3, p. 94-102.

Zerubavel, E. (1999). Social Mindscapes - An Invitation to Cognitive Sociology, Cambridge, Harvard University Press.

Zhaо, X. et Zhu, L. (2012). "Schema Theory and College English Reading Teaching ", English Language Teaching, vol. 5, nº 11, p. 111-117.

Zhong, B., Hardin, M. et Sun, T. (2011). « Less effortful thinking leads to more social networking ? The associations between the use of social network sites and personality traits », Computers in Human Behavior, vol. 27, $\mathrm{n}^{\circ} 3$, p. 1265-1271.

ZIPF, G.K. (1949). Human behavior and the principle of least effort, Cambridge, Addison-Wesley.

ZolA, É. (1888). « Le journalisme [préface à La Morasse] », Le Figaro supplément littéraire, $\mathrm{n}^{\circ}$ 47, 24 novembre 1888, p. 1 .

Zwann, R.A. et Radvansky, G.A. (1998). "Situation Models in Language Comprehension and Memory", Psychological Bulletin, vol. 123, $\mathrm{n}^{\circ}$ 2, p. $162-185$.

ZwaAn, R.A. (1994). "Effect of genre expectations on text comprehension ", Journal of Experimental Psychology : Learning, Memory, and Cognition, vol. $20, \mathrm{n}^{\circ} 4$, p. $920-933$.

ZwAAN, R.A. (2016). "Situation models, mental simulations, and abstract concepts in discourse comprehension ", Psychonomic Bulletin $\mathcal{E}$ Review, vol. 23, no 4, p. 1028-1034. 
Page blanche conservée intentionnellement 


\section{Table des matières}

Préface : Pour comprendre (enfin ?) les médias ....................... v

Introduction : À l'abordage d'un « grand mystère » ..................

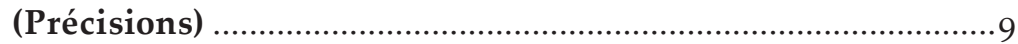

\section{Première partie - Pourquoi les contenus qui nous plaisent} nous plaisent-ils ?

1. De quelques convulsions du marché discursif ................. 17

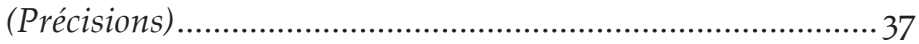

2. La pertinence des discours................................................. 43

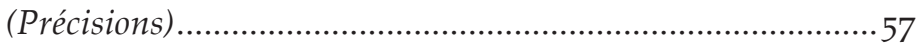

3. L'appel des choses simples ………………......................61

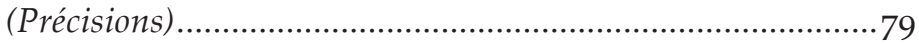

4. Le trouble jeu de l'effet cognitif ....................................... 83

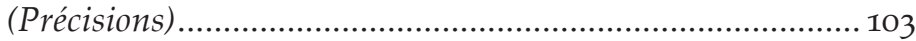

5. Des images plein la tête .................................................... 117

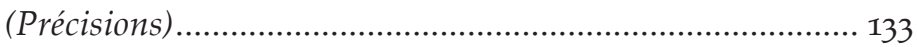

6. Triomphe et déroute de l'homo pertinensis.........................141

(Précisions) ....................................................................... 155

7. Les errances de la convenance ......................................... 161

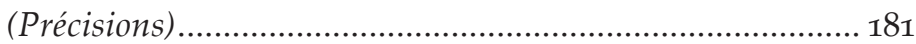

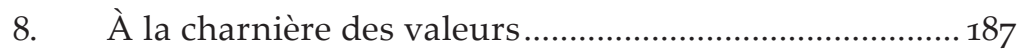

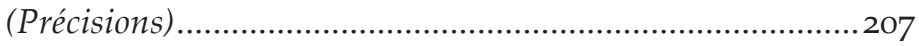

9. Sur les étals du marché discursif..................................... 215

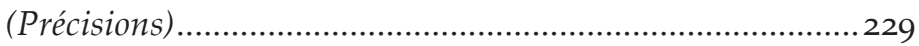

10. Des idées « libres » dans un poulailler libre ....................249

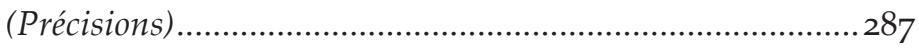




\section{Seconde Partie - Occurrences}

1. Ce que les traductions trahissent ....................................299

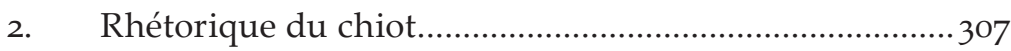

3. Le mystère de la pyramide inversée................................. 315

4. L'art de l'agonistique lexicale ........................................... 335

5. Souffrances et amertumes de la critique ......................... 343

6. Sur les pouvoirs du récit.................................................. 361

7. Vertus martiales des images salaces ................................ 369

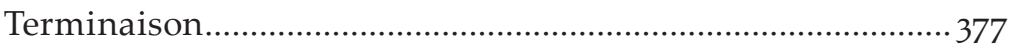

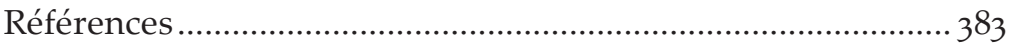

\section{Liste des figures}

Figure 1 : Les principales composantes de l'effort cognitif .......74

Figure 2 : Les principales composantes de l'effet cognitif .........96

Figure 3 : Un modèle de la pertinence cognitive .....................142

Figure 4 : L'articulation de l'adéquation discursive ................. 151

Figure 4 b : L'articulation (complétée) de l'adéquation discursive ........................................................... 195

Figure 5 : Une typologie oppositionnelle de la convenance sociale ................................................................ 197

Figure 6 : Le modèle général de l'adéquation sociocognitive.... 206

Figure 7 : Quelques représentations du modèle pyramidal.....323 
" Le tour de force d'être accessible à la fois aux spécialistes (avec une érudition remarquable) et à un public fort large. »

\section{- Nicolas Froeliger, Université Paris Diderot}

Pourquoi les messages qui nous plaisent nous plaisent-ils ? Dans notre société ultracommunicante, comment se déterminent les préférences de chacun?

Derrière de telles questions se cache l'un des plus vieux problèmes théoriques de la communication, mais aussi l'un des plus importants face au bouillonnement contemporain des contenus culturels, politiques, médiatiques et distractifs.

Au fil d'un cheminement méthodique dont la rigueur n'exclut pas l'humour, La valeur des informations s'attaque à ce défi sous un angle nouveau. Son approche interdisciplinaire montre avec clarté comment des facteurs psychologiques et normatifs connus de longue date, mais rarement rapprochés jusqu'alors, orientent conjointement la production et la réception des contenus triviaux ou érudits.

Menée dans des domaines discursifs aussi variés que le journalisme, la littérature, la communication scientifique ou les vidéos en ligne, cette exploration captivante révèle pas à pas les ressorts cognitifs et sociaux qui sous-tendent la concurrence de ces informations et qui contribuent à façonner, pour le meilleur ou le pire, notre espace du débat public.

" Le simple fait de savoir s'il s'agit bien d'un seul problème - et d'un problème soluble - plutôt que d'une multitude de problèmes distincts est encore loin d'aller de soi. C'est pourtant ce que ce livre se propose de montrer. La modestie d'une telle entreprise saute aux yeux... "

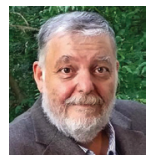

Bertrand Labasse est professeur aux départements de français et de communication de l'Université d'Ottawa et professeur invité à l'ESJ Lille. Ancien responsable scientifique de l'unité de recherche-développement de la presse française, il est l'auteur de plusieurs ouvrages, dont Une dynamique de l'insignifiance... (Enssib, 2002).

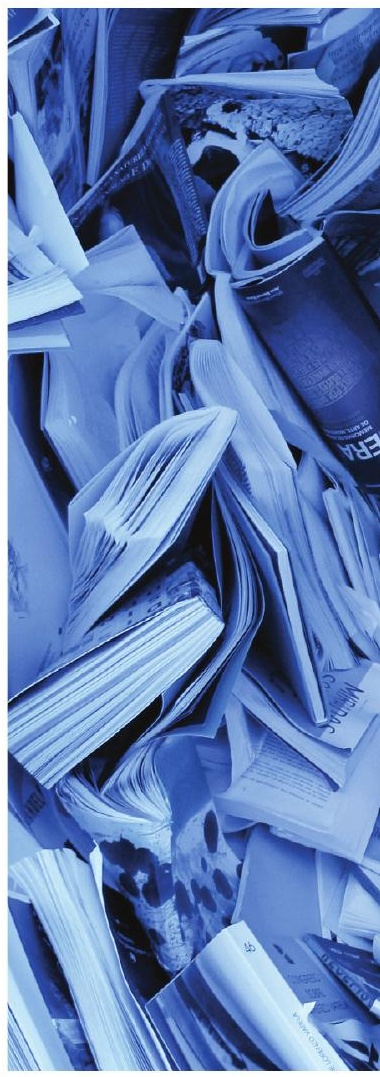

